\title{
Ethynylbenziodazolones (EBZ) as Electrophilic Alkynylation Reagents for the Highly Enantioselective Copper-Catalyzed Oxy- Alkynylation of Diazo Compounds
}

\author{
Durga Prasad Hari, ${ }^{[a]}$ Lionel Schouwey, ${ }^{[a]}$ Verity Barber,${ }^{[a]}$ Rosario Scopelliti, ${ }^{[b]}$ Farzaneh Fadaei-Tirani ${ }^{[b]}$ \\ and Jerome Waser ${ }^{*[a]}$
}

\begin{abstract}
Ethynylbenziodoxol(on)es (EBXs) cyclic hypervalent iodine reagents are now established reagents for the alkynylation of radicals and nucleophiles, yet they present limited possibilities for further structure and reactivity modification. Herein, we report the first synthesis of the corresponding Ethynylbenziodazolones (EBZs) reagents, in which the oxygen atom in the iodoheterocycle is replaced by a nitrogen. The substituent on the nitrogen enables further finetuning of the reagent structure and reactivity. EBZ reagents were easily obtained from the corresponding benzamides using a one-step procedure, and displayed a reactivity comparable to that of EBX reagents. In particular, they were applied in an asymmetric coppercatalyzed oxyalkynylation of diazo compounds, which proceeded in high yield and enantioselectivity for a broad range of substituents on the diazo compounds and the alkyne.
\end{abstract}

\section{Introduction}

Alkynes are among the most versatile functional groups in synthetic chemistry. ${ }^{[1]}$ They have also found widespread applications in chemical biology and material sciences. ${ }^{[2]}$ Therefore, synthetic methods to make or transfer triple bonds are of high importance. As terminal acetylenes are easily deprotonated, acetylide additions and cross-coupling reactions have been most often used for the alkynylation of electrophiles. More recently, alternative electrophilic alkynylation reagents have been developed for the functionalization of nucleophiles. ${ }^{[3]}$ The use of hypervalent iodine reagents has been especially successful in this context and our group used for the first time ethynylbenziodoxole (EBX) reagents for the direct or metalmediated alkynylation of nucleophiles and radicals. ${ }^{[4]}$ EBX reagents are now widely used in synthetic chemistry. ${ }^{[5]}$ They are particularly well-suited for transition metal based catalytic processes, due to their unique combination of stability and reactivity (Scheme 1A). ${ }^{[4 a, 4 b, 6]}$ Examples of successful processes include the gold or platinum-catalyzed synthesis of alkynylated

[a] Dr. Durga Prasad Hari, Lionel Schouwey, Verity Barber and Prof. Dr. Jerome Waser

Laboratory of Catalysis and Organic Synthesis

Ecole Polytechnique Fédérale de Lausanne

EPFL SB ISIC LCSO, BCH 4306, 1015 Lausanne, CH

E-mail: jerome.waser@epfl.ch

[b] Dr. Rosario Scopelliti, Dr. Farzaneh Fadaei Tirani

Institute of Chemical Sciences and Engineering

Ecole Polytechnique Fédérale de Lausanne

EPFL SB ISIC GE, BCH 2111, 1015 Lausanne, CH

heterocycles, ${ }^{[4 a, 6 a]}$ the palladium-catalyzed functionalization of olefins, ${ }^{[4 b]}$ the rhodium-catalyzed alkynylation of $\mathrm{C}-\mathrm{H}$ bonds ${ }^{[6 \mathrm{~b}-\mathrm{d}]}$ and the copper-catalyzed oxyalkynylation of diazo compounds. ${ }^{[6 e-}$ $\mathrm{f}, 7]$ The latter is particularly interesting, as it allows to transfer both parts of the reagent (iodobenzoic acid and alkyne) to the product. ${ }^{[8]}$ Using a copper catalyst with a chiral bisoxazoline (BOX) ligand gave access to propargylic esters with high enantiopurity. ${ }^{[6]]}$ These new transformations further extended the scope of multiple-functionalization of metal-carbene intermediates, a rapidly expanding field of organic chemistry. ${ }^{[9]}$

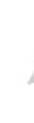

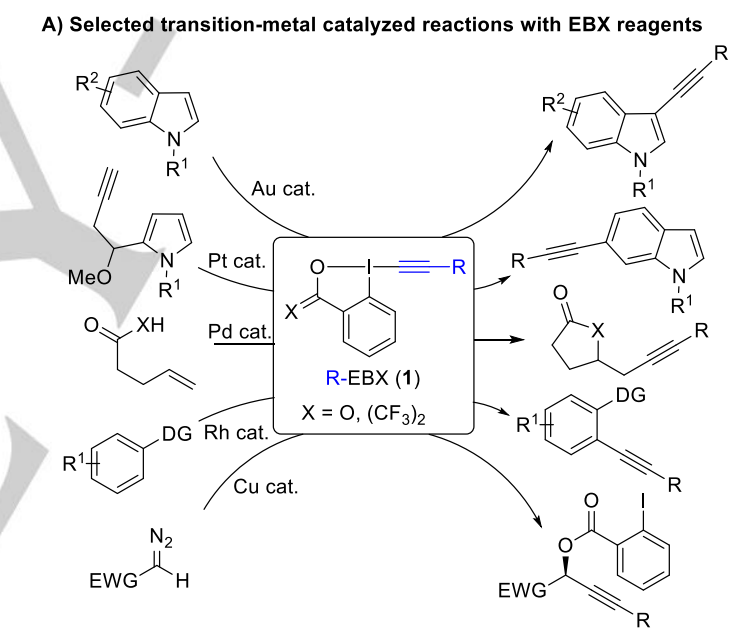

B) Ethynylbenziodiazolones (EBZ): fine-tuning of reactivity

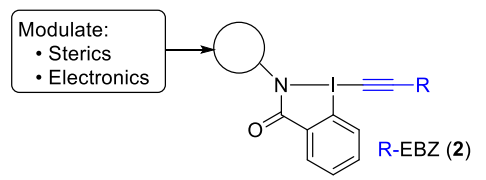

C) Highly enantioselective oxyalkynylation with $E B Z$ reagents

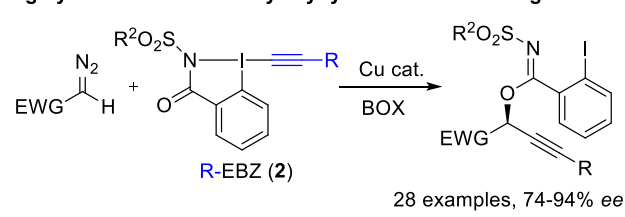

Scheme 1. Established EBX reagents $(\mathbf{A})$, newly designed EBZ reagents $(\mathbf{B})$, and their application in the enantioselective oxyalkynylation of diazo compounds (C).

Supporting information for this article is given via a link at the end of the document.

EBX reagents also offer the possibility of fine-tuning the reactivity by modifying the structure of the heterocyclic core. This has already proven essential for success in several transformations. ${ }^{[5]}$ 
The heteroatom connected to the iodine atom is expected to have the strongest impact on reactivity, yet so far only oxygen has been investigated. We were therefore highly interested in developing new reagents based on the benziodazolone core, in which the oxygen coordinated to iodine is replaced by a nitrogen atom. The extra substituent on nitrogen will allow to further modulate the electronic and steric environment of the reactive iodine atom (Scheme 1B). Benziodazolone-based hypervalent iodine reagents have been studied in the past, especially by Zhdankin and co-workers, ${ }^{[10]}$ but alkynylation reagents have never been reported to the best of our knowledge.

Herein, we report the first synthesis of ethynylbenziodazolone reagents (EBZ). EBZ bearing either a carbamide or a sulfonyl amide could be synthesized in high yield starting from the corresponding iodide. We further demonstrate that EBZ and EBX reagents have similar reactivities in both metal-free and metalcatalyzed transformations. In particular, they allowed the oxyalkynylation of diazo compounds with high enantioselectivities for aliphatic diazo esters as substrates (Scheme 1C).

\section{Results and Discussion}

\section{Synthesis and structure of EBZ reagents}

In order to access stable reagents, low electron-density on the nitrogen substituent next to the iodine center was expected to be essential. Therefore, we first selected tosyl substituted benzamide $\mathbf{3 a}$ as starting material, which had already been used for the synthesis of cyclic hypervalent iodine reagents before. ${ }^{[11]}$ In fact, the corresponding azidobenziodazolone had displayed enhanced stability when compared to the benziodoxolone reagent. The first EBZ reagent, TIPS-Ts-EBZ (2a) could be obtained in $96 \%$ yield on a gram scale using a slightly modified one-pot oxidation/alkynylation protocol develop by Olofsson and coworkers. ${ }^{[12]}$ In contrast to Olofsson's work, the most convenient trimethylsilyl acetylene $4 \mathrm{a}$ could be used as precursor instead of the corresponding boronic ester.
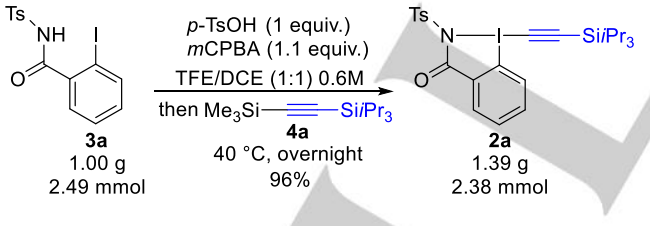

Scheme 2. Synthesis of TIPS-Ts-EBZ (2a).

Good quality crystals of $\mathbf{2 a}$ were obtained, and the structure of TIPS-EBX (1a) and TIPS-Ts-EBZ (2a) were compared by X-ray analysis (Figure 1 and 2). ${ }^{[13]}$ Bond lengths and angles around the iodine atom were similar for both reagents. The iodine alkyne bond, as well as the iodine-heteroatom bond were slightly longer in 2a when compared to 1a (2.060(9) vs 2.054(2) A and 2.387(6) vs 2.338(1), Å respectively. As for TIPS-EBX (1a), the hypervalent bond in $\mathbf{2 a}$ was close to linearity and in the plane of the aromatic ring. An interesting feature of the structure of $2 a$ was a possible interaction between one of the two oxygen atoms of the sulfonamide ( $\mathrm{O} 3$ in Figure 2) and the hypervalent iodine center (distance 3.281(5) $\AA$ ).

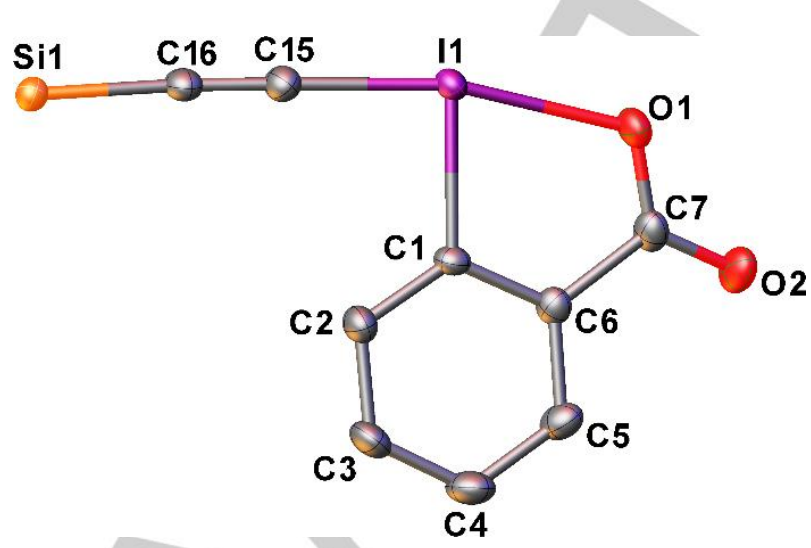

Figure 1. Structure of TIPS-EBX (1a) as determined by X-ray diffraction. Selected bond lengths and angles: I1-C15: 2.054(2) $\AA$; I1-O1 2.338(1) $\AA$; Angle O1-I1-C15: 166.11(6) ${ }^{\circ}$; C15-I1-C1: 91.37(7) ${ }^{\circ}$; Torsion C15-I1-C1-C2: -8.3(2) . $\mathrm{H}$-atom and substituents on $\mathrm{Si}$ are omitted for clarity.

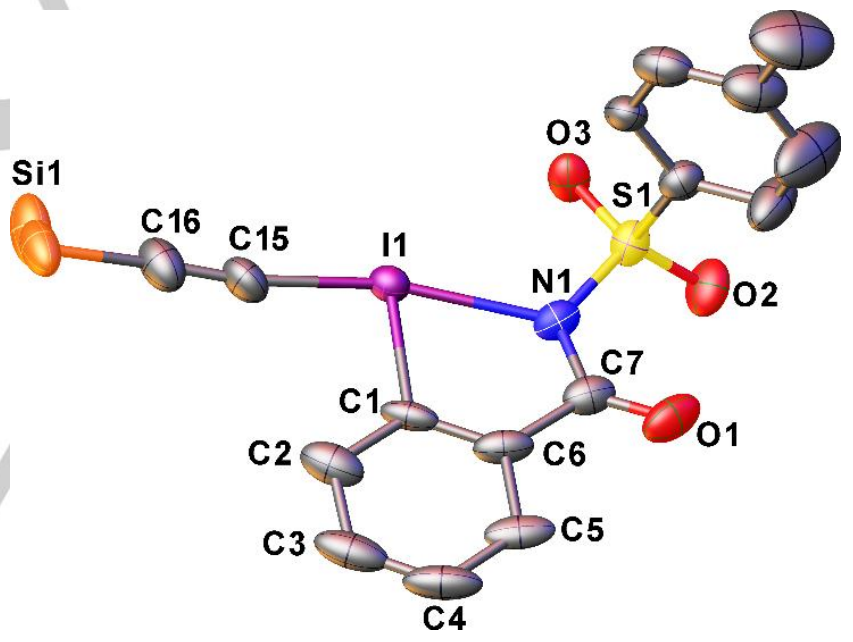

Figure 2. Structure of TIPS-Ts-EBZ (2a) as determined by X-ray diffraction

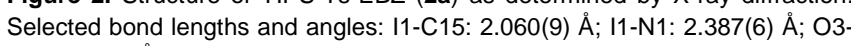
11: 3.281(5) Å; Angle N1-I1-C15: 165.8(3) ' ; C15-I1-C1: 92.1(3) ${ }^{\circ}$; Torsion C2C1-I1-C15: 6.6(7) ${ }^{\circ} ;$ I1-N1-S1-O3: -17.6(4) ${ }^{\circ}$. H-atom and substituents on $\mathrm{Si}$ are omitted for clarity.

Different EBZ sulfonyl reagents were synthesized next (Scheme $3 A$ ). A phenylethynyl substituent could be introduced to give reagent $\mathbf{2 b}$ in $52 \%$ yield. In this case, good results were obtained when adding directly the terminal alkyne after in situ oxidation to iodine(III). Tosyl-derived reagents $\mathbf{2 a}$ and $\mathbf{2 b}$ displayed a lower solubility in commonly used organic solvents. Therefore, the mesitylenesulfonyl (Mts) 2c and 2d were synthesized. Indeed, these reagents were more soluble, although the yields obtained for their synthesis were lower. Reagent $2 e$ derived from an aliphatic camphorsulphonamide could be also obtained in high yield. Reagents $\mathbf{2 f}$ and $\mathbf{2} \mathbf{g}$ bearing an acetyl group instead of the 
sulfonyl group on the nitrogen atom could also be accessed, but only in $21 \%$ yield (Scheme $3 \mathrm{~B}$ ). Furthermore, these reagents were less stable and decomposition was observed upon storage at $4{ }^{\circ} \mathrm{C}$ Further modification of the mesitylenesulfonyl derived reagents were then investigated, as they gave best results in the enantioselective oxyalkynylation of diazo compounds ( vide infra).
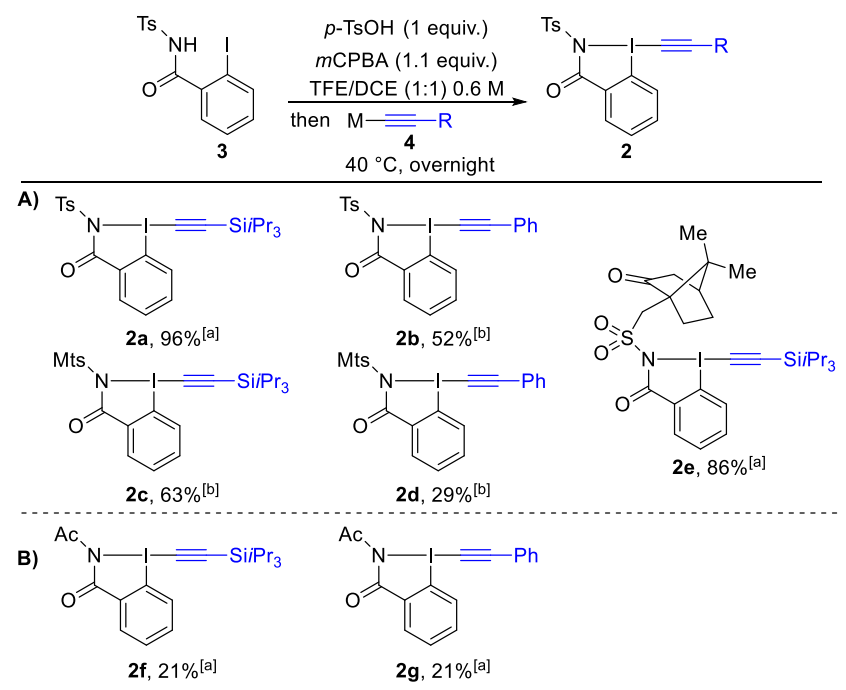

C)

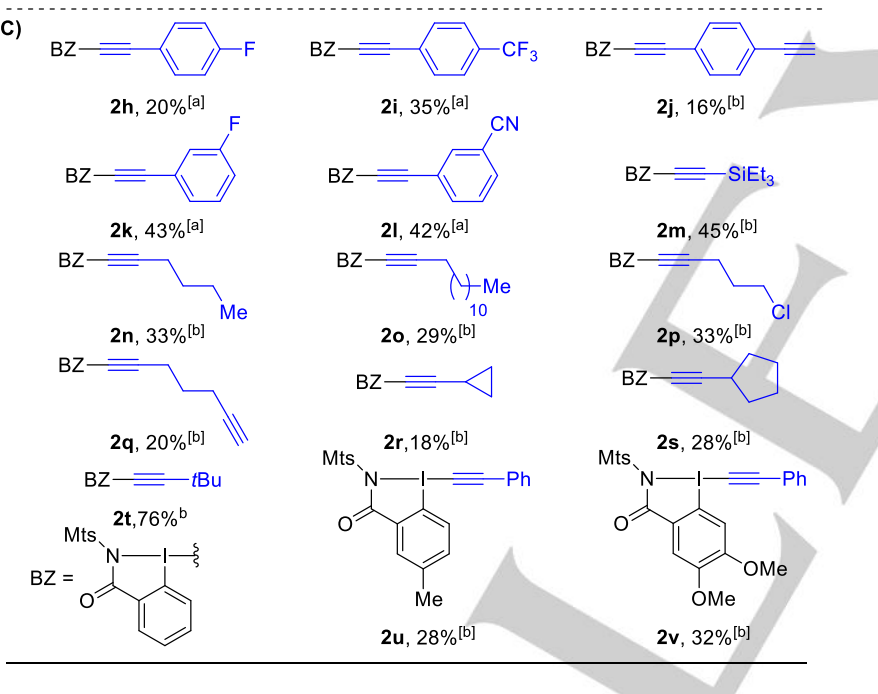

Scheme 3. Scope of EBZ reagents. Mts = Mesitylenesulfonyl [a] The trimethylsilylalkyne was used for the synthesis. ${ }^{[b]}$ The terminal alkyne was used for the synthesis.

Arylalkynyl derivatives $\mathbf{2} \mathbf{h}-\mathbf{l}$ bearing fluoro, trifluoromethyl, alkynyl or cyano groups in para or meta positions could be obtained in $20-42 \%$ yield. Triethylsilylethynyl reagent $2 \mathrm{~m}$ could be also synthesized in $45 \%$ yield. The protocol could be also extended to aliphatic reagents without further optimization. Primary $(\mathbf{2 n - q})$, secondary (2r and $2 \mathbf{s})$ and tertiary (2t) alkyl groups could be all introduced successfully. Finally, derivatives $2 \mathbf{u}$ and $\mathbf{2 v}$ bearing methyl or methoxy substituents on the benzamide core could also be accessed. Although the yields obtained for the synthesis of the reagent are moderate, the single step procedure is very convenient and both terminal or silyl alkynes can be used depending on their availability. No attempt was made to reoptimize the reaction conditions for specific reagents.

\section{Comparison of the reactivity of EBZ and EBX reagents}

As the structure of the EBZ reagents was very similar to the one of the highly successful EBXs, a few standard transformations reported for the latter were then examined (Scheme 4). The alkynylation of ketoesters was first investigated (eq. 1). ${ }^{[4 c]}$ TIPSTs-EBZ (2a) was as efficient as TMS-EBX in this transformation, giving the alkynylated product 6 in quantitative yield. With thiol nucleophile 7, 2a was slightly less efficient than TIPS-EBX, but the desired product 8 was still obtained in $79 \%$ yield (eq. 2). ${ }^{[4 d}$ Finally, 2a could be also used in the gold-catalyzed alkynylation of indole (9) (eq. 3). ${ }^{[4 a]}$ These preliminary results are promising for the use of EBZ reagents in electrophilic alkynylation reactions.

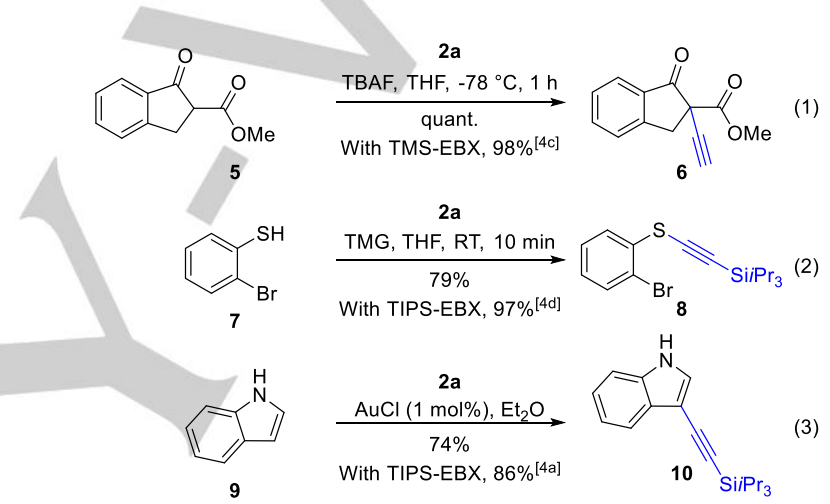

Scheme 4. Alkynylation of keto ester $\mathbf{5}$, thiol 7 and indole (9) with TIPS-Ts-EBZ (2a).

\section{Copper-catalyzed enantioselective oxyalkynylation of diazo compounds}

Having established that EBZ reagents and EBXs have similar reactivity, we decided to investigate their use in the functionalization of diazo compounds. ${ }^{[6 e-f]}$ In this transformation, the benzoic acid core of the reagent is incorporated in the product. Our goal was therefore to achieve an aminoalkynylation of diazo compounds to access important amino acid derivatives (Scheme 5). Although no product was observed with silylated alkynes, $17 \%$ of an addition product could be obtained when using Ph-Ts-EBZ (2b). The NMR and mass data were in good accordance with the desired aminoalkynylation product 13a. Nevertheless, X-ray diffraction on the more crystalline derivative $14 \mathrm{n}$ later showed that oxyalkynylation had occurred to give imidate $14 a^{[14]}$ In fact, full selectivity for reaction at the oxygen atom of EBZ reagents was always observed. This would be in agreement with a reaction mechanism involving first a nucleophilic attack of the more accessible oxygen atom onto an electrophilic carbene intermediate, followed by an alkyne transfer, as previously proposed in our work with EBX reagents. ${ }^{[6 e-f]}$ 


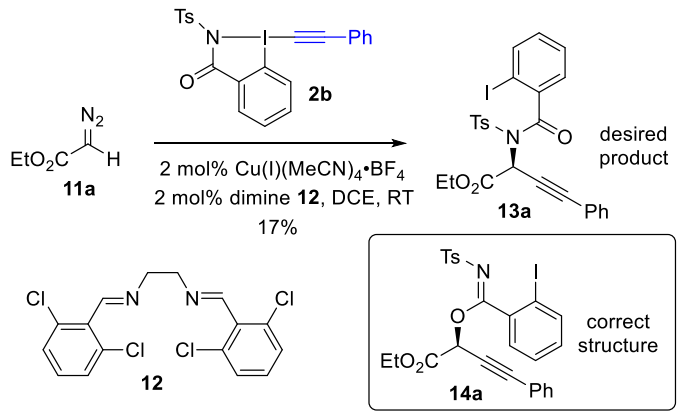

Scheme 5. Attempt of aminoalkynylation of diazo compound 11a with $\mathrm{Ph}$-TsEBZ $(\mathbf{2 b})$ resulting in oxyalkynylation instead.

We then turned to the optimization of an enantioselective variation of the oxyalkynylation reaction (Table 1). When the oxyalkynylation of ethyl diazoacetate (11a) was attempted with Ph-Ts-EBZ (2b) and 2 mol\% CuOAC as catalyst with bisoxazoline (BOX) ligand 15a, which had given high yield and enantioselectivity in the oxyalkynylation reaction with EBX reagents, ${ }^{[6]]}$ 14a could be obtained in an improved yield of $55 \%$ (entry 1). However, 14a was formed with only $33 \%$ ee. We therefore examined other BOX ligands. The use of PyBOX ligand 15b led to low yield and enantioselectivity (entry 2). With simple valine-derived BOX ligand 15b, oxyalkynylation product 14a was formed with $55 \%$ yield and $40 \%$ ee (entry 3 ). Better results were obtained with BOX 15d bearing more bulky tert-butyl groups $(65 \%$ yield, $69 \%$ ee, entry 4). Cyclopropane- and cyclopentane- derived BOX ligands $15 \mathrm{e}$ and $\mathbf{1 5 f}$ led to a very fast reaction, but lower yields and enantioselectivities (entries 5 and 6). At this stage, solvent effects were investigated. Acetonitrile, xylene and chlorobenzene gave lower yields and enantioselectivities (entries 7-9). The results in dichloromethane were similar to dichloroethane, but the reaction could be conducted at $35{ }^{\circ} \mathrm{C}$ instead of $60^{\circ} \mathrm{C}$ without increasing the reaction time (entry 10). Further attempts to improve the yield or enantioselectivity by tuning the reaction conditions were not successful. Therefore, modification of the EBZ reagents was investigated. Gratifyingly, the use of mesitylenesulfonyl derived reagent $2 \mathbf{d}$ led to the formation of $14 \mathrm{a}$ in $81 \%$ yield and $80 \%$ ee, although the reaction was slower (entry 11). As a final factor, we investigated counter ion effects on the copper catalyst, whereas the active catalysts where generated in situ by combining $\mathrm{CuCl}$ with a silver salt. $\mathrm{A}$ triflimide counter ion led to a sluggish reaction (entry 12). In contrast, the use of tosylate allows further increasing the yield to $91 \%$ (entry 13). In this case, the reaction could also be run at room temperature, allowing improving the ee to $88 \%$ (entry 14). Finally, by using $3 \mathrm{~mol} \%$ of copper catalyst, the desired product 14 a could be obtained in $98 \%$ yield and $88 \%$ ee after one hour at room temperature (entry 15$)$

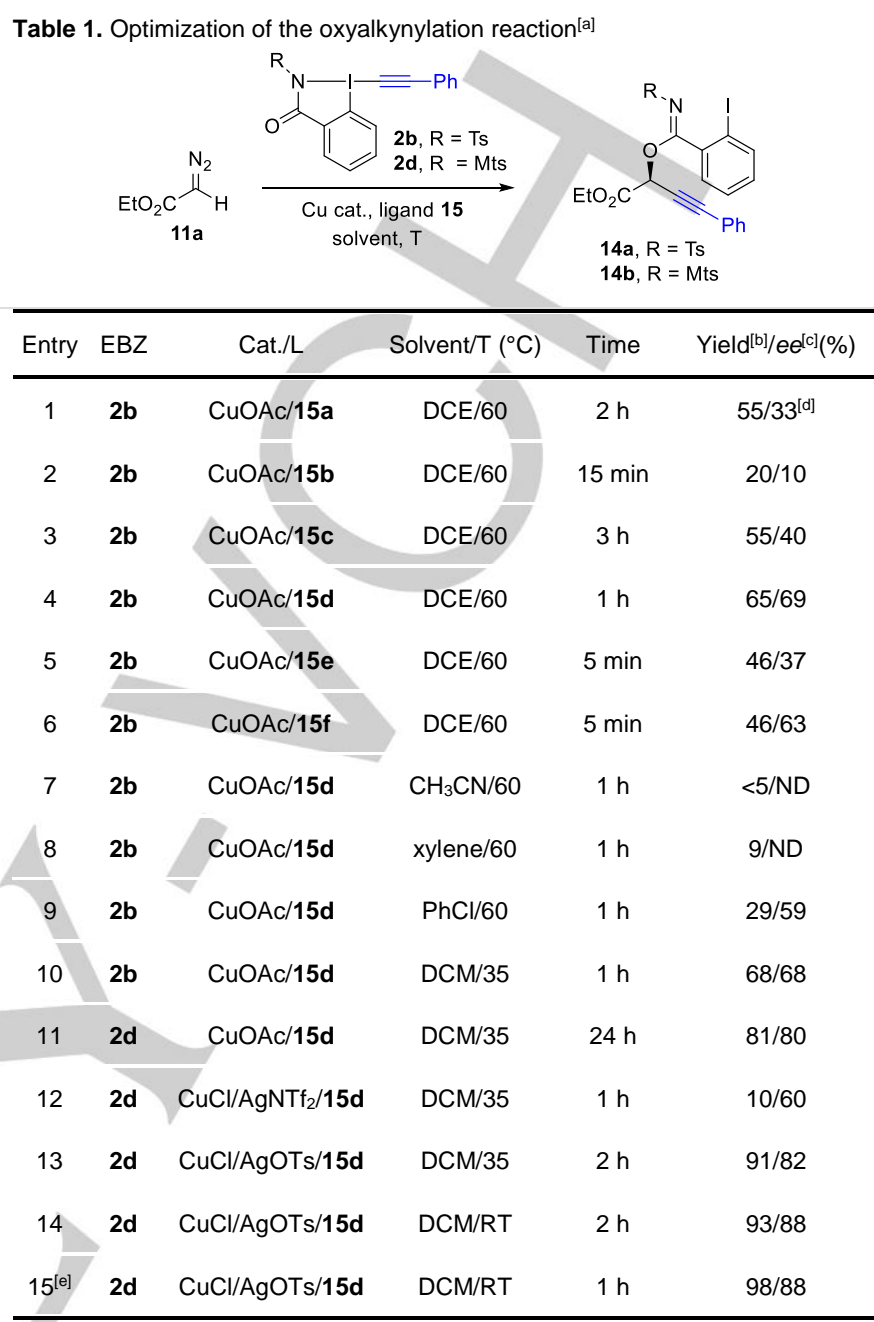

[a] Reaction conditions: $0.20 \mathrm{mmol}$ ethyldiazoacetate $(\mathbf{1 1 a}), 0.10 \mathrm{mmol}$ of $\mathbf{2 b}$ or $\mathbf{2 d}$ copper catalyst $(2.0 \mathrm{~mol} \%)$, silver catalyst $(2.0 \mathrm{~mol} \%$, if indicated), ligand 15 (2.5 mol\%), solvent $(0.05 \mathrm{M})$. [b]Yield after purification by column chromatography. [c] Obtained by chiral HPLC. [d] The opposite enantiomer of the product was obtained. ${ }^{[e]}$ Copper catalyst $(3.0 \mathrm{~mol} \%)$, ligand $15 \mathrm{~d}$ (3.75 mol\%).

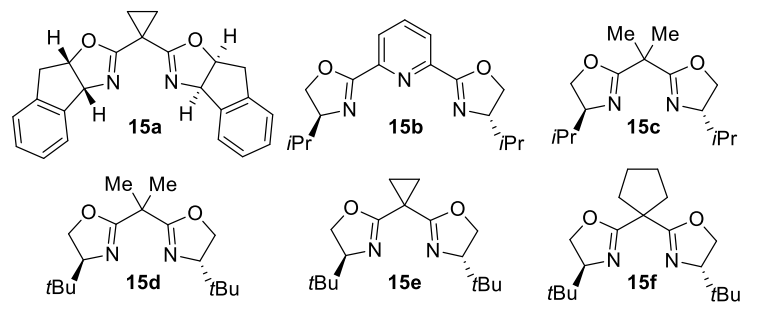

Next the scope of diazo compounds was examined (Scheme 6A). Oxyalkynylation products $\mathbf{1 4 c}$ and $\mathbf{1 4 d}$ bearing benzyl and tertbutyl esters respectively were obtained with lower enantioselectivity. More bulky alkyl groups in contrast led to ee over $90 \%$ (products $14 \mathrm{e}-\mathrm{i}$ ) with the best yield and ee (94\%) reached for dimethylbenzylester 14i. In contrast, bulky aryl esters 14j and 14k, which gave best results with EBX reagents, ${ }^{[6]}$ were obtained with lower enantioselectivities. Interestingly, good 
diastereoselectivities could be achieved with chiral menthol and cholesterol derivatives (14l and $\mathbf{1 4 m}$ ).

We then turned to modification of the alkyne substituent on the EBZ reagent using dimethylbenzylester-derived diazo compounds (Scheme 6B). Aryl EBZs gave access to the corresponding oxyalkynylation products $14 \mathrm{n}$-r bearing fluoro, trifluoromethyl, alkynyl or cyano group in para- or meta- position of the aryl group in $60-91 \%$ yield and $85-93 \%$ ee. Gratifyingly, silylated alkynes could be now used successfully to give products $14 \mathrm{~s}$ and $14 \mathrm{t}$ with more than $90 \%$ ee. Alkyl substituents on the alkyne were investigated next. Excellent results were obtained with primary alkyl groups bearing a simple aliphatic chain (14u and $14 v$ ) or a chloride/alkyne substituent (14w and 14x). Secondary alkyl groups, such as cyclopropane (14y) and cyclopentane (14z) were also well tolerated. Tert-butyl alkyne 14aa was obtained in $63 \%$ yield and $86 \%$ ee. Finally, modification of the aromatic core of the EBZ reagent was also possible to give products $14 \mathrm{ab}$ and 14ac in excellent yield and enantioselectivity. Overall, EBZ reagents gave therefore superior or comparable results than EBXs, without the need of a bulky aryl ester substituent to reach high enantioselectivity. Unfortunately, all attempts to isomerize the obtained imidate to the originally targeted amide, or to initiate an Overman-type rearrangement for transferring the nitrogen atom to the triple bond were unsuccessful.

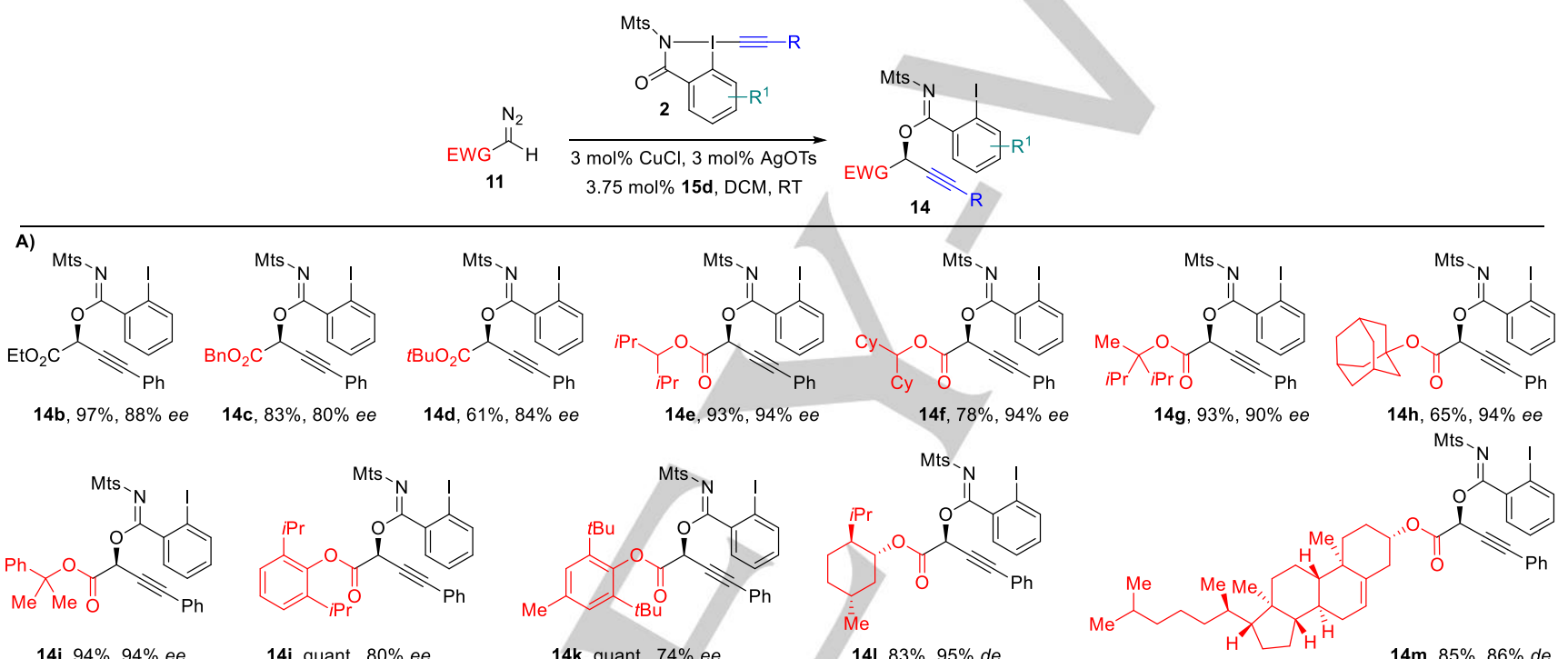

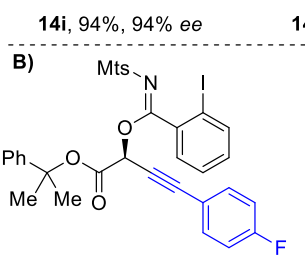

$14 \mathrm{n}, 91 \%, 92 \%$ ee<smiles>CC(C)(OC(=O)C(C#CC[SeH])OC(=NS(C)(C)c1ccccc1)c1ccccc1I)c1ccccc1</smiles>

14s, $71 \%, 90 \%$ ee

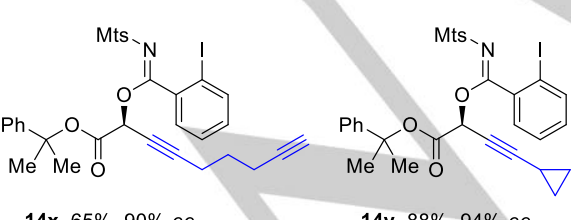<smiles>CN=C(O[C@H](C#Cc1ccc(C(F)(F)F)cc1)C(=O)OC(C)(C)c1ccccc1)c1ccccc1I</smiles>

$140,75 \%, 85 \%$ ee<smiles>CCOC#CC(OC(=O)C(C)(C)c1ccccc1)c1ccccc1I</smiles>

14t, $70 \%, 92 \%$ ee

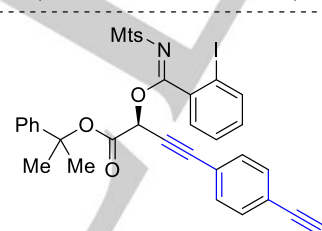

14p, $60 \%$, $93 \%$ ee<smiles>[3H]C(C)([NH3+])OC(=O)[C@H](C#CCCCC)OC(=NS(C)(=O)=O)c1ccccc1I</smiles><smiles>CN=C(O[C@H](C#Cc1cccc(F)c1)C(=O)OC(C)(C)c1ccccc1)c1ccccc1I</smiles>

$14 q, 83 \%, 92 \%$ ee<smiles>CCCC#CC(OC(=NS(C)(=O)=O)c1ccccc1I)C(=O)OC(C)(c1ccccc1)c1ccccc1</smiles>

$14 \mathrm{~m}, 85 \%, 86 \%$ de<smiles>CC(C)(OC(=O)C(C#Cc1cccc(C#N)c1)OC(=NN)c1ccccc1I)c1ccccc1</smiles>

$14 r, 81 \%, 87 \%$ ee<smiles>CC(C)(OC(=O)[C@H](C#CCCCCl)OC(=NS(C)(=O)=O)c1ccccc1I)c1ccccc1</smiles>

14u, $70 \%, 93 \%$ ee

$14 \mathrm{v}, 75 \%, 93 \%$ ee

$14 w, 52 \%, 90 \%$ ee<smiles>CC(C)(OC(=O)[C@H](C#CC1CCCC1)O/C(=N\N)c1ccccc1I)c1ccccc1</smiles><smiles>CC(C)(C)C#C[C@@H](OC(=NS(C)(=O)=O)c1ccccc1I)C(=O)OC(C)(C)c1ccccc1</smiles> 
Based on the well-established reactivity of electrophilic metal carbenes towards heteroatom nucleophiles, ${ }^{[9]}$ a tentative reaction mechanism can be proposed (Scheme 7A). Reaction of the copper catalyst with diazo compound $\mathbf{1 1}$ leads to copper carbene complex I. Nucleophilic attack of EBZ 2 gives then copper ylide II. Interestingly, the exclusive formation of the oxyalkynylation product supports a direct attack of the heteroatom not coordinated to the iodine, a fact that could not be established when using EBX reagents. Finally, electrophilic alkynylation of the copper ylide by the iodonium would give product 14. Further studies would be needed to propose an exact mechanism for this intriguing alkyne transfer. Concerning stereoinduction, the model proposed in our previous work with EBX reagents ${ }^{[6]}$ remains valid (Scheme 7B). We assume a $90^{\circ}$ angle between carbene and bisoxazoline ${ }^{[15]}$ and attack of EBZ 2 on carbene $\mathbf{I}$ in the plane opposite to the bulky tert-butyl group. Attack on the $R e$ face of I (Scheme 7B1, transition state III) is unhindered and favored, leading to the observed absolute stereochemistry of $\mathbf{1 4}$ if the alkynylation occurs with retention of the configuration. Attack on the Si face of I (Scheme 7B2, transition state IV) leads to steric interactions with the ester group on carbene I and is therefore disfavored.

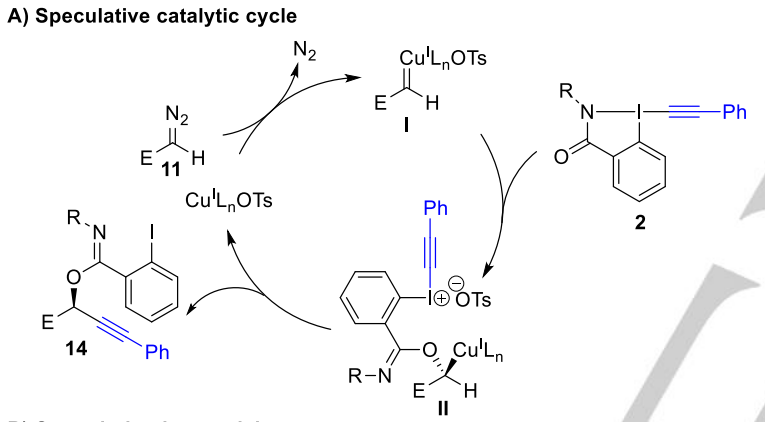

B) Stereoinduction model

B1) Favored transtion state III leading to II

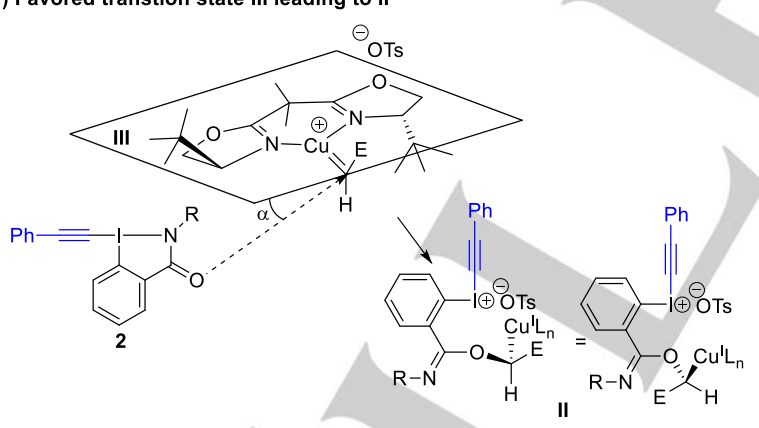

B2) Disfavored transtion state IV leading to II'

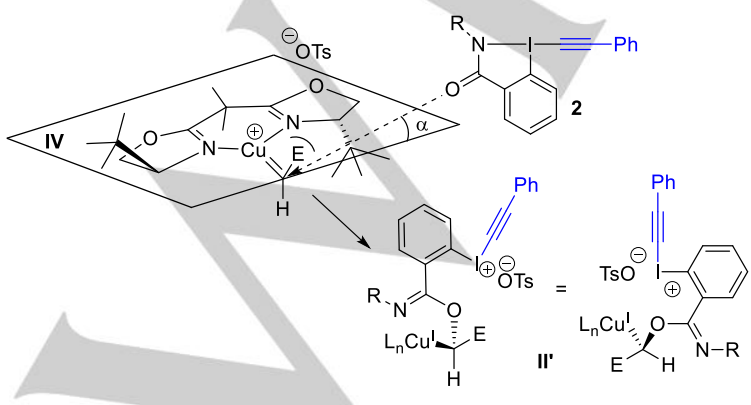

Scheme 7. Speculative mechanism and stereoinduction model for the oxyalkynylation reaction.

\section{Conclusions}

In summary, the first synthesis of ethynylbenziodazolone (EBZ) reagents has been described. The reagents can be easily obtained using a one-step procedure. Preliminary structure and stability studies showed their similarity with the more established ethynylbenziodoxolones (EBXs). In addition, the nitrogen substituent gave further flexibility to fine-tune the physical properties and reactivity of the reagent. The latter was instrumental in the development of a highly enantioselective oxyalkynylation of diazo compounds. This reaction proceeded with high yield and enantioselectivity for a broad range of diazo compounds and EBZ reagents. Our research is now focusing on further synthetic applications of the obtained enantioenriched imidate products, as well as on alternative strategies to implement the initially targeted aminoalkynylation process.

\section{Acknowledgements}

We thank European Research Council (ERC; Starting Grant iTools4MC, number 334840) and the EPFL for financial support.

Keywords: carbenes $\cdot$ diazo compounds $\cdot$ enantioselective transformation $\cdot$ catalysis $\cdot$ hypervalent iodine

[1] B. M. Trost, C. J. Li, Modern alkyne chemistry: catalytic and atomeconomic transformations., Wiley, 2014.

[2] F. Diederich, P. J. Stang, R. R. Tykwinski, Acetylene Chemistry: Chemistry, Biology and Material Science, Wiley-VCH, 2005.

[3] J. P. Brand, J. Waser, Chem. Soc. Rev. 2012, 41, 4165.

[4] Selected references: a) J. P. Brand, J. Charpentier, J. Waser, Angew. Chem., Int. Ed. 2009, 48, 9346; b) S. Nicolai, S. Erard, D. F. Gonzalez, J. Waser, Org. Lett. 2010, 12, 384; c) D. Fernandez Gonzalez, J. P. Brand, J. Waser, Chem. Eur. J. 2010, 16, 9457; d) R. Frei, J. Waser, J. Am. Chem. Soc. 2013, 135, 9620; e) F. Le Vaillant, T. Courant, J. Waser, Angew. Chem., Int. Ed. 2015, 54, 11200.

[5] Reviews: a) J. Kaschel, D. B. Werz, Angew. Chem., Int. Ed. 2015, 54, 8876; b) Y. Li, D. P. Hari, M. V. Vita, J. Waser, Angew. Chem., Int. Ed. 2016, 55, 4436; c) D. P. Hari, S. Nicolai, J. Waser, PATAl'S Chem. Funct. Groups 2018, DOI doi:10.1002/9780470682531.pat0951; d) D. P. Hari, P. Caramenti, J. Waser, Acc. Chem. Res. 2018, 51, 3112.

[6] a) Y. Li, J. Waser, Angew. Chem., Int. Ed. 2015, 54, 5438; b) C. Feng, T.-P. Loh, Angew. Chem., Int. Ed. 2014, 53, 2722; c) F. Xie, Z. Qi, S. Yu X. Li, J. Am. Chem. Soc. 2014, 136, 4780; d) K. D. Collins, F. Lied, F. Glorius, Chem. Commun. 2014, 50, 4459; e) D. P. Hari, J. Waser, J. Am Chem. Soc. 2016, 138, 2190; f) D. P. Hari, J. Waser, J. Am. Chem. Soc. 2017, 139, 8420.

[7] Selected examples of multi-functionalization reaction involving diazo compounds and hypervalent iodine reagents: a) J. S. Tao, R. Tran, G. K. Murphy, J. Am. Chem. Soc. 2013, 135, 16312; b) W. M. Yuan, L. Eriksson, K. J. Szabo, Angew. Chem., Int. Ed. 2016, 55, 8410; c) Z. Zhao, K. G. Kulkarni, G. K. Murphy, Adv. Synth. Catal. 2017, 359, 2222; d) N Döben, H. Yan, M. Kischkewitz, J. Mao, A. Studer, Org. Lett. 2018, 20, 7933 
[8] Other examples of atom-economic transformations involving hypervalent iodine reagents: a) S. G. Modha, M. F. Greaney, J. Am. Chem. Soc. 2015 137, 1416; b) J. Buendia, B. Darses, P. Dauban, Angew. Chem., Int. Ed. 2015, 54, 5697. Review: c) A. Boelke, P. Finkbeiner, B. J. Nachtsheim, Beilstein J. Org. Chem. 2018, 14, 1263.

[9] Selected examples: a) H. Huang, X. Guo, W. Hu, Angew. Chem., Int. Ed. 2007, 46, 1337; b) W. Hu, X. Xu, J. Zhou, W.-J. Liu, H. Huang, J. Hu, L. Yang, L.-Z. Gong, J. Am. Chem. Soc. 2008, 130, 7782; c) C.-Y. Zhou, J. C. Wang, J. Wei, Z.-J. Xu, Z. Guo, K.-H. Low, C.-M. Che, Angew. Chem., Int. Ed. 2012, 51, 11376; d) S. K. Jia, D. Xing, D. Zhang, W. H. Hu, Angew. Chem., Int. Ed. 2014, 53, 13098; e) D. F. Chen, F. Zhao, Y. Hu, L. Z. Gong, Angew. Chem., Int. Ed. 2014, 53, 10763; f) Y. Xia, S. Feng, Z. Liu, Y. Zhang, J. Wang, Angew. Chem., Int. Ed. 2015, 54, 7891; Reviews: g) X. Zhao, Y. Zhang, J. Wang, Chem. Commun. 2012, 48, 10162; h) X Guo, W. Hu, Acc. Chem. Res. 2013, 46, 2427; i) Y. Xia, D. Qiu, J. B. Wang, Chem. Rev 2017, 117, 13810.

[10] a) W. Wolf, Steinber.L, Chem. Commun. (London) 1965, 449; b) T. M. Balthazor, D. E. Godar, B. R. Stults, J. Org. Chem. 1979, 44, 1447; c) V. V. Zhdankin, R. M. Arbit, M. McSherry, B. Mismash, V. G. Young, J. Am. Chem. Soc. 1997, 119, 7408; d) V. V. Zhdankin, R. M. Arbit, B. J. Lynch, P. Kiprof, J. Org. Chem. 1998, 63, 6590; e) V. V. Zhdankin, J. T. Smart P. Zhao, P. Kiprof, Tetrahedron Lett. 2000, 41, 5299; f) V. V. Zhdankin
A. E. Koposov, J. T. Smart, R. R. Tykwinski, R. McDonald, A. MoralesIzquierdo, J. Am. Chem. Soc. 2001, 123, 4095; g) V. V. Zhdankin, A. Y Koposov, L. S. Su, V. V. Boyarskikh, B. C. Netzel, V. G. Young, Org. Lett. 2003, 5, 1583; h) M. Ochiai, T. Sueda, K. Miyamoto, P. Kiprof, V. V. Zhdankin, Angew. Chem., Int. Ed. 2006, 45, 8203; i) A. Yoshimura, M. T. Shea, C. L. Makitalo, M. E. Jarvi, G. T. Rohde, A. Saito, M. S. Yusubov, V. V. Zhdankin, Beilstein J. Org. Chem. 2018, 14, 1016.

[11] S. Alazet, J. Preindl, R. Simonet-Davin, S. Nicolai, A. Nanchen, T. Meyer, J. Waser, J. Org. Chem. 2018, 83, 12334.

[12] M. J. Bouma, B. Olofsson, Chem. Eur. J. 2012, 18, 14242.

[13] The structures of $\mathbf{2} \mathbf{a}$ and $\mathbf{2 e}$ were determined by $\mathrm{X}$-ray diffraction. The data are available from the Cambridge Crystallographic Data Center (CCDC numbers 1896879 (2a) and $1896878(\mathbf{2 e})$ ). The structure of $\mathbf{1 a}$ is already available (CCDC 863342), a new refined structure has been recently submitted (CCDC 1900537).

[14] The structure of $\mathbf{1 4 n}$ was determined by X-ray diffraction. The data are available from the Cambridge Crystallographic Data Center (CCDC number 1896880).

[15] J. M. Fraile, J. I. García, V. Martínez-Merino, J. A. Mayoral, L. Salvatella, J. Am. Chem. Soc. 2001, 123, 7616. 
Entry for the Table of Contents (Please choose one layout)

\section{FULL PAPER}

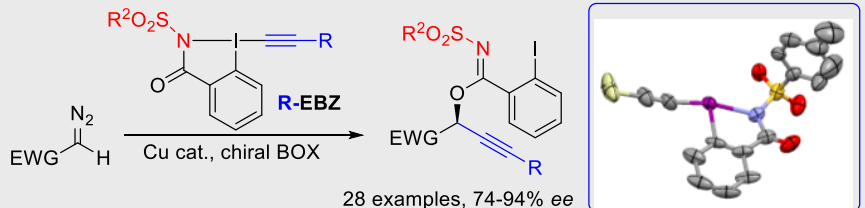

Ethynylbenziodazolone (EBZ) cyclic hypervalent iodine reagents have been synthesized for the first time and displayed similar structure and reactivity when compared to the established ethynylbenziodoxolone (EBX) reagents. They were used in the oxyalkynylation of diazo compounds to access imidate derivatives in high yield and enantioselectivity for a broad scope of diazo compounds and alkynes.
Durga Prasad Hari, Lionel Schouwey, Verity Barber, Rosario Scopelliti, Farzaneh Fadaei Tirani and Jerome Waser*

Page No. - Page No.

Ethynylbenziodazolones (EBZ) as Electrophilic Alkynylation Reagents for the Highly Enantioselective Copper-Catalyzed Oxy-Alkynylation of Diazo Compounds 


\section{Ethynylbenziodazolones (EBZ) as Electrophilic Alkynylation Reagents for the Highly Enantioselective Copper-Catalyzed Oxy- Alkynylation of Diazo Compounds}

Durga Prasad Hari, Lionel Schouwey, Verity Barber, Rosario Scopelliti, Farzaneh Fadaei Tirani and Jerome Waser*

Laboratory of Catalysis and Organic Synthesis, Ecole Polytechnique Fédérale de Lausanne, EPFL SB ISIC LCSO, BCH 4306, 1015 Lausanne, Switzerland

Supporting Information

(262 pages)

\section{Table of Contents}

1. General methods 


\section{General Methods}

All reactions were carried out in oven dried glassware under an atmosphere of nitrogen, unless stated otherwise. For quantitative flash chromatography technical grade solvents were used. For flash chromatography for analysis, HPLC grade solvents from Sigma-Aldrich were used. THF, $\mathrm{Et}_{2} \mathrm{O}, \mathrm{CH}_{3} \mathrm{CN}$, toluene, hexane and $\mathrm{CH}_{2} \mathrm{Cl}_{2}$ were dried by passage over activated alumina under nitrogen atmosphere $\left(\mathrm{H}_{2} \mathrm{O}\right.$ content $<10 \mathrm{ppm}$, Karl-Fischer titration $)$. The solvents were degassed by Freeze-Pump-Thaw method when mentioned. All chemicals were purchased from Acros, Aldrich, Fluka, VWR, Aplichem or Merck and used as such unless stated otherwise. Chromatographic purification was performed as flash chromatography using Macherey-Nagel silica 40-63, $60 \AA$ A, using the solvents indicated as eluent with 0.1-0.5 bar pressure. TLC was performed on Merck silica gel $60 \mathrm{~F}_{254}$ TLC glass plates or aluminium plates and visualized with UV light, permanganate stain, CAN stain or Anisaldehyde stain. Melting points were measured on a Büchi B-540 melting point apparatus using open glass capillaries, the data is uncorrected. ${ }^{1} \mathrm{H}-\mathrm{NMR}$ spectra were recorded on a Brucker DPX-400 $400 \mathrm{MHz}$ spectrometer in chloroform-d, DMSO- $d_{6}$ or $\mathrm{CD}_{3} \mathrm{OD}$, all signals are reported in ppm with the internal chloroform signal at $7.26 \mathrm{ppm}$, the internal DMSO signal at $2.50 \mathrm{ppm}$ or the internal methanol signal at $3.30 \mathrm{ppm}$ as standard. The data is being reported as $(\mathrm{s}=$ singlet, $\mathrm{d}=$ doublet, $\mathrm{t}=$ triplet, $\mathrm{q}=$ quadruplet, $\mathrm{qi}=$ quintet, $\mathrm{m}=$ multiplet or unresolved, $\mathrm{br}=$ broad signal, $\mathrm{app}=$ apparent, coupling constant(s) in $\mathrm{Hz}$, integration, interpretation). ${ }^{13} \mathrm{C}-\mathrm{NMR}$ spectra were recorded with ${ }^{1} \mathrm{H}$-decoupling on a Brucker DPX-400 $100 \mathrm{MHz}$ spectrometer in chloroform-d, DMSO- $d_{6}$ or $\mathrm{CD}_{3} \mathrm{OD}$, all signals are reported in ppm with the internal chloroform signal at 77.0 ppm, the internal DMSO signal at $39.5 \mathrm{ppm}$ or the internal methanol signal at $49.0 \mathrm{ppm}$ as standard. Infrared spectra were recorded on a JASCO FT-IR B4100 spectrophotometer with an ATR PRO410-S and a ZnSe prisma and are reported as $\mathrm{cm}^{-1}(\mathrm{w}=$ weak, $\mathrm{m}=$ medium, $\mathrm{s}=$ strong, br = broad). High resolution mass spectrometric measurements were performed by the mass spectrometry service of ISIC at the EPFL on a MICROMASS (ESI) Q-TOF Ultima API. HPLC measurements were done on a Agilent 1260 Infinity autosampler using a CHIRALPAK IA, IB, IC or ID column from DAICEL Chemical. Optical rotations were measured on a polarimeter using a $10 \mathrm{~cm}$ cell with a $\mathrm{Na} 589 \mathrm{~nm}$ filter. The specific solvents and concentrations (in $\mathrm{g} / 100 \mathrm{~mL}$ ) are indicated. 


\section{Preparation of EBZ reagents}

\section{4-Methylbenzenesulfonamide (17)}

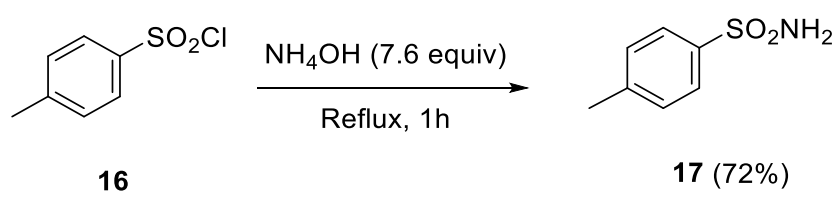

Following a slightly modified reported procedure, ${ }^{[1]}$ in a $100 \mathrm{~mL}$ round-bottom flask, a solution of 4-methylbenzene-1-sulfonyl chloride (4.00 g, $21.0 \mathrm{mmol}, 1.00$ equiv) in ammonium hydroxide solution $25 \% \mathrm{w} / \mathrm{w}(25.0 \mathrm{~mL}, 161 \mathrm{mmol}, 7.70$ equiv) was heated to reflux for $1 \mathrm{~h}$. After reaction completion, the reaction mixture was cooled down and filtered. The crude product was recrystallized in water to afford 4-methylbenzenesulfonamide (17) as white crystalline solid (2.81 g, $16.4 \mathrm{mmol}, 78 \%) .{ }^{1} \mathbf{H}$ NMR (400 MHz, $\left.\mathrm{CDCl}_{3}\right): \delta 7.82(\mathrm{~d}, J=8.3 \mathrm{~Hz}$, 2H, $\operatorname{Ar} H$ ), $7.32(\mathrm{~d}, J=8.0 \mathrm{~Hz}, 2 \mathrm{H}, \operatorname{Ar} H), 4.77$ (br s, 2H, NH$\left.H_{2}\right), 2.43$ (s, 3H, ArCH$)_{3}$. The characterization data corresponded to the reported values. ${ }^{[2]}$

\section{2-Iodo- $N$-tosylbenzamide (3a)}

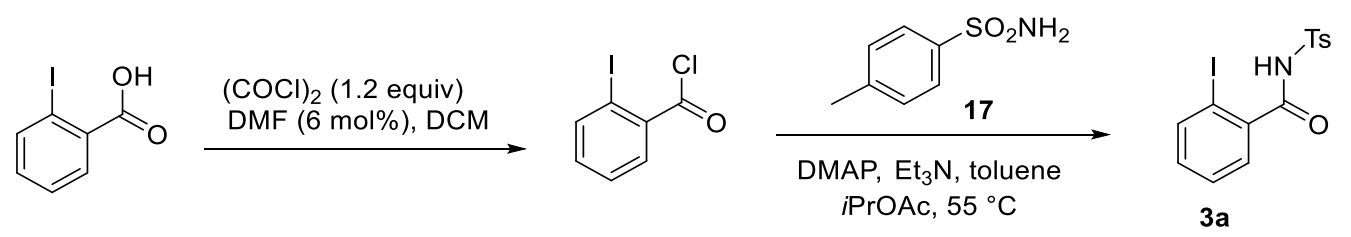

In a $25 \mathrm{~mL}$ round-bottom flask, 2-iodobenzoic acid (2.48 g, $10.0 \mathrm{mmol}, 1.00$ eqiv) and DMF (1 drop, 6 mol\%) were suspended in DCM (7.0 mL). Oxalyl chloride (1.1 mL, $12 \mathrm{mmol}, 98$ $\%, 1.2$ equiv) was added dropwise at $0{ }^{\circ} \mathrm{C}$. After the addition, the reaction was warmed up to RT and stirred for $3 \mathrm{~h}$. The solvent and the oxalyl chloride excess were removed in vacuum. The crude 2-iodobenzoyl chloride was dissolved in toluene $(6.5 \mathrm{~mL})$ and transferred to a solution of 4-methylbenzenesulfonamide (17) ( $1.50 \mathrm{~g}, 8.15 \mathrm{mmol}, 0.820$ equiv), $N, N$-dimethyl4-amino-pyridine ( $5.5 \mathrm{mg}, 0.05 \mathrm{mmol}, 0.005$ equiv), and $\mathrm{Et}_{3} \mathrm{~N}$ (3.2 mL, $23 \mathrm{mmol}, 2.3$ equiv) in isopropyl acetate $(20 \mathrm{~mL})$. The reaction mixture was heated to $55{ }^{\circ} \mathrm{C}$ and stirred for $1 \mathrm{~h}$. Water $(10 \mathrm{~mL})$ was added to quench the excess of acyl chloride. The organic layer was washed with $0.7 \mathrm{M} \mathrm{HCl}$ solution $(70 \mathrm{~mL})$ and the aqueous layer was back extracted with ethyl acetate (2 X $100 \mathrm{~mL}$ ). The organic layers were combined, dried and the solvent was removed under reduced pressure. The crude product was purified by flash chromatography using EtOAc:Pentane 1:3.5 as mobile phase to afford 3a as a slightly brown gum (2.92 g, $7.27 \mathrm{mmol}$, 80\%). TLC (EtOAc:Pentane 1:3.5): $\mathrm{R}_{\mathrm{f}}=0.26, \mathrm{KMnO}_{4} ;{ }^{1} \mathrm{H}$ NMR (400 MHz, $\left.\mathrm{CDCl}_{3}\right): \delta 9.04$ (s, 1H, NH), $7.98(\mathrm{~d}, J=8.3 \mathrm{~Hz}, 2 \mathrm{H}, \operatorname{Ar} H), 7.76(\mathrm{~d}, J=7.9 \mathrm{~Hz}, 1 \mathrm{H}, \operatorname{Ar} H), 7.34(\mathrm{~m}, 4 \mathrm{H}, \operatorname{Ar} H)$, $7.10-7.03(\mathrm{~m}, 1 \mathrm{H}, \mathrm{ArH}), 2.43$ (s, 3H, $\left.\mathrm{ArCH}_{3}\right) ;{ }^{13} \mathrm{C} \mathrm{NMR}\left(101 \mathrm{MHz}, \mathrm{CDCl}_{3}\right): \delta 165.9,145.4$, 
140.2, 138.4, 135.3, 135.0, 132.5, 129.6, 128.9, 128.3, 91.8, 21.8; IR v 3057 (w), 2363 (w), 1711 (m), 1594 (w), 1434 (m), 1353 (m), 1270 (s), 1172 (m), 1126 (w), 1081 (w), 1041 (w), 896 (w), 834 (m); HRMS (ESI) calcd. for $\mathrm{C}_{14} \mathrm{H}_{13} \mathrm{INO}_{3} \mathrm{~S}^{+}[\mathrm{M}+\mathrm{H}]^{+} 401.9655$; found 401.9669. General procedure 1 (GP1)
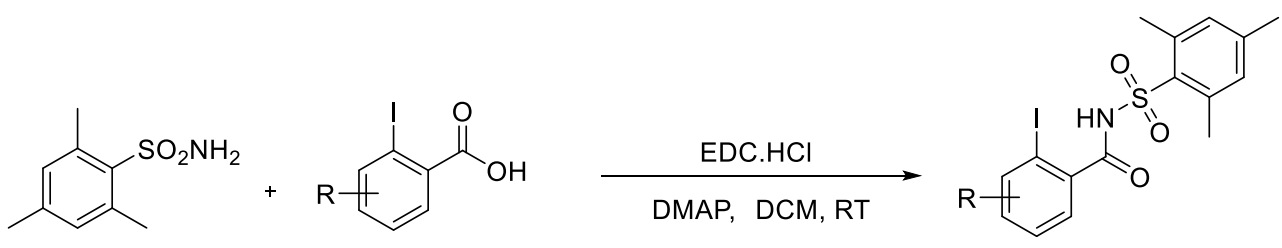

A solution of 2-iodobenzoic acid (16.6 mmol, 1.10 equiv), $N$-(3-dimethylaminopropyl)- $N$ 'ethylcarbodiimide hydrochloride ( $22.6 \mathrm{mmol}, 1.50$ equiv) and 4-dimethylaminopyridine (22.6 mmol, 1.50 equiv) in DCM $(0.5 \mathrm{M})$ was stirred for $1 \mathrm{~h}$ at RT. 2,4,6Trimethylbenzenesulfonamide ( $15 \mathrm{mmol}, 1.0$ equiv) was added in one portion to the solution and the reaction mixture was stirred overnight. The reaction mixture was quenched with $1 \mathrm{M}$ $\mathrm{HCl}(50 \mathrm{~mL})$ solution and stirred for $1 \mathrm{~h}$. The organic layer was washed with $1 \mathrm{M} \mathrm{HCl}(2 \mathrm{X} 50$ $\mathrm{mL}$ ) followed by saturated $\mathrm{NaCl}$ solution $(50 \mathrm{~mL})$. The combined aqueous layers were extracted with DCM (2 X $50 \mathrm{~mL}$ ). The organic layers were combined and dried over $\mathrm{MgSO}_{4}$, filtered and the solvent removed under reduced pressure. The product was purified by flash column chromatography using EtOAc:pentane (1:3 v/v).

2-Iodo- $N$-[2,4,6-trimethylbenzenesulfonamide]benzamide (3b)

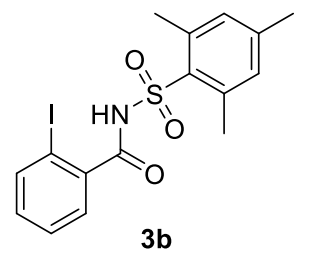

Following GP1, 2-iodobenzoic acid (4.11 g, 16.6 mmol, 1.10 equiv), $N$-(3dimethylaminopropyl)- $N$ '-ethylcarbodiimide hydrochloride (4.33 g, $22.6 \mathrm{mmol}, 1.50$ equiv), 4dimethylaminopyridine $(2.76 \mathrm{~g}, 22.6 \mathrm{mmol}, 1.50$ equiv $)$ and 2,4,6trimethylbenzenesulfonamide $(3.00 \mathrm{~g}, 15.0 \mathrm{mmol}, 1.00$ equiv) were stirred for $14 \mathrm{~h}$. The crude reaction mixture was concentrated under reduced pressure and purified by flash chromatography using EtOAc:pentane 1:3 as mobile phase to afford $\mathbf{3 b}$ as a white solid (5.12 g, 11.9 mmol, 79\%). Mp: 206-209 ${ }^{\circ} \mathrm{C}$; TLC (EtOAc:pentane 1:2 v/v): $\mathrm{R}_{\mathrm{f}}=0.55, \mathrm{KMnO}_{4} ;{ }^{1} \mathrm{H}$ NMR (400 MHz, $\left.\mathrm{CDCl}_{3}\right): \delta 8.44(\mathrm{~s}, 1 \mathrm{H}, \mathrm{NH}), 7.86(\mathrm{~d}, J=7.9 \mathrm{~Hz}, 1 \mathrm{H}, \mathrm{ArH}), 7.44-7.32(\mathrm{~m}$, 2H, ArH), $7.15-7.12$ (m, , 1H, ArH), 7.01 (s, 2H, ArH), 2.74 (s, 6H, ArCH3), 2.33 (s, 3H, $\left.\mathrm{ArCH}_{3}\right) ;{ }^{13} \mathrm{C} \mathrm{NMR}\left(100 \mathrm{MHz}, \mathrm{CDCl}_{3}\right): \delta$ 166.1, 144.0, 140.9, 140.3, 138.9, 132.4, 132.1, 132.0, 128.6, 128.3, 91.5, 23.1, 21.1; IR v 3210 (w), 2850 (w), 1710 (m), 1603 (w), 1468 (m), 1430 
(s), 1343 (m), 1277 (w), 1239 (w), 1165 (s), 1098 (m), 1054 (w), 1037 (w), 1017 (w), 887 (w), 822 (m); HRMS (ESI) calcd. for $\mathrm{C}_{16} \mathrm{H}_{17} \mathrm{INO}_{3} \mathrm{~S}^{+}[\mathrm{M}+\mathrm{H}]^{+} 429.9968$; found 429.9972.

\section{2-Iodo- $N$-(mesitylsulfonyl)-5-methylbenzamide (3c)}

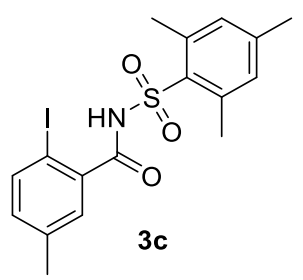

Following GP1, 2-iodo-5-methylbenzoic acid (0.725 g, $2.76 \mathrm{mmol}, 1.10$ equiv), $N$-(3dimethylaminopropyl)- $N$ '-ethylcarbodiimide hydrochloride $(0.721 \mathrm{~g}, 3.76 \mathrm{mmol}, 1.50$ equiv), 4-dimethylaminopyridine $(0.460 \mathrm{~g}, \quad 3.76 \mathrm{mmol}, \quad 1.50 \mathrm{equiv})$ and 2,4,6trimethylbenzenesulfonamide $(0.50 \mathrm{~g}, 2.5 \mathrm{mmol}, 1.0$ equiv) were stirred for $14 \mathrm{~h}$. The crude reaction mixture was concentrated under reduced pressure and purified by flash chromatography using EtOAc:pentane 1:3 as mobile phase to afford $\mathbf{3 c}$ as a white solid (0.864 g, 1.95 mmol, 78\%). Mp: 194-198 ${ }^{\circ} \mathrm{C}$; TLC (EtOAc:pentane 1:3 v/v): $\mathrm{R}_{\mathrm{f}}=0.39, \mathrm{KMnO}_{4} ;{ }^{1} \mathrm{H}$ NMR (400 MHz, $\left.\mathrm{CDCl}_{3}\right): \delta 8.42(\mathrm{~s}, 1 \mathrm{H}, \mathrm{NH}), 7.71(\mathrm{~d}, J=8.1 \mathrm{~Hz}, 1 \mathrm{H}, \mathrm{ArH}), 7.23-7.22(\mathrm{~m}$, 1H, $\mathrm{ArH}), 7.01$ (s, 2H, $\mathrm{ArH}), 6.97(\mathrm{dd}, J=8.1,2.1 \mathrm{~Hz}, 1 \mathrm{H}, \mathrm{ArH}), 2.74\left(\mathrm{~s}, 6 \mathrm{H}, 2 \mathrm{X} \mathrm{ArCH}_{3}\right)$, 2.32 (s, 3H, $\left.\mathrm{ArCH}_{3}\right), 2.30$ (s, 3H, $\left.\mathrm{ArCH}_{3}\right) ;{ }^{13} \mathrm{C} \mathrm{NMR}\left(100 \mathrm{MHz}, \mathrm{CDCl}_{3}\right.$ ): $\delta 166.1,143.9,140.9$, 140.1, 138.7, 138.6, 133.4, 132.1, 129.6, 87.3, 23.1, 21.1, 20.8; IR v 3225 (w), 2943 (w), 1711 (m), 1602 (w), 1566 (w), 1427 (m), 1341 (m), 1283 (w), 1245 (w), 1194 (w), 1160 (s), 1097 (m), 1053 (w), 1016 (w), 852 (m). One carbon was not resolved at $100 \mathrm{MHz}$. Couldn't identify the mass of the compound in HRMS. However, the product (14ab) of the next step is completely characterized.

\section{2-Iodo- $N$-(mesitylsulfonyl)-4,5-dimethoxybenzamide (3d)}

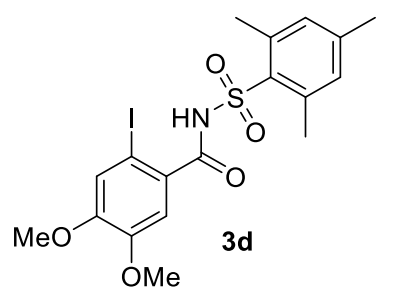

Following GP1, 2-iodo-4,5-dimethoxybenzoic acid (0.850 g, $2.76 \mathrm{mmol}, 1.00$ equiv), $\mathrm{N}$-(3dimethylaminopropyl)- $N$ '-ethylcarbodiimide hydrochloride $(0.721 \mathrm{~g}, 3.76 \mathrm{mmol}, 1.50$ equiv), 4-dimethylaminopyridine $(0.460 \mathrm{~g}, \quad 3.76 \mathrm{mmol}, \quad 1.50 \mathrm{equiv})$ and 2,4,6trimethylbenzenesulfonamide $(0.50 \mathrm{~g}, 2.5 \mathrm{mmol}, 1.0$ equiv) were stirred for $14 \mathrm{~h}$. The crude reaction mixture was concentrated under reduced pressure and purified by flash chromatography using EtOAc:pentane 1:3 as mobile phase to afford as a white solid $(0.450 \mathrm{~g}$, 
$0.920 \mathrm{mmol}, 37 \%$ ). Mp: 223-226 ${ }^{\circ} \mathrm{C}$; TLC (EtOAc:pentane 1:3 v/v): $\mathrm{R}_{\mathrm{f}}=0.3, \mathrm{KMnO}_{4} ;{ }^{1} \mathrm{H}$ NMR (400 MHz, $\mathrm{CDCl}_{3}$ ): $\delta 8.75(\mathrm{~s}, 1 \mathrm{H}, \mathrm{NH}), 7.22$ (s, 1H, $\left.\operatorname{Ar} H\right), 7.08$ (s, 1H, ArH), 7.01 (s, 2H, $\mathrm{ArH}$ ), 3.89 (s, 3H, $\left.\mathrm{ArOCH}_{3}\right), 3.84$ (s, 3H, $\left.\mathrm{ArOCH}_{3}\right), 2.76$ (s, 6H, $\left.2 \mathrm{X} \mathrm{ArCH}_{3}\right), 2.65$ (s, 3H, $\left.\mathrm{ArCH}_{3}\right) ;{ }^{13} \mathrm{C} \mathrm{NMR}\left(100 \mathrm{MHz}, \mathrm{CDCl}_{3}\right): \delta 165.2,151.7,149.3,143.9,140.8,132.1,131.9,130.2$, 122.5, 112.7, 80.9, 56.4, 56.2, 23.1, 21.1; IR v 3241 (w), 2938 (w), 2846 (w), 1699 (w), 1594 (w), 1565 (w), 1505 (w), 1422 (m), 1369 (w), 1336 (m), 1261 (m), 1207 (w), 1159 (s), 1094 (m), 1031 (w), 951 (w), 854 (m); HRMS (ESI) calcd. for $\mathrm{C}_{18} \mathrm{H}_{21} \mathrm{INO}_{5} \mathrm{~S}^{+}[\mathrm{M}+\mathrm{H}]^{+} 490.0180$; found 490.0179 .

(1S)-(+)-Camphorsulfonamide (18)
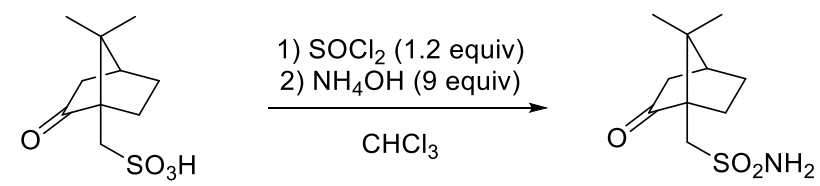

18

In a $20 \mathrm{ml}$ round-bottom flask, thionyl chloride $(0.42 \mathrm{~mL}, 5.7 \mathrm{mmol}, 1.2$ equiv) was slowly added to a refluxing solution of (1S)-(+)-camphorsulfonic acid (1.10 g, $4.74 \mathrm{mmol}, 1.00$ equiv) in chloroform $(5.0 \mathrm{~mL})$. The reflux was maintained overnight. The reaction was cooled down to $0{ }^{\circ} \mathrm{C}$ and added over $1 \mathrm{~h}$ to $25 \%$ w/w ammonium hydroxide solution $(11.0 \mathrm{~mL}, 78.0 \mathrm{mmol}$, 16.6 equiv) at $0{ }^{\circ} \mathrm{C}$. The reaction mixture was then heated to $\mathrm{RT}$ and stirred for $4 \mathrm{~h}$. The aqueous layer was extracted with DCM ( 3 X $20 \mathrm{~mL})$ and the combined organic layers were washed with brine $(20 \mathrm{~mL})$. The organic layers were dried with $\mathrm{Na}_{2} \mathrm{SO}_{4}$ and the solvent was removed under reduced pressure. The crude product was purified by flash chromatography using EtOAc:pentane 1:2 as mobile phase to afford 18 as a white solid (574 mg, $2.48 \mathrm{mmol}, 53 \%$ ). TLC (EtOAc:pentane 1:2 v/v): $\mathrm{R}_{\mathrm{f}}=0.27, \mathrm{KMnO}_{4} ;{ }^{1} \mathrm{H}$ NMR $\left(400 \mathrm{MHz}, \mathrm{CDCl}_{3}\right): \delta 5.36(\mathrm{~s}, 2 \mathrm{H}$, $\mathrm{NH}_{2}$ ), 3.14 and 3.53 (AB quartet, $J=15.1 \mathrm{~Hz}, 2 \mathrm{H}, \mathrm{CH}_{2} \mathrm{SO}_{2} \mathrm{NH}_{2}$ ), 2.43-1.43 (m, 7H, C(O)-C$\left.\mathrm{CH}_{2}-\mathrm{CH}_{2}-\mathrm{CH}-\mathrm{CH}_{2}\right), 1.01\left(\mathrm{~s}, 3 \mathrm{H}, \mathrm{CH}_{3}\right), 0.93\left(\mathrm{~s}, 3 \mathrm{H}, \mathrm{CH}_{3}\right)$. The characterization data corresponded to the reported values. ${ }^{[3]}$

\section{2-Iodo- $N$-camphorsulfonylbenzamide (3e)}

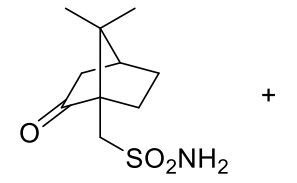

18

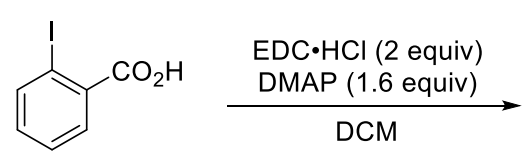

CS = Camphorsulfonyl

A solution of DMAP (450 mg, $3.68 \mathrm{mmol}, 1.60$ equiv), EDC ( $882 \mathrm{mg}, 4.60 \mathrm{mmol}, 2.00$ equiv) and 2-iodobenzoic acid (570 mg, $2.30 \mathrm{mmol}, 1.00$ equiv) in DCM (40 mL) was stirred $1 \mathrm{~h}$ at RT. (1S)-(+)-Camphorsulfonamide (18) (532 mg, $2.32 \mathrm{mmol}, 1.00$ equiv) was added in one 
portion to the solution and the reaction mixture was stirred overnight at RT. The reaction mixture was diluted with DCM $(100 \mathrm{~mL})$ and washed with $1 \mathrm{M} \mathrm{HCl}$ solution $(2 \mathrm{X} 50 \mathrm{~mL})$ and water $(50 \mathrm{~mL})$. The organic layer was dried with $\mathrm{Na}_{2} \mathrm{SO}_{4}$, filtered, and the solvent was removed under reduced pressure. The crude product was purified by flash chromatography using MeOH:DCM 5:95 as mobile phase to afford 3e as a pale yellow solid (833 $\mathrm{mg}, 1.81 \mathrm{mmol}$, 79\%). Mp: 89.8-90.7 ${ }^{\circ} \mathrm{C}$; TLC (MeOH:DCM 5:95 v/v): $\mathrm{R}_{\mathrm{f}}=0.19, \mathrm{KMnO}_{4} ;{ }^{1} \mathrm{H}$ NMR (400 $\left.\mathrm{MHz}_{\mathrm{CDCl}}\right): \delta 8.51(\mathrm{~s}, 1 \mathrm{H}, \mathrm{NH}), 7.88(\mathrm{~d}, J=7.9 \mathrm{~Hz}, 1 \mathrm{H}, \mathrm{Ar} H), 7.61(\mathrm{~d}, J=7.4 \mathrm{~Hz}, 1 \mathrm{H}, \operatorname{Ar} H)$, $7.37(\mathrm{t}, J=7.4 \mathrm{~Hz}, 1 \mathrm{H}, \operatorname{Ar} H$ ), $7.12(\mathrm{t}, J=7.4 \mathrm{~Hz}, 1 \mathrm{H}, \operatorname{Ar} H$ ), 4.06 and 3.46 (AB quartet, 14.0 $\mathrm{Hz}, 2 \mathrm{H}, \mathrm{CH}_{2} \mathrm{SO}_{2} \mathrm{NH}$ ), $2.46-1.25$ (m, 7H, C(O)-C-CH $\left.\mathrm{CH}_{2}-\mathrm{CH}_{2}-\mathrm{CH}-\mathrm{CH}_{2}\right), 1.06$ (s, 3H, CH $), 0.89$ (s, 3H, $\left.\mathrm{CH}_{3}\right) ;{ }^{13} \mathrm{C}$ NMR (100 MHz, $\left.\mathrm{CDCl}_{3}\right): \delta 215.5,167.1,140.8,138.6,132.8,129.0,128.5$, 92.4, 59.2, 52.0, 49.0, 43.0, 43.0, 27.2, 26.5, 20.1, 19.8; IR v 3667 (w), 2979 (s), 2903 (s), 2361 (w), 1748 (w), 1698 (w), 1627 (w), 1402 (m), 1251 (w), 1058 (s), 889 (w); HRMS (ESI) calcd. for $\mathrm{C}_{17} \mathrm{H}_{20} \mathrm{INO}_{4} \mathrm{SNa}^{+}[\mathrm{M}+\mathrm{Na}]^{+} 484.0050$; found 484.0063 .

\section{2-Iodo- $N$-acetylbenzamide (3f)}

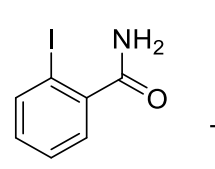<smiles>CC(=O)OC(C)=O</smiles><smiles>CC(C)(C)OOP(=O)(O)O</smiles><smiles>CC(=O)NC(=O)c1ccccc1I</smiles>

In a 2-5 ml microwave reaction vial, 2-iodobenzamide (247 mg, $1.00 \mathrm{mmol}, 1.00$ equiv) suspended in acetic anhydride $(0.3 \mathrm{ml}, 3 \mathrm{mmol}, 3$ equiv). Sulfuric acid (2 drops) was added and the tube was sealed. The vial was kept in microwave for $3 \mathrm{~min}$ at $100{ }^{\circ} \mathrm{C}(100 \mathrm{~W})$. The reaction mixture was diluted with ethyl acetate $(20 \mathrm{~mL})$, washed with a saturated $\mathrm{NaHCO}_{3}$ solution $(10 \mathrm{~mL})$ and brine $(10 \mathrm{~mL})$. The aqueous layers were back extracted with ethyl acetate (2 X $10 \mathrm{~mL}$ ). The organic layers were combined, dried with $\mathrm{Na}_{2} \mathrm{SO}_{4}$ and the solvent was removed under reduced pressure. The crude product was purified by flash chromatography using EtOAc:pentane 1:3 as mobile phase to afford $\mathbf{3 f}$ as a white solid $(0.267 \mathrm{~g}, 0.924 \mathrm{mmol}$, 92\%). Mp: 102.2-103.8 ${ }^{\circ} \mathrm{C}$; TLC (EtOAc:pentane 1:3.5 v/v): $\mathrm{R}_{\mathrm{f}}=0.29, \mathrm{KMnO}_{4} ;{ }^{1} \mathrm{H} \mathrm{NMR}(400$ $\left.\mathrm{MHz}, \mathrm{CDCl}_{3}\right): \delta 8.27(\mathrm{~s}, 1 \mathrm{H}, \mathrm{NH}), 7.92(\mathrm{~d}, J=7.9 \mathrm{~Hz}, 1 \mathrm{H}, \operatorname{Ar} H), 7.44(\mathrm{~m}, 2 \mathrm{H}, \operatorname{Ar} H), 7.25-$ $7.12(\mathrm{~m}, 1 \mathrm{H}, \mathrm{ArH}), 2.58$ (s, 3H, $\left.\left.\mathrm{COCH}_{3}\right) ;{ }^{13} \mathrm{C} \mathrm{NMR} \mathrm{(100} \mathrm{MHz,} \mathrm{CDCl}_{3}\right): \delta 172.4,167.6,140.6$, 140.2, 132.5, 128.6, 128.4, 92.1, 25.6; IR v 3269 (m), 3177 (w), 2961 (w), 1732 (s), 1694 (s), 1592 (w), 1495 (s), 1429 (m), 1375 (m), 1269 (s), 1226 (s), 1127 (w), 1019 (m), 953 (w), 887 (w), 839 (w); HRMS (ESI) calcd. for $\mathrm{C}_{9} \mathrm{H}_{9} \mathrm{INO}_{2}{ }^{+}[\mathrm{M}+\mathrm{H}]^{+} 289.9672$; found 289.9683. 
General procedure 2 (GP2)<smiles>[X][M]C(=O)c1ccccc1I</smiles>

3

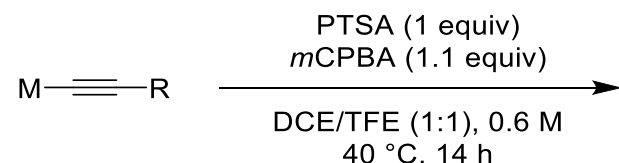

$40^{\circ} \mathrm{C}, 14 \mathrm{~h}$

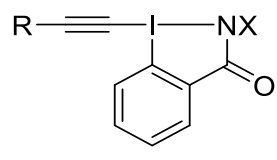

In a 2-5 mL microwave reaction vial, 2-iodo- $N$-substituted benzamide ( $2.5 \mathrm{mmol}, 1.0$ equiv), $p$-toluenesulfonic acid (1.0 equiv) and meta-chloroperbenzoic acid (1.1 equiv) were dissolved in dichloroethane - trifluoroethanol mixture $(1: 1$ ratio, $0.6 \mathrm{M})$. The solution was stirred for $1 \mathrm{~h}$ at $40{ }^{\circ} \mathrm{C}$. The corresponding terminal or silylated alkyne (1.4 equiv) was then added and the reaction mixture was stirred at $40{ }^{\circ} \mathrm{C}$ overnight. The precipitate was dissolved in DCM $(25 \mathrm{~mL})$ and the organic layer was washed with $\mathrm{NaHCO}_{3}$ sat. solution $(2 \mathrm{X} 20 \mathrm{~mL})$ and with brine (15 $\mathrm{mL})$. The combined aqueous layers were back-extracted with DCM (2 X $20 \mathrm{~mL})$. The organic layers were combined, dried with $\mathrm{Na}_{2} \mathrm{SO}_{4}$, filtrated and the solvent was removed under reduced pressure. The crude product was purified using the described method to afford the corresponding EBZ compound.

\section{$N$-[Tolylsulfonyl]-1-[triisopropylsilylethynyl]-1,2-benziodazol-3(1H)-one (2a)}

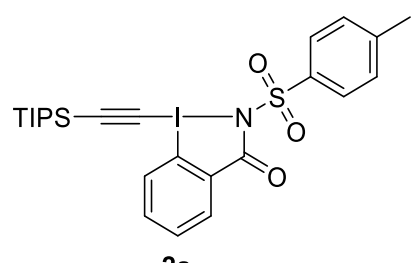

2a

Following GP2, 2-iodo- $N$-tosylbenzamide 3a (1.00 g, $2.49 \mathrm{mmol}, 1.00$ equiv) and triisopropyl((trimethylsilyl)ethynyl) silane $(0.888 \mathrm{~g}, 3.49 \mathrm{mmol}, 1.40$ equiv) were stirred for $14 \mathrm{~h}$. The crude reaction mixture was purified by recrystallization in ethyl acetate $(35 \mathrm{~mL})$ to afford 2a as white crystals (1.39 g, $2.38 \mathrm{mmol}, 96 \%)$. Mp: 241.0-243.6 ${ }^{\circ} \mathrm{C}$ (dec.); ${ }^{1} \mathrm{H}$ NMR $\left(400 \mathrm{MHz}, \mathrm{CDCl}_{3}\right): \delta 8.40-8.34(\mathrm{~m}, 1 \mathrm{H}, \mathrm{ArH}), 8.32-8.27(\mathrm{~m}, 1 \mathrm{H}, \mathrm{Ar} H), 8.01(\mathrm{~d}, J=8.3 \mathrm{~Hz}$, 2H, $\operatorname{Ar} H), 7.70(\mathrm{dd}, J=6.5,3.5 \mathrm{~Hz}, 2 \mathrm{H}, \operatorname{Ar} H), 7.28(\mathrm{~d}, J=8.2 \mathrm{~Hz}, 2 \mathrm{H}, \operatorname{Ar} H), 2.38(\mathrm{~s}, 3 \mathrm{H}$, $\left.\mathrm{CH}_{3}\right), 1.15$ (m, 21H, TIPS); ${ }^{13} \mathrm{C}$ NMR (100 MHz, $\left.\mathrm{CDCl}_{3}\right): \delta 160.7,143.4,137.9,135.2,134.3$, 132.0, 131.6, 129.2, 128.0, 127.1, 115.0, 114.0, 70.6, 21.6, 18.6, 11.2; IR v 2950 (w), $2867(\mathrm{w})$, 1661 (s), 1463 (w), 1440 (w), 1384 (w), 1287 (s), 1249 (w), 1148 (s), 1089 (m), 1002 (w), 894 (m), 862 (s), 819 (w); HRMS (ESI) calcd. for $\mathrm{C}_{25} \mathrm{H}_{33} \mathrm{INO}_{3} \mathrm{SSi}^{+}[\mathrm{M}+\mathrm{H}]^{+}$582.0990; found 582.0995 . 


\section{$N$-[Tolylsulfonyl]-1-[phenylethynyl]-1,2-benziodazol-3(1H)-one (2b)}

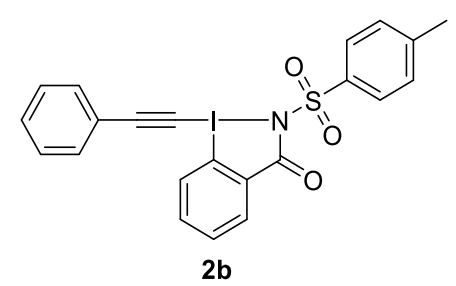

Following GP2, 2-iodo- $N$-tosylbenzamide 3a (1.00 g, $2.49 \mathrm{mmol}, 1.00$ equiv) and phenylacetylene $(0.39 \mathrm{~mL}, 3.5 \mathrm{mmol}, 1.4$ equiv) were stirred for $14 \mathrm{~h}$. The crude reaction mixture was concentrated under reduced pressure and purified by flash chromatography using EtOAc:DCM 1:24 as mobile phase followed by recrystallization by evaporation of DCM from a mixture of ethyl acetate / DCM $(0.5 \mathrm{~mL} / 10 \mathrm{~mL})$ to afford $\mathbf{2 b}$ as white crystals $(0.654 \mathrm{~g}, 1.31$ mmol, 52\%). Mp: 157.6-160.1 ${ }^{\circ} \mathrm{C}$ (dec.); TLC (EtOAc:DCM 1:24 v/v): $\mathrm{R}_{\mathrm{f}}=0.21, \mathrm{KMnO}_{4} ;{ }^{1} \mathrm{H}$ NMR (400 MHz, $\left.\mathrm{CDCl}_{3}\right): \delta 8.34(\mathrm{dd}, J=7.9,1.3 \mathrm{~Hz}, 1 \mathrm{H}, \mathrm{ArH}), 8.30(\mathrm{dd}, J=7.0,2.2 \mathrm{~Hz}, 1 \mathrm{H}$, $\operatorname{Ar} H), 8.03(\mathrm{~d}, J=8.3 \mathrm{~Hz}, 2 \mathrm{H}, \operatorname{Ar} H), 7.75-7.67(\mathrm{~m}, 2 \mathrm{H}, \operatorname{Ar} H), 7.60(\mathrm{~d}, J=8.2 \mathrm{~Hz}, 2 \mathrm{H}, \operatorname{Ar} H)$, $7.53-7.41(\mathrm{~m}, 3 \mathrm{H}, \mathrm{ArH}), 7.29(\mathrm{~d}, J=8.1 \mathrm{~Hz}, 2 \mathrm{H}, \mathrm{ArH}), 2.39$ (s, 3H, $\left.\mathrm{ArCH}_{3}\right) ;{ }^{13} \mathrm{C} \mathrm{NMR}(100$ $\left.\mathrm{MHz}_{2} \mathrm{CDCl}_{3}\right): \delta 160.8,143.5,137.9,135.4,134.2,132.9,132.0,131.6,130.8,129.3,128.8$, 128.0, 127.3, 120.5, 115.5, 106.5, 56.3, 21.6; IR v 2360 (w), $2327(w), 2146(w), 1652(\mathrm{~s})$, 1521 (w), 1490 (w), 1439 (m), 1314 (s), 1294 (s), 1247 (w), 1150 (s), 1087 (m), 1038 (w), 897 (m), 857 (s), 799 (w); HRMS (ESI) calcd. for $\mathrm{C}_{22} \mathrm{H}_{17} \mathrm{INO}_{3} \mathrm{~S}^{+}[\mathrm{M}+\mathrm{H}]^{+} 507.9968$; found 501.9974 .

\section{$N$-[Mesitylsulfonyl]-1-[(triisopropylsilyl)ethynyl]-1,2-benziodazol-3(1H)-one (2c)}

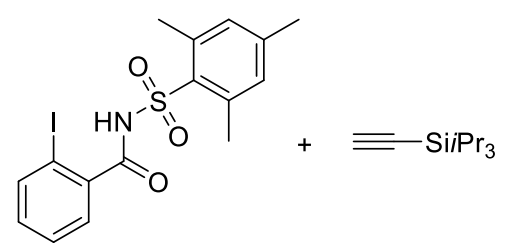

$3 \mathbf{b}$ $\frac{m \text { CPBA, TsOH. }{ }_{2} \mathrm{O}}{\text { DCE:TFE, } \mathrm{NaHCO}_{3}}$

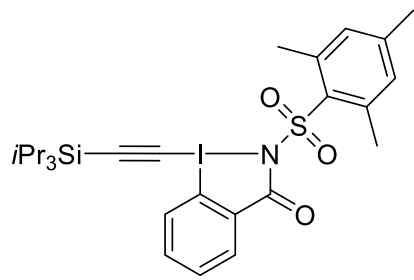

2c

In a $5 \mathrm{~mL}$ sealed tube, 2-iodo- $N$-(mesitylsulfonyl)benzamide (3b) $(0.500 \mathrm{~g}, 1.17 \mathrm{mmol}, 1.00$ equiv), 4-methylbenzenesulfonic acid hydrate ( $0.201 \mathrm{~g}, 1.17 \mathrm{mmol}, 1.00$ equiv) and $m$ CPBA (0.287 g, 77\% purity, $2.56 \mathrm{mmol}, 1.10$ equiv) were suspended in DCE:TFE 1:1 (2 mL) and stirred for $1 \mathrm{~h}$ at $40{ }^{\circ} \mathrm{C}$. Ethynyltriisopropylsilane $(0.297 \mathrm{~g}, 1.63 \mathrm{mmol}, 1.40$ equiv) was then added and the reaction mixture was stirred at $40{ }^{\circ} \mathrm{C}$ for $16 \mathrm{~h}$. The solvent was evaporated and the residue was dissolved in $\mathrm{CH}_{2} \mathrm{Cl}_{2}(10 \mathrm{~mL})$ and stirred vigorously with saturated solution of $\mathrm{NaHCO}_{3}(10 \mathrm{~mL})$. After $1 \mathrm{~h}$, the reaction mixture was transferred into a separating funnel and the layers were separated. The aqueous layer was extracted with $\mathrm{CH}_{2} \mathrm{Cl}_{2}(2 \mathrm{X} 10 \mathrm{~mL})$. The 
combined organic layers were washed with a saturated solution of $\mathrm{NaHCO}_{3}(15 \mathrm{~mL})$, brine (15 $\mathrm{mL}$ ), dried over $\mathrm{MgSO}_{4}$, filtered and concentrated under reduced pressure. The crude product was purified by flash chromatography using EtOAc:Pentane 1:4 as mobile phase followed by recrystallization in EtOAc ( $c$ a $10 \mathrm{~mL})$ to afford $2 \mathrm{c}$ as white crystals $(0.450 \mathrm{~g}, 0.738 \mathrm{mmol}$, 63\%). Mp: 219-223 ${ }^{\circ} \mathrm{C}$; TLC (EtOAc:Pentane 1:3): $\mathrm{R}_{\mathrm{f}}=0.73, \mathrm{KMnO}_{4} ;{ }^{1} \mathrm{H}$ NMR (400 MHz, $\left.\mathrm{CDCl}_{3}\right): \delta 8.41(\mathrm{~d}, J=8.9 \mathrm{~Hz}, 1 \mathrm{H}, \mathrm{ArH}), 8.32-8.25(\mathrm{~m}, 1 \mathrm{H}, \mathrm{ArH}), 7.79-7.64(\mathrm{~m}, 2 \mathrm{H}, \mathrm{ArH})$, 6.92 (s, 2H, $\mathrm{ArH}$ ), 2.78 (s, 6H, $\left.2 \mathrm{X} \mathrm{ArCH}_{3}\right), 2.26$ (s, 3H, $\left.\mathrm{ArCH}_{3}\right), 1.36-0.83$ (m, 21H, TIPS); ${ }^{13} \mathrm{C} \mathrm{NMR}\left(101 \mathrm{MHz}, \mathrm{CDCl}_{3}\right): \delta 161.2,142.2,139.8,135.1,134.9,134.4,131.9,131.6,131.6$, 127.0, 115.0, 113.9, 70.7, 23.2, 21.0, 18.5, 11.2; IR v 2940 (m), 2865 (m), $1656(\mathrm{~s}), 1606(\mathrm{w})$, 1465 (w), 1440 (w), 1380 (w), 1290 (s), 1249 (w), 1141 (s), 1055 (w), 998 (w), $892(\mathrm{~m}), 849$ (s); HRMS (ESI) calcd. for $\mathrm{C}_{27} \mathrm{H}_{37} \mathrm{INO}_{3} \mathrm{SSi}^{+}[\mathrm{M}+\mathrm{H}]^{+}$610.1303; found 610.1305.

\section{$N$-[Mesitylsulfonyl]-1-[phenylethynyl]-1,2-benziodazol-3(1H)-one (2d)}

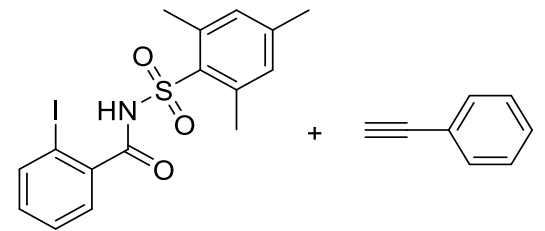

$3 \mathbf{b}$

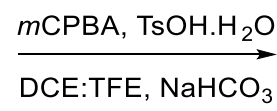

DCE:TFE, $\mathrm{NaHCO}_{3}$

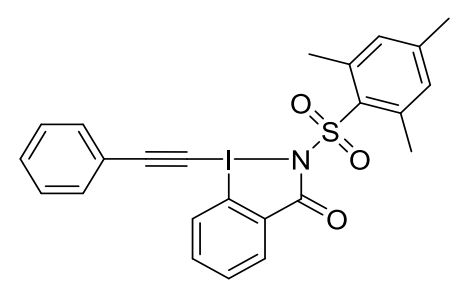

2d

In a $5 \mathrm{~mL}$ sealed tube, 2-iodo- $N$-(mesitylsulfonyl)benzamide (3b) $(1.00 \mathrm{~g}, 2.33 \mathrm{mmol}, 1.00$ equiv), 4-methylbenzenesulfonic acid hydrate ( $0.401 \mathrm{~g}, 2.33 \mathrm{mmol}, 1.00$ equiv) and $m \mathrm{CPBA}$ (0.574 g, 77\% purity, $2.56 \mathrm{mmol}, 1.10$ equiv) were suspended in DCE:TFE 1:1 (4 mL) and stirred for $1 \mathrm{~h}$ at $40{ }^{\circ} \mathrm{C}$. Ethynylbenzene $(0.36 \mathrm{~mL}, 3.3 \mathrm{mmol}, 1.4$ equiv) was then added and the reaction mixture was stirred at $40{ }^{\circ} \mathrm{C}$ for $16 \mathrm{~h}$. The solvent was evaporated and the residue was dissolved in $\mathrm{CH}_{2} \mathrm{Cl}_{2}(20 \mathrm{~mL})$ and stirred vigorously with saturated solution of $\mathrm{NaHCO}_{3}$ $(20 \mathrm{~mL})$. After $1 \mathrm{~h}$, the reaction mixture was transferred into a separating funnel and the layers were separated. The aqueous layer was extracted with $\mathrm{CH}_{2} \mathrm{Cl}_{2}(2 \times 20 \mathrm{~mL})$. The combined organic layers were washed with a saturated solution of $\mathrm{NaHCO}_{3}(30 \mathrm{~mL})$, brine $(30 \mathrm{~mL})$, dried over $\mathrm{MgSO}_{4}$, filtered and concentrated under reduced pressure. The crude product was purified by flash chromatography using EtOAc:DCM 1:25 as mobile phase followed by washing with EtOAc (3 X $3 \mathrm{~mL})$ to afford $\mathbf{2 d}$ as a white solid (0.352 g, $0.665 \mathrm{mmol}, 29 \%)$. Mp: 204-206 ${ }^{\circ} \mathrm{C}$; TLC (EtOAc:DCM 1:25): $\mathrm{R}_{\mathrm{f}}=0.18, \mathrm{KMnO}_{4} ;{ }^{1} \mathrm{H}$ NMR (400 MHz, $\left.\mathrm{CDCl}_{3}\right): \delta 8.37(\mathrm{dd}, J=8.0$, $1.1 \mathrm{~Hz}, 1 \mathrm{H}, \operatorname{Ar} H), 8.29(\mathrm{dd}, J=7.2,2.1 \mathrm{~Hz}, 1 \mathrm{H}, \operatorname{Ar} H), 7.82-7.64(\mathrm{~m}, 2 \mathrm{H}, \operatorname{Ar} H), 7.64-7.56$ (m, 2H, ArH), $7.53-7.37$ (m, 3H, ArH), 6.93 (s, 2H ArH), 2.80 (s, 6H, 2 X ArCH Ar $_{3}, 2.27$ (s, $\left.3 \mathrm{H}, \mathrm{ArCH}_{3}\right) ;{ }^{13} \mathrm{C} \mathrm{NMR}\left(100 \mathrm{MHz}, \mathrm{CDCl}_{3}\right): \delta 161.3,142.3,139.8,135.2,134.9,134.4,132.9$, 131.9, 131.7, 130.8, 128.8, 127.2, 120.6, 115.4, 106.5, 56.5, 23.2, 21.0; IR v 3072 (w), 2937 
(w), 2146 (w), $1655(\mathrm{~s}), 1525(\mathrm{w}), 1441$ (m), 1294 (s), 1248 (w), 1142 (s), $1054(\mathrm{w}), 897(\mathrm{~m})$, 849 (s); HRMS (ESI) calcd. for $\mathrm{C}_{24} \mathrm{H}_{21} \mathrm{INO}_{3} \mathrm{~S}^{+}[\mathrm{M}+\mathrm{H}]^{+}$530.0281; found 530.0283. One carbon was not resolved at $100 \mathrm{MHz}$.

\section{2-((((1S,4S)-7,7-Dimethyl-2-oxobicyclo[2.2.1]heptan-1-yl)methyl)sulfonyl)-1-}

((triisopropylsilyl)ethynyl)- 1,2-benziodazol-3(1H)-one (2e)

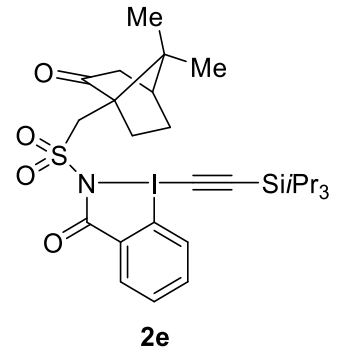

Following GP2, 2-iodo- $N$-camphorsulfonylbenzamide $3 \mathbf{e}$ (138 mg, $0.300 \mathrm{mmol}, 1.00$ equiv) and ethynyltriisopropylsilane (107 $\mathrm{mg}, 0.420 \mathrm{mmol}, 1.40$ equiv) were stirred for $14 \mathrm{~h}$. The crude product was purified by flash chromatography using EtOAc:pentane 1:1.5 as mobile phase to afford $2 \mathrm{e}$ as a white solid (0.166 g, $0.259 \mathrm{mmol}, 86 \%)$. Mp: $167.3-170.0{ }^{\circ} \mathrm{C}$; TLC (EtOAc:pentane 1:1.5): $\mathrm{R}_{\mathrm{f}}=0.26, \mathrm{KMnO}_{4} ;{ }^{1} \mathrm{H} \mathrm{NMR}\left(400 \mathrm{MHz}, \mathrm{CDCl}_{3}\right): \delta 8.43-8.32(\mathrm{~m}, 2 \mathrm{H}$, $\mathrm{ArH}$ ), $7.77-7.67$ (m, 2H, $\mathrm{ArH}$ ), 3.90 and 3.37 (AB quartet, $14.9 \mathrm{~Hz}, 2 \mathrm{H}, \mathrm{CH}_{2} \mathrm{SO}_{2} \mathrm{~N}$ ), 2.46$1.42\left(\mathrm{~m}, 7 \mathrm{H}, \mathrm{C}(\mathrm{O})-\mathrm{C}-\mathrm{CH}_{2}-\mathrm{CH}_{2}-\mathrm{CH}-\mathrm{CH}_{2}\right), 1.18-1.08$ (m, 21H, TIPS), 1.08 (s, 3H, $\mathrm{CH}_{3}$ ), 0.86 (s, 3H, $\left.\mathrm{CH}_{3}\right) ;{ }^{13} \mathrm{C}$ NMR (100 MHz, $\left.\mathrm{CDCl}_{3}\right): \delta 215.3,162.0,135.3,134.3,132.0,131.7,127.2$, 114.9, 113.9, 70.6, 59.0, 50.1, 48.5, 42.8, 42.7, 27.2, 25.6, 19.9, 18.6, 18.4, 11.2; IR v 3667 (w), 3500 (w), 2963 (s), 2361 (w), 1744 (m), 1650 (m), 1582 (w), 1528 (w), 1449 (m), 1401 (m), 1313 (s), 1255 (m), 1134 (s), 1057 (s), 892 (m), 856 (m); HRMS (ESI) calcd. for $\mathrm{C}_{28} \mathrm{H}_{41} \mathrm{INO}_{4} \mathrm{SSi}^{+}[\mathrm{M}+\mathrm{H}]^{+}$642.1565; found 642.1570.

$N$-[Acetyl]-1-[(triisopropylsilyl)ethynyl]-1,2-benziodazol-3(1H)-one (2f)

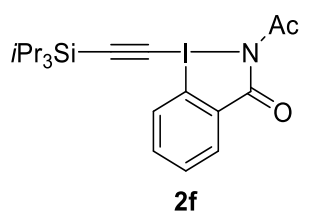

Following GP2, 2-iodo- $N$-acetylbenzamide $3 f(289 \mathrm{mg}, 1.00 \mathrm{mmol}, 1.00$ equiv) and ethynyltriisopropylsilane ( $356 \mathrm{mg}, 1.40 \mathrm{mmol}, 1.40$ equiv) were stirred for $14 \mathrm{~h}$. The crude product was purified by flash chromatography using EtOAc:pentane 1:9 as mobile phase to afford $2 \mathbf{f}$ as a white solid (0.102 g, $0.217 \mathrm{mmol}, 21 \%)$. Mp: 109.6-111.3 ${ }^{\circ} \mathrm{C}$; TLC (EtOAc:pentane 1:9): $\mathrm{R}_{\mathrm{f}}=0.10, \mathrm{KMnO}_{4} ;{ }^{1} \mathrm{H} \mathrm{NMR}\left(400 \mathrm{MHz}, \mathrm{CDCl}_{3}\right): \delta 8.56-8.51(\mathrm{~m}, 1 \mathrm{H}$, $\operatorname{ArH}), 8.39-8.34(\mathrm{~m}, 1 \mathrm{H}, \operatorname{ArH}), 7.76-7.71$ (m, 2H, $\mathrm{ArH}), 2.55$ (s, 3H, CH $), 1.13$ (m, 21H, TIPS); ${ }^{13} \mathrm{C}$ NMR (100 MHz, $\left.\mathrm{CDCl}_{3}\right): \delta$ 179.9, 162.1, 135.3, 135.1, 131.4, 131.3 127.4, 115.4, 
109.2, 81.1, 26.8, 18.5, 11.1; IR v 2944 (w), 2867 (w), 2324 (w), 1666 (s), 1617 (s), 1464 (w), 1441 (w), 1375 (s), 1344 (s), 1237 (w), 1158 (w), 1116 (w), 1021 (w), 885 (w); HRMS (ESI) calcd. for $\mathrm{C}_{20} \mathrm{H}_{29} \mathrm{INO}_{2} \mathrm{Si}^{+}[\mathrm{M}+\mathrm{H}]^{+} 470.1007$; found 470.1037 .

\section{$N$-[Acetyl]-1-[phenylethynyl]-1,2-benziodazol-3(1H)-one $(2 \mathrm{~g})$}

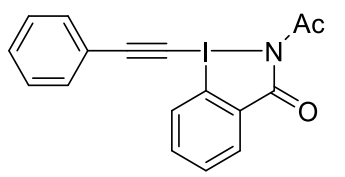

$2 \mathrm{~g}$

Following GP2, 2-iodo- $N$-acetylbenzamide $3 f(725 \mathrm{mg}, 2.51 \mathrm{mmol}, 1.00$ equiv) and phenylacetylene $(0.40 \mathrm{~mL}, 3.5 \mathrm{mmol}, 1.4$ equiv) were stirred for $14 \mathrm{~h}$. The crude product was purified by flash chromatography using EtOAc:DCM 1:25 as mobile phase to afford $\mathbf{2 g}$ as a white solid (0.204 g, 0.524 mmol, 21\%). Mp: 173.6-176.0 ${ }^{\circ} \mathrm{C}$; TLC (EtOAc:DCM 1:25): $\mathrm{R}_{\mathrm{f}}=$ 0.24, $\mathrm{KMnO}_{4} ;{ }^{1} \mathrm{H}$ NMR (400 MHz, $\left.\mathrm{CDCl}_{3}\right): \delta 8.48$ (d, $\left.J=9.3 \mathrm{~Hz}, 1 \mathrm{H}, \mathrm{ArH}\right), 8.37(\mathrm{~d}, J=9.2$ $\mathrm{Hz}, 1 \mathrm{H}, \operatorname{ArH}), 7.75(\mathrm{~d}, J=4.2 \mathrm{~Hz}, 2 \mathrm{H}, \operatorname{ArH}), 7.56(\mathrm{~d}, J=7.7 \mathrm{~Hz}, 2 \mathrm{H}, \operatorname{ArH}), 7.46-7.36(\mathrm{~m}$, $3 \mathrm{H}, \mathrm{ArH}), 2.57\left(\mathrm{~s}, 3 \mathrm{H}, \mathrm{CH}_{3}\right) ;{ }^{13} \mathrm{C} \mathrm{NMR}\left(100 \mathrm{MHz}, \mathrm{CDCl}_{3}\right): \delta 179.8,162.2,135.4,135.3,132.6$, 131.6, 131.4, 130.0, 128.7, 127.5, 121.7, 115.8, 103.3, 65.8, 26.9; IR v $2986(w), 2908$ (w), 2324 (w), 2141 (w), 1658 (s), 1616 (s), 1489 (w), 1443 (w), 1379 (s), 1344 (s), 1237 (w), 1211 (w), 1157 (w), 1118 (w), 1023 (w), 905 (w), 860 (w); HRMS (ESI) calcd. for $\mathrm{C}_{17} \mathrm{H}_{12} \mathrm{INNaO}_{2} \mathrm{~S}^{+}$ $[\mathrm{M}+\mathrm{Na}]^{+} 411.9805$; found 411.9810 .

\section{$N$-[Mesitylsulfonyl]-1-[(4-fluorophenyl)ethynyl]-1,2-benziodazol-3(1H)-one (2h)}
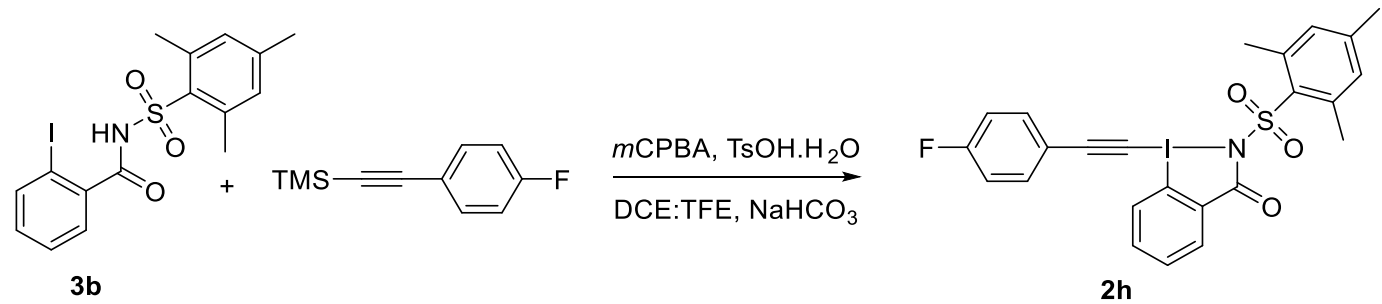

2h

In a $5 \mathrm{~mL}$ sealed tube, 2-iodo- $N$-(mesitylsulfonyl)benzamide (3b) $(0.500 \mathrm{~g}, 1.17 \mathrm{mmol}, 1.00$ equiv), 4-methylbenzenesulfonic acid hydrate (0.201 g, $1.17 \mathrm{mmol}, 1.00$ equiv) and $m$ CPBA (0.287 g, 77\% purity, $2.56 \mathrm{mmol}, 1.10$ equiv) were suspended in DCE:TFE 1:1 (2 mL) and stirred for $1 \mathrm{~h}$ at $40{ }^{\circ} \mathrm{C}$. ((3-Fluorophenyl)ethynyl)trimethylsilane $(0.314 \mathrm{~g}, 1.63 \mathrm{mmol}, 1.40$ equiv) was then added and the reaction mixture was stirred at $40{ }^{\circ} \mathrm{C}$ for $16 \mathrm{~h}$. The solvent was evaporated and the residue was dissolved in $\mathrm{CH}_{2} \mathrm{Cl}_{2}(10 \mathrm{~mL})$ and stirred vigorously with saturated solution of $\mathrm{NaHCO}_{3}(10 \mathrm{~mL})$. After $1 \mathrm{~h}$, the reaction mixture was transferred into a separating funnel and the layers were separated. The aqueous layer was extracted with $\mathrm{CH}_{2} \mathrm{Cl}_{2}$ ( 2 X $10 \mathrm{~mL}$ ). The combined organic layers were washed with saturated solution of $\mathrm{NaHCO}_{3}$ $(15 \mathrm{~mL})$, brine $(15 \mathrm{~mL})$, dried over $\mathrm{MgSO}_{4}$, filtered and concentrated under reduced pressure. 
The crude product was purified by flash chromatography using EtOAc:DCM 1:20 as mobile phase followed by washing with EtOAc $(3 \times 3 \mathrm{~mL})$ to afford $\mathbf{2 h}$ as a white solid $(0.125 \mathrm{~g}$, $0.228 \mathrm{mmol}, 20 \%)$. Mp: 205-209 ${ }^{\circ} \mathrm{C}$; TLC (EtOAc:DCM 1:20): $\mathrm{R}_{\mathrm{f}}=0.43, \mathrm{KMnO}_{4} ;{ }^{1} \mathrm{H} \mathrm{NMR}$ (400 MHz, $\left.\mathrm{CDCl}_{3}\right): \delta 8.34(\mathrm{dd}, 1 \mathrm{H}, \mathrm{ArH}), 8.29$ (dd, 1H, $\left.\mathrm{ArH}\right), 7.72(\mathrm{pd}, J=7.2,1.6 \mathrm{~Hz}, 2 \mathrm{H}$, $\operatorname{ArH}), 7.63-7.55$ (m, 2H, ArH), $7.19-7.08$ (m, 2H, ArH), 6.93 (s, 2H, ArH), 2.80 (s, 6H, 2 $\left.\mathrm{X} \mathrm{ArCH} H_{3}\right), 2.26\left(\mathrm{~s}, 3 \mathrm{H}, \mathrm{ArCH}_{3}\right) ;{ }^{13} \mathrm{C} \mathrm{NMR}\left(100 \mathrm{MHz}, \mathrm{CDCl}_{3}\right): \delta 163.9(\mathrm{~d}, J=253.8 \mathrm{~Hz}), 161.2$, $142.3,139.8,135.2,135.1$ (d, $J=8.8 \mathrm{~Hz}), 134.8,134.4,131.9,131.7,127.2,116.7$ (d, $J=3.5$ Hz), 116.3 (d, $J=22.4$ Hz), 115.4, 105.3, 56.8, 23.2, 21.0; IR v $3074(w), 2920(w), 2145(w)$, 1655 (m), 1600 (m), 1505 (m), 1438 (w), 1294 (s), 1239 (m), 1131 (s), 1054 (w), 898 (m), 859 (s), 839 (m); HRMS (ESI) calcd. for $\mathrm{C}_{24} \mathrm{H}_{19} \mathrm{FINNaO}_{3} \mathrm{~S}^{+}[\mathrm{M}+\mathrm{Na}]^{+}$570.0007; found 570.0013. One carbon was not resolved at $100 \mathrm{MHz}$.

\section{$N$-[Mesitylsulfonyl]-1-[(4-(trifluoromethyl)phenyl)ethynyl]-1,2-benziodazol-3(1H)-one}

(2i)

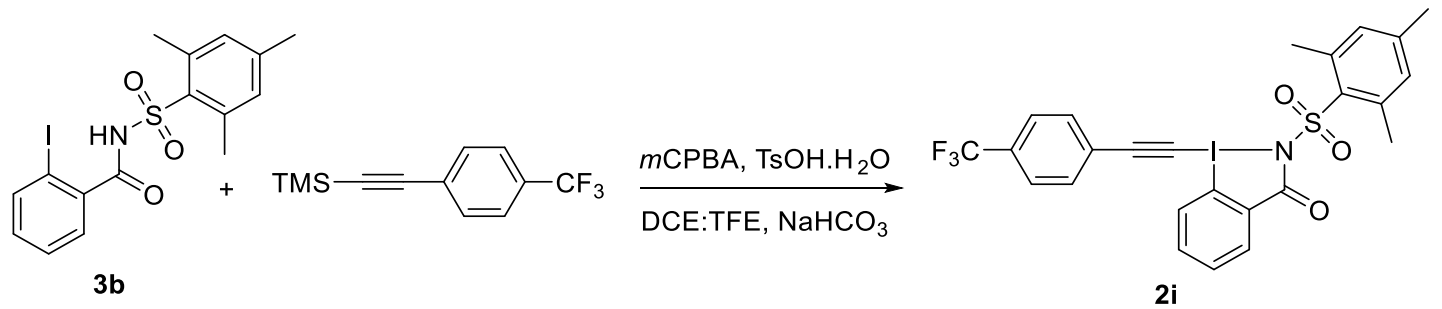

In a $5 \mathrm{~mL}$ sealed tube, 2-iodo- $N$-(mesitylsulfonyl)benzamide (3b) $(0.500 \mathrm{~g}, 1.17 \mathrm{mmol}, 1.00$ equiv), 4-methylbenzenesulfonic acid hydrate (0.201 g, $1.17 \mathrm{mmol}, 1.00$ equiv) and $m \mathrm{CPBA}$ (0.287 g, 77\% purity, $2.56 \mathrm{mmol}, 1.10$ equiv) were suspended in DCE:TFE 1:1 (2 mL) and stirred for $1 \mathrm{~h}$ at $40{ }^{\circ} \mathrm{C}$. Trimethyl((4-(trifluoromethyl)phenyl)ethynyl)silane (0.395 g, 1.63 mmol, 1.40 equiv) was then added and the reaction mixture was stirred at $40{ }^{\circ} \mathrm{C}$ for $16 \mathrm{~h}$. The solvent was evaporated and the residue was dissolved in $\mathrm{CH}_{2} \mathrm{Cl}_{2}(10 \mathrm{~mL})$ and stirred vigorously with saturated solution of $\mathrm{NaHCO}_{3}(10 \mathrm{~mL})$. After $1 \mathrm{~h}$, the reaction mixture was transferred into a separating funnel and the layers were separated. The aqueous layer was extracted with $\mathrm{CH}_{2} \mathrm{Cl}_{2}(2 \mathrm{X} 10 \mathrm{~mL})$. The combined organic layers were washed with saturated solution of $\mathrm{NaHCO}_{3}(15 \mathrm{~mL})$, brine $(15 \mathrm{~mL})$, dried over $\mathrm{MgSO}_{4}$, filtered and concentrated under reduced pressure. The crude product was purified by flash chromatography using EtOAc:DCM 1:25 as mobile phase followed by washing with EtOAc $(3 \mathrm{X} 3 \mathrm{~mL})$ to afford $2 \mathbf{i}$ as a white solid $(0.240$ g, 0.402 mmol, 35\%). Mp: 237-240 ${ }^{\circ} \mathrm{C}$; TLC (EtOAc:DCM 1:15): $\mathrm{R}_{\mathrm{f}}=0.56, \mathrm{KMnO}_{4} ;{ }^{1} \mathrm{H}$ NMR $\left(400 \mathrm{MHz}, \mathrm{CDCl}_{3}\right): \delta 8.35(\mathrm{dd}, J=8.0,1.2 \mathrm{~Hz}, 1 \mathrm{H}, \mathrm{Ar} H), 8.30(\mathrm{dd}, J=7.2,2.1 \mathrm{~Hz}, 1 \mathrm{H}, \mathrm{Ar} H)$, $7.78-7.71(\mathrm{~m}, 6 \mathrm{H}, \mathrm{ArH}), 6.94$ (s, 2H, $\mathrm{ArH}), 2.80$ (s, 6H, $\left.2 \mathrm{X} \mathrm{ArCH}_{3}\right), 2.27$ (s, 3H, $\mathrm{ArCH}_{3}$ ); ${ }^{13} \mathrm{C}$ NMR $\left(100 \mathrm{MHz}, \mathrm{CDCl}_{3}\right): \delta 161.1,142.4,139.9,135.4,134.7,134.3,133.0,132.2\left(\mathrm{q}, J_{\mathrm{C}-\mathrm{F}}\right.$ 
$=33.5 \mathrm{~Hz}), 132.0,131.8,131.7,127.2,125.7\left(\mathrm{q}, J_{\mathrm{C}-\mathrm{F}}=3.8 \mathrm{~Hz}\right), 124.4,123.5\left(\mathrm{q}, J_{\mathrm{C}-\mathrm{F}}=272.4\right.$ Hz), 115.3, 104.0, 60.3, 23.2, 21.0; IR v 3299 (w), 2935 (w), 2159 (w), 1762 (w), 1619 (m), 1573 (m), 1537 (m), 1435 (w), 1361 (m), 1324 (s), 1251 (m), 1169 (s), 1122 (s), 1060 (w), $1013(\mathrm{w}), \quad 902 \quad(\mathrm{w}), \quad 852 \quad(\mathrm{~m}), \quad 815 \quad(\mathrm{~m}) ; \quad$ HRMS $\quad(\mathrm{ESI}) \quad$ calcd. for $\mathrm{C}_{25} \mathrm{H}_{20} \mathrm{~F}_{3} \mathrm{INO}_{3} \mathrm{~S}^{+}[\mathrm{M}+\mathrm{H}]^{+}$598.0155; found 598.0167.

\section{$N$-[Mesitylsulfonyl]-1-[(4-ethynylphenyl)ethynyl]-1,2-benziodazol-3(1H)-one (2j)}

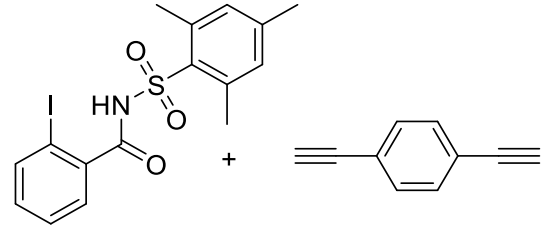

$3 b$

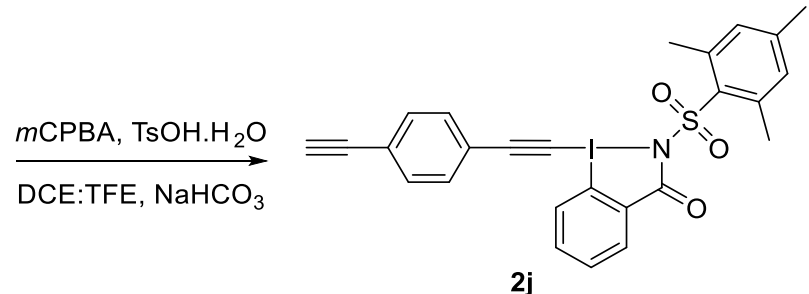

2j

In a $5 \mathrm{~mL}$ sealed tube, 2-iodo- $N$-(mesitylsulfonyl)benzamide (3b) $(0.500 \mathrm{~g}, 1.17 \mathrm{mmol}, 1.00$ equiv), 4-methylbenzenesulfonic acid hydrate (0.201 g, $1.17 \mathrm{mmol}, 1.00$ equiv) and $m \mathrm{CPBA}$ (0.287 g, 77\% purity, $2.56 \mathrm{mmol}, 1.10$ equiv) were suspended in DCE:TFE 1:1 (2 mL) and stirred for $1 \mathrm{~h}$ at $40{ }^{\circ} \mathrm{C}$. 1,4-Diethynylbenzene ( $0.206 \mathrm{~g}, 1.63 \mathrm{mmol}, 1.40$ equiv) was then added and the reaction mixture was stirred at $40{ }^{\circ} \mathrm{C}$ for $16 \mathrm{~h}$. The solvent was evaporated and the residue was dissolved in $\mathrm{CH}_{2} \mathrm{Cl}_{2}(10 \mathrm{~mL})$ and stirred vigorously with saturated solution of $\mathrm{NaHCO}_{3}(10 \mathrm{~mL})$. After $1 \mathrm{~h}$, the reaction mixture was transferred into a separating funnel and the layers were separated. The aqueous layer was extracted with $\mathrm{CH}_{2} \mathrm{Cl}_{2}(2 \mathrm{X} 10 \mathrm{~mL})$. The combined organic layers were washed with saturated solution of $\mathrm{NaHCO}_{3}(15 \mathrm{~mL})$, brine $(15$ $\mathrm{mL}$ ), dried over $\mathrm{MgSO}_{4}$, filtered and concentrated under reduced pressure. The crude product was purified by flash chromatography using EtOAc:DCM 1:20 as mobile phase followed by washing with EtOAc to afford $\mathbf{2} \mathbf{j}$ as a pale yellow solid $(0.100 \mathrm{~g}, 0.181 \mathrm{mmol}, 16 \%)$. Mp: $205-$ $209{ }^{\circ} \mathrm{C}$; TLC (EtOAc:DCM 1:20): $\mathrm{R}_{\mathrm{f}}=0.29, \mathrm{KMnO}_{4} ;{ }^{1} \mathrm{H} \mathrm{NMR}\left(400 \mathrm{MHz}, \mathrm{CDCl}_{3}\right): \delta 8.35$ (dd, $J=7.9,1.4 \mathrm{~Hz}, 1 \mathrm{H}, \operatorname{Ar} H), 8.29(\mathrm{dd}, J=7.2,2.2 \mathrm{~Hz}, 1 \mathrm{H}, \operatorname{Ar} H), 7.77-7.68(\mathrm{~m}, 2 \mathrm{H}, \operatorname{Ar} H), 7.54$ (s, 4H, ArH), 6.93 (s, 2H, ArH), 3.27 (s, 1H, CCH), 2.80 (s, 6H, $\left.2 \mathrm{X} \mathrm{ArCH}_{3}\right), 2.27$ (s, 3H, $\left.\mathrm{ArCH}_{3}\right) ;{ }^{13} \mathrm{C} \mathrm{NMR}\left(100 \mathrm{MHz}, \mathrm{CDCl}_{3}\right): \delta$ 161.2, 142.3, 139.9, 135.3, 134.8, 134.4, 132.7, 132.4, 132.0, 131.7, 131.7, 127.2, 124.6, 120.8, 115.4, 105.4, 83.0, 81.0, 59.0, 23.2, 21; IR v 3074 (w), $2920(\mathrm{w}), 2145$ (w), 1655 (m), 1600 (m), 1505 (m), 1438 (w), 1294 (s), 1239 (m), 1131 (s), $1054 \quad(\mathrm{w}), \quad 898 \quad(\mathrm{~m}), \quad 859 \quad(\mathrm{~s}), \quad 839 \quad(\mathrm{~m}) ; \quad$ HRMS $\quad$ (ESI) calcd. for $\mathrm{C}_{26} \mathrm{H}_{21} \mathrm{INO}_{3} \mathrm{~S}^{+}[\mathrm{M}+\mathrm{H}]^{+}$554.0281; found 554.0281. 


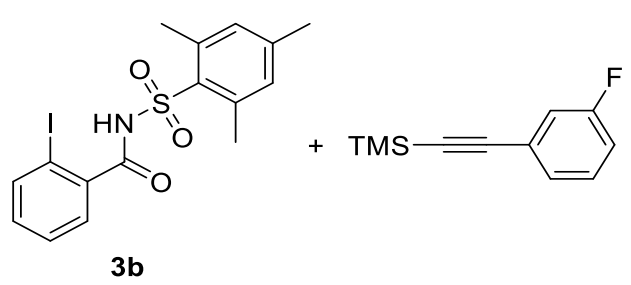

$\underset{\text { DCE:TFE, } \mathrm{NaHCO}_{3}}{\stackrel{m \text { CPBA, } \mathrm{TsOH} . \mathrm{H}_{2} \mathrm{O}}{\longrightarrow}}$

3b

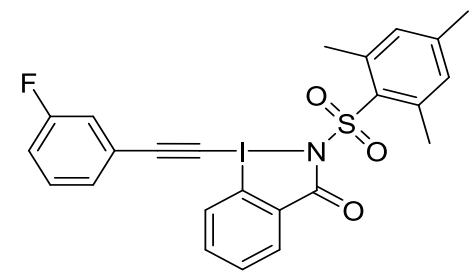

$2 \mathbf{k}$

In a $5 \mathrm{~mL}$ sealed tube, 2-iodo- $N$-(mesitylsulfonyl)benzamide (3b) $(0.500 \mathrm{~g}, 1.17 \mathrm{mmol}, 1.00$ equiv), 4-methylbenzenesulfonic acid hydrate ( $0.201 \mathrm{~g}, 1.17 \mathrm{mmol}, 1.00$ equiv) and $m \mathrm{CPBA}$ (0.287 g, 77\% purity, $2.56 \mathrm{mmol}, 1.10$ equiv) were suspended in DCE:TFE 1:1 (2 mL) and stirred for $1 \mathrm{~h}$ at $40{ }^{\circ} \mathrm{C}$. ((3-Fluorophenyl)ethynyl)trimethylsilane $(0.314 \mathrm{~g}, 1.63 \mathrm{mmol}, 1.40$ equiv) was then added and the reaction mixture was stirred at $40{ }^{\circ} \mathrm{C}$ for $16 \mathrm{~h}$. The solvent was evaporated and the residue was dissolved in $\mathrm{CH}_{2} \mathrm{Cl}_{2}(10 \mathrm{~mL})$ and stirred vigorously with saturated solution of $\mathrm{NaHCO}_{3}(10 \mathrm{~mL})$. After $1 \mathrm{~h}$, the reaction mixture was transferred into a separating funnel and the layers were separated. The aqueous layer was extracted with $\mathrm{CH}_{2} \mathrm{Cl}_{2}$ ( 2 X $10 \mathrm{~mL}$ ). The combined organic layers were washed with saturated solution of $\mathrm{NaHCO}_{3}$ $(15 \mathrm{~mL})$, brine $(15 \mathrm{~mL})$, dried over $\mathrm{MgSO}_{4}$, filtered and concentrated under reduced pressure. The crude product was purified by flash chromatography using EtOAc:DCM 1:20 as mobile phase followed by washing with EtOAc ( $3 \mathrm{X} 3 \mathrm{~mL})$ to afford $\mathbf{2 k}$ as a white solid $(0.275 \mathrm{~g}$, 0.487 mmol, 43\%). Mp: $221-225{ }^{\circ} \mathrm{C}$; TLC (EtOAc:DCM 1:20): $\mathrm{R}_{\mathrm{f}}=0.48, \mathrm{KMnO}_{4} ;{ }^{1} \mathrm{H}$ NMR $\left(400 \mathrm{MHz}, \mathrm{CDCl}_{3}\right): \delta 8.35$ (dd, $\left.J=8.1,1.0 \mathrm{~Hz}, 1 \mathrm{H}, \mathrm{Ar} H\right), 8.29(\mathrm{dd}, J=7.3,2.0 \mathrm{~Hz}, 1 \mathrm{H}, \operatorname{Ar} H$ ), 7.79 - $7.66(\mathrm{~m}, 2 \mathrm{H}, \mathrm{ArH}), 7.43$ - 7.36 (m, 2H, ArH), 7.27 - 7.25 (m, 1H, ArH) 7.24 - 7.14 (m, 1H, $\mathrm{ArH}$ ), 6.92 (s, 2H, $\mathrm{ArH}), 2.79$ (s, 6H, $\left.2 \mathrm{X} \mathrm{ArCH}_{3}\right), 2.26$ (s, 3H, $\left.\mathrm{ArCH}_{3}\right) ;{ }^{13} \mathrm{C} \mathrm{NMR} \mathrm{(100}$ $\left.\mathrm{MHz}, \mathrm{CDCl}_{3}\right): \delta 162.2(\mathrm{~d}, J=248.7 \mathrm{~Hz}), 161.3,142.3,139.8,135.3,134.8,134.3,131.9,131.7$, 131.7, $130.5(\mathrm{~d}, J=8.4 \mathrm{~Hz}), 128.7(\mathrm{~d}, J=3.2 \mathrm{~Hz}), 127.3,122.3(\mathrm{~d}, J=9.2 \mathrm{~Hz}), 119.5(\mathrm{~d}, J=$ $23.3 \mathrm{~Hz}), 118.2$ (d, $J=21.4 \mathrm{~Hz}), 115.3,104.4$ (d, $J=2.9 \mathrm{~Hz}), 58.3,23.2,21.0$; IR v $2972(\mathrm{w})$, 2922 (w), 2145 (w), 1654 (m), 1604 (w), 1580 (w), 1480 (w), 1438 (w), 1293 (s), 1137 (s), $1055 \quad(w), \quad 940 \quad(w), \quad 895 \quad(m), \quad 851 \quad$ (s); HRMS $\quad$ (ESI) calcd. for $\mathrm{C}_{24} \mathrm{H}_{20} \mathrm{FINO}_{3} \mathrm{~S}^{+}[\mathrm{M}+\mathrm{H}]^{+}$548.0187; found 548.0188. 
$N$-[Mesitylsulfonyl]-1-[(3-cyanophenyl)ethynyl]-1,2-benziodazol-3(1H)-one (2l)

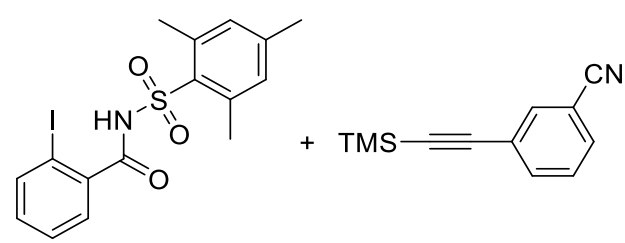

$3 \mathbf{b}$

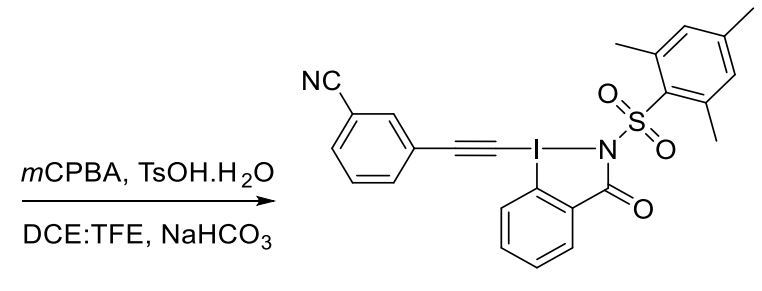

21

In a $5 \mathrm{~mL}$ sealed tube, 2-iodo- $N$-(mesitylsulfonyl)benzamide (3b) $(0.500 \mathrm{~g}, 1.17 \mathrm{mmol}, 1.00$ equiv), 4-methylbenzenesulfonic acid hydrate ( $0.201 \mathrm{~g}, 1.17 \mathrm{mmol}, 1.00$ equiv) and $m \mathrm{CPBA}$ (0.287 g, 77\% purity, $2.56 \mathrm{mmol}, 1.10$ equiv) were suspended in DCE:TFE 1:1 (2 mL) and stirred for $1 \mathrm{~h}$ at $40{ }^{\circ} \mathrm{C}$. 3-((Trimethylsilyl)ethynyl)benzonitrile $(0.325 \mathrm{~g}, 1.63 \mathrm{mmol}, 1.40$ equiv) was then added and the reaction mixture was stirred at $40{ }^{\circ} \mathrm{C}$ for $16 \mathrm{~h}$. The solvent was evaporated and the residue was dissolved in $\mathrm{CH}_{2} \mathrm{Cl}_{2}(10 \mathrm{~mL})$ and stirred vigorously with saturated solution of $\mathrm{NaHCO}_{3}(10 \mathrm{~mL})$. After $1 \mathrm{~h}$, the reaction mixture was transferred into a separating funnel and the layers were separated. The aqueous layer was extracted with $\mathrm{CH}_{2} \mathrm{Cl}_{2}$ (2 X $10 \mathrm{~mL}$ ). The combined organic layers were washed with saturated solution of $\mathrm{NaHCO}_{3}$ $(15 \mathrm{~mL})$, brine $(15 \mathrm{~mL})$, dried over $\mathrm{MgSO}_{4}$, filtered and concentrated under reduced pressure. The crude product was purified by flash chromatography using EtOAc:DCM 1:20 as mobile phase followed by washing with EtOAc $(3 \mathrm{X} 3 \mathrm{~mL})$ to afford $2 \mathbf{l}$ as a white solid $(0.270 \mathrm{~g}, 0.487$ mmol, 42\%). Mp: 230-234 ${ }^{\circ} \mathrm{C}$; TLC (EtOAc:DCM 1:20): $\mathrm{R}_{\mathrm{f}}=0.29, \mathrm{KMnO}_{4} ;{ }^{1} \mathrm{H}$ NMR (400 $\left.\mathrm{MHz}, \mathrm{CDCl}_{3}\right): \delta 8.33(\mathrm{dd}, J=8.0,1.3 \mathrm{~Hz}, 1 \mathrm{H}, \mathrm{ArH}), 8.29(\mathrm{dd}, J=7.2,2.2 \mathrm{~Hz}, 1 \mathrm{H}, \mathrm{ArH}), 7.86$ - 7.82 (m, 1H, ArH), 7.82 - 7.69 (m, 4H, ArH), 7.60 - 7.54 (m, 1H, ArH), 6.93 (s, 2H, ArH), 2.79 (s, 6H, $\left.2 \mathrm{X} \mathrm{ArCH}_{3}\right), 2.27$ (s, 3H, $\left.\left.\mathrm{ArCH}_{3}\right) ;{ }^{13} \mathrm{C} \mathrm{NMR} \mathrm{(100} \mathrm{MHz,} \mathrm{CDCl}_{3}\right): \delta 161.1,142.5$, 139.9, 136.6, 135.9, 135.4, 134.6, 134.3, 133.6, 132.1, 131.9, 131.7, 129.8, 127.2, 122.4, 117.4, 115.2, 113.5, 102.7, 61.0, 23.2, 21.0; IR v 3068 (w), 2235 (w), 2138 (w), 1763 (w), 1654 (m), 1477 (w), 1438 (w), 1295 (s), 1247 (w), 1133 (s), 1053 (w), 899 (m), 852 (s); HRMS (ESI) calcd. for $\mathrm{C}_{25} \mathrm{H}_{19} \mathrm{IN}_{2} \mathrm{NaO}_{3} \mathrm{~S}^{+}[\mathrm{M}+\mathrm{Na}]^{+}$577.0053; found 577.0055 .

\section{$N$-[Mesitylsulfonyl]-1-[(triethylsilyl)ethynyl]-1,2-benziodazol-3(1H)-one (2m)}

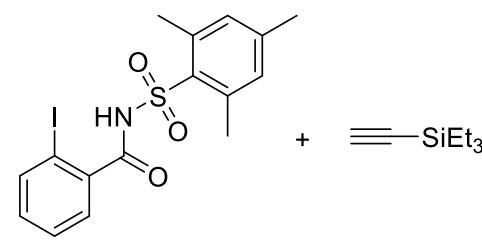

3b

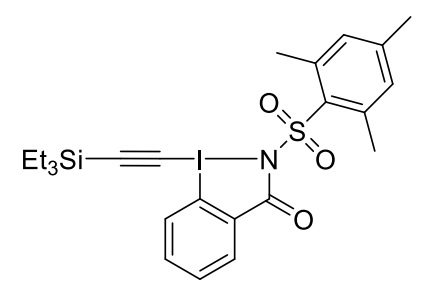

$2 m$

In a $5 \mathrm{~mL}$ sealed tube, 2-iodo- $N$-(mesitylsulfonyl)benzamide (3b) $(0.500 \mathrm{~g}, 1.17 \mathrm{mmol}, 1.00$ equiv), 4-methylbenzenesulfonic acid hydrate ( $0.201 \mathrm{~g}, 1.17 \mathrm{mmol}, 1.00$ equiv) and $m \mathrm{CPBA}$ 
(0.287 g, 77\% purity, $2.56 \mathrm{mmol}, 1.10$ equiv) were suspended in DCE:TFE 1:1 (2 mL) and stirred for $1 \mathrm{~h}$ at $40{ }^{\circ} \mathrm{C}$. Triethyl(ethynyl)silane $(0.229 \mathrm{~g}, 1.63 \mathrm{mmol}, 1.40$ equiv) was then added and the reaction mixture was stirred at $40{ }^{\circ} \mathrm{C}$ for $16 \mathrm{~h}$. The solvent was evaporated and the residue was dissolved in $\mathrm{CH}_{2} \mathrm{Cl}_{2}(10 \mathrm{~mL})$ and stirred vigorously with saturated solution of $\mathrm{NaHCO}_{3}(10 \mathrm{~mL})$. After $1 \mathrm{~h}$, the reaction mixture was transferred into a separating funnel and the layers were separated. The aqueous layer was extracted with $\mathrm{CH}_{2} \mathrm{Cl}_{2}(2 \mathrm{X} 10 \mathrm{~mL})$. The combined organic layers were washed with saturated solution of $\mathrm{NaHCO}_{3}(15 \mathrm{~mL})$, brine (15 $\mathrm{mL}$ ), dried over $\mathrm{MgSO}_{4}$, filtered and concentrated under reduced pressure. The crude product was purified by flash chromatography using EtOAc:Pentane 1:4.5 as mobile phase to afford $\mathbf{2 m}$ as a white solid (295 mg, $0.520 \mathrm{mmol}, 45 \%)$. Mp: 196-200 ${ }^{\circ} \mathrm{C}$; TLC (EtOAc:Pentane 1:4.5): $\mathrm{R}_{\mathrm{f}}=0.3, \mathrm{KMnO}_{4} ;{ }^{1} \mathrm{H} \mathrm{NMR}\left(400 \mathrm{MHz}, \mathrm{CDCl}_{3}\right): \delta 8.38-8.35(\mathrm{~m}, 1 \mathrm{H}, \mathrm{ArH}), 8.29-8.27$ (m, 1H, ArH),7.84- 7.58 (m, 2H, ArH), 6.92 (s, 2H, ArH), 2.78 (s, 6H, $\left.2 \mathrm{X} \mathrm{ArCH}_{3}\right), 2.26$ (s, $\left.3 \mathrm{H}, \mathrm{ArCH}_{3}\right), 1.08$ (t, $\left.J=7.9 \mathrm{~Hz}, 9 \mathrm{H}, 3 \mathrm{X} \mathrm{CH}_{2} \mathrm{CH}_{3}\right), 0.74\left(\mathrm{q}, J=7.9 \mathrm{~Hz}, 6 \mathrm{H}, 3 \mathrm{X} \mathrm{CH}_{2} \mathrm{CH}_{3}\right) ;{ }^{13} \mathrm{C}$ NMR (100 MHz, $\left.\mathrm{CDCl}_{3}\right): \delta 161.1,142.2,139.8,135.1,134.9,134.4,131.9,131.6,131.6$, 127.0, 114.8, 114.7, 70.6, 23.2, 21.0, 7.4, 4.1; IR v 2936 (w), 2868 (w), $2165(\mathrm{w}), 1653(\mathrm{~m})$, 1603 (w), 1438 (w), 1380 (w), 1290 (s), 1246 (w), 1169 (w), 1134 (s), 1055 (w), 1003 (w), 895 (m), 854 (s); HRMS (ESI) calcd. for $\mathrm{C}_{24} \mathrm{H}_{30} \mathrm{INNaO}_{3} \mathrm{SSi}^{+}$[M+Na] $]^{+}$590.0653; found 590.0652.

\section{$N$-[Mesitylsulfonyl]-1-[(butyl)ethynyl]-1,2-benziodazol-3(1H)-one (2n)}

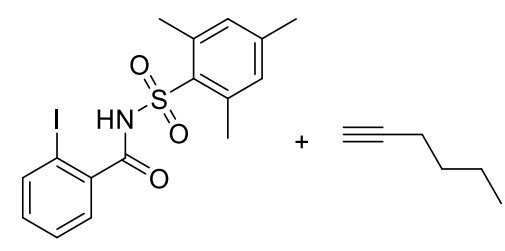

3b

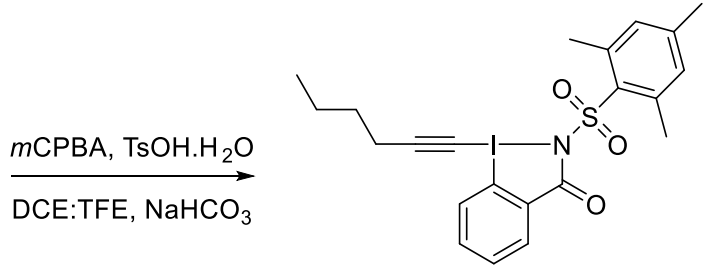

2n

In a $5 \mathrm{~mL}$ sealed tube, 2-iodo- $N$-(mesitylsulfonyl)benzamide (3b) $(0.500 \mathrm{~g}, 1.17 \mathrm{mmol}, 1.00$ equiv), 4-methylbenzenesulfonic acid hydrate ( $0.201 \mathrm{~g}, 1.17 \mathrm{mmol}, 1.00$ equiv) and $m \mathrm{CPBA}$ (0.287 g, 77\% purity, $2.56 \mathrm{mmol}, 1.10$ equiv) were suspended in DCE:TFE 1:1 (2 mL) and stirred for $1 \mathrm{~h}$ at $40{ }^{\circ} \mathrm{C}$. Hex-1-yne $(0.134 \mathrm{~g}, 0.190 \mathrm{~mL}, 1.63 \mathrm{mmol}, 1.40$ equiv) was then added and the reaction mixture was stirred at $40{ }^{\circ} \mathrm{C}$ for $16 \mathrm{~h}$. The solvent was evaporated and the residue was dissolved in $\mathrm{CH}_{2} \mathrm{Cl}_{2}(10 \mathrm{~mL})$ and stirred vigorously with saturated solution of $\mathrm{NaHCO}_{3}(10 \mathrm{~mL})$. After $1 \mathrm{~h}$, the reaction mixture was transferred into a separating funnel and the layers were separated. The aqueous layer was extracted with $\mathrm{CH}_{2} \mathrm{Cl}_{2}(2 \mathrm{X} 10 \mathrm{~mL})$. The combined organic layers were washed with saturated solution of $\mathrm{NaHCO}_{3}(15 \mathrm{~mL})$, brine $(15$ $\mathrm{mL}$ ), dried over $\mathrm{MgSO}_{4}$, filtered and concentrated under reduced pressure. The crude product 
was purified by flash chromatography using EtOAc:Pentane 1:4 as mobile phase followed by recrystallization in ethyl acetate $(4 \mathrm{~mL})$ to afford $\mathbf{2 n}$ as a white solid $(194 \mathrm{mg}, 0.380 \mathrm{mmol}$, 33\%). Mp: 205-210 ${ }^{\circ} \mathrm{C}$; TLC (EtOAc:Pentane 1:4): $\mathrm{R}_{\mathrm{f}}=0.3, \mathrm{KMnO}_{4} ;{ }^{1} \mathrm{H}$ NMR (400 MHz, $\left.\mathrm{CDCl}_{3}\right): \delta 8.30-8.25(\mathrm{~m}, 2 \mathrm{H}, \mathrm{ArH}), 7.75-7.65(\mathrm{~m}, 2 \mathrm{H}, \mathrm{ArH}), 6.91$ (s, 2H, ArH), $2.78(\mathrm{~s}, 6 \mathrm{H}$, $\left.2 \mathrm{X} \mathrm{ArCH}_{3}\right), 2.58\left(\mathrm{t}, J=7.1 \mathrm{~Hz}, 2 \mathrm{H}, \mathrm{CCCH}_{2}\right), 2.25\left(\mathrm{~s}, 3 \mathrm{H}, \mathrm{ArCH}_{3}\right), 1.69-1.60(\mathrm{~m}, 2 \mathrm{H}$, $\left.\mathrm{CCCH}_{2} \mathrm{CH}_{2}\right), 1.57-1.42\left(\mathrm{~m}, 2 \mathrm{H}, \mathrm{CH}_{2} \mathrm{CH}_{3}\right), 0.98\left(\mathrm{t}, J=7.3 \mathrm{~Hz}, 3 \mathrm{H}, \mathrm{CH}_{2} \mathrm{CH}_{3}\right) ;{ }^{13} \mathrm{C} \mathrm{NMR}(100$ $\left.\mathrm{MHz}, \mathrm{CDCl}_{3}\right): \delta 161.3,142.1,139.7,135.0,135.0,134.4,131.8,131.6,131.5,127.1,114.8$, 109.7, 45.5, 30.1, 23.2, 22.0, 21.0, 20.1, 13.5; IR v 2933 (w), 2868 (w), 2166 (w), 1653 (m), 1603 (w), 1438 (w), $1380(w), 1290$ (s), 1246 (w), 1168 (w), 1134 (s), 1053 (w), 1003 (w), 895 (m), 854 (s); HRMS (ESI) calcd. for $\mathrm{C}_{22} \mathrm{H}_{25} \mathrm{INO}_{3} \mathrm{~S}^{+}[\mathrm{M}+\mathrm{H}]^{+}$510.0594; found 510.0609.

\section{$\mathrm{N}$-[Mesitylsulfonyl]-1-[(dodecyl)ethynyl]-1,2-benziodazol-3(1H)-one (2o)}

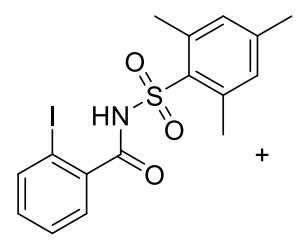

3b

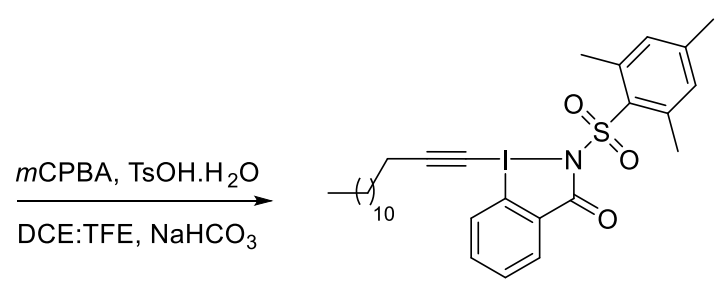

20

In a $5 \mathrm{~mL}$ sealed tube, 2-iodo- $N$-(mesitylsulfonyl)benzamide (3b) $(0.500 \mathrm{~g}, 1.17 \mathrm{mmol}, 1.00$ equiv), 4-methylbenzenesulfonic acid hydrate (0.201 g, $1.17 \mathrm{mmol}, 1.00$ equiv) and $m \mathrm{CPBA}$ (0.287 g, 77\% purity, $2.56 \mathrm{mmol}, 1.10$ equiv) were suspended in DCE:TFE 1:1 (2 mL) and stirred for $1 \mathrm{~h}$ at $40{ }^{\circ} \mathrm{C}$. Tetradec-1-yne $(0.317 \mathrm{~g}, 0.400 \mathrm{~mL}, 1.63 \mathrm{mmol}, 1.40$ equiv) was then added and the reaction mixture was stirred at $40{ }^{\circ} \mathrm{C}$ for $16 \mathrm{~h}$. The solvent was evaporated and the residue was dissolved in $\mathrm{CH}_{2} \mathrm{Cl}_{2}(10 \mathrm{~mL})$ and stirred vigorously with saturated solution of $\mathrm{NaHCO}_{3}(10 \mathrm{~mL})$. After $1 \mathrm{~h}$, the reaction mixture was transferred into a separating funnel and the layers were separated. The aqueous layer was extracted with $\mathrm{CH}_{2} \mathrm{Cl}_{2}(2 \mathrm{X} 10 \mathrm{~mL})$. The combined organic layers were washed with saturated solution of $\mathrm{NaHCO}_{3}(15 \mathrm{~mL})$, brine (15 $\mathrm{mL}$ ), dried over $\mathrm{MgSO}_{4}$, filtered and concentrated under reduced pressure. The crude product was purified by flash chromatography using EtOAc:Pentane 1:4 as mobile phase followed by recrystallization in ethyl acetate $(5 \mathrm{~mL})$ to afford 20 as a white solid $(0.207 \mathrm{~g}, 0.333 \mathrm{mmol}$, 29\%). Mp: 182-188 ${ }^{\circ} \mathrm{C}$; TLC (EtOAc:Pentane 1:2): $\mathrm{R}_{\mathrm{f}}=0.6, \mathrm{KMnO}_{4} ;{ }^{1} \mathrm{H}$ NMR (400 MHz, $\left.\mathrm{CDCl}_{3}\right): \delta 8.32-8.24(\mathrm{~m}, 2 \mathrm{H}, \mathrm{ArH}), 7.74-7.65(\mathrm{~m}, 2 \mathrm{H}, \mathrm{ArH}), 6.92(\mathrm{~s}, 2 \mathrm{H}, \mathrm{ArH}), 2.77(\mathrm{~s}, 6 \mathrm{H}$, $\left.2 \mathrm{X} \mathrm{ArCH}_{3}\right), 2.58\left(\mathrm{t}, J=7.1 \mathrm{~Hz}, 2 \mathrm{H}, \mathrm{CCCH}_{2}\right), 2.25\left(\mathrm{~s}, 3 \mathrm{H}, \mathrm{ArCH}_{3}\right), 1.65$ (p, J=7.1 Hz, 2H, $\left.\mathrm{CCCH}_{2} \mathrm{CH}_{2} \mathrm{CH}_{2}\right), 1.51-1.41\left(\mathrm{~m}, 2 \mathrm{H}, \mathrm{CH}_{2}\right), 1.38-1.22\left(\mathrm{~m}, 16 \mathrm{H}, 10 \mathrm{X} \mathrm{CH}_{2}\right), 0.87(\mathrm{t}, 3 \mathrm{H}$, $\left.\mathrm{CH}_{2} \mathrm{CH}_{3}\right) ;{ }^{13} \mathrm{C} \mathrm{NMR}\left(101 \mathrm{MHz}, \mathrm{CDCl}_{3}\right): \delta 161.3,142.1,139.8,135.0,135.0,134.5,131.8$, 131.6, 131.5, 127.1, 114.9, 109.8, 45.6, 31.9, 29.7, 29.6, 29.5, 29.3, 29.1, 28.9, 28.2, 27.7, 23.2, 
22.7, 21.0, 20.4, 14.1; IR v 3056 (w), 2920 (s), 2850 (m), 2166 (w), 1652 (m), 1606 (w), 1470 (w), 1441 (w), 1298 (s), 1247 (w), 1165 (w), 1139 (s), 1056 (w), 898 (w), 856 (s); HRMS (ESI) calcd. for $\mathrm{C}_{30} \mathrm{H}_{41} \mathrm{INO}_{3} \mathrm{~S}^{+}[\mathrm{M}+\mathrm{H}]^{+}$622.1846; found 622.1845 .

\section{$N$-[Mesitylsulfonyl]-1-[(3-chloropropyl)ethynyl]-1,2-benziodazol-3(1H)-one (2p)}

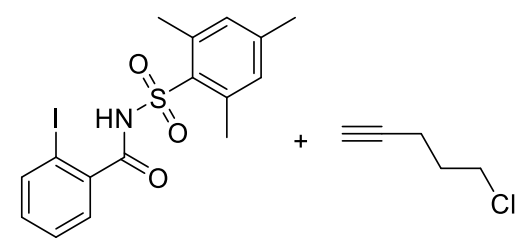

3b

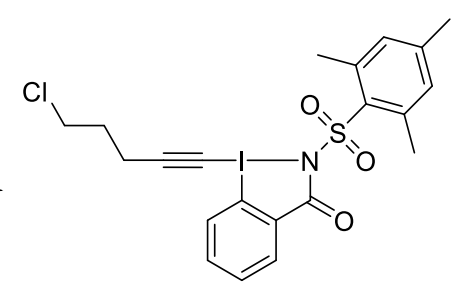

$2 p$

In a $5 \mathrm{~mL}$ sealed tube, 2-iodo- $N$-(mesitylsulfonyl)benzamide (3b) (0.500 g, $1.17 \mathrm{mmol}, 1.00$ equiv), 4-methylbenzenesulfonic acid hydrate (0.201 g, $1.17 \mathrm{mmol}, 1.00$ equiv) and $m \mathrm{CPBA}$ (0.287 g, 77\% purity, $2.56 \mathrm{mmol}, 1.10$ equiv) were suspended in DCE:TFE 1:1 (2 mL) and stirred for $1 \mathrm{~h}$ at $40{ }^{\circ} \mathrm{C}$. 5-Chloropent-1-yne $(0.167 \mathrm{~g}, 0.180 \mathrm{~mL}, 1.63 \mathrm{mmol}, 1.40$ equiv) was then added and the reaction mixture was stirred at $40{ }^{\circ} \mathrm{C}$ for $16 \mathrm{~h}$. The solvent was evaporated and the residue was dissolved in $\mathrm{CH}_{2} \mathrm{Cl}_{2}(10 \mathrm{~mL})$ and stirred vigorously with saturated solution of $\mathrm{NaHCO}_{3}(10 \mathrm{~mL})$. After $1 \mathrm{~h}$, the reaction mixture was transferred into a separating funnel and the layers were separated. The aqueous layer was extracted with $\mathrm{CH}_{2} \mathrm{Cl}_{2}(2 \mathrm{X} 10 \mathrm{~mL})$. The combined organic layers were washed with saturated solution of $\mathrm{NaHCO}_{3}(15 \mathrm{~mL})$, brine (15 $\mathrm{mL}$ ), dried over $\mathrm{MgSO}_{4}$, filtered and concentrated under reduced pressure. The crude product was purified by flash chromatography using EtOAc:pentane 1:1 as mobile phase to afford $\mathbf{2 p}$ as a white solid (186 mg, $0.350 \mathrm{mmol}, 30 \%)$. Mp: 209-212 ${ }^{\circ} \mathrm{C}$; TLC (EtOAc:pentane 1:1): $\mathrm{R}_{\mathrm{f}}$ $=0.5, \mathrm{KMnO}_{4} ;{ }^{1} \mathrm{H} \mathrm{NMR}\left(400 \mathrm{MHz}, \mathrm{CDCl}_{3}\right): \delta 8.32-8.24(\mathrm{~m}, 2 \mathrm{H}, \mathrm{ArH}), 7.77-7.65(\mathrm{~m}, 2 \mathrm{H}$, $\operatorname{ArH}), 6.92(\mathrm{~s}, 2 \mathrm{H}, \mathrm{ArH}), 3.71\left(\mathrm{t}, J=6.1 \mathrm{~Hz}, 2 \mathrm{H}, \mathrm{CH}_{2} \mathrm{CH}_{2} \mathrm{Cl}\right), 2.81(\mathrm{t}, J=6.9 \mathrm{~Hz}, 2 \mathrm{H}$, $\mathrm{CCCH}_{2} \mathrm{CH}_{2}$ ), 2.77 (s, 6H, $\left.2 \mathrm{X} \mathrm{ArCH}_{3}\right), 2.26\left(\mathrm{~s}, 3 \mathrm{H}, \mathrm{ArCH}_{3}\right), 2.11(\mathrm{p}, J=6.8 \mathrm{~Hz}, 2 \mathrm{H}$, $\left.\mathrm{CH}_{2} \mathrm{CH}_{2} \mathrm{CH}_{2} \mathrm{Cl}\right) ;{ }^{13} \mathrm{C}$ NMR $\left(100 \mathrm{MHz}, \mathrm{CDCl}_{3}\right): \delta 161.2,142.2,139.8,135.1,134.9,134.4$, 131.9, 131.6, 131.6, 127.1, 114.8, 107.0, 47.5, 43.2, 30.6, 23.2, 21.0, 17.8; IR v 2942 (w), 2161 (w), 1657 (m), 1602 (w), 1586 (w), 1438 (w), 1313 (m), 1290 (s), 1247 (w), 1170 (w), 1133 (s), 1055 (w), 898 (m), 857 (s); HRMS (ESI) calcd. for $\mathrm{C}_{21} \mathrm{H}_{22} \mathrm{ClINO}_{3} \mathrm{~S}^{+}[\mathrm{M}+\mathrm{H}]^{+} 530.0048$; found 530.0050 . 


\section{$N$-[Mesitylsulfonyl]-1-[hepta-1,6-diynyl]-1,2-benziodazol-3(1H)-one (2q)}

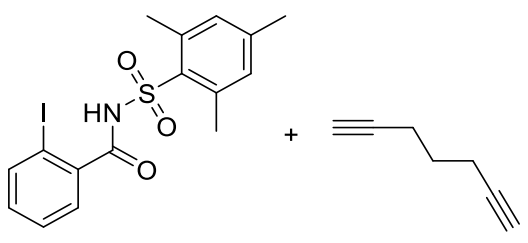

$3 \mathbf{b}$

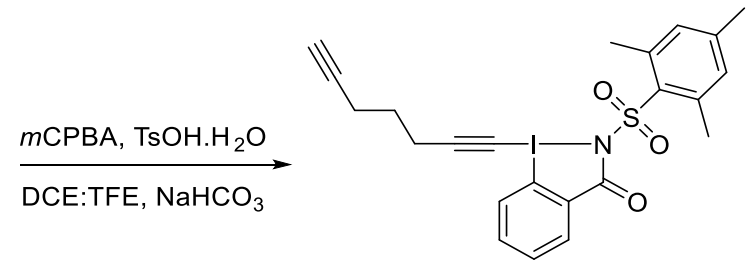

$2 q$

In a $5 \mathrm{~mL}$ sealed tube, 2-iodo- $N$-(mesitylsulfonyl)benzamide (3b) $(0.500 \mathrm{~g}, 1.17 \mathrm{mmol}, 1.00$ equiv), 4-methylbenzenesulfonic acid hydrate (0.201 g, $1.17 \mathrm{mmol}, 1.00$ equiv) and $m$ CPBA (0.287 g, 77\% purity, $2.56 \mathrm{mmol}, 1.10$ equiv) were suspended in DCE:TFE 1:1 (2 mL) and stirred for $1 \mathrm{~h}$ at $40{ }^{\circ} \mathrm{C}$. Hepta-1,6-diyne $(0.150 \mathrm{~g}, 0.190 \mathrm{~mL}, 1.63 \mathrm{mmol}, 1.40$ equiv $)$ was then added and the reaction mixture was stirred at $40{ }^{\circ} \mathrm{C}$ for $16 \mathrm{~h}$. The solvent was evaporated and the residue was dissolved in $\mathrm{CH}_{2} \mathrm{Cl}_{2}(10 \mathrm{~mL})$ and stirred vigorously with saturated solution of $\mathrm{NaHCO}_{3}(10 \mathrm{~mL})$. After $1 \mathrm{~h}$, the reaction mixture was transferred into a separating funnel and the layers were separated. The aqueous layer was extracted with $\mathrm{CH}_{2} \mathrm{Cl}_{2}(2 \mathrm{X} 10 \mathrm{~mL})$. The combined organic layers were washed with saturated solution of $\mathrm{NaHCO}_{3}(15 \mathrm{~mL})$, brine (15 $\mathrm{mL}$ ), dried over $\mathrm{MgSO}_{4}$, filtered and concentrated under reduced pressure. The crude product was purified by flash chromatography using EtOAc:Pentane 1:2 as mobile phase followed by washing with EtOAc (2X $3 \mathrm{~mL})$ to afford $\mathbf{2 q}$ as a white solid $(0.120 \mathrm{~g}, 0.231 \mathrm{mmol}, 20 \%)$. Mp: $178-182{ }^{\circ} \mathrm{C}$; TLC (EtOAc:Pentane 1:2): $\mathrm{R}_{\mathrm{f}}=0.4, \mathrm{KMnO}_{4} ;{ }^{1} \mathrm{H}$ NMR (400 MHz, $\left.\mathrm{CDCl}_{3}\right)$ : $\delta 8.32-8.24(\mathrm{~m}, 2 \mathrm{H}, \operatorname{ArH}), 7.76-7.65(\mathrm{~m}, 2 \mathrm{H}, \operatorname{Ar} H), 6.92(\mathrm{~s}, 2 \mathrm{H}, \operatorname{Ar} H), 2.77$ (s, 6H, 2 X $\left.\mathrm{ArCH}_{3}\right), 2.74\left(\mathrm{~d}, J=7.1 \mathrm{~Hz}, 2 \mathrm{H}, \mathrm{CH}_{2}\right), 2.41\left(\mathrm{td}, J=6.8,2.7 \mathrm{~Hz}, 2 \mathrm{H}, \mathrm{CH}_{2}\right), 2.26\left(\mathrm{~s}, 3 \mathrm{H}, \mathrm{ArCH}_{3}\right)$, $2.05(\mathrm{t}, J=2.7 \mathrm{~Hz}, 1 \mathrm{H}, \mathrm{CH}), 1.88\left(\mathrm{p}, J=7.0 \mathrm{~Hz}, 2 \mathrm{H}, \mathrm{CH}_{2}\right) ;{ }^{13} \mathrm{C} \mathrm{NMR}\left(101 \mathrm{MHz}, \mathrm{CDCl}_{3}\right): \delta$ $161.3,142.2$, 139.8, 135.1, 134.9, 134.4, 131.8, 131.6, 131.5, 127.1, 114.8, 108.0, 82.5, 69.8, 46.8, 26.8, 23.2, 21.0, 19.3, 17.6; IR v 3232 (w), 2166 (w), 1648 (s), 1604 (w), 1438 (w), 1315 (s), 1295 (s), 1247 (w), 1176 (w), 1136 (s), 1057 (w), 1004 (w), 900 (m), 858 (s); HRMS (ESI) calcd. for $\mathrm{C}_{23} \mathrm{H}_{23} \mathrm{INO}_{3} \mathrm{~S}^{+}[\mathrm{M}+\mathrm{H}]^{+}$520.0438; found 520.0444.

\section{$N$-[Mesitylsulfonyl]-1-[(cyclopropyl)ethynyl]-1,2-benziodazol-3(1H)-one (2r)}

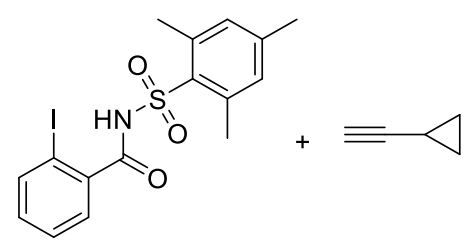

3b

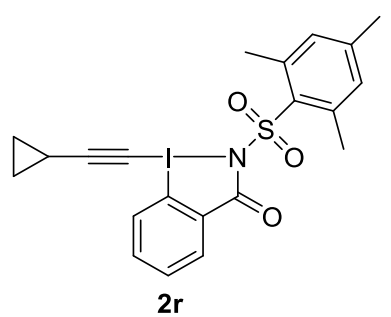

$2 r$

In a $5 \mathrm{~mL}$ sealed tube, 2-iodo- $N$-(mesitylsulfonyl)benzamide (3b) $(0.500 \mathrm{~g}, 1.17 \mathrm{mmol}, 1.00$ equiv), 4-methylbenzenesulfonic acid hydrate (0.201 g, $1.17 \mathrm{mmol}, 1.00$ equiv) and $m$ CPBA 
(0.287 g, 77\% purity, $2.56 \mathrm{mmol}, 1.10$ equiv) were suspended in DCE:TFE 1:1 (2 mL) and stirred for $1 \mathrm{~h}$ at $40{ }^{\circ} \mathrm{C}$. Ethynylcyclopropane $(0.108 \mathrm{~g}, 0.140 \mathrm{~mL}, 1.63 \mathrm{mmol}, 1.40$ equiv) was then added and the reaction mixture was stirred at $40{ }^{\circ} \mathrm{C}$ for $16 \mathrm{~h}$. The solvent was evaporated and the residue was dissolved in $\mathrm{CH}_{2} \mathrm{Cl}_{2}(10 \mathrm{~mL})$ and stirred vigorously with saturated solution of $\mathrm{NaHCO}_{3}(10 \mathrm{~mL})$. After $1 \mathrm{~h}$, the reaction mixture was transferred into a separating funnel and the layers were separated. The aqueous layer was extracted with $\mathrm{CH}_{2} \mathrm{Cl}_{2}(2 \mathrm{X} 10 \mathrm{~mL})$. The combined organic layers were washed with saturated solution of $\mathrm{NaHCO}_{3}(15 \mathrm{~mL})$, brine (15 $\mathrm{mL}$ ), dried over $\mathrm{MgSO}_{4}$, filtered and concentrated under reduced pressure. The crude product was purified by flash chromatography using EtOAc:Pentane 1:1 as mobile phase followed by washing with EtOAc (2 X $3 \mathrm{~mL})$ to afford $2 \mathbf{r}$ as a white solid $(0.100 \mathrm{~g}, 0.203 \mathrm{mmol}, 18 \%)$. Mp: 212-217 ${ }^{\circ} \mathrm{C}$; TLC (EtOAc:Pentane 1:1): $\mathrm{R}_{\mathrm{f}}=0.4, \mathrm{KMnO}_{4} ;{ }^{1} \mathrm{H}$ NMR $\left(400 \mathrm{MHz}, \mathrm{CDCl}_{3}\right)$ : $\delta 8.27(\mathrm{td}, J=7.8,1.5 \mathrm{~Hz}, 2 \mathrm{H}, \operatorname{ArH}), 7.80-7.55(\mathrm{~m}, 2 \mathrm{H}, \operatorname{ArH}), 6.91$ (s, 2H, ArH), 2.77 (s, 6H, $\left.2 \mathrm{X} \mathrm{ArCH}_{3}\right), 2.25\left(\mathrm{~s}, 3 \mathrm{H}, \mathrm{ArCH}_{3}\right), 1.84-1.50\left(\mathrm{~m}, 1 \mathrm{H}, \mathrm{CCH}\left(\mathrm{CH}_{2}\right)_{2}\right), 1.17-0.85(\mathrm{~m}, 4 \mathrm{H}, 2 \mathrm{X}$ $\left.\mathrm{CH}_{2}\right) ;{ }^{13} \mathrm{C} \mathrm{NMR}\left(100 \mathrm{MHz}, \mathrm{CDCl}_{3}\right): \delta 161.3,142.1,139.7,135.0,135.0,134.5,131.8,131.6$, 131.5, 127.0, 115.1, 113.4, 41.3, 23.2, 21.0, 9.8, 1.0; IR v 3010 (w), 2853 (w), 2164 (w), 1709 (w), 1653 (m), 1605 (w), 1476 (w), 1437 (w), 1313 (m), 1292 (s), 1247 (w), 1167 (w), 1131 (s), 1055 (w), 965 (w), 896 (m), 858 (s), 821 (w); HRMS (ESI) calcd. for $\mathrm{C}_{21} \mathrm{H}_{21} \mathrm{INO}_{3} \mathrm{~S}^{+}[\mathrm{M}+\mathrm{H}]^{+}$494.0281; found 494.0289.

\section{$N$-[Mesitylsulfonyl]-1-[(cyclopentyl)ethynyl]-1,2-benziodazol-3(1H)-one (2s)}

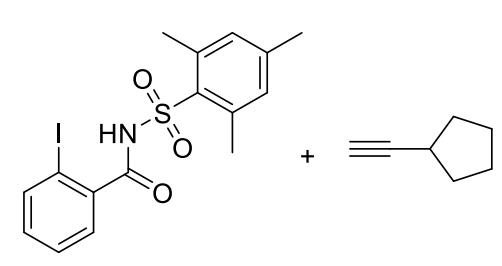

3b

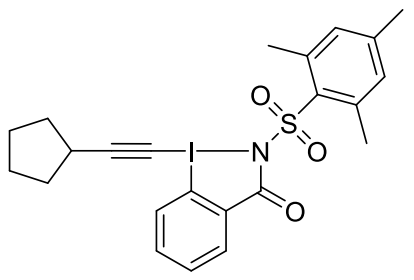

2s

In a $5 \mathrm{~mL}$ sealed tube, 2-iodo- $N$-(mesitylsulfonyl)benzamide (3b) $(0.500 \mathrm{~g}, 1.17 \mathrm{mmol}, 1.00$ equiv), 4-methylbenzenesulfonic acid hydrate (0.201 g, $1.17 \mathrm{mmol}, 1.00$ equiv) and $m \mathrm{CPBA}$ (0.287 g, 77\% purity, $2.56 \mathrm{mmol}, 1.10$ equiv) were suspended in DCE:TFE 1:1 (2 mL) and stirred for $1 \mathrm{~h}$ at $40{ }^{\circ} \mathrm{C}$. Ethynylcyclopentane $(0.154 \mathrm{~g}, 0.190 \mathrm{~mL}, 1.63 \mathrm{mmol}, 1.40$ equiv) was then added and the reaction mixture was stirred at $40{ }^{\circ} \mathrm{C}$ for $16 \mathrm{~h}$. The solvent was evaporated and the residue was dissolved in $\mathrm{CH}_{2} \mathrm{Cl}_{2}(10 \mathrm{~mL})$ and stirred vigorously with saturated solution of $\mathrm{NaHCO}_{3}(10 \mathrm{~mL})$. After $1 \mathrm{~h}$, the reaction mixture was transferred into a separating funnel and the layers were separated. The aqueous layer was extracted with $\mathrm{CH}_{2} \mathrm{Cl}_{2}(2 \mathrm{X} 10 \mathrm{~mL})$. The combined organic layers were washed with saturated solution of $\mathrm{NaHCO}_{3}(15 \mathrm{~mL})$, brine $(15$ 
$\mathrm{mL}$ ), dried over $\mathrm{MgSO}_{4}$, filtered and concentrated under reduced pressure. The crude product was purified by flash chromatography using EtOAc:Pentane 1:1 as mobile phase followed by washing with EtOAc to afford $2 \mathrm{~s}$ as a white solid (0.167 g, $0.320 \mathrm{mmol}, 28 \%)$. Mp: 235-239 ${ }^{\circ} \mathrm{C}$; TLC (EtOAc:Pentane 1:1): $\mathrm{R}_{\mathrm{f}}=0.4, \mathrm{KMnO}_{4} ;{ }^{1} \mathrm{H}_{\mathrm{NMR}}\left(400 \mathrm{MHz}, \mathrm{CDCl}_{3}\right): \delta 8.36-8.11$ (m, 2H, ArH), $7.81-7.55$ (m, 2H, ArH), 6.91 (s, 2H, ArH), $3.06-2.91$ (m, 1H, CH), 2.78 (s, $\left.6 \mathrm{H}, 2 \mathrm{X} \mathrm{ArCH}_{3}\right), 2.25$ (s, 3H, $\left.\mathrm{ArCH}_{3}\right), 2.13-1.99\left(\mathrm{~m}, 2 \mathrm{H}, \mathrm{CH}_{2}\right), 1.88-1.73\left(\mathrm{~m}, 4 \mathrm{H}, 2 \mathrm{X} \mathrm{CH}_{2}\right)$, $1.71-1.64\left(\mathrm{~m}, 2 \mathrm{H}, \mathrm{CH}_{2}\right) ;{ }^{13} \mathrm{C}$ NMR $\left(100 \mathrm{MHz} \mathrm{CDCl}_{3}\right): \delta 161.3,142.1,139.7,135.1,135.0$, 134.5, 131.8, 131.6, 131.5, 127.0, 114.9, 114.1, 45.0, 33.8, 31.5, 25.2, 23.2, 21.0; IR v 2966 (w), 2869 (w), $2167(\mathrm{w}), 1656(\mathrm{~m}), 1605$ (w), $1438(\mathrm{w}), 1294$ (s), 1247 (w), 1136 (s), 1055 (w), 897 (w), 854 (s); HRMS (ESI) calcd. for $\mathrm{C}_{23} \mathrm{H}_{24} \mathrm{INNaO}_{3} \mathrm{~S}^{+}[\mathrm{M}+\mathrm{Na}]^{+}$544.0414; found 544.0414.

\section{$N$-[Mesitylsulfonyl]-1-[(t-butyl)ethynyl]-1,2-benziodazol-3(1H)-one (2t)}

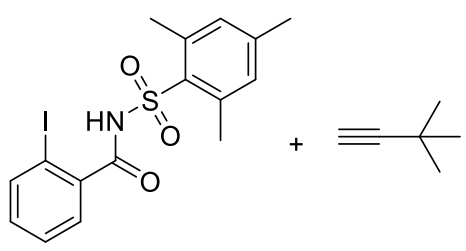

$3 \mathbf{b}$ $\underset{\text { DCE:TFE, } \mathrm{NaHCO}_{3}}{\stackrel{m \mathrm{CPBA}, \mathrm{TsOH} . \mathrm{H}_{2} \mathrm{O}}{\longrightarrow}}$

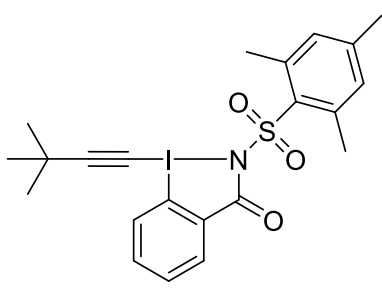

$2 \mathrm{t}$

In a $5 \mathrm{~mL}$ sealed tube, 2-iodo- $N$-(mesitylsulfonyl)benzamide (3b) $(0.500 \mathrm{~g}, 1.17 \mathrm{mmol}, 1.00$ equiv), 4-methylbenzenesulfonic acid hydrate (0.201 g, $1.17 \mathrm{mmol}, 1.00$ equiv) and $m$ CPBA (0.287 g, 77\% purity, $2.56 \mathrm{mmol}, 1.10$ equiv) were suspended in DCE:TFE 1:1 (2 mL) and stirred for $1 \mathrm{~h}$ at $40{ }^{\circ} \mathrm{C} .3,3-D i m e t h y l b u t-1-y n e(0.134 \mathrm{~g}, 0.200 \mathrm{~mL}, 1.63 \mathrm{mmol}, 1.40$ equiv) was then added and the reaction mixture was stirred at $40{ }^{\circ} \mathrm{C}$ for $16 \mathrm{~h}$. The solvent was evaporated and the residue was dissolved in $\mathrm{CH}_{2} \mathrm{Cl}_{2}(10 \mathrm{~mL})$ and stirred vigorously with saturated solution of $\mathrm{NaHCO}_{3}(10 \mathrm{~mL})$. After $1 \mathrm{~h}$, the reaction mixture was transferred into a separating funnel and the layers were separated. The aqueous layer was extracted with $\mathrm{CH}_{2} \mathrm{Cl}_{2}$ $(2 \mathrm{X} 10 \mathrm{~mL})$. The combined organic layers were washed with saturated solution of $\mathrm{NaHCO}_{3}$ (15 mL), brine (15 mL), dried over $\mathrm{MgSO}_{4}$, filtered and concentrated under reduced pressure. The crude product was purified by flash chromatography using EtOAc:DCM 1:20 as mobile phase followed by washing with EtOAc ( $3 \mathrm{X} 3 \mathrm{~mL})$ to afford $\mathbf{2 t}$ as a white solid $(0.453 \mathrm{~g}, 0.888$ mmol, 76\%). Mp: 256-260 ${ }^{\circ} \mathrm{C}$; TLC (EtOAc:DCM 1:20): $\mathrm{R}_{\mathrm{f}}=0.4, \mathrm{KMnO}_{4} ;{ }^{1} \mathrm{H}$ NMR (400 $\left.\mathrm{MHz}, \mathrm{CDCl}_{3}\right): \delta 8.27(\mathrm{dd}, J=7.3,2.0 \mathrm{~Hz}, 1 \mathrm{H}, \mathrm{ArH}), 8.24(\mathrm{dd}, J=8.1,1.0 \mathrm{~Hz}, 1 \mathrm{H}), 7.82-7.54$ (m, 2H, $\mathrm{ArH}$ ), 6.92 (s, 2H, $\mathrm{ArH}$ ), 2.78 (s, 6H, 2 x $\left.\mathrm{ArCH}_{3}\right), 2.25$ (s, 3H, $\left.\mathrm{ArCH}_{3}\right), 1.38$ (s, 9H, $t \mathrm{Bu}) ;{ }^{13} \mathrm{C} \mathrm{NMR}\left(100 \mathrm{MHz}, \mathrm{CDCl}_{3}\right): \delta 161.3,142.1,139.8,135.0,135.0,134.5,131.8,131.6$, 131.5, 126.9, 117.5, 114.9, 44.5, 30.5, 29.6, 23.2, 21.0; IR $v 2971(w), 2927(w), 2171(w)$, 
$2140(\mathrm{w}), 1652$ (m), 1604 (w), 1438 (w), 1293 (s), 1247 (w), 1135 (s), 1054 (w), 897 (m), 852

(s); HRMS (ESI) calcd. for $\mathrm{C}_{22} \mathrm{H}_{24} \mathrm{INNaO}_{3} \mathrm{~S}^{+}[\mathrm{M}+\mathrm{Na}]^{+}$532.0414; found 532.0394.

\section{$N$-(Mesitylsulfonyl)-5-methyl-1-[phenylethynyl]-1,2-benziodazol-3(1H)-one (2u)}

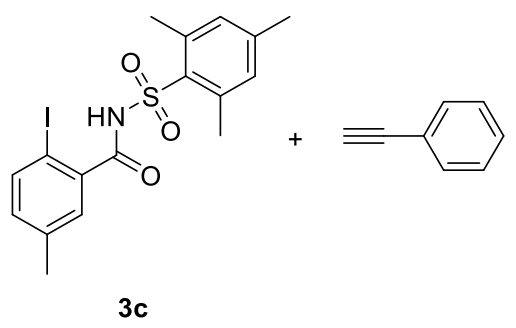

$\underset{\text { DCE:TFE, } \mathrm{NaHCO}_{3}}{\stackrel{m \mathrm{CPBA}, \mathrm{TsOH} \cdot \mathrm{H}_{2} \mathrm{O}}{\longrightarrow}}$

$3 c$

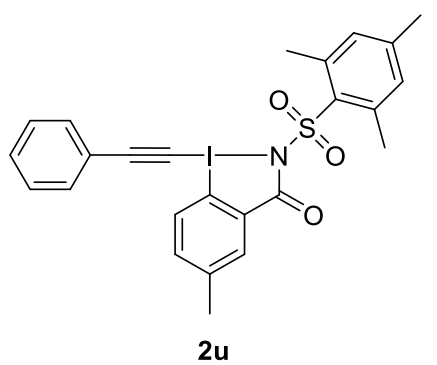

$2 \mathrm{u}$

In a $5 \mathrm{~mL}$ sealed tube, 2-iodo- $N$-(mesitylsulfonyl)-5-methylbenzamide (3c) $(0.700 \mathrm{~g}, 1.58$ mmol, 1.00 equiv), 4-methylbenzenesulfonic acid hydrate ( $0.272 \mathrm{~g}, 1.58 \mathrm{mmol}, 1.00$ equiv) and $m$ CPBA ( $0.389 \mathrm{~g}, 77 \%$ purity, $1.74 \mathrm{mmol}, 1.10$ equiv) were suspended in DCE:TFE 1:1 $(2.8 \mathrm{~mL})$ and stirred for $1 \mathrm{~h}$ at $40^{\circ} \mathrm{C}$. Ethynylbenzene $(0.25 \mathrm{~mL}, 2.2 \mathrm{mmol}, 1.4$ equiv) was then added and the reaction mixture was stirred at $40{ }^{\circ} \mathrm{C}$ for $16 \mathrm{~h}$. The solvent was evaporated and the residue was dissolved in $\mathrm{CH}_{2} \mathrm{Cl}_{2}(15 \mathrm{~mL})$ and stirred vigorously with saturated solution of $\mathrm{NaHCO}_{3}(15 \mathrm{~mL})$. After $1 \mathrm{~h}$, the reaction mixture was transferred into a separating funnel and the layers were separated. The aqueous layer was extracted with $\mathrm{CH}_{2} \mathrm{Cl}_{2}(2 \mathrm{X} 10 \mathrm{~mL})$. The combined organic layers were washed with saturated solution of $\mathrm{NaHCO}_{3}(20 \mathrm{~mL})$, brine $(20$ $\mathrm{mL}$ ), dried over $\mathrm{MgSO}_{4}$, filtered and concentrated under reduced pressure. The crude product was purified by flash chromatography using EtOAc:DCM 1:25 as mobile phase followed by washing with EtOAc ( 3 X $3 \mathrm{~mL})$ to afford $\mathbf{2 u}$ as a white solid $(0.240 \mathrm{~g}, 0.442 \mathrm{mmol}, 28 \%)$. Mp: 190-194 ${ }^{\circ} \mathrm{C}$; TLC (EtOAc:DCM 1:25): $\mathrm{R}_{\mathrm{f}}=0.18, \mathrm{KMnO}_{4} ;{ }^{1} \mathrm{H}_{\mathrm{NMR}}\left(400 \mathrm{MHz}, \mathrm{CDCl}_{3}\right)$ : $\delta 8.19(\mathrm{~d}, J=8.5 \mathrm{~Hz}, 1 \mathrm{H}, \operatorname{Ar} H), 8.10(\mathrm{~d}, J=2.0 \mathrm{~Hz}, 1 \mathrm{H}, \operatorname{Ar} H), 7.62-7.57(\mathrm{~m}, 2 \mathrm{H}, \operatorname{Ar} H), 7.55$ - 7.41 (m, 4H, ArH), 6.92 (s, 2H, $\mathrm{ArH}$ ), 2.79 (s, 6H, $2 \mathrm{X} \mathrm{ArCH}_{3}$ ), 2.43 (s, 3H, $\mathrm{ArCH}_{3}$ ), 2.26 (s, 3H, $\left.\mathrm{ArCH}_{3}\right) ;{ }^{13} \mathrm{C} \mathrm{NMR}\left(100 \mathrm{MHz}, \mathrm{CDCl}_{3}\right): \delta 161.5,142.5,142.1,139.8,136.1,134.9$, 134.0, 132.8, 132.5, 131.6, 130.7, 128.7, 127.0, 120.6, 111.5, 106.1, 56.2, 23.2, 21.0, 20.8; IR v 3055 (w), 2975 (w), 2936 (w), 2144 (w), 1649 (s), 1526 (w), 1452 (m), 1403 (w), 1293 (s), 1250 (m), 1215 (w), 1138 (s), 1054 (m), 1002 (w), 869 (s); HRMS (ESI) calcd. for $\mathrm{C}_{25} \mathrm{H}_{22} \mathrm{INNaO}_{3} \mathrm{~S}^{+}[\mathrm{M}+\mathrm{Na}]^{+}$566.0257; found 566.0232. 


\section{$N$-(Mesitylsulfonyl)-5,6-dimethoxy-1-[phenylethynyl]-1,2-benziodazol-3(1H)-one (2v)}
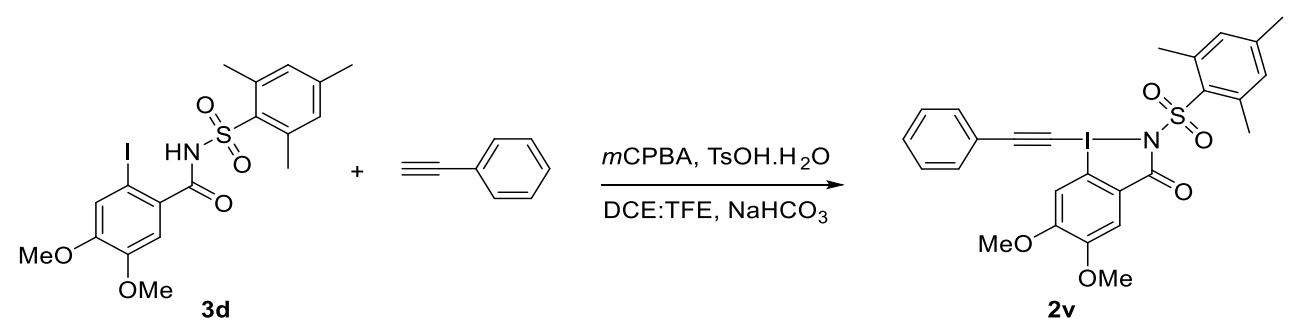

In a $5 \mathrm{~mL}$ sealed tube, 2-iodo- $N$-(mesitylsulfonyl)-4,5-dimethoxybenzamide (3d) $(0.400 \mathrm{~g}$, $0.817 \mathrm{mmol}, 1.00$ equiv), 4-methylbenzenesulfonic acid hydrate $(0.141 \mathrm{~g}, 0.817 \mathrm{mmol}, 1.00$ equiv) and $m \mathrm{CPBA}(0.202 \mathrm{~g}, 77 \%$ purity, $0.899 \mathrm{mmol}, 1.10$ equiv) were suspended in DCE:TFE 1:1 (1.4 mL) and stirred for $1 \mathrm{~h}$ at $40^{\circ} \mathrm{C}$. Ethynylbenzene $(0.130 \mathrm{~mL}, 1.15 \mathrm{mmol}$, 1.40 equiv) was then added and the reaction mixture was stirred at $40{ }^{\circ} \mathrm{C}$ for $16 \mathrm{~h}$. The solvent was evaporated and the residue was dissolved in $\mathrm{CH}_{2} \mathrm{Cl}_{2}(10 \mathrm{~mL})$ and stirred vigorously with saturated solution of $\mathrm{NaHCO}_{3}(10 \mathrm{~mL})$. After $1 \mathrm{~h}$, the reaction mixture was transferred into a separating funnel and the layers were separated. The aqueous layer was extracted with $\mathrm{CH}_{2} \mathrm{Cl}_{2}$ ( 2 X $20 \mathrm{~mL}$ ). The combined organic layers were washed with saturated solution of $\mathrm{NaHCO}_{3}$ $(15 \mathrm{~mL})$, brine $(15 \mathrm{~mL})$, dried over $\mathrm{MgSO}_{4}$, filtered and concentrated under reduced pressure. The crude product was purified by flash chromatography using EtOAc:DCM 1:25 as mobile phase followed by washing with EtOAc $(3 \mathrm{X} 3 \mathrm{~mL})$ to afford $2 \mathbf{v}$ as a white solid $(0.155 \mathrm{~g}$, 0.263 mmol, 32\%). Mp: 204-206 ${ }^{\circ} \mathrm{C}$; TLC (EtOAc:DCM 1:25): $\mathrm{R}_{\mathrm{f}}=0.31, \mathrm{KMnO}_{4} ;{ }^{1} \mathrm{H}$ NMR $\left(400 \mathrm{MHz}, \mathrm{CDCl}_{3}\right): \delta 7.81(\mathrm{~s}, 1 \mathrm{H}, \mathrm{ArH}), 7.73(\mathrm{~s}, 1 \mathrm{H}, \mathrm{ArH}), 7.59-7.54(\mathrm{~m}, 2 \mathrm{H}, \mathrm{ArH}), 7.52$ -

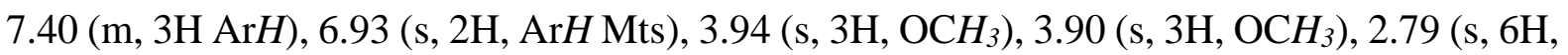
$\left.2 \mathrm{X} \mathrm{ArCH}_{3}\right), 2.26\left(\mathrm{~s}, 3 \mathrm{H}, \mathrm{ArCH}_{3}\right) ;{ }^{13} \mathrm{C} \mathrm{NMR}\left(100 \mathrm{MHz}, \mathrm{CDCl}_{3}\right): \delta 161.6,154.9,152.0,142.2$, 139.8, 135.0, 132.6, 131.7, 130.8, 128.9, 127.3, 120.5, 112.8, 108.9, 106.1, 104.6, 57.4, 56.6, 56.5, 23.2, 21.0; IR v 2942 (w), $2141(\mathrm{w}), 1634$ (w), 1600 (w), 1500 (m), 1443 (w), 1396 (m), 1288 (s), 1219 (w), 1182 (w), 1141 (s), 1053 (w), 1029 (w), 879 (m), 812 (w); HRMS (ESI) calcd. for $\mathrm{C}_{26} \mathrm{H}_{25} \mathrm{INO}_{5} \mathrm{~S}^{+}[\mathrm{M}+\mathrm{H}]^{+}$590.0493; found 590.0485. 


\section{Preparation of Diazo-compounds}

Ethyl 2-diazoacetate (11a), benzyl 2-diazoacetate (11b) and tert-butyl 2-diazoacetate (11c) were directly purchased from Sigma Aldrich. The synthesis of diazo compounds (11d-11l) had been already described before. The procedures are taken here from the indicated publications to facilitate reproduction of the results by having all the data in the same file.
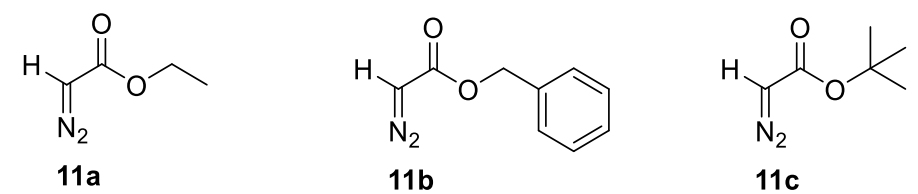

\section{2,4-Dimethylpentan-3-yl 2-diazoacetate (11d)}

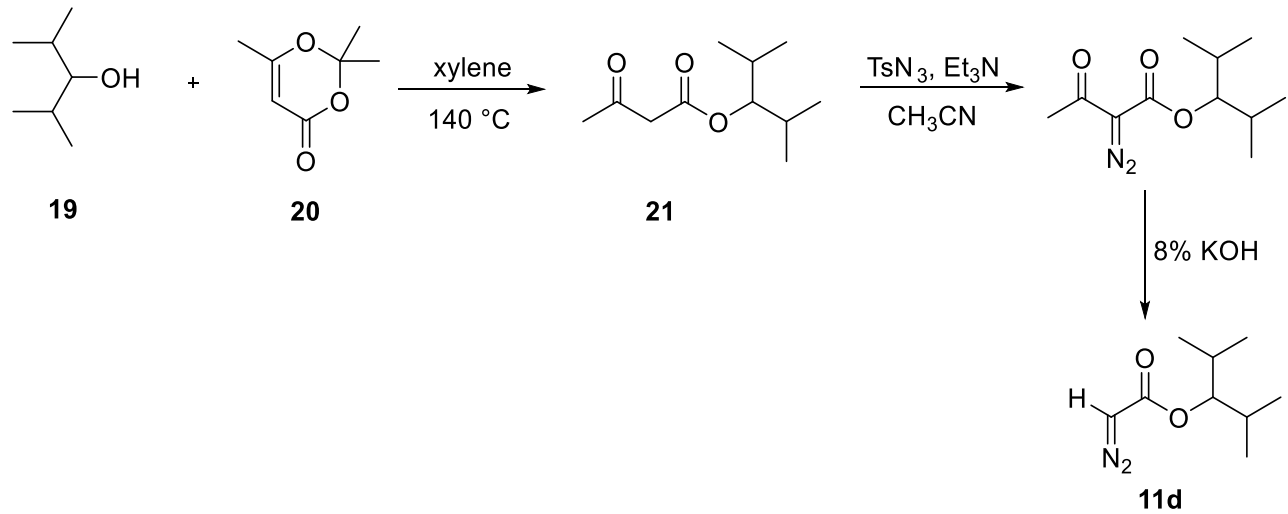

Following a slightly modified procedure, ${ }^{[4]}$ a mixture of 2,4-dimethylpentan-3-ol (19) (3.50 $\mathrm{mL}, 25.0$ mmol, 1.00 equiv), 2,2,6-trimethyl-4H-1,3-dioxin-4-one (20) (3.55 g, 25.0 mmol, 1.00 equiv), and xylene $(5 \mathrm{~mL})$ was stirred at $140^{\circ} \mathrm{C}$ for $2 \mathrm{~h}$. After cooling to room temperature, the xylene was evaporated and the residue was purified by flash column chromatography using 1:50 EtOAc:pentane as mobile phase to afford 2,4-dimethylpentan-3-yl 3-oxobutanoate (21) as a colorless oil (4.2 g, $21 \mathrm{mmol}, 84 \%)$. TLC (EtOAc:pentane, 1:20 v/v): $\mathrm{R}_{\mathrm{f}}=0.36, \mathrm{KMnO}_{4} ;{ }^{1} \mathrm{H}$ NMR (400 MHz, $\mathrm{CDCl}_{3}$ ): $\delta 12.17$ (s, $0.08 \mathrm{H}, \mathrm{OH}$ of enol form), 4.97 (s, $0.08 \mathrm{H}$, vinyl $H$ of enol form), $4.60(\mathrm{t}, J=6.1 \mathrm{~Hz}, 1 \mathrm{H}, \mathrm{OCH}), 3.46$ (s, $1.84 \mathrm{H}, \mathrm{CH}_{3} \mathrm{COCH}_{2}$ of keto form), 2.26 (s, $2.76 \mathrm{H}$, $\mathrm{CH}_{3} \mathrm{COCH}_{2}$ of keto form), 1.92 (s, $0.24 \mathrm{H}, \mathrm{CH}_{3}$ of enol form), 1.87 (dq, $J=13.3,6.8 \mathrm{~Hz}, 2 \mathrm{H}, 2$ $\left.\mathrm{X} \mathrm{CH}\left(\mathrm{CH}_{3}\right)_{2}\right), 0.86\left(\mathrm{~d}, J=6.8 \mathrm{~Hz}, 6 \mathrm{H}, \mathrm{CH}\left(\mathrm{CH}_{3}\right)_{2}\right), 0.83\left(\mathrm{~d}, J=6.7 \mathrm{~Hz}, 6 \mathrm{H}, \mathrm{CH}\left(\mathrm{CH}_{3}\right)_{2}\right) ;{ }^{13} \mathrm{C}$ NMR (100 MHz, $\left.\mathrm{CDCl}_{3}\right): \delta 200.7,167.0,84.0,50.1,30.3,29.2,19.4,17.1$. Enol form, ${ }^{13} \mathrm{C}$ NMR (100 MHz, $\left.\mathrm{CDCl}_{3}\right): \delta 175.2,172.7,89.6,81.9$. Some carbons of enol form were not resolved at $100 \mathrm{MHz}$. The characterization data of keto form corresponded to the reported values. $^{[4]}$ 
Following a slightly modified procedure, ${ }^{[4]}$ to a solution of 2,4-dimethylpentan-3-yl 3oxobutanoate $(\mathbf{2 1})(1.0 \mathrm{~g}, 5.0 \mathrm{mmol}, 1.0$ equiv) in acetonitrile $(6 \mathrm{~mL})$ was added triethylamine (660 mg, $6.50 \mathrm{mmol}, 1.30$ equiv). The reaction mixture was cooled in an ice bath and a solution of tosyl azide ( $1.1 \mathrm{~g}, 5.5 \mathrm{mmol}, 1.1 \mathrm{equiv})$ in acetonitrile $(6 \mathrm{~mL})$ was added slowly. The reaction mixture was allowed to warm to room temperature. After stirring for $20 \mathrm{~h}$, the solvent was removed under reduced pressure. The residue was dissolved in ether $(30 \mathrm{~mL})$ and washed with $8 \%$ aqueous $\mathrm{KOH}$ solution. The organic layer was separated, dried over $\mathrm{MgSO}_{4}$, and evaporated. The crude product was re-dissolved in acetonitrile $(15 \mathrm{~mL})$ and $8 \%$ aqueous $\mathrm{KOH}$ $(25 \mathrm{~mL})$ solution was added at room temperature. After vigorous stirring for $4 \mathrm{~h}$, water $(15 \mathrm{~mL})$ was added and the reaction mixture was extracted with diethyl ether ( 2 X $30 \mathrm{~mL})$. The combined organic layers were dried over $\mathrm{MgSO}_{4}$, and concentrated under reduced pressure. The crude product was purified by column chromatography using 1:20 $\mathrm{Et}_{2} \mathrm{O}$ :pentane as mobile phase to afford 2,4-dimethylpentan-3-yl 2-diazoacetate (11d) as a yellow oil (800 mg, 4.35 mmol, 87\%). TLC $\left(\mathrm{Et}_{2} \mathrm{O}:\right.$ pentane, $\left.1: 9 \mathrm{v} / \mathrm{v}\right): \mathrm{R}_{\mathrm{f}}=0.55, \mathrm{KMnO}_{4} ;{ }^{1} \mathrm{H} \mathrm{NMR}\left(400 \mathrm{MHz}, \mathrm{CDCl}_{3}\right): \delta$ 4.73 (br s, 1H, CHN $\left.\mathrm{N}_{2}\right), 4.62(\mathrm{t}, J=6.2 \mathrm{~Hz}, 1 \mathrm{H}, \mathrm{OCH}), 1.88(\mathrm{dq}, J=13.4,6.7 \mathrm{~Hz}, 2 \mathrm{H}, 2 \mathrm{X}$ $\left.\mathrm{CH}\left(\mathrm{CH}_{3}\right)_{2}\right), 0.88\left(\mathrm{~d}, J=6.8 \mathrm{~Hz}, 6 \mathrm{H}, \mathrm{CH}\left(\mathrm{CH}_{3}\right)_{2}\right), 0.85\left(\mathrm{~d}, J=6.7 \mathrm{~Hz}, 6 \mathrm{H}, \mathrm{CH}\left(\mathrm{CH}_{3}\right)_{2}\right) ;{ }^{13} \mathrm{C} \mathrm{NMR}$ $\left(100 \mathrm{MHz}, \mathrm{CDCl}_{3}\right): \delta 167.0,83.1,45.8,29.4,19.5,17.1$. The characterization data corresponded to the reported values. ${ }^{[4]}$

\section{Dicyclohexylmethyl 2-diazoacetate (11e)}

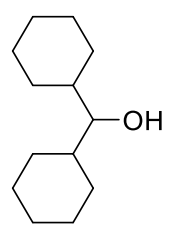

22

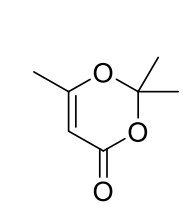

20

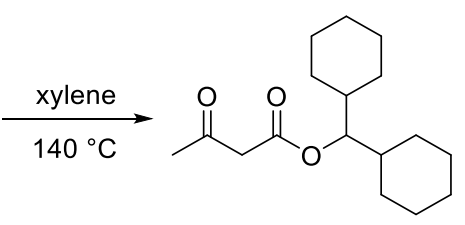

23

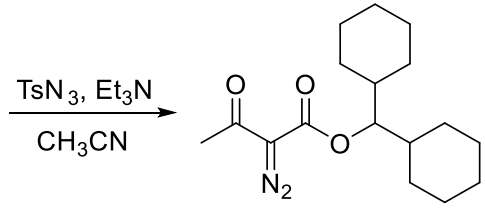

$\downarrow 8 \% \mathrm{KOH}$

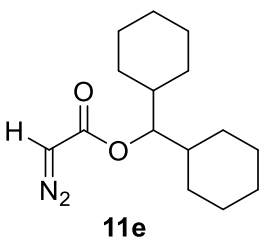

Following a slightly modified procedure, ${ }^{[4]}$ a mixture of dicyclohexylmethanol (22) $(2.45 \mathrm{~g}$, 12.5 mmol, 1.00 equiv), 2,2,6-trimethyl-4H-1,3-dioxin-4-one (20) (1.78 g, 12.5 mmol, 1.00 equiv), and xylene $(2.5 \mathrm{~mL})$ was stirred at $140{ }^{\circ} \mathrm{C}$ for $2 \mathrm{~h}$. After cooling to room temperature, the xylene was evaporated and the residue was purified by flash column chromatography using 1:50 EtOAc:pentane as mobile phase to afford dicyclohexylmethyl 3-oxobutanoate (23) as a white solid (3.00 g, $10.7 \mathrm{mmol}, 86 \%)$. Mp: 64.5-66.8 ${ }^{\circ} \mathrm{C}$; TLC (EtOAc:pentane, 1:20 v/v): $\mathrm{R}_{\mathrm{f}}$ 
$=0.36, \mathrm{KMnO}_{4} ;{ }^{1} \mathrm{H} \mathrm{NMR}\left(400 \mathrm{MHz}, \mathrm{CDCl}_{3}\right): \delta 12.20(\mathrm{~s}, 0.09 \mathrm{H}, \mathrm{OH}$ of enol form), 4.99 (s, $0.09 \mathrm{H}$, vinyl $H$ of enol form), $4.67(\mathrm{t}, J=5.8 \mathrm{~Hz}, 1 \mathrm{H}, \mathrm{OCH}), 3.47$ (s, $1.8 \mathrm{H}, \mathrm{CH}_{3} \mathrm{COCH}_{2}$ of keto form), 2.29 (s, 2.7H, $\mathrm{CH}_{3} \mathrm{COCH}_{2}$ of keto form), 1.95 (s, $0.27 \mathrm{H}, \mathrm{CH}_{3}$ of enol form), 1.81-1.42 $\left(\mathrm{m}, 12 \mathrm{H}, 2 \mathrm{X} \mathrm{Cy}-\mathrm{CH}\right.$ and $\left.5 \mathrm{X} \mathrm{Cy}-\mathrm{CH}_{2}\right), 1.34-0.83\left(\mathrm{~m}, 10 \mathrm{H}, 5 \mathrm{X} \mathrm{Cy}-\mathrm{CH}_{2}\right) ;{ }^{13} \mathrm{C} \mathrm{NMR}(100$ $\left.\mathrm{MHz}, \mathrm{CDCl}_{3}\right): \delta 200.9,167.0,82.8,50.2,38.2,30.4,29.8,27.4,26.3,26.2,26.0$. Enol form, ${ }^{13} \mathrm{C} \mathrm{NMR}\left(100 \mathrm{MHz}, \mathrm{CDCl}_{3}\right): \delta 175.2,172.8,89.8,80.8,38.3,21.2$, some carbons of enol form were not resolved at $100 \mathrm{MHz}$; IR v 2976 (s), 2928 (s), 2862 (m), 2109 (w), 1725 (m), 1646 (w), 1447 (m), 1403 (m), 1313 (m), 1246 (m), 1188 (m), 1152 (m), 1056 (s), 891 (w); HRMS (ESI) calcd. for $\mathrm{C}_{17} \mathrm{H}_{28} \mathrm{NaO}_{3}{ }^{+}[\mathrm{M}+\mathrm{Na}]^{+}$303.1931; found 303.1928.

Following a slightly modified procedure, ${ }^{[4]}$ to a solution of dicyclohexylmethyl 3-oxobutanoate (23) (1.4 g, $5.0 \mathrm{mmol}, 1.0$ equiv) in acetonitrile $(6 \mathrm{~mL})$ was added triethylamine $(660 \mathrm{mg}, 6.50$ mmol, 1.30 equiv). The reaction mixture was cooled in an ice bath and a solution of tosyl azide $(1.1 \mathrm{~g}, 5.5 \mathrm{mmol}, 1.1$ equiv $)$ in acetonitrile $(6 \mathrm{~mL})$ was added slowly. The reaction mixture was allowed to warm to room temperature. After stirring for $20 \mathrm{~h}$, the solvent was removed under reduced pressure. The residue was dissolved in ether $(30 \mathrm{~mL})$ and washed with $8 \%$ aqueous $\mathrm{KOH}$ solution. The organic layer was separated, dried over $\mathrm{MgSO}_{4}$, and evaporated. The crude product was re-dissolved in acetonitrile $(15 \mathrm{~mL})$ and $8 \%$ aqueous $\mathrm{KOH}(25 \mathrm{~mL})$ solution was added at room temperature. After vigorous stirring for $4 \mathrm{~h}$, water $(15 \mathrm{~mL})$ was added and the reaction mixture was extracted with diethyl ether $(2 \times 30 \mathrm{~mL})$. The combined organic layers were dried over $\mathrm{MgSO}_{4}$, and concentrated under reduced pressure. The crude product was purified by column chromatography using 1:40 $\mathrm{Et}_{2} \mathrm{O}$ :pentane as mobile phase to afford dicyclohexylmethyl 2-diazoacetate (11e) as a yellow solid (1.10 g, $4.16 \mathrm{mmol}, 83 \%)$. Mp (Dec.): 81.2-83.2 ${ }^{\circ} \mathrm{C}$; TLC (Et $2 \mathrm{O}$ :pentane, 1:25 v/v): $\mathrm{R}_{\mathrm{f}}=0.52, \mathrm{KMnO}_{4} ;{ }^{1} \mathrm{H}$ NMR $(400 \mathrm{MHz}$, $\left.\mathrm{CDCl}_{3}\right): \delta 4.73\left(\mathrm{bs}, 1 \mathrm{H}, \mathrm{CHN}_{2}\right), 4.67(\mathrm{t}, J=5.9 \mathrm{~Hz}, 1 \mathrm{H}, \mathrm{OCH}), 1.84-1.45(\mathrm{~m}, 12 \mathrm{H}, 2 \mathrm{X} \mathrm{Cy}-\mathrm{CH}$ and $\left.\left.5 \mathrm{X} \mathrm{Cy}-\mathrm{CH}_{2}\right), 1.31-0.88\left(\mathrm{~m}, 10 \mathrm{H}, 5 \mathrm{X} \mathrm{Cy}-\mathrm{CH}_{2}\right) ;{ }^{13} \mathrm{C} \mathrm{NMR} \mathrm{(100} \mathrm{MHz,} \mathrm{CDCl}_{3}\right): \delta 167.0$, 82.0, 45.9, 38.4, 29.8, 27.4, 26.3, 26.2, 26.0; IR v 2929 (s), 2855 (m), 2110 (s), 1692 (s), 1451 (w), 1377 (m), 1242 (m), 1191 (s), 1099 (w), 991 (w), 931 (w); HRMS (ESI) calcd. for $\mathrm{C}_{15} \mathrm{H}_{24} \mathrm{~N}_{2} \mathrm{O}_{2}[\mathrm{M}+]$ 264.1832; found 264.1836. 
2,3,4-Trimethylpentan-3-yl 2-diazoacetate (11f)

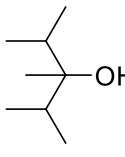

24

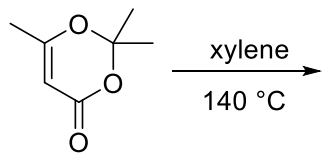

20

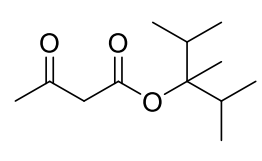

25

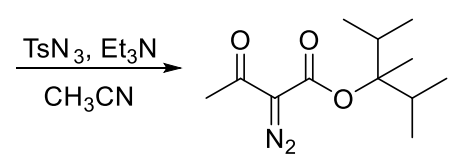

$\mid 8 \% \mathrm{KOH}$

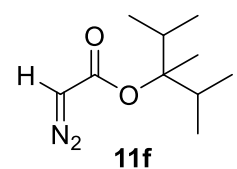

Following a slightly modified procedure, ${ }^{[4]}$ a mixture of 2,3,4-trimethylpentan-3-ol (24) (1.63 g, 12.5 mmol, 1.00 equiv), 2,2,6-trimethyl-4H-1,3-dioxin-4-one (20) (1.78 g, 12.5 mmol, 1.00 equiv), and xylene $(2.5 \mathrm{~mL})$ was stirred at $140^{\circ} \mathrm{C}$ for $2 \mathrm{~h}$. After cooling to room temperature, the xylene was evaporated and the residue was purified by flash column chromatography using 1:50 EtOAc:pentane as mobile phase to afford 2,3,4-trimethylpentan-3-yl 3-oxobutanoate (25) as a colorless oil (1.5 g, $7.0 \mathrm{mmol}, 56 \%)$. TLC (EtOAc:pentane, 1:20 v/v): $\mathrm{R}_{\mathrm{f}}=0.36, \mathrm{KMnO}_{4}$; ${ }^{1} \mathrm{H}$ NMR (400 MHz, $\mathrm{CDCl}_{3}$ ): 12.28 (s, $0.05 \mathrm{H}, \mathrm{OH}$ of enol form), 4.94 (s, 0.05H, vinyl $H$ of enol form), $\delta 3.40\left(\mathrm{~s}, 1.9 \mathrm{H}, \mathrm{CH}_{3} \mathrm{COCH}_{2}\right.$ of keto form), 2.33-2.21 (m, $4.85 \mathrm{H}, \mathrm{CH}_{3} \mathrm{COCH}_{2}$ of

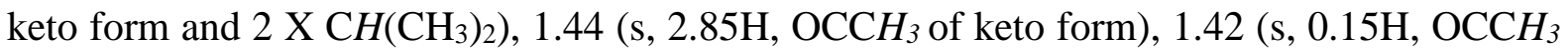
of enol form) $\left.0.95\left(\mathrm{~m}, 12 \mathrm{H}, 2 \mathrm{X} \mathrm{CH}\left(\mathrm{CH}_{3}\right)_{2}\right) ;{ }^{13} \mathrm{C} \mathrm{NMR} \mathrm{(100} \mathrm{MHz}, \mathrm{CDCl}_{3}\right): \delta 201.2,166.3$, 92.8, 51.5, 34.3, 30.2, 18.0, 17.8; Enol form, $\left.{ }^{13} \mathrm{C} \mathrm{NMR} \mathrm{(100} \mathrm{MHz}, \mathrm{CDCl}_{3}\right)$ : $\delta 174.5,172.8$, 91.2, 76.0, some carbons of enol form were not resolved at $100 \mathrm{MHz}$. The characterization data corresponded to the reported values. ${ }^{[5]}$

Following a slightly modified procedure, to a solution of 2,3,4-trimethylpentan-3-yl 3oxobutanoate (25) (1.07 g, $5.00 \mathrm{mmol}, 1.00$ equiv) in acetonitrile (6 mL) was added triethylamine ( $660 \mathrm{mg}, 6.50 \mathrm{mmol}, 1.30$ equiv). The reaction mixture was cooled in an ice bath and a solution of tosyl azide ( $1.1 \mathrm{~g}, 5.5 \mathrm{mmol}, 1.1$ equiv) in acetonitrile $(6 \mathrm{~mL})$ was added slowly. The reaction mixture was allowed to warm to room temperature. After stirring for 20 $\mathrm{h}$, the solvent was removed under reduced pressure. The residue was dissolved in ether (30 $\mathrm{mL}$ ) and washed with $8 \%$ aqueous $\mathrm{KOH}$ solution. The organic layer was separated, dried over $\mathrm{MgSO}_{4}$, and evaporated. The crude product was re-dissolved in acetonitrile $(15 \mathrm{~mL})$ and $8 \%$ aqueous $\mathrm{KOH}(25 \mathrm{~mL})$ solution was added at room temperature. After vigorous stirring for 4 $\mathrm{h}$, water $(15 \mathrm{~mL})$ was added and the reaction mixture was extracted with diethyl ether $(2 \mathrm{X} 30$ $\mathrm{mL}$ ). The combined organic layers were dried over $\mathrm{MgSO}_{4}$, and concentrated under reduced pressure. The crude product was purified by column chromatography using 1:35 $\mathrm{Et}_{2} \mathrm{O}$ :pentane 
as mobile phase to afford 2,3,4-trimethylpentan-3-yl 2-diazoacetate (11f) as a yellow oil (800 $\mathrm{mg}, 4.05 \mathrm{mmol}, 81 \%)$. TLC (Et $2 \mathrm{O}$ :pentane, 1:25 v/v): $\mathrm{R}_{\mathrm{f}}=0.5, \mathrm{KMnO}_{4} ;{ }^{1} \mathrm{H}^{\mathrm{NMR}}(400 \mathrm{MHz}$, $\mathrm{CDCl}_{3}$ ): $\delta 4.60$ (br s, $1 \mathrm{H}, \mathrm{CHN}_{2}$ ), 2.26 (hept, $\left.J=6.9 \mathrm{~Hz}, 2 \mathrm{H}, 2 \mathrm{X} \mathrm{CH}\left(\mathrm{CH}_{3}\right)_{2}\right), 1.41(\mathrm{~s}, 3 \mathrm{H}$, $\left.\mathrm{OCCH}_{3}\right), 0.93\left(\mathrm{~m}, 12 \mathrm{H}, 2 \mathrm{X} \mathrm{CH}\left(\mathrm{CH}_{3}\right)_{2}\right) ;{ }^{13} \mathrm{C} \mathrm{NMR}\left(100 \mathrm{MHz}, \mathrm{CDCl}_{3}\right): \delta 166.1,91.9,46.5$, $34.5,18.1,18.0,17.8$. The ${ }^{1} \mathrm{H}$ NMR data corresponded to the reported values. ${ }^{[5]}$

\section{Adamantan-1-yl 2-diazoacetate (11g)}

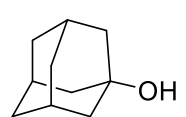

26

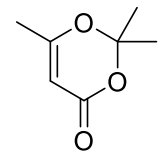

20

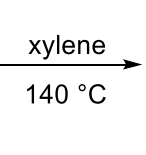

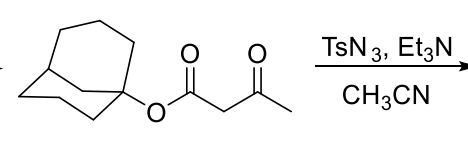

27

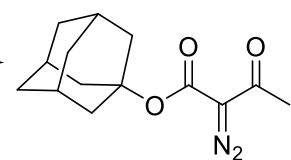

$8 \% \mathrm{KOH}$

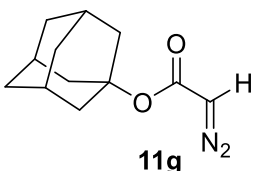

Following a slightly modified procedure, ${ }^{[4]}$ a mixture of adamantan-1-ol (26) (3.81 g, 25.0 mmol, 1.00 equiv), 2,2,6-trimethyl-4H-1,3-dioxin-4-one (20) (3.55 g, 25.0 mmol, 1.00 equiv), and xylene $(5 \mathrm{~mL})$ was stirred at $140{ }^{\circ} \mathrm{C}$ for $2 \mathrm{~h}$. After cooling to room temperature, the xylene was evaporated and the residue was purified by flash column chromatography using 1:50 EtOAc:pentane as mobile phase to afford adamantan-1-yl 3-oxobutanoate (27) as a colorless oil (5.2 g, 22 mmol, 88\%). TLC (EtOAc:pentane, 1:20 v/v): $\mathrm{R}_{\mathrm{f}}=0.35, \mathrm{KMnO}_{4} ;{ }^{1} \mathrm{H}$ NMR (400 $\mathrm{MHz}_{\mathrm{CDCl}}$ ): $\delta 12.20$ (s, $0.07 \mathrm{H}, \mathrm{OH}$ of enol form), 4.85 (s, $0.07 \mathrm{H}$, vinyl $H$ of enol form), 3.32 (s, $1.85 \mathrm{H}, \mathrm{CH}_{3} \mathrm{COCH}_{2}$ of keto form), 2.22 (s, 2.8H, $\mathrm{CH}_{3} \mathrm{COCH}_{2}$ of keto form), 2.17-2.04 (m, 9H, $3 \mathrm{X} \mathrm{CH}$ and $3 \mathrm{X} \mathrm{CH}_{2}$ of adamantly group), 1.87 (s, $0.2 \mathrm{H}, \mathrm{CH}_{3}$ of enol form), 1.69-1.55 (m, $6 \mathrm{H}, 3 \mathrm{X} \mathrm{CH}_{2}$ of adamantly group); $\left.{ }^{13} \mathrm{C} \mathrm{NMR} \mathrm{(100} \mathrm{MHz,} \mathrm{CDCl}_{3}\right): \delta 201.2,166.0,81.9,51.6$, 41.0, 35.9, 30.7, 29.9. Enol form, ${ }^{13} \mathrm{C} \mathrm{NMR}\left(100 \mathrm{MHz}, \mathrm{CDCl}_{3}\right): \delta 174.6,172.3,91.1,80.7,41.4$, 36.0, 21.1, One carbon of enol form was not resolved at $100 \mathrm{MHz}$. The characterization data corresponded to the reported values. ${ }^{[6]}$

Following a slightly modified procedure, ${ }^{[4]}$ to a solution of adamantan-1-yl 3-oxobutanoate (27) (1.18 g, $5.00 \mathrm{mmol}, 1.00$ equiv) in acetonitrile $(6 \mathrm{~mL})$ was added triethylamine (660 mg, $6.50 \mathrm{mmol}, 1.30$ equiv). The reaction mixture was cooled in an ice bath and a solution of tosyl azide $(1.1 \mathrm{~g}, 5.5 \mathrm{mmol}, 1.1$ equi) in acetonitrile $(6 \mathrm{~mL})$ was added slowly. The reaction mixture was allowed to warm to room temperature. After stirring for $20 \mathrm{~h}$, the solvent was removed under reduced pressure. The residue was dissolved in ether $(30 \mathrm{~mL})$ and washed with $8 \%$ aqueous $\mathrm{KOH}$ solution. The organic layer was separated, dried over $\mathrm{MgSO}_{4}$, and evaporated. 
The crude product was re-dissolved in acetonitrile $(15 \mathrm{~mL})$ and $8 \%$ aqueous $\mathrm{KOH}(25 \mathrm{~mL})$ solution was added at room temperature. After vigorous stirring for $4 \mathrm{~h}$, water $(15 \mathrm{~mL})$ was added and the reaction mixture was extracted with diethyl ether $(2 \times 30 \mathrm{~mL})$. The combined organic layers were dried over $\mathrm{MgSO}_{4}$, and concentrated under reduced pressure. The crude product was purified by column chromatography using 1:20 $\mathrm{Et}_{2} \mathrm{O}$ :pentane as mobile phase to afford adamantan-1-yl 2-diazoacetate (11g) as a yellow solid (960 mg, $4.36 \mathrm{mmol}, 87 \%)$. TLC $\left(\mathrm{Et}_{2} \mathrm{O}\right.$ :pentane, 1:10 v/v): $\mathrm{R}_{\mathrm{f}}=0.54, \mathrm{KMnO}_{4} ;{ }^{1} \mathrm{H} \mathrm{NMR}\left(400 \mathrm{MHz}, \mathrm{CDCl}_{3}\right): \delta 4.60(\mathrm{~s}, 1 \mathrm{H}$, $\left.\mathrm{CHN}_{2}\right), 2.22-2.06\left(\mathrm{~m}, 9 \mathrm{H}, 3 \mathrm{X} \mathrm{CH}\right.$ and $\left.3 \mathrm{X} \mathrm{CH}_{2}\right), 1.69-1.61\left(\mathrm{~m}, 6 \mathrm{H}, 3 \mathrm{X} \mathrm{CH}_{2}\right) ;{ }^{13} \mathrm{C} \mathrm{NMR} \mathrm{(100}$ $\left.\mathrm{MHz}, \mathrm{CDCl}_{3}\right): \delta 165.9,81.5,46.7,41.6,36.1,30.8$. The characterization data corresponded to the reported values. ${ }^{[6]}$

\section{2-Phenylpropan-2-yl 2-diazoacetate (11h)}

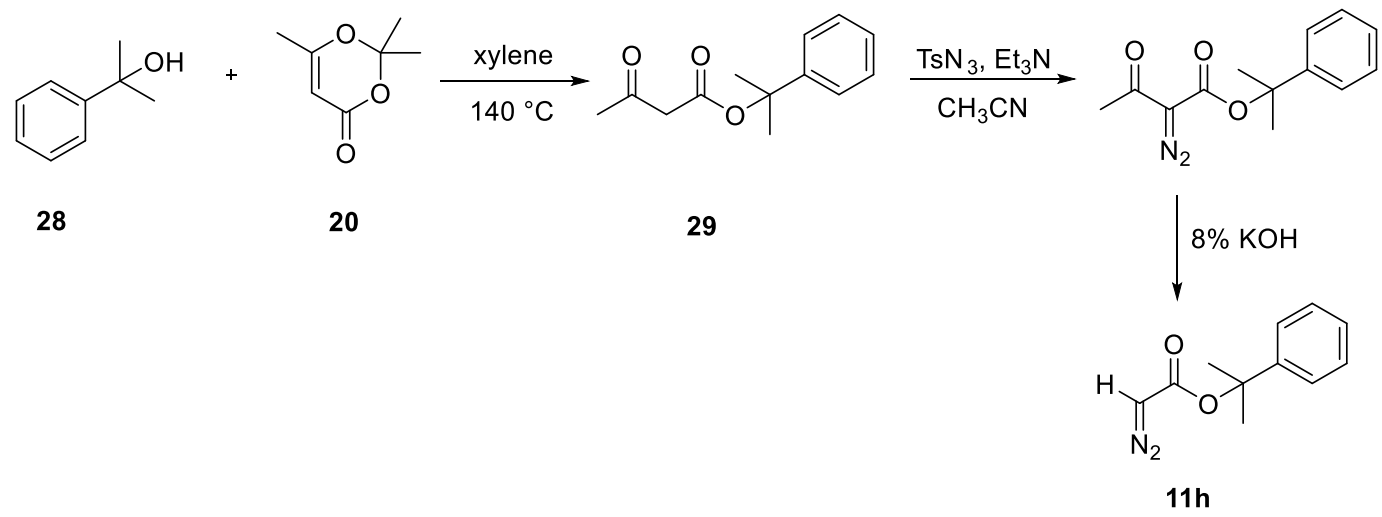

Following a slightly modified procedure, ${ }^{[4]}$ a mixture of 2-phenylpropan-2-ol (28) (3.4 g, 25 mmol, 1.0 equiv), 2,2,6-trimethyl-4H-1,3-dioxin-4-one (20) (3.55 g, 25.0 mmol, 1.00 equiv), and xylene $(5 \mathrm{~mL})$ was stirred at $140{ }^{\circ} \mathrm{C}$ for $2 \mathrm{~h}$. After cooling to room temperature, the xylene was evaporated and the residue was purified by flash column chromatography using 1:5 EtOAc:pentane as mobile phase to afford 2-phenylpropan-2-yl 3-oxobutanoate (29) as a colorless oil (2.60 g, $11.8 \mathrm{mmol}, 48 \%)$. TLC (EtOAc:pentane, $1: 4 \mathrm{v} / \mathrm{v}): \mathrm{R}_{\mathrm{f}}=0.4, \mathrm{KMnO}_{4} ;{ }^{1} \mathrm{H}$ NMR (400 MHz, $\left.\mathrm{CDCl}_{3}\right): \delta 11.98$ (s, 0.1H, OH of enol form), 7.41-7.31 (m, 4H, ArH), 7.307.22 (m, $1 \mathrm{H}, \mathrm{Ar} H$ ), 5.06 (s, $0.1 \mathrm{H}$, vinyl $\mathrm{H}$ of enol form), 3.43 (s, $1.8 \mathrm{H}, \mathrm{CH}_{3} \mathrm{COCH}_{2}$ of keto form), 2.24 (s, 2.7H, $\mathrm{CH}_{3} \mathrm{COCH}_{2}$ of keto form), 1.93 (s, $0.3 \mathrm{H}, \mathrm{CH}_{3}$ of enol form), 1.80 (s, 5.4H, $\mathrm{OC}\left(\mathrm{CH}_{3}\right)_{2} \mathrm{Ar}$ of keto form), 1.79 (s, $0.6 \mathrm{H}, \mathrm{OC}\left(\mathrm{CH}_{3}\right)_{2} \mathrm{Ar}$ of enol form); ${ }^{13} \mathrm{C} \mathrm{NMR}(100 \mathrm{MHz}$, $\left.\mathrm{CDCl}_{3}\right): \delta 200.9,165.5,145.0,128.2,127.1,124.2,82.9,51.1,30.1,28.3$; Enol form, ${ }^{13} \mathrm{C}$ NMR $\left(100 \mathrm{MHz}, \mathrm{CDCl}_{3}\right) \delta 175.2,171.7,145.7,128.1,126.8,123.9,90.6,81.5,28.9,21.1$. The characterization data corresponded to the reported values. ${ }^{[7]}$

Following a slightly modified procedure, ${ }^{[4]}$ to a solution of 2-phenylpropan-2-yl 3oxobutanoate (29) $(1.1 \mathrm{~g}, 5.0 \mathrm{mmol}, 1.0$ equiv) in acetonitrile $(6 \mathrm{~mL})$ was added triethylamine 
(660 mg, $6.50 \mathrm{mmol}, 1.30$ equiv). The reaction mixture was cooled in an ice bath and a solution of tosyl azide ( $1.1 \mathrm{~g}, 5.5 \mathrm{mmol}, 1.1 \mathrm{equiv})$ in acetonitrile $(6 \mathrm{~mL})$ was added slowly. The reaction mixture was allowed to warm to room temperature. After stirring for $20 \mathrm{~h}$, the solvent was removed under reduced pressure. The residue was dissolved in ether $(30 \mathrm{~mL})$ and washed with $8 \%$ aqueous $\mathrm{KOH}$ solution. The organic layer was separated, dried over $\mathrm{MgSO}_{4}$, and evaporated. The crude product was re-dissolved in acetonitrile $(15 \mathrm{~mL})$ and $8 \%$ aqueous $\mathrm{KOH}$ $(25 \mathrm{~mL})$ solution was added at room temperature. After vigorous stirring for $4 \mathrm{~h}$, water $(15 \mathrm{~mL})$ was added and the reaction mixture was extracted with diethyl ether ( $2 \times 30 \mathrm{~mL})$. The combined organic layers were dried over $\mathrm{MgSO}_{4}$, and concentrated under reduced pressure. The crude product was purified by column chromatography using 1:10 $\mathrm{Et}_{2} \mathrm{O}$ :pentane as mobile phase to afford 2-phenylpropan-2-yl 2-diazoacetate (11h) as a yellow oil (820 mg, $4.02 \mathrm{mmol}$, $80 \%)$. TLC (Et $2 \mathrm{O}:$ pentane, 1:10 v/v): $\mathrm{R}_{\mathrm{f}}=0.17, \mathrm{KMnO}_{4} ;{ }^{1} \mathrm{H} \mathrm{NMR}\left(400 \mathrm{MHz}, \mathrm{CDCl}_{3}\right): \delta 7.41-$ 7.32 (m, 4H, $\mathrm{ArH}$ ), 7.28-7.24 (m, 1H, $\mathrm{ArH}), 4.72$ (br s, $1 \mathrm{H}, \mathrm{CHN} 2), 1.81$ (s, 6H, OC(CH3) $\left.)_{2} \mathrm{Ar}\right)$; ${ }^{13} \mathrm{C}$ NMR (100 MHz, $\left.\mathrm{CDCl}_{3}\right): \delta 165.5,145.6,128.2,127.0,124.1,82.4,46.9,28.9$. The ${ }^{1} \mathrm{H}$ NMR data corresponded to the reported values. ${ }^{[7]}$

\section{2,6-Diisopropylphenyl 2-diazoacetate (11i)}

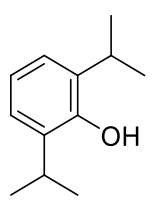

30

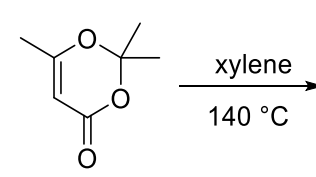

20

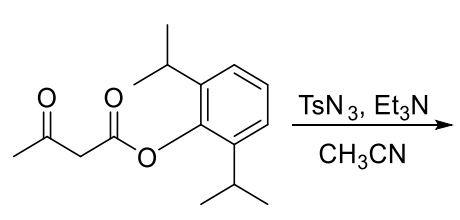

31
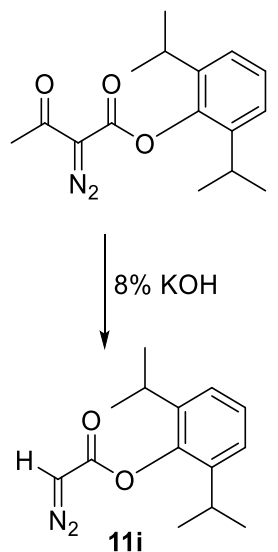

Following a slightly modified procedure, ${ }^{[4]}$ a mixture of 2,6-diisopropylphenol (30) (4.46 g, 25.0 mmol, 1.00 equiv), 2,2,6-trimethyl-4H-1,3-dioxin-4-one (20) (3.55 g, 25.0 mmol, 1.00 equiv), and xylene ( $5 \mathrm{~mL}$ ) was stirred at $140{ }^{\circ} \mathrm{C}$ for $2 \mathrm{~h}$. After cooling to room temperature, the xylene was evaporated and the residue was purified by flash column chromatography using 1:50 EtOAc:pentane as mobile phase to afford 2,6-diisopropylphenyl 3-oxobutanoate (31) as a colorless oil (5.00 g, $19.1 \mathrm{mmol}, 76 \%)$. TLC (EtOAc:pentane, 1:20 v/v): $\mathrm{R}_{\mathrm{f}}=0.35, \mathrm{KMnO}_{4}$; ${ }^{1} \mathrm{H}$ NMR (400 MHz, $\left.\mathrm{CDCl}_{3}\right): \delta 12.08(\mathrm{~s}, 0.22 \mathrm{H}, \mathrm{OH}$ of enol form), 7.31-7.24 (m, 1H, $\mathrm{ArH})$, 7.24-7.18 (m, 2H, $\mathrm{ArH}), 3.81\left(\mathrm{~s}, 1.56 \mathrm{H}, \mathrm{CH}_{3} \mathrm{COCH}_{2}\right.$ of keto form), $3.03(\mathrm{~m}, 2 \mathrm{H}, 2 \mathrm{X}$ $\left.\mathrm{CH}\left(\mathrm{CH}_{3}\right)_{2}\right), 2.41\left(\mathrm{~s}, 2.32 \mathrm{H}, \mathrm{CH}_{3} \mathrm{COCH}_{2}\right.$ of keto form), 1.28-1.21 (m, $\left.12 \mathrm{H}, 2 \mathrm{X} \mathrm{CH}\left(\mathrm{CH}_{3}\right)_{2}\right) ;{ }^{13} \mathrm{C}$ 
NMR (100 MHz, $\left.\mathrm{CDCl}_{3}\right): \delta 199.9,165.7,145.1,140.2,126.8,124.0,49.6,30.4,27.4,27.3$; Enol form, ${ }^{13} \mathrm{C} \mathrm{NMR}\left(100 \mathrm{MHz}, \mathrm{CDCl}_{3}\right): \delta 177.7,171.5,144.5,140.5,126.5,123.9,88.7,23.7$, 22.7, 21.4; IR v 2966 (m), 2876 (w), 1760 (m), 1723 (m), 1634 (w), 1447 (m), 1410 (w), 1360 (m), 1315 (m), 1222 (s), 1140 (s), 1102 (m), 1053 (w), 976 (w); HRMS (ESI) calcd. for $\mathrm{C}_{16} \mathrm{H}_{22} \mathrm{NaO}_{3}{ }^{+}[\mathrm{M}+\mathrm{Na}]^{+}$285.1461; found 285.1467.

Following a slightly modified procedure, ${ }^{[4]}$ to a solution of 2,6-diisopropylphenyl 3oxobutanoate (31) (1.31 g, $5.00 \mathrm{mmol}, 1.00$ equiv) in acetonitrile (6 mL) was added triethylamine ( $660 \mathrm{mg}, 6.50 \mathrm{mmol}, 1.30$ equiv). The reaction mixture was cooled in an ice bath and a solution of tosyl azide ( $1.1 \mathrm{~g}, 5.5 \mathrm{mmol}, 1.1$ equiv) in acetonitrile $(6 \mathrm{~mL})$ was added slowly. The reaction mixture was allowed to warm to room temperature. After stirring for 20 $\mathrm{h}$, the solvent was removed under reduced pressure. The residue was dissolved in ether (30 $\mathrm{mL}$ ) and washed with $8 \%$ aqueous $\mathrm{KOH}$ solution. The organic layer was separated, dried over $\mathrm{MgSO}_{4}$, and evaporated. The crude product was re-dissolved in acetonitrile $(15 \mathrm{~mL})$ and $8 \%$ aqueous $\mathrm{KOH}(25 \mathrm{~mL})$ solution was added at room temperature. After vigorous stirring for 4 $\mathrm{h}$, water $(15 \mathrm{~mL})$ was added and the reaction mixture was extracted with diethyl ether $(2 \mathrm{X} 30$ $\mathrm{mL}$ ). The combined organic layers were dried over $\mathrm{MgSO}_{4}$, and concentrated under reduced pressure. The crude product was purified by column chromatography using 1:30 $\mathrm{Et}_{2} \mathrm{O}$ :pentane as mobile phase to afford 2,6-diisopropylphenyl 2-diazoacetate (11i) as a yellow oil (620 mg, $2.52 \mathrm{mmol}, 50 \%)$. TLC (Et $2 \mathrm{O}$ :pentane, 1:30 v/v): $\mathrm{R}_{\mathrm{f}}=0.36, \mathrm{KMnO}_{4} ;{ }^{1} \mathrm{H}$ NMR $(400 \mathrm{MHz}$, $\left.\mathrm{CDCl}_{3}\right): \delta$ 7.32-7.25 (m, 1H, $\mathrm{ArH}$ ), 7.23-7.20 (m, 2H, $\mathrm{ArH}$ ), 5.09 (br s, $1 \mathrm{H}, \mathrm{CHN}_{2}$ ), 3.05 (sept, $\left.J=6.9 \mathrm{~Hz}, 2 \mathrm{H}, 2 \times \mathrm{X} \mathrm{CH}\left(\mathrm{CH}_{3}\right)_{2}\right), 1.27\left(\mathrm{~d}, J=6.9 \mathrm{~Hz}, 12 \mathrm{H}, 2 \mathrm{X} \mathrm{CH}\left(\mathrm{CH}_{3}\right)_{2}\right) ;{ }^{13} \mathrm{C} \mathrm{NMR} \mathrm{(100}$ $\left.\mathrm{MHz}, \mathrm{CDCl}_{3}\right): \delta 165.6,145.1,140.8,126.7,123.9,46.3,27.5,23.4$. The characterization data slightly differ from the reported values. ${ }^{[8]}$

\section{2,6-Di-tert-butyl-4-methylphenyl 2-diazoacetate (11j)}

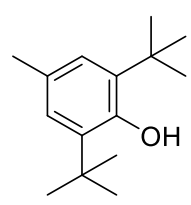

32<smiles>CC1=CC(=O)OC(C)(C)O1</smiles>

20<smiles>CC(=O)CC(=O)Oc1c(C(C)(C)C)cc(C)cc1C(C)(C)C</smiles>

33

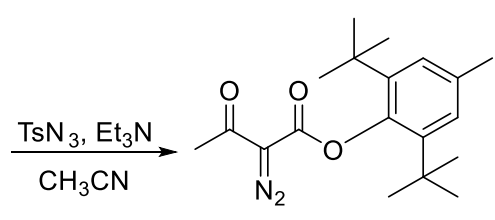

$8 \% \mathrm{KOH}$

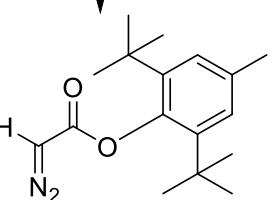

11j 
Following a slightly modified procedure, ${ }^{[4]}$ a mixture of 2,6-di-tert-butyl-4-methylphenol (32) (5.91 g, 25.0 mmol, 1.00 equiv), 2,2,6-trimethyl-4H-1,3-dioxin-4-one (20) (3.55 g, 25.0 mmol, 1.00 equiv), and xylene ( $5 \mathrm{~mL}$ ) was stirred at $140^{\circ} \mathrm{C}$ for $2 \mathrm{~h}$. After cooling to room temperature, the xylene was evaporated and the residue was purified by flash column chromatography using 1:50 EtOAc:pentane as mobile phase to afford 2,6-di-tert-butyl-4-methylphenyl 3oxobutanoate (33) as a white solid (6.40 g, $21.0 \mathrm{mmol}, 84 \%)$. Mp: 96.5-99.6 ${ }^{\circ} \mathrm{C}$; TLC (EtOAc:pentane, 1:50 v/v): $\mathrm{R}_{\mathrm{f}}=0.34, \mathrm{KMnO}_{4} ;{ }^{1} \mathrm{H} \mathrm{NMR}\left(400 \mathrm{MHz}, \mathrm{CDCl}_{3}\right): 12.16(\mathrm{~s}, 0.55 \mathrm{H}$, $\mathrm{OH}$ of enol form), $\delta 7.13$ (s, $2 \mathrm{H}, \mathrm{Ar} H$ ), 5.39-5.24 (m, $0.55 \mathrm{H}$, vinyl $H$ of enol form), 3.73 (s, $1 \mathrm{H}, 0.9 \mathrm{H}, \mathrm{CH}_{3} \mathrm{COCH}_{2}$ of keto form), 2.40 (s, $1 \mathrm{H}, 1.35 \mathrm{H}, \mathrm{CH}_{3} \mathrm{COCH}_{2}$ of keto form), 2.33 (s, $\left.3 \mathrm{H}, \mathrm{ArCH}_{3}\right), 2.07$ (s, $1.65 \mathrm{H}, \mathrm{CH}_{3}$ of enol form), 1.33 (s, 8.1H, $\mathrm{C}\left(\mathrm{CH}_{3}\right)_{3}$ of keto form), 1.32 (s, 9.9H, $\mathrm{C}\left(\mathrm{CH}_{3}\right)_{3}$ of enol form); ${ }^{13} \mathrm{C} \mathrm{NMR}\left(100 \mathrm{MHz} \mathrm{CDCl}_{3}\right): \delta 200.2,167.7,145.3,141.8,135.0$, 127.2, 50.7, 35.2, 31.4, 30.8, 21.5; Enol form, $\left.{ }^{13} \mathrm{C} \mathrm{NMR} \mathrm{(100} \mathrm{MHz,} \mathrm{CDCl}_{3}\right)$ : $\delta 177.4,173.3$, 144.9, 142.2, 134.6, 126.9, 90.4, 35.2, 31.4, 21.5, 21.5; IR v 2964 (m), 2919 (m), 2880 (w), 2110 (w), 1757 (m), 1726 (m), 1633 (s), 1408 (m), 1369 (m), 1318 (m), 1219 (s), 1199 (s), 1143 (s), 1113 (m), 1030 (w), 978 (w), 924 (w); HRMS (ESI) calcd. for $\mathrm{C}_{19} \mathrm{H}_{28} \mathrm{NaO}_{3}{ }^{+}[\mathrm{M}+\mathrm{Na}]^{+}$ 327.1931; found 327.1933.

Following a slightly modified procedure, ${ }^{[4]}$ to a solution of 2,6-di-tert-butyl-4-methylphenyl 3oxobutanoate (33) (1.52 g, $5.00 \mathrm{mmol}, 1.00$ equiv) in acetonitrile (6 mL) was added triethylamine ( $660 \mathrm{mg}, 6.50 \mathrm{mmol}, 1.30$ equiv). The reaction mixture was cooled in an ice bath and a solution of tosyl azide (1.1 g, $5.5 \mathrm{mmol}, 1.1$ equiv) in acetonitrile $(6 \mathrm{~mL})$ was added slowly. The reaction mixture was allowed to warm to room temperature. After stirring for 20 $\mathrm{h}$, the solvent was removed under reduced pressure. The residue was dissolved in ether (30 $\mathrm{mL}$ ) and washed with $8 \%$ aqueous $\mathrm{KOH}$ solution. The organic layer was separated, dried over $\mathrm{MgSO}_{4}$, and evaporated. The crude product was re-dissolved in acetonitrile (15 mL) and 8\% aqueous $\mathrm{KOH}(25 \mathrm{~mL})$ solution was added at room temperature. After vigorous stirring for 4 $\mathrm{h}$, water $(15 \mathrm{~mL})$ was added and the reaction mixture was extracted with diethyl ether $(2 \mathrm{X} 30$ $\mathrm{mL}$ ). The combined organic layers were dried over $\mathrm{MgSO}_{4}$, and concentrated under reduced pressure. The crude product was purified by column chromatography using 1:30 $\mathrm{Et}_{2} \mathrm{O}$ :pentane as mobile phase to afford 2,6-di-tert-butyl-4-methylphenyl 2-diazoacetate (11j) as a yellow solid (1.20 g, $4.16 \mathrm{mmol}, 83 \%)$. TLC (Et $2 \mathrm{O}$ :pentane, 1:30 v/v): $\mathrm{R}_{\mathrm{f}}=0.36, \mathrm{KMnO}_{4} ;{ }^{1} \mathrm{H} \mathrm{NMR}$ $\left(400 \mathrm{MHz}, \mathrm{CDCl}_{3}\right): \delta 7.12(\mathrm{~s}, 2 \mathrm{H}, \mathrm{ArH}), 5.00\left(\mathrm{~s}, 1 \mathrm{H}, \mathrm{CHN}_{2}\right), 2.32\left(\mathrm{~s}, 3 \mathrm{H}, \mathrm{ArCH}_{3}\right), 1.36(\mathrm{~s}, 18 \mathrm{H}$, $\left.2 \mathrm{X} \mathrm{C}\left(\mathrm{CH}_{3}\right)_{3}\right) ;{ }^{13} \mathrm{C} \mathrm{NMR}\left(100 \mathrm{MHz}, \mathrm{CDCl}_{3}\right): \delta 166.3,145.1,142.4,134.8,127.0,47.3,35.3$, 31.5, 21.5. The ${ }^{1} \mathrm{H}$ NMR data corresponded to the reported values. ${ }^{[5]}$ 


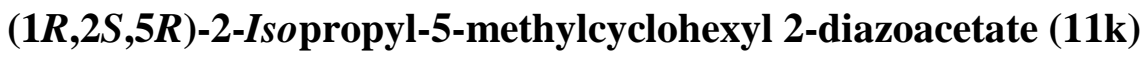

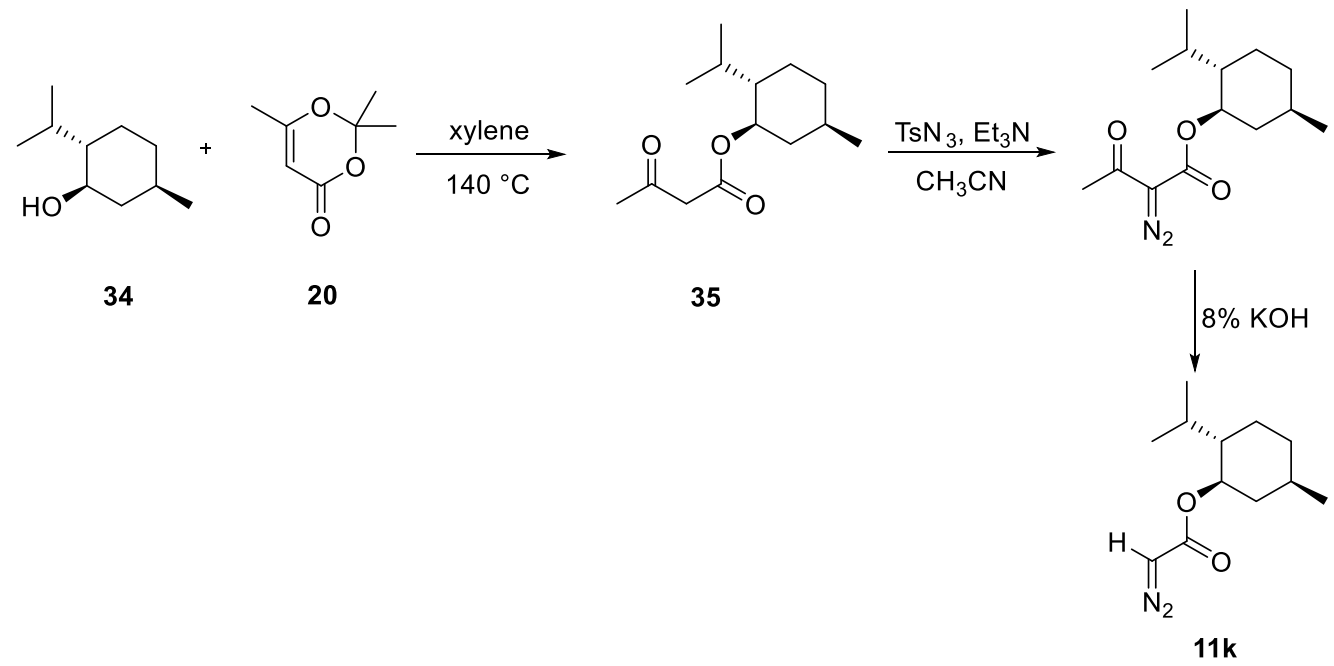

Following a slightly modified procedure, ${ }^{[4]}$ a mixture of $(1 R, 2 S, 5 R)$-2-isopropyl-5methylcyclohexanol (34) (1.95 g, 12.5 mmol, 1.00 equiv), 2,2,6-trimethyl-4H-1,3-dioxin-4one (20) (1.78 g, $12.5 \mathrm{mmol}, 1.00$ equiv), and xylene $(2.5 \mathrm{~mL})$ was stirred at $140{ }^{\circ} \mathrm{C}$ for $2 \mathrm{~h}$. After cooling to room temperature, the xylene was evaporated and the residue was purified by flash column chromatography using 1:50 EtOAc:pentane as mobile phase to afford $(1 R, 2 S, 5 R)$ 2-isopropyl-5-methylcyclohexyl 3-oxobutanoate (35) as a colorless liquid (1.70 g, $7.07 \mathrm{mmol}$, 57\%). TLC (EtOAc:pentane, 1:25 v/v): $\mathrm{R}_{\mathrm{f}}=0.3, \mathrm{KMnO}_{4} ;{ }^{1} \mathrm{H} \mathrm{NMR}\left(400 \mathrm{MHz}, \mathrm{CDCl}_{3}\right): \delta 12.18$ (s, $0.08 \mathrm{H}, \mathrm{OH}$ of enol form), 4.94 (s, $0.08 \mathrm{H}$, vinyl $H$ of enol form), 4.71 (td, $J=10.9,4.4 \mathrm{~Hz}$, $1 \mathrm{H}, \mathrm{OCH}$ ), 3.41 (s, $1.84 \mathrm{H}, \mathrm{CH}_{3} \mathrm{COCH}_{2}$ of keto form), 2.24 (s, 2.75H, $\mathrm{CH}_{3} \mathrm{COCH}_{2}$ of keto form), 2.04-1.95 (m, 1H), 1.93 (s, 0.025H, $\mathrm{CH}_{3}$ of enol form), 1.90-1.79 (m, 1H), 1.71-1.61 (m, 2H), $1.54-1.40(\mathrm{~m}, 1 \mathrm{H}), 1.39-1.29(\mathrm{~m}, 1 \mathrm{H}), 1.10-0.92(\mathrm{~m}, 2 \mathrm{H}), 0.91-0.83(\mathrm{~m}, 7 \mathrm{H}), 0.74(\mathrm{~d}, J=7.0$ $\left.\mathrm{Hz}, 3 \mathrm{H}, \mathrm{CH}_{3}\right) ;{ }^{13} \mathrm{C} \mathrm{NMR}\left(100 \mathrm{MHz}, \mathrm{CDCl}_{3}\right): \delta 200.7,166.7,75.4,50.5,46.7,40.6,34.0,31.3$, 30.0, 26.0, 23.1, 21.9, 20.7, 16.0; Enol form, ${ }^{13} \mathrm{C} \mathrm{NMR}$ (100 MHz, $\left.\mathrm{CDCl}_{3}\right)$ : $\delta$ 175.2, 172.3, 90.0, 73.6, 46.9, 40.9, 34.1, 26.2, 23.4, 22.0, 21.2, 20.6, 16.3. One carbon of enol form was not resolved at $100 \mathrm{MHz}$. The ${ }^{1} \mathrm{H}$ NMR data corresponded to the reported values. ${ }^{[9]}$

Following a slightly modified procedure, ${ }^{[9]}$ to a solution of $(1 R, 2 S, 5 R)-2$-isopropyl-5methylcyclohexyl 3-oxobutanoate (35) (0.72 g, $3.0 \mathrm{mmol}, 1.0$ equiv) in acetonitrile $(3.0 \mathrm{~mL})$ was added triethylamine $(0.33 \mathrm{~g}, 3.3 \mathrm{mmol}, 1.1$ equiv). The reaction mixture was cooled in an ice bath and a solution of tosyl azide $(0.77 \mathrm{~g}, 3.9 \mathrm{mmol}, 1.3$ equiv) in acetonitrile $(3.0 \mathrm{~mL})$ was added slowly. The reaction mixture was allowed to warm to room temperature. After stirring for $6 \mathrm{~h}$, the reaction mixture was treated with a solution of $\mathrm{LiOH} \cdot \mathrm{H}_{2} \mathrm{O}(0.38 \mathrm{~g}, 9.0 \mathrm{mmol}, 3.0$ equiv) in water $(3 \mathrm{~mL})$ and stirred for another $6 \mathrm{~h}$. The resulting mixture was extracted with diethyl ether ( 2 X $15 \mathrm{~mL})$. The combined organic layers were washed with brine $(15 \mathrm{~mL})$ and 
dried over anhydrous $\mathrm{MgSO}_{4}$. The solvent was removed under reduced pressure, and the residue was purified by silica gel column chromatography using 1:30 $\mathrm{Et}_{2} \mathrm{O}$ :pentane as mobile phase to afford (1R,2S,5R)-2-isopropyl-5-methylcyclohexyl 2-diazoacetate (11k) as a yellow solid (0.60 g, $2.7 \mathrm{mmol}, 89 \%)$. TLC (Et $2 \mathrm{O}:$ pentane, 1:30 v/v): $\mathrm{R}_{\mathrm{f}}=0.2, \mathrm{KMnO}_{4} ;{ }^{1} \mathrm{H} \mathrm{NMR}(400$ $\left.\mathrm{MHz}, \mathrm{CDCl}_{3}\right): \delta 4.80-4.67\left(\mathrm{~m}, 2 \mathrm{H}, \mathrm{CHN} \mathrm{N}_{2}\right.$ and $\left.\mathrm{OCH}\right), 2.10-1.96(\mathrm{~m}, 1 \mathrm{H}), 1.93-1.79(\mathrm{~m}, 1 \mathrm{H})$, 1.73-1.60 (m, 2H), 1.56-1.42 (m, 1H), 1.40-1.30 (m, 1H), 1.12-0.93 (m, 2H), 0.92-0.86 (m, $7 \mathrm{H}), 0.77\left(\mathrm{~d}, J=7.0 \mathrm{~Hz}, 3 \mathrm{H}, \mathrm{CH}_{3}\right) ;{ }^{13} \mathrm{C} \mathrm{NMR}\left(100 \mathrm{MHz}, \mathrm{CDCl}_{3}\right): \delta 166.6,74.7,47.1,46.2$, $41.2,34.1,31.4,26.3,23.5,22.0,20.7,16.4$. The characterization data corresponded to the reported values (except one peak in ${ }^{1} \mathrm{H}$ NMR at $\left.4.67 \mathrm{ppm}\right){ }^{[10]}$

$3 S, 8 S, 9 S, 10 R, 13 R, 14 S, 17 R)-10,13-D i m e t h y l-17-((R)-6-m e t h y l h e p t a n-2-y l)-$

2,3,4,7,8,9,10,11,12,13,14,15,16,17-tetradecahydro- $1 H$-cyclopenta[a]phenanthren-3-yl 2diazoacetate (111)

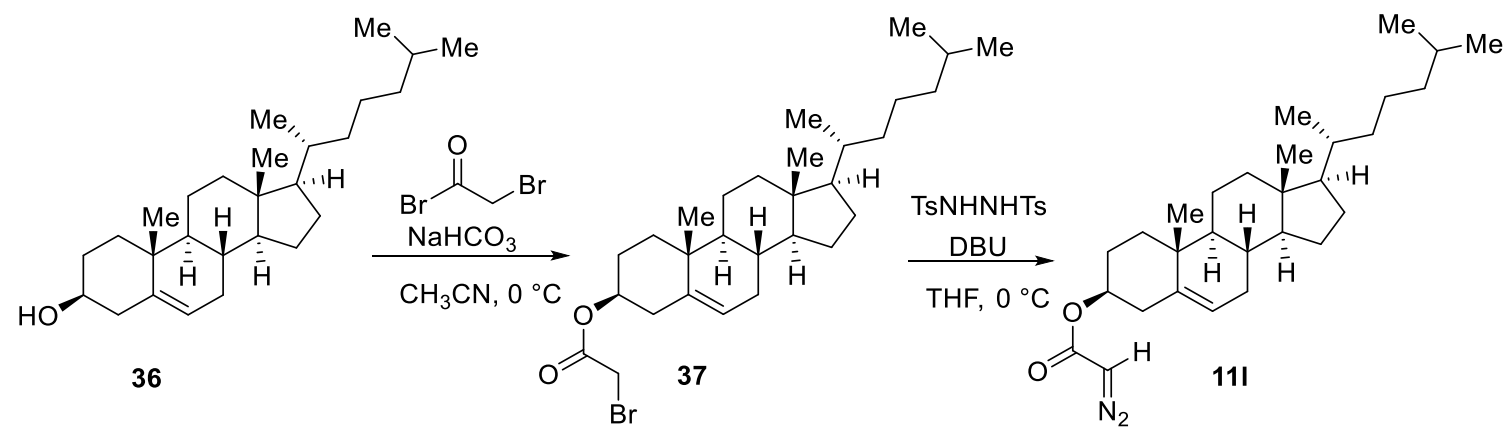

Following a reported procedure, ${ }^{[11]}$ cholesterol $36(773 \mathrm{mg}, 2.00 \mathrm{mmol}, 1.00$ equiv) and $\mathrm{NaHCO}_{3}\left(840 \mathrm{mg}, 10.0 \mathrm{mmol}, 5.00\right.$ equiv) were dissolved in dry $\mathrm{CH}_{2} \mathrm{Cl}_{2}(10 \mathrm{~mL})$ and bromoacetyl bromide $\left(0.53 \mathrm{~mL}, 6.0 \mathrm{mmol}, 3.0\right.$ equiv) was added slowly at $0{ }^{\circ} \mathrm{C}$ and stirred for $6 \mathrm{~h}$ at room temperature, the reaction was quenched with $\mathrm{H}_{2} \mathrm{O}(25 \mathrm{~mL})$ and the solution was extracted with $\mathrm{CH}_{2} \mathrm{Cl}_{2}(3 \times 50 \mathrm{~mL})$. After washing with water $(50 \mathrm{~mL})$ and drying over $\mathrm{MgSO}_{4}$, the solvent was evaporated and the residue was used in the next step without further purification. The resulting crude bromoacetamide 37 and $N, N^{\prime}$-ditosylhydrazine (1.36 g, 4.00 mmol, 2.00 equiv) were dissolved in dry THF $(10 \mathrm{~mL})$ and cooled down to $0{ }^{\circ} \mathrm{C}$, then DBU (1.5 mL, $10 \mathrm{mmol}, 5.0$ equiv) was added dropwise and stirred at room temperature for $1 \mathrm{~h}$. After quenching with saturated solution of $\mathrm{NaHCO}_{3}(20 \mathrm{~mL})$ and extracting with diethyl ether (3 X $50 \mathrm{~mL}$ ), the organic layer was dried over $\mathrm{MgSO}_{4}$, filtered and concentrated under reduced pressure. The resulting crude product was purified by flash chromatography using $\mathrm{Et}_{2} \mathrm{O}$ :pentane 1:20 as mobile phase to afford $\mathbf{1 1 l}$ as a pale yellow solid $(750 \mathrm{mg}, 1.65 \mathrm{mmol}$, $82 \%)$. TLC (Et $2 \mathrm{O}$ :pentane, 1:20 v/v): $\mathrm{R}_{\mathrm{f}}=0.4, \mathrm{KMnO}_{4} ;{ }^{1} \mathrm{H} \mathrm{NMR}\left(400 \mathrm{MHz}, \mathrm{CDCl}_{3}\right): \delta 5.38$ (d, $J=5.1 \mathrm{~Hz}, 1 \mathrm{H}$, olefinic $H), 4.75-4.65\left(\mathrm{~m}, 2 \mathrm{H}, \mathrm{N}_{2} \mathrm{CH}\right.$ and $\left.\mathrm{OCH}\right), 2.45-2.23(\mathrm{~m}, 2 \mathrm{H}), 2.08-$ 
$1.76(\mathrm{~m}, 5 \mathrm{H}), 1.64-0.80(\mathrm{~m}, 33 \mathrm{H}), 0.68(\mathrm{~s}, 3 \mathrm{H}) ;{ }^{13} \mathrm{C} \mathrm{NMR}\left(100 \mathrm{MHz}, \mathrm{CDCl}_{3}\right): \delta 166.3,139.5$, 122.8, 74.6, 56.7, 56.1, 50.0, 46.3, 42.3, 39.7, 39.5, 38.3, 36.9, 36.5, 36.2, 35.8, 31.9, 31.8, $28.2,28.0,28.0,24.3,23.8,22.8,22.5,21.0,19.3,18.7,11.8$. The characterization data corresponded to the reported values. ${ }^{[11]}$ 


\section{Synthesis of ligands}

The synthesis of ligands (15a, 15e-i) had been already described before. The procedures are taken here from the indicated publications to facilitate reproduction of the results by having all the data in the same file.

(3aR,3a' $\left.R, 8 \mathrm{a} S, 8 \mathrm{a}^{\prime} S\right)$-2,2' -(Cyclopropane-1,1-diyl)bis(8,8a-dihydro-3a $H$-indeno[1,2d]oxazole) (15a)

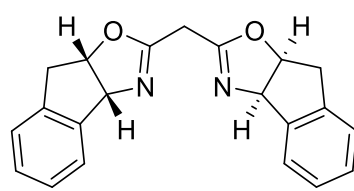

38

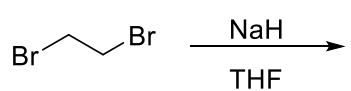

39

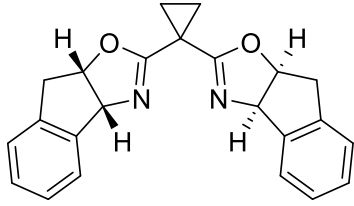

$15 a$

Following a reported procedure, ${ }^{[12]}$ to a solution of dihydrobisoxazoline $\mathbf{3 8}$ (330 mg, 1.00 mmol, 1.00 equiv) in THF (4 mL), was added $\mathrm{NaH}$ (120 mg, 60\% dispersion in paraffin liquid, $3.00 \mathrm{mmol}, 3.00$ equiv) in portions at $0{ }^{\circ} \mathrm{C}$. After complete addition, the mixture was stirred for $30 \mathrm{~min}$. at that temperature. A solution of dibromoethane (39) $(130 \mu \mathrm{L}, 1.50 \mathrm{mmol}, 1.50$ equiv) in THF ( $1 \mathrm{~mL})$ was then added dropwise at $0{ }^{\circ} \mathrm{C}$ over $10 \mathrm{~min}$. After the addition, the ice bath was removed and the reaction mixture was heated to $50^{\circ} \mathrm{C}$ for an additional $2 \mathrm{~h}$. The reaction was quenched with sat. $\mathrm{NH}_{4} \mathrm{Cl}(10 \mathrm{~mL})$ and extracted with $\mathrm{CH}_{2} \mathrm{Cl}_{2}(3 \mathrm{X} 20 \mathrm{~mL})$. The combined organic phases were dried over $\mathrm{MgSO}_{4}$, and removed under reduced pressure. The crude product was purified by chromatography on silica gel using 2\% MeOH/EtOAc followed by recrystallization (EtOAc/hexane, 1:4, $15 \mathrm{~mL}$ ) to afford (3aR,3a'R,8aS,8a'S)-2,2'(cyclopropane-1,1-diyl)bis(8,8a-dihydro-3aH-indeno[1,2-d]oxazole) (15a) (220 mg, 0.617 mmol, 62\%) as a white solid. TLC (EtOAc:MeOH, 9:1 v/v): $\mathrm{R}_{\mathrm{f}}=0.50, \mathrm{KMnO}_{4} ;{ }^{1} \mathrm{H}$ NMR (400 $\left.\mathrm{MHz}, \mathrm{CDCl}_{3}\right): \delta$ 7.48-7.41 (m, 2H, $\left.\mathrm{ArH}\right), 7.25-7.17(\mathrm{~m}, 6 \mathrm{H}, \mathrm{ArH}), 5.52(\mathrm{~d}, J=7.9 \mathrm{~Hz}, 2 \mathrm{H}, 2$ x N-CH), 5.41-5.23 (m, 2H, 2 X O-CH), $3.38\left(\mathrm{dd}, J=17.9,7.0 \mathrm{~Hz}, 2 \mathrm{H}, 2\right.$ X ArCH $\left.H_{a}\right), 3.19$ (dd, $\left.J=17.9,1.9 \mathrm{~Hz}, 2 \mathrm{H}, 2 \times \mathrm{ArCH}_{b}\right), 1.44-1.15\left(\mathrm{~m}, 4 \mathrm{H}, \mathrm{CH}_{2} \mathrm{CH} 2\right) ;{ }^{13} \mathrm{C} \mathrm{NMR}\left(100 \mathrm{MHz}, \mathrm{CDCl}_{3}\right)$ : $\delta 166.0,141.9,139.8,128.5,127.5,125.7,125.3,83.5,76.5,39.8,18.5,16.0$. The characterization data corresponded to the reported values. ${ }^{[13]}$

\section{Bis((S)-4-(tert-butyl)-4,5-dihydrooxazol-2-yl)methane (15g)}

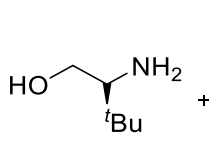

40

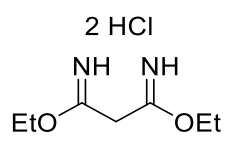

41

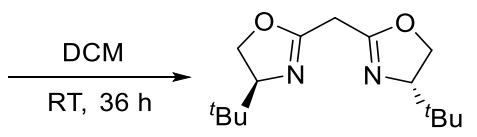

$15 \mathrm{~g}$

Following a reported procedure, ${ }^{[14]}$ to a solution of $(S)$-tert-leucinol (40) (0.94 g, $8.0 \mathrm{mmol}, 2.0$ equiv) in $\mathrm{CH}_{2} \mathrm{Cl}_{2}(40 \mathrm{~mL}$ ) was added imidate 41 (0.93 g, $4.0 \mathrm{mmol}, 1.0$ equiv). The resulting 
cloudy solution was stirred at room temperature for $36 \mathrm{~h}$. The reaction mixture was diluted with water $(8 \mathrm{~mL})$ and extracted with $\mathrm{CH}_{2} \mathrm{Cl}_{2}(3 \times 20 \mathrm{~mL})$. The combined organic layers were washed with brine $(40 \mathrm{~mL})$, dried over $\mathrm{MgSO}_{4}$, and concentrated. The resulting oily residue was distilled bulb-to-bulb (Kugelrohr distillation, $150{ }^{\circ} \mathrm{C}$ at $0.2 \mathrm{mbar}$ ) to afford bis $((S)-4-($ tertbutyl)-4,5-dihydrooxazol-2-yl)methane (15g) (0.600 g, $2.84 \mathrm{mmol}, 71 \%)$ as a white solid: TLC (EtOAc:pentane, 1:1 v/v): $\mathrm{R}_{\mathrm{f}}=0.16, \mathrm{KMnO}_{4} ;{ }^{1} \mathrm{H} \mathrm{NMR}\left(400 \mathrm{MHz}, \mathrm{CDCl}_{3}\right): \delta 4.13(\mathrm{dd}, J=$ 10.1, 8.7 Hz, 2H, $\left.2 \times \mathrm{OCH}_{a}\right), 4.02\left(\mathrm{dd}, J=8.7,7.7 \mathrm{~Hz}, 2 \mathrm{H}, 2 \mathrm{X} \mathrm{C}\left(\mathrm{CH}_{3}\right)_{3} \mathrm{CH}\right), 3.81$ (ddt, $J=$ $\left.10.1,7.8,1.1 \mathrm{~Hz}, 2 \mathrm{H}, 2 \mathrm{X} \mathrm{OCH} H_{b}\right), 3.27\left(\mathrm{t}, J=1.2 \mathrm{~Hz}, 2 \mathrm{H}, \mathrm{O}(\mathrm{C}=\mathrm{N}) \mathrm{CH}_{2}\right), 0.82(\mathrm{~s}, 18 \mathrm{H}, 2 \mathrm{X}$ $\left.\mathrm{C}\left(\mathrm{CH}_{3}\right)_{3}\right) ;{ }^{13} \mathrm{C} \mathrm{NMR}\left(100 \mathrm{MHz}, \mathrm{CDCl}_{3}\right): \delta 161.5,76.0,69.1,34.0,28.4,26.0$. The characterization data corresponded to the reported values. ${ }^{[14]}$

(4S,4'S)-2,2'-(Cyclopropane-1,1-diyl)bis(4-(tert-butyl)-4,5-dihydrooxazole) (15e)

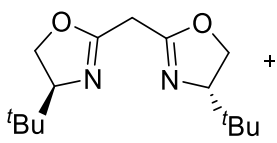

$15 \mathrm{~g}$

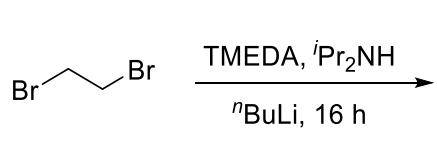

39

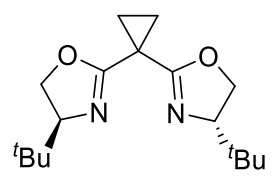

$15 e$

Following a reported procedure, ${ }^{[14]}$ to a solution of bis((S)-4-(tert-butyl)-4,5-dihydrooxazol-2yl)methane (15g) (75 mg, $0.28 \mathrm{mmol}, 1.0$ equiv) in THF (5 mL) in a $20 \mathrm{~mL}$ microwave vial, was added TMEDA ( $85 \mu \mathrm{L}, 0.56 \mathrm{mmol}, 2.0$ equiv) and $i$ - $\operatorname{Pr}_{2} \mathrm{NH}$ (40 mL, $0.28 \mathrm{mmol}, 1.0$ equiv). The solution was cooled to $-78{ }^{\circ} \mathrm{C}$ and $n$-BuLi $(0.38 \mathrm{~mL}, 1.5 \mathrm{M}$ in hexane, $0.56 \mathrm{mmol}, 2.0$ equiv) was added. The reaction mixture was warmed to $-20^{\circ} \mathrm{C}$ and stirred at that temperature for $30 \mathrm{~min}$. The solution was cooled back to $-78{ }^{\circ} \mathrm{C}$ and 1,2 dibromoethane (39) $(25 \mu \mathrm{L}, 0.28$ mmol, 2.0 equiv) was added in $10 \mathrm{~min}$. After the addition, the cold bath was removed and the reaction mixture was allowed to stir at room temperature for an additional $16 \mathrm{~h}$. The reaction mixture was quenched by the addition of sat. aq. $\mathrm{NH}_{4} \mathrm{Cl}(2.5 \mathrm{~mL})$ and diluted with water (2 $\mathrm{mL})$ to dissolve the resulting salts. The mixture was extracted with diethylether $(3 \mathrm{X} 10 \mathrm{~mL})$. The combined organic layers were washed with brine $(10 \mathrm{~mL})$, dried over $\mathrm{MgSO}_{4}$, and concentrated. The resulting oily residue was purified by column chromatography using 1:2 to 1:1 EtOAc:pentane as mobile phase to afford $(4 S, 4 ' S)$-2,2'-(cyclopropane-1,1-diyl)bis(4-(tertbutyl)-4,5-dihydrooxazole) (15e) as a white solid. TLC (EtOAc:pentane, 1:2 v/v): $\mathbf{R}_{\mathrm{f}}=0.15$, $\mathrm{KMnO}_{4} ;{ }^{1} \mathrm{H} \mathrm{NMR}\left(400 \mathrm{MHz}, \mathrm{CDCl}_{3}\right): \delta 4.18\left(\mathrm{dd}, J=10.0,8.6 \mathrm{~Hz}, 2 \mathrm{H}, 2 \mathrm{X} \mathrm{OCH}_{a}\right), 4.10(\mathrm{dd}$, $\left.J=8.7,7.3 \mathrm{~Hz}, 2 \mathrm{H}, 2 \times \mathrm{C}\left(\mathrm{CH}_{3}\right)_{3} \mathrm{CH}\right), 3.82\left(\mathrm{dd}, J=10.0,7.2 \mathrm{~Hz}, 2 \mathrm{H}, 2 \times \mathrm{OCH}_{b}\right), 1.52-1.47$ (m, 2H, $2 \mathrm{X} \mathrm{CH}_{a}$ of CyP), 1.30-1.24 (m, 2H, $2 \mathrm{X} \mathrm{CH}_{b}$ of $\left.\mathrm{CyP}\right), 0.86\left(\mathrm{~s}, 18 \mathrm{H}, 2 \mathrm{X} \mathrm{C}\left(\mathrm{CH}_{3}\right)_{3}\right)$; ${ }^{13} \mathrm{C} \mathrm{NMR}\left(100 \mathrm{MHz}, \mathrm{CDCl}_{3}\right): \delta 165.4,75.2,69.1,33.8,25.7,18.2,15.1$. The characterization data corresponded to the reported values. ${ }^{[14]}$ 
(4S,4'S)-2,2'-(Cyclopentane-1,1-diyl)bis(4-(tert-butyl)-4,5-dihydrooxazole) (15f)

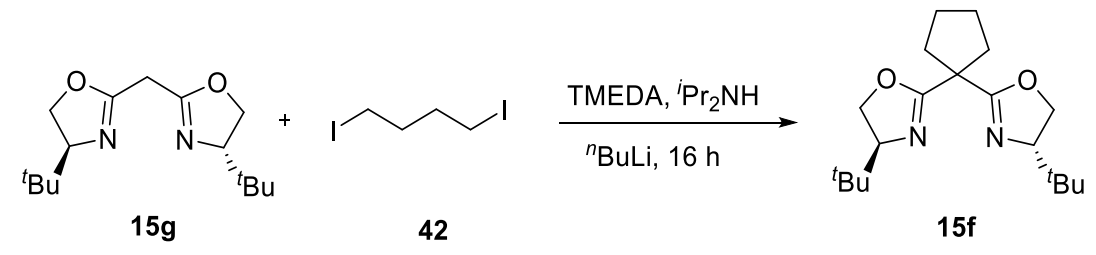

Following a reported procedure, ${ }^{[14]}$ to a solution of bis((S)-4-(tert-butyl)-4,5-dihydrooxazol-2yl)methane (15g) (75 mg, $0.28 \mathrm{mmol}, 1.0$ equiv) in THF (5 mL) in a $20 \mathrm{~mL}$ microwave vial was added TMEDA ( $85 \mu \mathrm{L}, 0.56 \mathrm{mmol}, 2.0$ equiv) and $i-\operatorname{Pr}_{2} \mathrm{NH}(40 \mathrm{~mL}, 0.28 \mathrm{mmol}, 1.0$ equiv). The solution was cooled to $-78{ }^{\circ} \mathrm{C}$ and $n$-BuLi $(0.38 \mathrm{~mL}, 1.5 \mathrm{M}$ in hexane, $0.56 \mathrm{mmol}, 2.0$ equiv) was added. The reaction mixture was warmed to $-20{ }^{\circ} \mathrm{C}$ and stirred at that temperature for $30 \mathrm{~min}$. The solution was cooled back to $-78{ }^{\circ} \mathrm{C}$ and 1,4 diiodobutane (42) $(37 \mu \mathrm{L}, 0.28$ mmol, 2.0 equiv) was added in $10 \mathrm{~min}$. After the addition. the cold bath was removed and the reaction mixture was allowed to stir at room temperature for an additional $16 \mathrm{~h}$. The reaction mixture was quenched by the addition of sat. aq. $\mathrm{NH}_{4} \mathrm{Cl}(2.5 \mathrm{~mL})$ and diluted with water (2 $\mathrm{mL})$ to dissolve the resulting salts. The mixture was extracted with diethylether $(3 \mathrm{X} 10 \mathrm{~mL})$. The combined organic layers were washed with brine $(10 \mathrm{~mL})$, dried over $\mathrm{MgSO}_{4}$, and concentrated. The resulting oily residue was purified by column chromatography using 1:4 EtOAc:pentane as mobile phase to afford (4S,4'S)-2,2'-(cyclopentane-1,1-diyl)bis(4-(tertbutyl)-4,5-dihydrooxazole) (15f) as a white solid. TLC (EtOAc:pentane, 1:2 v/v): $\mathrm{R}_{\mathrm{f}}=0.6$, $\mathrm{KMnO}_{4} ;{ }^{1} \mathrm{H}$ NMR (400 MHz, $\left.\mathrm{CDCl}_{3}\right): \delta 4.15\left(\mathrm{dd}, J=10.1,8.6 \mathrm{~Hz}, 2 \mathrm{H}, 2 \mathrm{X} \mathrm{OCH}_{a}\right), 4.07(\mathrm{dd}$, $\left.J=8.7,7.1 \mathrm{~Hz}, 2 \mathrm{H}, 2 \mathrm{X} \mathrm{C}\left(\mathrm{CH}_{3}\right)_{3} \mathrm{CH}\right), 3.84\left(\mathrm{dd}, J=10.0,7.1 \mathrm{~Hz}, 2 \mathrm{H}, 2 \mathrm{X} \mathrm{OCH}_{b}\right), 2.43-2.33$ (m, 2H, $\left.2 \mathrm{X} \mathrm{CCH}_{a} \mathrm{CH}_{2}\right), 2.20-2.05\left(\mathrm{~m}, 2 \mathrm{H}, 2 \mathrm{X} \mathrm{CCH}_{b} \mathrm{CH}_{2}\right), 1.81-1.62\left(\mathrm{~m}, 4 \mathrm{H}, 2 \mathrm{X} \mathrm{CCH}_{2} \mathrm{CH}_{2}\right.$ ), $0.86\left(\mathrm{~s}, 18 \mathrm{H}, 2 \mathrm{X} \mathrm{C}\left(\mathrm{CH}_{3}\right)_{3}\right) ;{ }^{13} \mathrm{C} \mathrm{NMR}\left(100 \mathrm{MHz}, \mathrm{CDCl}_{3}\right): \delta 168.0,75.3,69.1,49.1,35.4,33.9$, $25.7,25.0$. The characterization data corresponded to the reported values. ${ }^{[14]}$

(3aR,3a' $\left.R, 8 \mathrm{a} S, 8 \mathrm{a}^{\prime} S\right)$-2,2' -(Propane-2,2-diyl)bis(8,8a-dihydro-3a $H$-indeno[1,2-d]oxazole) (15h)

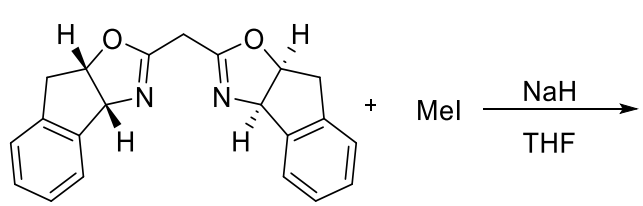

38

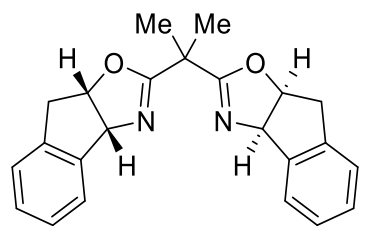

$15 \mathrm{~h}$

Following a reported procedure, ${ }^{[15]}$ a Schlenk tube was charged with dry THF (2 mL), TMEDA (46 $\mu \mathrm{L}, 0.30 \mathrm{mmol}, 2.0$ equiv) and $i-\mathrm{Pr}_{2} \mathrm{NH}(43 \mu \mathrm{L}, 0.30 \mathrm{mmol}, 2.0$ equiv). The solution was cooled to $-20{ }^{\circ} \mathrm{C}$ and $n$-BuLi $(0.20 \mathrm{~mL}, 1.5 \mathrm{M}$ in hexane, $0.30 \mathrm{mmol}, 2.0$ equiv) was added. The reaction mixture was stirred for $1 \mathrm{~h}$ at that temperature and $\mathbf{3 8}$ (50 mg, $0.15 \mathrm{mmol}, 1.0$ 
equiv) in THF ( $2 \mathrm{~mL})$ was added. The mixture was stirred for $3 \mathrm{~h}$. Then, MeI ( $38 \mu \mathrm{L}, 0.6 \mathrm{mmol}$, 4.0 equiv) was added at $-20^{\circ} \mathrm{C}$. After the addition, the cold bath was removed and the reaction mixture was heated to $60^{\circ} \mathrm{C}$ for an additional $24 \mathrm{~h}$. The solution was cooled, washed with sat. $\mathrm{NH}_{4} \mathrm{Cl}(20 \mathrm{~mL})$ and extracted with EtOAc $(3 \times 30 \mathrm{~mL})$. The combined organic phases were dried over $\mathrm{MgSO}_{4}$, and removed under reduced pressure, to afford (3aR,3a'R,8aS,8a'S)-2,2'(propane-2,2-diyl)bis(8,8a-dihydro-3aH-indeno[1,2-d]oxazole) (15h) $(53.5 \mathrm{mg}, 0.15 \mathrm{mmol}$, quant.) as a white solid. No purification was needed. TLC (EtOAc: $\mathrm{MeOH}, 9: 1 \mathrm{v} / \mathrm{v}$ ): $\mathrm{R}_{\mathrm{f}}=0.53$, $\mathrm{KMnO}_{4} ;{ }^{1} \mathrm{H}$ NMR $\left(400 \mathrm{MHz}, \mathrm{CDCl}_{3}\right): \delta$ 7.54-7.45 (m, 2H, $\left.\mathrm{ArH}\right), 7.30-7.18(\mathrm{~m}, 6 \mathrm{H}, \mathrm{ArH})$, $5.52(\mathrm{~d}, J=7.9 \mathrm{~Hz}, 2 \mathrm{H}, 2 \mathrm{X} \mathrm{N}-\mathrm{CH}), 5.28-5.25(\mathrm{~m}, 2 \mathrm{H}, 2$ X O-CH), 3.30 (dd, $J=17.9,7.1 \mathrm{~Hz}$, $\left.2 \mathrm{H}, 2 \mathrm{X} \mathrm{ArCH}_{a}\right), 2.95\left(\mathrm{dd}, J=17.9,1.9 \mathrm{~Hz}, 2 \mathrm{H}, 2 \mathrm{X} \mathrm{ArCH}_{b}\right), 1.42\left(\mathrm{~s}, 6 \mathrm{H}, 2 \mathrm{X} \mathrm{CH}_{3}\right) ;{ }^{13} \mathrm{C} \mathrm{NMR}$ $\left(100 \mathrm{MHz}, \mathrm{CDCl}_{3}\right): \delta 169.1,141.8,139.7,128.3,127.3,125.6,125.0,83.2,76.5,39.6,38.4$, 23.9. The characterization data corresponded to the reported values. ${ }^{[15]}$

\section{(4S,4'S)-2,2'-(Ethane-1,1-diyl)bis(4-(tert-butyl)-4,5-dihydrooxazole) (15i)}

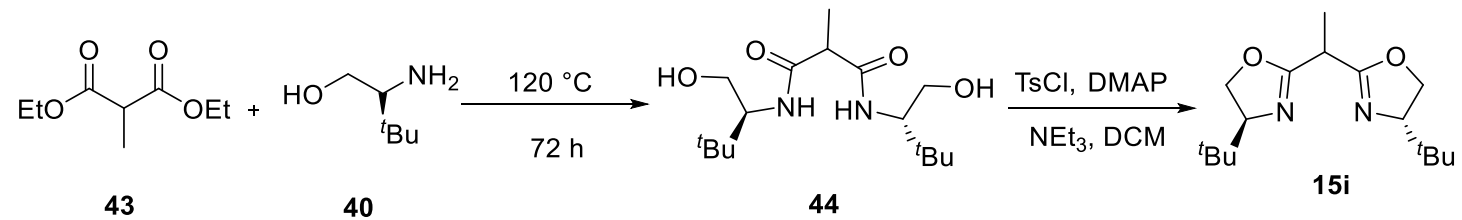

Following a reported procedure, ${ }^{[16]}$ diethyl methylmalonate $(\mathbf{4 3})(0.850 \mathrm{~mL}, 5.00 \mathrm{mmol}, 1.00$ equiv) and (S)-tert-leucinol (40) (1.23 g, $10.5 \mathrm{mmol}, 2.10$ equiv) were added to a Schlenk tube. The mixture was stirred for 3 days at $120^{\circ} \mathrm{C}$. The reaction mixture was cooled down to room temperature to obatine the product, which was used in the following step without further purification.

Following a reported procedure, ${ }^{[17]}$ a $50 \mathrm{~mL}$ Schlenk flask was charged with bis $((S)$-1-hydroxy3,3-dimethylbutan-2-yl)-2-methylmalonamide (44) (1.44 g, $4.54 \mathrm{mmol}, 1.00$ equiv), 4(dimethylamino)-pyridine (0.06 g, $0.05 \mathrm{mmol}, 0.100$ equiv), and $\mathrm{CH}_{2} \mathrm{Cl}_{2}(40 \mathrm{~mL})$. Triethylamine (3.00 mL, $21.5 \mathrm{mmol}, 4.75$ equiv) was then added. A solution of $p$ toluenesulfonyl chloride (1.88 g, $9.90 \mathrm{mmol}, 2.10$ equiv) in $\mathrm{CH}_{2} \mathrm{Cl}_{2}(10 \mathrm{~mL})$ was added slowly. The resulting bright yellow solution was stirred at room temperature for $24 \mathrm{~h}$. It was diluted with $\mathrm{CH}_{2} \mathrm{Cl}_{2}(10 \mathrm{~mL})$ and washed with sat. $\mathrm{NH}_{4} \mathrm{Cl}(15 \mathrm{~mL})$. The aqueous layer was backextracted with $\mathrm{CH}_{2} \mathrm{Cl}_{2}$ (3 X $15 \mathrm{~mL}$ ). The combined organic extracts were washed with sat. $\mathrm{NaHCO}_{3}(30 \mathrm{~mL})$. The organic layer was dried over $\mathrm{MgSO}_{4}$, filtered, and concentrated under vacuum. The crude product was purified by column chromatography using 98:2 $\mathrm{CH}_{2} \mathrm{Cl}_{2}: \mathrm{MeOH}$ to afford (4S,4'S)-2,2'-(ethane-1,1-diyl)bis(4-(tert-butyl)-4,5-dihydrooxazole) (15i) $(1.00 \mathrm{~g}, 3.57 \mathrm{mmol}, 79 \%)$ as a colorless thick liquid. TLC (EtOAc:MeOH, 9:1 v/v): $\mathrm{R}_{\mathrm{f}}=$ 
0.53, $\mathrm{KMnO}_{4} ;{ }^{1} \mathrm{H}$ NMR (400 MHz, $\left.\mathrm{CDCl}_{3}\right): \delta 4.21-4.13(\mathrm{~m}, 2 \mathrm{H}, 2 \mathrm{X} \mathrm{OCH}$ ), 4.12-4.01 (m, $\left.2 \mathrm{H}, 2 \times \mathrm{X}\left(\mathrm{CH}_{3}\right)_{3} \mathrm{CH}\right), 3.97-3.76\left(\mathrm{~m}, 2 \mathrm{H}, 2 \mathrm{X} \mathrm{OCH}_{b}\right), 3.61-3.45\left(\mathrm{~m}, 1 \mathrm{H}, \mathrm{CH}_{3} \mathrm{CH}\right), 1.46(\mathrm{~d}, J=$ $\left.7.3 \mathrm{~Hz}, 3 \mathrm{H}, \mathrm{CH}_{3} \mathrm{CH}\right), 0.88\left(\mathrm{~s}, 9 \mathrm{H}, \mathrm{C}\left(\mathrm{CH}_{3}\right)_{3}\right), 0.87$ (s, 9H, $\left.\mathrm{C}\left(\mathrm{CH}_{3}\right)_{3}\right) ;{ }^{13} \mathrm{C} \mathrm{NMR}(100 \mathrm{MHz}$, $\left.\mathrm{CDCl}_{3}\right): \delta 165.5,165.3,75.4,68.9,34.0,33.8,25.7,25.6,15.3$. The characterization data corresponded to the reported values. ${ }^{[17]}$ 


\section{Preliminary studies of EBZ reactivity}

\section{Methyl 1-oxo-2,3-dihydro-1H-indene-2-carboxylate (5)}

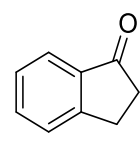

45

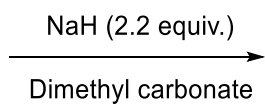

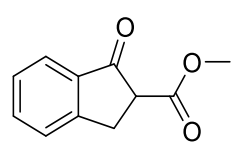

5

Following a reported procedure, ${ }^{[18]}$ a solution of 2,3-dihydro-1H-inden-1-one (45) (1.00 g, 7.57 mmol, 1.00 equiv) in dimethyl carbonate $(5 \mathrm{~mL})$ was added dropwise to a suspension of $60 \%$ $\mathrm{NaH}(0.666 \mathrm{~g}, 16.7 \mathrm{mmol}, 2.20$ equiv $)$ in dimethyl carbonate $(2 \mathrm{~mL})$ at $0{ }^{\circ} \mathrm{C}$. Once the addition complete, the solution was heated up to $80{ }^{\circ} \mathrm{C}$. After $2 \mathrm{~h}$ the reaction mixture was cooled to room temperature, and water $(35 \mathrm{~mL})$ was added. The aqueous layer was extracted with DCM ( 3 X $100 \mathrm{~mL}$ ). The combined organic layers were dried with $\mathrm{Na}_{2} \mathrm{SO}_{4}$ and the solvent was removed under reduced pressure. The crude product was purified by flash chromatography using EtOAc:pentane 1:9 as mobile phase to afford methyl 1-oxo-2,3-dihydro- $1 H$-indene-2carboxylate (5) as an orange solid (0.913 g, $4.80 \mathrm{mmol}, 64 \%) .{ }^{1} \mathrm{H}$ NMR (400 $\left.\mathrm{MHz}, \mathrm{CDCl}_{3}\right)$ : Keto-enol (6:1) $\delta 10.36$ (br s, 0.15H, OH enol), 7.78 (d, $J=7.7 \mathrm{~Hz}, 1 \mathrm{H}, \operatorname{Ar} H$ ), 7.63 (t, $J=7.4$ $\mathrm{Hz}, 1 \mathrm{H}, \operatorname{Ar} H), 7.51(\mathrm{~d}, J=7.7 \mathrm{~Hz}, 1 \mathrm{H}, \operatorname{Ar} H), 7.40(\mathrm{t}, J=7.4 \mathrm{~Hz}, 1 \mathrm{H}, \operatorname{Ar} H), 3.86(\mathrm{~s}, 0.5 \mathrm{H}$, $\mathrm{OCH}_{3}$-enol), $3.80\left(\mathrm{~s}, 3 \mathrm{H}, \mathrm{OCH}_{3}\right), 3.74(\mathrm{~d}, J=8.3 \mathrm{~Hz}, 1 \mathrm{H}, \mathrm{CHCO}), 3.55$ (d, $J=4.0 \mathrm{~Hz}, 1 \mathrm{H}$ $\left.\mathrm{CH}_{2}\right), 3.40\left(\mathrm{~d}, \mathrm{~J}=8.3 \mathrm{~Hz}, 1 \mathrm{H}, \mathrm{CH}_{2}\right)$. The ${ }^{1} \mathrm{H}$ NMR data corresponds to literature data. ${ }^{[18]}$

\section{Methyl 2-ethynyl-1-oxo-2,3-dihydro-1H-indene-2-carboxylate (6)}

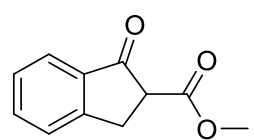

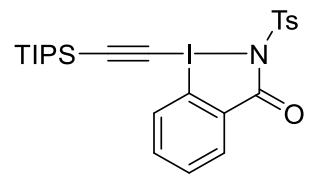

2a

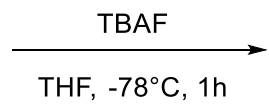

$\mathrm{THF},-78^{\circ} \mathrm{C}, 1 \mathrm{~h}$

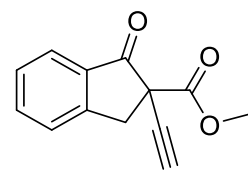

6

A solution of TIPS-Ts-EBZ (2a) (227 mg, $0.390 \mathrm{mmol}, 1.30$ equiv) and methyl 1-oxo-2,3dihydro- $1 H$-indene-2-carboxylate $(5)(57.1 \mathrm{mg}, 0.300 \mathrm{mmol}, 1.00$ equiv) was cooled down to $-78{ }^{\circ} \mathrm{C}$ and stirred for $5 \mathrm{~min}$. After this time, tetrabutylammonium fluoride ( $1 \mathrm{M}$ in THF, 0.40 $\mathrm{mL}, 0.40 \mathrm{mmol}, 1.3$ equiv) was added. After $1 \mathrm{~h}$, the solvent was removed under reduced pressure and the crude product was purified by flash chromatography using EtOAc:pentane 1:5 as mobile phase to afford methyl 2-ethynyl-1-oxo-2,3-dihydro- $1 H$-indene-2-carboxylate (6) as a yellow oil (65 mg, $0.30 \mathrm{mmol}$, quant.). ${ }^{1} \mathrm{H} \mathrm{NMR}$ (400 $\left.\mathrm{MHz}, \mathrm{CDCl}_{3}\right): \delta 7.83$ (d, $J=8.1 \mathrm{~Hz}$, 1H, ArH), $7.67(\mathrm{td}, J=7.7,1.2 \mathrm{~Hz}, 1 \mathrm{H}, \operatorname{ArH}), 7.55-7.48(\mathrm{~m}, 1 \mathrm{H}, \operatorname{Ar} H), 7.44(\mathrm{t}, J=7.5 \mathrm{~Hz}$, 1H, $\mathrm{ArH}), 3.94\left(\mathrm{~d}, J=17.1 \mathrm{~Hz}, 1 \mathrm{H}, \mathrm{CH}_{2}\right), 3.80\left(\mathrm{~s}, 3 \mathrm{H}, \mathrm{CH}_{3}\right), 3.53\left(\mathrm{~d}, J=17.1 \mathrm{~Hz}, 1 \mathrm{H}, \mathrm{CH}_{2}\right)$, 2.43 (s, $1 \mathrm{H}, \mathrm{C} \equiv \mathrm{CH})$. The ${ }^{1} \mathrm{H}$ NMR data corresponds to literature data. ${ }^{[19]}$ 
(((2-Bromophenyl)thio)ethynyl)triisopropylsilane (8)

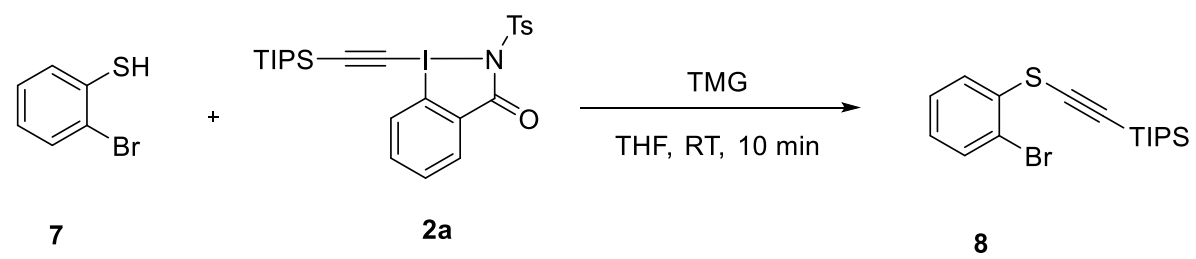

In a $10 \mathrm{~mL}$ flask TIPS-Ts-EBZ (2a) (0.206 g, $0.354 \mathrm{mmol}, 1.00$ equiv) was added in one portion to a solution of 1,1,3,3-tetramethylguanidine (41 $\mathrm{mg}, 0.35 \mathrm{mmol}, 1.0$ equiv) and 2bromobenzenethiol (7) (67 mg, $0.35 \mathrm{mmol}, 1.0$ equiv) in THF (2.5 ml). The solution was stirred at room temperature for $5 \mathrm{~min}$. The reaction was quenched with water $(5 \mathrm{~mL})$. The aqueous layer was extracted with ethyl acetate $(3 \times 10 \mathrm{~mL})$. The combined organic layers were dried with $\mathrm{Na}_{2} \mathrm{SO}_{4}$ and the solvent was removed under reduced pressure. The crude product was purified by flash chromatography using pentane as mobile phase to afford (((2bromophenyl)thio)ethynyl)triisopropylsilane (8) as a colourless oil (104 $\mathrm{mg}, 0.282 \mathrm{mmol}$, 79\%). ${ }^{1} \mathrm{H}$ NMR $\left(400 \mathrm{MHz}, \mathrm{CDCl}_{3}\right): \delta 7.67(\mathrm{dd}, J=8.0,1.5 \mathrm{~Hz}, 1 \mathrm{H}, \mathrm{Ar} H), 7.39(\mathrm{dd}, J=7.9$, $1.3 \mathrm{~Hz}, 1 \mathrm{H}, \mathrm{ArH}), 7.29-7.22$ (m, 1H, ArH), 6.98 (td, $J=7.7,1.6 \mathrm{~Hz}, 1 \mathrm{H}, \operatorname{Ar} H), 1.05$ (m, 21H, TIPS). The ${ }^{1} \mathrm{H}$ NMR data corresponds to literature data. ${ }^{[20]}$

\section{3-((Triisopropylsilyl)ethynyl)-1H-indole (10)}

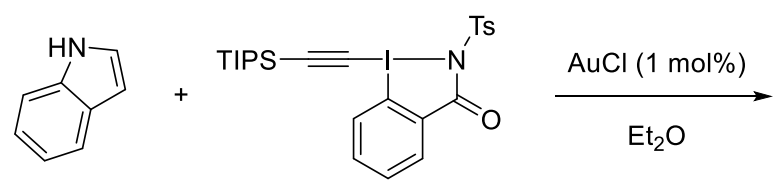

9

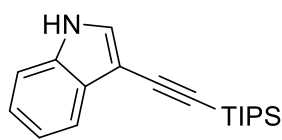

10

In a $10 \mathrm{~mL}$ flask TIPS-Ts-EBZ (2a) $(0.135 \mathrm{~g}, 0.232 \mathrm{mmol}, 1.16$ equiv) was added to a solution of gold(I) chloride ( $0.5 \mathrm{mg}, 2 \mu \mathrm{mol}, 0.01$ equiv) and indole (9) (23 mg, $0.20 \mathrm{mmol}, 1.0$ equiv) in diethylether $(3.3 \mathrm{~mL})$. The solution was stirred at room temperature for $19 \mathrm{~h}$. The reaction mixture was diluted with $\mathrm{Et}_{2} \mathrm{O}(5 \mathrm{~mL})$, washed with $0.1 \mathrm{M} \mathrm{NaOH}(2 \times 5 \mathrm{~mL})$. The combined aqueous layers were back-extracted with $\mathrm{Et}_{2} \mathrm{O}(5 \mathrm{~mL})$. The combined organic layers were washed with saturated $\mathrm{NaHCO}_{3}$ solution $(5 \mathrm{~mL})$, brine $(5 \mathrm{~mL})$, dried with $\mathrm{Na}_{2} \mathrm{SO}_{4}$. The solvent was removed under reduced pressure. The crude product was purified by flash chromatography using $\mathrm{Et}_{2} \mathrm{O}$ :pentane 1:4 as mobile phase to afford 3-((triisopropylsilyl)ethynyl)-1H-indole (10) as colorless oil (44 mg, $0.15 \mathrm{mmol}, 74 \%) .{ }^{1} \mathrm{H} \mathrm{NMR}$ (400 MHz, $\mathrm{CDCl}_{3}$ ): 8.05 (s, $1 \mathrm{H}, \mathrm{ArH}$ ), $7.79(\mathrm{~m}, 1 \mathrm{H}, \operatorname{Ar} H), 7.38$ (d, J=2.6 Hz, 1H, ArH), 7.34 (m, 1H, ArH), 7.28 - 7.24 (m, 2H, ArH), $1.22(\mathrm{~m}, 21 \mathrm{H}, \mathrm{TIPS})$. The ${ }^{1} \mathrm{H}$ NMR data corresponds to literature data. ${ }^{[21]}$ 


\section{Optimization of the reaction conditions.}

\section{a) Screening of copper catalysts}

A flame dried $5 \mathrm{~mL}$ microwave vial was charged under nitrogen with catalyst $(2.00 \mu \mathrm{mol}, 0.02$ equiv), ligand 12 (2.25 $\mu \mathrm{mol}, 0.025$ equiv) and dry DCE $(0.5 \mathrm{~mL})$. The resulting solution was stirred at room temperature for $30 \mathrm{~min}$. To this solution was added a mixture of $N$-[tosyl]-1[phenylethynyl]-1,2-benziodazol-3(1H)-one (2b) (0.10 mmol, 1.0 equiv) and ethyl 2diazoacetate (11a) $(0.20 \mathrm{mmol}, 2.0$ equiv) in dry DCE $(1.5 \mathrm{~mL})$ in $2 \mathrm{~min}$ and the resulting reaction mixture was stirred at $60{ }^{\circ} \mathrm{C}$ until the reaction was completed (monitored by TLC, EtOAc:pentane, 1:4 v/v), the solvent was evaporated under reduced pressure and the crude product was purified by column chromatography (EtOAc:pentane, 1:4 v/v) directly without any further work-up.
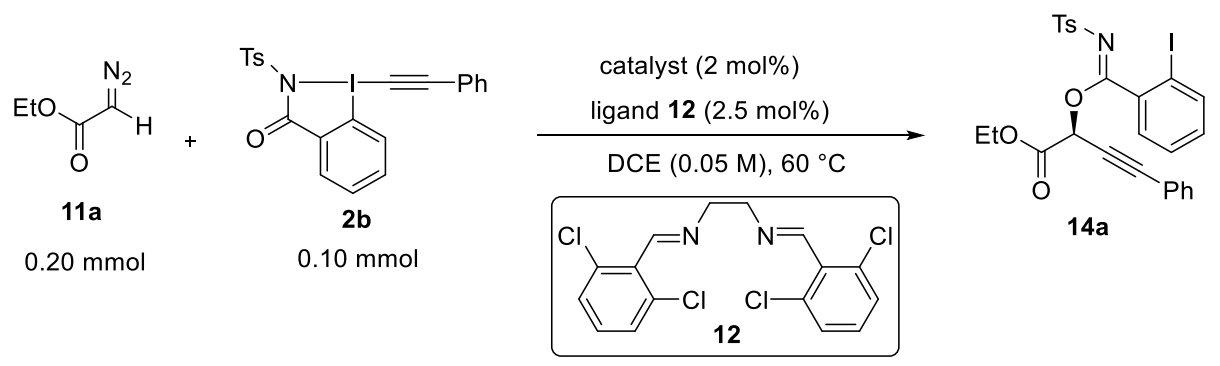

\begin{tabular}{cccc}
\hline Entry & Catalyst & Time & Yield \\
\hline 1 & $\mathrm{Cu}(\mathrm{OTf})_{2}$ & $2.5 \mathrm{~h}$ & $24 \%$ \\
2 & $\mathrm{CuOTf}$ & $2.5 \mathrm{~h}$ & $27 \%$ \\
$\mathbf{3}$ & $\mathrm{CuOAc}$ & $\mathbf{2 . 0 ~ h}$ & $\mathbf{3 8 \%}$ \\
4 & $\mathrm{Cu}(\mathrm{OAc})_{2}$ & $2.0 \mathrm{~h}$ & $7 \%$ \\
5 & $\mathrm{CuCl}$ & $3.0 \mathrm{~h}$ & $9 \%$ \\
6 & $\mathrm{AuBr}_{3}$ & $16 \mathrm{~h}$ & $<5 \%$ \\
7 & $\mathrm{AuCl}$ & $18 \mathrm{~h}$ & $<5 \%$ \\
8 & $\mathrm{Rh}(\mathrm{OAc})_{2}$ & $18 \mathrm{~h}$ & $<5 \%$ \\
\hline
\end{tabular}

\section{b) Screening of ligands}

A flame dried $5 \mathrm{~mL}$ microwave vial was charged under nitrogen with $\mathrm{CuOAc}(2.00 \mu \mathrm{mol}, 0.02$ equiv), ligand 15 ( $2.25 \mu \mathrm{mol}, 0.025$ equiv) and dry DCE $(0.5 \mathrm{~mL})$. The resulting solution was stirred at room temperature for $30 \mathrm{~min}$. To this solution was added a mixture of $N$-[tosyl]-1[phenylethynyl]-1,2-benziodazol-3(1H)-one (2b) (0.10 mmol, 1.0 equiv) and ethyl 2diazoacetate (11a) $(0.20 \mathrm{mmol}, 2.0$ equiv) in dry DCE $(1.5 \mathrm{~mL})$ in $2 \mathrm{~min}$ and the resulting reaction mixture was stirred at $60{ }^{\circ} \mathrm{C}$ until the reaction was completed (monitored by TLC, EtOAc:pentane, 1:4 v/v), the solvent was evaporated under reduced pressure and the crude 
product was purified by column chromatography (EtOAc:pentane, 1:4 v/v) directly without any further work-up.

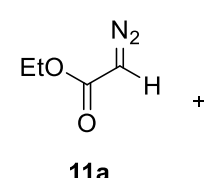

$0.20 \mathrm{mmol}$

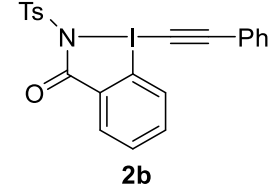

$0.10 \mathrm{mmol}$

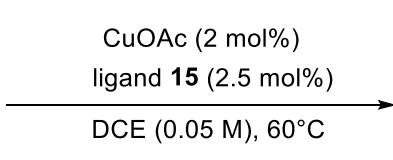

DCE $(0.05 \mathrm{M}), 60^{\circ} \mathrm{C}$

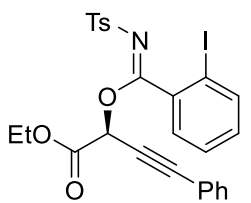

$14 a$

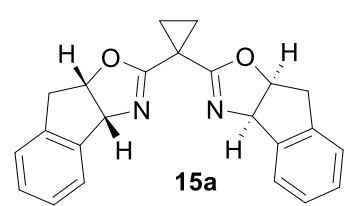

$1 \mathrm{~h}, 55 \%, 33 \%$ ee

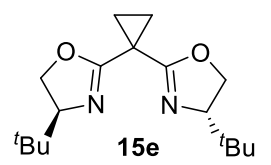

$5 \min , 46 \%, 37 \%$ ee

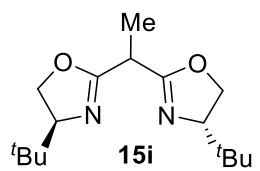

5 h, $49 \%, 27 \%$ ee

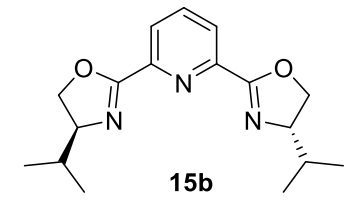

$15 \min , 20 \%$, $10 \%$ ee
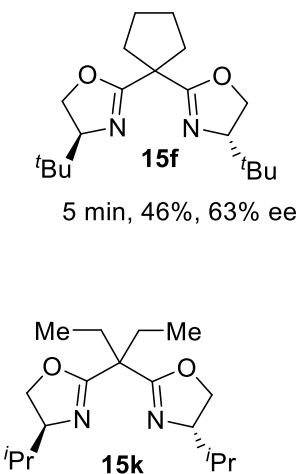

$1 \mathrm{~h}, 41 \%,,<5 \%$ ee

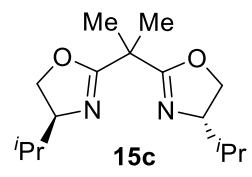

$3 \mathrm{~h}, 55 \%, 40 \%$ ee

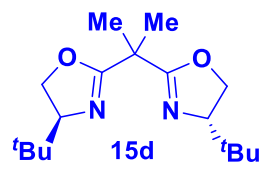

$0.5 \mathrm{~h}, 65 \%, 69 \%$ ee

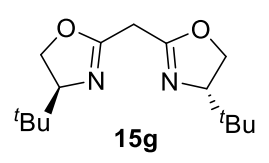

$1 \mathrm{~h}, 25 \%,<5 \%$ ee

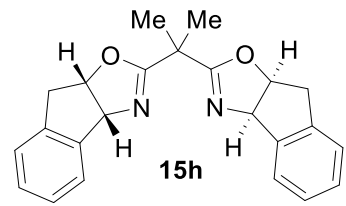

2 h, $38 \%, 10 \%$ ee

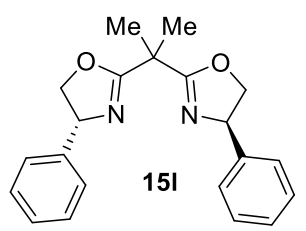

$1.5 \mathrm{~h}, 41 \%, 70 \%$ ee

\section{c) Screening of solvents}

A flame dried $5 \mathrm{~mL}$ microwave vial was charged under nitrogen with $\mathrm{CuOAc}(2.00 \mu \mathrm{mol}, 0.02$ equiv), ligand 15d $(2.25 \mu \mathrm{mol}, 0.025$ equiv) and solvent $(0.5 \mathrm{~mL})$. The resulting solution was stirred at room temperature for $30 \mathrm{~min}$. To this solution was added a mixture of $N$-[tosyl]-1[phenylethynyl]-1,2-benziodazol-3(1H)-one (2b) (0.10 mmol, 1.0 equiv) and ethyl 2diazoacetate (11a) $(0.20 \mathrm{mmol}, 2.0$ equiv) in solvent $(1.5 \mathrm{~mL})$ in $2 \mathrm{~min}$ and the resulting reaction mixture was stirred until the reaction was completed (monitored by TLC, EtOAc:pentane, 1:4 v/v), the solvent was evaporated under reduced pressure and the crude product was purified by column chromatography (EtOAc:pentane, 1:4 v/v) directly without any further work-up. 

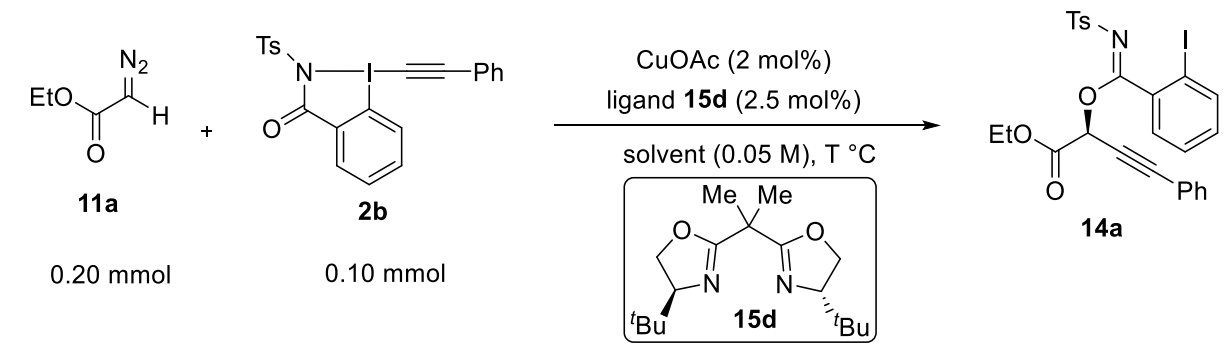

\begin{tabular}{cccccc}
\hline Entry & solvent & $\mathbf{T}^{\circ} \mathbf{C}$ & Time & Yield (\%) & ee \\
\hline 1 & DCE & $60{ }^{\circ} \mathrm{C}$ & $0.5 \mathrm{~h}$ & 65 & 69 \\
\hline $\mathbf{3}$ & $\mathrm{DCM}$ & $\mathrm{RT}$ & $1.0 \mathrm{~h}$ & 70 & 31 \\
4 & $\mathbf{D C M}$ & $\mathbf{3 5}{ }^{\circ} \mathbf{C}$ & $\mathbf{1 . 0 ~ h}$ & $\mathbf{6 8}$ & $\mathbf{6 8}$ \\
5 & $\mathrm{CHCl}_{3}$ & $30^{\circ} \mathrm{C}$ & $1.0 \mathrm{~h}$ & 43 & 63 \\
6 & $\mathrm{CH}_{3} \mathrm{CN}$ & $60^{\circ} \mathrm{C}$ & $1.0 \mathrm{~h}$ & $<5$ & $<5$ \\
7 & xylene & $60{ }^{\circ} \mathrm{C}$ & $1.0 \mathrm{~h}$ & 29 & 59 \\
8 & cholorobenzene & $60^{\circ} \mathrm{C}$ & $1.0 \mathrm{~h}$ & 49 & 53 \\
\hline
\end{tabular}

\section{d) Replacing the tosyl group with mesitylsulfonyl group on the nitrogen atom}

A flame dried $5 \mathrm{~mL}$ microwave vial was charged under nitrogen with $\mathrm{CuOAc}(2.00 \mu \mathrm{mol}, 0.02$ equiv), ligand 15d (2.25 $\mu \mathrm{mol}, 0.025$ equiv) and dry DCM $(0.5 \mathrm{~mL})$. The resulting solution was stirred at room temperature for $30 \mathrm{~min}$. To this solution was added a mixture of $\mathrm{N}-[$ mesitylsulfonyl]-1-[phenylethynyl]-1,2-benziodazol-3(1H)-one (2d) (0.10 mmol, 1.0 equiv) and ethyl 2-diazoacetate (11a) $(0.20 \mathrm{mmol}, 2.0$ equiv) in dry DCM (1.5 mL) in $2 \mathrm{~min}$ and the resulting reaction mixture was stirred until the reaction was completed (monitored by TLC, EtOAc:pentane, 1:7 v/v), the solvent was evaporated under reduced pressure and the crude product was purified by column chromatography (EtOAc:pentane, 1:7 v/v) directly without any further work-up.

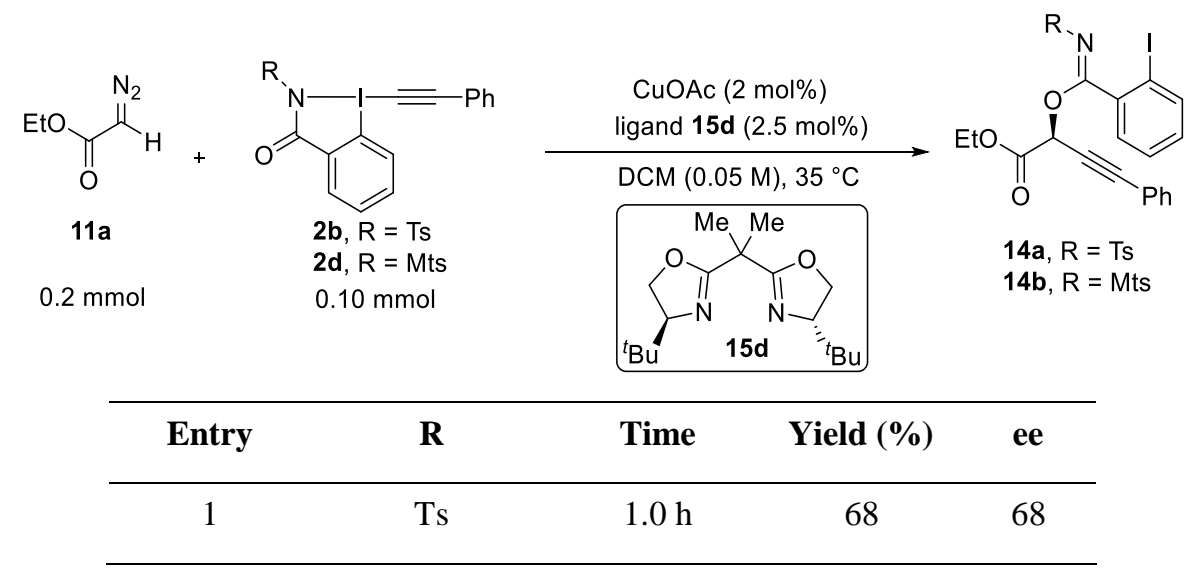




\begin{tabular}{lllll}
\hline 2 & Mts & $24 \mathrm{~h}$ & 81 & 80 \\
\hline
\end{tabular}

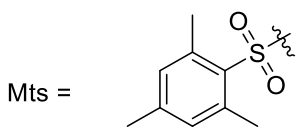

\section{e) Screening of solvents using Ph-Mts-EBZ (2d)}

A flame dried $5 \mathrm{~mL}$ microwave vial was charged under nitrogen with $\mathrm{CuOAc}(2.00 \mu \mathrm{mol}, 0.02$ equiv), ligand $15 d$ ( $2.25 \mu \mathrm{mol}, 0.025$ equiv) and solvent $(0.5 \mathrm{~mL})$. The resulting solution was stirred at room temperature for $30 \mathrm{~min}$. To this solution was added a mixture of $\mathrm{N}$-[ mesitylsulfonyl]-1-[phenylethynyl]-1,2-benziodazol-3(1H)-one (2d) $(0.10 \mathrm{mmol}, 1.0$ equiv) and ethyl 2-diazoacetate (11a) $(0.20 \mathrm{mmol}, 2.0$ equiv) in solvent $(1.5 \mathrm{~mL})$ in $2 \mathrm{~min}$ and the resulting reaction mixture was stirred until the reaction was completed (monitored by TLC, EtOAc:pentane, 1:7 v/v), the solvent was evaporated under reduced pressure and the crude product was purified by column chromatography (EtOAc:pentane, 1:7 v/v) directly without any further work-up.

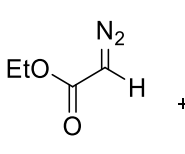

$11 \mathrm{a}$

$0.20 \mathrm{mmol}$

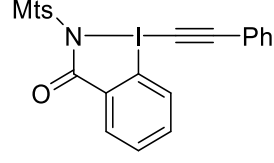

2d

$0.10 \mathrm{mmol}$
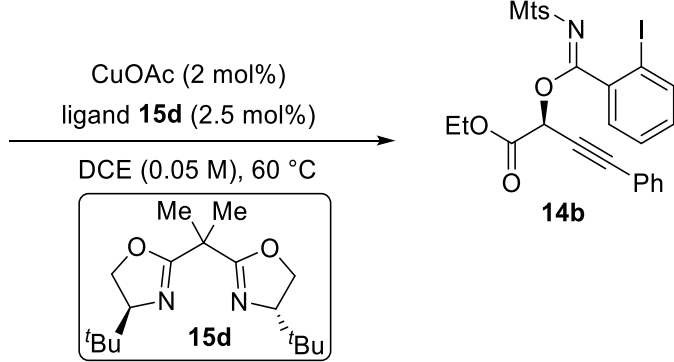

\begin{tabular}{cccccc}
\hline Entry & Solvent & T ${ }^{\circ} \mathbf{C}$ & Time & Yield (\%) & ee \\
\hline 1 & DCE & $60^{\circ} \mathrm{C}$ & $1.0 \mathrm{~h}$ & 76 & 79 \\
\hline 2 & cholorobenzene & $60^{\circ} \mathrm{C}$ & $6.0 \mathrm{~h}$ & 25 & 69 \\
\hline 3 & $o$-dicholorobenzene & $60^{\circ} \mathrm{C}$ & $3.0 \mathrm{~h}$ & 63 & 72 \\
\hline $\mathbf{4}$ & DCM & $\mathbf{R T}$ & $\mathbf{2 4 ~ h}$ & $\mathbf{8 1}$ & $\mathbf{8 0}$
\end{tabular}




\section{f) Screening of copper catalysts using Ph-Mts-EBZ (2d)}

A flame dried $5 \mathrm{~mL}$ microwave vial was charged under nitrogen with catalyst $(2.00 \mu \mathrm{mol}, 0.02$ equiv), ligand 15d (2.25 $\mu \mathrm{mol}, 0.025$ equiv) and dry DCM $(0.5 \mathrm{~mL})$. The resulting solution was stirred at room temperature for $30 \mathrm{~min}$. To this solution was added a mixture of $\mathrm{N}$ [mesitylsulfonyl]-1-[phenylethynyl]-1,2-benziodazol-3(1H)-one (2d) $(0.10 \mathrm{mmol}, 1.0$ equiv) and ethyl 2-diazoacetate (11a) $(0.20 \mathrm{mmol}, 2.0$ equiv) in dry DCM $(1.5 \mathrm{~mL})$ in $2 \mathrm{~min}$ and the resulting reaction mixture was stirred until the reaction was completed (monitored by TLC, EtOAc:pentane, 1:7 v/v), the solvent was evaporated under reduced pressure and the crude product was purified by column chromatography (EtOAc:pentane, 1:7 v/v) directly without any further work-up.
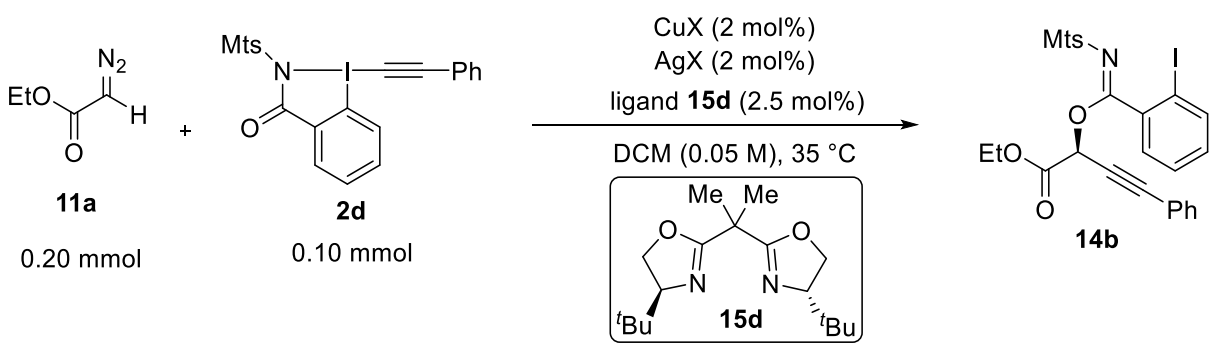

\begin{tabular}{|c|c|c|c|c|}
\hline Entry & CuX/AgX & Time & Yield (\%) & ee \\
\hline 1 & $\mathrm{CuCl} / \mathrm{AgNTf}_{2}$ & $1.0 \mathrm{~h}$ & 10 & 60 \\
\hline 2 & $\mathrm{CuCl} / \mathrm{AgOTf}$ & $1.0 \mathrm{~h}$ & 10 & 33 \\
\hline 3 & $\mathrm{CuCl} / \mathrm{AgBF}_{4}$ & $0.5 \mathrm{~h}$ & $<5$ & nd \\
\hline 4 & $\mathrm{CuCl} / \mathrm{AgPF}_{6}$ & $0.5 \mathrm{~h}$ & $<5$ & nd \\
\hline 5 & $\mathrm{CuCl} / \mathrm{AgSbF}_{6}$ & $0.5 \mathrm{~h}$ & $<5$ & nd \\
\hline 6 & $\mathrm{CuCl} / \mathrm{AgClO}_{4}$ & $24 \mathrm{~h}$ & 90 & 82 \\
\hline 7 & $\mathrm{CuCl} / \mathrm{AgOTs}$ & $2.0 \mathrm{~h}$ & 91 & 82 \\
\hline 8 & $\mathrm{CuBr} / \mathrm{NaBARF}$ & $20 \mathrm{~h}$ & $<5$ & nd \\
\hline 9 & $\mathrm{CuBr} / \mathrm{KOPiv}$ & $18 \mathrm{~h}$ & 76 & 78 \\
\hline
\end{tabular}

\section{g) Screening of temperatures using Ph-Mts-EBZ (2d)}

A flame dried $5 \mathrm{~mL}$ microwave vial was charged under nitrogen with $\mathrm{CuCl}(2.00 \mu \mathrm{mol}, 0.02$ equiv), AgOTs ( $2.00 \mu \mathrm{mol}, 0.02$ equiv), ligand 15d ( $2.25 \mu \mathrm{mol}, 0.025$ equiv) and dry DCM $(0.5 \mathrm{~mL})$. The resulting solution was stirred at room temperature for $30 \mathrm{~min}$. To this solution was added a mixture of $\mathrm{N}$-[mesitylsulfonyl]-1-[phenylethynyl]-1,2-benziodazol-3(1H)-one (2d) (0.10 mmol, 1.0 equiv) and ethyl 2-diazoacetate (11a) $(0.20 \mathrm{mmol}, 2.0$ equiv) in dry DCM $(1.5 \mathrm{~mL})$ in $2 \mathrm{~min}$ and the resulting reaction mixture was stirred until the reaction was 
completed (monitored by TLC, EtOAc:pentane, 1:7 v/v), the solvent was evaporated under reduced pressure and the crude product was purified by column chromatography (EtOAc:pentane, 1:7 v/v) directly without any further work-up.
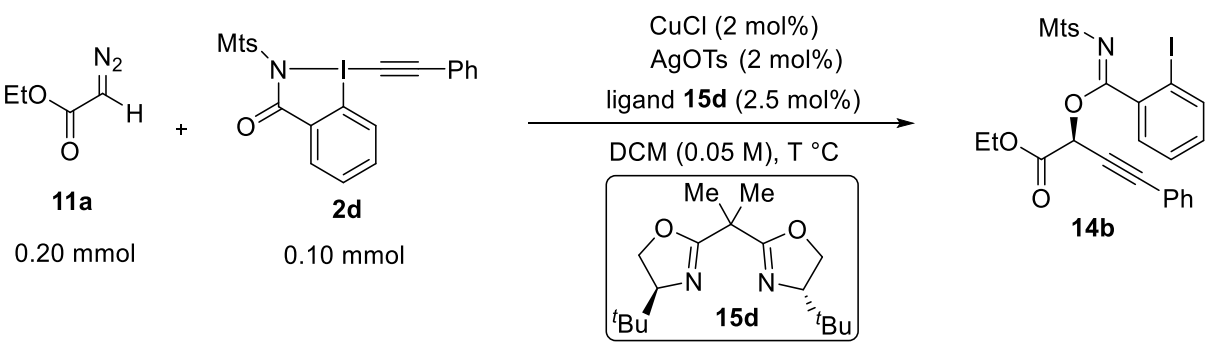

\begin{tabular}{ccccc}
\hline Entry & Temperature & Time & Yield (\%) & ee \\
\hline 1 & $35^{\circ} \mathrm{C}$ & $2.0 \mathrm{~h}$ & 91 & 82 \\
2 & $\mathbf{R T}$ & $\mathbf{2 . 0 ~ h}$ & $\mathbf{9 3}$ & $\mathbf{8 8}$ \\
3 & $0{ }^{\circ} \mathrm{C}$ & $20 \mathrm{~h}$ & 90 & 88
\end{tabular}

\section{h) Screening of catalyst loading}

A flame dried $5 \mathrm{~mL}$ microwave vial was charged under nitrogen with $\mathrm{CuCl}$ (x mol\%), AgOTs (x mol\%), ligand 15d (2.25 $\mu \mathrm{mol}, 0.025$ equiv) and dry DCM $(0.5 \mathrm{~mL})$. The resulting solution was stirred at room temperature for $30 \mathrm{~min}$. To this solution was added a mixture of $\mathrm{N}$ [mesitylsulfonyl]-1-[phenylethynyl]-1,2-benziodazol-3(1H)-one (2d) (0.10 mmol, 1.0 equiv) and ethyl 2-diazoacetate (11a) $(0.20 \mathrm{mmol}, 2.0$ equiv) in dry DCM $(1.5 \mathrm{~mL})$ in $2 \mathrm{~min}$ and the resulting reaction mixture was stirred until the reaction was completed (monitored by TLC, EtOAc:pentane, 1:7 v/v), the solvent was evaporated under reduced pressure and the crude product was purified by column chromatography (EtOAc:pentane, 1:7 v/v) directly without any further work-up.

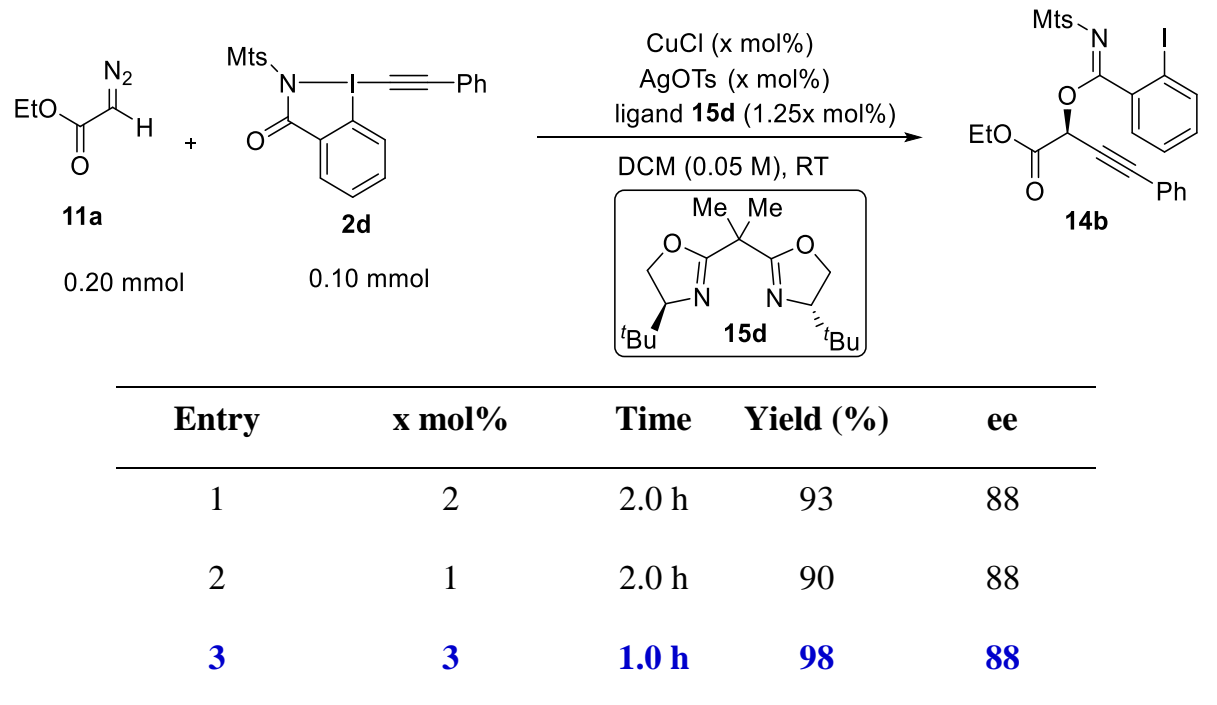


7. Copper catalyzed oxy-alkynylation using EBZ reagents

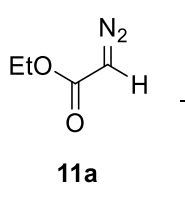

$0.20 \mathrm{mmol}$

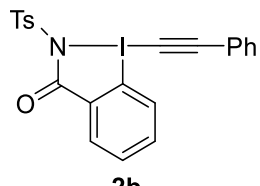

$0.10 \mathrm{mmol}$
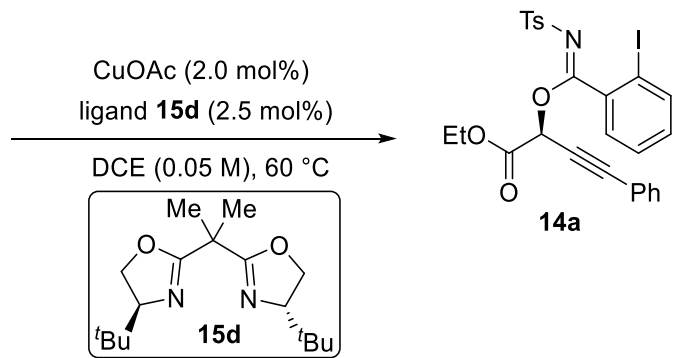

A flame dried $5 \mathrm{~mL}$ microwave vial was charged under nitrogen with $\mathrm{CuOAc}(0.3 \mathrm{mg}, 2 \mu \mathrm{mol}$, 0.02 equiv), and ligand $\mathbf{1 5 d}(0.8 \mathrm{mg}, 3 \mu \mathrm{mol}, 0.03$ equiv) and dry DCE ( $1 \mathrm{~mL})$. The resulting solution was stirred at room temperature for $1 \mathrm{~h}$ and then added to a mixture of Ph-Ts-EBZ (2b) (50 mg, $0.10 \mathrm{mmol}, 1.0$ equiv) and diazo compound 11a ( $25 \mu \mathrm{L}, 0.20 \mathrm{mmol}, 2.0$ equiv) in dry DCE $(1 \mathrm{~mL})$ in $2 \mathrm{~min}$ and the resulting reaction mixture was stirred $60{ }^{\circ} \mathrm{C}$. After $1 \mathrm{~h}$, the solvent was evaporated under reduced pressure and the crude product was purified by flash chromatography using EtOAc:pentane 1:4 as mobile phase to afford 14a as a pale yellow oil (38.0 mg, 0.065mmol, 65\%). TLC (EtOAc:Pentane 1:4): $\mathrm{R}_{\mathrm{f}}=0.28, \mathrm{KMnO}_{4} ;{ }^{1} \mathrm{H} \mathrm{NMR}$ (400 $\left.\mathrm{MHz}, \mathrm{CDCl}_{3}\right): \delta 7.84(\mathrm{~d}, J=8.0 \mathrm{~Hz}, 1 \mathrm{H}, \mathrm{Ar} H), 7.73(\mathrm{~d}, J=8.3 \mathrm{~Hz}, 2 \mathrm{H}, \mathrm{Ar} H), 7.49-7.43(\mathrm{~m}$, 2H, ArH), $7.43-7.38$ (m, 2H, ArH), $7.36-7.32(\mathrm{~m}, 1 \mathrm{H}, \operatorname{Ar} H), 7.31(\mathrm{~d}, J=7.5 \mathrm{~Hz}, 2 \mathrm{H}, \operatorname{Ar} H)$, $7.23(\mathrm{~d}, J=8.1 \mathrm{~Hz}, 2 \mathrm{H}, \operatorname{Ar} H), 7.21-7.15(\mathrm{~m}, 1 \mathrm{H}, \mathrm{Ar} H), 6.04(\mathrm{~s}, 1 \mathrm{H}, \mathrm{C} \equiv \mathrm{CH}), 4.23$ (qd, $J=$ 7.2, $\left.4.0 \mathrm{~Hz}, 2 \mathrm{H}, \mathrm{OCH}_{2} \mathrm{CH}_{3}\right), 2.40\left(\mathrm{~s}, 3 \mathrm{H}, \mathrm{Ar}-\mathrm{CH}_{3}\right), 1.29$ (t, $\left.J=7.1 \mathrm{~Hz}, 3 \mathrm{H}, \mathrm{OCH}_{2} \mathrm{CH}_{3}\right) ;{ }^{13} \mathrm{C}$ NMR (100 MHz, $\left.\mathrm{CDCl}_{3}\right): \delta 168.0,164.6,143.7,138.9,137.6,137.2,132.0,131.8,129.3$, 129.2, 128.2, 127.6, 127.4, 121.2, 92.6, 88.6, 79.4, 67.4, 62.7, 21.6, 14.0; IR v 3063 (w), 2983 (w), 2926 (w), 2867 (w), 2239 (w), 1755 (s), 1714 (w), 1626 (s), 1581 (m), 1492 (w), 1464 (m), 1334 (s), 1285 (s), 1255 (m), 1190 (m), 1168 (s), 1152 (s), 1092 (s), 1022 (m), 993 (w), 914 (w), 816 (w); HRMS (ESI) calcd. for $\mathrm{C}_{26} \mathrm{H}_{23} \mathrm{INO}_{5} \mathrm{~S}^{+}[\mathrm{M}+\mathrm{H}]^{+} 588.0336$; found 588.0345; Chiral HPLC conditions: $e e=69 \%$; Chiralpak IB 80:20 Hexane $/ i \mathrm{PrOH}, 0.8 \mathrm{~mL} / \mathrm{min}, 31 \mathrm{~min}$. $\mathrm{t}_{\mathrm{r}}($ minor $)=15.4$ min. and $\mathrm{t}_{\mathrm{r}}($ major $)=19.5 \min . \lambda=254 \mathrm{~cm}^{-1} ;[\alpha]_{\mathrm{D}}{ }^{25.0}=+20.2\left(\mathrm{c}=0.5, \mathrm{CHCl}_{3}\right)$. One carbon was not resolved at $100 \mathrm{MHz}$.

General procedure 3 (GP3)

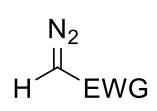

11

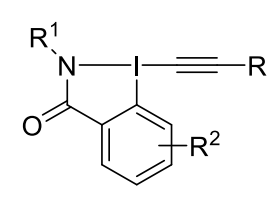

2

( $0.30 \mathrm{mmol}, 2.0$ equiv) $(0.15 \mathrm{mmol}, 1.0$ equiv)

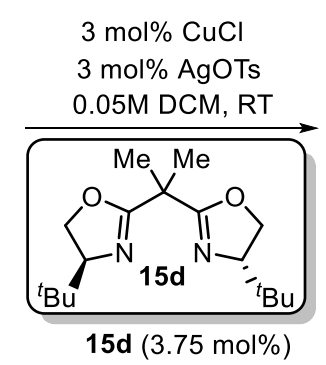<smiles>[R]C#C[C@@H]([14CH2][R])OC(=N[R1])c1ccccc1I</smiles> 
A flame dried $5 \mathrm{~mL}$ microwave vial was charged under nitrogen with $\mathrm{CuCl}(0.3 \mathrm{mg}, 3 \mu \mathrm{mol}$, 0.03 equiv), AgOTs ( $0.9 \mathrm{mg}, 3 \mu \mathrm{mol}, 0.03$ equiv), ligand $\mathbf{1 5 d}(1.1 \mathrm{mg}, 3.8 \mu \mathrm{mol}, 0.0375$ equiv) and dry dichloromethane $(1 \mathrm{~mL})$. The resulting solution was stirred at room temperature for 1 $\mathrm{h}$ and then added to a mixture of R-EBZ 2 ( $0.1 \mathrm{mmol}, 1.0$ equiv) and diazo compound 11 (0.20 mmol, 2.0 equiv) in dry dichloromethane $(1 \mathrm{~mL})$ in 2 min and the resulting reaction mixture was stirred at room temperature. After the reaction was completed (monitored by TLC, EtOAc;pentane or $\mathrm{Et}_{2} \mathrm{O}$ :pentane), the solvent was evaporated under reduced pressure and the crude product was purified by column chromatography (EtOAc:pentane or $\mathrm{Et}_{2} \mathrm{O}$ :pentane) directly without any further work-up.

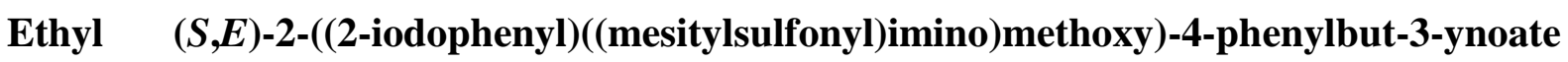
(14b)

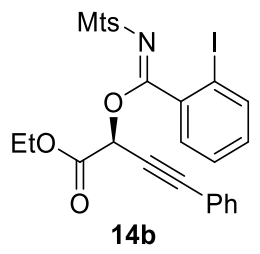

Following GP3, $N$-[mesitylsulfonyl]-1-[phenylethynyl]-1,2-benziodazol-3(1H)-one (2d) (53 $\mathrm{mg}, 0.10 \mathrm{mmol}, 1.0$ equiv) and ethyl 2-diazoacetate (11a) $(25 \mu \mathrm{L}, 0.20 \mathrm{mmol}, 13 \mathrm{wt}$ \% dichloromethane, 2.0 equiv) were stirred for $18 \mathrm{~h}$. The crude reaction mixture was concentrated under reduced pressure and purified by flash chromatography using EtOAc:pentane 1:7 as mobile phase to afford $\mathbf{1 4 b}$ as a pale yellow solid (60.0 mg, $0.097 \mathrm{mmol}, 97 \%)$. Mp: 51.4-56.1 ${ }^{\circ} \mathrm{C}$; TLC (EtOAc:Pentane 1:7): $\mathrm{R}_{\mathrm{f}}=0.35, \mathrm{KMnO}_{4} ;{ }^{1} \mathrm{H} \mathrm{NMR}\left(400 \mathrm{MHz}, \mathrm{CDCl}_{3}\right): \delta 7.85$ (d, $J=$ $7.9 \mathrm{~Hz}, 1 \mathrm{H}, \mathrm{ArH}), 7.46-7.28$ (m, 7H, ArH), $7.22-7.13$ (m, 1H, ArH), 6.89 (s, 2H, ArH), 6.07 (s, 1H, OCH), $4.29-4.14\left(\mathrm{~m}, 2 \mathrm{H}, \mathrm{CH}_{2} \mathrm{CH}_{3}\right), 2.55$ (s, 6H, $\left.2 \mathrm{X} \mathrm{ArCH}_{3}\right), 2.28$ (s, 3H, $\left.\mathrm{ArCH}_{3}\right)$, $1.28\left(\mathrm{t}, J=7.1 \mathrm{~Hz}, 3 \mathrm{H}, \mathrm{CH}_{2} \mathrm{CH}_{3}\right) ;{ }^{13} \mathrm{C} \mathrm{NMR}\left(100 \mathrm{MHz}, \mathrm{CDCl}_{3}\right): \delta 168.1,164.7,142.5,139.6$, 139.03, 137.4, 134.9, 132.1, 131.8, 131.6, 129.6, 129.3, 128.3, 127.5, 121.2, 92.6, 88.5, 79.6, 67.1, 62.8, 22.8, 21.0, 14.0; IR v 3059 (w), 2981 (w), 2938 (w), 2236 (w), 1763 (m), $1621(\mathrm{~s})$, 1580 (m), 1493 (w), 1464 (w), 1331 (s), 1282 (s), 1254 (m), 1194 (m), 1165 (s), 1144 (s), 1104 (w), 1017 (m), 999 (m), 952 (w); HRMS (ESI) calcd. for $\mathrm{C}_{28} \mathrm{H}_{26} \mathrm{INNaO}_{5} \mathrm{~S}^{+}[\mathrm{M}+\mathrm{Na}]^{+}$638.0469; found 638.0474; Chiral HPLC conditions: $e e=88 \%$; Chiralpak IA 80:20 Hexane/iPrOH, 1.0 $\mathrm{mL} / \mathrm{min}, 31 \mathrm{~min} . \mathrm{t}_{\mathrm{r}}($ minor $)=13.8 \mathrm{~min}$. and $\mathrm{t}_{\mathrm{r}}($ major $)=16.1 \mathrm{~min} . \lambda=250 \mathrm{~cm}^{-1} ;[\alpha]_{\mathrm{D}}{ }^{25.0}=+28.8$ $\left(\mathrm{c}=0.5, \mathrm{CHCl}_{3}\right)$. 


\section{Benzyl (S,E)-2-((2-iodophenyl)((mesitylsulfonyl)imino)methoxy)-4-phenylbut-3-ynoate}

(14c)

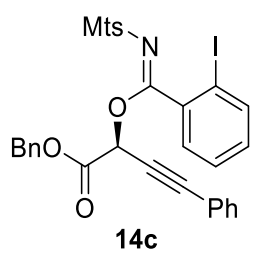

Following GP3, $N$-[mesitylsulfonyl]-1-[phenylethynyl]-1,2-benziodazol-3(1H)-one (2d) (53 $\mathrm{mg}, 0.10 \mathrm{mmol}, 1.0$ equiv) and benzyl 2-diazoacetate (11b) $(34 \mu \mathrm{L}, 0.20 \mathrm{mmol}, 10 \mathrm{wt}$. \% dichloromethane, 2.0 equiv) were stirred for $20 \mathrm{~h}$. The crude reaction mixture was concentrated under reduced pressure and purified by flash chromatography using $\mathrm{Et}_{2} \mathrm{O}$ :pentane 1:4 as mobile phase to afford $14 \mathrm{c}$ as a pale yellow solid (56.0 $\mathrm{mg}, 0.083 \mathrm{mmol}, 83 \%)$. Mp: $73.3-75.8{ }^{\circ} \mathrm{C}$; TLC (Et $2 \mathrm{O}:$ Pentane 1:4): $\mathrm{R}_{\mathrm{f}}=0.17, \mathrm{KMnO}_{4} ;{ }^{1} \mathrm{H}$ NMR (400 MHz, $\left.\mathrm{CDCl}_{3}\right): \delta 7.83(\mathrm{~d}, J=8.0$ $\mathrm{Hz}, 1 \mathrm{H}, \mathrm{ArH}), 7.40-7.30(\mathrm{~m}, 12 \mathrm{H}, \operatorname{Ar} H), 7.16(\mathrm{td}, J=7.8,1.8 \mathrm{~Hz}, 1 \mathrm{H}, \operatorname{Ar} H), 6.87(\mathrm{~s}, 2 \mathrm{H}$, $\mathrm{ArH}), 6.13(\mathrm{~s}, 1 \mathrm{H}, \mathrm{OCH}), 5.23\left(\mathrm{~d}, J=12.2 \mathrm{~Hz}, 1 \mathrm{H}, \mathrm{CH}^{a}{ }_{2} \mathrm{Ph}\right), 5.12\left(\mathrm{~d}, J=12.2 \mathrm{~Hz}, 1 \mathrm{H}, \mathrm{CH}^{b}{ }_{2} \mathrm{Ph}\right)$, 2.55 (s, 6H, $\left.2 \times \mathrm{XrCH}_{3}\right), 2.27$ (s, 3H, $\left.\mathrm{ArCH}_{3}\right) ;{ }^{13} \mathrm{C} \mathrm{NMR}\left(100 \mathrm{MHz}, \mathrm{CDCl}_{3}\right): \delta 168.1,164.5$, $142.5,139.5,139.0,137.3,134.9,134.6,132.1,131.7,131.6,129.6,129.4,128.6,128.6,128.3$, 128.3, 127.4, 121.1, 92.6, 88.7, 79.4, 68.2, 67.0, 22.8, 21.0; IR v $3066(\mathrm{w}), 3032(\mathrm{w}), 2938(\mathrm{w})$, 2237 (w), 1762 (s), 1622 (s), 1580 (m), 1460 (m), 1330 (s), 1282 (s), 1254 (s), 1189 (m), 1146 (s), $1021(\mathrm{~m}), 854(\mathrm{w})$; HRMS (ESI) calcd. for $\mathrm{C}_{33} \mathrm{H}_{28} \mathrm{INNaO}_{5} \mathrm{~S}^{+}[\mathrm{M}+\mathrm{Na}]^{+} 700.0625$; found 700.0635; Chiral HPLC conditions: $e e=80 \%$; Chiralpak IB 90:10 Hexane/ $i \mathrm{PrOH}, 1.0 \mathrm{~mL} / \mathrm{min}$, 31 min. $\mathrm{t}_{\mathrm{r}}($ minor $)=13.4 \mathrm{~min}$. and $\mathrm{t}_{\mathrm{r}}($ major $)=14.4 \mathrm{~min} . \lambda=254 \mathrm{~cm}^{-1} ;[\alpha]_{\mathrm{D}}{ }^{25.0}=+13.2(\mathrm{c}=0.5$, $\left.\mathrm{CHCl}_{3}\right)$.

Tert-butyl (S,E)-2-((2-iodophenyl)((mesitylsulfonyl)imino)methoxy)-4-phenylbut-3ynoate (14d)

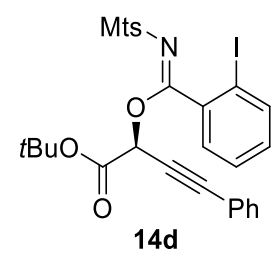

Following GP3, $N$-[mesitylsulfonyl]-1-[phenylethynyl]-1,2-benziodazol-3(1H)-one (2d) (53 $\mathrm{mg}, 0.10 \mathrm{mmol}, 1.0$ equiv) and tert-butyl 2-diazoacetate (11c) $(33 \mu \mathrm{L}, 0.20 \mathrm{mmol}, 15$ wt. \% dichloromethane, 2.0 equiv) were stirred for $20 \mathrm{~h}$. The crude reaction mixture was concentrated under reduced pressure and purified by flash chromatography using EtOAc:pentane 1:7 as mobile phase to afford 14d as a pale yellow solid (39 mg, $0.06 \mathrm{mmol}, 61 \%$ ). Mp: 55.2-56.8 ${ }^{\circ} \mathrm{C}$; TLC (EtOAc:Pentane 1:7): $\mathrm{R}_{\mathrm{f}}=0.35, \mathrm{KMnO}_{4} ;{ }^{1} \mathrm{H}$ NMR $\left(400 \mathrm{MHz}, \mathrm{CDCl}_{3}\right): \delta 7.83(\mathrm{~d}, J=$ 
$8.0 \mathrm{~Hz}, 1 \mathrm{H}, \operatorname{Ar} H), 7.45-7.39(\mathrm{~m}, 4 \mathrm{H}, \mathrm{ArH}), 7.38-7.27$ (m, 3H, ArH), 7.17 (s, 1H, ArH), 6.88 (s, 2H, ArH), 5.98 (s, 1H, OCH), 2.56 (s, 6H, $\left.2 \mathrm{X} \mathrm{ArCH}_{3}\right), 2.27$ (s, 3H, ArCH $\left.H_{3}\right), 1.48$ (s, 9H, $t \mathrm{Bu}) ;{ }^{13} \mathrm{C} \mathrm{NMR}\left(100 \mathrm{MHz}, \mathrm{CDCl}_{3}\right): \delta 168.1,163.5,142.4,139.5,139.0,137.6,135.0,132.0$, 131.7, 131.6, 129.6, 129.2, 128.3, 127.4, 121.4, 92.6, 87.9, 84.1, 67.5, 53.4, 27.8, 22.9, 21.0; IR v 2979 (w), 2935 (w), 2237 (w), 1752 (m), 1621 (m), 1580 (w), 1462 (w), 1371 (m), 1330 (m), 1283 (m), 1254 (m), 1145 (s), 1043 (w), 1016 (w), 844 (w); HRMS (ESI) calcd. for $\mathrm{C}_{30} \mathrm{H}_{31} \mathrm{INO}_{5} \mathrm{~S}^{+}[\mathrm{M}+\mathrm{H}]^{+}$644.0962; found 644.0965; Chiral HPLC conditions: $e e=84 \%$; Chiralpak IA 95:5 Hexane $/ \mathrm{PrOH}, 1.0 \mathrm{~mL} / \mathrm{min}, 31 \mathrm{~min} . \mathrm{t}_{\mathrm{r}}($ minor $)=18.9 \mathrm{~min}$. and $\mathrm{t}_{\mathrm{r}}($ major $)=$ $21.4 \min . \lambda=254 \mathrm{~cm}^{-1} ;[\alpha]_{\mathrm{D}}{ }^{25.0}=+17.9\left(\mathrm{c}=0.5, \mathrm{CHCl}_{3}\right)$.

\section{2,4-Dimethylpentan-3-yl (S,E)-2-((2-iodophenyl)((mesitylsulfonyl)imino)methoxy)-4- phenylbut-3-ynoate (14e)}

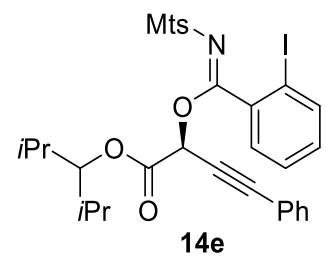

Following GP3, $N$-[mesitylsulfonyl]-1-[phenylethynyl]-1,2-benziodazol-3(1H)-one (2d) (53 $\mathrm{mg}, 0.10 \mathrm{mmol}, 1.0$ equiv) and 2,4-dimethylpentan-3-yl 2-diazoacetate (11d) (37 mg, 0.20 mmol, 2.0 equiv) were stirred for $18 \mathrm{~h}$. The crude reaction mixture was concentrated under reduced pressure and purified by flash chromatography using $\mathrm{Et}_{2} \mathrm{O}$ :pentane 1:12 as mobile phase to afford 14e as a white solid (64.0 mg, $0.093 \mathrm{mmol}, 93 \%)$ Mp: $51.4-56.8{ }^{\circ} \mathrm{C}$; TLC $\left(\mathrm{Et}_{2} \mathrm{O}:\right.$ Pentane 1:10): $\mathrm{R}_{\mathrm{f}}=0.4, \mathrm{KMnO}_{4} ;{ }^{1} \mathrm{H}$ NMR $\left(400 \mathrm{MHz}, \mathrm{CDCl}_{3}\right): \delta 7.83(\mathrm{~d}, J=7.8 \mathrm{~Hz}, 1 \mathrm{H}$, $\operatorname{ArH}), 7.45-7.27(\mathrm{~m}, 7 \mathrm{H}, \operatorname{Ar} H), 7.20-7.11(\mathrm{~m}, 1 \mathrm{H}, \operatorname{Ar} H), 6.86(\mathrm{~s}, 2 \mathrm{H}, \operatorname{Ar} H), 6.21(\mathrm{~s}, 1 \mathrm{H}$, $\mathrm{OCH}), 4.75-4.65$ (m, 1H, CH, $i \mathrm{Pr}), 2.55$ (s, 6H, $\left.2 \mathrm{X} \mathrm{ArCH}_{3}\right), 2.27$ (s, 3H, $\left.\mathrm{ArCH}_{3}\right), 2.02-1.85$ $\left(\mathrm{m}, 2 \mathrm{H}, 2 \mathrm{X} \mathrm{CHCH}_{3}\right), 0.93\left(\mathrm{dd}, J=6.8,4.2 \mathrm{~Hz}, 6 \mathrm{H}, 2 \mathrm{X} \mathrm{CHCH}_{3}\right), 0.83(\mathrm{dd}, J=18.2,6.8 \mathrm{~Hz}$, $\left.6 \mathrm{H}, 2 \mathrm{X} \mathrm{CHCH}_{3}\right) ;{ }^{13} \mathrm{C} \mathrm{NMR}\left(100 \mathrm{MHz}, \mathrm{CDCl}_{3}\right): \delta 168.4,164.6,142.4,139.4,139.0,137.4$, 135.0, 131.9, 131.7, 131.6, 129.7, 129.3, 128.3, 127.4, 121.3, 92.7, 88.1, 86.1, 66.6, 53.4, 29.6, 29.5, 22.9, 21.0, 19.5, 19.3, 17.4, 16.8; IR v 3059 (w), 2968 (m), 2238 (w), 1760 (s), 1620 (s), 1580 (m), 1465 (m), 1332 (s), 1283 (s), 1255 (m), 1203 (m), 1165 (m), 1148 (s), 1016 (m), 930 (w), 890 (w); HRMS (ESI) calcd. for $\mathrm{C}_{33} \mathrm{H}_{37} \mathrm{INO}_{5} \mathrm{~S}^{+}[\mathrm{M}+\mathrm{H}]^{+}$686.1432; found 686.1433; Chiral HPLC conditions: $e e=94 \%$; Chiralpak IA 95:5 Hexane/iPrOH, $1.0 \mathrm{~mL} / \mathrm{min}, 31 \mathrm{~min}$. $\mathrm{t}_{\mathrm{r}}$ (minor) $=16.6 \min$. and $\mathrm{t}_{\mathrm{r}}($ major $)=20.9 \min . \lambda=254 \mathrm{~cm}^{-1} ;[\alpha]_{\mathrm{D}}{ }^{25.0}=+36.0\left(\mathrm{c}=0.5, \mathrm{CHCl}_{3}\right)$. 


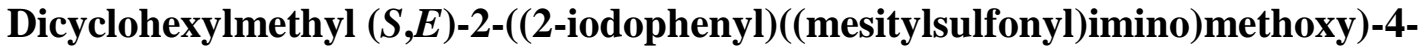 phenylbut-3-ynoate (14f)}

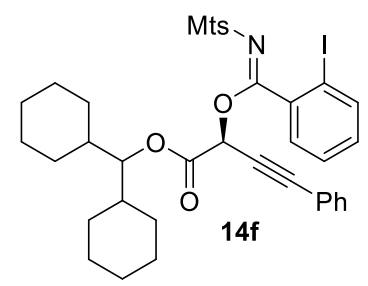

Following GP3, $N$-[mesitylsulfonyl]-1-[phenylethynyl]-1,2-benziodazol-3(1H)-one (2d) (53 $\mathrm{mg}, 0.10 \mathrm{mmol}, 1.0$ equiv) and dicyclohexylmethyl 2-diazoacetate (11e) (53 mg, $0.20 \mathrm{mmol}$, 2.0 equiv) were stirred for $20 \mathrm{~h}$. The crude reaction mixture was concentrated under reduced pressure and purified by flash chromatography using $\mathrm{Et}_{2} \mathrm{O}$ :pentane 1:4 as mobile phase to afford $14 f$ as a white solid $(60.0 \mathrm{mg}, 0.078 \mathrm{mmol}, 78 \%)$. Mp: $67.6-71.5^{\circ} \mathrm{C}$; TLC (Et $2 \mathrm{O}$ :Pentane 1:4): $\mathrm{R}_{\mathrm{f}}=0.4, \mathrm{KMnO}_{4} ;{ }^{1} \mathrm{H}$ NMR $\left(400 \mathrm{MHz}, \mathrm{CDCl}_{3}\right): \delta 7.82(\mathrm{~d}, J=7.9 \mathrm{~Hz}, 1 \mathrm{H}, \mathrm{ArH}), 7.43-$ 7.37 (m, 4H, ArH), $7.37-7.27$ (m, 3H, ArH), 7.15 (ddd, $J=8.0,6.3,2.9 \mathrm{~Hz}, 1 \mathrm{H}, \operatorname{Ar} H), 6.86$ (s, 2H, ArH), $6.22(\mathrm{~s}, 1 \mathrm{H}, \mathrm{OCH}), 4.75\left(\mathrm{t}, J=5.9 \mathrm{~Hz}, 1 \mathrm{H},(\mathrm{Cy})_{2} \mathrm{CH}\right), 2.54(\mathrm{~s}, 6 \mathrm{H}, 2 \mathrm{X} \mathrm{ArCH})_{3}$, $2.27\left(\mathrm{~s}, 3 \mathrm{H}, \mathrm{ArCH}_{3}\right), 1.79-1.59\left(\mathrm{~m}, 11 \mathrm{H}, \mathrm{Cy}-\mathrm{CH}_{2}\right), 1.25-0.95\left(\mathrm{~m}, 11 \mathrm{H}, \mathrm{Cy}-\mathrm{CH}_{2}\right) ;{ }^{13} \mathrm{C} \mathrm{NMR}$ $\left(100 \mathrm{MHz}, \mathrm{CDCl}_{3}\right): \delta 168.4,164.5,142.4,139.5,139.0,137.3,135.0,131.9,131.6,131.6$, $129.7,129.3,128.3,127.4,121.3,92.7,88.2$, 84.8, 80.5, 66.6, 53.4, 38.6, 38.3, 29.8, 29.6, 27.7, 26.9, 26.2, 26.2, 26.1, 26.0, 25.9, 22.9, 21.0; IR v 3058 (w), 2929 (s), 2853 (m), 2237 (w), 1760 (s), 1620 (s), 1580 (m), 1449 (m), 1332 (s), 1283 (s), 1255 (m), 1192 (m), 1166 (s), 1148 (s), 1045 (w), $1015 \quad(\mathrm{~m}), \quad 935$ (w), $854 \quad$ (w); HRMS $\quad$ (ESI) calcd. for $\mathrm{C}_{39} \mathrm{H}_{44} \mathrm{INNaO}_{5} \mathrm{~S}^{+}[\mathrm{M}+\mathrm{Na}]^{+} 788.1877$; found 788.1887; Chiral HPLC conditions: $e e=94 \%$; Chiralpak IA 95:5 Hexane $/ \mathrm{PrOH}, 1.0 \mathrm{~mL} / \mathrm{min}, 31 \mathrm{~min} . \mathrm{t}_{\mathrm{r}}($ minor $)=17.8 \mathrm{~min}$. and $\mathrm{t}_{\mathrm{r}}($ major $)=$ $25.5 \min . \lambda=260 \mathrm{~cm}^{-1} ;[\alpha]_{\mathrm{D}}{ }^{25.0}=+41.4\left(\mathrm{c}=0.5, \mathrm{CHCl}_{3}\right)$.

\section{2,3,4-Trimethylpentan-3-yl (S,E)-2-((2-iodophenyl)((mesitylsulfonyl)imino)methoxy)-4-} phenylbut-3-ynoate (14g)

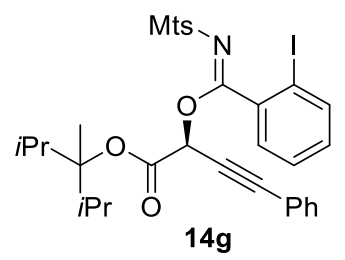

Following GP3, $\mathrm{N}$-[mesitylsulfonyl]-1-[phenylethynyl]-1,2-benziodazol-3(1H)-one (2d) (53 $\mathrm{mg}, 0.10 \mathrm{mmol}, 1.0$ equiv) and 2,3,4-trimethylpentan-3-yl 2-diazoacetate (11f) (40 mg, 0.20 mmol, 2.0 equiv) were stirred for $20 \mathrm{~h}$. The crude reaction mixture was concentrated under reduced pressure and purified by flash chromatography using $\mathrm{Et}_{2} \mathrm{O}$ :pentane 1:4 as mobile phase 
to afford $14 \mathrm{~g}$ as a white solid (64.0 $\mathrm{mg}, 0.093 \mathrm{mmol}, 93 \%)$. Mp: $131.5-132.9{ }^{\circ} \mathrm{C}$; TLC (Et $2 \mathrm{O}:$ Pentane 1:4): $\mathrm{R}_{\mathrm{f}}=0.37, \mathrm{KMnO}_{4} ;{ }^{1} \mathrm{H} \mathrm{NMR}\left(400 \mathrm{MHz}, \mathrm{CDCl}_{3}\right): \delta 7.83(\mathrm{~d}, J=7.9 \mathrm{~Hz}$, 1H, ArH), $7.47-7.27$ (m, 7H, ArH), 7.15 (d, J=1.4 Hz, 1H, ArH), 6.87 (s, 2H, ArH), 6.04 (s, $1 \mathrm{H}, \mathrm{OCH}), 2.56\left(\mathrm{~s}, 6 \mathrm{H}, 2 \mathrm{X} \mathrm{ArCH}_{3}\right), 2.29-2.17\left(\mathrm{~m}, 5 \mathrm{H}, \mathrm{ArCH}_{3}\right.$ and $\left.2 \mathrm{X} \mathrm{CHCH}_{3}\right), 1.44(\mathrm{~s}, 3 \mathrm{H}$, $\left.i \mathrm{PrCCH}_{3}\right), 0.97\left(\mathrm{~d}, J=6.9 \mathrm{~Hz}, 3 \mathrm{H}, \mathrm{CHCH}_{3}\right), 0.95\left(\mathrm{~d}, J=7.0 \mathrm{~Hz}, 3 \mathrm{H}, \mathrm{CHCH}_{3}\right), 0.90$ (d, $J=$ $\left.6.8 \mathrm{~Hz}, 6 \mathrm{H}, 2 \mathrm{X} \mathrm{CHCH}_{3}\right) ;{ }^{13} \mathrm{C} \mathrm{NMR}\left(100 \mathrm{MHz} \mathrm{CDCl}_{3}\right): \delta 168.4,163.2,142.4,139.4,139.0$, 137.5, 135.1, 131.9, 131.6, 131.6, 129.8, 129.2, 128.3, 127.4, 121.5, 95.0, 92.7, 87.8, 80.6, 67.2, 34.6, 34.4, 22.9, 21.0, 17.9, 17.9, 17.7, 17.6, 17.5; IR v 2972 (m), 2237 (w), 1757 (s), 1619 (s), 1580 (m), 1465 (m), 1380 (w), 1331 (s), 1283 (s), 1251 (m), 1204 (m), 1148 (s), 1060 (m), 1015 (m), $940(\mathrm{w})$; HRMS (ESI) calcd. for $\mathrm{C}_{34} \mathrm{H}_{38} \mathrm{INNaO}_{5} \mathrm{~S}^{+}[\mathrm{M}+\mathrm{Na}]^{+}$722.1408; found 722.1409; Chiral HPLC conditions: $e e=90 \%$; Chiralpak IA 95:5 Hexane/iPrOH, $1.0 \mathrm{~mL} / \mathrm{min}$, 31 min. $t_{r}($ minor $)=15.1 \mathrm{~min}$. and $\mathrm{t}_{\mathrm{r}}($ major $)=16.7 \mathrm{~min} . \lambda=254 \mathrm{~cm}^{-1} ;[\alpha]_{\mathrm{D}}{ }^{25.0}=+33.4(\mathrm{c}=0.5$, $\left.\mathrm{CHCl}_{3}\right)$.

Adamantan-1-yl (S,E)-2-((2-iodophenyl)((mesitylsulfonyl)imino)methoxy)-4-phenylbut3-ynoate (14h)

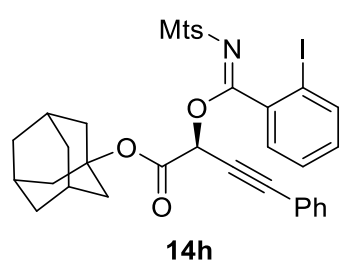

Following GP3, $N$-[mesitylsulfonyl]-1-[phenylethynyl]-1,2-benziodazol-3(1H)-one (2d) (53 mg, 0.10 mmol, 1.0 equiv) and adamantan-1-yl 2-diazoacetate (11g) (44 mg, 0.20 mmol, 2.0 equiv) were stirred for $20 \mathrm{~h}$. The crude reaction mixture was concentrated under reduced pressure and purified by flash chromatography using $\mathrm{Et}_{2} \mathrm{O}$ :pentane 1:4 as mobile phase to afford $14 \mathrm{~h}$ as a white solid $(47.0 \mathrm{mg}, 0.065 \mathrm{mmol}, 65 \%)$. Mp: 78.4-84.6 ${ }^{\circ} \mathrm{C}$; TLC (Et $2 \mathrm{O}:$ Pentane 1:4): $\mathrm{R}_{\mathrm{f}}=0.32, \mathrm{KMnO}_{4} ;{ }^{1} \mathrm{H} \mathrm{NMR}\left(400 \mathrm{MHz}, \mathrm{CDCl}_{3}\right): \delta 7.84(\mathrm{~d}, J=7.9 \mathrm{~Hz}$, 1H, ArH), 7.45 - 7.38 (m, 4H, ArH), 7.37 - 7.28 (m, 3H, ArH), 7.20 - 7.14 (m, 1H, ArH), 6.89 (s, 2H, ArH), 5.95 (s, 1H, OCH), 2.58 (s, 6H, $\left.2 \mathrm{X} \mathrm{ArCH}_{3}\right), 2.28$ (s, 3H, $\left.\mathrm{ArCH}_{3}\right), 2.15$ (s, 3H, 3 $\mathrm{X}$ Ad-CH), $2.11-1.99$ (m, 6H, 3 X Ad-CH $), 1.64$ (s, 6H, 3 X Ad-CH$\left.H_{2}\right) ;{ }^{13} \mathrm{C}$ NMR $(100 \mathrm{MHz}$, $\left.\mathrm{CDCl}_{3}\right): \delta 168.1,163.1,142.4,139.6,139.0,137.7,135.1,132.0,131.6,131.6,129.5,129.2$, 128.3, 127.4, 121.5, 92.7, 87.9, 84.0, 80.2, 67.6, 40.9, 35.9, 30.9, 22.9, 21.0; IR v 3057 (w), 2914 (m), 2854 (w), 2236 (w), 1753 (m), 1620 (s), 1580 (m), 1460 (m), 1330 (s), 1283 (s), 1254 (s), 1192 (s), 1166 (s), 1147 (s), 1049 (s), 1015 (m), 856 (w); HRMS (ESI) calcd. for $\mathrm{C}_{36} \mathrm{H}_{37} \mathrm{INO}_{5} \mathrm{~S}^{+}[\mathrm{M}+\mathrm{H}]^{+} 722.1432$; found 722.1427; Chiral HPLC conditions: $e e=94 \%$; 
Chiralpak IA 90:10 Hexane/iPrOH, $1.0 \mathrm{~mL} / \mathrm{min}, 31 \mathrm{~min} . \mathrm{t}_{\mathrm{r}}$ (major) $=16.8 \mathrm{~min}$. and $\mathrm{t}_{\mathrm{r}}$ (minor) $=21.7 \min . \lambda=254 \mathrm{~cm}^{-1} ;[\alpha]_{\mathrm{D}}{ }^{25.0}=+8.1\left(\mathrm{c}=0.5, \mathrm{CHCl}_{3}\right)$.

2-Phenylpropan-2-yl (S,E)-2-((2-iodophenyl)((mesitylsulfonyl)imino)methoxy)-4phenylbut-3-ynoate (14i)

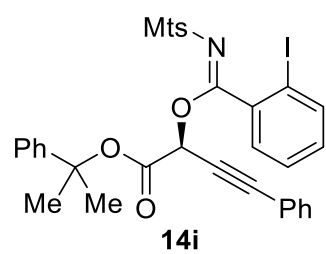

Following GP3, $N$-[mesitylsulfonyl]-1-[phenylethynyl]-1,2-benziodazol-3(1H)-one (2d) (53 $\mathrm{mg}, 0.10 \mathrm{mmol}, 1.0$ equiv) and 2-phenylpropan-2-yl 2-diazoacetate (11h) (41 mg, $0.20 \mathrm{mmol}$, 2.0 equiv) were stirred for $20 \mathrm{~h}$. The crude reaction mixture was concentrated under reduced pressure and purified by flash chromatography using $\mathrm{Et}_{2} \mathrm{O}$ :pentane 1:4 as mobile phase to afford 14i as a white solid $(66.0 \mathrm{mg}, 0.094 \mathrm{mmol}, 94 \%)$. Mp: 86.8-87.7 ${ }^{\circ} \mathrm{C}$; TLC (Et $2 \mathrm{O}$ :Pentane 1:2): $\mathrm{R}_{\mathrm{f}}=0.35, \mathrm{KMnO}_{4} ;{ }^{1} \mathrm{H} \mathrm{NMR}\left(400 \mathrm{MHz}, \mathrm{CDCl}_{3}\right): \delta 7.81(\mathrm{~d}, J=8.3 \mathrm{~Hz}, 1 \mathrm{H}, \mathrm{ArH}), 7.48-$ $7.27(\mathrm{~m}, 12 \mathrm{H}, \operatorname{Ar} H), 7.14$ (ddd, $J=8.0,6.7,2.5 \mathrm{~Hz}, 1 \mathrm{H}, \operatorname{Ar} H), 6.87$ (s, 2H, ArH), 6.08 (s, 1H, $\mathrm{OCH}), 2.55$ (s, 6H, $\left.2 \mathrm{X} \mathrm{ArCH}_{3}\right), 2.28\left(\mathrm{~s}, 3 \mathrm{H}, \mathrm{ArCH}_{3}\right), 1.83\left(\mathrm{~s}, 3 \mathrm{H}, \mathrm{OCCH}_{3}\right), 1.76(\mathrm{~s}, 3 \mathrm{H}$, $\left.\mathrm{OCCH}_{3}\right) ;{ }^{13} \mathrm{C} \mathrm{NMR}\left(100 \mathrm{MHz}, \mathrm{CDCl}_{3}\right): \delta 168.2,162.6,144.6,142.5,139.6,138.9,137.4$, 135.0, 132.0, 131.6, 131.6, 129.6, 129.3, 128.4, 127.4, 124.3, 121.3, 92.6, 88.4, 85.0, 80.0, 67.4, 53.4, 29.1, 27.4, 22.9, 21.0; IR v 3059 (w), 2981 (w), 2938 (w), 2236 (w), 1763 (m), 1621 (s), 1580 (m), 1493 (w), 1464 (w), 1331 (s), 1282 (s), 1254 (m), 1194 (m), 1165 (s), 1143 (s), 1104 (w), 1017 (m), 995 (m), 952 (w), 943 (w), 852 (w); HRMS (ESI) calcd. for $\mathrm{C}_{35} \mathrm{H}_{32} \mathrm{INNaO}_{5} \mathrm{~S}^{+}[\mathrm{M}+\mathrm{Na}]^{+} 728.0938$; found 728.0948; Chiral HPLC conditions: $e e=94 \%$; Chiralpak IA 90:10 Hexane/iPrOH, $1.0 \mathrm{~mL} / \mathrm{min}, 31 \mathrm{~min} . \mathrm{t}_{\mathrm{r}}$ (minor) $=15.3 \mathrm{~min}$. and $\mathrm{t}_{\mathrm{r}}$ (major) $=17.5 \min . \lambda=254 \mathrm{~cm}^{-1} ;[\alpha]_{\mathrm{D}}{ }^{25.0}=+40.8\left(\mathrm{c}=0.5, \mathrm{CHCl}_{3}\right)$. One carbon was not resolved at 100 $\mathrm{MHz}$.

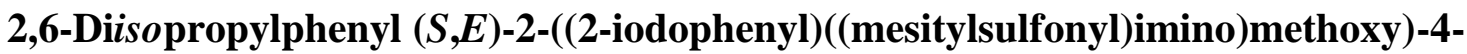
phenylbut-3-ynoate $(\mathbf{1 4 j})$

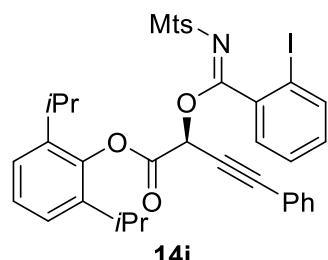

Following GP3, $N$-[mesitylsulfonyl]-1-[phenylethynyl]-1,2-benziodazol-3(1H)-one (2d) (53 $\mathrm{mg}, 0.10 \mathrm{mmol}, 1.0$ equiv) and 2,6-diisopropylphenyl 2-diazoacetate (11i) (49.5 mg, 0.200 mmol, 2.00 equiv) were stirred for $18 \mathrm{~h}$. The crude reaction mixture was concentrated under 
reduced pressure and purified by flash chromatography using $\mathrm{Et}_{2} \mathrm{O}$ :pentane 1:6 as mobile phase to afford $\mathbf{1 4} \mathbf{j}$ as a white solid (76 $\mathrm{mg}, 0.10 \mathrm{mmol}$, quant.). Mp: 73.6-82.0 ${ }^{\circ} \mathrm{C}$; TLC (Et $2 \mathrm{O}:$ Pentane 1:6): $\mathrm{R}_{\mathrm{f}}=0.21, \mathrm{KMnO}_{4} ;{ }^{1} \mathrm{H} \mathrm{NMR}\left(400 \mathrm{MHz}, \mathrm{CDCl}_{3}\right): \delta 7.82(\mathrm{~d}, J=8.0 \mathrm{~Hz}$, 1H, ArH), $7.49-7.44$ (m, 2H, ArH), $7.43-7.32$ (m, 5H, ArH), 7.24 (d, J=7.6 Hz, 1H, ArH), $7.22-7.12(\mathrm{~m}, 3 \mathrm{H}, \mathrm{ArH}), 6.88(\mathrm{~s}, 2 \mathrm{H}, \mathrm{ArH}), 6.53(\mathrm{~s}, 1 \mathrm{H}, \mathrm{CH}), 3.19-3.03(\mathrm{~m}, 2 \mathrm{H}, 2 \mathrm{X}$ $\left.\left.\mathrm{CH}\left(\mathrm{CH}_{3}\right)_{2}\right), 2.56(\mathrm{~s}, 6 \mathrm{H}, 2 \mathrm{X} \mathrm{ArCH})_{3}\right), 2.28\left(\mathrm{~s}, 3 \mathrm{H}, \mathrm{ArCH}_{3}\right), 1.17(\mathrm{~d}, J=6.8 \mathrm{~Hz}, 12 \mathrm{H}, 2 \mathrm{X}$ $\left.\mathrm{CH}\left(\mathrm{CH}_{3}\right)_{2}\right) ;{ }^{13} \mathrm{C} \mathrm{NMR}\left(100 \mathrm{MHz}, \mathrm{CDCl}_{3}\right): \delta 168.3,163.3,145.0,142.6,140.5,139.5,139.0$, 137.0, 134.9, 132.0, 131.8, 131.67, 129.9, 129.6, 128.4, 127.4, 127.1, 124.1, 121.1, 92.7, 89.0, 79.5, 66.4, 27.3, 23.5, 22.9, 21.0; IR v 3062 (w), 2966 (w), 2870 (w), 2237 (w), 1779 (m), 1619 (s), 1579 (w), 1465 (w), 1332 (s), 1281 (m), 1252 (m), 1166 (s), 1142 (s), 1094 (w), 1040 (w), 1000 (m), 939 (w), 851 (w); HRMS (ESI) calcd. for $\mathrm{C}_{38} \mathrm{H}_{38} \mathrm{INNaO}_{5} \mathrm{~S}^{+}[\mathrm{M}+\mathrm{Na}]^{+} 770.1408$; found 770.1421; Chiral HPLC conditions: $e e=80 \%$; Chiralpak IA 80:20 Hexane/ $i \mathrm{PrOH}, 1.0$ $\mathrm{mL} / \mathrm{min}, 31 \mathrm{~min} . \mathrm{t}_{\mathrm{r}}($ minor $)=8.6 \mathrm{~min}$. and $\mathrm{t}_{\mathrm{r}}($ major $)=17.9 \mathrm{~min} . \lambda=254 \mathrm{~cm}^{-1} ;[\alpha]_{\mathrm{D}}{ }^{25.0}=+49.9(\mathrm{c}$ $\left.=0.5, \mathrm{CHCl}_{3}\right)$.

\section{2,6-Di-tert-butyl-4-methylphenyl $(S, E)-2-((2-$}

\section{iodophenyl)((mesitylsulfonyl)imino)methoxy)-4-phenylbut-3-ynoate (14k)}

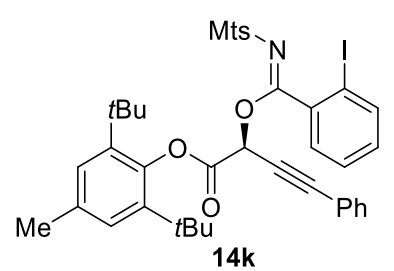

Following GP3, $N$-[mesitylsulfonyl]-1-[phenylethynyl]-1,2-benziodazol-3(1H)-one (2d) (53 $\mathrm{mg}, 0.10 \mathrm{mmol}, 1.0$ equiv) and 2,6-di-tert-butyl-4-methylphenyl 2-diazoacetate (11j) (58 mg, $0.20 \mathrm{mmol}, 2.0$ equiv) were stirred for $18 \mathrm{~h}$. The crude reaction mixture was concentrated under reduced pressure and purified by flash chromatography using $\mathrm{Et}_{2} \mathrm{O}$ :pentane 1:6 as mobile phase to afford $14 \mathbf{k}$ as a white solid (80 $\mathrm{mg}, 0.10 \mathrm{mmol}$, quant.). Mp: 84.2-89.9 ${ }^{\circ} \mathrm{C}$; TLC $\left(\mathrm{Et}_{2} \mathrm{O}:\right.$ Pentane 1:5): $\mathrm{R}_{\mathrm{f}}=0.31, \mathrm{KMnO}_{4} ;{ }^{1} \mathrm{H}$ NMR $\left(400 \mathrm{MHz}, \mathrm{CDCl}_{3}\right): \delta 7.83(\mathrm{~d}, J=8.0 \mathrm{~Hz}$, 1H, ArH), $7.46-7.30$ (m, 7H, ArH), $7.19-7.13$ (m, 1H, ArH), $7.13-7.08$ (m, 2H, ArH), 6.87 (s, 2H, $\mathrm{ArH}), 6.68(\mathrm{~s}, 1 \mathrm{H}, \mathrm{OCH}), 2.57$ (s, 6H, $\left.2 \mathrm{X} \mathrm{ArCH}_{3}\right), 2.31$ (s, 3H, $\left.\mathrm{ArCH}_{3}\right), 2.28$ (s, 3H, $\left.\mathrm{ArCH}_{3}, \mathrm{Mts}\right), 1.31$ (s, 9H, $\left.t \mathrm{Bu}\right), 1.27$ (s, 9H, $\left.t \mathrm{Bu}\right) ;{ }^{13} \mathrm{C} \mathrm{NMR}\left(100 \mathrm{MHz}, \mathrm{CDCl}_{3}\right): \delta 168.1,164.3$, 145.6, 142.5, 142.1, 141.8, 139.6, 139.1, 137.2, 135.3, 135.0, 132.0, 131.7, 131.6, 129.7, 129.4, 128.4, 127.4, 127.3, 127.0, 121.3, 92.5, 89.9, 79.6, 67.0, 53.4, 35.3, 35.2, 31.6, 31.3, 22.9, 21.5, 21.0; IR v 3058 (w), 2964 (m), 2238 (w), 1778 (m), 1622 (s), 1580 (m), 1464 (m), 1421 (w), 1365 (w), 1331 (s), 1279 (m), 1254 (m), 1146 (s), 1102 (m), 1038 (w), 991 (m), 904 (w), 859 
(w), 838 (w); HRMS (ESI) calcd. for $\mathrm{C}_{41} \mathrm{H}_{45} \mathrm{INO}_{5} \mathrm{~S}^{+}[\mathrm{M}+\mathrm{H}]^{+}$790.2058; found 790.2063; Chiral HPLC conditions: $e e=74 \%$; Chiralpak IA 95:5 Hexane $/ \mathrm{PrOH}, 1.0 \mathrm{~mL} / \mathrm{min}, 31 \mathrm{~min}$. $\mathrm{t}_{\mathrm{r}}$ (minor) $=12.5 \mathrm{~min}$. and $\mathrm{t}_{\mathrm{r}}($ major $)=15.7 \mathrm{~min} . \lambda=254 \mathrm{~cm}^{-1} ;[\alpha]_{\mathrm{D}}{ }^{25.0}=+59.8\left(\mathrm{c}=0.5, \mathrm{CHCl}_{3}\right) . \underline{t} \mathrm{Bu}$ groups of BHT and Me groups of Mts were not identical and gave separate signals.

$(1 R, 2 S, 5 R)-2$-isopropyl-5-methylcyclohexyl $(S)-2-((E)-(2-$

iodophenyl)((mesitylsulfonyl)imino)methoxy)-4-phenylbut-3-ynoate (14l)

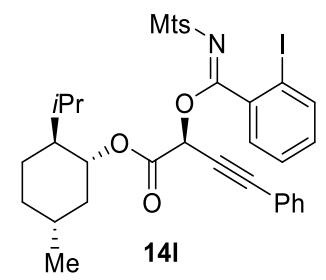

Following GP3, $N$-[mesitylsulfonyl]-1-[phenylethynyl]-1,2-benziodazol-3(1H)-one (2d) (53 $\mathrm{mg}, 0.10 \mathrm{mmol}, 1.0$ equiv) and (1R,2S,5R)-2-isopropyl-5-methylcyclohexyl 3-oxobutanoate (11k) (45 mg, $0.20 \mathrm{mmol}, 2.0$ equiv) were stirred for $18 \mathrm{~h}$. The crude reaction mixture was concentrated under reduced pressure and purified by flash chromatography using $\mathrm{Et}_{2} \mathrm{O}$ :pentane 1:6 as mobile phase to afford $\mathbf{1 4 l}$ as a white solid (60.0 mg, $0.083 \mathrm{mmol}, 83 \%)$. Mp: 56.6-61.2 ${ }^{\circ} \mathrm{C}$; TLC $\left(\mathrm{Et}_{2} \mathrm{O}:\right.$ Pentane 1:6): $\mathrm{R}_{\mathrm{f}}=0.23, \mathrm{KMnO}_{4} ;{ }^{1} \mathrm{H}$ NMR $\left(400 \mathrm{MHz}, \mathrm{CDCl}_{3}\right): \delta 7.84(\mathrm{~d}, J=$ $7.9 \mathrm{~Hz}, 1 \mathrm{H}, \mathrm{ArH}), 7.45-7.28(\mathrm{~m}, 7 \mathrm{H}, \mathrm{ArH}), 7.17$ (ddd, J=8.0, 6.3, $2.8 \mathrm{~Hz}, 1 \mathrm{H}, \operatorname{ArH}), 6.88$ (s, $2 \mathrm{H}, \operatorname{Ar} H), 6.11(\mathrm{~s}, 1 \mathrm{H}, \mathrm{OCH}), 4.81\left(\mathrm{td}, J=10.9,4.4 \mathrm{~Hz}, 1 \mathrm{H}, \mathrm{OCHCH}_{2}\right), 2.57(\mathrm{~s}, 6 \mathrm{H}, 2 \mathrm{X}$ $\left.\mathrm{ArCH}_{3}\right), 2.28\left(\mathrm{~s}, 3 \mathrm{H}, \mathrm{ArCH}_{3}\right), 2.08-1.86(\mathrm{~m}, 2 \mathrm{H}), 1.77-1.63(\mathrm{~m}, 2 \mathrm{H}), 1.54-1.43(\mathrm{~m}, 2 \mathrm{H})$, $1.12-1.05(\mathrm{~m}, 2 \mathrm{H}), 0.97-0.81(\mathrm{~m}, 7 \mathrm{H}), 0.75\left(\mathrm{~d}, J=6.9 \mathrm{~Hz}, 3 \mathrm{H}, \mathrm{CH}_{3}\right.$ of menthol); ${ }^{13} \mathrm{C} \mathrm{NMR}$ $\left(100 \mathrm{MHz} \mathrm{CDCl}_{3}\right): \delta 168.2,164.2,142.4,139.5,139.0,137.5,134.9,132.0,131.7,131.6$, 129.6, 129.3, 128.3, 127.4, 121.3, 92.6, 88.3, 80.0, 67.1, 47.0, 40.2, 34.1, 31.4, 26.2, 23.5, 22.9, 21.9, 21.0, 20.7, 16.3; IR v 2957 (s), 2928 (m), 2870 (m), 2241 (w), 1751 (m), 1621 (s), 1581 (m), 1491 (w), 1461 (m), 1377 (w), 1332 (s), 1282 (s), 1255 (s), 1205 (m), 1164 (s), 1152 (s), 1053 (m), 1018 (m), 912 (m); HRMS (ESI) calcd. for $\mathrm{C}_{36} \mathrm{H}_{40} \mathrm{INNaO}_{5} \mathrm{~S}^{+}[\mathrm{M}+\mathrm{Na}]^{+}$748.1564; found 748.1571; Chiral HPLC conditions: $d r=2.5: 97.5$; Chiralpak IA 95:5 Hexane/iPrOH, 1.0 $\mathrm{mL} / \mathrm{min}, 31 \mathrm{~min} . \mathrm{t}_{\mathrm{r}}($ minor $)=21.0 \mathrm{~min}$. and $\mathrm{t}_{\mathrm{r}}$ (major) $=23.8 \mathrm{~min} . \lambda=254 \mathrm{~cm}^{-1} ;[\alpha]_{\mathrm{D}}{ }^{25.0}=+2.0(\mathrm{c}$ $\left.=0.5, \mathrm{CHCl}_{3}\right)$. 
$(3 S, 8 S, 9 S, 10 R, 13 R, 14 S)-10,13-d i m e t h y l-17-((R)-6-m e t h y l h e p t a n-2-y l)-$

2,3,4,7,8,9,10,11,12,13,14,15,16,17-tetradecahydro-1H-cyclopenta[a]phenanthren-3-yl (2S)-2-((E)-(2-iodophenyl)((mesitylsulfonyl)imino)methoxy)-4-phenylbut-3-ynoate (14m)

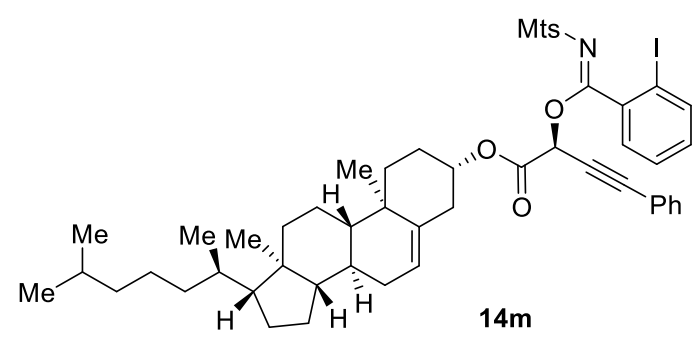

Following GP3, $N$-[mesitylsulfonyl]-1-[phenylethynyl]-1,2-benziodazol-3(1H)-one (2d) (53 mg, 0.10 mmol, 1.0 equiv) and $(3 S, 8 S, 9 S, 10 R, 13 R, 14 S, 17 R)$-10,13-dimethyl-17-((R)-6methylheptan-2-yl)-2,3,4,7,8,9,10,11,12,13,14,15,16,17-tetradecahydro- $1 \mathrm{H}$ -

cyclopenta[a]phenanthren-3-yl 2-diazoacetate (11l) $(91 \mathrm{mg}, 0.20 \mathrm{mmol}, 2.0$ equiv) were stirred for $18 \mathrm{~h}$. The crude reaction mixture was concentrated under reduced pressure and purified by flash chromatography using $\mathrm{Et}_{2} \mathrm{O}$ :pentane 1:6.5 as mobile phase to afford $\mathbf{1 4 m}$ as a white solid (81.0 mg, $0.085 \mathrm{mmol}, 85 \%)$. Mp: 96.4-100.4 ${ }^{\circ} \mathrm{C} ; \mathrm{TLC}\left(\mathrm{Et}_{2} \mathrm{O}:\right.$ Pentane 1:5): $\mathrm{R}_{\mathrm{f}}=0.4, \mathrm{KMnO}_{4}$; ${ }^{1} \mathrm{H}$ NMR (400 MHz, $\mathrm{CDCl}_{3}$ ): $\delta 7.85(\mathrm{~d}, J=8.0 \mathrm{~Hz}, 1 \mathrm{H}, \mathrm{ArH}), 7.46-7.39$ (m, 4H, ArH), 7.38 - 7.28 (m, 3H, ArH), 7.18 (ddd, $J=9.2,7.9,4.2 \mathrm{~Hz}, 1 \mathrm{H}, \mathrm{ArH}), 6.88$ (s, 2H, ArH), 6.03 (s, 1H, OCHCC), 5.37 (d, $J=3.9 \mathrm{~Hz}, 1 \mathrm{H}$, olefinic $H$ ), 4.63 (dtd, $J=12.5,8.6,4.5 \mathrm{~Hz}, 1 \mathrm{H}, \mathrm{OCHCH}_{2}$ ), $2.57\left(\mathrm{~s}, 6 \mathrm{H}, 2 \mathrm{X} \mathrm{ArCH}_{3}\right), 2.39-2.31$ (m, 2H), 2.28 (s, 3H, $\left.\mathrm{ArCH}_{3}\right), 2.05-1.95$ (m, 2H), 1.89 $-1.74(\mathrm{~m}, 3 \mathrm{H}), 1.64-0.97(\mathrm{~m}, 24 \mathrm{H}), 0.93(\mathrm{~d}, J=6.5 \mathrm{~Hz}, 3 \mathrm{H}), 0.88$ (d, $J=1.8 \mathrm{~Hz}, 3 \mathrm{H}), 0.86$ $(\mathrm{d}, J=1.9 \mathrm{~Hz}, 3 \mathrm{H}), 0.69$ (s, 3H); ${ }^{13} \mathrm{C} \mathrm{NMR}\left(100 \mathrm{MHz}, \mathrm{CDCl}_{3}\right): \delta 168.1,164.2,142.4,139.5$, 139.0, 137.5, 135.0, 132.1, 131.7, 131.6, 129.5, 129.3, 128.3, 127.4, 123.2, 121.3, 92.7, 88.4, $79.7,76.8,67.2,56.7,56.1,50.0,42.3,39.7,39.5,37.5,36.9,36.5,36.2,35.8,31.9,31.8,28.2$, 28.0, 27.3, 24.3, 23.8, 22.9, 22.8, 22.6, 21.0, 19.3, 18.7, 11.9; IR v 2942 (s), 2868 (m), 2238 (w), $1759(\mathrm{~m}), 1623$ (s), 1580 (m), 1465 (m), 1333 (s), 1283 (s), 1255 (s), 1193 (m), 1147 (s), 1016 (m), 852 (w); HRMS (ESI) calcd. for $\mathrm{C}_{53} \mathrm{H}_{66} \mathrm{INNaO}_{5} \mathrm{~S}^{+}[\mathrm{M}+\mathrm{Na}]^{+}$978.3599; found 978.3599; Chiral HPLC conditions: $d r=7: 93$; Chiralpak IA 80:20 Hexane/ $i \mathrm{PrOH}, 1.0 \mathrm{~mL} / \mathrm{min}$, 31 min. $t_{r}($ minor $)=10.5 \min$. and $t_{r}($ major $)=14.8 \min . \lambda=254 \mathrm{~cm}^{-1} ;[\alpha]_{\mathrm{D}}{ }^{25.0}=+5.7(\mathrm{c}=0.5$, $\mathrm{CHCl}_{3}$ ). One carbon was not resolved at $100 \mathrm{MHz}$. 


\section{2-Phenylpropan-2-yl (S,E)-4-(4-fluorophenyl)-2-((2-}

\section{iodophenyl)((mesitylsulfonyl)imino)methoxy)but-3-ynoate (14n)}

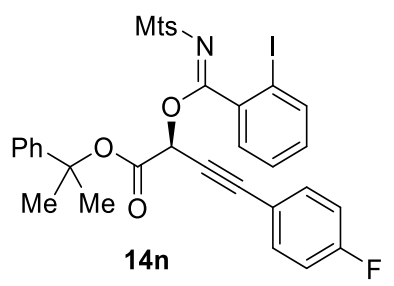

Following GP3, $N$-[mesitylsulfonyl]-1-[(4-fluorophenyl)ethynyl]-1,2-benziodazol-3(1H)-one (2h) (55 mg, $0.10 \mathrm{mmol}, 1.0$ equiv) and 2-phenylpropan-2-yl 2-diazoacetate (11h) (41 mg, 0.20 mmol, 2.0 equiv) were stirred for $20 \mathrm{~h}$. The crude reaction mixture was concentrated under reduced pressure and purified by flash chromatography using $\mathrm{Et}_{2} \mathrm{O}$ :pentane 1:5 as mobile phase to afford 14n as a white solid (66.0 $\mathrm{mg}, 0.091 \mathrm{mmol}, 91 \%)$. Mp: 96.8-98.9 ${ }^{\circ} \mathrm{C}$; TLC $\left(\mathrm{Et}_{2} \mathrm{O}:\right.$ Pentane 1:4): $\mathrm{R}_{\mathrm{f}}=0.33, \mathrm{KMnO}_{4} ;{ }^{1} \mathrm{H}$ NMR $\left(400 \mathrm{MHz}, \mathrm{CDCl}_{3}\right): \delta 7.81(\mathrm{dd}, J=8.0,0.9$ $\mathrm{Hz}, 1 \mathrm{H}, \operatorname{Ar} H), 7.47-7.26$ (m, 9H, ArH), 7.15 (ddd, $J=8.0,7.0,2.2 \mathrm{~Hz}, 1 \mathrm{H}, \operatorname{Ar} H), 7.07-7.00$ (m, 2H, ArH), 6.88 (s, 2H, ArH), 6.06 (s, 1H, OCH), 2.55 (s, 6H, $\left.2 \mathrm{X} \mathrm{ArCH}_{3}\right), 2.28(\mathrm{~s}, 3 \mathrm{H}$, $\left.\mathrm{ArCH}_{3}\right), 1.83$ (s, 3H, $\left.\mathrm{OCCH}_{3}\right), 1.76\left(\mathrm{~s}, 3 \mathrm{H}, \mathrm{OCCH}_{3}\right) ;{ }^{13} \mathrm{C} \mathrm{NMR}\left(100 \mathrm{MHz}, \mathrm{CDCl}_{3}\right): \delta 168.2$, $163.1(\mathrm{~d}, J=251.5 \mathrm{~Hz}), 162.6,144.6,142.5,139.6,138.9,137.3,134.9,134.1\left(\mathrm{~d}, J_{\mathrm{C}-\mathrm{F}}=8.6\right.$ $\mathrm{Hz}), 131.7,131.6,129.6,128.4,127.4,127.4,124.3,117.4\left(\mathrm{~d}, J_{\mathrm{C}-\mathrm{F}}=3.5 \mathrm{~Hz}\right), 115.8\left(\mathrm{~d}, J_{\mathrm{C}-\mathrm{F}}=\right.$ $22.2 \mathrm{~Hz}$ ), 92.6, 87.4, 85.1, 79.8, 67.3, 29.1, 27.4, 22.9, 21.0; IR v 3063 (w), 2982 (w), 2938 (w), 2240 (w), 1763 (m), 1622 (s), 1508 (m), 1464 (w), 1331 (s), 1284 (s), 1238 (s), 1145 (s), 1017 (m), 841 (m); HRMS (ESI) calcd. for $\mathrm{C}_{35} \mathrm{H}_{31} \mathrm{FINNaO}_{5} \mathrm{~S}^{+}[\mathrm{M}+\mathrm{Na}]^{+}$746.0844; found 746.0839; Chiral HPLC conditions: $e e=92 \%$; Chiralpak IB 90:10 Hexane $/ \mathrm{PrOH}, 1.0 \mathrm{~mL} / \mathrm{min}$, 31 min. $t_{\mathrm{r}}($ minor $)=10.6 \min$. and $\mathrm{t}_{\mathrm{r}}($ major $)=16.5 \mathrm{~min} . \lambda=254 \mathrm{~cm}^{-1} ;[\alpha]_{\mathrm{D}}{ }^{25.0}=+32.5(\mathrm{c}=$ $\left.0.5, \mathrm{CHCl}_{3}\right)$.

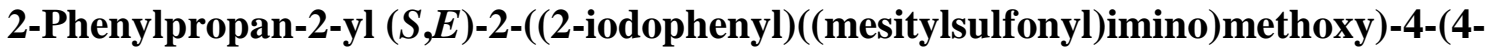
(trifluoromethyl)phenyl)but-3-ynoate (140)

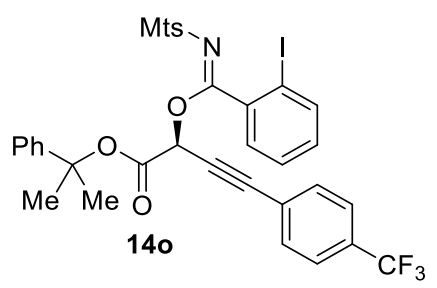

Following GP3, $N$-[mesitylsulfonyl]-1-[(4-(trifluoromethyl)phenyl)ethynyl]-1,2-benziodazol3(1H)-one (2i) (60 mg, $0.10 \mathrm{mmol}, 1.0$ equiv) and 2-phenylpropan-2-yl 2-diazoacetate (11h) (41 $\mathrm{mg}, 0.20 \mathrm{mmol}, 2.0$ equiv) were stirred for $20 \mathrm{~h}$. The crude reaction mixture was concentrated under reduced pressure and purified by flash chromatography using $\mathrm{Et}_{2} \mathrm{O}$ :pentane 
1:4 as mobile phase to afford $\mathbf{1 4 0}$ as a white solid (58.0 mg, $0.075 \mathrm{mmol}, 75 \%)$. Mp: 65.7-71.9 ${ }^{\circ} \mathrm{C}$; TLC (Et $2 \mathrm{O}:$ Pentane 1:4): $\mathrm{R}_{\mathrm{f}}=0.23, \mathrm{KMnO}_{4} ;{ }^{1} \mathrm{H}$ NMR $\left(400 \mathrm{MHz}, \mathrm{CDCl}_{3}\right): \delta 7.82$ (dd, $J=$ 8.0, 1.0 Hz, 1H, ArH), $7.60(\mathrm{~d}, J=8.2 \mathrm{~Hz}, 2 \mathrm{H}, \operatorname{Ar} H), 7.54$ (d, $J=8.2 \mathrm{~Hz}, 2 \mathrm{H}, \operatorname{Ar} H), 7.42-$ 7.27 (m, 7H, ArH), 7.16 (ddd, $J=8.0,7.0,2.1 \mathrm{~Hz}, 1 \mathrm{H}, \operatorname{Ar} H), 6.87$ (s, 2H, ArH), 6.09 (s, 1H, $\mathrm{OCH}$ ), 2.55 (s, 6H, $\left.2 \mathrm{X} \mathrm{ArCH}_{3}\right), 2.27$ (s, 3H, $\mathrm{ArCH}_{3}$ ), 1.84 (s, 3H, OCCH $\mathrm{OH}_{3}, 1.78$ (s, 3H, $\left.\mathrm{OCCH}_{3}\right) ;{ }^{13} \mathrm{C} \mathrm{NMR}\left(100 \mathrm{MHz}, \mathrm{CDCl}_{3}\right): \delta 168.0,162.3,144.4,142.6,139.6,139.0,137.3$, $134.9,132.2,131.8,131.6,131.3\left(\mathrm{~d}, J_{\mathrm{C}-\mathrm{F}}=33.3 \mathrm{~Hz}\right), 129.6,128.4,127.5,127.4,125.3\left(\mathrm{q}, J_{\mathrm{C}-\mathrm{F}}\right.$ $=3.6 \mathrm{~Hz}), 125.1,124.3,92.6,86.8,85.3,82.4,67.1,29.0,27.4,22.9,21.0$; IR v $3061(\mathrm{w}), 2982$ (w), 2938 (w), 1763 (m), 1620 (s), 1580 (w), 1464 (w), 1325 (s), 1282 (m), 1255 (m), 1167 (s), 1132 (s), 1069 (w), 1016 (m), 953 (w), 846 (m); HRMS (ESI) calcd. for $\mathrm{C}_{36} \mathrm{H}_{31} \mathrm{~F}_{3} \mathrm{INNaO}_{5} \mathrm{~S}^{+}[\mathrm{M}+\mathrm{Na}]^{+}$796.0812; found 796.0818; Chiral HPLC conditions: $e e=85 \%$; Chiralpak IB 90:10 Hexane/iPrOH, $1.0 \mathrm{~mL} / \mathrm{min}, 31 \mathrm{~min}$. $\mathrm{t}_{\mathrm{r}}($ minor $)=8.8 \mathrm{~min}$. and $\mathrm{t}_{\mathrm{r}}$ (major) $=$ $16.1 \min . \lambda=254 \mathrm{~cm}^{-1} ;[\alpha]_{\mathrm{D}}{ }^{25.0}=+27.6\left(\mathrm{c}=0.5, \mathrm{CHCl}_{3}\right) . \mathrm{CF}_{3}$ carbon was not resolved at 100 $\mathrm{MHz}$.

\section{2-Phenylpropan-2-yl $(S, E)-4-(4-e t h y n y l p h e n y l)-2-((2-$}

\section{iodophenyl)((mesitylsulfonyl)imino)methoxy)but-3-ynoate (14p)}

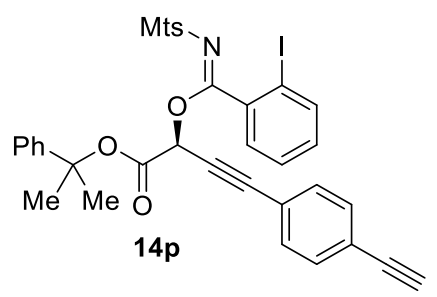

Following GP3, $N$-[mesitylsulfonyl]-1-[(4-ethynylphenyl)ethynyl]-1,2-benziodazol-3(1H)one (2j) (55 mg, $0.10 \mathrm{mmol}, 1.0$ equiv) and 2-phenylpropan-2-yl 2-diazoacetate (11h) (41 mg, $0.20 \mathrm{mmol}, 2.0$ equiv) were stirred for $20 \mathrm{~h}$. The crude reaction mixture was concentrated under reduced pressure and purified by flash chromatography using $\mathrm{Et}_{2} \mathrm{O}$ :pentane 1:4 as mobile phase to afford $14 \mathrm{p}$ as a white solid (44 mg, $0.06 \mathrm{mmol}, 60 \%)$. Mp: $68.5-71.8^{\circ} \mathrm{C}$; TLC (Et ${ }_{2} \mathrm{O}$ :Pentane 1:3): $\mathrm{R}_{\mathrm{f}}=0.29, \mathrm{KMnO}_{4} ;{ }^{1} \mathrm{H} \mathrm{NMR}\left(400 \mathrm{MHz}, \mathrm{CDCl}_{3}\right): \delta 7.82(\mathrm{~d}, J=7.9 \mathrm{~Hz}, 1 \mathrm{H}, \mathrm{ArH}), 7.48-$ $7.26(\mathrm{~m}, 11 \mathrm{H}, \operatorname{Ar} H), 7.15(\mathrm{ddd}, J=8.0,6.9,2.3 \mathrm{~Hz}, 1 \mathrm{H}, . \operatorname{Ar} H), 6.87$ (s, 2H, ArH), 6.07 (s, 1H, $\mathrm{OCH}), 3.19$ (s, $1 \mathrm{H}, \mathrm{CCH}), 2.55$ (s, 6H, $\left.2 \mathrm{X} \mathrm{ArCH}_{3}\right), 2.28$ (s, 3H, $\left.\mathrm{ArCH}_{3}\right), 1.83\left(\mathrm{~s}, 3 \mathrm{H}, \mathrm{OCCH}_{3}\right)$, $1.77\left(\mathrm{~s}, 3 \mathrm{H}, \mathrm{OCCH}_{3}\right) ;{ }^{13} \mathrm{C} \mathrm{NMR}\left(100 \mathrm{MHz}, \mathrm{CDCl}_{3}\right): \delta 168.1,162.5,144.6,142.5,139.6,138.9$, 137.3, 134.9, 132.1, 131.9, 131.7, 131.6, 129.6, 128.4, 127.4, 127.4, 124.3, 123.1, 121.7, 92.6, 87.7, 85.2, 82.9, 81.9, 79.5, 67.3, 29.0, 27.4, 22.9, 21.0; IR v $3290(\mathrm{w}), 3058(\mathrm{w}), 3035(\mathrm{w})$, 2927 (w), 2853 (w), 2241 (w), 1762 (m), 1622 (s), 1581 (m), 1500 (w), 1464 (m), 1445 (w), 1368 (w), 1331 (s), 1284 (s), 1257 (m), 1194 (m), 1164 (s), 1145 (s), 1105 (w), 1018 (m), 997 
(m), 953 (w), 913 (m); HRMS (ESI) calcd. for $\mathrm{C}_{37} \mathrm{H}_{32} \mathrm{INNaO}_{5} \mathrm{~S}^{+}[\mathrm{M}+\mathrm{Na}]^{+}$752.0938; found 752.0950; Chiral HPLC conditions: $e e=93 \%$; Chiralpak IA 90:10 Hexane $/ i \mathrm{PrOH}, 1.0 \mathrm{~mL} / \mathrm{min}$, 31 min. $t_{\mathrm{r}}($ minor $)=18.4 \mathrm{~min}$. and $\mathrm{t}_{\mathrm{r}}($ major $)=21.2 \mathrm{~min} . \lambda=254 \mathrm{~cm}^{-1} ;[\alpha]_{\mathrm{D}}{ }^{25.0}=+50.9(\mathrm{c}=$ $\left.0.5, \mathrm{CHCl}_{3}\right)$.

\section{2-Phenylpropan-2-yl $(S, E)-4-(3-f l u o r o p h e n y l)-2-((2-$}

\section{iodophenyl)((mesitylsulfonyl)imino)methoxy)but-3-ynoate (14q)}

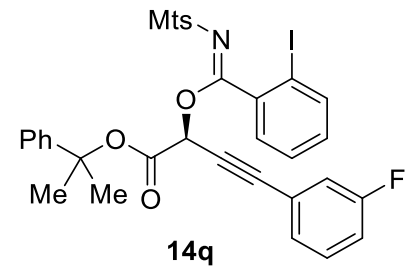

Following GP3, $N$-[mesitylsulfonyl]-1-[(3-fluorophenyl)ethynyl]-1,2-benziodazol-3(1H)-one (2k) (55 mg, $0.10 \mathrm{mmol}, 1.0$ equiv) and 2-phenylpropan-2-yl 2-diazoacetate (11h) (41 mg, 0.20 mmol, 2.0 equiv) were stirred for $20 \mathrm{~h}$. The crude reaction mixture was concentrated under reduced pressure and purified by flash chromatography using $\mathrm{Et}_{2} \mathrm{O}$ :pentane 1:5 as mobile phase to afford $\mathbf{1 4 q}$ as a white solid $(60.0 \mathrm{mg}, 0.083 \mathrm{mmol}, 83 \%)$. Mp: 63.8-66.1 ${ }^{\circ} \mathrm{C}$; TLC $\left(\mathrm{Et}_{2} \mathrm{O}:\right.$ Pentane 1:4): $\mathrm{R}_{\mathrm{f}}=0.34, \mathrm{KMnO}_{4} ;{ }^{1} \mathrm{H}$ NMR $\left(400 \mathrm{MHz}, \mathrm{CDCl}_{3}\right): \delta 7.82(\mathrm{dd}, J=8.0,1.0$ Hz, 1H, ArH), $7.43-7.27$ (m, 8H, ArH), 7.22 (dt, J= 7.7, $1.3 \mathrm{~Hz}, 1 \mathrm{H}, \operatorname{Ar} H$ ), $7.18-7.06$ (m, 3H, $\mathrm{ArH}$ ), 6.88 (s, 2H, $\mathrm{ArH}), 6.07$ (s, 1H, OCH), 2.55 (s, 6H, $\left.2 \mathrm{X} \mathrm{ArCH}_{3}\right), 2.28$ (s, 3H, $\mathrm{ArCH}_{3}$ ), $1.83\left(\mathrm{~s}, 3 \mathrm{H}, \mathrm{OCCH}_{3}\right), 1.77$ (s, 3H, $\left.\mathrm{OCCH}_{3}\right) ;{ }^{13} \mathrm{C} \mathrm{NMR}\left(100 \mathrm{MHz}, \mathrm{CDCl}_{3}\right): \delta 168.1,162.4$, $162.2(\mathrm{~d}, J=247.3 \mathrm{~Hz}), 144.5,142.6,139.6,138.9$, 137.3, 134.9, 131.7, 131.6, 130.0 (d, $J=$ 8.7 Hz), 129.6, 128.4, 127.9 (d, $J=3.2 \mathrm{~Hz}), 127.5,127.4,124.3,123.1(\mathrm{~d}, J=9.6 \mathrm{~Hz}), 118.8$ (d, $J=23.1 \mathrm{~Hz}), 116.8$ (d, $J=21.3 \mathrm{~Hz}), 92.6,87.0$ (d, $J=3.3 \mathrm{~Hz}), 85.2,80.9$, 67.2, 29.0, 27.4, 22.9, 21.0; IR v 3064 (w), 2934 (w), 2851 (w), 2242 (w), 1763 (m), 1624 (s), 1469 (w), 1435 (m), 1332 (s), 1289 (s), 1250 (s), 1148 (s), 1001 (w), 945 (w), 873 (w); HRMS (ESI) calcd. for $\mathrm{C}_{35} \mathrm{H}_{31} \mathrm{FINNaO}_{5} \mathrm{~S}^{+}[\mathrm{M}+\mathrm{Na}]^{+}$746.0844; found 746.0857; Chiral HPLC conditions: $e e=92 \%$; Chiralpak IB 90:10 Hexane/iPrOH, $1.0 \mathrm{~mL} / \mathrm{min}, 31 \mathrm{~min} . \mathrm{t}_{\mathrm{r}}$ (minor) = $10.6 \mathrm{~min}$. and $\mathrm{t}_{\mathrm{r}}$ (major) $=15.9 \min . \lambda=230 \mathrm{~cm}^{-1} ;[\alpha]_{\mathrm{D}}{ }^{25.0}=+29.6\left(\mathrm{c}=0.5, \mathrm{CHCl}_{3}\right)$.

2-Phenylpropan-2-yl (S,E)-4-(3-cyanophenyl)-2-((2iodophenyl)((mesitylsulfonyl)imino)methoxy)but-3-ynoate (14r)

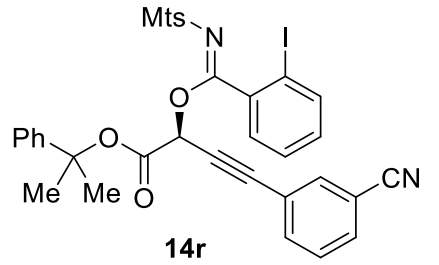


Following GP3, $N$-[mesitylsulfonyl]-1-[(3-cyanophenyl)ethynyl]-1,2-benziodazol-3(1H)-one (2) $(55.5 \mathrm{mg}, 0.100 \mathrm{mmol}, 1.00$ equiv) and 2-phenylpropan-2-yl 2-diazoacetate (11h) (41 mg, $0.20 \mathrm{mmol}, 2.0$ equiv) were stirred for $20 \mathrm{~h}$. The crude reaction mixture was concentrated under reduced pressure and purified by flash chromatography using $\mathrm{Et}_{2} \mathrm{O}$ :pentane 1:2 as mobile phase to afford $14 \mathrm{r}$ as a white solid (59.0 $\mathrm{mg}, 0.081 \mathrm{mmol}, 81 \%)$. Mp: 83.9-85.6 ${ }^{\circ} \mathrm{C}$; TLC (Et $2 \mathrm{O}:$ Pentane 1:2): $\mathrm{R}_{\mathrm{f}}=0.18, \mathrm{KMnO}_{4} ;{ }^{1} \mathrm{H}$ NMR (400 MHz, $\left.\mathrm{CDCl}_{3}\right): \delta 7.85(\mathrm{dd}, J=8.0,1.0$ Hz, 1H, ArH), $7.72-7.64(\mathrm{~m}, 3 \mathrm{H}, \operatorname{Ar} H), 7.49$ (td, $J=7.8,0.7 \mathrm{~Hz}, 1 \mathrm{H}, \operatorname{Ar} H), 7.45-7.29$ (m, 7H, ArH), 7.19 (ddd, $J=8.0,7.2,2.0 \mathrm{~Hz}, 1 \mathrm{H}, \operatorname{ArH}), 6.93-6.87$ (m, 2H, ArH), 6.09 (s, 1H, $\mathrm{OCH}), 2.57$ (s, 6H, $\left.2 \mathrm{X} \mathrm{ArCH}_{3}\right), 2.31\left(\mathrm{~s}, 3 \mathrm{H}, \mathrm{ArCH}_{3}\right), 1.86\left(\mathrm{~s}, 3 \mathrm{H}, \mathrm{OCCH}_{3}\right), 1.81$ (s, 3H, $\left.\mathrm{OCCH}_{3}\right) ;{ }^{13} \mathrm{C} \mathrm{NMR}\left(100 \mathrm{MHz}, \mathrm{CDCl}_{3}\right): \delta 168.0,162.2,144.4,142.7,139.6,139.0,137.2$, 136.0, 135.2, 134.8, 132.5, 131.8, 131.6, 129.6, 129.4, 128.4, 127.6, 127.5, 124.3, 123.0, 117.7, 113.1, 92.5, 85.7, 85.4, 82.6, 67.0, 28.9, 27.5, 22.9, 21.0; IR v 2984 (m), 2904 (m), 2233 (w), 1763 (m), 1625 (s), 1580 (w), 1464 (w), 1408 (w), 1385 (w), 1332 (s), 1279 (m), 1253 (s), 1165 (s), 1147 (s), 1061 (s), 907 (w); HRMS (ESI) calcd. for $\mathrm{C}_{36} \mathrm{H}_{31} \mathrm{IN}_{2} \mathrm{NaO}_{5} \mathrm{~S}^{+}[\mathrm{M}+\mathrm{Na}]^{+} 753.0891$; found 753.0892; Chiral HPLC conditions: $e e=87 \%$; Chiralpak IA 80:20 Hexane/iPrOH, 1.0 $\mathrm{mL} / \mathrm{min}, 40$ min. $\mathrm{t}_{\mathrm{r}}($ minor $)=22.9 \mathrm{~min}$. and $\mathrm{t}_{\mathrm{r}}$ (major $)=29.2 \mathrm{~min} . \lambda=254 \mathrm{~cm}^{-1} ;[\alpha]_{\mathrm{D}}{ }^{25.0}=+31.8$ (c $\left.=0.5, \mathrm{CHCl}_{3}\right)$.

\section{2-Phenylpropan-2-yl (S,E)-2-((2-iodophenyl)((mesitylsulfonyl)imino)methoxy)-4-} (triisopropylsilyl)but-3-ynoate (14s)

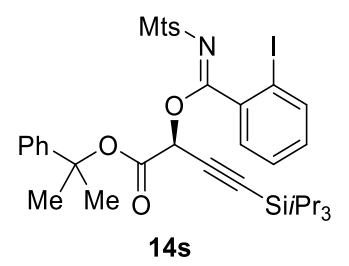

Following GP3, N-[mesitylsulfonyl]-1-[(triisopropylsilyl)ethynyl]-1,2-benziodazol-3(1H)one (2c) (61 mg, $0.10 \mathrm{mmol}, 1.0$ equiv) and 2-phenylpropan-2-yl 2-diazoacetate (11h) (41 mg, $0.20 \mathrm{mmol}, 2.0$ equiv) were stirred for $20 \mathrm{~h}$. The crude reaction mixture was concentrated under reduced pressure and purified by flash chromatography using $\mathrm{Et}_{2} \mathrm{O}$ :pentane 1:7 as mobile phase to afford $14 \mathrm{~s}$ as a white solid (56.0 mg, $0.071 \mathrm{mmol}, 71 \%)$. Mp: $116.4-121.6{ }^{\circ} \mathrm{C}$; TLC $\left(\mathrm{Et}_{2} \mathrm{O}:\right.$ Pentane 1:5): $\mathrm{R}_{\mathrm{f}}=0.25, \mathrm{KMnO}_{4} ;{ }^{1} \mathrm{H} \mathrm{NMR}\left(400 \mathrm{MHz}, \mathrm{CDCl}_{3}\right): \delta 7.81(\mathrm{dd}, J=8.0,1.1$ $\mathrm{Hz}, 1 \mathrm{H}, \mathrm{Ar} H$ ), $7.40-7.26$ (m, 7H, ArH), 7.13 (ddd, $J=8.0,7.3,1.9 \mathrm{~Hz}, 1 \mathrm{H}, \operatorname{Ar} H$ ), 6.88 (s, $2 \mathrm{H}, \mathrm{ArH}), 6.02$ (s, 1H, OCH), 2.52 (s, 6H, $\left.2 \mathrm{X} \mathrm{ArCH}_{3}\right), 2.28$ (s, 3H, $\left.\mathrm{ArCH}_{3}\right), 1.81$ (s, 3H, $\left.\mathrm{OCCH}_{3}\right), 1.75$ (s, 3H, $\left.\mathrm{OCCH}_{3}\right), 1.08$ (s, 21H, TIPS); $\left.{ }^{13} \mathrm{C} \mathrm{NMR} \mathrm{(100} \mathrm{MHz,} \mathrm{CDCl}_{3}\right): \delta 168.4$, 162.5, 144.7, 142.4, 139.5, 138.9, 137.4, 135.1, 131.6, 131.6, 129.6, 128.4, 127.3, 124.1, 97.0, 92.6, 91.1, 84.9, 66.8, 28.9, 27.6, 22.9, 21.0, 18.6, 11.1; IR v 2943 (m), 2866 (m), 1764 (m), 
1620 (s), 1580 (w), 1464 (m), 1385 (w), 1332 (s), 1274 (s), 1252 (s), 1194 (m), 1144 (s), 1104 (w), 1059 (m), 1016 (m), 930 (w), 885 (w), 809 (w); HRMS (ESI) calcd. for $\mathrm{C}_{38} \mathrm{H}_{48} \mathrm{INNaO}_{5} \mathrm{SSi}^{+}[\mathrm{M}+\mathrm{Na}]^{+}$808.1959; found 808.1924; Chiral HPLC conditions: $e e=90 \%$; Chiralpak IA 95:5 Hexane $/ \mathrm{PrOH}, 1.0 \mathrm{~mL} / \mathrm{min}, 31 \mathrm{~min} . \mathrm{t}_{\mathrm{r}}($ minor $)=9.9 \mathrm{~min}$. and $\mathrm{t}_{\mathrm{r}}$ (major) $=$ $16.4 \min . \lambda=254 \mathrm{~cm}^{-1} ;[\alpha]_{\mathrm{D}}{ }^{25.0}=+34.0\left(\mathrm{c}=0.5, \mathrm{CHCl}_{3}\right)$. One carbon was not resolved at 100 MHz.

\section{2-Phenylpropan-2-yl (S,E)-2-((2-iodophenyl)((mesitylsulfonyl)imino)methoxy)-4- (triethylsilyl)but-3-ynoate (14t)}

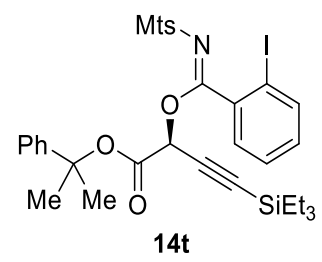

Following GP3, $N$-[mesitylsulfonyl]-1-[(triethylsilyl)ethynyl]-1,2-benziodazol-3(1H)-one (2m) (57 mg, $0.10 \mathrm{mmol}, 1.0$ equiv) and 2-phenylpropan-2-yl 2-diazoacetate (11h) (41 mg, $0.20 \mathrm{mmol}, 2.0$ equiv) were stirred for $18 \mathrm{~h}$. The crude reaction mixture was concentrated under reduced pressure and purified by flash chromatography using $\mathrm{Et}_{2} \mathrm{O}$ :pentane 1:6.5 as mobile phase to afford 14t as a white solid (52 mg, $0.07 \mathrm{mmol}, 70 \%)$. Mp: 123.0-126.6 ${ }^{\circ} \mathrm{C}$; TLC $\left(\mathrm{Et}_{2} \mathrm{O}\right.$ :Pentane 1:6.5): $\mathrm{R}_{\mathrm{f}}=0.17, \mathrm{KMnO}_{4} ;{ }^{1} \mathrm{H}$ NMR (400 MHz, $\left.\mathrm{CDCl}_{3}\right): \delta 7.82-7.79(\mathrm{~m}, 1 \mathrm{H}$, $\operatorname{Ar} H), 7.42-7.26$ (m, 7H, ArH), 7.13 (ddd, $J=8.0,7.1,2.1 \mathrm{~Hz}, 1 \mathrm{H}, \operatorname{Ar} H), 6.88$ (s, 2H, ArH), $5.95(\mathrm{~s}, 1 \mathrm{H}, \mathrm{OCH}), 2.53\left(\mathrm{~s}, 6 \mathrm{H}, 2 \mathrm{X} \mathrm{ArCH}_{3}\right), 2.28\left(\mathrm{~s}, 3 \mathrm{H}, \mathrm{ArCH}_{3}\right), 1.80\left(\mathrm{~s}, 3 \mathrm{H}, \mathrm{OCCH}_{3}\right), 1.74$ (s, 3H, $\mathrm{OCCH}_{3}$ ), 0.99 (t, $J=7.9 \mathrm{~Hz}, 9 \mathrm{H}, 3 \mathrm{X} \mathrm{CH}_{2} \mathrm{CH}_{3}$ ), 0.63 (q, $J=7.9 \mathrm{~Hz}, 6 \mathrm{H}, 2 \mathrm{X} \mathrm{CH}_{3} \mathrm{CH}_{2}$ ); ${ }^{13} \mathrm{C}$ NMR $\left(100 \mathrm{MHz}, \mathrm{CDCl}_{3}\right): \delta 168.3,162.5,144.7,142.5,139.5,138.9,137.4,135.0,131.6$, 131.6, 129.6, 128.3, 127.3, 124.1, 96.3, 92.6, 92.2, 84.9, 67.0, 29.0, 27.5, 22.9, 21.0, 7.4, 4.0; IR v $3061(w), 2955$ (w), 2876 (w), 1766 (m), 1623 (s), 1581 (w), 1465 (w), 1334 (s), 1276 (m), 1253 (m), 1194 (w), 1166 (m), 1145 (s), 1105 (w), 1059 (w), 1017 (m); HRMS (ESI) calcd. for $\mathrm{C}_{35} \mathrm{H}_{42} \mathrm{INNaO}_{5} \mathrm{SSi}^{+}[\mathrm{M}+\mathrm{Na}]^{+}$766.1490; found 766.1496; Chiral HPLC conditions: $e e=92 \%$; Chiralpak IA 95:5 Hexane $/ \mathrm{PrOH}, 1.0 \mathrm{~mL} / \mathrm{min}, 31 \mathrm{~min} . \mathrm{t}_{\mathrm{r}}$ (minor) $=10.8 \mathrm{~min}$. and $\mathrm{t}_{\mathrm{r}}($ major $)=15.8 \min . \lambda=254 \mathrm{~cm}^{-1} ;[\alpha]_{\mathrm{D}}{ }^{25.0}=+24.7\left(\mathrm{c}=0.5, \mathrm{CHCl}_{3}\right)$. One carbon was not resolved at $100 \mathrm{MHz}$. 


\section{2-Phenylpropan-2-yl (S,E)-2-((2-iodophenyl)((mesitylsulfonyl)imino)methoxy)oct-3- ynoate (14u)}

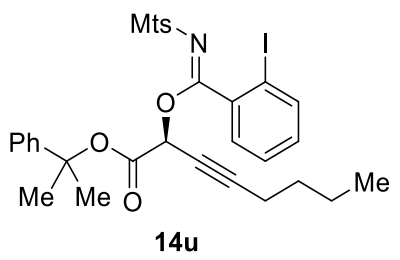

Following GP3, $N$-[mesitylsulfonyl]-1-[(butyl)ethynyl]-1,2-benziodazol-3(1H)-one (2n) (51 $\mathrm{mg}, 0.10 \mathrm{mmol}, 1.0$ equiv) and 2-phenylpropan-2-yl 2-diazoacetate (11h) (41 mg, $0.20 \mathrm{mmol}$, 2.0 equiv) were stirred for $20 \mathrm{~h}$. The crude reaction mixture was concentrated under reduced pressure and purified by flash chromatography using $\mathrm{Et}_{2} \mathrm{O}$ :pentane 1:4 as mobile phase to afford $14 \mathbf{u}$ as a thick colourless gel $(48 \mathrm{mg}, 0.07 \mathrm{mmol}, 70 \%)$. TLC $\left(\mathrm{Et}_{2} \mathrm{O}\right.$ :Pentane 1:4): $\mathrm{R}_{\mathrm{f}}=$ 0.37, $\mathrm{KMnO}_{4} ;{ }^{1} \mathrm{H}$ NMR (400 MHz, $\left.\mathrm{CDCl}_{3}\right): \delta 7.82-7.77(\mathrm{~m}, 1 \mathrm{H}, \mathrm{ArH}), 7.41-7.27(\mathrm{~m}, 7 \mathrm{H}$, ArH), 7.13 (ddd, $J=7.9,7.3,2.0 \mathrm{~Hz}, 1 \mathrm{H}, \operatorname{Ar} H), 6.87$ (s, 2H, ArH), 5.84 (t, $J=2.3 \mathrm{~Hz}, 1 \mathrm{H}$, $\mathrm{OCH}), 2.53\left(\mathrm{~s}, 6 \mathrm{H}, 2 \mathrm{X} \mathrm{ArCH}_{3}\right), 2.29-2.22\left(\mathrm{~m}, 5 \mathrm{H}, \mathrm{ArCH}_{3}\right.$ and $\left.\mathrm{CCCH}_{2}\right), 1.79\left(\mathrm{~s}, 3 \mathrm{H}, \mathrm{OCCH}_{3}\right)$, $1.74\left(\mathrm{~s}, 3 \mathrm{H}, \mathrm{OCCH}_{3}\right), 1.56-1.47\left(\mathrm{~m}, 2 \mathrm{H}, \mathrm{CCCH}_{2} \mathrm{CH}_{2}\right), 1.47-1.36\left(\mathrm{~m}, 2 \mathrm{H}, \mathrm{CH}_{2} \mathrm{CH}_{3}\right), 0.90$ (t, $\left.J=7.2 \mathrm{~Hz}, 3 \mathrm{H}, \mathrm{CH}_{2} \mathrm{CH}_{3}\right) ;{ }^{13} \mathrm{C} \mathrm{NMR}\left(100 \mathrm{MHz}, \mathrm{CDCl}_{3}\right): \delta 168.3,163.1,144.8,142.4,139.5$, 138.9, 137.5, 135.0, 131.6, 129.6, 128.3, 127.3, 127.3, 124.2, 92.6, 90.1, 84.7, 71.2, 67.4, 30.1, 28.9, 27.4, 22.9, 21.9, 21.0, 18.5, 13.5; IR v 3061 (w), 2936 (w), 2869 (w), 2241 (w), 1764 (m), 1622 (s), 1580 (w), 1464 (w), 1333 (s), 1278 (s), 1255 (m), 1203 (m), 1150 (s), 1105 (w), 1016 (w), 854 (w); HRMS (ESI) calcd for $\mathrm{C}_{33} \mathrm{H}_{36} \mathrm{INNaO}_{5} \mathrm{~S}^{+}[\mathrm{M}+\mathrm{Na}]^{+}$708.1251; found 708.1235; Chiral HPLC conditions: $e e=93 \%$; Chiralpak IA 90:10 Hexane $/ i \mathrm{PrOH}, 1.0 \mathrm{~mL} / \mathrm{min}, 31 \mathrm{~min}$. $\mathrm{t}_{\mathrm{r}}($ minor $)=11.3 \mathrm{~min}$. and $\mathrm{t}_{\mathrm{r}}($ major $)=13.9 \min . \lambda=254 \mathrm{~cm}^{-1} ;[\alpha]_{\mathrm{D}}{ }^{25.0}=-5.1\left(\mathrm{c}=0.5, \mathrm{CHCl}_{3}\right)$. One carbon was not resolved at $100 \mathrm{MHz}$.

2-Phenylpropan-2-yl (S,E)-2-((2-iodophenyl)((mesitylsulfonyl)imino)methoxy)octadec-3ynoate (14v)

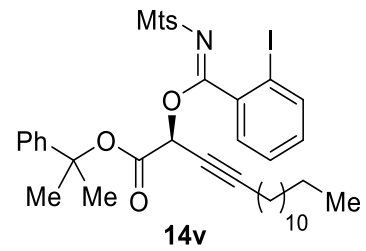

Following GP3, $N$-[mesitylsulfonyl]-1-[(dodecyl)ethynyl]-1,2-benziodazol-3(1H)-one (2o) (63 mg, $0.10 \mathrm{mmol}, 1.0$ equiv) and 2-phenylpropan-2-yl 2-diazoacetate (11h) (41 mg, 0.20 mmol, 2.0 equiv) were stirred for $18 \mathrm{~h}$. The crude reaction mixture was concentrated under reduced pressure and purified by flash chromatography using $\mathrm{Et}_{2} \mathrm{O}$ :pentane 1:5 as mobile phase to afford $14 \mathbf{v}$ as a colourless thick gel (60.0 mg, $0.075 \mathrm{mmol}, 75 \%)$. TLC (Et ${ }_{2} \mathrm{O}$ :Pentane 1:5): 
$\mathrm{R}_{\mathrm{f}}=0.3, \mathrm{KMnO}_{4} ;{ }^{1} \mathrm{H} \mathrm{NMR}\left(400 \mathrm{MHz}, \mathrm{CDCl}_{3}\right): \delta 7.80(\mathrm{~d}, J=7.9 \mathrm{~Hz}, 1 \mathrm{H}, \mathrm{Ar} H), 7.40-7.27$ $(\mathrm{m}, 7 \mathrm{H}, \operatorname{Ar} H), 7.13(\mathrm{td}, J=7.6,2.0 \mathrm{~Hz}, 1 \mathrm{H}, \mathrm{ArH}), 6.88(\mathrm{~s}, 2 \mathrm{H}, \mathrm{Ar} H), 5.84(\mathrm{t}, J=2.3 \mathrm{~Hz}, 1 \mathrm{H}$, $\mathrm{OCH}), 2.54\left(\mathrm{~s}, 6 \mathrm{H}, 2 \mathrm{X} \mathrm{ArCH}_{3}\right), 2.29-2.22\left(\mathrm{~m}, 5 \mathrm{H}, \mathrm{ArCH}_{3}\right.$ and $\left.\mathrm{CCCH}_{2}\right), 1.79\left(\mathrm{~s}, 3 \mathrm{H}, \mathrm{OCCH}_{3}\right)$, $1.74\left(\mathrm{~s}, 3 \mathrm{H}, \mathrm{OCCH}_{3}\right), 1.56-1.48\left(\mathrm{~m}, 2 \mathrm{H}, \mathrm{CCCH}_{2} \mathrm{CH}_{2} \mathrm{CH}_{2}\right), 1.42-1.33\left(\mathrm{~m}, 2 \mathrm{H}, \mathrm{CH}_{2}\right), 1.31-$ $1.21(\mathrm{~m}, 16 \mathrm{H}), 0.88\left(\mathrm{t}, J=6.8 \mathrm{~Hz}, 3 \mathrm{H}, \mathrm{CH}_{2} \mathrm{CH}_{3}\right) ;{ }^{13} \mathrm{C} \mathrm{NMR}\left(100 \mathrm{MHz}, \mathrm{CDCl}_{3}\right): \delta 168.3,163.1$, $144.8,142.4,139.5,138.8,137.5,135.0,131.5,130.1,129.6,128.3,127.3,127.3,124.2$, 92.6, 90.2, 84.7, 71.1, 67.4, 31.9, 29.6, 29.6, 29.5, 29.3, 29.1, 28.9, 28.9, 28.3, 28.1, 27.4, 22.9, 22.7, 21.0, 18.8, 14.1; IR v 2927 (m), 2854 (w), 2244 (w), 1764 (m), 1622 (s), 1580 (w), 1464 (m), 1334 (s), 1278 (s), 1255 (m), 1204 (m), 1150 (s), 1000 (w), 951 (w); HRMS (ESI) calcd. for $\mathrm{C}_{41} \mathrm{H}_{52} \mathrm{INNaO}_{5} \mathrm{~S}^{+}[\mathrm{M}+\mathrm{Na}]^{+}$820.2503; found 820.2510; Chiral HPLC conditions: $e e=93 \%$; Chiralpak IA 95:5 Hexane $/ \mathrm{PrOH}, 1.0 \mathrm{~mL} / \mathrm{min}, 31 \mathrm{~min} . \mathrm{t}_{\mathrm{r}}($ minor $)=12.3 \mathrm{~min}$. and $\mathrm{t}_{\mathrm{r}}($ major $)=$ $15.7 \min . \lambda=254 \mathrm{~cm}^{-1} ;[\alpha]_{\mathrm{D}}{ }^{25.0}=-1.2\left(\mathrm{c}=0.5, \mathrm{CHCl}_{3}\right)$.

\section{2-Phenylpropan-2-yl (S,E)-7-chloro-2-((2-}

\section{iodophenyl)((mesitylsulfonyl)imino)methoxy)hept-3-ynoate (14w)}

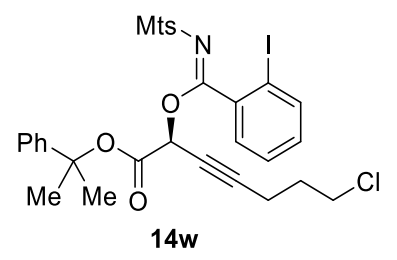

Following GP3, $N$-[mesitylsulfonyl]-1-[(3-chloropropyl)ethynyl]-1,2-benziodazol-3(1H)-one (2p) (53 mg, $0.10 \mathrm{mmol}, 1.0$ equiv) and 2-phenylpropan-2-yl 2-diazoacetate (11h) (41 mg, 0.20 mmol, 2.0 equiv) were stirred for $20 \mathrm{~h}$. The crude reaction mixture was concentrated under reduced pressure and purified by flash chromatography using $\mathrm{Et}_{2} \mathrm{O}$ :pentane 1:4 as mobile phase to afford $14 \mathrm{w}$ as a white solid (37.0 mg, $0.052 \mathrm{mmol}, 52 \%)$. Mp: $42.8-47.3{ }^{\circ} \mathrm{C}$; TLC $\left(\mathrm{Et}_{2} \mathrm{O}:\right.$ Pentane 1:4): $\mathrm{R}_{\mathrm{f}}=0.37, \mathrm{KMnO}_{4} ;{ }^{1} \mathrm{H} \mathrm{NMR}\left(400 \mathrm{MHz}, \mathrm{CDCl}_{3}\right): \delta 7.81(\mathrm{dd}, J=8.0,1.0$ $\mathrm{Hz}, 1 \mathrm{H}, \mathrm{Ar} H), 7.40-7.26(\mathrm{~m}, 7 \mathrm{H}, \mathrm{ArH}), 7.14(\mathrm{td}, J=7.6,1.9 \mathrm{~Hz}, 1 \mathrm{H}, \mathrm{Ar} H), 6.88(\mathrm{~s}, 2 \mathrm{H}, \operatorname{Ar} H)$, $5.85(\mathrm{t}, J=2.2 \mathrm{~Hz}, 1 \mathrm{H}, \mathrm{OCH}), 3.62\left(\mathrm{t}, J=6.3 \mathrm{~Hz}, 2 \mathrm{H}, \mathrm{CH}_{2} \mathrm{CH}_{2} \mathrm{Cl}\right), 2.54\left(\mathrm{~s}, 6 \mathrm{H}, 2 \mathrm{X} \mathrm{ArCH}_{3}\right)$, $2.47\left(\mathrm{td}, J=6.8,2.3 \mathrm{~Hz}, 2 \mathrm{H}, \mathrm{CCCH}_{2}\right), 2.28\left(\mathrm{~s}, 3 \mathrm{H}, \mathrm{ArCH}_{3}\right), 1.97(\mathrm{p}, J=6.6 \mathrm{~Hz}, 2 \mathrm{H}$, $\mathrm{CH}_{2} \mathrm{CH}_{2} \mathrm{CH}_{2} \mathrm{Cl}$ ), 1.80 (s, 3H, OCCH$\left.H_{3}\right), 1.75$ (s, 3H, $\left.\left.\mathrm{OCCH}_{3}\right) ;{ }^{13} \mathrm{C} \mathrm{NMR} \mathrm{(100} \mathrm{MHz,} \mathrm{CDCl}_{3}\right): \delta$ 168.2, 162.9, 144.6, 142.5, 139.5, 138.9, 137.4, 135.0, 131.6, 131.6, 129.6, 128.3, 127.4, 127.4, 124.2, 92.6, 87.9, 84.9, 72.3, 67.1, 43.4, 30.7, 28.8, 27.4, 22.9, 21.0, 16.2; IR v 3060 (w), 2981 (w), 2939 (w), 2245 (w), 1761 (m), 1619 (s), 1580 (m), 1460 (m), 1329 (s), 1276 (s), 1254 (s), $1203(\mathrm{~m}), 1140$ (s), 1104 (m), 1000 (m), 853 (w); HRMS (ESI) calcd. for $\mathrm{C}_{32} \mathrm{H}_{33} \mathrm{ClINNaO}_{5} \mathrm{~S}^{+}[\mathrm{M}+\mathrm{Na}]^{+} 728.0705$; found 728.0698; Chiral HPLC conditions: $e e=90 \%$; 
Chiralpak IA 90:10 Hexane/iPrOH, $1.0 \mathrm{~mL} / \mathrm{min}, 31$ min. $\mathrm{t}_{\mathrm{r}}$ (minor) $=17.9 \mathrm{~min}$. and $\mathrm{t}_{\mathrm{r}}$ (major) $=23.2 \min . \lambda=254 \mathrm{~cm}^{-1} ;[\alpha]_{\mathrm{D}}{ }^{25.0}=-1.9\left(\mathrm{c}=0.5, \mathrm{CHCl}_{3}\right)$.

2-Phenylpropan-2-yl (S,E)-2-((2-iodophenyl)((mesitylsulfonyl)imino)methoxy)nona-3,8diynoate $(14 x)$

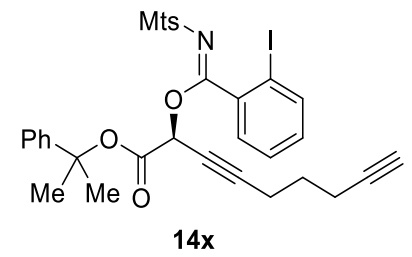

Following GP3, $N$-[mesitylsulfonyl]-1-[hepta-1,6-diynyl]-1,2-benziodazol-3(1H)-one (2q) (52 mg, $0.10 \mathrm{mmol}, 1.0$ equiv) and 2-phenylpropan-2-yl 2-diazoacetate (11h) (41 mg, 0.20 mmol, 2.0 equiv) were stirred for $20 \mathrm{~h}$. The crude reaction mixture was concentrated under reduced pressure and purified by flash chromatography using $\mathrm{Et}_{2} \mathrm{O}$ :pentane 1:3 as mobile phase to afford $14 \mathbf{x}$ as a white semi-solid (45.0 $\mathrm{mg}, 0.065 \mathrm{mmol}, 65 \%)$. TLC (Et $2 \mathrm{O}:$ Pentane 1:3): $\mathrm{R}_{\mathrm{f}}$ $=0.18, \mathrm{KMnO}_{4} ;{ }^{1} \mathrm{H} \mathrm{NMR}\left(400 \mathrm{MHz}, \mathrm{CDCl}_{3}\right): \delta 7.80(\mathrm{dd}, J=8.0,1.0 \mathrm{~Hz}, 1 \mathrm{H}, \mathrm{Ar} H), 7.41-$ $7.27(\mathrm{~m}, 7 \mathrm{H}, \operatorname{Ar} H), 7.13(\mathrm{td}, J=7.6,1.9 \mathrm{~Hz}, 1 \mathrm{H}, \operatorname{Ar} H), 6.88(\mathrm{~s}, 2 \mathrm{H}, \operatorname{Ar} H), 5.84(\mathrm{t}, J=2.3 \mathrm{~Hz}$, $1 \mathrm{H}, \mathrm{OCH}), 2.53\left(\mathrm{~s}, 6 \mathrm{H}, 2 \mathrm{X} \mathrm{ArCH}_{3}\right), 2.41\left(\mathrm{td}, J=7.0,2.3 \mathrm{~Hz}, 2 \mathrm{H}, \mathrm{OCHCCCH}_{2}\right), 2.33-2.26$ $\left(\mathrm{m}, 5 \mathrm{H}, \mathrm{CHCCH}_{2}\right.$ and $\left.\mathrm{ArCH}_{3}\right), 1.97(\mathrm{t}, J=2.7 \mathrm{~Hz}, 1 \mathrm{H}, \mathrm{CCH}), 1.79\left(\mathrm{~s}, 3 \mathrm{H}, \mathrm{OCCH}_{3}\right), 1.78-$ $1.71\left(\mathrm{~m}, 5 \mathrm{H}, \mathrm{OCCH}_{3}\right.$ and $\left.\mathrm{CH}_{2} \mathrm{CH}_{2} \mathrm{CH}_{2}\right) ;{ }^{13} \mathrm{C} \mathrm{NMR}\left(100 \mathrm{MHz}, \mathrm{CDCl}_{3}\right): \delta 168.3,163.0,144.7$, $142.5,139.5,138.9,137.5,135.0,131.6,129.6$, 128.4, 127.4, 124.3, 92.6, 88.7, 84.8, 83.1, 72.0, 69.2, 67.2, 28.9, 27.5, 27.0, 22.9, 21.0, 17.8, 17.6; IR v 3300 (w), $2982(\mathrm{w}), 2939$ (w), 2244 (w), 1761 (m), 1619 (s), 1463 (w), 1329 (s), 1274 (s), 1203 (m), 1144 (s), 1104 (m), 999 (m), 854 (w); HRMS (ESI) calcd. for $\mathrm{C}_{34} \mathrm{H}_{35} \mathrm{INO}_{5} \mathrm{~S}^{+}[\mathrm{M}+\mathrm{H}]^{+}$696.1275; found 696.1276; Chiral HPLC conditions: $e e=90 \%$; Chiralpak IA 90:10 Hexane/ $i \mathrm{PrOH}, 1.0 \mathrm{~mL} / \mathrm{min}, 31 \mathrm{~min}$. $\mathrm{t}_{\mathrm{r}}($ minor $)=16.8 \mathrm{~min}$. and $\mathrm{t}_{\mathrm{r}}($ major $)=20.4 \mathrm{~min} . \lambda=254 \mathrm{~cm}^{-1} ;[\alpha]_{\mathrm{D}}{ }^{25.0}=-3.6\left(\mathrm{c}=0.5, \mathrm{CHCl}_{3}\right)$. Two carbons were not resolved at $100 \mathrm{MHz}$.

\section{2-Phenylpropan-2-yl (S,E)-4-cyclopropyl-2-((2-}

iodophenyl)((mesitylsulfonyl)imino)methoxy)but-3-ynoate (14y)

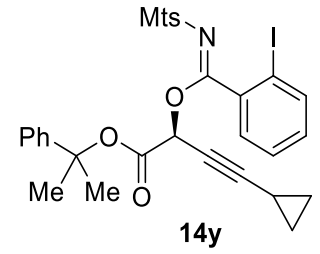

Following GP3, $N$-[mesitylsulfonyl]-1-[(cyclopropyl)ethynyl]-1,2-benziodazol-3(1H)-one (2r) (49.5 mg, $0.100 \mathrm{mmol}, 1.00$ equiv) and 2-phenylpropan-2-yl 2-diazoacetate (11h) (41 mg, $0.20 \mathrm{mmol}, 2.0$ equiv) were stirred for $20 \mathrm{~h}$. The crude reaction mixture was concentrated under 
reduced pressure and purified by flash chromatography using $\mathrm{Et}_{2} \mathrm{O}$ :pentane 1:3 as mobile phase to afford $14 \mathbf{y}$ as a white solid (59.0 $\mathrm{mg}, 0.088 \mathrm{mmol}, 88 \%)$. Mp: 51.4-56.8 ${ }^{\circ} \mathrm{C}$; TLC $\left(\mathrm{Et}_{2} \mathrm{O}\right.$ :Pentane 1:3): $\mathrm{R}_{\mathrm{f}}=0.22, \mathrm{KMnO}_{4} ;{ }^{1} \mathrm{H}$ NMR $\left(400 \mathrm{MHz}, \mathrm{CDCl}_{3}\right): \delta 7.80(\mathrm{dd}, J=7.9,1.0$ $\mathrm{Hz}, 1 \mathrm{H}, \mathrm{Ar} H$ ), $7.40-7.27$ (m, 7H, ArH), 7.13 (ddd, $J=8.0,7.2,1.9 \mathrm{~Hz}, 1 \mathrm{H}, \mathrm{Ar} H), 6.88$ (s, 2H, $\mathrm{ArH}), 5.79(\mathrm{~d}, J=2.1 \mathrm{~Hz}, 1 \mathrm{H}, \mathrm{OCH}), 2.53\left(\mathrm{~s}, 6 \mathrm{H}, 2 \mathrm{X} \mathrm{ArCH}_{3}\right), 2.28\left(\mathrm{~s}, 3 \mathrm{H}, \mathrm{ArCH}_{3}\right), 1.78$ $\left(\mathrm{s}, 3 \mathrm{H}, \mathrm{OCCH}_{3}\right), 1.72\left(\mathrm{~s}, 3 \mathrm{H}, \mathrm{OCCH}_{3}\right), 1.34-1.26\left(\mathrm{~m}, 1 \mathrm{H}, \mathrm{CCH}\left(\mathrm{CH}_{2}\right)_{2}\right), 0.86-0.78(\mathrm{~m}, 2 \mathrm{H}$, $\left.\mathrm{CH}_{2}\right), 0.78-0.71\left(\mathrm{~m}, 2 \mathrm{H}, \mathrm{CH}_{2}\right) ;{ }^{13} \mathrm{C} \mathrm{NMR}\left(100 \mathrm{MHz}, \mathrm{CDCl}_{3}\right): \delta 168.3,163.0,144.8,142.4$, $139.5,138.8,137.5,135.0,131.6,129.5,128.3,127.3,124.2$, 93.2, 92.6, 84.6, 67.5, 66.3, 53.4, 29.1, 27.4, 22.9, 21.0, 8.4, 8.4, -0.5; IR $v 2981(\mathrm{w}), 2936$ (w), 2245 (w), 1762 (m), 1620 (s), 1580 (w), 1465 (w), 1330 (s), 1277 (s), 1255 (m), 1191 (w), 1145 (s), 1000 (w), 891 (w), 817 (w); HRMS (ESI) calcd for $\mathrm{C}_{32} \mathrm{H}_{32} \mathrm{INNaO}_{5} \mathrm{~S}^{+}[\mathrm{M}+\mathrm{Na}]^{+}$692.0938; found 692.0944; Chiral HPLC conditions: $e e=94 \%$; Chiralpak IA 90:10 Hexane $/ \mathrm{PrOH}, 1.0 \mathrm{~mL} / \mathrm{min}, 31 \mathrm{~min} . \mathrm{t}_{\mathrm{r}}$ $($ minor $)=14.5 \mathrm{~min}$. and $\mathrm{t}_{\mathrm{r}}($ major $)=17.6 \mathrm{~min} . \lambda=254 \mathrm{~cm}^{-1} ;[\alpha]_{\mathrm{D}}{ }^{25.0}=+2.9\left(\mathrm{c}=0.5, \mathrm{CHCl}_{3}\right)$. One carbon was not resolved at $100 \mathrm{MHz}$.

\section{2-Phenylpropan-2-yl (S,E)-4-cyclopentyl-2-((2-}

\section{iodophenyl)((mesitylsulfonyl)imino)methoxy)but-3-ynoate (14z)}

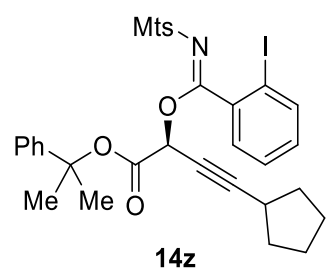

Following GP3, $N$-[mesitylsulfonyl]-1-[(cyclopentyl)ethynyl]-1,2-benziodazol-3(1H)-one (2s) (52 mg, $0.10 \mathrm{mmol}, 1.0$ equiv) and 2-phenylpropan-2-yl 2-diazoacetate (11h) (41 mg, 0.20 mmol, 2.0 equiv) were stirred for $20 \mathrm{~h}$. The crude reaction mixture was concentrated under reduced pressure and purified by flash chromatography using $\mathrm{Et}_{2} \mathrm{O}$ :pentane 1:4 as mobile phase to afford $14 \mathrm{z}$ as a white solid (49 mg, $0.07 \mathrm{mmol}, 70 \%)$. Mp: 54.4-57.8 ${ }^{\circ} \mathrm{C}$; TLC (Et ${ }_{2} \mathrm{O}$ :Pentane 1:4): $\mathrm{R}_{\mathrm{f}}=0.21, \mathrm{KMnO}_{4} ;{ }^{1} \mathrm{H} \mathrm{NMR}\left(400 \mathrm{MHz}, \mathrm{CDCl}_{3}\right): \delta 7.80(\mathrm{dd}, J=8.0,1.0 \mathrm{~Hz}, 1 \mathrm{H}, \operatorname{Ar} H)$, $7.41-7.26(\mathrm{~m}, 7 \mathrm{H}, \mathrm{ArH}), 7.13(\mathrm{ddd}, J=8.0,7.1,2.1 \mathrm{~Hz}, 1 \mathrm{H}, \operatorname{Ar} H), 6.88$ (s, 2H, ArH), 5.85 $(\mathrm{d}, J=2.1 \mathrm{~Hz}, 1 \mathrm{H}, \mathrm{CH}), 2.68(\mathrm{pd}, J=7.4,2.1 \mathrm{~Hz}, 1 \mathrm{H}, \mathrm{CCCH}), 2.54\left(\mathrm{~s}, 6 \mathrm{H}, 2 \mathrm{X} \mathrm{ArCH}_{3}\right), 2.28$ (s, 3H, $\left.\mathrm{ArCH}_{3}\right), 1.97-1.87\left(\mathrm{~m}, 2 \mathrm{H}, \mathrm{CH}_{2}\right), 1.78$ (s, 3H, $\left.\mathrm{OCCH}_{3}\right), 1.73$ (s, 3H, $\left.\mathrm{OCCH}_{3}\right), 1.70-$ 1.51 (m, 6H, $\left.\left.3 \mathrm{X} \mathrm{CH}_{2}\right) ;{ }^{13} \mathrm{C} \mathrm{NMR} \mathrm{(100} \mathrm{MHz,} \mathrm{CDCl}_{3}\right): \delta 168.3,163.1,144.8,142.4,139.5$, 138.9, 137.6, 135.1, 131.6, 129.6, 128.3, 127.3, 127.3, 124.2, 94.2, 92.6, 84.5, 70.7, 67.4, 33.4, 33.3, 30.1, 29.1, 27.4, 25.0, 22.9, 21.0; IR v 3061 (w), 2958 (w), $2870(\mathrm{w}), 2243(\mathrm{w}), 1764(\mathrm{~m})$, 1622 (s), 1580 (w), 1464 (w), 1333 (s), 1280 (m), 1255 (m), 1201 (w), 1148 (s), 1001 (w), 954 
(w), 837 (w); HRMS (ESI) calcd. for $\mathrm{C}_{34} \mathrm{H}_{36} \mathrm{INNaO}_{5} \mathrm{~S}^{+}[\mathrm{M}+\mathrm{Na}]^{+} 720.1251$; found 720.1256; Chiral HPLC conditions: $e e=92 \%$; Chiralpak IA 90:10 Hexane/ $i \mathrm{PrOH}, 1.0 \mathrm{~mL} / \mathrm{min}, 31 \mathrm{~min}$. $\mathrm{t}_{\mathrm{r}}($ minor $)=11.8 \mathrm{~min}$. and $\mathrm{t}_{\mathrm{r}}($ major $)=14.6 \mathrm{~min} . \lambda=254 \mathrm{~cm}^{-1} ;[\alpha]_{\mathrm{D}}{ }^{25.0}=+1.6\left(\mathrm{c}=0.5, \mathrm{CHCl}_{3}\right)$.

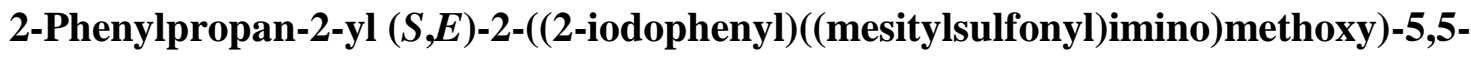

dimethylhex-3-ynoate (14aa)

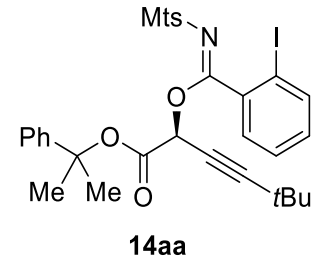

Following GP3, $N$-[mesitylsulfonyl]-1-[(t-butyl)ethynyl]-1,2-benziodazol-3(1H)-one (2t) (51 $\mathrm{mg}, 0.10 \mathrm{mmol}, 1.0$ equiv) and 2-phenylpropan-2-yl 2-diazoacetate (11h) (41 mg, $0.20 \mathrm{mmol}$, 2.0 equiv) were stirred for $20 \mathrm{~h}$. The crude reaction mixture was concentrated under reduced pressure and purified by flash chromatography using $\mathrm{Et}_{2} \mathrm{O}$ :pentane 1:4 as mobile phase to afford 14aa as a colourless thick gel (43.0 mg, $0.063 \mathrm{mmol}, 63 \%)$. TLC (Et ${ }_{2} \mathrm{O}$ :Pentane 1:4): $\mathrm{R}_{\mathrm{f}}$ $=0.22, \mathrm{KMnO}_{4} ;{ }^{1} \mathrm{H} \mathrm{NMR}\left(400 \mathrm{MHz}, \mathrm{CDCl}_{3}\right): \delta 7.81(\mathrm{dd}, J=8.0,1.0 \mathrm{~Hz}, 1 \mathrm{H}, \operatorname{Ar} H), 7.43-$ $7.26(\mathrm{~m}, 7 \mathrm{H}, \operatorname{Ar} H), 7.13$ (ddd, $J=8.0,7.0,2.2 \mathrm{~Hz}, 1 \mathrm{H}, \operatorname{Ar} H), 6.88(\mathrm{~s}, 2 \mathrm{H}, \operatorname{Ar} H), 5.86(\mathrm{~s}, 1 \mathrm{H}$, $\mathrm{OCH}$ ), 2.54 (s, 6H, $\left.2 \mathrm{X} \mathrm{ArCH}_{3}\right), 2.28$ (s, 3H, $\left.\mathrm{ArCH}_{3}\right), 1.78\left(\mathrm{~s}, 3 \mathrm{H}, \mathrm{OCCH}_{3}\right), 1.72(\mathrm{~s}, 3 \mathrm{H}$, $\left.\mathrm{OCCH}_{3}\right), 1.24(\mathrm{~s}, 9 \mathrm{H}, \mathrm{tBu}) ;{ }^{13} \mathrm{C} \mathrm{NMR}\left(100 \mathrm{MHz}, \mathrm{CDCl}_{3}\right): \delta 168.3,163.1,144.8,142.4,139.5$, 138.9, 137.6, 135.1, 131.6, 129.6, 128.3, 127.3, 127.3, 124.1, 97.6, 92.6, 84.5, 69.9, 67.2, 53.4, 30.5, 29.1, 27.6, 27.4, 22.9, 21.0; IR v 3061 (w), 2975 (w), 2250 (w), 1765 (m), 1621 (s), 1581 (w), 1463 (w), 1333 (s), 1280 (s), 1256 (m), 1203 (w), 1146 (s), 1015 (w), 862 (w); HRMS (ESI) calcd. for $\mathrm{C}_{33} \mathrm{H}_{36} \mathrm{INNaO}_{5} \mathrm{~S}^{+}[\mathrm{M}+\mathrm{Na}]^{+} 708.1251$; found 708.1257; Chiral HPLC conditions: $e e=86 \%$; Chiralpak IA 90:10 Hexane $/ \mathrm{PrOH}, 1.0 \mathrm{~mL} / \mathrm{min}, 31 \mathrm{~min} . \mathrm{t}_{\mathrm{r}}$ (minor) $=9.5$ $\min$. and $\mathrm{t}_{\mathrm{r}}($ major $)=12.7 \min . \lambda=254 \mathrm{~cm}^{-1} ;[\alpha]_{\mathrm{D}}{ }^{25.0}=+4.5\left(\mathrm{c}=0.5, \mathrm{CHCl}_{3}\right)$.

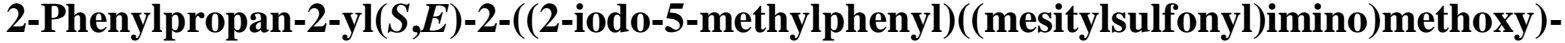
4-phenylbut-3-ynoate (14ab)

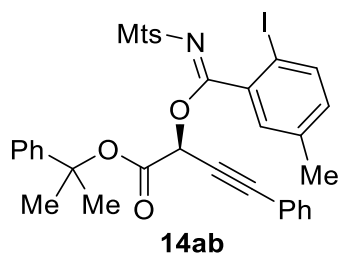

Following GP3, $N$-(mesitylsulfonyl)-5-methyl-1-[phenylethynyl]-1,2-benziodazol-3(1H)-one (2u) (54.5 mg, $0.100 \mathrm{mmol}, 1.00$ equiv) and 2-phenylpropan-2-yl 2-diazoacetate (11h) (41 mg, $0.20 \mathrm{mmol}, 2.0$ equiv) were stirred for $18 \mathrm{~h}$. The crude reaction mixture was concentrated under 
reduced pressure and purified by flash chromatography using $\mathrm{Et}_{2} \mathrm{O}$ :pentane 1:5 as mobile phase to afford 14ab as a white solid (62.0 mg, $0.086 \mathrm{mmol}, 86 \%)$. Mp: $61.2-66.8{ }^{\circ} \mathrm{C}$; TLC (Et $2 \mathrm{O}:$ Pentane 1:5): $\mathrm{R}_{\mathrm{f}}=0.25, \mathrm{KMnO}_{4} ;{ }^{1} \mathrm{H} \mathrm{NMR}\left(400 \mathrm{MHz}, \mathrm{CDCl}_{3}\right): \delta 7.65(\mathrm{~d}, J=8.1 \mathrm{~Hz}$, 1H, ArH), $7.48-7.26(\mathrm{~m}, 10 \mathrm{H}, \mathrm{ArH}), 6.98(\mathrm{~d}, J=2.2 \mathrm{~Hz}, 1 \mathrm{H}, \mathrm{ArH}), 6.95-6.90$ (m, 1H, ArH), 6.86 (s, 2H, $\mathrm{ArH}), 6.11$ (s, 1H, OCH), 2.52 (s, 6H, 2 X $\left.\mathrm{ArCH}_{3}\right), 2.27$ (s, 3H, $\left.\mathrm{ArCH}_{3}\right), 2.22$ (s, $\left.3 \mathrm{H}, \mathrm{ArCH}_{3}\right), 1.86\left(\mathrm{~s}, 3 \mathrm{H}, \mathrm{OCCH}_{3}\right), 1.77\left(\mathrm{~s}, 3 \mathrm{H}, \mathrm{OCCH}_{3}\right) ;{ }^{13} \mathrm{C} \mathrm{NMR}\left(100 \mathrm{MHz}, \mathrm{CDCl}_{3}\right): \delta$ 168.4, 162.7, 144.7, 142.4, 139.6, 138.7, 137.6, 137.0, 135.0, 132.6, 132.0, 131.5, 130.0, 129.3, $128.4,127.4,124.3,121.4,88.6,88.3,85.0,80.0,67.3,29.2,27.4,22.9$, 21.0, 20.9; IR v 3058 (w), 2981 (w), 2940 (w), 2235 (w), 1762 (m), 1620 (s), 1566 (w), 1493 (w), 1464 (m), 1448 (m), 1387 (w), 1330 (s), 1288 (s), 1253 (s), 1206 (s), 1162 (s), 1136 (s), 1104 (m), 1016 (m), $1000(\mathrm{~m}), 964(\mathrm{w}), 849(\mathrm{~m}), 817(\mathrm{~m})$; HRMS (ESI) calcd. for $\mathrm{C}_{36} \mathrm{H}_{35} \mathrm{INO}_{5} \mathrm{~S}^{+}[\mathrm{M}+\mathrm{H}]^{+} 720.1275$; found 720.1281; Chiral HPLC conditions: $e e=93 \%$; Chiralpak IB 95:5 Hexane $/ \mathrm{PrOH}, 1.0$ $\mathrm{mL} / \mathrm{min}, 31 \mathrm{~min} . \mathrm{t}_{\mathrm{r}}($ minor $)=11.7 \mathrm{~min}$. and $\mathrm{t}_{\mathrm{r}}($ major $)=16.5 \mathrm{~min} . \lambda=254 \mathrm{~cm}^{-1} ;[\alpha]_{\mathrm{D}}{ }^{25.0}=+34.6$ $\left(\mathrm{c}=0.5, \mathrm{CHCl}_{3}\right)$. One carbon was not resolved at $100 \mathrm{MHz}$.

\section{2-Phenylpropan-2-yl (S,E)-2-((2-iodo-4,5-dimethoxyphenyl)}

((mesitylsulfonyl)imino)methoxy)-4-phenylbut-3-ynoate (14ac)

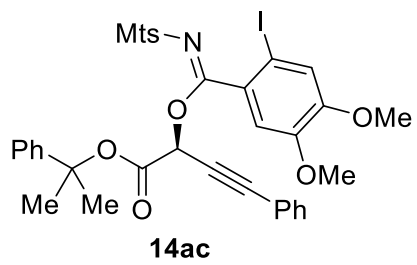

Following GP3, $N$-(mesitylsulfonyl)-5,6-dimethoxy-1-[phenylethynyl]-1,2-benziodazol3(1H)-one (2v) (59 mg, $0.10 \mathrm{mmol}, 1.0$ equiv) and 2-phenylpropan-2-yl 2-diazoacetate (11h) (41 mg, $0.20 \mathrm{mmol}, 2.0$ equiv) were stirred for $18 \mathrm{~h}$. The crude reaction mixture was concentrated under reduced pressure and purified by flash chromatography using $\mathrm{Et}_{2} \mathrm{O}$ :pentane 1:1 as mobile phase to afford $14 a c$ as a whitesolid (65.0 $\mathrm{mg}, 0.085 \mathrm{mmol}, 85 \%)$. Mp: $73.5-$ $76.3{ }^{\circ} \mathrm{C}$; TLC (Et $2 \mathrm{O}:$ Pentane 1:1): $\mathrm{R}_{\mathrm{f}}=0.3, \mathrm{KMnO}_{4} ;{ }^{1} \mathrm{H}$ NMR $\left(400 \mathrm{MHz}, \mathrm{CDCl}_{3}\right): \delta 7.47-$ $7.44(\mathrm{~m}, 2 \mathrm{H}, \operatorname{ArH}), 7.43-7.40(\mathrm{~m}, 5 \mathrm{H}, \mathrm{ArH}), 7.39-7.35$ (m, 2H, $\operatorname{Ar} H), 7.29-7.27(\mathrm{~m}, 1 \mathrm{H}$, $\operatorname{ArH}), 7.18$ (s, 1H, $\operatorname{ArH}$ ), 6.88 (s, 2H, $\operatorname{ArH}), 6.80$ (s, 1H, $\operatorname{ArH}), 6.05$ (s, 1H, CCH), 3.87 (s, 3H, $\left.\mathrm{OCH}_{3}\right), 3.77\left(\mathrm{~s}, 3 \mathrm{H}, \mathrm{OCH}_{3}\right), 2.56\left(\mathrm{~s}, 6 \mathrm{H}, 2 \mathrm{X} \mathrm{ArCH}_{3}\right), 2.27$ (s, 3H, $\left.\mathrm{ArCH}_{3}\right), 1.83$ (s, 3H, $\left.\mathrm{OCCH}_{3}\right), 1.75\left(\mathrm{~s}, 3 \mathrm{H}, \mathrm{OCCH}_{3}\right) ;{ }^{13} \mathrm{C} \mathrm{NMR}\left(100 \mathrm{MHz}, \mathrm{CDCl}_{3}\right): \delta 168.2,162.7,150.9,148.5$, 144.7, 142.4, 139.5, 135.2, 132.0, 131.6, 129.4, 129.3, 128.4, 127.4, 124.2, 121.4, 121.2 , 112.8, 88.3, 85.0, 81.8, 80.1, 67.4, 56.2, 56.0, 29.2, 27.4, 22.9, 21.0; IR v 2976 (w), 2938 (w), 2237 (w), 1764 (m), 1619 (s), 1566 (w), 1508 (m), 1464 (w), 1445 (m), 1380 (m), 1334 (s), 1268 (s), 
1213 (s), 1183 (m), 1163 (m), 1142 (m), 1102 (w), 1079 (w), 1022 (m), 976 (w), 944 (w); HRMS (ESI) calcd. for $\mathrm{C}_{37} \mathrm{H}_{36} \mathrm{INNaO}_{7} \mathrm{~S}^{+}[\mathrm{M}+\mathrm{Na}]^{+} 788.1149$; found 788.1142; Chiral HPLC conditions: $e e=94 \%$; Chiralpak IB 90:10 Hexane $/ \mathrm{PrOH}, 1.0 \mathrm{~mL} / \mathrm{min}, 31 \mathrm{~min} . \mathrm{t}_{\mathrm{r}}$ (minor) = 19.2 min. and $t_{\mathrm{r}}($ major $)=21.7 \min . \lambda=254 \mathrm{~cm}^{-1} ;[\alpha]_{\mathrm{D}}{ }^{25.0}=+29.8\left(\mathrm{c}=0.5, \mathrm{CHCl}_{3}\right)$. One carbon was not resolved at $100 \mathrm{MHz}$. 


\section{Crystal Data}

$N$-[Tolylsulfonyl]-1-[triisopropylsilylethynyl]-1,2-benziodazol-3(1H)-one (2a)

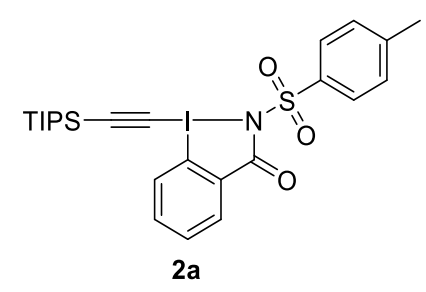

Table S1. Crystal data and structure refinement for $\mathbf{2 a}$.

Identification code

$1 s 01-022$

Empirical formula

$\mathrm{C}_{25} \mathrm{H}_{32} \mathrm{INO}_{3} \mathrm{SSi}$

Formula weight

581.56

Temperature

100(2) K

Wavelength

$0.71073 \AA$

Crystal system

Monoclinic

Space group

$P 2{ }_{1} / c$

Unit cell dimensions

$\mathrm{a}=16.4417(18) \AA \quad \square=90^{\circ}$.

$\mathrm{b}=7.7306(5) \AA \quad \square=94.776(8)^{\circ}$.

$\mathrm{c}=20.8819(15) \AA \quad \square=90^{\circ}$.

Volume

2645.0(4) $\AA^{3}$

Z

4

Density (calculated)

$1.460 \mathrm{Mg} / \mathrm{m}^{3}$

Absorption coefficient

$1.361 \mathrm{~mm}^{-1}$

$\mathrm{F}(000)$

1184

Crystal size

$0.412 \times 0.341 \times 0.236 \mathrm{~mm}^{3}$

$\square$ range for data collection

1.243 to $27.499^{\circ}$.

Index ranges

$-21 \leq \mathrm{h} \leq 21,-10 \leq \mathrm{k} \leq 10,-27 \leq 1 \leq 24$

Reflections collected

32240

Independent reflections

Completeness to $\square=25.242^{\circ}$

$6050\left[R_{\text {int }}=0.0740\right]$

$99.9 \%$

Absorption correction

Semi-empirical from equivalents

Max. and min. transmission

0.7456 and 0.3507

Refinement method

Data / restraints / parameters

Goodness-of-fit on $\mathrm{F}^{2}$

Full-matrix least-squares on $\mathrm{F}^{2}$

6050 / 577 / 393

Final R indices [I > 2 $\square(\mathrm{I})]$

1.101

$\mathrm{R}$ indices (all data)

$R_{1}=0.0848, w R_{2}=0.2155$

$R_{1}=0.1081, w R_{2}=0.2482$

Largest diff. peak and hole

4.365 and -2.428 e. $\AA^{-3}$ 
Table S2. Atomic coordinates (x 104) and equivalent isotropic displacement parameters $\left(\AA^{2} \mathrm{x}\right.$ $10^{3}$ ) for $\mathbf{2 a}$. $U(\mathrm{eq})$ is defined as one third of the trace of the orthogonalized $\mathrm{U}^{\mathrm{ij}}$ tensor.

\begin{tabular}{|c|c|c|c|c|}
\hline & $\mathrm{x}$ & $\mathrm{y}$ & $\mathrm{z}$ & $\mathrm{U}(\mathrm{eq})$ \\
\hline $\mathrm{I}(1)$ & $772(1)$ & $-566(1)$ & $6527(1)$ & $31(1)$ \\
\hline $\mathrm{S}(1)$ & $-991(1)$ & $-1693(2)$ & $7250(1)$ & $34(1)$ \\
\hline $\operatorname{Si}(1)$ & $3257(7)$ & $2524(14)$ & $5810(5)$ & $57(2)$ \\
\hline $\operatorname{Si}(2)$ & $3408(7)$ & $2513(16)$ & $6022(5)$ & $67(2)$ \\
\hline $\mathrm{O}(1)$ & $-1603(4)$ & $-3051(8)$ & $5943(3)$ & $55(2)$ \\
\hline $\mathrm{O}(2)$ & $-1438(4)$ & $-3211(8)$ & $7414(3)$ & $50(1)$ \\
\hline $\mathrm{O}(3)$ & $-379(3)$ & $-1052(8)$ & $7724(2)$ & $40(1)$ \\
\hline $\mathrm{N}(1)$ & $-511(4)$ & $-1953(7)$ & $6613(3)$ & $34(1)$ \\
\hline $\mathrm{C}(1)$ & $443(5)$ & $-1704(9)$ & $5610(3)$ & $38(1)$ \\
\hline$C(2)$ & $970(7)$ & $-1635(11)$ & $5134(4)$ & $51(2)$ \\
\hline $\mathrm{C}(3)$ & 703(8) & $-2395(11)$ & $4533(4)$ & $60(2)$ \\
\hline$C(4)$ & $-53(8)$ & $-3099(11)$ & $4425(4)$ & $62(2)$ \\
\hline$C(5)$ & $-577(7)$ & $-3133(9)$ & $4906(4)$ & $49(2)$ \\
\hline$C(6)$ & $-327(6)$ & $-2435(9)$ & $5519(3)$ & $41(2)$ \\
\hline$C(7)$ & $-909(5)$ & $-2530(8)$ & $6036(4)$ & $38(2)$ \\
\hline$C(8)$ & $-1712(5)$ & $-15(11)$ & $7059(4)$ & $37(2)$ \\
\hline$C(9)$ & $-1433(5)$ & $1647(10)$ & $7046(4)$ & $37(2)$ \\
\hline$C(10)$ & $-1984(6)$ & $2985(11)$ & $6868(4)$ & $50(2)$ \\
\hline $\mathrm{C}(11)$ & $-2803(7)$ & $2626(13)$ & $6698(5)$ & $64(2)$ \\
\hline$C(12)$ & $-3054(8)$ & $947(17)$ & $6728(8)$ & $95(5)$ \\
\hline$C(13)$ & $-2517(6)$ & $-420(12)$ & $6901(6)$ & $63(3)$ \\
\hline$C(14)$ & $-3374(10)$ & $4046(19)$ & $6495(8)$ & $101(5)$ \\
\hline$C(15)$ & $1818(5)$ & $496(11)$ & $6214(4)$ & $44(2)$ \\
\hline$C(16)$ & $2427(6)$ & $1262(12)$ & $6102(5)$ & $53(2)$ \\
\hline$C(17)$ & $2936(15)$ & $4900(30)$ & $5775(11)$ & $78(3)$ \\
\hline $\mathrm{C}(18)$ & 2316(19) & $5310(40)$ & $6216(15)$ & $79(5)$ \\
\hline$C(19)$ & $2770(20)$ & $5630(40)$ & $5136(12)$ & $106(7)$ \\
\hline$C(20)$ & $4131(12)$ & $2260(40)$ & $6473(10)$ & $81(3)$ \\
\hline$C(21)$ & $3850(18)$ & $2690(50)$ & $7106(13)$ & $101(5)$ \\
\hline$C(22)$ & $4859(17)$ & $3270(50)$ & $6349(16)$ & $115(7)$ \\
\hline$C(23)$ & $3460(14)$ & $1580(30)$ & 4993(11) & $66(3)$ \\
\hline
\end{tabular}




$\begin{array}{llllr}\mathrm{C}(24) & 3479(16) & -360(20) & 5028(14) & 73(5) \\ \mathrm{C}(25) & 4195(14) & 2220(30) & 4717(15) & 76(5) \\ \mathrm{C}(26) & 3259(12) & 4650(20) & 6424(13) & 81(3) \\ \mathrm{C}(27) & 2495(12) & 5610(30) & 6186(18) & 77(5) \\ \mathrm{C}(28) & 3978(13) & 5870(40) & 6450(20) & 117(8) \\ \mathrm{C}(29) & 4301(13) & 1370(30) & 6484(10) & 85(3) \\ \mathrm{C}(30) & 4280(18) & 1500(40) & 7198(10) & 99(5) \\ \mathrm{C}(31) & 4390(18) & -460(30) & 6297(13) & 102(6) \\ \mathrm{C}(32) & 3558(11) & 2630(30) & 5137(8) & 71(3) \\ \mathrm{C}(33) & 3310(16) & 1010(30) & 4798(14) & 85(5) \\ \mathrm{C}(34) & 4398(13) & 3140(40) & 4986(14) & 89(5)\end{array}$


Table S3. Bond lengths $[\AA]$ and angles $\left[^{\circ}\right]$ for $\mathbf{2 a}$.

\begin{tabular}{|c|c|}
\hline $\mathrm{I}(1)-\mathrm{C}(15)$ & $2.060(9)$ \\
\hline $\mathrm{I}(1)-\mathrm{C}(1)$ & $2.135(7)$ \\
\hline $\mathrm{I}(1)-\mathrm{N}(1)$ & $2.387(6)$ \\
\hline $\mathrm{S}(1)-\mathrm{O}(2)$ & $1.441(6)$ \\
\hline $\mathrm{S}(1)-\mathrm{O}(3)$ & $1.441(5)$ \\
\hline $\mathrm{S}(1)-\mathrm{N}(1)$ & $1.614(7)$ \\
\hline$S(1)-C(8)$ & $1.779(8)$ \\
\hline $\mathrm{Si}(1)-\mathrm{C}(16)$ & $1.823(15)$ \\
\hline $\mathrm{Si}(1)-\mathrm{C}(23)$ & $1.911(18)$ \\
\hline $\operatorname{Si}(1)-C(17)$ & $1.914(17)$ \\
\hline $\mathrm{Si}(1)-\mathrm{C}(20)$ & $1.921(17)$ \\
\hline $\mathrm{Si}(2)-\mathrm{C}(26)$ & $1.877(18)$ \\
\hline $\operatorname{Si}(2)-C(32)$ & $1.886(16)$ \\
\hline $\mathrm{Si}(2)-\mathrm{C}(16)$ & $1.901(16)$ \\
\hline $\operatorname{Si}(2)-C(29)$ & $1.906(17)$ \\
\hline $\mathrm{O}(1)-\mathrm{C}(7)$ & $1.210(10)$ \\
\hline $\mathrm{N}(1)-\mathrm{C}(7)$ & $1.396(9)$ \\
\hline $\mathrm{C}(1)-\mathrm{C}(2)$ & $1.372(11)$ \\
\hline$C(1)-C(6)$ & $1.386(13)$ \\
\hline$C(2)-C(3)$ & $1.422(13)$ \\
\hline $\mathrm{C}(2)-\mathrm{H}(2)$ & 0.9500 \\
\hline$C(3)-C(4)$ & $1.359(16)$ \\
\hline $\mathrm{C}(3)-\mathrm{H}(3)$ & 0.9500 \\
\hline$C(4)-C(5)$ & $1.378(14)$ \\
\hline $\mathrm{C}(4)-\mathrm{H}(4)$ & 0.9500 \\
\hline$C(5)-C(6)$ & $1.418(10)$ \\
\hline $\mathrm{C}(5)-\mathrm{H}(5)$ & 0.9500 \\
\hline$C(6)-C(7)$ & $1.502(12)$ \\
\hline $\mathrm{C}(8)-\mathrm{C}(9)$ & $1.365(11)$ \\
\hline $\mathrm{C}(8)-\mathrm{C}(13)$ & $1.375(12)$ \\
\hline $\mathrm{C}(9)-\mathrm{C}(10)$ & $1.405(12)$ \\
\hline $\mathrm{C}(9)-\mathrm{H}(9)$ & 0.9500 \\
\hline $\mathrm{C}(10)-\mathrm{C}(11)$ & $1.391(15)$ \\
\hline $\mathrm{C}(10)-\mathrm{H}(10)$ & 0.9500 \\
\hline $\mathrm{C}(11)-\mathrm{C}(12)$ & $1.365(17)$ \\
\hline $\mathrm{C}(11)-\mathrm{C}(14)$ & $1.483(15)$ \\
\hline
\end{tabular}




\begin{tabular}{|c|c|}
\hline $\mathrm{C}(12)-\mathrm{C}(13)$ & $1.404(14)$ \\
\hline $\mathrm{C}(12)-\mathrm{H}(12)$ & 0.9500 \\
\hline $\mathrm{C}(13)-\mathrm{H}(13)$ & 0.9500 \\
\hline $\mathrm{C}(14)-\mathrm{H}(14 \mathrm{~A})$ & 0.9800 \\
\hline $\mathrm{C}(14)-\mathrm{H}(14 \mathrm{~B})$ & 0.9800 \\
\hline $\mathrm{C}(14)-\mathrm{H}(14 \mathrm{C})$ & 0.9800 \\
\hline $\mathrm{C}(15)-\mathrm{C}(16)$ & $1.203(13)$ \\
\hline $\mathrm{C}(17)-\mathrm{C}(19)$ & $1.451(17)$ \\
\hline$C(17)-C(18)$ & $1.463(16)$ \\
\hline $\mathrm{C}(17)-\mathrm{H}(17)$ & 1.0000 \\
\hline $\mathrm{C}(18)-\mathrm{H}(18 \mathrm{~A})$ & 0.9800 \\
\hline $\mathrm{C}(18)-\mathrm{H}(18 \mathrm{~B})$ & 0.9800 \\
\hline $\mathrm{C}(18)-\mathrm{H}(18 \mathrm{C})$ & 0.9800 \\
\hline $\mathrm{C}(19)-\mathrm{H}(19 \mathrm{~A})$ & 0.9800 \\
\hline C(19)-H(19B) & 0.9800 \\
\hline $\mathrm{C}(19)-\mathrm{H}(19 \mathrm{C})$ & 0.9800 \\
\hline$C(20)-C(22)$ & $1.469(18)$ \\
\hline $\mathrm{C}(20)-\mathrm{C}(21)$ & $1.473(18)$ \\
\hline $\mathrm{C}(20)-\mathrm{H}(20)$ & 1.0000 \\
\hline $\mathrm{C}(21)-\mathrm{H}(21 \mathrm{~A})$ & 0.9800 \\
\hline $\mathrm{C}(21)-\mathrm{H}(21 \mathrm{~B})$ & 0.9800 \\
\hline $\mathrm{C}(21)-\mathrm{H}(21 \mathrm{C})$ & 0.9800 \\
\hline $\mathrm{C}(22)-\mathrm{H}(22 \mathrm{~A})$ & 0.9800 \\
\hline $\mathrm{C}(22)-\mathrm{H}(22 \mathrm{~B})$ & 0.9800 \\
\hline $\mathrm{C}(22)-\mathrm{H}(22 \mathrm{C})$ & 0.9800 \\
\hline $\mathrm{C}(23)-\mathrm{C}(25)$ & $1.469(17)$ \\
\hline$C(23)-C(24)$ & $1.498(17)$ \\
\hline $\mathrm{C}(23)-\mathrm{H}(23)$ & 1.0000 \\
\hline $\mathrm{C}(24)-\mathrm{H}(24 \mathrm{~A})$ & 0.9800 \\
\hline $\mathrm{C}(24)-\mathrm{H}(24 \mathrm{~B})$ & 0.9800 \\
\hline $\mathrm{C}(24)-\mathrm{H}(24 \mathrm{C})$ & 0.9800 \\
\hline $\mathrm{C}(25)-\mathrm{H}(25 \mathrm{~A})$ & 0.9800 \\
\hline $\mathrm{C}(25)-\mathrm{H}(25 \mathrm{~B})$ & 0.9800 \\
\hline $\mathrm{C}(25)-\mathrm{H}(25 \mathrm{C})$ & 0.9800 \\
\hline$C(26)-C(27)$ & $1.509(13)$ \\
\hline$C(26)-C(28)$ & $1.510(13)$ \\
\hline $\mathrm{C}(26)-\mathrm{H}(26)$ & 1.0000 \\
\hline $\mathrm{C}(27)-\mathrm{H}(27 \mathrm{~A})$ & 0.9800 \\
\hline
\end{tabular}




\begin{tabular}{|c|c|}
\hline $\mathrm{C}(27)-\mathrm{H}(27 \mathrm{~B})$ & 0.9800 \\
\hline $\mathrm{C}(27)-\mathrm{H}(27 \mathrm{C})$ & 0.9800 \\
\hline $\mathrm{C}(28)-\mathrm{H}(28 \mathrm{~A})$ & 0.9800 \\
\hline $\mathrm{C}(28)-\mathrm{H}(28 \mathrm{~B})$ & 0.9800 \\
\hline $\mathrm{C}(28)-\mathrm{H}(28 \mathrm{C})$ & 0.9800 \\
\hline $\mathrm{C}(29)-\mathrm{C}(31)$ & $1.479(16)$ \\
\hline $\mathrm{C}(29)-\mathrm{C}(30)$ & $1.498(16)$ \\
\hline $\mathrm{C}(29)-\mathrm{H}(29)$ & 1.0000 \\
\hline $\mathrm{C}(30)-\mathrm{H}(30 \mathrm{~A})$ & 0.9800 \\
\hline $\mathrm{C}(30)-\mathrm{H}(30 \mathrm{~B})$ & 0.9800 \\
\hline $\mathrm{C}(30)-\mathrm{H}(30 \mathrm{C})$ & 0.9800 \\
\hline $\mathrm{C}(31)-\mathrm{H}(31 \mathrm{~A})$ & 0.9800 \\
\hline $\mathrm{C}(31)-\mathrm{H}(31 \mathrm{~B})$ & 0.9800 \\
\hline $\mathrm{C}(31)-\mathrm{H}(31 \mathrm{C})$ & 0.9800 \\
\hline $\mathrm{C}(32)-\mathrm{C}(33)$ & $1.484(16)$ \\
\hline $\mathrm{C}(32)-\mathrm{C}(34)$ & $1.495(15)$ \\
\hline $\mathrm{C}(32)-\mathrm{H}(32)$ & 1.0000 \\
\hline $\mathrm{C}(33)-\mathrm{H}(33 \mathrm{~A})$ & 0.9800 \\
\hline $\mathrm{C}(33)-\mathrm{H}(33 \mathrm{~B})$ & 0.9800 \\
\hline $\mathrm{C}(33)-\mathrm{H}(33 \mathrm{C})$ & 0.9800 \\
\hline $\mathrm{C}(34)-\mathrm{H}(34 \mathrm{~A})$ & 0.9800 \\
\hline $\mathrm{C}(34)-\mathrm{H}(34 \mathrm{~B})$ & 0.9800 \\
\hline $\mathrm{C}(34)-\mathrm{H}(34 \mathrm{C})$ & 0.9800 \\
\hline $\mathrm{C}(15)-\mathrm{I}(1)-\mathrm{C}(1)$ & $92.1(3)$ \\
\hline $\mathrm{C}(15)-\mathrm{I}(1)-\mathrm{N}(1)$ & $165.8(3)$ \\
\hline $\mathrm{C}(1)-\mathrm{I}(1)-\mathrm{N}(1)$ & $73.9(3)$ \\
\hline $\mathrm{O}(2)-\mathrm{S}(1)-\mathrm{O}(3)$ & $117.2(3)$ \\
\hline $\mathrm{O}(2)-\mathrm{S}(1)-\mathrm{N}(1)$ & $112.9(4)$ \\
\hline $\mathrm{O}(3)-\mathrm{S}(1)-\mathrm{N}(1)$ & $104.3(3)$ \\
\hline $\mathrm{O}(2)-\mathrm{S}(1)-\mathrm{C}(8)$ & $107.7(4)$ \\
\hline $\mathrm{O}(3)-\mathrm{S}(1)-\mathrm{C}(8)$ & $108.5(4)$ \\
\hline $\mathrm{N}(1)-\mathrm{S}(1)-\mathrm{C}(8)$ & $105.6(3)$ \\
\hline $\mathrm{C}(16)-\mathrm{Si}(1)-\mathrm{C}(23)$ & $106.5(8)$ \\
\hline $\mathrm{C}(16)-\mathrm{Si}(1)-\mathrm{C}(17)$ & 108.4(9) \\
\hline $\mathrm{C}(23)-\mathrm{Si}(1)-\mathrm{C}(17)$ & $113.7(11)$ \\
\hline$C(16)-\operatorname{Si}(1)-C(20)$ & 103.8(9) \\
\hline$C(23)-\operatorname{Si}(1)-C(20)$ & $115.3(12)$ \\
\hline
\end{tabular}




$\begin{array}{ll}\mathrm{C}(17)-\mathrm{Si}(1)-\mathrm{C}(20) & 108.4(12) \\ \mathrm{C}(26)-\mathrm{Si}(2)-\mathrm{C}(32) & 115.3(12) \\ \mathrm{C}(26)-\mathrm{Si}(2)-\mathrm{C}(16) & 105.4(8) \\ \mathrm{C}(32)-\mathrm{Si}(2)-\mathrm{C}(16) & 107.0(8) \\ \mathrm{C}(26)-\mathrm{Si}(2)-\mathrm{C}(29) & 107.8(11) \\ \mathrm{C}(32)-\mathrm{Si}(2)-\mathrm{C}(29) & 111.2(10) \\ \mathrm{C}(16)-\mathrm{Si}(2)-\mathrm{C}(29) & 110.0(10) \\ \mathrm{C}(7)-\mathrm{N}(1)-\mathrm{S}(1) & 121.6(6) \\ \mathrm{C}(7)-\mathrm{N}(1)-\mathrm{I}(1) & 115.6(5) \\ \mathrm{S}(1)-\mathrm{N}(1)-\mathrm{I}(1) & 120.2(3) \\ \mathrm{C}(2)-\mathrm{C}(1)-\mathrm{C}(6) & 122.8(8) \\ \mathrm{C}(2)-\mathrm{C}(1)-\mathrm{I}(1) & 120.3(7) \\ \mathrm{C}(6)-\mathrm{C}(1)-\mathrm{I}(1) & 116.8(5) \\ \mathrm{C}(1)-\mathrm{C}(2)-\mathrm{C}(3) & 117.2(10) \\ \mathrm{C}(1)-\mathrm{C}(2)-\mathrm{H}(2) & 121.4 \\ \mathrm{C}(3)-\mathrm{C}(2)-\mathrm{H}(2) & 121.4 \\ \mathrm{C}(4)-\mathrm{C}(3)-\mathrm{C}(2) & 121.5(9) \\ \mathrm{C}(4)-\mathrm{C}(3)-\mathrm{H}(3) & 119.2 \\ \mathrm{C}(2)-\mathrm{C}(3)-\mathrm{H}(3) & 119.2 \\ \mathrm{C}(3)-\mathrm{C}(4)-\mathrm{C}(5) & 120.3(8) \\ \mathrm{C}(3)-\mathrm{C}(4)-\mathrm{H}(4) & 119.8 \\ \mathrm{C}(5)-\mathrm{C}(4)-\mathrm{H}(4) & 119.8 \\ \mathrm{C}(4)-\mathrm{C}(5)-\mathrm{C}(6) & 120.0(10) \\ \mathrm{C}(4)-\mathrm{C}(5)-\mathrm{H}(5) & 120.0 \\ \mathrm{C}(6)-\mathrm{C}(5)-\mathrm{H}(5) & 120.0 \\ \mathrm{C}(1)-\mathrm{C}(6)-\mathrm{C}(5) & 118.1(8) \\ \mathrm{C}(1)-\mathrm{C}(6)-\mathrm{C}(7) & 123.5(6) \\ \mathrm{C}(5)-\mathrm{C}(6)-\mathrm{C}(7) & 118.4(8) \\ \mathrm{O}(1)-\mathrm{C}(7)-\mathrm{N}(1) & 127.8(8) \\ \mathrm{O}(1)-\mathrm{C}(7)-\mathrm{C}(6) & 123.3(7) \\ \mathrm{N}(1)-\mathrm{C}(7)-\mathrm{C}(6) & 108.9(7) \\ \mathrm{C}(9)-\mathrm{C}(8)-\mathrm{C}(13) & 121.8(8) \\ \mathrm{C}(9)-\mathrm{C}(8)-\mathrm{S}(1) & 118.2(6) \\ \mathrm{C}(13)-\mathrm{C}(8)-\mathrm{S}(1) & 119.9(6) \\ \mathrm{C}(8)-\mathrm{C}(9)-\mathrm{C}(10) & 119.3(8) \\ \mathrm{C}(11)-\mathrm{C}(10)-\mathrm{C}(9) & 120.4 \\ & \end{array}$




\begin{tabular}{|c|c|}
\hline $\mathrm{C}(1)$ & .7 \\
\hline $\mathrm{C}(9)-\mathrm{C}(10)-\mathrm{H}(10)$ & 119.7 \\
\hline$C(12)-C(11)-C(10)$ & $117.9(9)$ \\
\hline$C(12)-C(11)-C(14)$ & $122.0(12)$ \\
\hline $\mathrm{C}(10)-\mathrm{C}(11)-\mathrm{C}(14)$ & $120.1(11)$ \\
\hline$C(11)-C(12)-C(13)$ & $122.8(11)$ \\
\hline $\mathrm{C}(11)-\mathrm{C}(12)-\mathrm{H}(12)$ & 118.6 \\
\hline $\mathrm{C}(13)-\mathrm{C}(12)-\mathrm{H}(12)$ & 118.6 \\
\hline $\mathrm{C}(8)-\mathrm{C}(13)-\mathrm{C}(12)$ & $117.5(9)$ \\
\hline $\mathrm{C}(8)-\mathrm{C}(13)-\mathrm{H}(13)$ & 121.2 \\
\hline $\mathrm{C}(12)-\mathrm{C}(13)-\mathrm{H}(13)$ & 121.2 \\
\hline $\mathrm{C}(11)-\mathrm{C}(14)-\mathrm{H}(14 \mathrm{~A})$ & 109.5 \\
\hline $\mathrm{C}(11)-\mathrm{C}(14)-\mathrm{H}(14 \mathrm{~B})$ & 109.5 \\
\hline $\mathrm{H}(14 \mathrm{~A})-\mathrm{C}(14)-\mathrm{H}(14 \mathrm{~B})$ & 109.5 \\
\hline $\mathrm{C}(11)-\mathrm{C}(14)-\mathrm{H}(14 \mathrm{C})$ & 109.5 \\
\hline $\mathrm{H}(14 \mathrm{~A})-\mathrm{C}(14)-\mathrm{H}(14 \mathrm{C})$ & 109.5 \\
\hline $\mathrm{H}(14 \mathrm{~B})-\mathrm{C}(14)-\mathrm{H}(14 \mathrm{C})$ & 109.5 \\
\hline $\mathrm{C}(16)-\mathrm{C}(15)-\mathrm{I}(1)$ & $171.2(8)$ \\
\hline$C(15)-C(16)-S i(1)$ & $170.8(9)$ \\
\hline$C(15)-C(16)-\operatorname{Si}(2)$ & $173.8(10)$ \\
\hline$C(19)-C(17)-C(18)$ & $114.1(17)$ \\
\hline $\mathrm{C}(19)-\mathrm{C}(17)-\mathrm{Si}(1)$ & $115.8(18)$ \\
\hline $\mathrm{C}(18)-\mathrm{C}(17)-\mathrm{Si}(1)$ & $112.6(14)$ \\
\hline $\mathrm{C}(19)-\mathrm{C}(17)-\mathrm{H}(17)$ & 104.2 \\
\hline $\mathrm{C}(18)-\mathrm{C}(17)-\mathrm{H}(17)$ & 104.2 \\
\hline $\mathrm{Si}(1)-\mathrm{C}(17)-\mathrm{H}(17)$ & 104.2 \\
\hline $\mathrm{C}(17)-\mathrm{C}(18)-\mathrm{H}(18 \mathrm{~A})$ & 109.5 \\
\hline $\mathrm{C}(17)-\mathrm{C}(18)-\mathrm{H}(18 \mathrm{~B})$ & 109.5 \\
\hline $\mathrm{H}(18 \mathrm{~A})-\mathrm{C}(18)-\mathrm{H}(18 \mathrm{~B})$ & 109.5 \\
\hline $\mathrm{C}(17)-\mathrm{C}(18)-\mathrm{H}(18 \mathrm{C})$ & 109.5 \\
\hline $\mathrm{H}(18 \mathrm{~A})-\mathrm{C}(18)-\mathrm{H}(18 \mathrm{C})$ & 109.5 \\
\hline $\mathrm{H}(18 \mathrm{~B})-\mathrm{C}(18)-\mathrm{H}(18 \mathrm{C})$ & 109.5 \\
\hline $\mathrm{C}(17)-\mathrm{C}(19)-\mathrm{H}(19 \mathrm{~A})$ & 109.5 \\
\hline $\mathrm{C}(17)-\mathrm{C}(19)-\mathrm{H}(19 \mathrm{~B})$ & 109.5 \\
\hline $\mathrm{H}(19 \mathrm{~A})-\mathrm{C}(19)-\mathrm{H}(19 \mathrm{~B})$ & 109.5 \\
\hline $\mathrm{C}(17)-\mathrm{C}(19)-\mathrm{H}(19 \mathrm{C})$ & 109.5 \\
\hline $\mathrm{H}(19 \mathrm{~A})-\mathrm{C}(19)-\mathrm{H}(19 \mathrm{C})$ & 109.5 \\
\hline $\mathrm{H}(19 \mathrm{~B})-\mathrm{C}(19)-\mathrm{H}(19 \mathrm{C})$ & 109.5 \\
\hline
\end{tabular}




\begin{tabular}{|c|c|}
\hline$-C(20)-C(21)$ & 9) \\
\hline$C(22)-C(20)-\operatorname{Si}(1)$ & $112.9(19)$ \\
\hline$C(21)-C(20)-\operatorname{Si}(1)$ & $110.5(19)$ \\
\hline $\mathrm{C}(22)-\mathrm{C}(20)-\mathrm{H}(20)$ & 107.3 \\
\hline $\mathrm{C}(21)-\mathrm{C}(20)-\mathrm{H}(20)$ & 107.3 \\
\hline $\mathrm{Si}(1)-\mathrm{C}(20)-\mathrm{H}(20)$ & 107.3 \\
\hline $\mathrm{C}(20)-\mathrm{C}(21)-\mathrm{H}(21 \mathrm{~A})$ & 109.5 \\
\hline $\mathrm{C}(20)-\mathrm{C}(21)-\mathrm{H}(21 \mathrm{~B})$ & 109.5 \\
\hline $\mathrm{H}(21 \mathrm{~A})-\mathrm{C}(21)-\mathrm{H}(21 \mathrm{~B})$ & 109.5 \\
\hline $\mathrm{C}(20)-\mathrm{C}(21)-\mathrm{H}(21 \mathrm{C})$ & 109.5 \\
\hline $\mathrm{H}(21 \mathrm{~A})-\mathrm{C}(21)-\mathrm{H}(21 \mathrm{C})$ & 109.5 \\
\hline $\mathrm{H}(21 \mathrm{~B})-\mathrm{C}(21)-\mathrm{H}(21 \mathrm{C})$ & 1 \\
\hline $\mathrm{C}(20)-\mathrm{C}(22)-\mathrm{H}(22 \mathrm{~A})$ & 109.5 \\
\hline $\mathrm{C}(20)-\mathrm{C}(22)-\mathrm{H}(22 \mathrm{~B})$ & 109.5 \\
\hline $\mathrm{H}(22 \mathrm{~A})-\mathrm{C}(22)-\mathrm{H}(22 \mathrm{~B})$ & 109.5 \\
\hline $\mathrm{C}(20)-\mathrm{C}(22)-\mathrm{H}(22 \mathrm{C})$ & 109.5 \\
\hline $\mathrm{H}(22 \mathrm{~A})-\mathrm{C}(22)-\mathrm{H}(22 \mathrm{C})$ & 109.5 \\
\hline $\mathrm{H}(22 \mathrm{~B})-\mathrm{C}(22)-\mathrm{H}(22 \mathrm{C})$ & 109.5 \\
\hline $\mathrm{C}(25)-\mathrm{C}(23)-\mathrm{C}(24)$ & $110.1(16)$ \\
\hline$C(25)-C(23)-S i(1)$ & $115.5(18)$ \\
\hline $\mathrm{C}(24)-\mathrm{C}(23)-\mathrm{Si}(1)$ & 110 \\
\hline $\mathrm{C}(25)-\mathrm{C}(23)-\mathrm{H}(23)$ & 106.9 \\
\hline $\mathrm{C}(24)-\mathrm{C}(23)-\mathrm{H}(23)$ & 106.9 \\
\hline $\mathrm{Si}(1)-\mathrm{C}(23)-\mathrm{H}(23)$ & 106.9 \\
\hline $\mathrm{C}(23)-\mathrm{C}(24)-\mathrm{H}(24 \mathrm{~A})$ & 109.5 \\
\hline $\mathrm{C}(23)-\mathrm{C}(24)-\mathrm{H}(24 \mathrm{~B})$ & 109.5 \\
\hline $\mathrm{H}(24 \mathrm{~A})-\mathrm{C}(24)-\mathrm{H}(24 \mathrm{~B})$ & 109.5 \\
\hline $\mathrm{C}(23)-\mathrm{C}(24)-\mathrm{H}(24 \mathrm{C})$ & 109.5 \\
\hline $\mathrm{H}(24 \mathrm{~A})-\mathrm{C}(24)-\mathrm{H}(24 \mathrm{C})$ & 109.5 \\
\hline $\mathrm{H}(24 \mathrm{~B})-\mathrm{C}(24)-\mathrm{H}(24 \mathrm{C})$ & 109.5 \\
\hline $\mathrm{C}(23)-\mathrm{C}(25)-\mathrm{H}(25 \mathrm{~A})$ & 109.5 \\
\hline $\mathrm{C}(23)-\mathrm{C}(25)-\mathrm{H}(25 \mathrm{~B})$ & 109.5 \\
\hline $\mathrm{H}(25 \mathrm{~A})-\mathrm{C}(25)-\mathrm{H}(25 \mathrm{~B})$ & 109.5 \\
\hline $\mathrm{C}(23)-\mathrm{C}(25)-\mathrm{H}(25 \mathrm{C})$ & 109.5 \\
\hline $\mathrm{H}(25 \mathrm{~A})-\mathrm{C}(25)-\mathrm{H}(25 \mathrm{C})$ & 109.5 \\
\hline $\mathrm{H}(25 \mathrm{~B})-\mathrm{C}(25)-\mathrm{H}(25 \mathrm{C})$ & 109.5 \\
\hline$C(27)-C(26)-C(28)$ & $109.3(13)$ \\
\hline$C(27)-C(26)-S i(2)$ & $114.9(17)$ \\
\hline
\end{tabular}




\begin{tabular}{|c|c|}
\hline$-C(26)-$ & $116(2)$ \\
\hline $\mathrm{C}(27)-\mathrm{C}(26)-\mathrm{H}(26)$ & 105.3 \\
\hline $\mathrm{C}(28)-\mathrm{C}(26)-\mathrm{H}(26)$ & 105.3 \\
\hline $\mathrm{Si}(2)-\mathrm{C}(26)-\mathrm{H}(26)$ & 105.3 \\
\hline $\mathrm{C}(26)-\mathrm{C}(27)-\mathrm{H}(27 \mathrm{~A})$ & 109.5 \\
\hline $\mathrm{C}(26)-\mathrm{C}(27)-\mathrm{H}(27 \mathrm{~B})$ & 109.5 \\
\hline $\mathrm{H}(27 \mathrm{~A})-\mathrm{C}(27)-\mathrm{H}(27 \mathrm{~B})$ & 109.5 \\
\hline $\mathrm{C}(26)-\mathrm{C}(27)-\mathrm{H}(27 \mathrm{C})$ & 109.5 \\
\hline $\mathrm{H}(27 \mathrm{~A})-\mathrm{C}(27)-\mathrm{H}(27 \mathrm{C})$ & 109.5 \\
\hline $\mathrm{H}(27 \mathrm{~B})-\mathrm{C}(27)-\mathrm{H}(27 \mathrm{C})$ & 109.5 \\
\hline $\mathrm{C}(26)-\mathrm{C}(28)-\mathrm{H}(28 \mathrm{~A})$ & 109.5 \\
\hline $\mathrm{C}(26)-\mathrm{C}(28)-\mathrm{H}(28 \mathrm{~B})$ & 109.5 \\
\hline $\mathrm{H}(28 \mathrm{~A})-\mathrm{C}(28)-\mathrm{H}(28 \mathrm{~B})$ & 109.5 \\
\hline $\mathrm{C}(26)-\mathrm{C}(28)-\mathrm{H}(28 \mathrm{C})$ & 109.5 \\
\hline $\mathrm{H}(28 \mathrm{~A})-\mathrm{C}(28)-\mathrm{H}(28 \mathrm{C})$ & 109.5 \\
\hline $\mathrm{H}(28 \mathrm{~B})-\mathrm{C}(28)-\mathrm{H}(28 \mathrm{C})$ & 109.5 \\
\hline $\mathrm{C}(31)-\mathrm{C}(29)-\mathrm{C}(30)$ & $110.0(16)$ \\
\hline$C(31)-C(29)-\operatorname{Si}(2)$ & $113.6(16)$ \\
\hline $\mathrm{C}(30)-\mathrm{C}(29)-\mathrm{Si}(2)$ & $113.0(16)$ \\
\hline $\mathrm{C}(31)-\mathrm{C}(29)-\mathrm{H}(29)$ & 106.6 \\
\hline $\mathrm{C}(30)-\mathrm{C}(29)-\mathrm{H}(29)$ & 106.6 \\
\hline $\mathrm{Si}(2)-\mathrm{C}(29)-\mathrm{H}(29)$ & 106.6 \\
\hline $\mathrm{C}(29)-\mathrm{C}(30)-\mathrm{H}(30 \mathrm{~A})$ & 109.5 \\
\hline $\mathrm{C}(29)-\mathrm{C}(30)-\mathrm{H}(30 \mathrm{~B})$ & 109.5 \\
\hline $\mathrm{H}(30 \mathrm{~A})-\mathrm{C}(30)-\mathrm{H}(30 \mathrm{~B})$ & 109.5 \\
\hline $\mathrm{C}(29)-\mathrm{C}(30)-\mathrm{H}(30 \mathrm{C})$ & 109.5 \\
\hline $\mathrm{H}(30 \mathrm{~A})-\mathrm{C}(30)-\mathrm{H}(30 \mathrm{C})$ & 109.5 \\
\hline $\mathrm{H}(30 \mathrm{~B})-\mathrm{C}(30)-\mathrm{H}(30 \mathrm{C})$ & 109.5 \\
\hline $\mathrm{C}(29)-\mathrm{C}(31)-\mathrm{H}(31 \mathrm{~A})$ & 109.5 \\
\hline $\mathrm{C}(29)-\mathrm{C}(31)-\mathrm{H}(31 \mathrm{~B})$ & 109.5 \\
\hline $\mathrm{H}(31 \mathrm{~A})-\mathrm{C}(31)-\mathrm{H}(31 \mathrm{~B})$ & 109.5 \\
\hline $\mathrm{C}(29)-\mathrm{C}(31)-\mathrm{H}(31 \mathrm{C})$ & 109.5 \\
\hline $\mathrm{H}(31 \mathrm{~A})-\mathrm{C}(31)-\mathrm{H}(31 \mathrm{C})$ & 109.5 \\
\hline $\mathrm{H}(31 \mathrm{~B})-\mathrm{C}(31)-\mathrm{H}(31 \mathrm{C})$ & 109.5 \\
\hline$C(33)-C(32)-C(34)$ & $110.1(15)$ \\
\hline $\mathrm{C}(33)-\mathrm{C}(32)-\mathrm{Si}(2)$ & $111.9(16)$ \\
\hline$C(34)-C(32)-\operatorname{Si}(2)$ & $114.7(16)$ \\
\hline $\mathrm{C}(33)-\mathrm{C}(32)-\mathrm{H}(32)$ & 106.5 \\
\hline
\end{tabular}




$\begin{array}{ll}\text { C(34)-C(32)-H(32) } & 106.5 \\ \text { Si(2)-C(32)-H(32) } & 106.5 \\ \text { C(32)-C(33)-H(33A) } & 109.5 \\ \text { C(32)-C(33)-H(33B) } & 109.5 \\ \text { H(33A)-C(33)-H(33B) } & 109.5 \\ \text { C(32)-C(33)-H(33C) } & 109.5 \\ \text { H(33A)-C(33)-H(33C) } & 109.5 \\ \text { H(33B)-C(33)-H(33C) } & 109.5 \\ \text { C(32)-C(34)-H(34A) } & 109.5 \\ \text { C(32)-C(34)-H(34B) } & 109.5 \\ \text { H(34A)-C(34)-H(34B) } & 109.5 \\ \text { C(32)-C(34)-H(34C) } & 109.5 \\ \text { H(34A)-C(34)-H(34C) } & 109.5 \\ \text { H(34B)-C(34)-H(34C) } & 109.5\end{array}$

Symmetry transformations used to generate equivalent atoms: 
Table S4. Anisotropic displacement parameters $\left(\AA^{2} \times 10^{3}\right)$ for 2a. The anisotropic displacement factor exponent takes the form: $-2 \square^{2}\left[h^{2} a^{*} U^{11}+\ldots+2 h k a^{*} b^{*} U^{12}\right]$

\begin{tabular}{|c|c|c|c|c|c|c|}
\hline & $\mathrm{U}^{11}$ & $\mathrm{U}^{22}$ & $\mathrm{U}^{33}$ & $\mathrm{U}^{23}$ & $\mathrm{U}^{13}$ & $\mathrm{U}^{12}$ \\
\hline $\mathrm{I}(1)$ & $37(1)$ & $29(1)$ & $26(1)$ & $2(1)$ & $5(1)$ & $3(1)$ \\
\hline$S(1)$ & $34(1)$ & $37(1)$ & $32(1)$ & $9(1)$ & $-2(1)$ & $-4(1)$ \\
\hline $\operatorname{Si}(1)$ & $48(4)$ & $42(3)$ & $87(5)$ & $12(4)$ & $33(4)$ & $2(3)$ \\
\hline $\operatorname{Si}(2)$ & $44(4)$ & $71(3)$ & $87(5)$ & $36(4)$ & $20(3)$ & $-1(3)$ \\
\hline $\mathrm{O}(1)$ & $58(3)$ & $45(3)$ & $59(4)$ & $-5(3)$ & $-24(3)$ & $-4(3)$ \\
\hline $\mathrm{O}(2)$ & $47(3)$ & $42(3)$ & $60(4)$ & $20(3)$ & $3(3)$ & $-9(3)$ \\
\hline $\mathrm{O}(3)$ & $36(3)$ & $58(3)$ & $25(2)$ & $5(2)$ & $-1(2)$ & $-3(2)$ \\
\hline $\mathrm{N}(1)$ & $42(3)$ & $29(3)$ & $31(3)$ & $3(2)$ & $-7(2)$ & $-1(2)$ \\
\hline $\mathrm{C}(1)$ & $72(4)$ & $24(3)$ & $17(3)$ & $2(2)$ & $9(3)$ & $16(3)$ \\
\hline$C(2)$ & $81(6)$ & $43(4)$ & $31(4)$ & $9(3)$ & $13(3)$ & $24(4)$ \\
\hline $\mathrm{C}(3)$ & $110(6)$ & $41(4)$ & $31(4)$ & $6(3)$ & $16(4)$ & $36(4)$ \\
\hline $\mathrm{C}(4)$ & $125(7)$ & $33(4)$ & $25(4)$ & $-3(3)$ & $-5(4)$ & $24(4)$ \\
\hline $\mathrm{C}(5)$ & $95(6)$ & $20(3)$ & $28(3)$ & $1(3)$ & $-17(3)$ & $16(4)$ \\
\hline$C(6)$ & $70(4)$ & $26(3)$ & $23(3)$ & $-1(2)$ & $-10(3)$ & $10(3)$ \\
\hline$C(7)$ & $51(4)$ & $27(3)$ & $33(3)$ & $3(3)$ & $-10(3)$ & $6(3)$ \\
\hline $\mathrm{C}(8)$ & $42(4)$ & $34(3)$ & $33(4)$ & $0(3)$ & $-2(3)$ & $-2(3)$ \\
\hline $\mathrm{C}(9)$ & $43(4)$ & $35(3)$ & $35(4)$ & $-7(3)$ & $9(3)$ & $-1(3)$ \\
\hline $\mathrm{C}(10)$ & $79(5)$ & $38(4)$ & $34(4)$ & $0(3)$ & $11(4)$ & $8(4)$ \\
\hline $\mathrm{C}(11)$ & $76(5)$ & $53(4)$ & $60(6)$ & $-5(4)$ & $-5(5)$ & $21(4)$ \\
\hline $\mathrm{C}(12)$ & $58(6)$ & $66(5)$ & 153(14) & $-13(7)$ & $-43(8)$ & $15(4)$ \\
\hline $\mathrm{C}(13)$ & $44(4)$ & $39(4)$ & 104(9) & $-2(5)$ & $-10(5)$ & $-3(3)$ \\
\hline$C(14)$ & $116(11)$ & $77(7)$ & $107(11)$ & $-10(8)$ & $-16(9)$ & $48(8)$ \\
\hline$C(15)$ & $47(4)$ & $49(4)$ & $37(4)$ & $3(3)$ & $16(3)$ & $7(3)$ \\
\hline$C(16)$ & $53(4)$ & $49(4)$ & $59(5)$ & $12(4)$ & $23(4)$ & $8(3)$ \\
\hline $\mathrm{C}(17)$ & $76(7)$ & $56(6)$ & $105(7)$ & $15(6)$ & $31(6)$ & $8(6)$ \\
\hline $\mathrm{C}(18)$ & 79(10) & $57(10)$ & 104(10) & $16(9)$ & $26(9)$ & $10(9)$ \\
\hline $\mathrm{C}(19)$ & $120(17)$ & $86(15)$ & $116(12)$ & $25(12)$ & $33(12)$ & $34(13)$ \\
\hline $\mathrm{C}(20)$ & $63(6)$ & $82(6)$ & $100(6)$ & $23(6)$ & $17(5)$ & $3(5)$ \\
\hline $\mathrm{C}(21)$ & $85(11)$ & $117(12)$ & 101(10) & $18(10)$ & $12(9)$ & $9(10)$ \\
\hline $\mathrm{C}(22)$ & $85(11)$ & $124(13)$ & $137(14)$ & 17(13) & $16(11)$ & $-17(10)$ \\
\hline $\mathrm{C}(23)$ & $55(6)$ & $61(6)$ & $87(7)$ & $10(6)$ & $30(6)$ & $2(6)$ \\
\hline
\end{tabular}




$\begin{array}{lcccccc}\mathrm{C}(24) & 70(11) & 64(8) & 88(12) & -1(9) & 36(10) & 11(8) \\ \mathrm{C}(25) & 65(8) & 75(9) & 92(10) & 15(9) & 37(8) & 4(8) \\ \mathrm{C}(26) & 70(6) & 67(6) & 106(7) & 17(6) & 13(6) & -4(5) \\ \mathrm{C}(27) & 76(9) & 49(9) & 107(11) & 11(8) & 18(9) & -2(8) \\ \mathrm{C}(28) & 89(12) & 97(14) & 161(18) & 19(15) & -5(14) & -26(11) \\ \mathrm{C}(29) & 63(6) & 94(7) & 100(7) & 34(7) & 16(6) & -1(6) \\ \mathrm{C}(30) & 84(11) & 111(11) & 102(8) & 35(9) & 5(9) & 12(10) \\ \mathrm{C}(31) & 81(13) & 99(10) & 123(14) & 40(10) & -8(13) & 24(11) \\ \mathrm{C}(32) & 57(5) & 73(5) & 88(6) & 25(5) & 29(5) & 4(5) \\ \mathrm{C}(33) & 80(9) & 85(9) & 93(10) & 15(8) & 26(9) & 7(8) \\ \mathrm{C}(34) & 68(8) & 103(11) & 100(11) & 23(10) & 33(9) & -3(8)\end{array}$



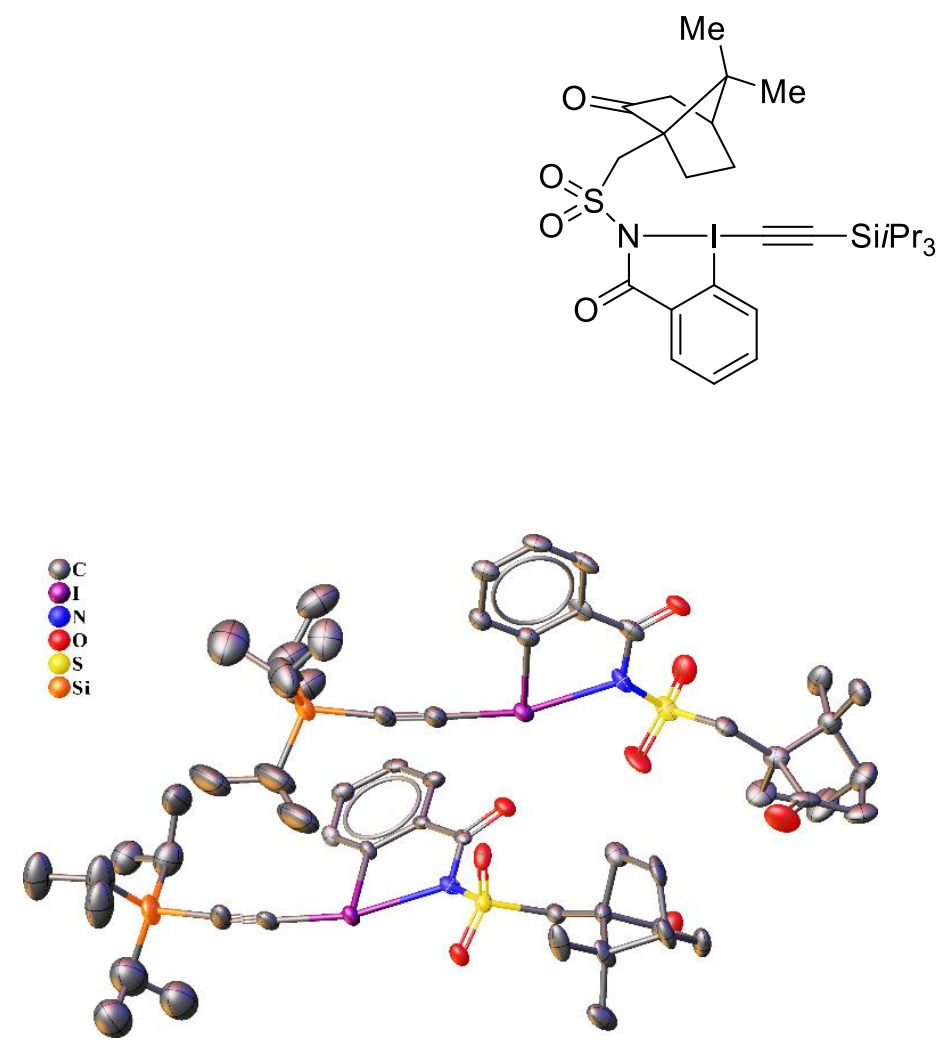

Experimental. Single colourless prism-shaped crystals of $\mathbf{2 e}$ were obtained by recrystallisation from SOLVENT at TEMPERATURE. A suitable crystal of $0.85 \times 0.19 \times 0.10 \mathrm{~mm}^{3}$ was selected and mounted on a suitable support on a Bruker P4 diffractometer. The crystal was kept at a steady $T=100(2) \mathrm{K}$ during data collection. The structure was solved with the ShelXT (Sheldrick, 2015) structure solution program using the dual solution method and by using Olex2 (Dolomanov et al., 2009) as the graphical interface. The model was refined with version 2018/3 of ShelXL (Sheldrick, 2015) using full matrix least squares on $|\boldsymbol{F}|^{2}$ minimisation.

Crystal Data. $\mathrm{C}_{28} \mathrm{H}_{40} \mathrm{INO}_{4} \mathrm{SSi}, \quad M_{r}=641.66$, orthorhombic, $P 22_{1} 2_{1} 2_{1}$ (No. 19), a = 12.8506(17) $\AA$, $\mathrm{b}=15.8182(15) \AA, \mathrm{c}=31.718(4) \AA, \square=\square=\square=90^{\circ}$, $V=6447.4(14) \AA^{3}, T=100(2) \mathrm{K}, Z=8, Z^{\prime}=2$, $\square(\mathrm{MoK} \square)=1.125,74240$ reflections measured, 11585 unique $\left(R_{\text {int }}=0.1052\right)$ which were used in all calculations. The final $w R_{2}$ was 0.1420 (all data) and $R_{l}$ was 0.0634 (I > 2(I)). 


\begin{tabular}{|c|c|}
\hline Compound & $2 \mathbf{e}$ \\
\hline Formula & $\mathrm{C}_{28} \mathrm{H}_{40} \mathrm{INO}_{4} \mathrm{SSi}$ \\
\hline$D_{\text {calc. }} / \mathrm{g} \mathrm{cm}^{-3}$ & 1.322 \\
\hline$\square / \mathrm{mm}^{-1}$ & 1.125 \\
\hline Formula Weight & 641.66 \\
\hline Colour & colourless \\
\hline Shape & prism \\
\hline Size $/ \mathrm{mm}^{3}$ & $0.85 \times 0.19 \times 0.10$ \\
\hline$T / \mathrm{K}$ & $100(2)$ \\
\hline Crystal System & orthorhombic \\
\hline Flack Parameter & $0.09(4)$ \\
\hline Hooft Parameter & $0.083(13)$ \\
\hline Space Group & $P 2{ }_{1}{ }_{1} 2_{1}$ \\
\hline$a / \AA$ & $12.8506(17)$ \\
\hline$b / \AA$ & $15.8182(15)$ \\
\hline$c / \AA$ & $31.718(4)$ \\
\hline$\square /^{\circ}$ & 90 \\
\hline$\square /^{\circ}$ & 90 \\
\hline$\square /^{\circ}$ & 90 \\
\hline $\mathrm{V} / \AA^{3}$ & $6447.4(14)$ \\
\hline$Z$ & 8 \\
\hline$Z^{\prime}$ & 2 \\
\hline Wavelength/Å & 0.71073 \\
\hline Radiation type & MoK $\square$ \\
\hline$\square_{\min } I^{\circ}$ & 1.710 \\
\hline$\left.\square_{\max }\right|^{\circ}$ & 25.200 \\
\hline Measured Refl. & 74240 \\
\hline \multicolumn{2}{|c|}{ Independent Refl. 11585} \\
\hline \multicolumn{2}{|c|}{$\begin{array}{l}\text { Reflections with I } 9479 \\
>2(\mathrm{I})\end{array}$} \\
\hline$R_{\text {int }}$ & 0.1052 \\
\hline Parameters & 666 \\
\hline Restraints & 682 \\
\hline \multicolumn{2}{|c|}{$\underset{3}{\text { Largest Peak/e } \AA^{-} 0.911}$} \\
\hline $\begin{array}{l}\text { Deepest Hole/e } \\
\AA^{-3}\end{array}$ & -1.007 \\
\hline GooF & 1.109 \\
\hline$w R_{2}$ (all data) & 0.1420 \\
\hline$w R_{2}$ & 0.1334 \\
\hline$R_{l}$ (all data) & 0.0811 \\
\hline$R_{1}$ & 0.0634 \\
\hline
\end{tabular}


A colourless prism-shaped crystal with dimensions of $0.85 \times 0.19 \times 0.10 \mathrm{~mm}^{3}$ was mounted on a suitable support. Data were collected using a Bruker P4 diffractometer operating at $T=100(2) \mathrm{K}$.

Data were measured using $\square$ scans using MoK $\square$ radiation. The total number of runs and images was based on the strategy calculation from the program XS (Sheldrick, 2008). The maximum resolution achieved was $\square=25.200^{\circ}(0.83 \AA)$.

The diffraction pattern was indexed. The total number of runs and images was based on the strategy calculation from the program XS (Sheldrick, 2008) and the unit cell was refined using EvalCCD (Duisenberg \& Schreurs, 1990-2000) on 179 reflections, 0\%\% of the observed reflections.

Data reduction, scaling and absorption corrections were performed using EvalCCD (Duisenberg \& Schreurs, 1990-2000). The final completeness is $99.80 \% \%$ out to $25.200^{\circ}$ in $\square$. A multi-scan absorption correction was performed using SADABS-2008/1 (Bruker,2008) was used for absorption correction. $w R_{2}$ (int) was 0.1094 before and 0.0953 after correction. The Ratio of minimum to maximum transmission is 0.6779 . The $\square / 2$ correction factor is 0.0015 . The absorption coefficient $\square$ of this material is $1.125 \mathrm{~mm}^{-1}$ at this wavelength ( $\square=0.711 \AA$ ) and the minimum and maximum transmissions are 0.505 and 0.745 .

The structure was solved and the space group $P 2{ }_{1} 2{ }_{1} 2_{1}$ (\# 19) determined by the ShelXT (Sheldrick, 2015) structure solution program using dual and refined by full matrix least squares on $|\boldsymbol{F}|^{2}$ using version 2018/3 of ShelXL (Sheldrick, 2015). All non-hydrogen atoms were refined anisotropically. Hydrogen atom positions were calculated geometrically and refined using the riding model. Hydrogen atom positions were calculated geometrically and refined using the riding model.

The structure was refined as a 2-component inversion twin.

The value of $Z^{\prime}$ is 2 . This means that there are two independent molecules in the asymmetric unit.

The Flack parameter was refined to 0.09(4). Determination of absolute structure using Bayesian statistics on Bijvoet differences using the Olex2 results in 0.083(13). Note: The Flack parameter is used to determine chirality of the crystal studied, the value should be near 0 , a value of 1 means that the stereochemistry is wrong and the model should be inverted. A value of 0.5 means that the crystal consists of a racemic mixture of the two enantiomers. 
Table S5: Fractional Atomic Coordinates $\left(\times 10^{4}\right)$ and Equivalent Isotropic Displacement Parameters $\left(\AA^{2} \times 10^{3}\right)$ for 2e. $U_{e q}$ is defined as $1 / 3$ of the trace of the orthogonalised $U_{i j}$.

\begin{tabular}{|c|c|c|c|c|}
\hline Atom & $\mathbf{x}$ & $\mathbf{y}$ & $\mathbf{z}$ & $U_{e q}$ \\
\hline $\mathrm{I} 1$ & $3420.0(6)$ & $6202.8(4)$ & $7335.8(2)$ & $24.34(19)$ \\
\hline $\mathrm{S} 1$ & $1648(3)$ & $5243.4(19)$ & $6628.0(10)$ & $31.5(7)$ \\
\hline Si1 & $6032(3)$ & $6838(3)$ & $8517.1(13)$ & $37.1(10)$ \\
\hline $\mathrm{O} 1$ & $41(6)$ & $6388(6)$ & $7017(3)$ & $32(2)$ \\
\hline $\mathrm{O} 2$ & $914(8)$ & $4635(6)$ & $6769(3)$ & $42(2)$ \\
\hline $\mathrm{O} 3$ & $2709(7)$ & $4975(6)$ & $6543(3)$ & $45(3)$ \\
\hline $\mathrm{O} 4$ & $1687(9)$ & $6368(7)$ & $5358(3)$ & $58(3)$ \\
\hline N1 & $1785(7)$ & $6010(6)$ & $6966(3)$ & $30(2)$ \\
\hline $\mathrm{C} 1$ & $2308(9)$ & $7003(8)$ & $7645(4)$ & $28(3)$ \\
\hline $\mathrm{C} 2$ & $2627(10)$ & $7538(8)$ & 7964(4) & $30(3)$ \\
\hline $\mathrm{C} 3$ & 1874(9) & $8088(8)$ & $8146(4)$ & $32(3)$ \\
\hline $\mathrm{C} 4$ & $861(10)$ & $8077(8)$ & $8003(4)$ & $34(3)$ \\
\hline $\mathrm{C} 5$ & 571(9) & $7551(8)$ & $7679(5)$ & $39(3)$ \\
\hline C6 & $1280(9)$ & $6982(7)$ & $7494(4)$ & $27(3)$ \\
\hline $\mathrm{C} 7$ & 963(9) & $6428(8)$ & $7147(4)$ & $32(3)$ \\
\hline $\mathrm{C} 8$ & $1143(10)$ & $5761(8)$ & $6173(4)$ & $27(3)$ \\
\hline C9 & $968(10)$ & $5209(8)$ & $5778(4)$ & $30(3)$ \\
\hline $\mathrm{C} 10$ & $1170(10)$ & $5707(9)$ & $5371(4)$ & $36(3)$ \\
\hline $\mathrm{C} 11$ & $694(12)$ & $5249(9)$ & $5022(4)$ & $45(3)$ \\
\hline $\mathrm{C} 12$ & $182(13)$ & $4505(10)$ & $5244(4)$ & $48(3)$ \\
\hline $\mathrm{C} 13$ & $-153(10)$ & $4886(8)$ & $5679(4)$ & $32(3)$ \\
\hline $\mathrm{C} 14$ & $-965(10)$ & $5581(9)$ & $5629(5)$ & $42(3)$ \\
\hline C15 & $-584(12)$ & $4224(9)$ & $5994(4)$ & $47(4)$ \\
\hline $\mathrm{C} 16$ & $1100(14)$ & $3899(10)$ & $5362(5)$ & $62(5)$ \\
\hline $\mathrm{C} 17$ & $1677(13)$ & $4400(8)$ & $5729(4)$ & $44(3)$ \\
\hline C18 & $4558(10)$ & $6504(9)$ & $7776(4)$ & $35(3)$ \\
\hline
\end{tabular}




\begin{tabular}{|c|c|c|c|c|}
\hline Atom & $\mathbf{x}$ & $\mathbf{y}$ & $\mathbf{z}$ & $U_{e q}$ \\
\hline$\overline{\mathrm{C} 19}$ & $5168(9)$ & $6654(8)$ & $8049(4)$ & $31(3)$ \\
\hline $\mathrm{C} 20$ & $5592(16)$ & $6056(12)$ & $8915(6)$ & $86(4)$ \\
\hline $\mathrm{C} 21$ & $5794(18)$ & $5160(12)$ & $8740(6)$ & $87(4)$ \\
\hline $\mathrm{C} 22$ & $4431(15)$ & $6189(14)$ & $9035(6)$ & $86(4)$ \\
\hline $\mathrm{C} 23$ & $5833(14)$ & 7993(14) & $8718(6)$ & $88(6)$ \\
\hline $\mathrm{C} 24$ & $4784(14)$ & $8387(11)$ & $8658(6)$ & $78(6)$ \\
\hline $\mathrm{C} 25$ & $6030(20)$ & $8013(16)$ & $9199(6)$ & 115(9) \\
\hline $\mathrm{C} 26$ & $7378(14)$ & $6732(18)$ & $8330(7)$ & $113(5)$ \\
\hline $\mathrm{C} 27$ & $7602(15)$ & $7323(16)$ & $7964(7)$ & $113(6)$ \\
\hline $\mathrm{C} 28$ & $8194(14)$ & $6856(17)$ & $8670(7)$ & $114(6)$ \\
\hline $\mathrm{I} 2$ & $8369.9(6)$ & $5363.3(4)$ & $7298.4(2)$ & $22.51(19)$ \\
\hline S2 & $6514(2)$ & $6296.4(18)$ & $6579.6(9)$ & $23.4(6)$ \\
\hline $\mathrm{Si} 2$ & $10788(3)$ & $4417(3)$ & $8490.8(14)$ & $51.5(13)$ \\
\hline $\mathrm{O} 5$ & $4986(6)$ & $5197(5)$ & $7018(2)$ & 27.4(19) \\
\hline O6 & $5881(7)$ & $6940(5)$ & $6776(3)$ & $29(2)$ \\
\hline $\mathrm{O} 7$ & $7531(6)$ & $6531(6)$ & $6428(3)$ & $29(2)$ \\
\hline $\mathrm{O} 8$ & $4614(8)$ & $5113(6)$ & $5502(3)$ & $47(3)$ \\
\hline $\mathrm{N} 2$ & $6727(7)$ & $5500(6)$ & $6901(3)$ & $23(2)$ \\
\hline $\mathrm{C} 29$ & $7287(8)$ & $4515(7)$ & $7595(4)$ & $20(2)$ \\
\hline C30 & $7574(9)$ & $3982(7)$ & 7904(4) & $20(2)$ \\
\hline C31 & $6852(9)$ & $3445(8)$ & $8097(4)$ & $28(3)$ \\
\hline C32 & $5837(10)$ & $3447(8)$ & $7945(4)$ & $26(3)$ \\
\hline C33 & $5540(9)$ & $3987(8)$ & $7626(4)$ & $29(3)$ \\
\hline C34 & $6259(8)$ & $4527(7)$ & $7443(3)$ & $18(2)$ \\
\hline $\mathrm{C} 35$ & $5925(9)$ & $5109(7)$ & 7101(3) & $18(2)$ \\
\hline $\mathrm{C} 36$ & $5805(9)$ & $5819(8)$ & $6152(4)$ & $27(3)$ \\
\hline C37 & 5193(9) & $6406(8)$ & $5855(4)$ & $28(2)$ \\
\hline C38 & $4704(10)$ & $5850(9)$ & $5498(4)$ & $32(3)$ \\
\hline C39 & $4379(11)$ & $6506(9)$ & $5157(4)$ & $42(3)$ \\
\hline
\end{tabular}




\begin{tabular}{lcccl}
\hline Atom & $\mathbf{x}$ & $\mathbf{y}$ & $\mathbf{z}$ & $\boldsymbol{U}_{\text {eq }}$ \\
\hline C40 & $4663(10)$ & $7313(9)$ & $5344(5)$ & $40(3)$ \\
C41 & $5697(10)$ & $7120(9)$ & $5595(4)$ & $37(3)$ \\
C42 & $6618(12)$ & $6802(10)$ & $5305(5)$ & $55(4)$ \\
C43 & $6081(12)$ & $7903(9)$ & $5849(5)$ & $52(4)$ \\
C44 & $3896(10)$ & $7520(9)$ & $5700(5)$ & $43(3)$ \\
C45 & $4199(10)$ & $6858(8)$ & $6053(4)$ & $33(3)$ \\
C46 & $9475(9)$ & $5066(8)$ & $7751(4)$ & $30(3)$ \\
C47 & $10009(11)$ & $4864(10)$ & $8039(4)$ & $42(4)$ \\
C48 & $10959(17)$ & $5257(12)$ & $8872(6)$ & $90(4)$ \\
C49 & $11281(17)$ & $6109(12)$ & $8693(6)$ & $91(4)$ \\
C50 & $11696(17)$ & $5066(13)$ & $9243(6)$ & $90(4)$ \\
C51 & $11996(16)$ & $3867(14)$ & $8306(7)$ & $99(4)$ \\
C52 & $12741(16)$ & $4515(14)$ & $8138(7)$ & $99(4)$ \\
C53 & $11735(17)$ & $3246(14)$ & $7949(6)$ & $99(4)$ \\
C54 & $9912(13)$ & $3530(10)$ & $8715(5)$ & $63(4)$ \\
C55 & $10431(14)$ & $2882(11)$ & $8989(5)$ & $67(5)$ \\
C56 & $8981(13)$ & $3870(13)$ & $8945(5)$ & $75(5)$
\end{tabular}

Table S6: Anisotropic Displacement Parameters $\left(\times 10^{4}\right)$ 2e. The anisotropic displacement factor exponent takes the form: $-2 \square^{2}\left[h^{2} a^{* 2} \times U_{11}+\ldots+2 h k a^{*} \times b^{*} \times U_{12}\right]$

\begin{tabular}{llllccc}
\hline Atom & \multicolumn{1}{c}{$\boldsymbol{U}_{\mathbf{1 1}}$} & $\boldsymbol{U}_{\mathbf{2 2}}$ & $\boldsymbol{U}_{\mathbf{3 3}}$ & $\boldsymbol{U}_{\mathbf{2 3}}$ & $\boldsymbol{U}_{\mathbf{1 3}}$ & $\boldsymbol{U}_{\mathbf{1 2}}$ \\
\hline I1 & $18.1(4)$ & $25.0(4)$ & $30.0(4)$ & $2.6(3)$ & $0.5(4)$ & $2.6(3)$ \\
S1 & $23.0(15)$ & $23.9(16)$ & $47.5(18)$ & $-1.1(14)$ & $-4.6(16)$ & $0.0(16)$ \\
Si1 & $20.5(19)$ & $51(3)$ & $40(2)$ & $7(2)$ & $-5.8(17)$ & $-5.4(18)$ \\
O1 & $17(4)$ & $35(5)$ & $44(5)$ & $1(4)$ & $3(4)$ & $-2(4)$ \\
O2 & $47(6)$ & $33(5)$ & $45(6)$ & $3(5)$ & $-8(5)$ & $-14(5)$ \\
O3 & $20(5)$ & $41(6)$ & $73(7)$ & $-17(5)$ & $-5(5)$ & $16(4)$ \\
O4 & $46(6)$ & $53(6)$ & $73(7)$ & $23(5)$ & $13(6)$ & $-10(5)$ \\
N1 & $15(5)$ & $28(5)$ & $46(6)$ & $1(4)$ & $-6(4)$ & $-2(4)$
\end{tabular}




\begin{tabular}{|c|c|c|c|c|c|c|}
\hline Atom & $U_{11}$ & $U_{22}$ & $U_{33}$ & $U_{23}$ & $U_{13}$ & $U_{12}$ \\
\hline$\overline{\mathrm{C} 1}$ & $23(5)$ & $29(6)$ & $33(6)$ & $2(5)$ & $4(5)$ & $1(5)$ \\
\hline $\mathrm{C} 2$ & $23(6)$ & $29(7)$ & $39(7)$ & $0(5)$ & $-1(5)$ & $1(5)$ \\
\hline $\mathrm{C} 3$ & $30(6)$ & $21(6)$ & $45(8)$ & $0(5)$ & $8(5)$ & $-3(5)$ \\
\hline $\mathrm{C} 4$ & $27(6)$ & $28(7)$ & $48(8)$ & $-3(6)$ & $15(6)$ & $5(6)$ \\
\hline $\mathrm{C} 5$ & $18(6)$ & $33(7)$ & $68(9)$ & $-6(7)$ & $10(6)$ & $-8(5)$ \\
\hline C6 & $23(6)$ & $13(6)$ & $45(7)$ & $1(5)$ & $3(4)$ & $-12(4)$ \\
\hline $\mathrm{C} 7$ & $20(5)$ & $23(7)$ & $52(7)$ & $5(5)$ & $5(5)$ & $-4(5)$ \\
\hline $\mathrm{C} 8$ & $20(6)$ & $23(7)$ & $37(6)$ & $0(5)$ & $2(5)$ & $5(5)$ \\
\hline C9 & $35(6)$ & $16(6)$ & $40(6)$ & $-3(5)$ & $6(5)$ & $-2(5)$ \\
\hline $\mathrm{C} 10$ & $31(7)$ & $35(6)$ & $43(7)$ & $9(5)$ & $20(5)$ & $11(5)$ \\
\hline $\mathrm{C} 11$ & $52(9)$ & $41(8)$ & $43(7)$ & $-4(6)$ & $20(6)$ & $12(6)$ \\
\hline C12 & $63(9)$ & $43(8)$ & $39(7)$ & $-10(6)$ & $-3(6)$ & $0(6)$ \\
\hline C13 & $32(6)$ & $29(7)$ & $35(6)$ & $3(5)$ & $1(5)$ & $-4(5)$ \\
\hline $\mathrm{C} 14$ & $32(7)$ & $44(8)$ & $48(9)$ & $-4(7)$ & $-8(6)$ & $-9(6)$ \\
\hline C15 & $56(10)$ & $44(9)$ & $40(8)$ & $5(6)$ & $-5(7)$ & $-27(7)$ \\
\hline C16 & $95(12)$ & $43(9)$ & $48(9)$ & $-19(7)$ & $-4(8)$ & $23(8)$ \\
\hline $\mathrm{C} 17$ & $55(8)$ & $27(7)$ & $50(8)$ & $-4(5)$ & $3(7)$ & $19(7)$ \\
\hline C18 & $24(6)$ & $47(9)$ & $35(7)$ & $3(6)$ & $2(5)$ & $-1(6)$ \\
\hline C19 & $19(6)$ & $36(8)$ & $37(7)$ & $14(6)$ & $1(5)$ & $-1(6)$ \\
\hline $\mathrm{C} 20$ & $119(10)$ & $79(8)$ & $60(7)$ & $2(6)$ & $16(7)$ & $11(8)$ \\
\hline $\mathrm{C} 21$ & $120(11)$ & $79(8)$ & $61(7)$ & $2(7)$ & $15(7)$ & $12(8)$ \\
\hline $\mathrm{C} 22$ & $118(11)$ & $80(8)$ & $60(7)$ & $3(7)$ & $17(7)$ & $10(8)$ \\
\hline $\mathrm{C} 23$ & $65(11)$ & $89(13)$ & 111(14) & $-39(12)$ & $-23(11)$ & $-4(10)$ \\
\hline $\mathrm{C} 24$ & $100(13)$ & $44(11)$ & $89(14)$ & $-5(10)$ & $-19(11)$ & $8(10)$ \\
\hline $\mathrm{C} 25$ & $130(20)$ & $100(20)$ & 111(14) & $-18(16)$ & $-15(14)$ & $37(17)$ \\
\hline $\mathrm{C} 26$ & $41(7)$ & $167(15)$ & 131(13) & $-33(11)$ & $21(7)$ & $-23(8)$ \\
\hline $\mathrm{C} 27$ & $43(7)$ & $167(15)$ & 131(13) & $-33(11)$ & $23(7)$ & $-22(8)$ \\
\hline $\mathrm{C} 28$ & $41(7)$ & $169(15)$ & $132(13)$ & $-32(11)$ & $19(7)$ & $-22(8)$ \\
\hline $\mathrm{I} 2$ & $15.7(3)$ & $24.6(4)$ & $27.3(4)$ & $4.7(3)$ & $-3.5(4)$ & $-1.9(3)$ \\
\hline
\end{tabular}




\begin{tabular}{|c|c|c|c|c|c|c|}
\hline$\overline{\text { Atom }}$ & $U_{11}$ & $U_{22}$ & $U_{33}$ & $U_{23}$ & $U_{13}$ & $U_{12}$ \\
\hline$\overline{\mathrm{S} 2}$ & $21.7(15)$ & $23.2(15)$ & $25.4(14)$ & $0.2(12)$ & $-5.2(14)$ & $3.1(15)$ \\
\hline $\mathrm{Si} 2$ & $33(2)$ & $77(4)$ & $44(2)$ & $11(2)$ & $-18.7(19)$ & $7(2)$ \\
\hline $\mathrm{O} 5$ & $18(4)$ & $31(5)$ & $33(5)$ & $-4(4)$ & $1(4)$ & $4(4)$ \\
\hline O6 & $37(5)$ & $19(5)$ & $32(5)$ & $-7(4)$ & $-15(4)$ & $8(4)$ \\
\hline O7 & $19(4)$ & $34(5)$ & $34(5)$ & $5(4)$ & $-4(4)$ & $-6(4)$ \\
\hline O8 & $68(7)$ & $37(5)$ & $37(5)$ & $0(4)$ & $-9(5)$ & $-7(5)$ \\
\hline N2 & $17(5)$ & $20(5)$ & $32(5)$ & $1(4)$ & $2(4)$ & $3(4)$ \\
\hline $\mathrm{C} 29$ & $14(5)$ & $21(6)$ & $27(6)$ & $0(4)$ & $-3(4)$ & $-1(4)$ \\
\hline $\mathrm{C} 30$ & $19(6)$ & $7(5)$ & $32(6)$ & $-4(4)$ & $-4(5)$ & $-1(4)$ \\
\hline C31 & $33(6)$ & $24(6)$ & $25(6)$ & $1(5)$ & $5(5)$ & $-3(5)$ \\
\hline $\mathrm{C} 32$ & $26(6)$ & $24(7)$ & $26(6)$ & $-8(5)$ & $13(5)$ & $-1(5)$ \\
\hline C33 & $25(6)$ & $37(7)$ & $25(6)$ & $-5(5)$ & $8(5)$ & $-7(5)$ \\
\hline C34 & $16(5)$ & $19(6)$ & $17(5)$ & $-6(4)$ & $4(4)$ & $-3(4)$ \\
\hline $\mathrm{C} 35$ & $16(5)$ & $18(6)$ & $21(6)$ & $-8(4)$ & $2(4)$ & $3(4)$ \\
\hline C36 & $20(6)$ & $30(7)$ & $30(6)$ & $-4(5)$ & $-1(5)$ & $2(5)$ \\
\hline C37 & $22(6)$ & $35(6)$ & $27(6)$ & $0(4)$ & $-7(4)$ & $3(5)$ \\
\hline C38 & $26(7)$ & $44(6)$ & $25(6)$ & $-3(5)$ & $-2(5)$ & $-1(5)$ \\
\hline C39 & $36(8)$ & $52(8)$ & $37(7)$ & $6(5)$ & $-14(6)$ & $-18(6)$ \\
\hline $\mathrm{C} 40$ & $23(6)$ & $44(7)$ & $52(7)$ & $18(6)$ & $-18(5)$ & $-8(6)$ \\
\hline $\mathrm{C} 41$ & $21(6)$ & $41(7)$ & $48(8)$ & $23(5)$ & $-12(5)$ & $2(5)$ \\
\hline $\mathrm{C} 42$ & $29(7)$ & $75(11)$ & $61(9)$ & $30(7)$ & $1(7)$ & $9(8)$ \\
\hline $\mathrm{C} 43$ & $45(9)$ & $44(8)$ & $66(10)$ & $28(7)$ & $-28(7)$ & $-26(7)$ \\
\hline C44 & $25(7)$ & $48(9)$ & $56(8)$ & $-5(6)$ & $-24(6)$ & $9(6)$ \\
\hline $\mathrm{C} 45$ & $30(7)$ & $26(7)$ & $45(7)$ & $-5(5)$ & $-3(5)$ & $7(5)$ \\
\hline C46 & $18(6)$ & $40(8)$ & $33(7)$ & $10(6)$ & $4(5)$ & $-5(5)$ \\
\hline $\mathrm{C} 47$ & $29(7)$ & $62(10)$ & $35(7)$ & $13(7)$ & $-4(5)$ & $-20(7)$ \\
\hline $\mathrm{C} 48$ & $108(10)$ & $84(8)$ & $77(8)$ & $38(7)$ & $-44(7)$ & $-27(8)$ \\
\hline C49 & $108(10)$ & $86(9)$ & $78(8)$ & $38(7)$ & $-45(7)$ & $-27(8)$ \\
\hline C50 & $108(10)$ & $84(9)$ & $77(8)$ & $38(7)$ & $-43(7)$ & $-28(8)$ \\
\hline
\end{tabular}




\begin{tabular}{lcrrcrc}
\hline Atom & $\boldsymbol{U}_{\mathbf{1 1}}$ & $\boldsymbol{U}_{\mathbf{2 2}}$ & $\boldsymbol{U}_{\mathbf{3 3}}$ & $\boldsymbol{U}_{\mathbf{2 3}}$ & $\boldsymbol{U}_{\mathbf{1 3}}$ & $\boldsymbol{U}_{\mathbf{1 2}}$ \\
\hline C51 & $79(8)$ & $122(12)$ & $95(10)$ & $27(8)$ & $-5(8)$ & $-3(8)$ \\
C52 & $80(9)$ & $122(12)$ & $96(10)$ & $27(8)$ & $-3(8)$ & $-5(8)$ \\
C53 & $80(9)$ & $122(12)$ & $95(10)$ & $27(8)$ & $-5(8)$ & $-2(8)$ \\
C54 & $77(11)$ & $57(10)$ & $54(10)$ & $10(8)$ & $-11(8)$ & $-2(8)$ \\
C55 & $79(13)$ & $65(12)$ & $57(11)$ & $5(9)$ & $-6(9)$ & $3(9)$ \\
C56 & $61(11)$ & $115(17)$ & $49(10)$ & $23(11)$ & $-5(8)$ & $-4(10)$
\end{tabular}

Table S7: Bond Lengths in $\AA$ for 2 e.

\begin{tabular}{|c|c|c|}
\hline$\overline{\text { Atom }}$ & Atom & 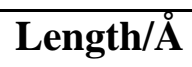 \\
\hline$\overline{\mathrm{I} 1}$ & N1 & $2.426(10)$ \\
\hline I1 & $\mathrm{C} 1$ & $2.145(12)$ \\
\hline I1 & $\mathrm{C} 18$ & $2.077(13)$ \\
\hline S1 & $\mathrm{O} 2$ & $1.420(10)$ \\
\hline S1 & $\mathrm{O} 3$ & $1.453(9)$ \\
\hline S1 & N1 & $1.627(11)$ \\
\hline S1 & $\mathrm{C} 8$ & $1.781(13)$ \\
\hline Si1 & C19 & $1.877(14)$ \\
\hline Sil & $\mathrm{C} 20$ & $1.85(2)$ \\
\hline Si1 & $\mathrm{C} 23$ & $1.95(2)$ \\
\hline Si1 & $\mathrm{C} 26$ & $1.84(2)$ \\
\hline $\mathrm{O} 1$ & $\mathrm{C} 7$ & $1.256(14)$ \\
\hline $\mathrm{O} 4$ & $\mathrm{C} 10$ & $1.240(16)$ \\
\hline N1 & $\mathrm{C} 7$ & $1.372(16)$ \\
\hline $\mathrm{C} 1$ & $\mathrm{C} 2$ & $1.381(18)$ \\
\hline $\mathrm{C} 1$ & C6 & $1.405(17)$ \\
\hline $\mathrm{C} 2$ & $\mathrm{C} 3$ & $1.424(17)$ \\
\hline $\mathrm{C} 3$ & $\mathrm{C} 4$ & $1.378(18)$ \\
\hline $\mathrm{C} 4$ & $\mathrm{C} 5$ & $1.374(19)$ \\
\hline C5 & C6 & $1.409(17)$ \\
\hline C6 & $\mathrm{C} 7$ & $1.466(18)$ \\
\hline
\end{tabular}




\begin{tabular}{|c|c|c|}
\hline$\overline{\text { Atom }}$ & Atom & Length/Å \\
\hline$\overline{\mathrm{C} 8}$ & C9 & $1.544(17)$ \\
\hline C9 & $\mathrm{C} 10$ & $1.532(18)$ \\
\hline C9 & $\mathrm{C} 13$ & $1.561(18)$ \\
\hline $\mathrm{C} 9$ & $\mathrm{C} 17$ & $1.580(17)$ \\
\hline $\mathrm{C} 10$ & $\mathrm{C} 11$ & $1.46(2)$ \\
\hline $\mathrm{C} 11$ & $\mathrm{C} 12$ & $1.52(2)$ \\
\hline $\mathrm{C} 12$ & $\mathrm{C} 13$ & $1.565(19)$ \\
\hline $\mathrm{C} 12$ & $\mathrm{C} 16$ & $1.57(2)$ \\
\hline C13 & $\mathrm{C} 14$ & $1.524(19)$ \\
\hline $\mathrm{C} 13$ & $\mathrm{C} 15$ & $1.550(18)$ \\
\hline C16 & $\mathrm{C} 17$ & $1.59(2)$ \\
\hline C18 & C19 & $1.192(17)$ \\
\hline $\mathrm{C} 20$ & $\mathrm{C} 21$ & $1.544(17)$ \\
\hline $\mathrm{C} 20$ & $\mathrm{C} 22$ & $1.555(17)$ \\
\hline $\mathrm{C} 23$ & $\mathrm{C} 24$ & $1.497(17)$ \\
\hline $\mathrm{C} 23$ & $\mathrm{C} 25$ & $1.546(18)$ \\
\hline $\mathrm{C} 26$ & $\mathrm{C} 27$ & $1.519(18)$ \\
\hline $\mathrm{C} 26$ & $\mathrm{C} 28$ & $1.517(17)$ \\
\hline $\mathrm{I} 2$ & $\mathrm{~N} 2$ & $2.469(9)$ \\
\hline $\mathrm{I} 2$ & $\mathrm{C} 29$ & $2.150(11)$ \\
\hline $\mathrm{I} 2$ & $\mathrm{C} 46$ & $2.074(12)$ \\
\hline S2 & O6 & $1.445(9)$ \\
\hline $\mathrm{S} 2$ & $\mathrm{O} 7$ & $1.441(9)$ \\
\hline $\mathrm{S} 2$ & $\mathrm{~N} 2$ & $1.642(9)$ \\
\hline S2 & C36 & $1.800(12)$ \\
\hline $\mathrm{Si} 2$ & $\mathrm{C} 47$ & $1.884(14)$ \\
\hline $\mathrm{Si} 2$ & $\mathrm{C} 48$ & $1.81(2)$ \\
\hline $\mathrm{Si} 2$ & C51 & $1.87(2)$ \\
\hline $\mathrm{Si} 2$ & C54 & $1.935(18)$ \\
\hline
\end{tabular}




\begin{tabular}{|c|c|c|}
\hline$\overline{\text { Atom }}$ & Atom & Length/A \\
\hline$\overline{\mathrm{O} 5}$ & C35 & $1.242(13)$ \\
\hline O8 & C38 & $1.172(16)$ \\
\hline N2 & C35 & $1.359(14)$ \\
\hline $\mathrm{C} 29$ & $\mathrm{C} 30$ & $1.345(16)$ \\
\hline $\mathrm{C} 29$ & C34 & $1.406(15)$ \\
\hline $\mathrm{C} 30$ & C31 & $1.398(16)$ \\
\hline C31 & C32 & $1.391(17)$ \\
\hline C32 & C33 & $1.377(17)$ \\
\hline C33 & C34 & $1.386(16)$ \\
\hline C34 & C35 & $1.486(16)$ \\
\hline C36 & C37 & $1.538(17)$ \\
\hline C37 & C38 & $1.566(17)$ \\
\hline C37 & $\mathrm{C} 41$ & $1.541(18)$ \\
\hline C37 & $\mathrm{C} 45$ & $1.592(17)$ \\
\hline C38 & C39 & $1.555(19)$ \\
\hline C39 & $\mathrm{C} 40$ & $1.45(2)$ \\
\hline $\mathrm{C} 40$ & C41 & $1.578(16)$ \\
\hline $\mathrm{C} 40$ & $\mathrm{C} 44$ & $1.53(2)$ \\
\hline C41 & $\mathrm{C} 42$ & $1.58(2)$ \\
\hline $\mathrm{C} 41$ & $\mathrm{C} 43$ & $1.56(2)$ \\
\hline $\mathrm{C} 44$ & $\mathrm{C} 45$ & $1.582(18)$ \\
\hline C46 & $\mathrm{C} 47$ & $1.187(17)$ \\
\hline C48 & C49 & $1.519(17)$ \\
\hline C48 & C50 & $1.541(16)$ \\
\hline C51 & C52 & $1.501(17)$ \\
\hline C51 & $\mathrm{C} 53$ & $1.535(18)$ \\
\hline C54 & C55 & $1.500(16)$ \\
\hline C54 & C56 & $1.501(16)$ \\
\hline
\end{tabular}




\begin{tabular}{|c|c|c|c|}
\hline Atom & Atom & Atom & Angle $/^{\circ}$ \\
\hline$\overline{\mathrm{C} 1}$ & I1 & N1 & $73.6(4)$ \\
\hline $\mathrm{C} 18$ & I1 & N1 & $164.5(4)$ \\
\hline C18 & $\mathrm{I} 1$ & $\mathrm{C} 1$ & $91.5(5)$ \\
\hline $\mathrm{O} 2$ & $\mathrm{~S} 1$ & $\mathrm{O} 3$ & $119.0(6)$ \\
\hline $\mathrm{O} 2$ & $\mathrm{~S} 1$ & N1 & $111.6(6)$ \\
\hline $\mathrm{O} 2$ & $\mathrm{~S} 1$ & $\mathrm{C} 8$ & 108.9(6) \\
\hline $\mathrm{O} 3$ & $\mathrm{~S} 1$ & N1 & $103.8(5)$ \\
\hline $\mathrm{O} 3$ & $\mathrm{~S} 1$ & $\mathrm{C} 8$ & $109.0(6)$ \\
\hline N1 & $\mathrm{S} 1$ & $\mathrm{C} 8$ & $103.3(6)$ \\
\hline C19 & Sil & $\mathrm{C} 23$ & 109.0(7) \\
\hline $\mathrm{C} 20$ & Sil & C19 & 104.7(8) \\
\hline $\mathrm{C} 20$ & Sil & $\mathrm{C} 23$ & 111.2(9) \\
\hline $\mathrm{C} 26$ & Si1 & C19 & 106.7(8) \\
\hline $\mathrm{C} 26$ & Si1 & $\mathrm{C} 20$ & $116.5(10)$ \\
\hline $\mathrm{C} 26$ & Si1 & $\mathrm{C} 23$ & $108.3(11)$ \\
\hline S1 & N1 & I1 & $120.4(5)$ \\
\hline $\mathrm{C} 7$ & N1 & I1 & $113.8(8)$ \\
\hline C7 & N1 & $\mathrm{S} 1$ & $123.5(8)$ \\
\hline $\mathrm{C} 2$ & $\mathrm{C} 1$ & I1 & 119.9(9) \\
\hline $\mathrm{C} 2$ & $\mathrm{C} 1$ & C6 & $122.8(12)$ \\
\hline C6 & $\mathrm{C} 1$ & I1 & 117.2(9) \\
\hline $\mathrm{C} 1$ & $\mathrm{C} 2$ & $\mathrm{C} 3$ & $118.1(12)$ \\
\hline $\mathrm{C} 4$ & $\mathrm{C} 3$ & $\mathrm{C} 2$ & $120.1(13)$ \\
\hline C5 & $\mathrm{C} 4$ & $\mathrm{C} 3$ & $120.6(13)$ \\
\hline $\mathrm{C} 4$ & $\mathrm{C} 5$ & C6 & $121.5(12)$ \\
\hline $\mathrm{C} 1$ & C6 & $\mathrm{C} 5$ & $116.9(12)$ \\
\hline $\mathrm{C} 1$ & C6 & $\mathrm{C} 7$ & $122.0(11)$ \\
\hline $\mathrm{C} 5$ & C6 & $\mathrm{C} 7$ & $121.0(11)$ \\
\hline
\end{tabular}




\begin{tabular}{|c|c|c|c|}
\hline$\overline{\text { Atom }}$ & Atom & Atom & Angle $/^{\circ}$ \\
\hline$\overline{\mathrm{O} 1}$ & $\mathrm{C} 7$ & N1 & $124.4(12)$ \\
\hline $\mathrm{O} 1$ & $\mathrm{C} 7$ & C6 & $122.6(12)$ \\
\hline N1 & C7 & C6 & $112.9(11)$ \\
\hline C9 & $\mathrm{C} 8$ & $\mathrm{~S} 1$ & 116.9(9) \\
\hline $\mathrm{C} 8$ & C9 & $\mathrm{C} 13$ & $118.8(11)$ \\
\hline $\mathrm{C} 8$ & C9 & $\mathrm{C} 17$ & $117.0(11)$ \\
\hline $\mathrm{C} 10$ & C9 & $\mathrm{C} 8$ & $111.7(10)$ \\
\hline $\mathrm{C} 10$ & C9 & $\mathrm{C} 13$ & $98.9(10)$ \\
\hline $\mathrm{C} 10$ & C9 & $\mathrm{C} 17$ & $103.7(10)$ \\
\hline $\mathrm{C} 13$ & C9 & $\mathrm{C} 17$ & $104.3(10)$ \\
\hline O4 & $\mathrm{C} 10$ & $\mathrm{C} 9$ & $123.6(13)$ \\
\hline $\mathrm{O} 4$ & $\mathrm{C} 10$ & C11 & $128.1(13)$ \\
\hline $\mathrm{C} 11$ & $\mathrm{C} 10$ & C9 & $108.3(12)$ \\
\hline $\mathrm{C} 10$ & $\mathrm{C} 11$ & $\mathrm{C} 12$ & $102.2(11)$ \\
\hline $\mathrm{C} 11$ & $\mathrm{C} 12$ & $\mathrm{C} 13$ & $103.3(11)$ \\
\hline C11 & C12 & C16 & $105.0(13)$ \\
\hline $\mathrm{C} 13$ & $\mathrm{C} 12$ & $\mathrm{C} 16$ & $103.4(11)$ \\
\hline C9 & $\mathrm{C} 13$ & $\mathrm{C} 12$ & $92.8(10)$ \\
\hline $\mathrm{C} 14$ & C13 & C9 & $114.6(11)$ \\
\hline $\mathrm{C} 14$ & $\mathrm{C} 13$ & $\mathrm{C} 12$ & $112.0(11)$ \\
\hline C14 & C13 & $\mathrm{C} 15$ & 108.1(12) \\
\hline C15 & C13 & C9 & $115.0(11)$ \\
\hline C15 & C13 & $\mathrm{C} 12$ & $113.9(12)$ \\
\hline $\mathrm{C} 12$ & $\mathrm{C} 16$ & $\mathrm{C} 17$ & $102.8(11)$ \\
\hline C9 & $\mathrm{C} 17$ & $\mathrm{C} 16$ & $101.9(11)$ \\
\hline C19 & $\mathrm{C} 18$ & I1 & $175.6(12)$ \\
\hline C18 & C19 & Si1 & $174.1(12)$ \\
\hline $\mathrm{C} 21$ & $\mathrm{C} 20$ & Sil & $108.5(13)$ \\
\hline $\mathrm{C} 21$ & $\mathrm{C} 20$ & $\mathrm{C} 22$ & $111.9(18)$ \\
\hline
\end{tabular}




\begin{tabular}{|c|c|c|c|}
\hline$\overline{\text { Atom }}$ & Atom & Atom & Angle $/^{\circ}$ \\
\hline$\overline{\mathrm{C} 22}$ & $\mathrm{C} 20$ & Si1 & $111.7(14)$ \\
\hline $\mathrm{C} 24$ & $\mathrm{C} 23$ & Sil & $117.8(13)$ \\
\hline $\mathrm{C} 24$ & $\mathrm{C} 23$ & $\mathrm{C} 25$ & $105.3(17)$ \\
\hline $\mathrm{C} 25$ & $\mathrm{C} 23$ & Si1 & $108.7(15)$ \\
\hline $\mathrm{C} 27$ & $\mathrm{C} 26$ & Si1 & $111.7(15)$ \\
\hline $\mathrm{C} 28$ & $\mathrm{C} 26$ & Si1 & $114.2(15)$ \\
\hline $\mathrm{C} 28$ & $\mathrm{C} 26$ & $\mathrm{C} 27$ & $109.5(19)$ \\
\hline $\mathrm{C} 29$ & $\mathrm{I} 2$ & $\mathrm{~N} 2$ & $74.0(4)$ \\
\hline $\mathrm{C} 46$ & $\mathrm{I} 2$ & $\mathrm{~N} 2$ & $163.6(4)$ \\
\hline $\mathrm{C} 46$ & $\mathrm{I} 2$ & $\mathrm{C} 29$ & $89.9(4)$ \\
\hline O6 & $\mathrm{S} 2$ & $\mathrm{~N} 2$ & $111.4(5)$ \\
\hline O6 & $\mathrm{S} 2$ & C36 & $109.6(5)$ \\
\hline O7 & $\mathrm{S} 2$ & O6 & $118.2(5)$ \\
\hline $\mathrm{O} 7$ & $\mathrm{~S} 2$ & $\mathrm{~N} 2$ & $104.7(5)$ \\
\hline $\mathrm{O} 7$ & $\mathrm{~S} 2$ & C36 & $108.4(6)$ \\
\hline N2 & $\mathrm{S} 2$ & C36 & $103.3(6)$ \\
\hline $\mathrm{C} 47$ & $\mathrm{Si} 2$ & C54 & $104.0(6)$ \\
\hline $\mathrm{C} 48$ & $\mathrm{Si} 2$ & $\mathrm{C} 47$ & 107.3(8) \\
\hline $\mathrm{C} 48$ & $\mathrm{Si} 2$ & C51 & $116.7(10)$ \\
\hline $\mathrm{C} 48$ & $\mathrm{Si} 2$ & C54 & $110.9(8)$ \\
\hline C51 & $\mathrm{Si} 2$ & C47 & $112.1(8)$ \\
\hline C51 & $\mathrm{Si} 2$ & C54 & $105.1(8)$ \\
\hline $\mathrm{S} 2$ & $\mathrm{~N} 2$ & $\mathrm{I} 2$ & $121.8(5)$ \\
\hline $\mathrm{C} 35$ & $\mathrm{~N} 2$ & $\mathrm{I} 2$ & $111.7(7)$ \\
\hline $\mathrm{C} 35$ & $\mathrm{~N} 2$ & $\mathrm{~S} 2$ & $120.9(8)$ \\
\hline C30 & $\mathrm{C} 29$ & $\mathrm{I} 2$ & $122.2(8)$ \\
\hline $\mathrm{C} 30$ & $\mathrm{C} 29$ & C34 & 121.1(11) \\
\hline C34 & $\mathrm{C} 29$ & $\mathrm{I} 2$ & $116.7(8)$ \\
\hline $\mathrm{C} 29$ & $\mathrm{C} 30$ & C31 & $121.1(11)$ \\
\hline
\end{tabular}




\begin{tabular}{llll}
\hline Atom & Atom & Atom & Angle $^{\circ}$ \\
\hline C32 & C31 & C30 & $118.0(12)$ \\
C33 & C32 & C31 & $121.0(12)$ \\
C32 & C33 & C34 & $120.3(11)$ \\
C29 & C34 & C35 & $122.0(10)$ \\
C33 & C34 & C29 & $118.3(11)$ \\
C33 & C34 & C35 & $119.7(10)$ \\
O5 & C35 & N2 & $125.9(11)$ \\
O5 & C35 & C34 & $120.2(10)$ \\
N2 & C35 & C34 & $113.8(9)$ \\
C37 & C36 & S2 & $117.9(9)$ \\
C36 & C37 & C38 & $108.1(10)$ \\
C33 36 & C37 & C41 & $123.7(10)$ \\
C37 37 & C41 & C42 & $113.5(11)$ \\
C36 & C37 & C45 & $116.0(10)$ \\
C38 & C37 & C45 & $102.5(10)$ \\
C41 & C37 & C38 & $101.1(10)$ \\
C41 34 & C37 & C45 & $102.6(10)$ \\
C39 34 & C38 & C37 & $126.1(12)$ \\
C39 34 & C38 $3408.4(12)$
\end{tabular}




\begin{tabular}{llll}
\hline Atom & Atom & Atom & Angle $^{\circ}$ \\
\hline C40 & C44 & C45 & $102.8(10)$ \\
C44 & C45 & C37 & $102.5(10)$ \\
C47 & C46 & I2 & $172.1(11)$ \\
C46 & C47 & Si2 & $173.3(14)$ \\
C49 & C48 & Si2 & $115.8(14)$ \\
C49 & C48 & C50 & $107.0(15)$ \\
C50 & C48 & Si2 & $116.2(15)$ \\
C52 & C51 & Si2 & $108.8(16)$ \\
C52 & C51 & C53 & $108.3(19)$ \\
C53 & C51 & Si2 & $110.3(15)$ \\
C55 & C54 & Si2 & $116.8(12)$ \\
C55 & C54 & C56 & $108.6(14)$ \\
C56 & C54 & Si2 & $112.5(12)$
\end{tabular}

Table S8: Hydrogen Fractional Atomic Coordinates $\left(\times 10^{4}\right)$ and Equivalent Isotropic Displacement Parameters $\left(\AA^{2} \times 10^{3}\right)$ for 2 e. $U_{e q}$ is defined $\begin{array}{llll}\mathbf{x} & \mathbf{y} & \mathbf{z} & \boldsymbol{U}_{\text {eq }}\end{array}$ as $1 / 3$ of the trace of the orthogonalised $U_{i j}$.

\section{Atom}

\begin{tabular}{lllll}
\hline H2 & 3327.91 & 7539.6 & 8058.61 & 36 \\
H3 & 2069.63 & 8462.67 & 8365.91 & 38 \\
H4 & 358.37 & 8437.24 & 8130.13 & 41 \\
H5 & -123.96 & 7571.91 & 7577.58 & 47 \\
H8A & 469.79 & 6022.56 & 6250.46 & 32 \\
H8B & 1624.99 & 6224.07 & 6096.85 & 32 \\
H11A & 1223.86 & 5056.36 & 4816.86 & 55
\end{tabular}


Table S8: Hydrogen Fractional

Atomic Coordinates $\left(\times 10^{4}\right)$

and Equivalent Isotropic

Displacement Parameters

$\left(\AA^{2} \times 10^{3}\right)$ for 2e. $U_{e q}$ is defined

$\begin{array}{llll}\mathbf{x} & \mathbf{y} & \mathbf{z} & \boldsymbol{U}_{e q}\end{array}$ as $1 / 3$ of the trace of the

orthogonalised $U_{i j}$.

\begin{tabular}{|c|c|c|c|c|}
\hline Atom & & & & \\
\hline$\overline{\mathrm{H} 11 \mathrm{~B}}$ & 172.38 & 5602.46 & 4874.13 & 55 \\
\hline H12 & -394.43 & 4231.56 & 5082.28 & 58 \\
\hline H14A & -1148.67 & 5805.92 & 5907.51 & 63 \\
\hline H14B & -1587.74 & 5348.65 & 5494.09 & 63 \\
\hline $\mathrm{H} 14 \mathrm{C}$ & -680.08 & 6036.76 & 5454.44 & 63 \\
\hline $\mathrm{H} 15 \mathrm{~A}$ & -87.19 & 3756.95 & 6020.89 & 70 \\
\hline H15B & -1249.98 & 4005.23 & 5889.37 & 70 \\
\hline $\mathrm{H} 15 \mathrm{C}$ & -688.36 & 4489.44 & 6269.48 & 70 \\
\hline H16A & 1567.04 & 3805.84 & 5118.8 & 74 \\
\hline H16B & 837.43 & 3346.63 & 5463.56 & 74 \\
\hline H17A & 1697.42 & 4065.78 & 5992.85 & 53 \\
\hline H17B & 2395.83 & 4553.28 & 5646.56 & 53 \\
\hline $\mathrm{H} 20$ & 6022.77 & 6131.9 & 9174.69 & 103 \\
\hline $\mathrm{H} 21 \mathrm{~A}$ & 5410.46 & 5085.55 & 8475.84 & 130 \\
\hline $\mathrm{H} 21 \mathrm{~B}$ & 5560.13 & 4739.39 & 8946.04 & 130 \\
\hline $\mathrm{H} 21 \mathrm{C}$ & 6540.48 & 5087.65 & 8687.88 & 130 \\
\hline $\mathrm{H} 22 \mathrm{~A}$ & 4275.18 & 6795.01 & 9042.85 & 129 \\
\hline $\mathrm{H} 22 \mathrm{~B}$ & 4299.34 & 5941.76 & 9313.77 & 129 \\
\hline $\mathrm{H} 22 \mathrm{C}$ & 3985.51 & 5912.62 & 8825.72 & 129 \\
\hline $\mathrm{H} 23$ & 6362.66 & 8362.55 & 8578.85 & 106 \\
\hline $\mathrm{H} 24 \mathrm{~A}$ & 4658.6 & 8476.83 & 8356.35 & 117 \\
\hline H24B & 4759.79 & 8931.3 & 8805.25 & 117 \\
\hline
\end{tabular}


Table S8: Hydrogen Fractional

Atomic Coordinates $\left(\times 10^{4}\right)$

and Equivalent Isotropic

Displacement Parameters

$\left(\AA^{2} \times 10^{3}\right)$ for 2e. $U_{e q}$ is defined

$\mathbf{X}$

$\mathbf{y}$

$\mathbf{z}$

$U_{e q}$ as $1 / 3$ of the trace of the

orthogonalised $U_{i j}$.

\begin{tabular}{|c|c|c|c|c|}
\hline Atom & & & & \\
\hline$\overline{\mathrm{H} 24 \mathrm{C}}$ & 4247.77 & 8011.44 & 8772.27 & 117 \\
\hline $\mathrm{H} 25 \mathrm{~A}$ & 5594.05 & 7585.97 & 9336.86 & 173 \\
\hline $\mathrm{H} 25 \mathrm{~B}$ & 5853.66 & 8573.91 & 9309.34 & 173 \\
\hline $\mathrm{H} 25 \mathrm{C}$ & 6763.89 & 7891.98 & 9255.36 & 173 \\
\hline $\mathrm{H} 26$ & 7457.87 & 6141.63 & 8221.96 & 136 \\
\hline $\mathrm{H} 27 \mathrm{~A}$ & 7138.65 & 7186.9 & 7727.79 & 170 \\
\hline $\mathrm{H} 27 \mathrm{~B}$ & 8327.33 & 7254.72 & 7874.39 & 170 \\
\hline $\mathrm{H} 27 \mathrm{C}$ & 7484.83 & 7908.88 & 8052.23 & 170 \\
\hline $\mathrm{H} 28 \mathrm{~A}$ & 8280.36 & 7460.8 & 8726.22 & 171 \\
\hline $\mathrm{H} 28 \mathrm{~B}$ & 8858.16 & 6617.67 & 8574.66 & 171 \\
\hline $\mathrm{H} 28 \mathrm{C}$ & 7972.4 & 6568.34 & 8928.46 & 171 \\
\hline H30 & 8279.86 & 3969.55 & 7993.02 & 23 \\
\hline H31 & 7048.84 & 3089.94 & 8324.65 & 33 \\
\hline H32 & 5340.1 & 3070.01 & 8062.47 & 31 \\
\hline H33 & 4838.9 & 3989.21 & 7530.89 & 35 \\
\hline H36A & 6305.54 & 5493.79 & 5979.25 & 32 \\
\hline H36B & 5308.1 & 5408.74 & 6274.59 & 32 \\
\hline H39A & 4760.23 & 6410.56 & 4890.18 & 50 \\
\hline H39B & 3622.02 & 6478.63 & 5101.2 & 50 \\
\hline $\mathrm{H} 40$ & 4739.24 & 7778.77 & 5132.98 & 48 \\
\hline $\mathrm{H} 42 \mathrm{~A}$ & 7282.65 & 6894.66 & 5449.76 & 82 \\
\hline $\mathrm{H} 42 \mathrm{~B}$ & 6612.42 & 7117.35 & 5039.26 & 82 \\
\hline
\end{tabular}


Table S8: Hydrogen Fractional

Atomic Coordinates $\left(\times 10^{4}\right)$

and Equivalent Isotropic

Displacement Parameters

$\left(\AA^{2} \times 10^{3}\right)$ for 2e. $U_{e q}$ is defined

$\mathbf{X}$

$\mathbf{y}$

$\mathbf{z}$

$U_{e q}$ as $1 / 3$ of the trace of the

orthogonalised $U_{i j}$.

\begin{tabular}{|c|c|c|c|c|}
\hline Atom & & & & \\
\hline$\overline{\mathrm{H} 42 \mathrm{C}}$ & 6530.92 & 6197.87 & 5247.13 & 82 \\
\hline $\mathrm{H} 43 \mathrm{~A}$ & 5481.42 & 8202.38 & 5968.36 & 77 \\
\hline $\mathrm{H} 43 \mathrm{~B}$ & 6464.73 & 8284.01 & 5661.44 & 77 \\
\hline $\mathrm{H} 43 \mathrm{C}$ & 6539.67 & 7716.49 & 6078.02 & 77 \\
\hline $\mathrm{H} 44 \mathrm{~A}$ & 3167.27 & 7441.26 & 5606.58 & 51 \\
\hline H44B & 3988.37 & 8106.9 & 5801.05 & 51 \\
\hline $\mathrm{H} 45 \mathrm{~A}$ & 3629.28 & 6448.97 & 6102.73 & 40 \\
\hline $\mathrm{H} 45 \mathrm{~B}$ & 4373.67 & 7143.62 & 6321.54 & 40 \\
\hline $\mathrm{H} 48$ & 10258.11 & 5348.39 & 9000.59 & 107 \\
\hline H49A & 11930.89 & 6046.68 & 8534.8 & 136 \\
\hline H49B & 11385.05 & 6510.88 & 8924.76 & 136 \\
\hline $\mathrm{H} 49 \mathrm{C}$ & 10734.03 & 6319.87 & 8505.33 & 136 \\
\hline H50A & 11386.96 & 4628.17 & 9422.44 & 135 \\
\hline H50B & 11802.59 & 5581.49 & 9409.14 & 135 \\
\hline H50C & 12366.17 & 4867.09 & 9134.38 & 135 \\
\hline H51 & 12323.2 & 3555.98 & 8546.37 & 118 \\
\hline $\mathrm{H} 52 \mathrm{~A}$ & 12863.67 & 4410.15 & 7837.22 & 149 \\
\hline H52B & 13400.64 & 4477.32 & 8291.23 & 149 \\
\hline H52C & 12443.82 & 5081.03 & 8174.41 & 149 \\
\hline H53A & 11102.54 & 2931.38 & 8021.89 & 148 \\
\hline H53B & 12313.59 & 2850.23 & 7911.49 & 148 \\
\hline H53C & 11620.85 & 3560.58 & 7687.21 & 148 \\
\hline
\end{tabular}


Table S8: Hydrogen Fractional

Atomic Coordinates $\left(\times 10^{4}\right)$

and Equivalent Isotropic

Displacement Parameters

$\left(\AA^{2} \times 10^{3}\right)$ for 2 e. $U_{e q}$ is defined

$\begin{array}{lllll}\mathbf{x} & \mathbf{y} & \mathbf{z} & \boldsymbol{U}_{e q}\end{array}$ as $1 / 3$ of the trace of the

orthogonalised $U_{i j}$.

\begin{tabular}{lllll} 
Atom & & & & \\
\hline H54 & 9634.43 & 3215.79 & 8466.16 & 75 \\
H55A & 10992.71 & 2608.36 & 8831.47 & 100 \\
H55B & 9919.26 & 2456.89 & 9075.85 & 100 \\
H55C & 10719.29 & 3158.83 & 9240.05 & 100 \\
H56A & 9212.04 & 4227.05 & 9179.67 & 112 \\
H56B & 8565.84 & 3399.45 & 9054.46 & 112 \\
H56C & 8556.96 & 4207.19 & 8750.68 & 112
\end{tabular}

Table S9: Solvent masking (Olex2) information for ls01-205.

\begin{tabular}{llllccl}
\hline No & $\mathbf{x}$ & $\mathbf{y}$ & $\mathbf{z}$ & $\mathbf{V}$ & $\mathbf{e}$ & Content \\
\hline 1 & 0.495 & 0.250 & 0.500 & 392.3 & 107.4 & $?$ \\
2 & 0.208 & 0.750 & 0.000 & 392.3 & 107.5 & $?$
\end{tabular}




\section{2-Phenylpropan-2-yl $(S, E)-4-(4-f l u o r o p h e n y l)-2-((2-$}

\section{iodophenyl)((mesitylsulfonyl)imino)methoxy)but-3-ynoate (14n)}

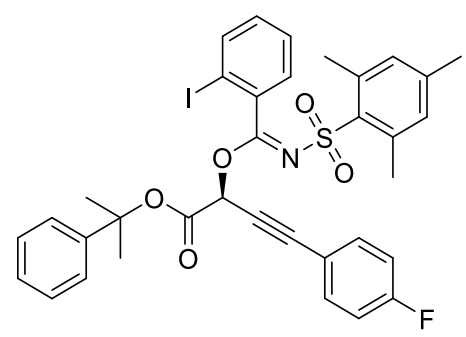

Table S10. Crystal data and structure refinement for $\mathbf{1 4 n}$.

Identification code ps-097

Empirical formula

$\mathrm{C}_{35} \mathrm{H}_{31} \mathrm{FINO}_{5} \mathrm{~S}$

Formula weight 723.57

Temperature 100.00(10) K

Wavelength $1.54184 \AA$

Crystal system Orthorhombic

Space group $P 2{ }_{1} 2_{1} 2_{1}$

Unit cell dimensions

$\mathrm{a}=9.45135(4) \AA \quad \square=90^{\circ}$.

$\mathrm{b}=14.02781(6) \AA \quad \square=90^{\circ}$.

$\mathrm{c}=24.20130(9) \AA \quad \square=90^{\circ}$.

Volume 3208.65(2) $\AA^{3}$

$\mathrm{Z}$

Density (calculated)

4

Absorption coefficient

$1.498 \mathrm{Mg} / \mathrm{m}^{3}$

$8.855 \mathrm{~mm}^{-1}$

$\mathrm{F}(000)$

1464

Crystal size

$0.649 \times 0.144 \times 0.094 \mathrm{~mm}^{3}$

$\square$ range for data collection 3.642 to $73.748^{\circ}$.

Index ranges $-11 \leq \mathrm{h} \leq 10,-17 \leq \mathrm{k} \leq 17,-30 \leq 1 \leq 30$

Reflections collected 53907

Independent reflections

Completeness to $\square=67.684^{\circ}$

$6449\left[R_{\text {int }}=0.0308\right]$

$100.0 \%$

Absorption correction

Gaussian

Max. and min. transmission

0.904 and 0.181

Refinement method

Full-matrix least-squares on $\mathrm{F}^{2}$

Data / restraints / parameters

Goodness-of-fit on $\mathrm{F}^{2}$

6449 / 0 / 403

Final R indices [I > 2 $\square(\mathrm{I})]$

1.065

$\mathrm{R}$ indices (all data)

$R_{1}=0.0156, w R 2=0.0405$

Absolute structure parameter

$R_{1}=0.0157, w R_{2}=0.0406$

$-0.0179(13)$ 
Extinction coefficient

Largest diff. peak and hole

0.00027(3)

0.302 and -0.605 e. $\AA^{-3}$

Table S11. Atomic coordinates $\left(\mathrm{x} 10^{4}\right.$ ) and equivalent isotropic displacement parameters $\left(\AA^{2} \times 10^{3}\right)$

for $\mathbf{1 4 n}$. U(eq) is defined as one third of the trace of the orthogonalized $\mathrm{U}^{\mathrm{ij}}$ tensor.

\begin{tabular}{|c|c|c|c|c|}
\hline & $\mathrm{x}$ & $\mathrm{y}$ & $\mathrm{z}$ & $\mathrm{U}(\mathrm{eq})$ \\
\hline $\mathrm{I}(1)$ & $-1150(1)$ & $5877(1)$ & $4872(1)$ & $21(1)$ \\
\hline$S(1)$ & $2569(1)$ & $4212(1)$ & $5601(1)$ & $14(1)$ \\
\hline $\mathrm{F}(1)$ & $-189(2)$ & $12378(1)$ & $7262(1)$ & $32(1)$ \\
\hline $\mathrm{O}(1)$ & $610(2)$ & $6424(1)$ & $6122(1)$ & $15(1)$ \\
\hline $\mathrm{O}(2)$ & $4079(2)$ & $4256(1)$ & $5564(1)$ & $21(1)$ \\
\hline $\mathrm{O}(3)$ & $1988(2)$ & $3531(1)$ & $5980(1)$ & $20(1)$ \\
\hline $\mathrm{O}(4)$ & $3012(2)$ & $6437(1)$ & $6822(1)$ & $18(1)$ \\
\hline $\mathrm{O}(5)$ & $4185(2)$ & $7232(1)$ & $6140(1)$ & $14(1)$ \\
\hline $\mathrm{N}(1)$ & $2116(2)$ & $5315(1)$ & $5789(1)$ & $14(1)$ \\
\hline $\mathrm{C}(1)$ & $1747(2)$ & $7099(2)$ & $6038(1)$ & $14(1)$ \\
\hline$C(2)$ & $870(2)$ & $5529(2)$ & $5949(1)$ & $14(1)$ \\
\hline $\mathrm{C}(3)$ & $-440(2)$ & $4943(2)$ & $5981(1)$ & $14(1)$ \\
\hline$C(4)$ & $-1443(2)$ & $4981(2)$ & $5559(1)$ & $17(1)$ \\
\hline$C(5)$ & $-2666(3)$ & $4431(2)$ & $5591(1)$ & $22(1)$ \\
\hline$C(6)$ & $-2896(3)$ & $3865(2)$ & $6049(1)$ & $24(1)$ \\
\hline$C(7)$ & $-1906(3)$ & $3827(2)$ & $6473(1)$ & $24(1)$ \\
\hline $\mathrm{C}(8)$ & $-688(3)$ & $4356(2)$ & $6440(1)$ & $20(1)$ \\
\hline $\mathrm{C}(9)$ & $1859(2)$ & $4063(2)$ & 4931(1) & $15(1)$ \\
\hline$C(10)$ & $2416(3)$ & $4644(2)$ & $4507(1)$ & $18(1)$ \\
\hline $\mathrm{C}(11)$ & 1912(3) & $4512(2)$ & $3972(1)$ & $22(1)$ \\
\hline $\mathrm{C}(12)$ & $886(3)$ & $3839(2)$ & $3848(1)$ & $25(1)$ \\
\hline$C(13)$ & $334(3)$ & $3295(2)$ & $4275(1)$ & $22(1)$ \\
\hline $\mathrm{C}(14)$ & $801(2)$ & $3376(2)$ & $4820(1)$ & $18(1)$ \\
\hline$C(15)$ & $3542(3)$ & $5397(2)$ & $4592(1)$ & $24(1)$ \\
\hline$C(16)$ & $390(4)$ & $3688(2)$ & $3261(1)$ & $37(1)$ \\
\hline $\mathrm{C}(17)$ & $165(3)$ & $2694(2)$ & $5235(1)$ & $25(1)$ \\
\hline $\mathrm{C}(18)$ & $1240(3)$ & $8035(2)$ & $6221(1)$ & $16(1)$ \\
\hline
\end{tabular}




$\begin{array}{lrrrr}\mathrm{C}(19) & 990(3) & 8806(2) & 6403(1) & 16(1) \\ \mathrm{C}(20) & 680(3) & 9735(2) & 6623(1) & 17(1) \\ \mathrm{C}(21) & -238(3) & 9833(2) & 7071(1) & 21(1) \\ \mathrm{C}(22) & -545(3) & 10724(2) & 7288(1) & 24(1) \\ \mathrm{C}(23) & 88(3) & 11501(2) & 7050(1) & 23(1) \\ \mathrm{C}(24) & 990(3) & 11443(2) & 6603(1) & 28(1) \\ \mathrm{C}(25) & 1287(3) & 10547(2) & 6391(1) & 24(1) \\ \mathrm{C}(26) & 3055(2) & 6851(2) & 6388(1) & 14(1) \\ \mathrm{C}(27) & 5599(3) & 7178(2) & 6404(1) & 16(1) \\ \mathrm{C}(28) & 5638(3) & 7819(2) & 6911(1) & 16(1) \\ \mathrm{C}(29) & 4601(3) & 8496(2) & 7018(1) & 22(1) \\ \mathrm{C}(30) & 4694(3) & 9083(2) & 7479(1) & 26(1) \\ \mathrm{C}(31) & 5837(3) & 9012(2) & 7837(1) & 26(1) \\ \mathrm{C}(32) & 6887(3) & 8357(2) & 7727(1) & 25(1) \\ \mathrm{C}(33) & 6792(3) & 7763(2) & 7268(1) & 21(1) \\ \mathrm{C}(34) & 6563(3) & 7567(2) & 5956(1) & 24(1) \\ \mathrm{C}(35) & 5962(3) & 6139(2) & 6529(1) & 22(1)\end{array}$


Table S12. Bond lengths $[\AA]$ and angles $\left[{ }^{\circ}\right]$ for $\mathbf{1 4 n}$.

\begin{tabular}{|c|c|}
\hline $\mathrm{I}(1)-\mathrm{C}(4)$ & $2.102(2)$ \\
\hline $\mathrm{S}(1)-\mathrm{O}(2)$ & $1.4317(17)$ \\
\hline $\mathrm{S}(1)-\mathrm{O}(3)$ & $1.4328(17)$ \\
\hline $\mathrm{S}(1)-\mathrm{N}(1)$ & $1.6682(19)$ \\
\hline$S(1)-C(9)$ & $1.768(2)$ \\
\hline $\mathrm{F}(1)-\mathrm{C}(23)$ & $1.358(3)$ \\
\hline $\mathrm{O}(1)-\mathrm{C}(2)$ & $1.348(3)$ \\
\hline $\mathrm{O}(1)-\mathrm{C}(1)$ & $1.446(3)$ \\
\hline $\mathrm{O}(4)-\mathrm{C}(26)$ & $1.201(3)$ \\
\hline $\mathrm{O}(5)-\mathrm{C}(26)$ & $1.336(3)$ \\
\hline $\mathrm{O}(5)-\mathrm{C}(27)$ & $1.483(3)$ \\
\hline $\mathrm{N}(1)-\mathrm{C}(2)$ & $1.275(3)$ \\
\hline $\mathrm{C}(1)-\mathrm{C}(18)$ & $1.465(3)$ \\
\hline$C(1)-C(26)$ & $1.538(3)$ \\
\hline $\mathrm{C}(1)-\mathrm{H}(1)$ & 1.0000 \\
\hline$C(2)-C(3)$ & $1.488(3)$ \\
\hline$C(3)-C(4)$ & $1.394(3)$ \\
\hline$C(3)-C(8)$ & $1.402(3)$ \\
\hline$C(4)-C(5)$ & 1.391(3) \\
\hline$C(5)-C(6)$ & $1.380(4)$ \\
\hline $\mathrm{C}(5)-\mathrm{H}(5)$ & 0.9500 \\
\hline$C(6)-C(7)$ & $1.389(4)$ \\
\hline $\mathrm{C}(6)-\mathrm{H}(6)$ & 0.9500 \\
\hline$C(7)-C(8)$ & $1.372(4)$ \\
\hline $\mathrm{C}(7)-\mathrm{H}(7)$ & 0.9500 \\
\hline $\mathrm{C}(8)-\mathrm{H}(8)$ & 0.9500 \\
\hline $\mathrm{C}(9)-\mathrm{C}(10)$ & $1.412(3)$ \\
\hline $\mathrm{C}(9)-\mathrm{C}(14)$ & $1.414(3)$ \\
\hline $\mathrm{C}(10)-\mathrm{C}(11)$ & $1.392(3)$ \\
\hline$C(10)-C(15)$ & $1.513(3)$ \\
\hline $\mathrm{C}(11)-\mathrm{C}(12)$ & $1.387(4)$ \\
\hline $\mathrm{C}(11)-\mathrm{H}(11)$ & 0.9500 \\
\hline$C(12)-C(13)$ & $1.386(4)$ \\
\hline$C(12)-C(16)$ & $1.510(4)$ \\
\hline$C(13)-C(14)$ & $1.397(3)$ \\
\hline $\mathrm{C}(13)-\mathrm{H}(13)$ & 0.9500 \\
\hline
\end{tabular}




\begin{tabular}{|c|c|}
\hline$C(14)-C(17)$ & $1.511(3)$ \\
\hline $\mathrm{C}(15)-\mathrm{H}(15 \mathrm{~A})$ & 0.9800 \\
\hline $\mathrm{C}(15)-\mathrm{H}(15 \mathrm{~B})$ & 0.9800 \\
\hline $\mathrm{C}(15)-\mathrm{H}(15 \mathrm{C})$ & 0.9800 \\
\hline $\mathrm{C}(16)-\mathrm{H}(16 \mathrm{~A})$ & 0.9800 \\
\hline $\mathrm{C}(16)-\mathrm{H}(16 \mathrm{~B})$ & 0.9800 \\
\hline $\mathrm{C}(16)-\mathrm{H}(16 \mathrm{C})$ & 0.9800 \\
\hline $\mathrm{C}(17)-\mathrm{H}(17 \mathrm{~A})$ & 0.9800 \\
\hline $\mathrm{C}(17)-\mathrm{H}(17 \mathrm{~B})$ & 0.9800 \\
\hline $\mathrm{C}(17)-\mathrm{H}(17 \mathrm{C})$ & 0.9800 \\
\hline C(18)-C(19) & 1.193(3) \\
\hline $\mathrm{C}(19)-\mathrm{C}(20)$ & $1.437(3)$ \\
\hline $\mathrm{C}(20)-\mathrm{C}(25)$ & $1.394(3)$ \\
\hline $\mathrm{C}(20)-\mathrm{C}(21)$ & $1.397(3)$ \\
\hline $\mathrm{C}(21)-\mathrm{C}(22)$ & $1.386(4)$ \\
\hline $\mathrm{C}(21)-\mathrm{H}(21)$ & 0.9500 \\
\hline $\mathrm{C}(22)-\mathrm{C}(23)$ & $1.371(4)$ \\
\hline $\mathrm{C}(22)-\mathrm{H}(22)$ & 0.9500 \\
\hline $\mathrm{C}(23)-\mathrm{C}(24)$ & $1.378(4)$ \\
\hline $\mathrm{C}(24)-\mathrm{C}(25)$ & $1.387(3)$ \\
\hline $\mathrm{C}(24)-\mathrm{H}(24)$ & 0.9500 \\
\hline $\mathrm{C}(25)-\mathrm{H}(25)$ & 0.9500 \\
\hline $\mathrm{C}(27)-\mathrm{C}(34)$ & $1.518(3)$ \\
\hline $\mathrm{C}(27)-\mathrm{C}(28)$ & $1.523(3)$ \\
\hline $\mathrm{C}(27)-\mathrm{C}(35)$ & $1.528(3)$ \\
\hline $\mathrm{C}(28)-\mathrm{C}(29)$ & $1.389(3)$ \\
\hline $\mathrm{C}(28)-\mathrm{C}(33)$ & $1.393(3)$ \\
\hline $\mathrm{C}(29)-\mathrm{C}(30)$ & $1.391(4)$ \\
\hline $\mathrm{C}(29)-\mathrm{H}(29)$ & 0.9500 \\
\hline $\mathrm{C}(30)-\mathrm{C}(31)$ & $1.387(4)$ \\
\hline $\mathrm{C}(30)-\mathrm{H}(30)$ & 0.9500 \\
\hline $\mathrm{C}(31)-\mathrm{C}(32)$ & $1.377(4)$ \\
\hline $\mathrm{C}(31)-\mathrm{H}(31)$ & 0.9500 \\
\hline $\mathrm{C}(32)-\mathrm{C}(33)$ & $1.392(4)$ \\
\hline $\mathrm{C}(32)-\mathrm{H}(32)$ & 0.9500 \\
\hline $\mathrm{C}(33)-\mathrm{H}(33)$ & 0.9500 \\
\hline $\mathrm{C}(34)-\mathrm{H}(34 \mathrm{~A})$ & 0.9800 \\
\hline $\mathrm{C}(34)-\mathrm{H}(34 \mathrm{~B})$ & 0.9800 \\
\hline
\end{tabular}




\begin{tabular}{|c|c|}
\hline $\mathrm{C}(34)-\mathrm{H}(34 \mathrm{C})$ & 0.9800 \\
\hline $\mathrm{C}(35)-\mathrm{H}(35 \mathrm{~A})$ & 0.9800 \\
\hline $\mathrm{C}(35)-\mathrm{H}(35 \mathrm{~B})$ & 0.9800 \\
\hline $\mathrm{C}(35)-\mathrm{H}(35 \mathrm{C})$ & 0.9800 \\
\hline $\mathrm{O}(2)-\mathrm{S}(1)-\mathrm{O}(3)$ & $116.75(10)$ \\
\hline $\mathrm{O}(2)-\mathrm{S}(1)-\mathrm{N}(1)$ & $103.47(10)$ \\
\hline $\mathrm{O}(3)-\mathrm{S}(1)-\mathrm{N}(1)$ & $110.20(10)$ \\
\hline $\mathrm{O}(2)-\mathrm{S}(1)-\mathrm{C}(9)$ & $109.04(10)$ \\
\hline $\mathrm{O}(3)-\mathrm{S}(1)-\mathrm{C}(9)$ & $111.33(11)$ \\
\hline $\mathrm{N}(1)-\mathrm{S}(1)-\mathrm{C}(9)$ & $105.18(10)$ \\
\hline $\mathrm{C}(2)-\mathrm{O}(1)-\mathrm{C}(1)$ & $115.57(17)$ \\
\hline $\mathrm{C}(26)-\mathrm{O}(5)-\mathrm{C}(27)$ & $120.38(17)$ \\
\hline $\mathrm{C}(2)-\mathrm{N}(1)-\mathrm{S}(1)$ & $122.52(16)$ \\
\hline $\mathrm{O}(1)-\mathrm{C}(1)-\mathrm{C}(18)$ & $107.53(19)$ \\
\hline $\mathrm{O}(1)-\mathrm{C}(1)-\mathrm{C}(26)$ & $111.82(17)$ \\
\hline$C(18)-C(1)-C(26)$ & $107.46(18)$ \\
\hline $\mathrm{O}(1)-\mathrm{C}(1)-\mathrm{H}(1)$ & 110.0 \\
\hline $\mathrm{C}(18)-\mathrm{C}(1)-\mathrm{H}(1)$ & 110.0 \\
\hline $\mathrm{C}(26)-\mathrm{C}(1)-\mathrm{H}(1)$ & 110.0 \\
\hline $\mathrm{N}(1)-\mathrm{C}(2)-\mathrm{O}(1)$ & $118.8(2)$ \\
\hline $\mathrm{N}(1)-\mathrm{C}(2)-\mathrm{C}(3)$ & $130.9(2)$ \\
\hline $\mathrm{O}(1)-\mathrm{C}(2)-\mathrm{C}(3)$ & $110.30(19)$ \\
\hline $\mathrm{C}(4)-\mathrm{C}(3)-\mathrm{C}(8)$ & $119.3(2)$ \\
\hline $\mathrm{C}(4)-\mathrm{C}(3)-\mathrm{C}(2)$ & $120.4(2)$ \\
\hline $\mathrm{C}(8)-\mathrm{C}(3)-\mathrm{C}(2)$ & $120.3(2)$ \\
\hline$C(5)-C(4)-C(3)$ & $120.2(2)$ \\
\hline$C(5)-C(4)-I(1)$ & $119.01(18)$ \\
\hline $\mathrm{C}(3)-\mathrm{C}(4)-\mathrm{I}(1)$ & $120.83(17)$ \\
\hline$C(6)-C(5)-C(4)$ & $119.7(2)$ \\
\hline $\mathrm{C}(6)-\mathrm{C}(5)-\mathrm{H}(5)$ & 120.2 \\
\hline $\mathrm{C}(4)-\mathrm{C}(5)-\mathrm{H}(5)$ & 120.2 \\
\hline$C(5)-C(6)-C(7)$ & $120.6(2)$ \\
\hline $\mathrm{C}(5)-\mathrm{C}(6)-\mathrm{H}(6)$ & 119.7 \\
\hline $\mathrm{C}(7)-\mathrm{C}(6)-\mathrm{H}(6)$ & 119.7 \\
\hline$C(8)-C(7)-C(6)$ & $120.1(2)$ \\
\hline $\mathrm{C}(8)-\mathrm{C}(7)-\mathrm{H}(7)$ & 119.9 \\
\hline $\mathrm{C}(6)-\mathrm{C}(7)-\mathrm{H}(7)$ & 119.9 \\
\hline
\end{tabular}




$\begin{array}{ll}\mathrm{C}(7)-\mathrm{C}(8)-\mathrm{C}(3) & 120.2(2) \\ \mathrm{C}(7)-\mathrm{C}(8)-\mathrm{H}(8) & 119.9 \\ \mathrm{C}(3)-\mathrm{C}(8)-\mathrm{H}(8) & 119.9 \\ \mathrm{C}(10)-\mathrm{C}(9)-\mathrm{C}(14) & 121.3(2) \\ \mathrm{C}(10)-\mathrm{C}(9)-\mathrm{S}(1) & 117.19(17) \\ \mathrm{C}(14)-\mathrm{C}(9)-\mathrm{S}(1) & 121.48(17) \\ \mathrm{C}(11)-\mathrm{C}(10)-\mathrm{C}(9) & 118.1(2) \\ \mathrm{C}(11)-\mathrm{C}(10)-\mathrm{C}(15) & 117.4(2) \\ \mathrm{C}(9)-\mathrm{C}(10)-\mathrm{C}(15) & 124.5(2) \\ \mathrm{C}(12)-\mathrm{C}(11)-\mathrm{C}(10) & 122.1(2) \\ \mathrm{C}(12)-\mathrm{C}(11)-\mathrm{H}(11) & 118.9 \\ \mathrm{C}(10)-\mathrm{C}(11)-\mathrm{H}(11) & 118.9 \\ \mathrm{C}(13)-\mathrm{C}(12)-\mathrm{C}(11) & 118.4(2) \\ \mathrm{C}(13)-\mathrm{C}(12)-\mathrm{C}(16) & 120.5(3) \\ \mathrm{C}(11)-\mathrm{C}(12)-\mathrm{C}(16) & 121.1(3) \\ \mathrm{C}(12)-\mathrm{C}(13)-\mathrm{C}(14) & 122.8(2) \\ \mathrm{C}(12)-\mathrm{C}(13)-\mathrm{H}(13) & 118.6 \\ \mathrm{C}(14)-\mathrm{C}(13)-\mathrm{H}(13) & 118.6 \\ \mathrm{C}(13)-\mathrm{C}(14)-\mathrm{C}(9) & 117.2(2) \\ \mathrm{C}(13)-\mathrm{C}(14)-\mathrm{C}(17) & 116.8(2) \\ \mathrm{C}(9)-\mathrm{C}(14)-\mathrm{C}(17) & 126.0(2) \\ \mathrm{C}(10)-\mathrm{C}(15)-\mathrm{H}(15 \mathrm{~A}) & 109.5 \\ \mathrm{C}(10)-\mathrm{C}(15)-\mathrm{H}(15 \mathrm{~B}) & 109.5 \\ \mathrm{H}(15 \mathrm{~A})-\mathrm{C}(15)-\mathrm{H}(15 \mathrm{~B}) & 109.5 \\ \mathrm{C}(10)-\mathrm{C}(15)-\mathrm{H}(15 \mathrm{C}) & 109.5 \\ \mathrm{H}(15 \mathrm{~A})-\mathrm{C}(15)-\mathrm{H}(15 \mathrm{C}) & 109.5 \\ \mathrm{H}(15 \mathrm{~B})-\mathrm{C}(15)-\mathrm{H}(15 \mathrm{C}) & 109.5 \\ \mathrm{C}(12)-\mathrm{C}(16)-\mathrm{H}(16 \mathrm{~A}) & 109.5 \\ \mathrm{C}(12)-\mathrm{C}(16)-\mathrm{H}(16 \mathrm{~B}) & 109.5 \\ \mathrm{H}(16 \mathrm{~A})-\mathrm{C}(16)-\mathrm{H}(16 \mathrm{~B}) & 109.5 \\ \mathrm{C}(12)-\mathrm{C}(16)-\mathrm{H}(16 \mathrm{C}) & 109.5 \\ \mathrm{H}(16 \mathrm{~A})-\mathrm{C}(16)-\mathrm{H}(16 \mathrm{C}) & 109.5 \\ \mathrm{H}(16 \mathrm{~B})-\mathrm{C}(16)-\mathrm{H}(16 \mathrm{C}) & 109.5 \\ \mathrm{C}(14)-\mathrm{C}(17)-\mathrm{H}(17 \mathrm{~A}) & 109.5 \\ \mathrm{C}(14)-\mathrm{C}(17)-\mathrm{H}(17 \mathrm{~B}) & 109.5 \\ \mathrm{H}(17 \mathrm{~A})-\mathrm{C}(17)-\mathrm{H}(17 \mathrm{~B}) & 109.5 \\ \mathrm{C}(14)-\mathrm{C}(17)-\mathrm{H}(17 \mathrm{C}) & 109.5 \\ \mathrm{H}(17 \mathrm{~A})-\mathrm{C}(17)-\mathrm{H}(17 \mathrm{C}) & 109.5 \\ & \end{array}$




$\begin{array}{ll}\mathrm{H}(17 \mathrm{~B})-\mathrm{C}(17)-\mathrm{H}(17 \mathrm{C}) & 109.5 \\ \mathrm{C}(19)-\mathrm{C}(18)-\mathrm{C}(1) & 171.5(3) \\ \mathrm{C}(18)-\mathrm{C}(19)-\mathrm{C}(20) & 179.7(3) \\ \mathrm{C}(25)-\mathrm{C}(20)-\mathrm{C}(21) & 119.2(2) \\ \mathrm{C}(25)-\mathrm{C}(20)-\mathrm{C}(19) & 120.6(2) \\ \mathrm{C}(21)-\mathrm{C}(20)-\mathrm{C}(19) & 120.2(2) \\ \mathrm{C}(22)-\mathrm{C}(21)-\mathrm{C}(20) & 120.8(2) \\ \mathrm{C}(22)-\mathrm{C}(21)-\mathrm{H}(21) & 119.6 \\ \mathrm{C}(20)-\mathrm{C}(21)-\mathrm{H}(21) & 119.6 \\ \mathrm{C}(23)-\mathrm{C}(22)-\mathrm{C}(21) & 117.9(2) \\ \mathrm{C}(23)-\mathrm{C}(22)-\mathrm{H}(22) & 121.1 \\ \mathrm{C}(21)-\mathrm{C}(22)-\mathrm{H}(22) & 121.1 \\ \mathrm{~F}(1)-\mathrm{C}(23)-\mathrm{C}(22) & 118.5(2) \\ \mathrm{F}(1)-\mathrm{C}(23)-\mathrm{C}(24) & 118.0(2) \\ \mathrm{C}(22)-\mathrm{C}(23)-\mathrm{C}(24) & 123.5(2) \\ \mathrm{C}(23)-\mathrm{C}(24)-\mathrm{C}(25) & 118.0(2) \\ \mathrm{C}(23)-\mathrm{C}(24)-\mathrm{H}(24) & 121.0 \\ \mathrm{C}(25)-\mathrm{C}(24)-\mathrm{H}(24) & 121.0 \\ \mathrm{C}(24)-\mathrm{C}(25)-\mathrm{C}(20) & 120.5(2) \\ \mathrm{C}(24)-\mathrm{C}(25)-\mathrm{H}(25) & 119.7 \\ \mathrm{C}(20)-\mathrm{C}(25)-\mathrm{H}(25) & 119.7 \\ \mathrm{O}(4)-\mathrm{C}(26)-\mathrm{O}(5) & 127.8(2) \\ \mathrm{O}(4)-\mathrm{C}(26)-\mathrm{C}(1) & 124.3(2) \\ \mathrm{O}(5)-\mathrm{C}(26)-\mathrm{C}(1) & 107.73(18) \\ \mathrm{O}(5)-\mathrm{C}(27)-\mathrm{C}(34) & 102.44(18) \\ \mathrm{O}(5)-\mathrm{C}(27)-\mathrm{C}(28) & 109.85(18) \\ \mathrm{C}(34)-\mathrm{C}(27)-\mathrm{C}(28) & 110.4(2) \\ \mathrm{O}(5)-\mathrm{C}(27)-\mathrm{C}(35) & 109.70(19) \\ \mathrm{C}(34)-\mathrm{C}(27)-\mathrm{C}(35) & 110.5(2) \\ \mathrm{C}(28)-\mathrm{C}(27)-\mathrm{C}(35) & 113.40(19) \\ \mathrm{C}(29)-\mathrm{C}(28)-\mathrm{C}(33) & 118.5(2) \\ \mathrm{C}(29)-\mathrm{C}(28)-\mathrm{C}(27) & 122.4(2) \\ \mathrm{C}(33)-\mathrm{C}(28)-\mathrm{C}(27) & 119.0(2) \\ \mathrm{C}(28)-\mathrm{C}(29)-\mathrm{C}(30) & 120.6(2) \\ \mathrm{C}(28)-\mathrm{C}(29)-\mathrm{H}(29) & 119.7 \\ \mathrm{C}(31)-\mathrm{C}(29)-\mathrm{H}(29) & 119.7 \\ & 120.5(2) \\ & 119.7 \\ & \end{array}$




$\begin{array}{ll}\mathrm{C}(29)-\mathrm{C}(30)-\mathrm{H}(30) & 119.7 \\ \mathrm{C}(32)-\mathrm{C}(31)-\mathrm{C}(30) & 119.2(2) \\ \mathrm{C}(32)-\mathrm{C}(31)-\mathrm{H}(31) & 120.4 \\ \mathrm{C}(30)-\mathrm{C}(31)-\mathrm{H}(31) & 120.4 \\ \mathrm{C}(31)-\mathrm{C}(32)-\mathrm{C}(33) & 120.5(2) \\ \mathrm{C}(31)-\mathrm{C}(32)-\mathrm{H}(32) & 119.8 \\ \mathrm{C}(33)-\mathrm{C}(32)-\mathrm{H}(32) & 119.8 \\ \mathrm{C}(32)-\mathrm{C}(33)-\mathrm{C}(28) & 120.7(2) \\ \mathrm{C}(32)-\mathrm{C}(33)-\mathrm{H}(33) & 119.6 \\ \mathrm{C}(28)-\mathrm{C}(33)-\mathrm{H}(33) & 119.6 \\ \mathrm{C}(27)-\mathrm{C}(34)-\mathrm{H}(34 \mathrm{~A}) & 109.5 \\ \mathrm{C}(27)-\mathrm{C}(34)-\mathrm{H}(34 \mathrm{~B}) & 109.5 \\ \mathrm{H}(34 \mathrm{~A})-\mathrm{C}(34)-\mathrm{H}(34 \mathrm{~B}) & 109.5 \\ \mathrm{C}(27)-\mathrm{C}(34)-\mathrm{H}(34 \mathrm{C}) & 109.5 \\ \mathrm{H}(34 \mathrm{~A})-\mathrm{C}(34)-\mathrm{H}(34 \mathrm{C}) & 109.5 \\ \mathrm{H}(34 \mathrm{~B})-\mathrm{C}(34)-\mathrm{H}(34 \mathrm{C}) & 109.5 \\ \mathrm{C}(27)-\mathrm{C}(35)-\mathrm{H}(35 \mathrm{~A}) & 109.5 \\ \mathrm{C}(27)-\mathrm{C}(35)-\mathrm{H}(35 \mathrm{~B}) & 109.5 \\ \mathrm{H}(35 \mathrm{~A})-\mathrm{C}(35)-\mathrm{H}(35 \mathrm{~B}) & 109.5 \\ \mathrm{C}(27)-\mathrm{C}(35)-\mathrm{H}(35 \mathrm{C}) & 109.5 \\ \mathrm{H}(35 \mathrm{~A})-\mathrm{C}(35)-\mathrm{H}(35 \mathrm{C}) & 109.5 \\ \mathrm{H}(35 \mathrm{~B})-\mathrm{C}(35)-\mathrm{H}(35 \mathrm{C}) & 109.5 \\ & \end{array}$

Symmetry transformations used to generate equivalent atoms: 
Table S13. Anisotropic displacement parameters $\left(\AA^{2} \times 10^{3}\right)$ for $14 \mathbf{n}$. The anisotropic displacement factor exponent takes the form: $-2 \square^{2}\left[\mathrm{~h}^{2} \mathrm{a}^{*} \mathrm{U}^{11}+\ldots+2 \mathrm{~h} \mathrm{k} \mathrm{a}^{*} \mathrm{~b}^{*} \mathrm{U}^{12}\right]$

\begin{tabular}{|c|c|c|c|c|c|c|}
\hline & $\mathrm{U}^{11}$ & $\mathrm{U}^{22}$ & $\mathrm{U}^{33}$ & $\mathrm{U}^{23}$ & $\mathrm{U}^{13}$ & $\mathrm{U}^{12}$ \\
\hline $\mathrm{I}(1)$ & $21(1)$ & $26(1)$ & $17(1)$ & $3(1)$ & $0(1)$ & $6(1)$ \\
\hline$S(1)$ & $13(1)$ & $12(1)$ & $18(1)$ & $-2(1)$ & $-2(1)$ & $1(1)$ \\
\hline $\mathrm{F}(1)$ & $45(1)$ & $18(1)$ & $35(1)$ & $-11(1)$ & $-6(1)$ & $10(1)$ \\
\hline $\mathrm{O}(1)$ & $12(1)$ & $12(1)$ & $20(1)$ & $-4(1)$ & $2(1)$ & $-1(1)$ \\
\hline $\mathrm{O}(2)$ & $13(1)$ & $21(1)$ & $29(1)$ & $-5(1)$ & $-3(1)$ & $4(1)$ \\
\hline $\mathrm{O}(3)$ & $23(1)$ & $16(1)$ & $20(1)$ & 1(1) & $-2(1)$ & $1(1)$ \\
\hline $\mathrm{O}(4)$ & $20(1)$ & $18(1)$ & $16(1)$ & $2(1)$ & $1(1)$ & $-3(1)$ \\
\hline $\mathrm{O}(5)$ & $13(1)$ & $16(1)$ & $15(1)$ & $0(1)$ & $0(1)$ & $-2(1)$ \\
\hline $\mathrm{N}(1)$ & $13(1)$ & $13(1)$ & $17(1)$ & $-4(1)$ & $-1(1)$ & $0(1)$ \\
\hline $\mathrm{C}(1)$ & $13(1)$ & $12(1)$ & $17(1)$ & $-1(1)$ & $1(1)$ & $-3(1)$ \\
\hline $\mathrm{C}(2)$ & $16(1)$ & $12(1)$ & $14(1)$ & $-1(1)$ & $-2(1)$ & $0(1)$ \\
\hline $\mathrm{C}(3)$ & $12(1)$ & $13(1)$ & $18(1)$ & $-2(1)$ & $0(1)$ & $0(1)$ \\
\hline $\mathrm{C}(4)$ & $15(1)$ & $15(1)$ & $20(1)$ & $-3(1)$ & $0(1)$ & $2(1)$ \\
\hline $\mathrm{C}(5)$ & $13(1)$ & $22(1)$ & $30(1)$ & $-6(1)$ & $-3(1)$ & $0(1)$ \\
\hline$C(6)$ & $13(1)$ & $18(1)$ & $42(2)$ & $-3(1)$ & $5(1)$ & $-2(1)$ \\
\hline $\mathrm{C}(7)$ & $23(1)$ & $18(1)$ & $30(1)$ & $6(1)$ & $7(1)$ & $4(1)$ \\
\hline $\mathrm{C}(8)$ & $18(1)$ & $20(1)$ & $23(1)$ & $0(1)$ & $-2(1)$ & $4(1)$ \\
\hline $\mathrm{C}(9)$ & $15(1)$ & $14(1)$ & $17(1)$ & $-2(1)$ & $1(1)$ & $3(1)$ \\
\hline $\mathrm{C}(10)$ & $16(1)$ & $18(1)$ & $21(1)$ & $-2(1)$ & $5(1)$ & $4(1)$ \\
\hline $\mathrm{C}(11)$ & $25(1)$ & $23(1)$ & $18(1)$ & $0(1)$ & $4(1)$ & $5(1)$ \\
\hline $\mathrm{C}(12)$ & $28(2)$ & $25(1)$ & $21(1)$ & $-5(1)$ & $-3(1)$ & $7(1)$ \\
\hline $\mathrm{C}(13)$ & $22(1)$ & $19(1)$ & $25(1)$ & $-8(1)$ & $-5(1)$ & $1(1)$ \\
\hline $\mathrm{C}(14)$ & $16(1)$ & $14(1)$ & $22(1)$ & $-5(1)$ & 1(1) & $0(1)$ \\
\hline $\mathrm{C}(15)$ & 21(1) & $24(1)$ & $26(1)$ & $0(1)$ & $6(1)$ & $-4(1)$ \\
\hline $\mathrm{C}(16)$ & $48(2)$ & $41(2)$ & $23(1)$ & $-5(1)$ & $-9(1)$ & $0(1)$ \\
\hline $\mathrm{C}(17)$ & $31(1)$ & $18(1)$ & $26(1)$ & $-4(1)$ & $0(1)$ & $-9(1)$ \\
\hline $\mathrm{C}(18)$ & $15(1)$ & $16(1)$ & $19(1)$ & $0(1)$ & $-2(1)$ & $-2(1)$ \\
\hline $\mathrm{C}(19)$ & $14(1)$ & $17(1)$ & $18(1)$ & 1(1) & $-1(1)$ & $-1(1)$ \\
\hline $\mathrm{C}(20)$ & $18(1)$ & $15(1)$ & $17(1)$ & $-3(1)$ & $-4(1)$ & $3(1)$ \\
\hline $\mathrm{C}(21)$ & $24(1)$ & $19(1)$ & $22(1)$ & $-1(1)$ & $2(1)$ & $0(1)$ \\
\hline $\mathrm{C}(22)$ & $25(1)$ & $25(1)$ & $23(1)$ & $-5(1)$ & $3(1)$ & $5(1)$ \\
\hline
\end{tabular}




$\begin{array}{lllllll}\mathrm{C}(23) & 28(1) & 15(1) & 25(1) & -6(1) & -8(1) & 7(1) \\ \mathrm{C}(24) & 42(2) & 15(1) & 26(1) & -1(1) & 2(1) & -3(1) \\ \mathrm{C}(25) & 31(2) & 19(1) & 24(1) & -2(1) & 6(1) & -2(1) \\ \mathrm{C}(26) & 16(1) & 11(1) & 16(1) & -6(1) & 0(1) & 0(1) \\ \mathrm{C}(27) & 11(1) & 17(1) & 19(1) & -1(1) & -1(1) & 1(1) \\ \mathrm{C}(28) & 17(1) & 13(1) & 17(1) & 1(1) & 1(1) & -3(1) \\ \mathrm{C}(29) & 19(1) & 20(1) & 26(1) & -5(1) & -5(1) & 1(1) \\ \mathrm{C}(30) & 24(1) & 22(1) & 34(1) & -11(1) & -2(1) & 4(1) \\ \mathrm{C}(31) & 33(2) & 23(1) & 24(1) & -7(1) & -3(1) & -4(1) \\ \mathrm{C}(32) & 27(2) & 28(1) & 21(1) & -1(1) & -8(1) & -2(1) \\ \mathrm{C}(33) & 21(1) & 20(1) & 22(1) & 0(1) & -3(1) & 1(1) \\ \mathrm{C}(34) & 16(1) & 32(1) & 23(1) & 1(1) & 3(1) & -1(1) \\ \mathrm{C}(35) & 22(1) & 16(1) & 29(1) & -4(1) & -2(1) & 3(1)\end{array}$


Table S14. Hydrogen coordinates (x 104) and isotropic displacement parameters $\left(\AA^{2} \times 10^{3}\right)$ for $\mathbf{1 4 n}$.

\begin{tabular}{|c|c|c|c|c|}
\hline & $\mathrm{x}$ & $\mathrm{y}$ & $\mathrm{z}$ & $\mathrm{U}(\mathrm{eq})$ \\
\hline $\mathrm{H}(1)$ & 2008 & 7124 & 5638 & 17 \\
\hline $\mathrm{H}(5)$ & -3338 & 4446 & 5300 & 26 \\
\hline $\mathrm{H}(6)$ & -3738 & 3498 & 6074 & 29 \\
\hline $\mathrm{H}(7)$ & -2074 & 3434 & 6786 & 28 \\
\hline $\mathrm{H}(8)$ & -10 & 4325 & 6729 & 24 \\
\hline $\mathrm{H}(11)$ & 2283 & 4896 & 3683 & 26 \\
\hline $\mathrm{H}(13)$ & -392 & 2848 & 4193 & 26 \\
\hline $\mathrm{H}(15 \mathrm{~A})$ & 3326 & 5765 & 4926 & 35 \\
\hline $\mathrm{H}(15 \mathrm{~B})$ & 3563 & 5824 & 4272 & 35 \\
\hline $\mathrm{H}(15 \mathrm{C})$ & 4466 & 5088 & 4634 & 35 \\
\hline $\mathrm{H}(16 \mathrm{~A})$ & -602 & 3884 & 3228 & 56 \\
\hline $\mathrm{H}(16 \mathrm{~B})$ & 477 & 3012 & 3164 & 56 \\
\hline $\mathrm{H}(16 \mathrm{C})$ & 972 & 4070 & 3009 & 56 \\
\hline $\mathrm{H}(17 \mathrm{~A})$ & 882 & 2229 & 5349 & 37 \\
\hline $\mathrm{H}(17 \mathrm{~B})$ & -635 & 2359 & 5066 & 37 \\
\hline $\mathrm{H}(17 \mathrm{C})$ & -164 & 3050 & 5558 & 37 \\
\hline $\mathrm{H}(21)$ & -658 & 9282 & 7230 & 26 \\
\hline $\mathrm{H}(22)$ & -1174 & 10794 & 7591 & 29 \\
\hline $\mathrm{H}(24)$ & 1396 & 12000 & 6446 & 33 \\
\hline $\mathrm{H}(25)$ & 1910 & 10486 & 6084 & 29 \\
\hline $\mathrm{H}(29)$ & 3820 & 8558 & 6773 & 26 \\
\hline $\mathrm{H}(30)$ & 3970 & 9535 & 7551 & 32 \\
\hline $\mathrm{H}(31)$ & 5896 & 9410 & 8153 & 32 \\
\hline $\mathrm{H}(32)$ & 7680 & 8311 & 7966 & 30 \\
\hline $\mathrm{H}(33)$ & 7523 & 7314 & 7197 & 25 \\
\hline $\mathrm{H}(34 \mathrm{~A})$ & 6487 & 7168 & 5625 & 36 \\
\hline $\mathrm{H}(34 \mathrm{~B})$ & 7544 & 7564 & 6088 & 36 \\
\hline $\mathrm{H}(34 \mathrm{C})$ & 6283 & 8222 & 5866 & 36 \\
\hline $\mathrm{H}(35 \mathrm{~A})$ & 5476 & 5940 & 6868 & 33 \\
\hline $\mathrm{H}(35 \mathrm{~B})$ & 6987 & 6076 & 6580 & 33 \\
\hline
\end{tabular}


$\mathrm{H}(35 \mathrm{C})$ 
Table S15.Torsion angles $\left[{ }^{\circ}\right]$ for $\mathbf{1 4 n}$.

\begin{tabular}{|c|c|}
\hline $\mathrm{O}(2)-\mathrm{S}(1)-\mathrm{N}(1)-\mathrm{C}(2)$ & $-168.80(19)$ \\
\hline $\mathrm{O}(3)-\mathrm{S}(1)-\mathrm{N}(1)-\mathrm{C}(2)$ & $-43.3(2)$ \\
\hline $\mathrm{C}(9)-\mathrm{S}(1)-\mathrm{N}(1)-\mathrm{C}(2)$ & $76.8(2)$ \\
\hline $\mathrm{C}(2)-\mathrm{O}(1)-\mathrm{C}(1)-\mathrm{C}(18)$ & $176.65(18)$ \\
\hline $\mathrm{C}(2)-\mathrm{O}(1)-\mathrm{C}(1)-\mathrm{C}(26)$ & $-65.6(2)$ \\
\hline $\mathrm{S}(1)-\mathrm{N}(1)-\mathrm{C}(2)-\mathrm{O}(1)$ & $176.35(15)$ \\
\hline $\mathrm{S}(1)-\mathrm{N}(1)-\mathrm{C}(2)-\mathrm{C}(3)$ & $-3.8(3)$ \\
\hline $\mathrm{C}(1)-\mathrm{O}(1)-\mathrm{C}(2)-\mathrm{N}(1)$ & $8.2(3)$ \\
\hline $\mathrm{C}(1)-\mathrm{O}(1)-\mathrm{C}(2)-\mathrm{C}(3)$ & $-171.68(18)$ \\
\hline $\mathrm{N}(1)-\mathrm{C}(2)-\mathrm{C}(3)-\mathrm{C}(4)$ & $-97.4(3)$ \\
\hline $\mathrm{O}(1)-\mathrm{C}(2)-\mathrm{C}(3)-\mathrm{C}(4)$ & $82.4(3)$ \\
\hline $\mathrm{N}(1)-\mathrm{C}(2)-\mathrm{C}(3)-\mathrm{C}(8)$ & $83.4(3)$ \\
\hline $\mathrm{O}(1)-\mathrm{C}(2)-\mathrm{C}(3)-\mathrm{C}(8)$ & $-96.7(2)$ \\
\hline$C(8)-C(3)-C(4)-C(5)$ & $-0.9(3)$ \\
\hline$C(2)-C(3)-C(4)-C(5)$ & $179.9(2)$ \\
\hline $\mathrm{C}(8)-\mathrm{C}(3)-\mathrm{C}(4)-\mathrm{I}(1)$ & $178.36(17)$ \\
\hline $\mathrm{C}(2)-\mathrm{C}(3)-\mathrm{C}(4)-\mathrm{I}(1)$ & $-0.8(3)$ \\
\hline$C(3)-C(4)-C(5)-C(6)$ & $1.5(3)$ \\
\hline $\mathrm{I}(1)-\mathrm{C}(4)-\mathrm{C}(5)-\mathrm{C}(6)$ & $-177.77(18)$ \\
\hline $\mathrm{C}(4)-\mathrm{C}(5)-\mathrm{C}(6)-\mathrm{C}(7)$ & $-1.1(4)$ \\
\hline$C(5)-C(6)-C(7)-C(8)$ & $0.1(4)$ \\
\hline$C(6)-C(7)-C(8)-C(3)$ & $0.5(4)$ \\
\hline$C(4)-C(3)-C(8)-C(7)$ & $-0.1(3)$ \\
\hline $\mathrm{C}(2)-\mathrm{C}(3)-\mathrm{C}(8)-\mathrm{C}(7)$ & 179.1(2) \\
\hline $\mathrm{O}(2)-\mathrm{S}(1)-\mathrm{C}(9)-\mathrm{C}(10)$ & $-45.3(2)$ \\
\hline $\mathrm{O}(3)-\mathrm{S}(1)-\mathrm{C}(9)-\mathrm{C}(10)$ & $-175.57(16)$ \\
\hline $\mathrm{N}(1)-\mathrm{S}(1)-\mathrm{C}(9)-\mathrm{C}(10)$ & $65.08(19)$ \\
\hline $\mathrm{O}(2)-\mathrm{S}(1)-\mathrm{C}(9)-\mathrm{C}(14)$ & $133.20(18)$ \\
\hline $\mathrm{O}(3)-\mathrm{S}(1)-\mathrm{C}(9)-\mathrm{C}(14)$ & $3.0(2)$ \\
\hline $\mathrm{N}(1)-\mathrm{S}(1)-\mathrm{C}(9)-\mathrm{C}(14)$ & $-116.38(18)$ \\
\hline $\mathrm{C}(14)-\mathrm{C}(9)-\mathrm{C}(10)-\mathrm{C}(11)$ & $-1.1(3)$ \\
\hline$S(1)-C(9)-C(10)-C(11)$ & $177.48(18)$ \\
\hline$C(14)-C(9)-C(10)-C(15)$ & $179.7(2)$ \\
\hline$S(1)-C(9)-C(10)-C(15)$ & $-1.7(3)$ \\
\hline $\mathrm{C}(9)-\mathrm{C}(10)-\mathrm{C}(11)-\mathrm{C}(12)$ & $0.4(4)$ \\
\hline $\mathrm{C}(15)-\mathrm{C}(10)-\mathrm{C}(11)-\mathrm{C}(12)$ & $179.7(2)$ \\
\hline
\end{tabular}




\begin{tabular}{|c|c|}
\hline$C(10)-C(11)-C(12)-C(13)$ & $1.1(4)$ \\
\hline $\mathrm{C}(10)-\mathrm{C}(11)-\mathrm{C}(12)-\mathrm{C}(16)$ & $-178.1(3)$ \\
\hline $\mathrm{C}(11)-\mathrm{C}(12)-\mathrm{C}(13)-\mathrm{C}(14)$ & $-2.1(4)$ \\
\hline$C(16)-C(12)-C(13)-C(14)$ & $177.1(2)$ \\
\hline$C(12)-C(13)-C(14)-C(9)$ & $1.5(4)$ \\
\hline$C(12)-C(13)-C(14)-C(17)$ & $-176.3(2)$ \\
\hline$C(10)-C(9)-C(14)-C(13)$ & $0.1(3)$ \\
\hline$S(1)-C(9)-C(14)-C(13)$ & $-178.34(17)$ \\
\hline$C(10)-C(9)-C(14)-C(17)$ & $177.7(2)$ \\
\hline$S(1)-C(9)-C(14)-C(17)$ & $-0.7(3)$ \\
\hline$C(25)-C(20)-C(21)-C(22)$ & $-0.2(4)$ \\
\hline$C(19)-C(20)-C(21)-C(22)$ & $179.9(2)$ \\
\hline $\mathrm{C}(20)-\mathrm{C}(21)-\mathrm{C}(22)-\mathrm{C}(23)$ & $-0.4(4)$ \\
\hline $\mathrm{C}(21)-\mathrm{C}(22)-\mathrm{C}(23)-\mathrm{F}(1)$ & $-179.2(2)$ \\
\hline $\mathrm{C}(21)-\mathrm{C}(22)-\mathrm{C}(23)-\mathrm{C}(24)$ & $1.1(4)$ \\
\hline $\mathrm{F}(1)-\mathrm{C}(23)-\mathrm{C}(24)-\mathrm{C}(25)$ & $179.2(3)$ \\
\hline $\mathrm{C}(22)-\mathrm{C}(23)-\mathrm{C}(24)-\mathrm{C}(25)$ & $-1.0(4)$ \\
\hline$C(23)-C(24)-C(25)-C(20)$ & $0.3(4)$ \\
\hline $\mathrm{C}(21)-\mathrm{C}(20)-\mathrm{C}(25)-\mathrm{C}(24)$ & $0.3(4)$ \\
\hline$C(19)-C(20)-C(25)-C(24)$ & $-179.9(3)$ \\
\hline $\mathrm{C}(27)-\mathrm{O}(5)-\mathrm{C}(26)-\mathrm{O}(4)$ & $-0.6(3)$ \\
\hline $\mathrm{C}(27)-\mathrm{O}(5)-\mathrm{C}(26)-\mathrm{C}(1)$ & $175.19(17)$ \\
\hline $\mathrm{O}(1)-\mathrm{C}(1)-\mathrm{C}(26)-\mathrm{O}(4)$ & $-28.5(3)$ \\
\hline $\mathrm{C}(18)-\mathrm{C}(1)-\mathrm{C}(26)-\mathrm{O}(4)$ & $89.3(3)$ \\
\hline $\mathrm{O}(1)-\mathrm{C}(1)-\mathrm{C}(26)-\mathrm{O}(5)$ & $155.53(17)$ \\
\hline $\mathrm{C}(18)-\mathrm{C}(1)-\mathrm{C}(26)-\mathrm{O}(5)$ & $-86.7(2)$ \\
\hline $\mathrm{C}(26)-\mathrm{O}(5)-\mathrm{C}(27)-\mathrm{C}(34)$ & $172.79(19)$ \\
\hline $\mathrm{C}(26)-\mathrm{O}(5)-\mathrm{C}(27)-\mathrm{C}(28)$ & $-69.9(2)$ \\
\hline $\mathrm{C}(26)-\mathrm{O}(5)-\mathrm{C}(27)-\mathrm{C}(35)$ & $55.4(3)$ \\
\hline $\mathrm{O}(5)-\mathrm{C}(27)-\mathrm{C}(28)-\mathrm{C}(29)$ & $-13.2(3)$ \\
\hline $\mathrm{C}(34)-\mathrm{C}(27)-\mathrm{C}(28)-\mathrm{C}(29)$ & $99.0(3)$ \\
\hline $\mathrm{C}(35)-\mathrm{C}(27)-\mathrm{C}(28)-\mathrm{C}(29)$ & $-136.4(2)$ \\
\hline $\mathrm{O}(5)-\mathrm{C}(27)-\mathrm{C}(28)-\mathrm{C}(33)$ & $170.2(2)$ \\
\hline $\mathrm{C}(34)-\mathrm{C}(27)-\mathrm{C}(28)-\mathrm{C}(33)$ & $-77.5(3)$ \\
\hline $\mathrm{C}(35)-\mathrm{C}(27)-\mathrm{C}(28)-\mathrm{C}(33)$ & $47.1(3)$ \\
\hline $\mathrm{C}(33)-\mathrm{C}(28)-\mathrm{C}(29)-\mathrm{C}(30)$ & $-1.9(4)$ \\
\hline $\mathrm{C}(27)-\mathrm{C}(28)-\mathrm{C}(29)-\mathrm{C}(30)$ & $-178.5(2)$ \\
\hline$C(28)-C(29)-C(30)-C(31)$ & $1.0(4)$ \\
\hline
\end{tabular}


$\mathrm{C}(29)-\mathrm{C}(30)-\mathrm{C}(31)-\mathrm{C}(32)$

$\mathrm{C}(30)-\mathrm{C}(31)-\mathrm{C}(32)-\mathrm{C}(33)$

$\mathrm{C}(31)-\mathrm{C}(32)-\mathrm{C}(33)-\mathrm{C}(28)$

$\mathrm{C}(29)-\mathrm{C}(28)-\mathrm{C}(33)-\mathrm{C}(32)$

$\mathrm{C}(27)-\mathrm{C}(28)-\mathrm{C}(33)-\mathrm{C}(32)$
0.4(4)

$-1.0(4)$

$0.1(4)$

178.1(2)

Symmetry transformations used to generate equivalent atoms:

\section{Citations}

A. J. M. Duisenberg, L. M. J. Kroon-Batenburg, A. M. M. Schreurs, J. Appl. Crystallogr. (2003), 36, 220-229.

R. H. Blessing, Acta Cryst., (1995), A51, 33-38.

Sheldrick, G.M., ShelXT-Integrated space-group and crystal-structure determination, Acta Cryst., (2015), A71, 3-8.

Sheldrick, G.M., Crystal structure refinement with ShelXL, Acta Cryst., (2015), C71, 3-8.

O.V. Dolomanov, L.J. Bourhis, R.J. Gildea, J.A.K. Howard, H. Puschmann, Olex2: A complete structure solution, refinement and analysis program, J. Appl. Cryst., (2009), 42, 339-341. 


\section{References}

[1] C. Wu, E. R. Decker, N. Blok, H. Bui, Q. Chen, B. Raju, A. R. Bourgoyne, V. Knowles, R. J. Biediger, R. V. Market, et al., J. Med. Chem. 1999, 42, 4485-4499.

[2] A. S. Deeming, C. J. Russell, M. C. Willis, Angew. Chemie - Int. Ed. 2016, 55, 747750.

[3] J. C. Towson, F. A. Davis, S. Lal, P. J. Carroll, M. C. Weismiller, J. Am. Chem. Soc. 2005, 110, 8477-8482.

[4] P. Müller, P. Polleux, Helv. Chim. Acta 1994, 77, 645-654.

[5] M. P. Doyle, V. Bagheri, T. J. Wandless, N. K. Ham, D. A. Brinker, C. T. Eagle, K. L. Loh, J. Am. Chem. Soc. 1990, 112, 1906-1912.

[6] K. Fukushima, Y. Q. Lu, T. Ibata, Bull. Chem. Soc. Jpn. 1996, 69, 3289-3295.

[7] M. P. Doyle, A. van Oeveren, L. J. Westrum, T. W. Clayton, M. N. Protopopova, J. Am. Chem. Soc. 1991, 113, 8982-8984.

[8] D. A. Nicewicz, J. S. Johnson, J. Am. Chem. Soc. 2005, 127, 6170-6171.

[9] H. W. Xu, G. Y. Li, M. K. Wong, C. M. Che, Org. Lett. 2005, 7, 5349-5352.

[10] T. Torna, J. Shimokawa, T. Fukuyama, Org. Lett. 2007, 9, 3195-3197.

[11] D. P. Hari, J. Waser, J. Am. Chem. Soc. 2016, 138, 2190-2193.

[12] M. P. Sibi, M. Liu, Org. Lett. 2000, 2, 3393-3396.

[13] A. H. Cherney, S. E. Reisman, J. Am. Chem. Soc. 2014, 136, 14365-14368.

[14] S. E. Denmark, G. M. Stiff, J. Org. Chem. 2000, 65, 5875-5878.

[15] G. Chollet, F. Rodriguez, E. Schulz, Org. Lett. 2006, 8, 539-542.

[16] G. Chollet, M. G. Guillerez, E. Schulz, Chem. - A Eur. J. 2007, 13, 992-1000.

[17] S. S. Lee, S. Hadinoto, J. Y. Ying, Adv. Synth. Catal. 2006, 348, 1248-1254.

[18] D. F. González, J. P. Brand, J. Waser, Chem. - A Eur. J. 2010, 16, 9457-9461.

[19] D. Fernández González, J. P. Brand, R. Mondière, J. Waser, Adv. Synth. Catal. 2013, $355,1631-1639$. 
[20] R. Frei, J. Waser, J. Am. Chem. Soc. 2013, 135, 9620-9623.

[21] J. P. Brand, J. Charpentier, J. Waser, Angew. Chemie - Int. Ed. 2009, 48, 9346-9349. 
10. Spectra of new compounds.

${ }^{\mathbf{1}} \mathbf{H}-\mathbf{N M R}\left(400 \mathrm{MHz}, \mathrm{CDCl}_{3}\right)$ of compound 3a

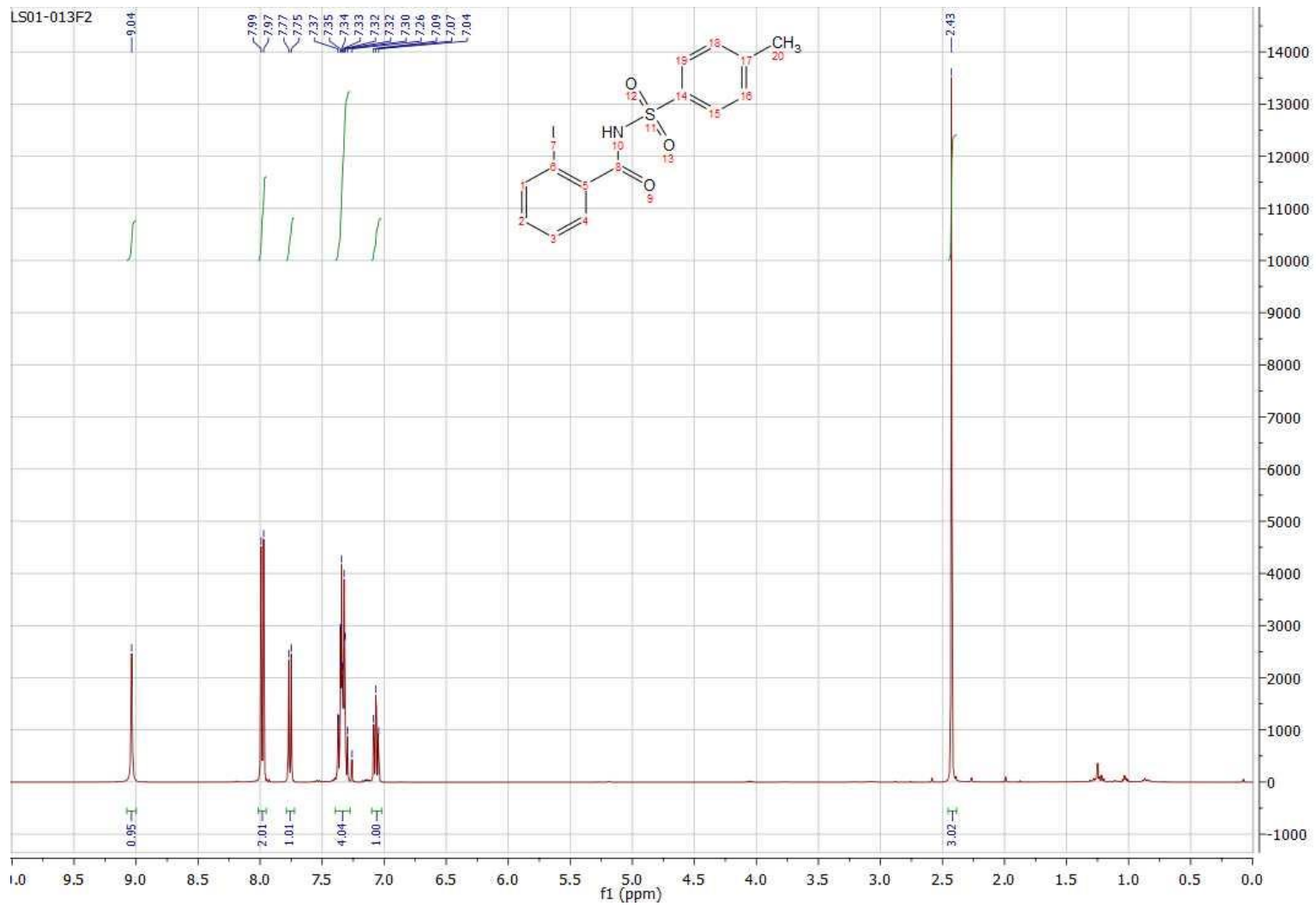

${ }^{13} \mathbf{C}$-NMR $\left(100 \mathrm{MHz}, \mathrm{CDCl}_{3}\right)$ of compound 3a

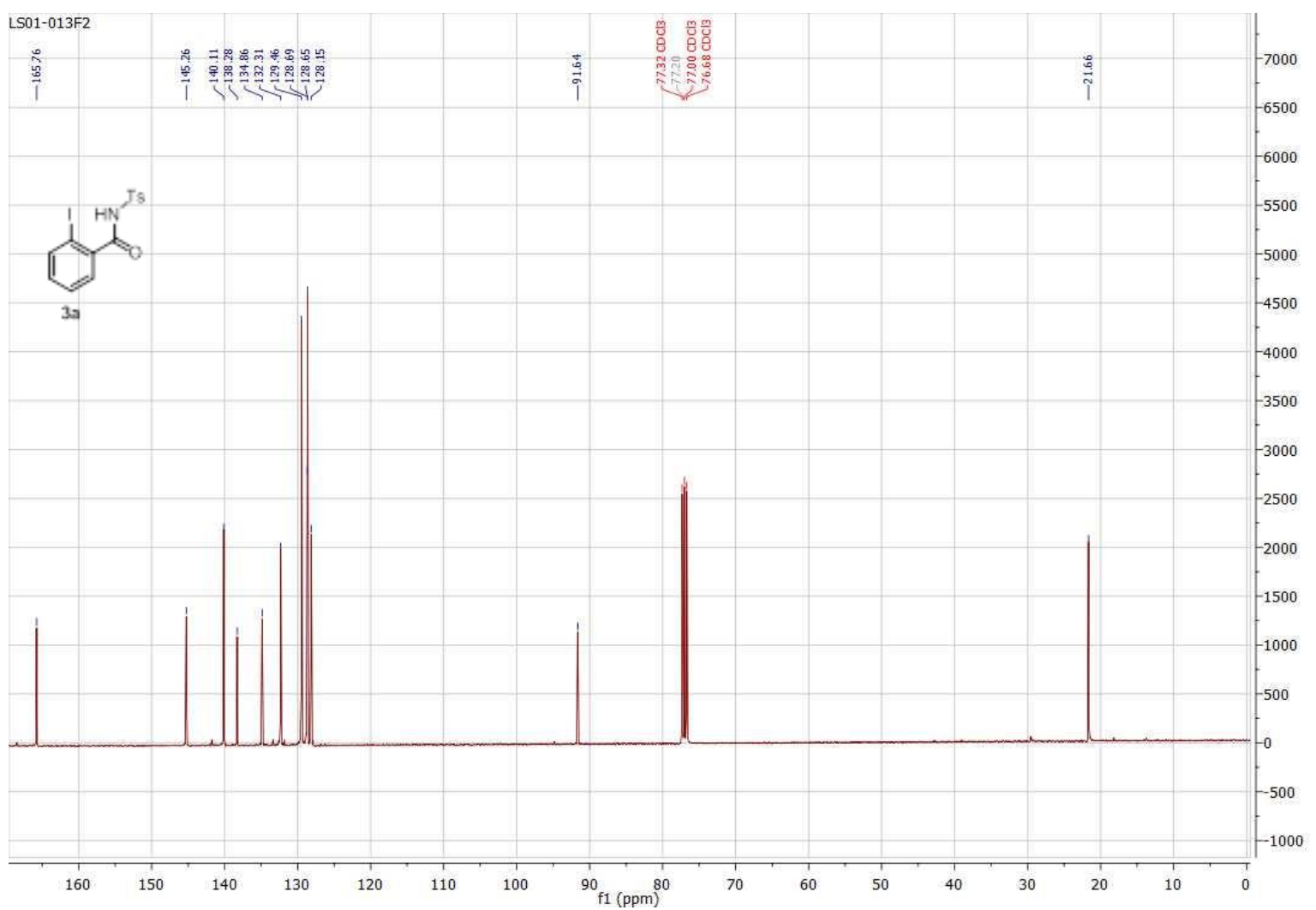




\section{IR of compound $\mathbf{3 a}$}

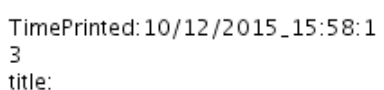<smiles>O=C(N[In])c1ccccc1I</smiles>
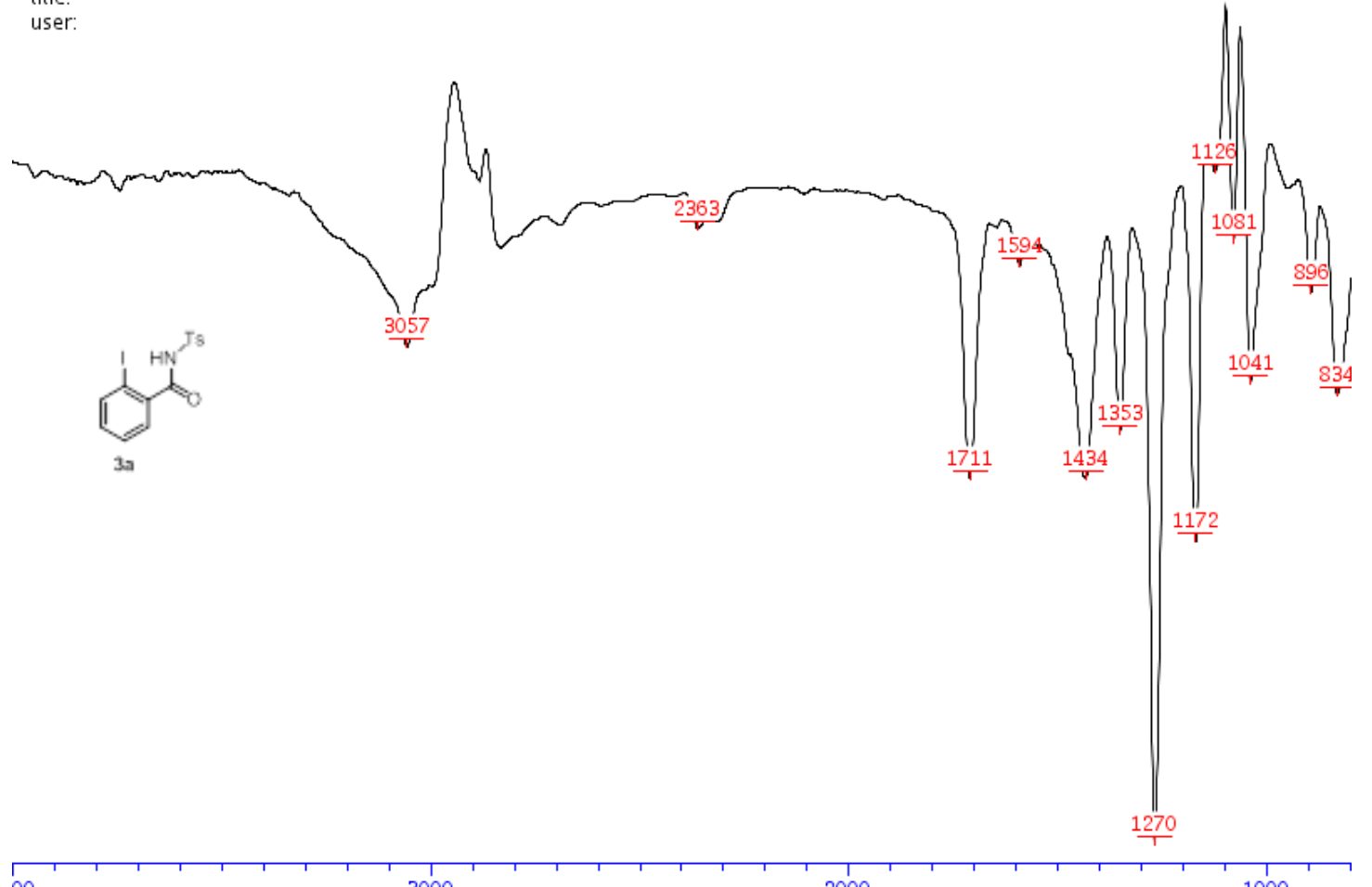
${ }^{\mathbf{1}} \mathbf{H}-\mathbf{N M R}\left(400 \mathrm{MHz}, \mathrm{CDCl}_{3}\right)$ of compound $\mathbf{3 b}$

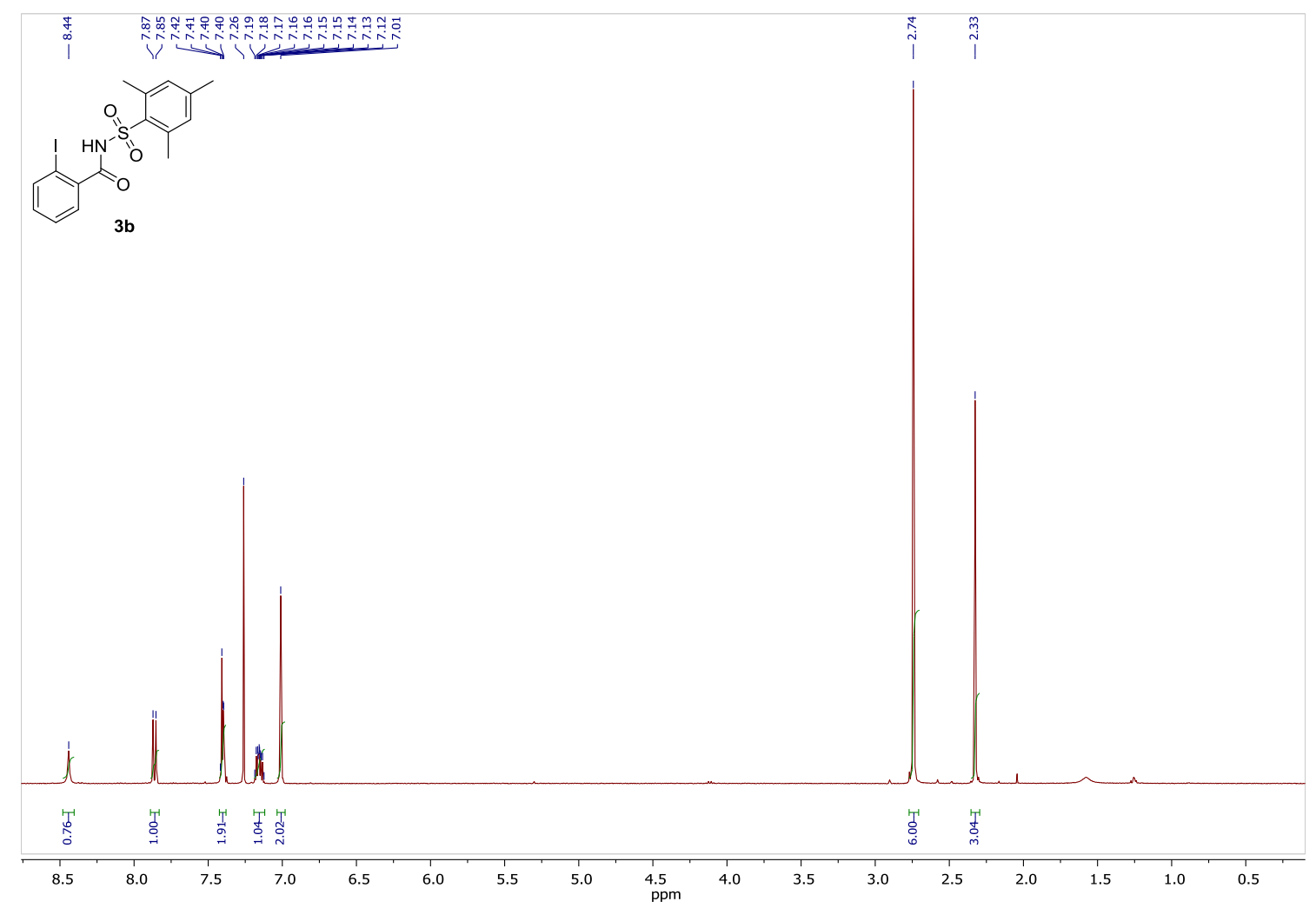

${ }^{13} \mathbf{C}$-NMR (100 MHz, $\left.\mathrm{CDCl}_{3}\right)$ of compound 3b

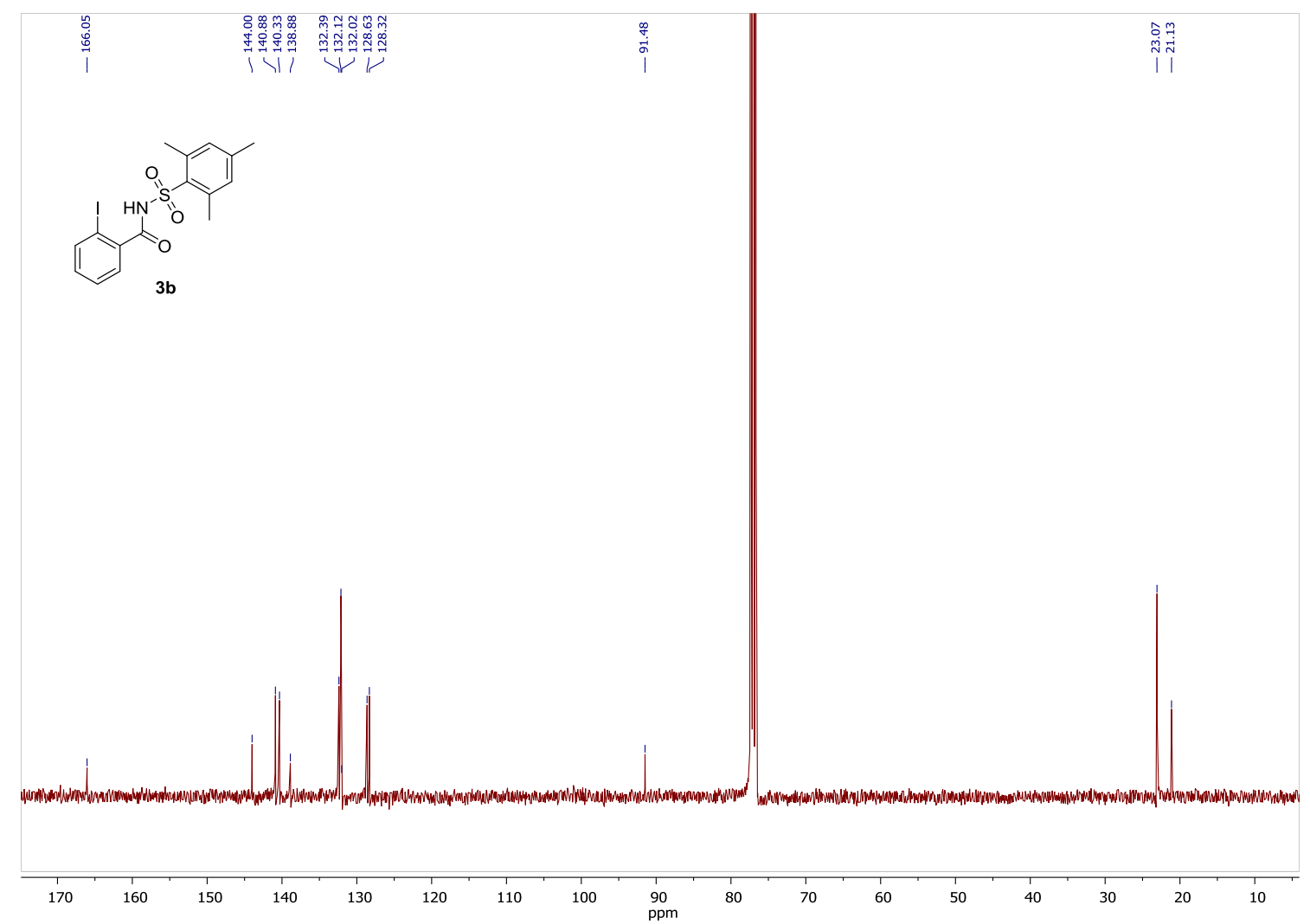


IR of compound $\mathbf{3 b}$

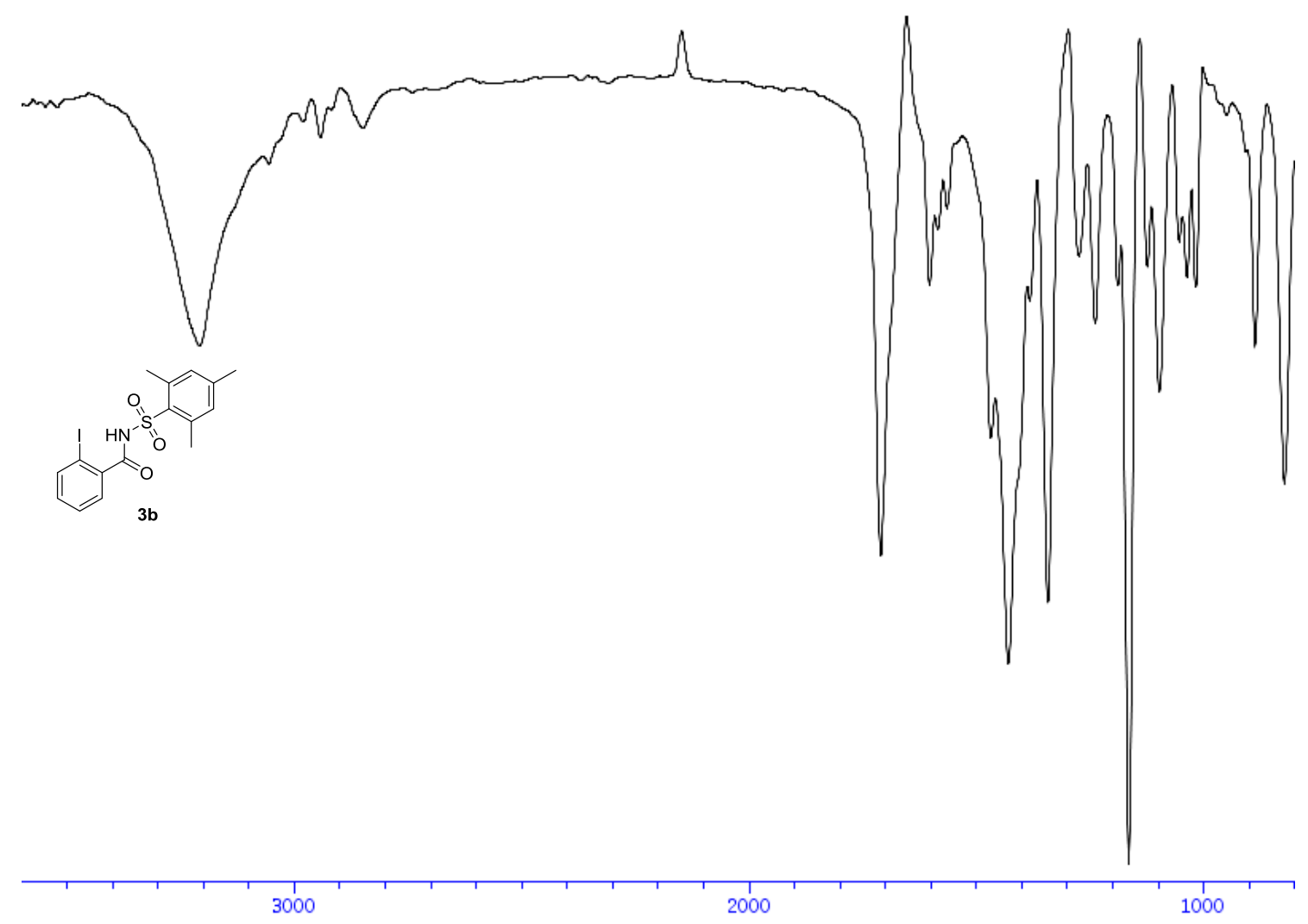


${ }^{1} \mathbf{H}-\mathbf{N M R}\left(400 \mathrm{MHz}, \mathrm{CDCl}_{3}\right)$ of compound 3c

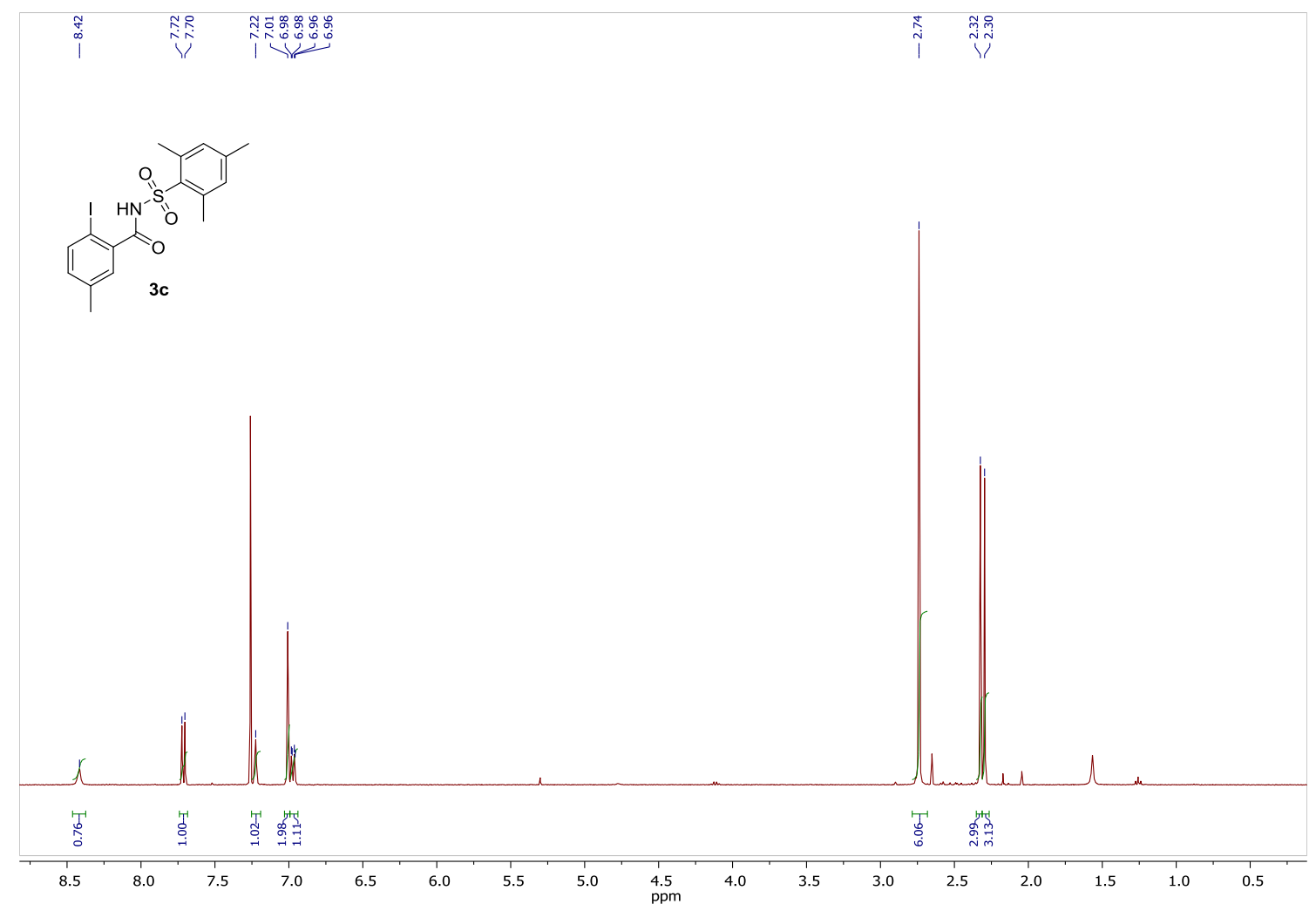

${ }^{13} \mathbf{C}$-NMR $\left(100 \mathrm{MHz}, \mathrm{CDCl}_{3}\right)$ of compound 3c

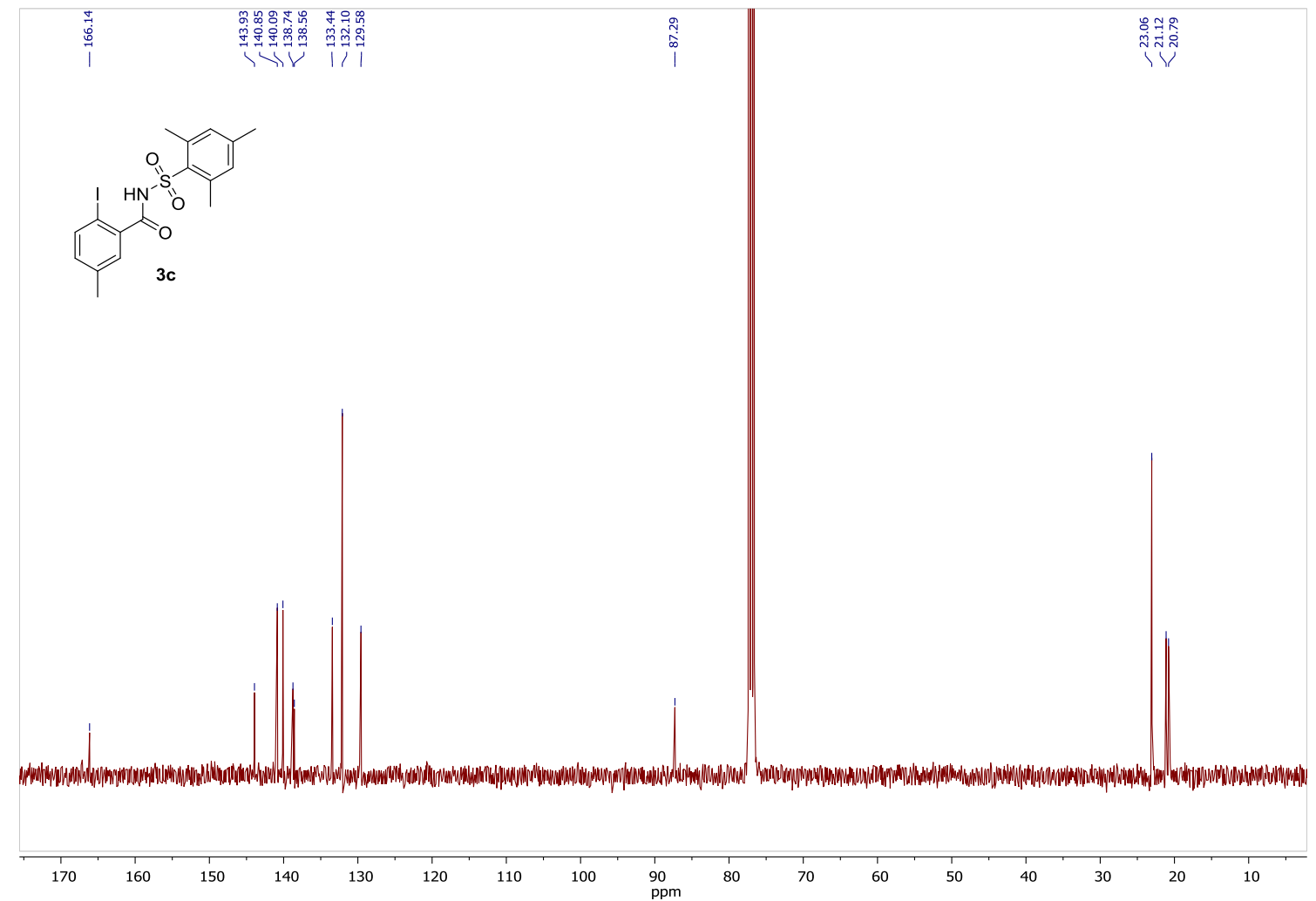


IR of compound $\mathbf{3 c}$

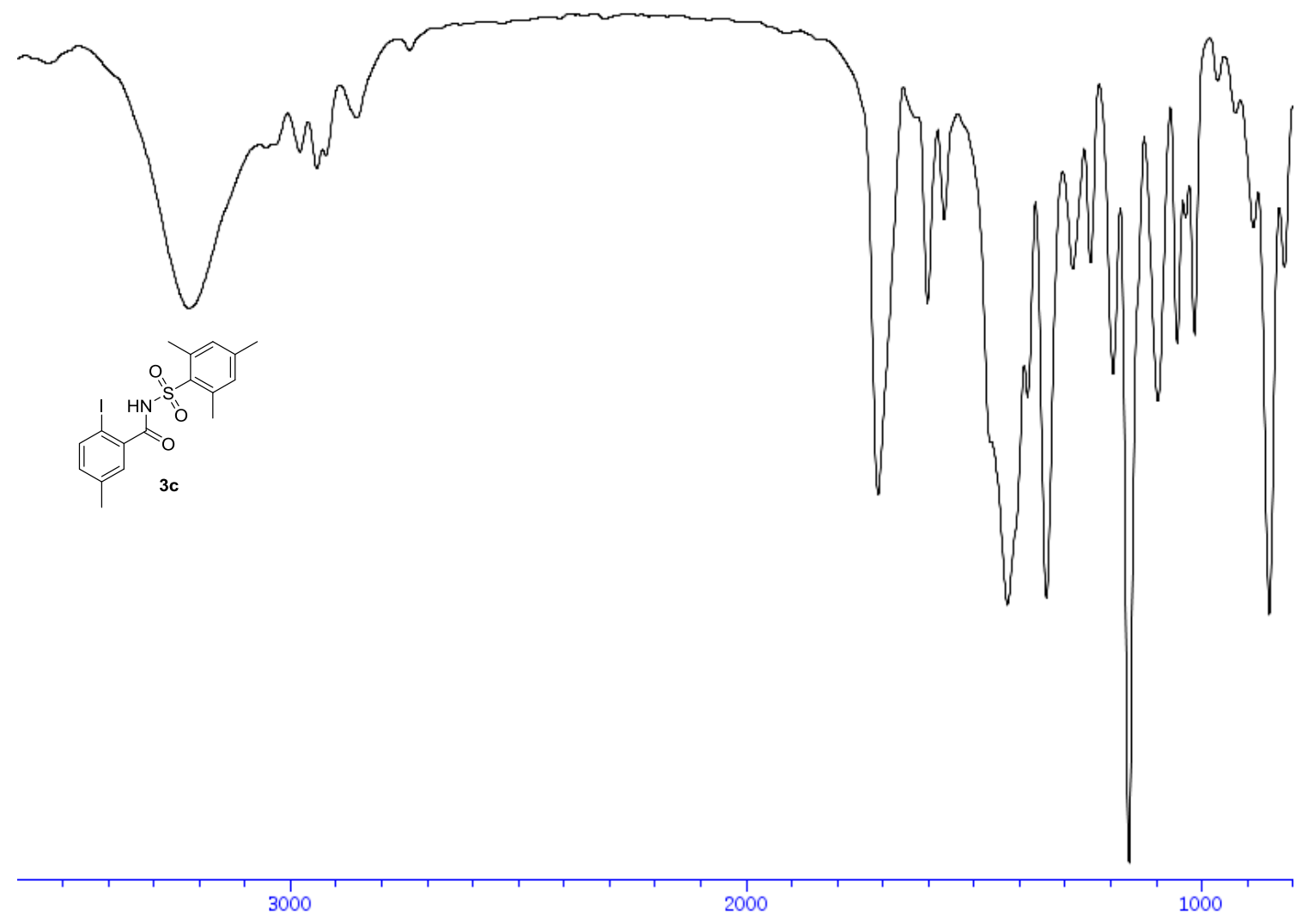


${ }^{\mathbf{1}} \mathbf{H}-\mathbf{N M R}\left(400 \mathrm{MHz}, \mathrm{CDCl}_{3}\right)$ of compound $\mathbf{3 d}$

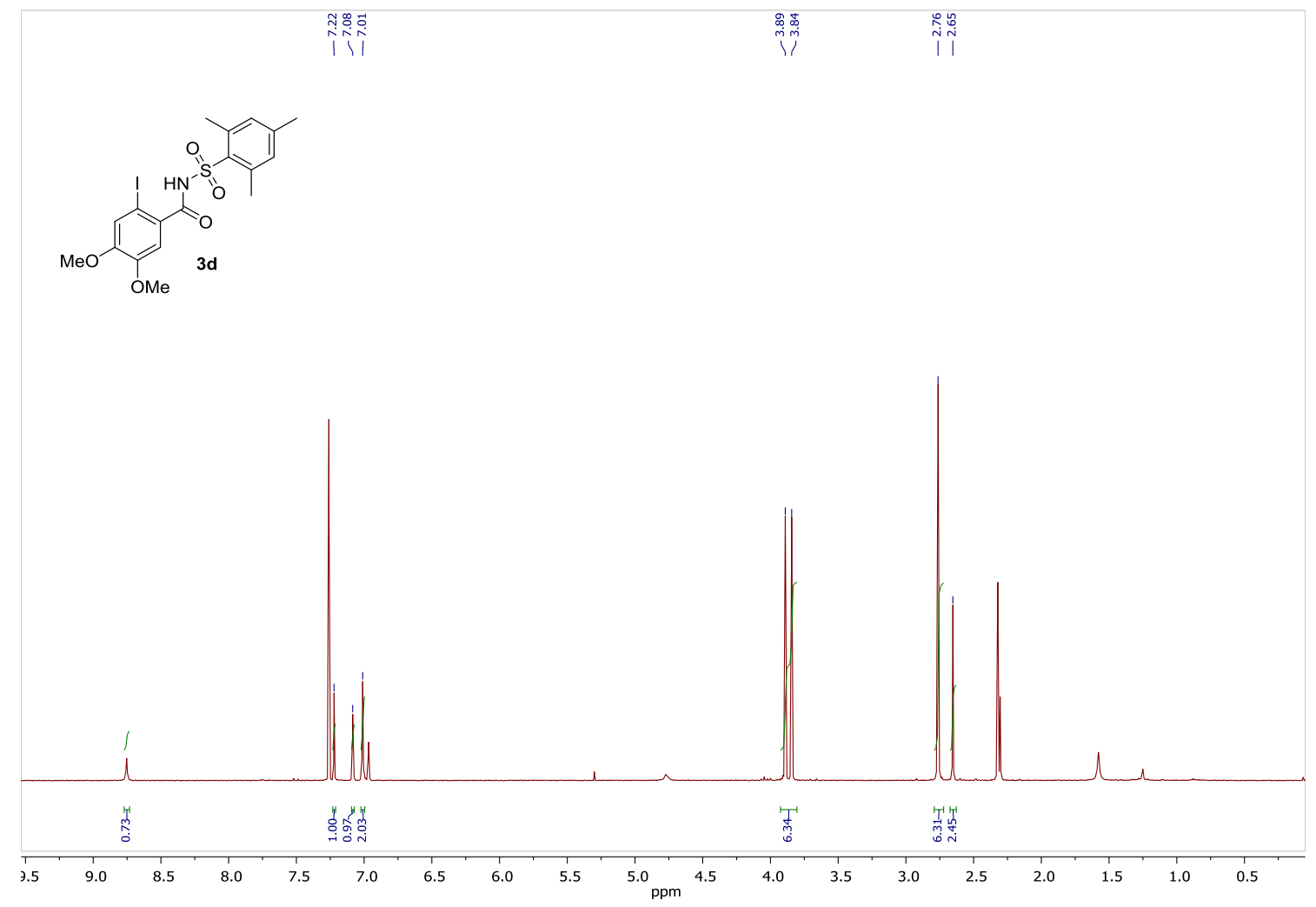

${ }^{13} \mathbf{C}$-NMR (100 MHz, $\left.\mathrm{CDCl}_{3}\right)$ of compound 3d

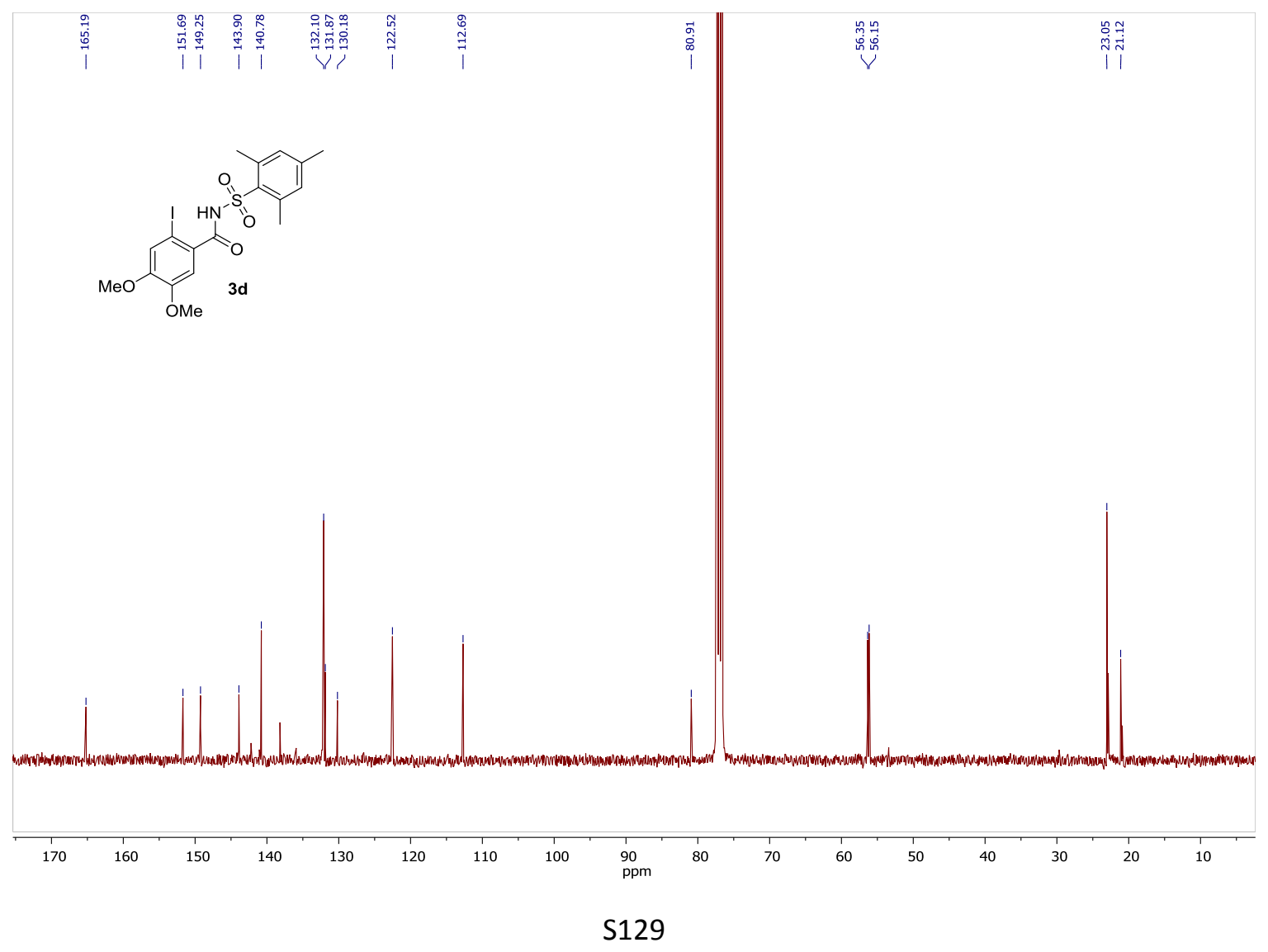


IR of compound $\mathbf{3 d}$

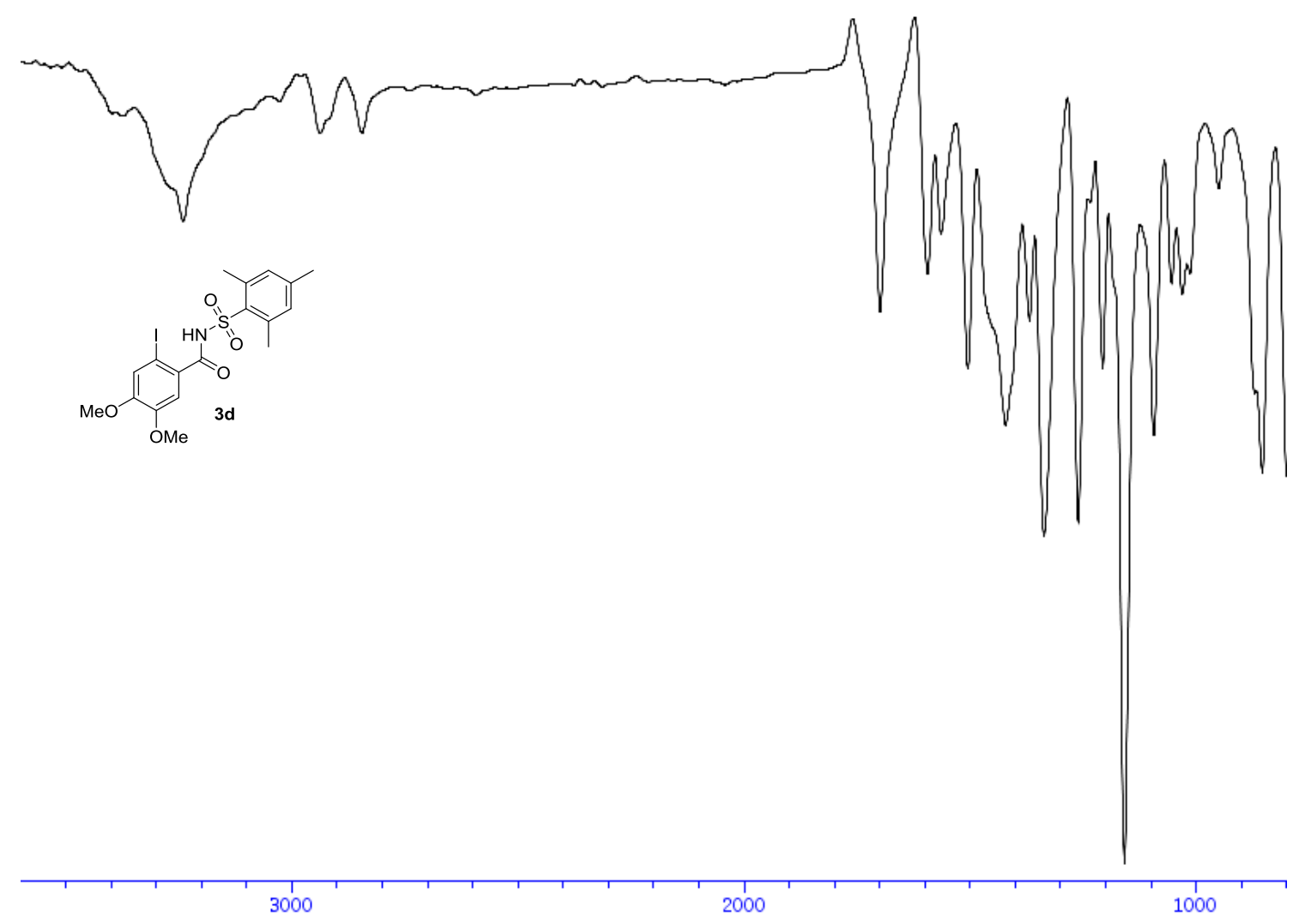


${ }^{1} \mathbf{H}-\mathbf{N M R}\left(400 \mathrm{MHz}, \mathrm{CDCl}_{3}\right)$ of compound $\mathbf{3 e}$

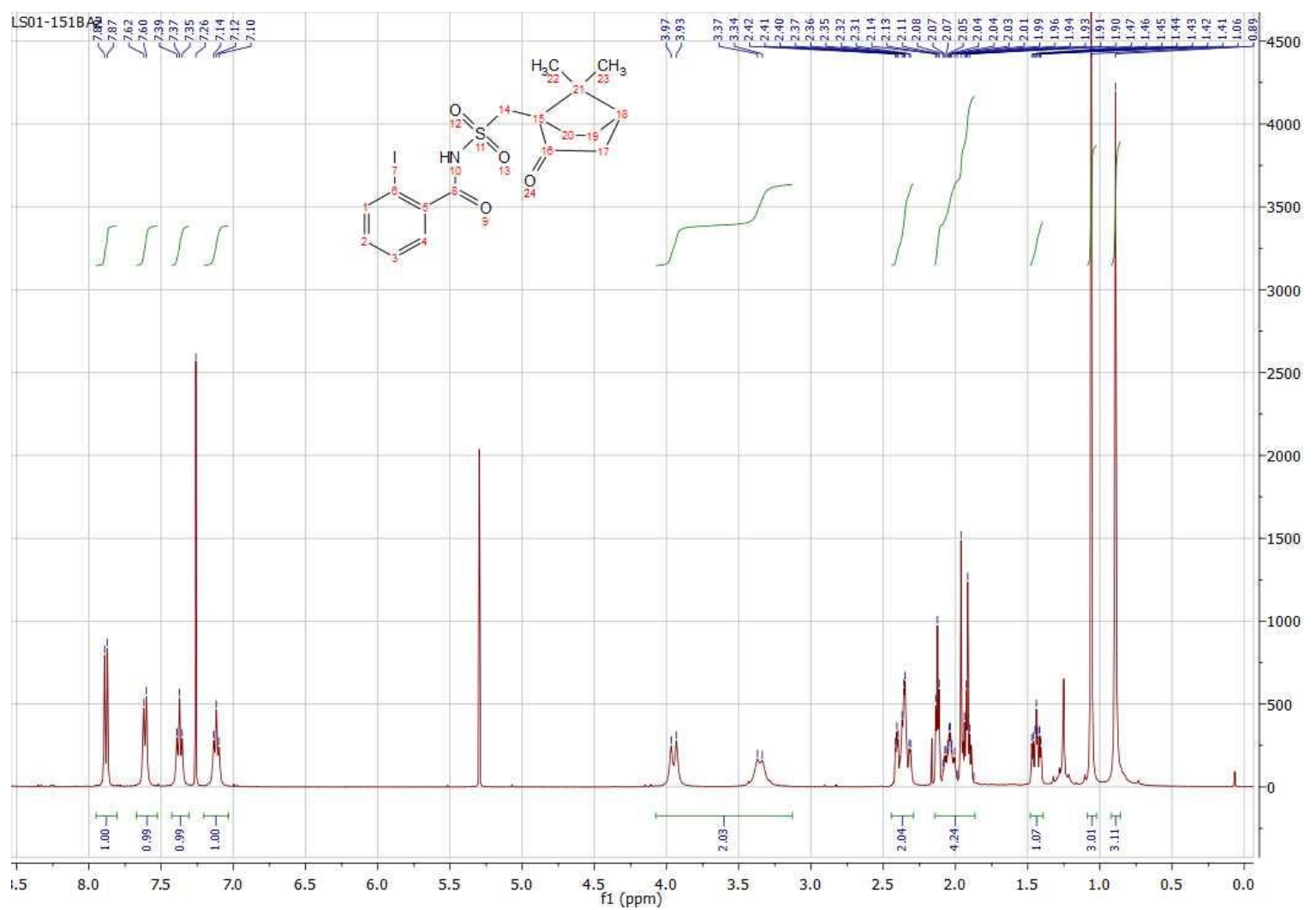

${ }^{13} \mathbf{C}$-NMR $\left(100 \mathrm{MHz}, \mathrm{CDCl}_{3}\right)$ of compound $\mathbf{3 e}$

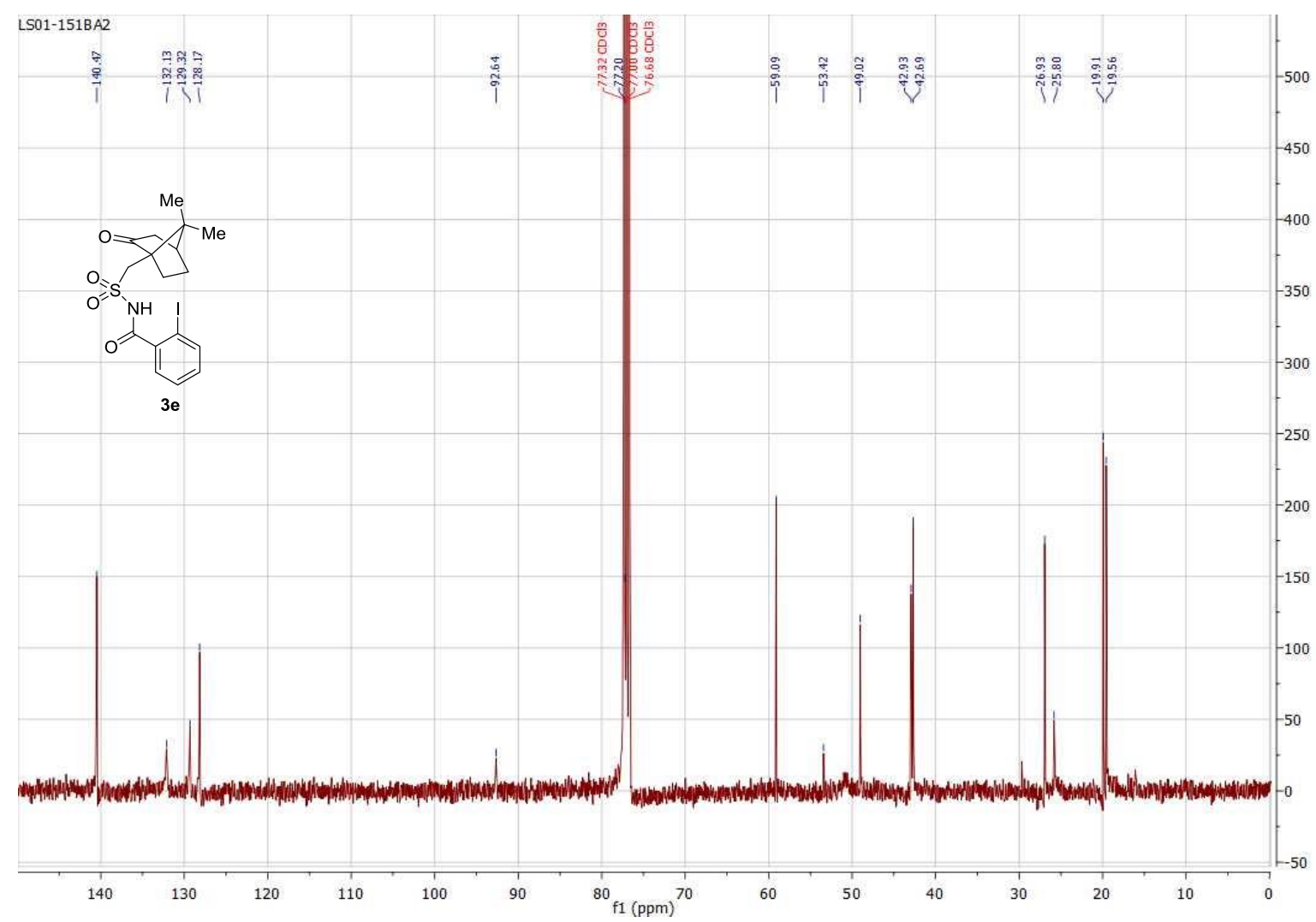


IR of compound $\mathbf{3 e}$

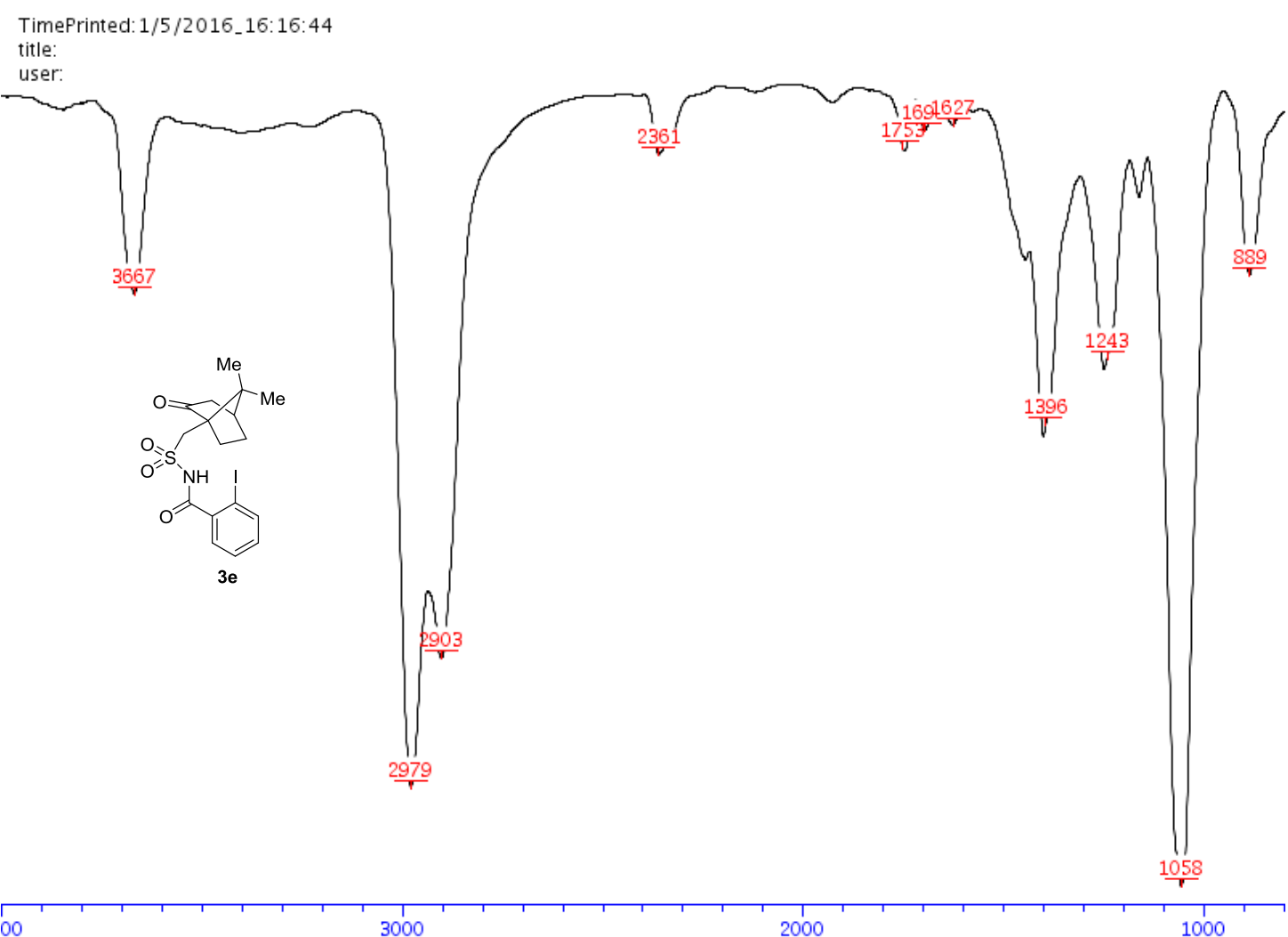


${ }^{1} \mathbf{H}-\mathbf{N M R}\left(400 \mathrm{MHz}, \mathrm{CDCl}_{3}\right)$ of compound $\mathbf{3 f}$

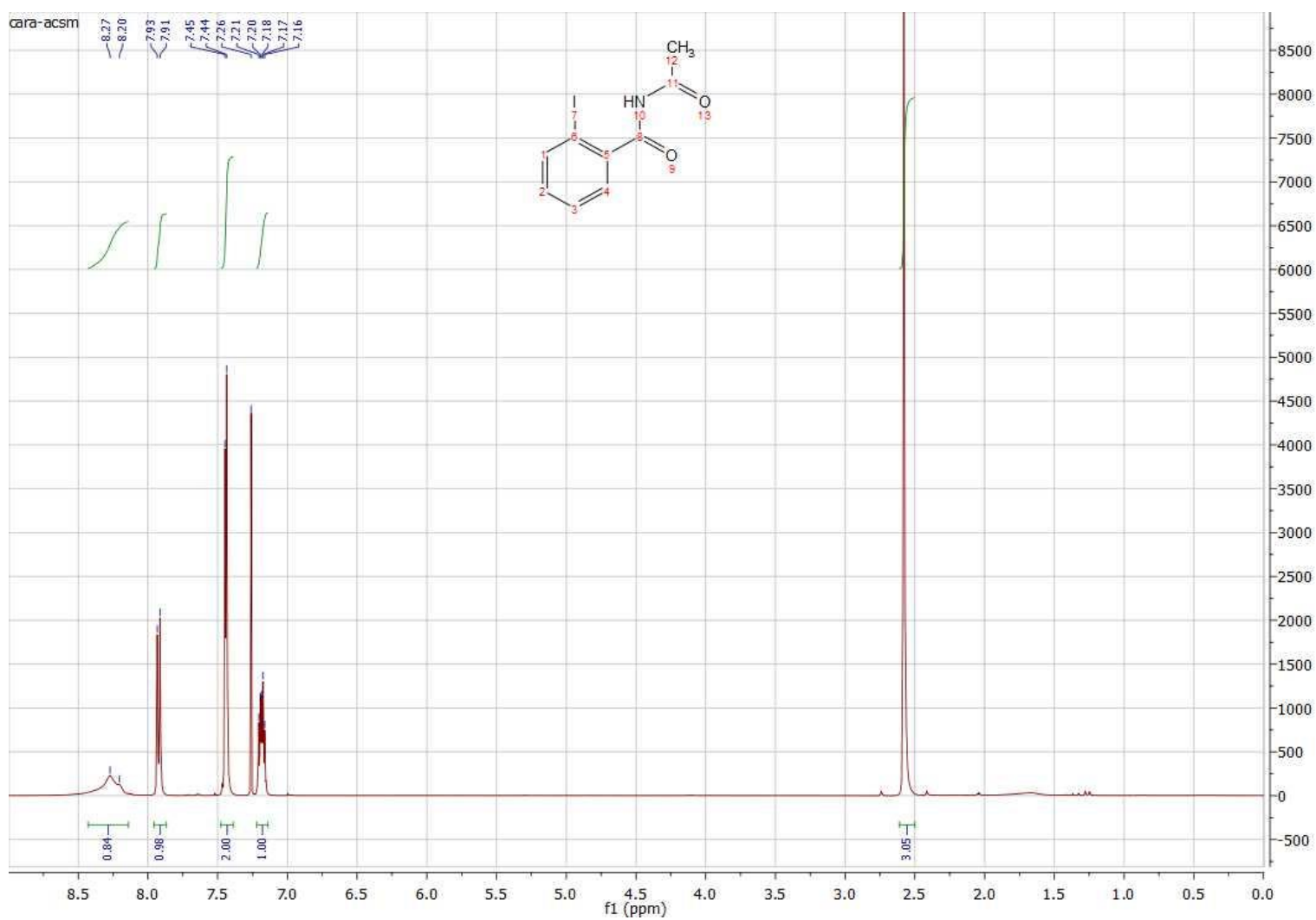

${ }^{13} \mathbf{C}$-NMR $\left(100 \mathrm{MHz}, \mathrm{CDCl}_{3}\right)$ of compound $\mathbf{3 f}$

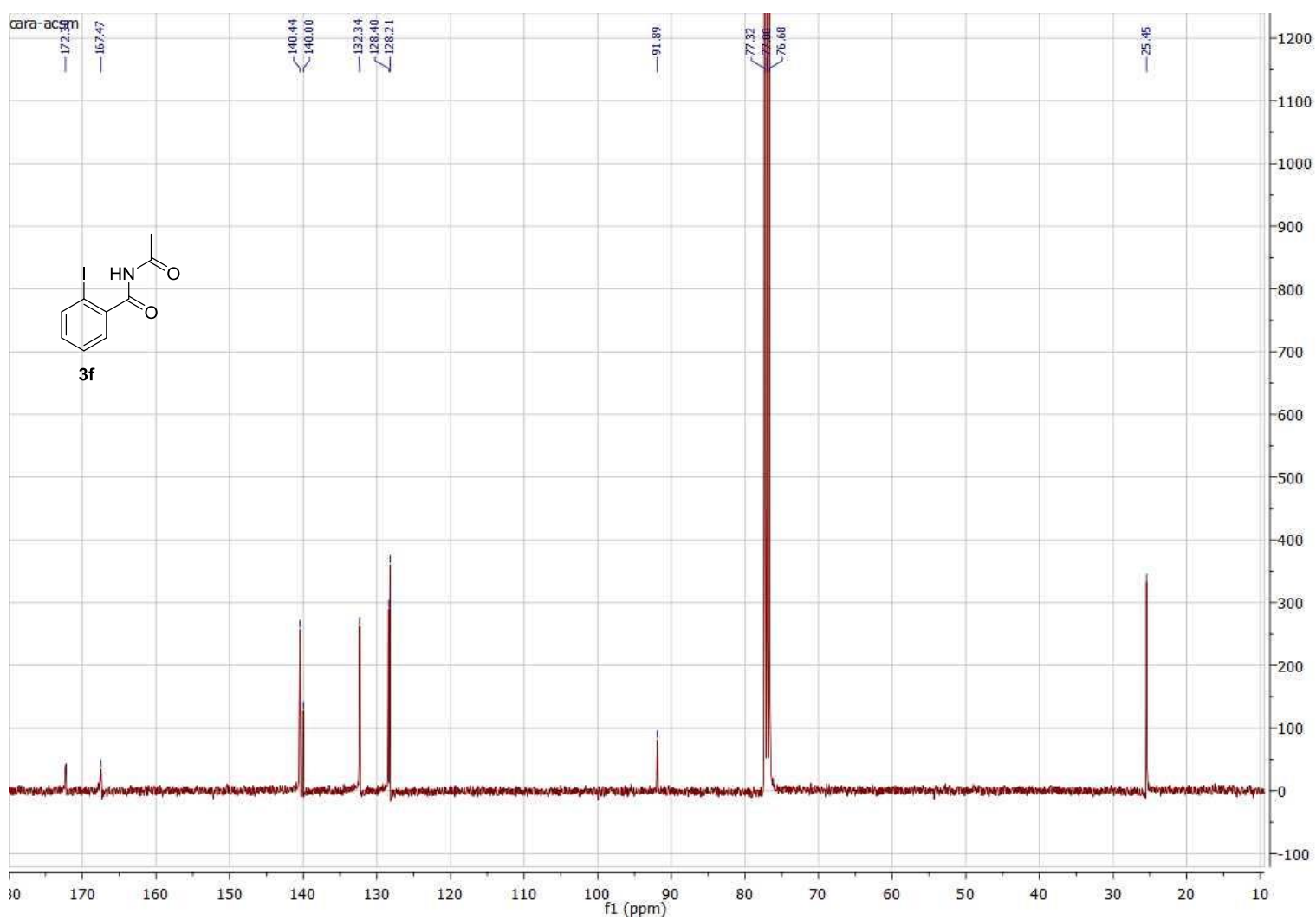


IR of compound $\mathbf{3 f}$

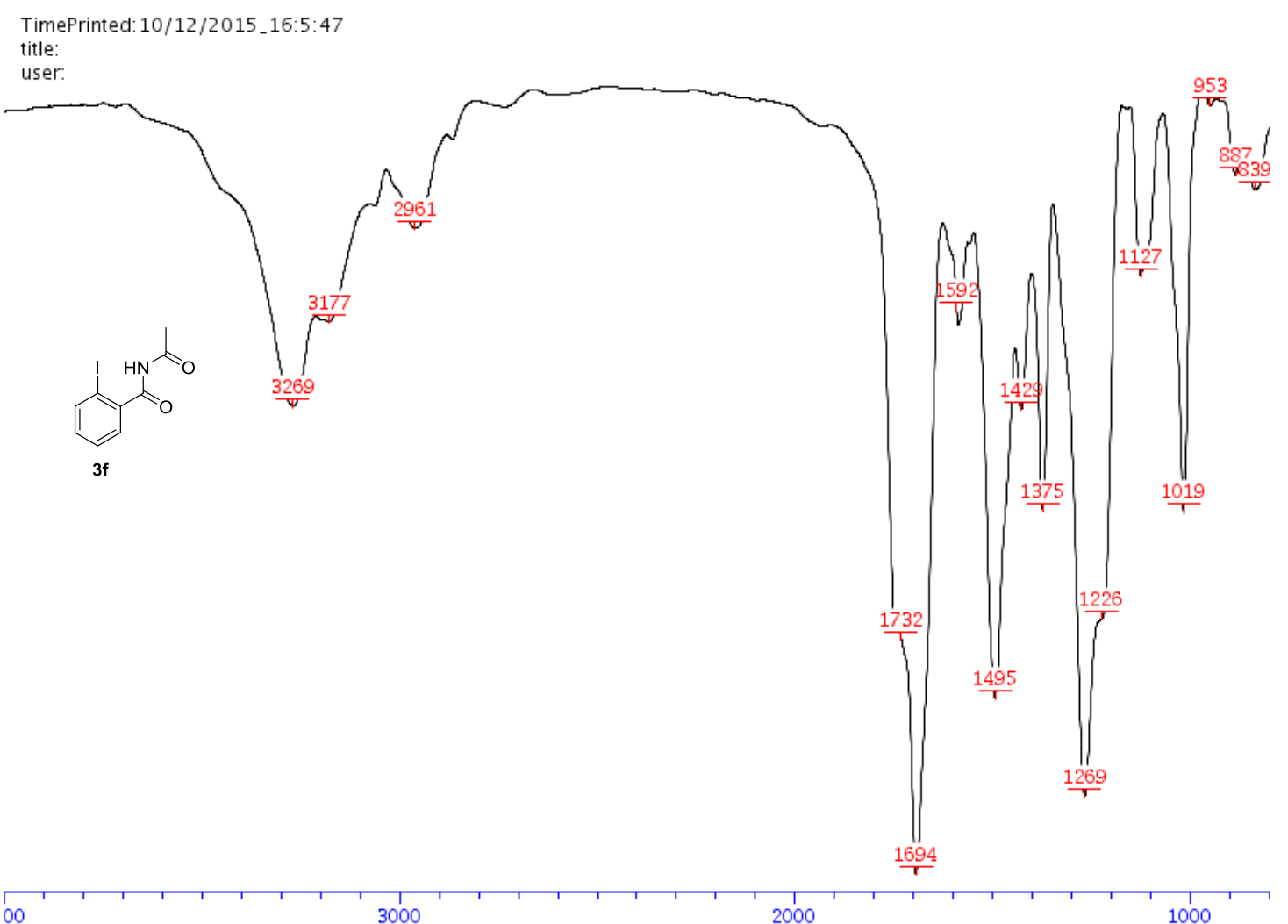


${ }^{1} \mathbf{H}-\mathbf{N M R}\left(400 \mathrm{MHz}, \mathrm{CDCl}_{3}\right)$ of compound $\mathbf{2 a}$

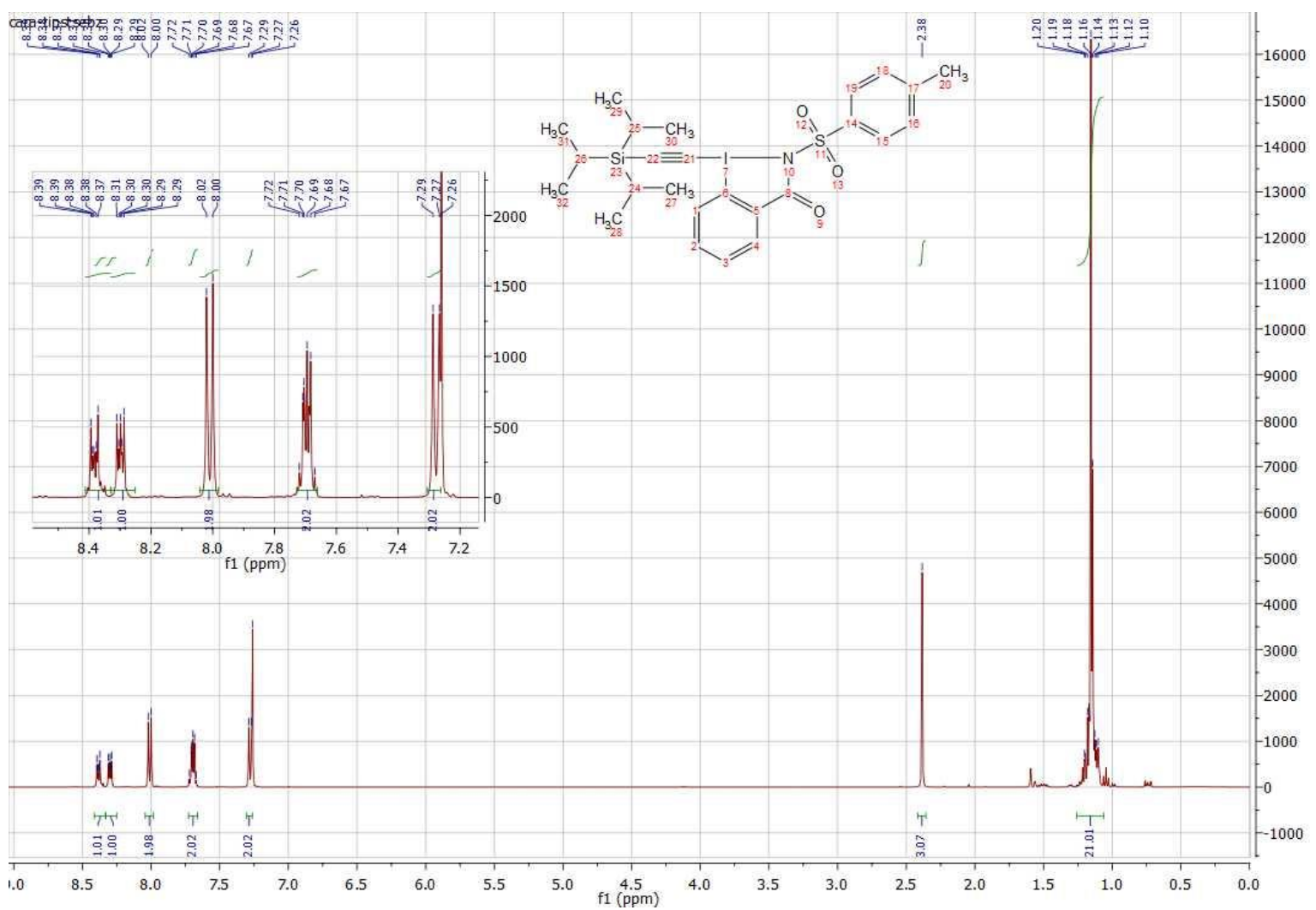

${ }^{13} \mathbf{C}$-NMR $\left(100 \mathrm{MHz}, \mathrm{CDCl}_{3}\right)$ of compound $\mathbf{2 a}$

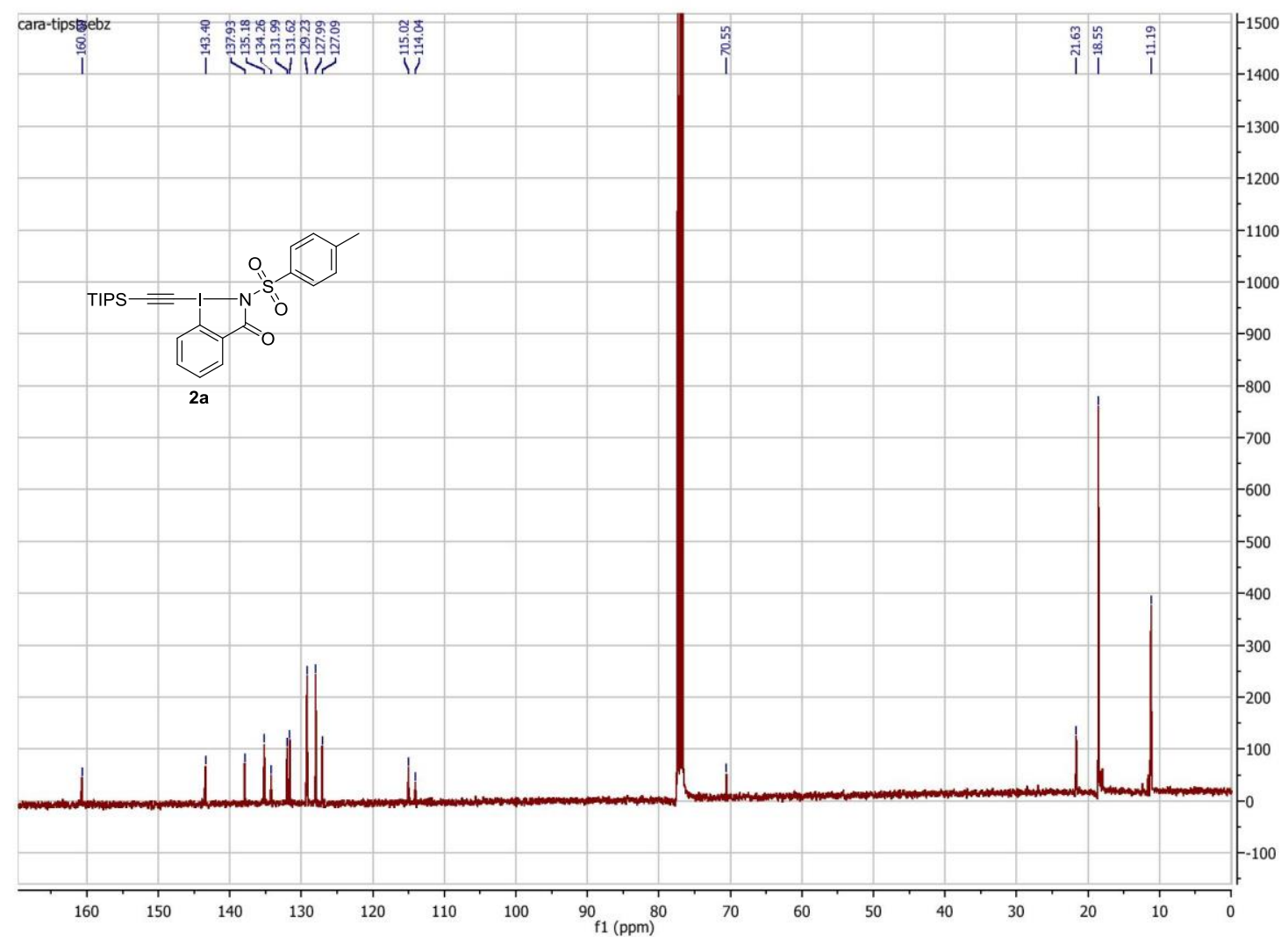


IR of compound $\mathbf{2 a}$

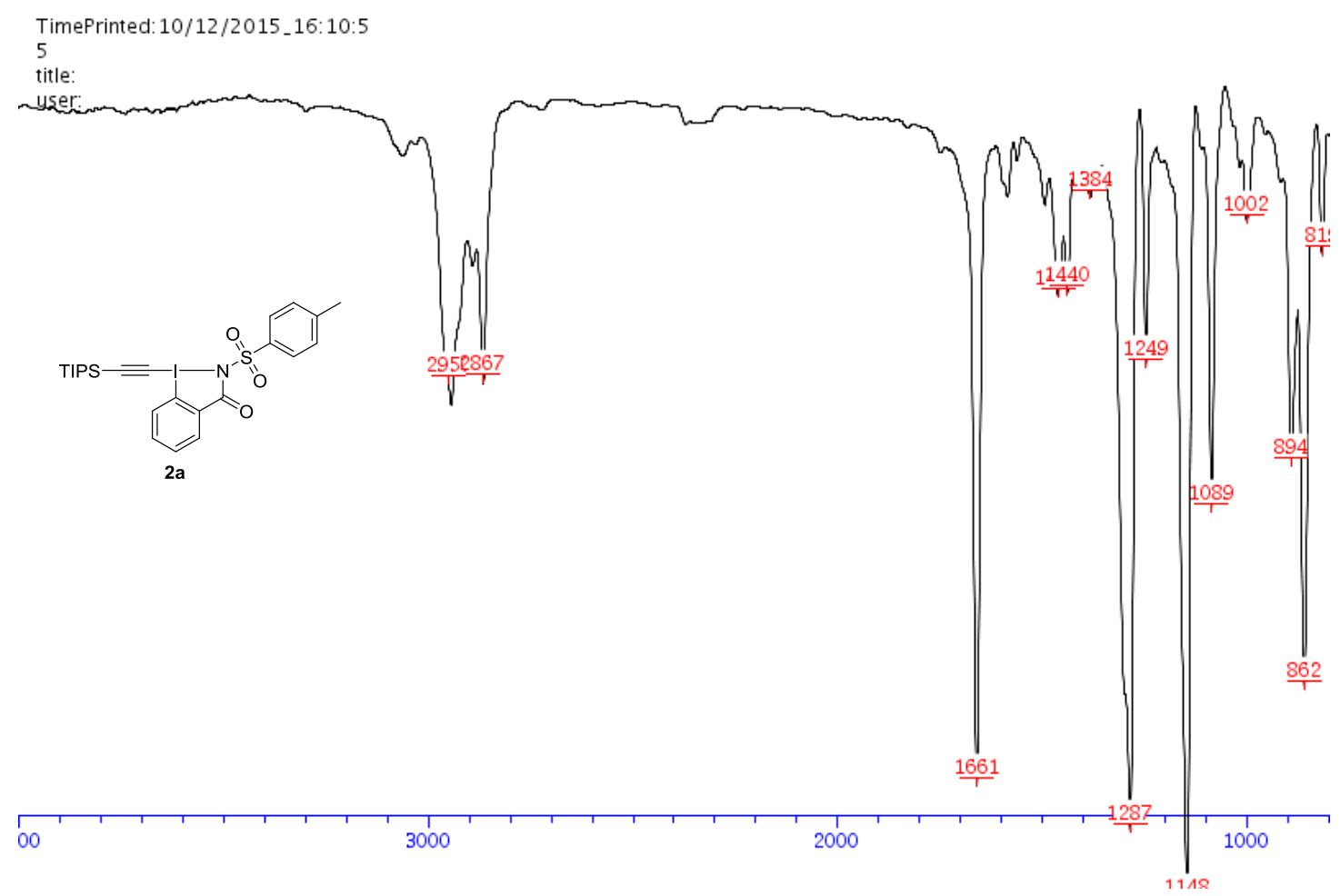


${ }^{\mathbf{1}} \mathbf{H}-\mathbf{N M R}\left(400 \mathrm{MHz}, \mathrm{CDCl}_{3}\right.$ ) of compound $\mathbf{2 b}$

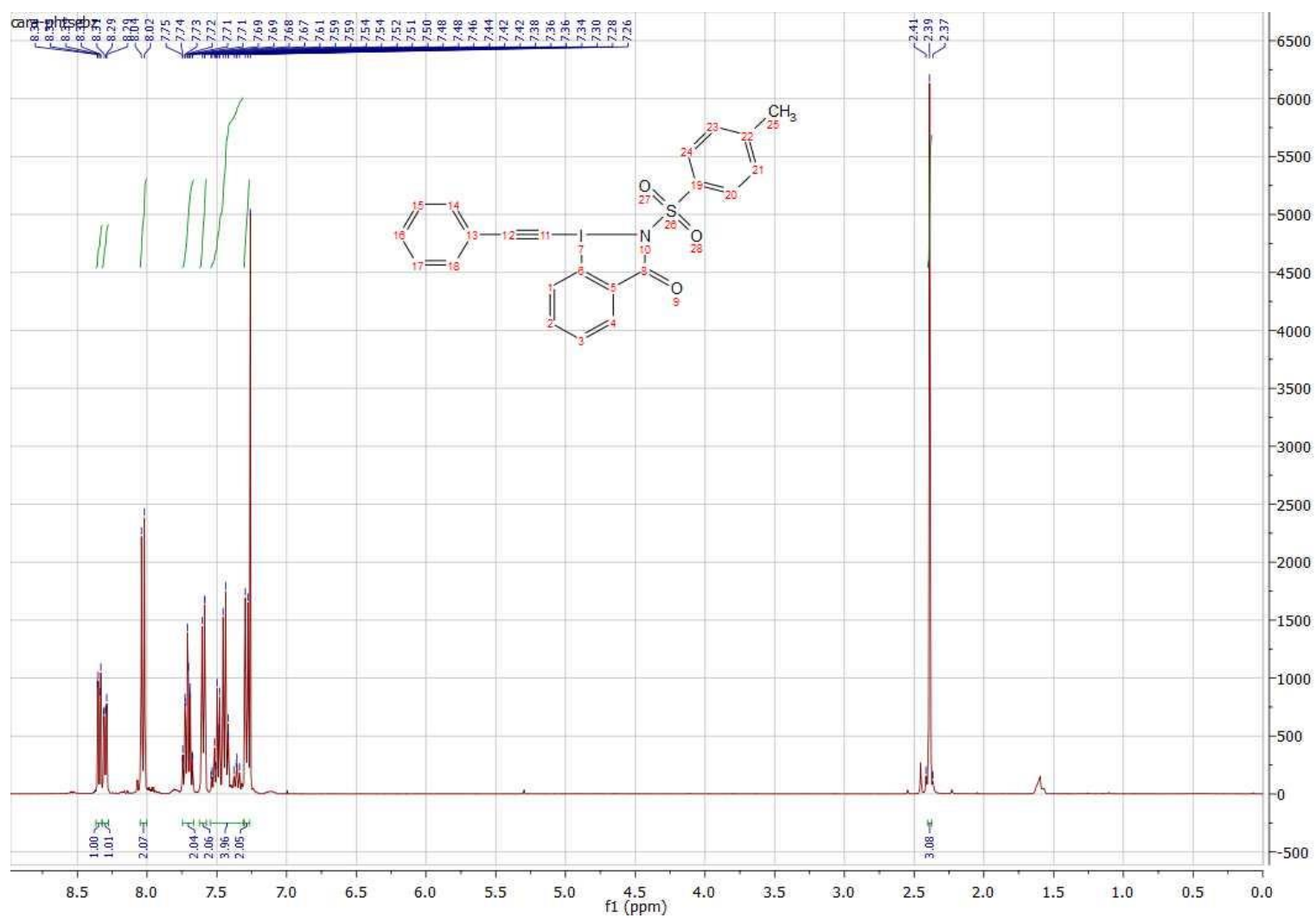

${ }^{13} \mathbf{C}-\mathbf{N M R}\left(100 \mathrm{MHz}, \mathrm{CDCl}_{3}\right)$ of compound $\mathbf{2 b}$

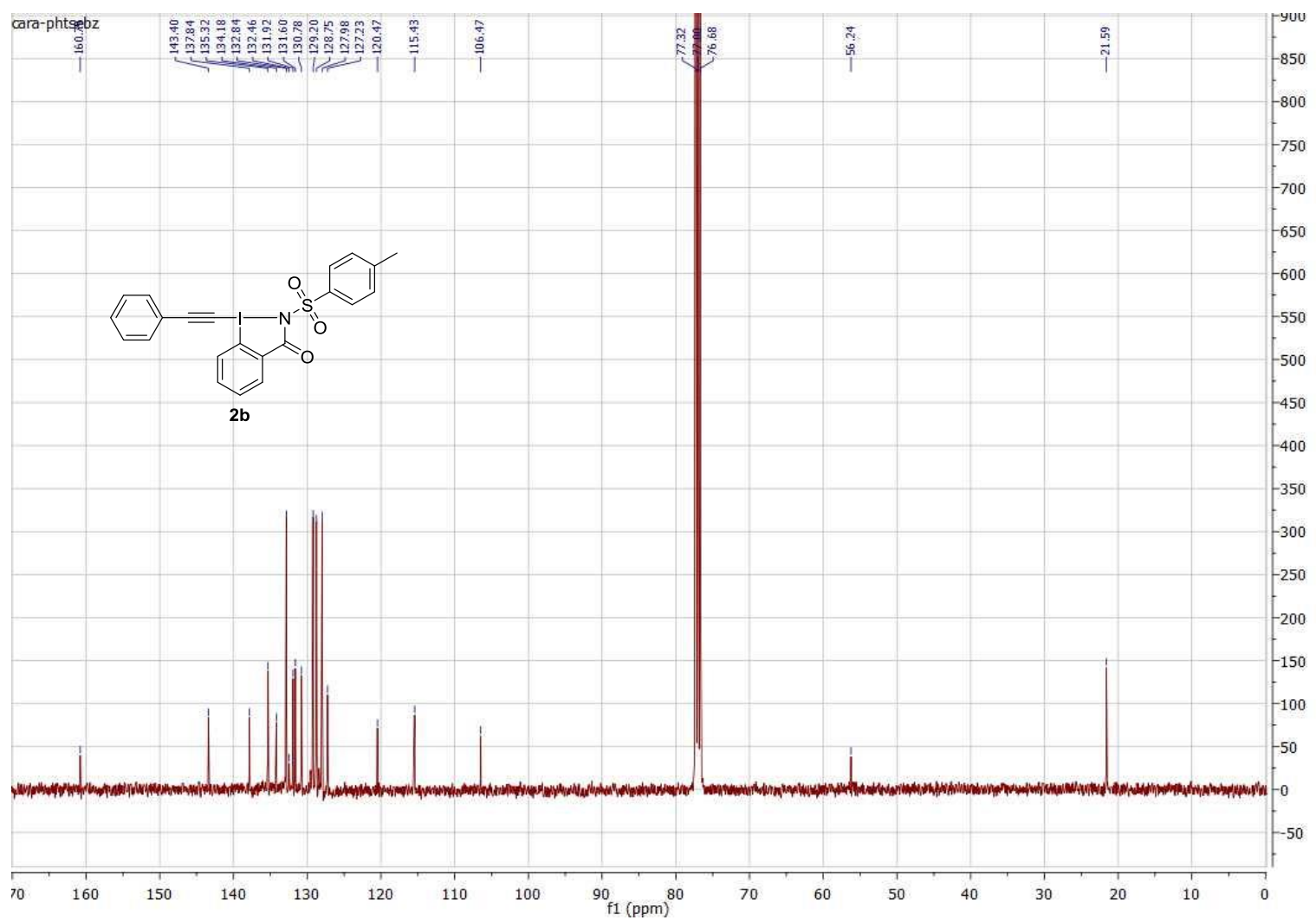


IR of compound $\mathbf{2 b}$

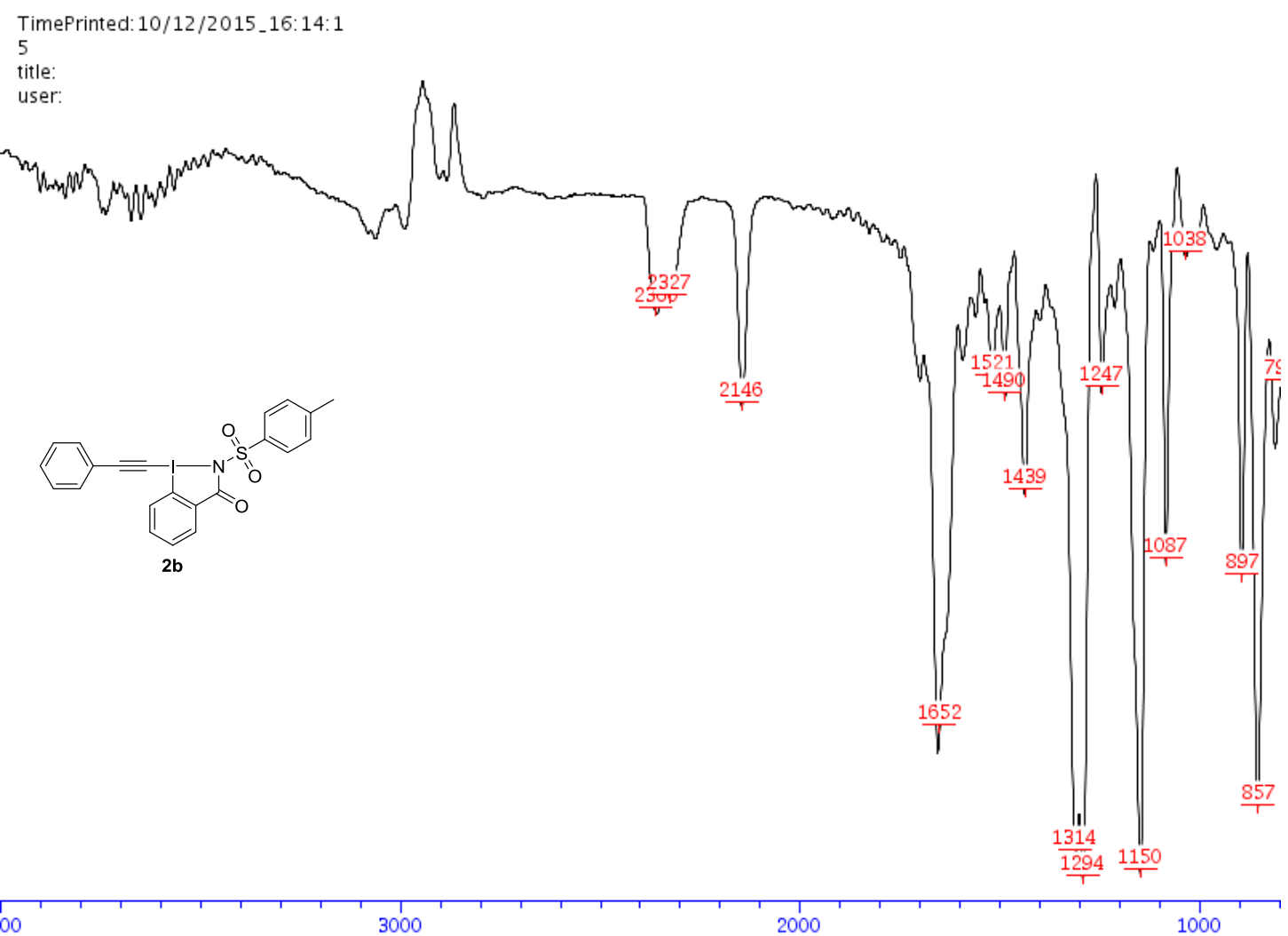


${ }^{1} \mathbf{H}-\mathbf{N M R}\left(400 \mathrm{MHz}, \mathrm{CDCl}_{3}\right)$ of compound 2c

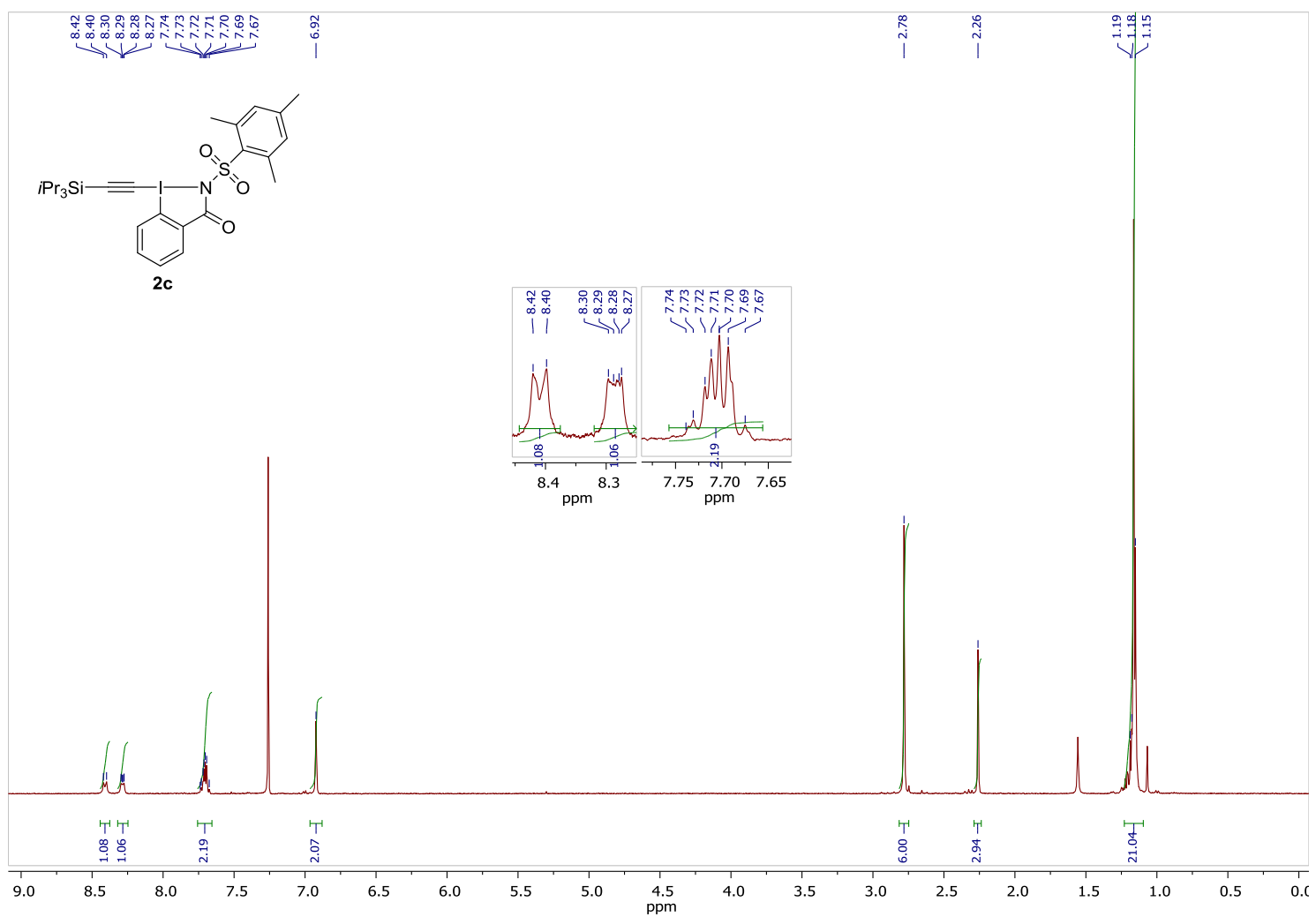

${ }^{13} \mathbf{C}$-NMR (100 MHz, $\left.\mathrm{CDCl}_{3}\right)$ of compound 2c

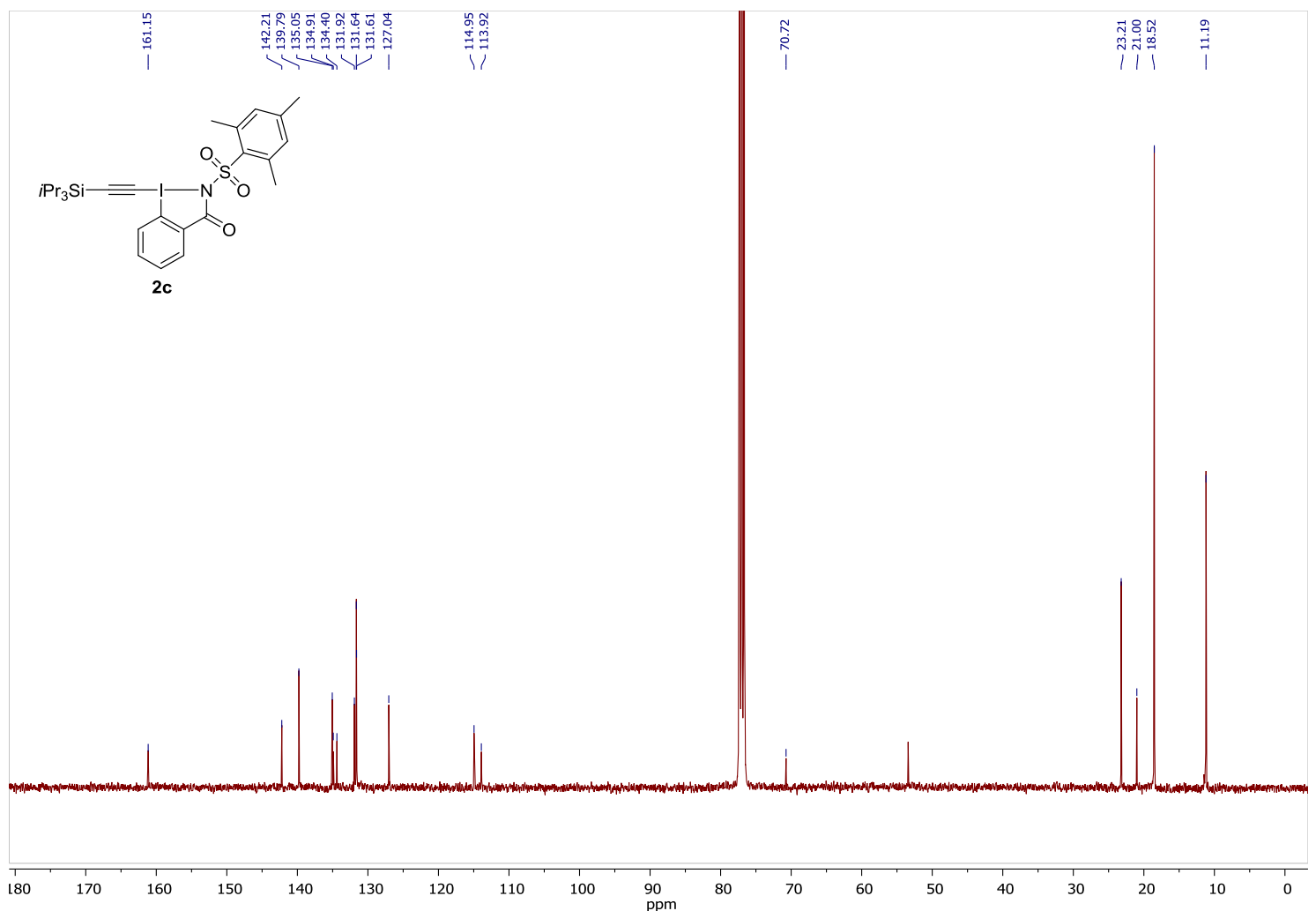


IR of compound $\mathbf{2 c}$

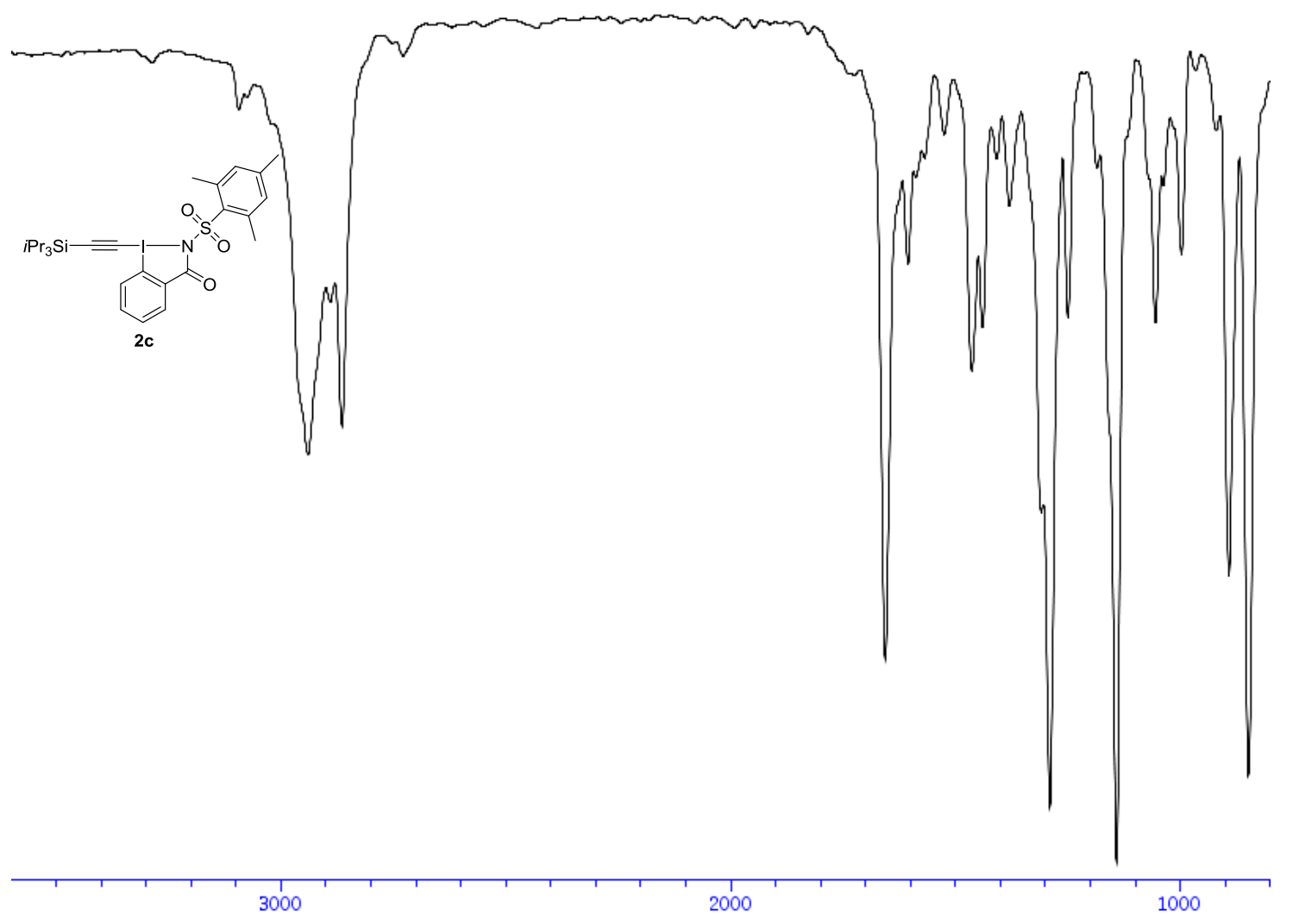


${ }^{\mathbf{1}} \mathbf{H}-\mathbf{N M R}\left(400 \mathrm{MHz}, \mathrm{CDCl}_{3}\right)$ of compound $\mathbf{2 d}$

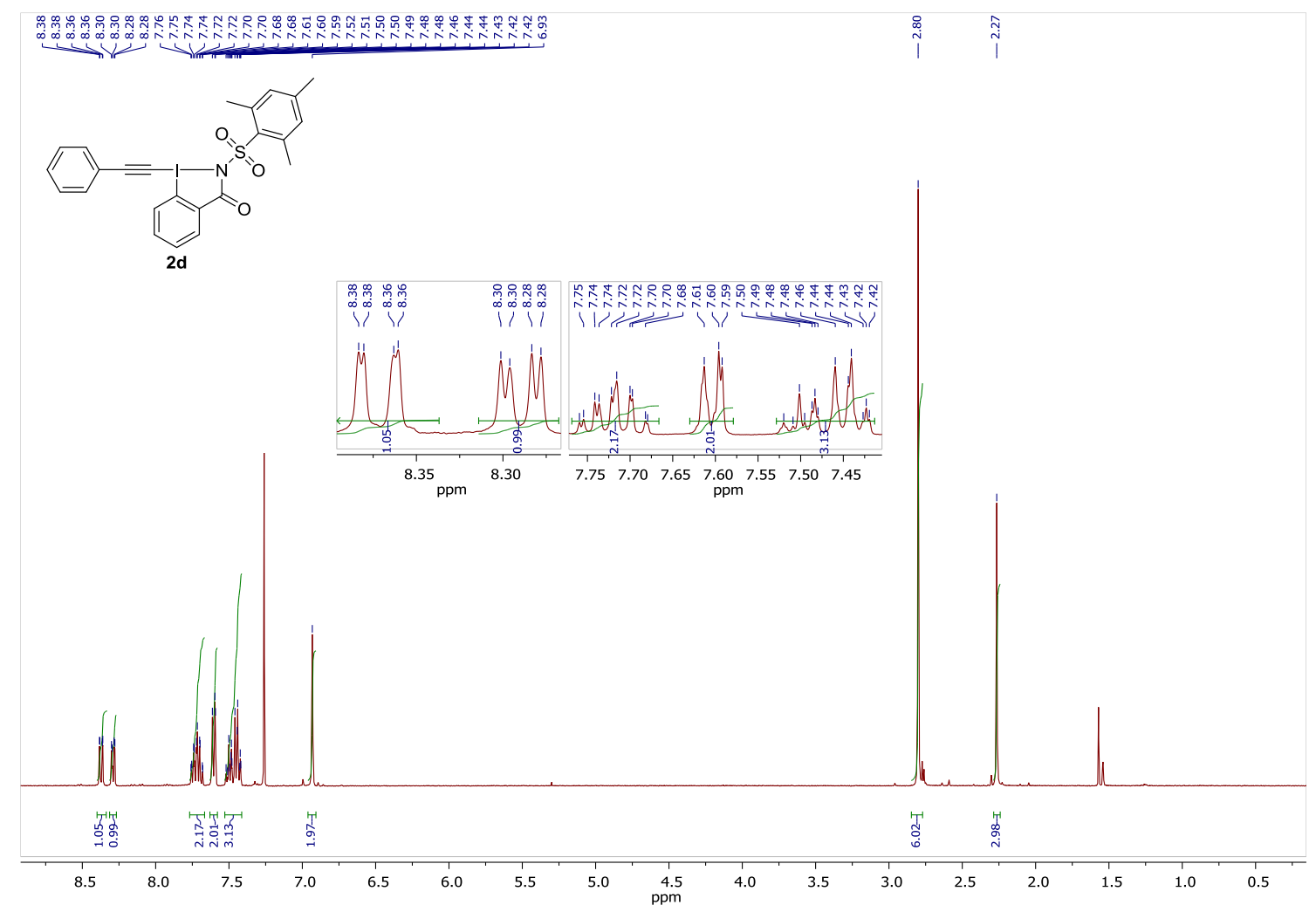

${ }^{13} \mathbf{C}-\mathbf{N M R}\left(100 \mathrm{MHz}, \mathrm{CDCl}_{3}\right)$ of compound 2d

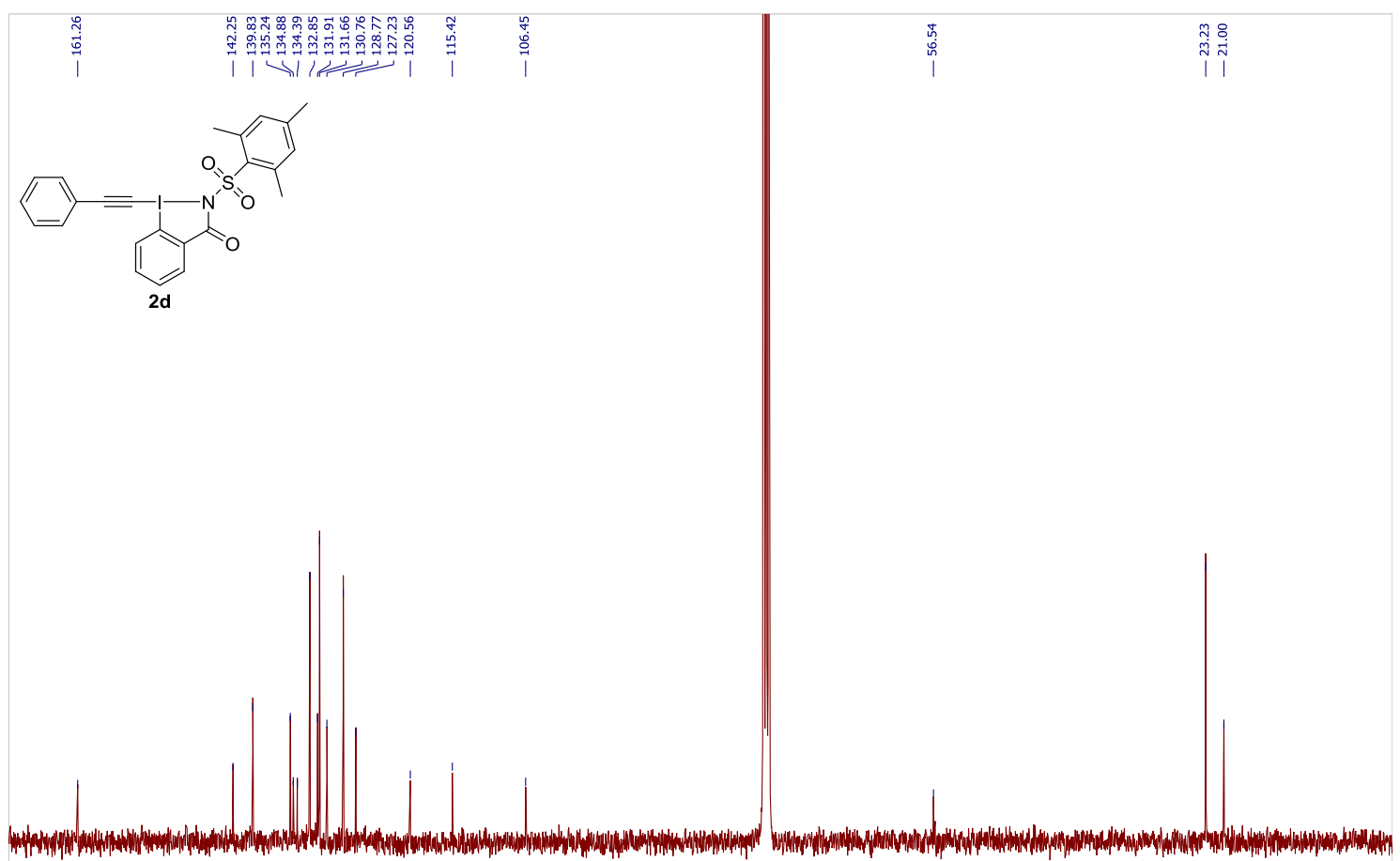

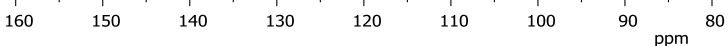


IR of compound $\mathbf{2 d}$

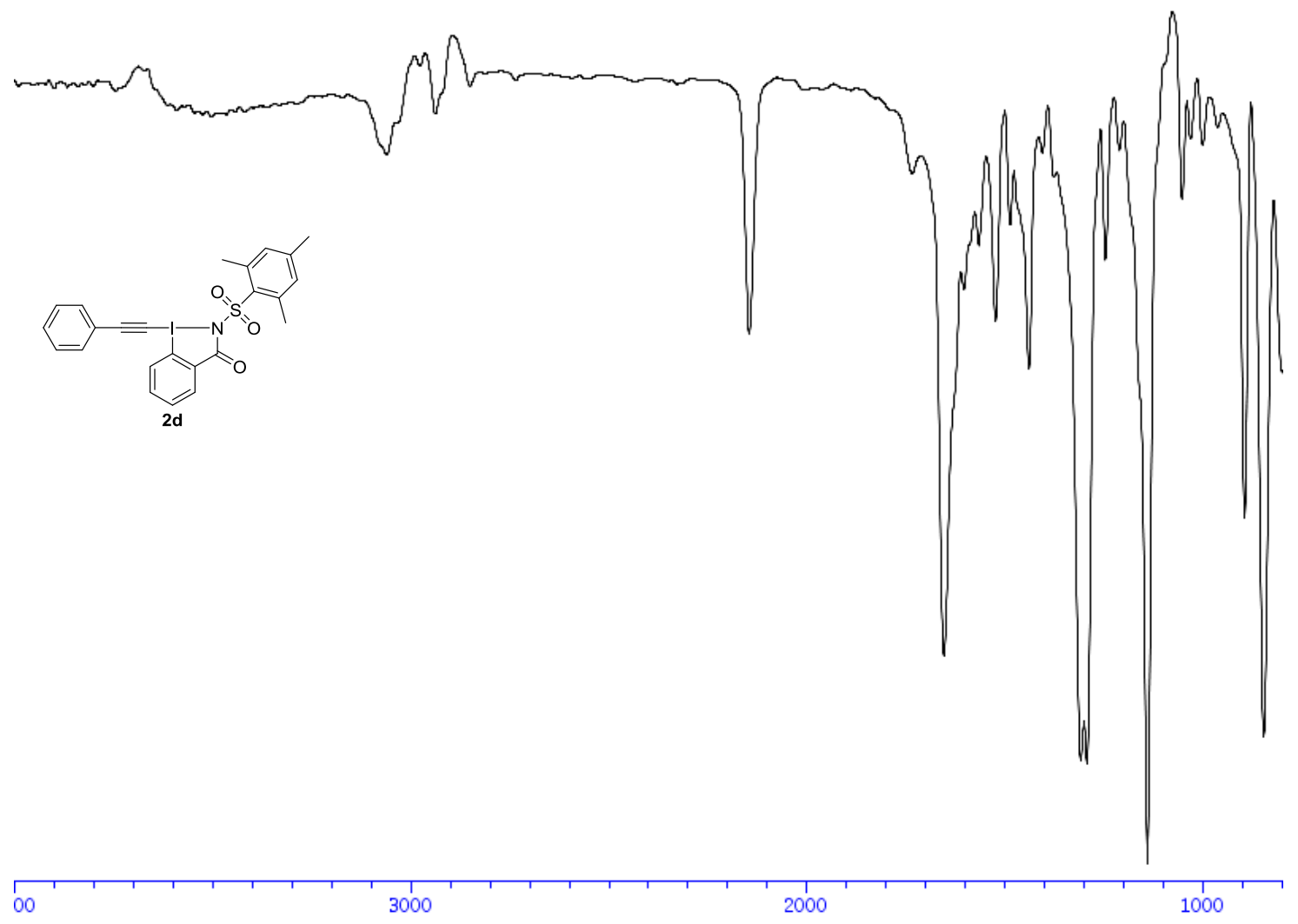


${ }^{1} \mathbf{H}-\mathbf{N M R}\left(400 \mathrm{MHz}, \mathrm{CDCl}_{3}\right)$ of compound $\mathbf{2 e}$

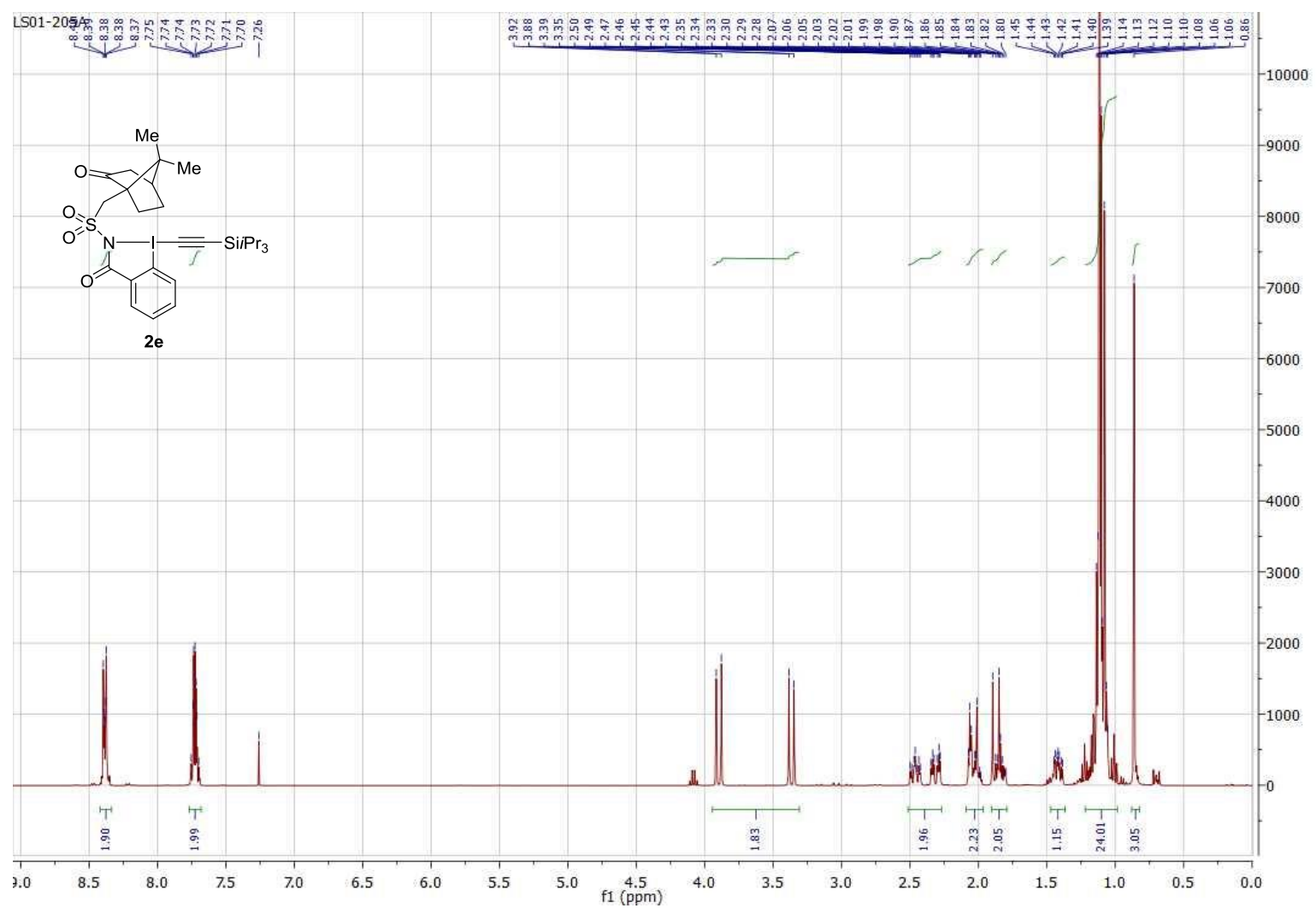

${ }^{13} \mathbf{C}$-NMR $\left(100 \mathrm{MHz}, \mathrm{CDCl}_{3}\right)$ of compound $\mathbf{2 e}$

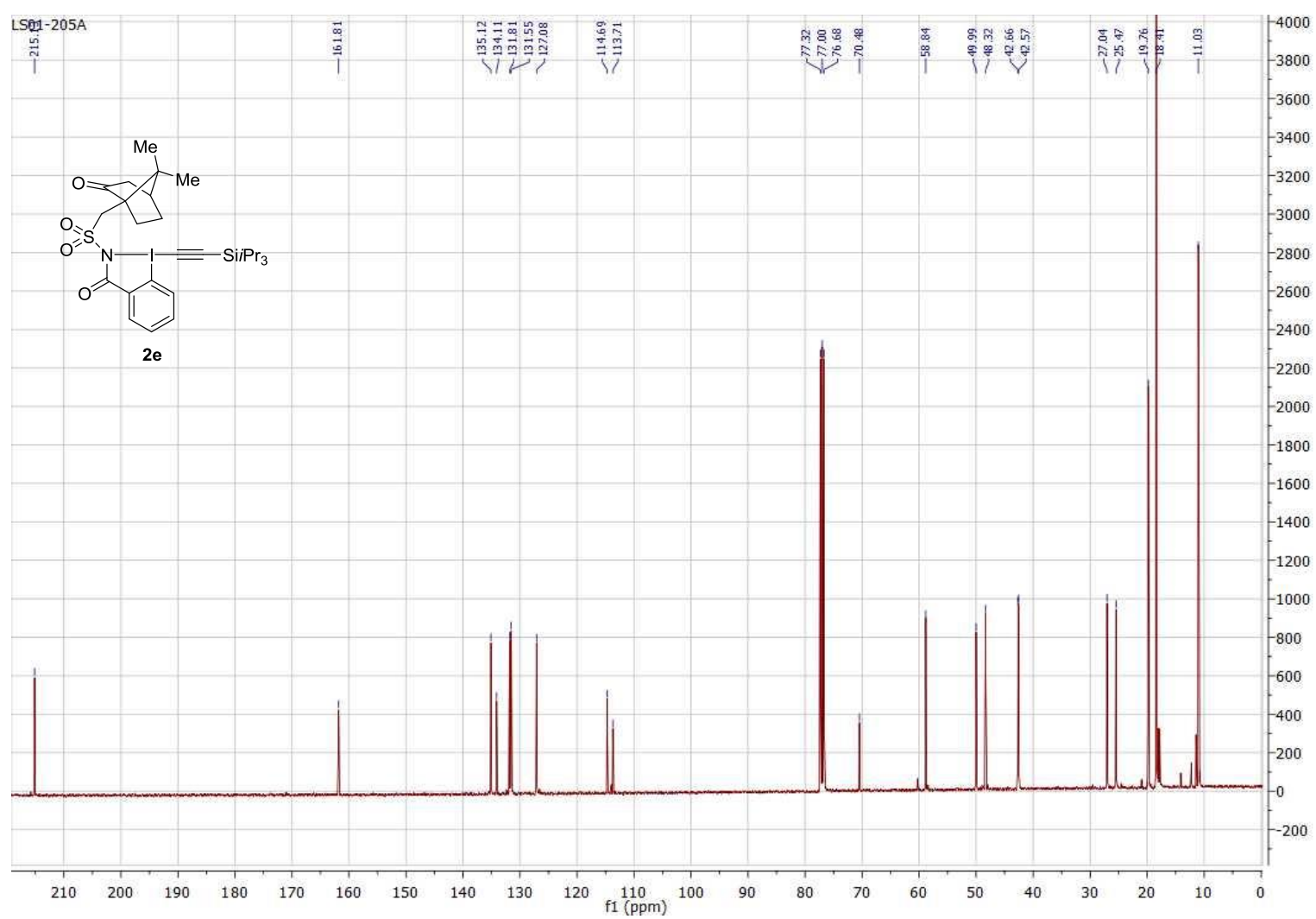


IR of compound $\mathbf{2 e}$

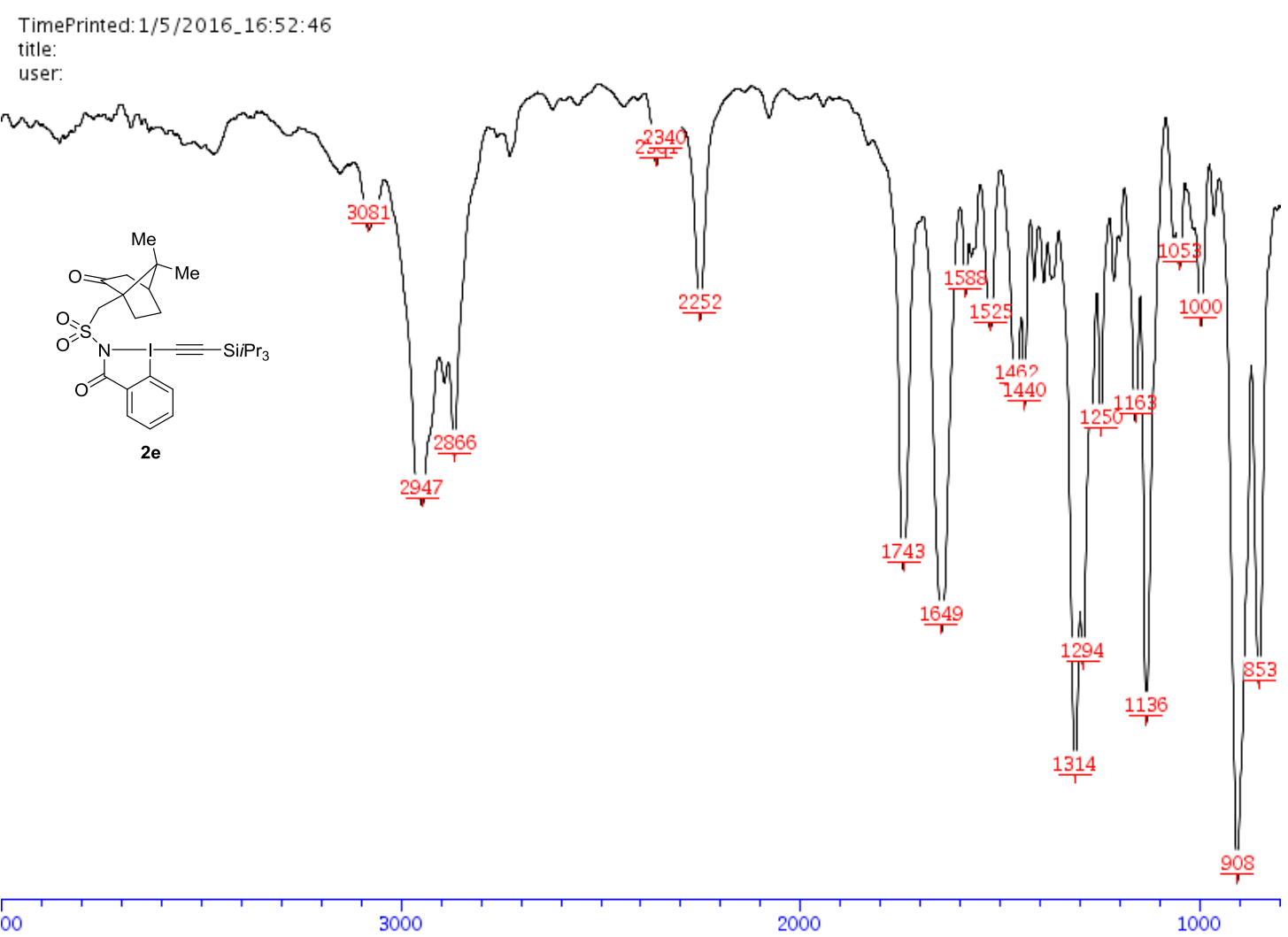


${ }^{1} \mathbf{H}-\mathbf{N M R}\left(400 \mathrm{MHz}, \mathrm{CDCl}_{3}\right)$ of compound $\mathbf{2 f}$

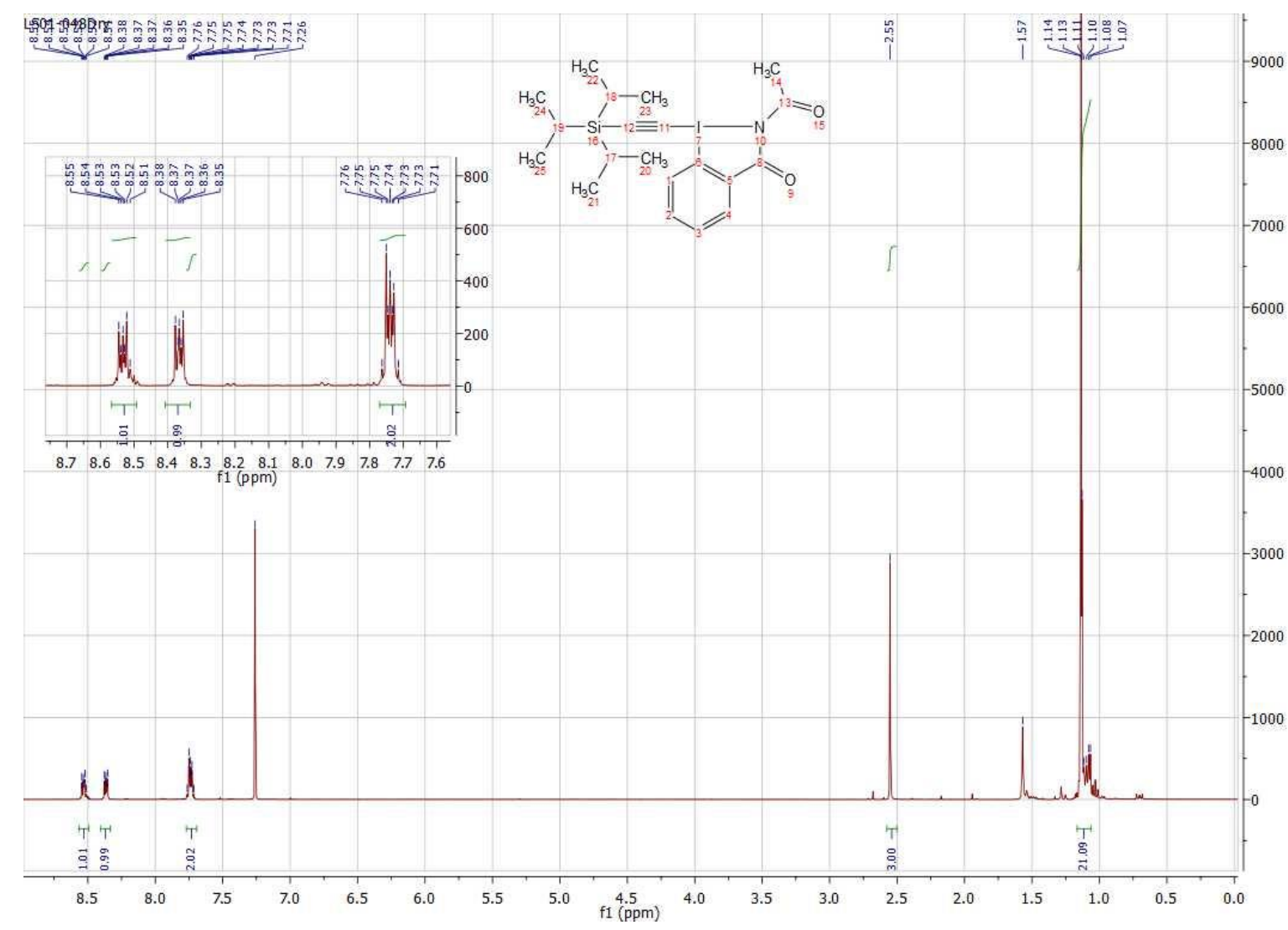

${ }^{13} \mathbf{C}$-NMR $\left(100 \mathrm{MHz}, \mathrm{CDCl}_{3}\right)$ of compound $\mathbf{2 f}$

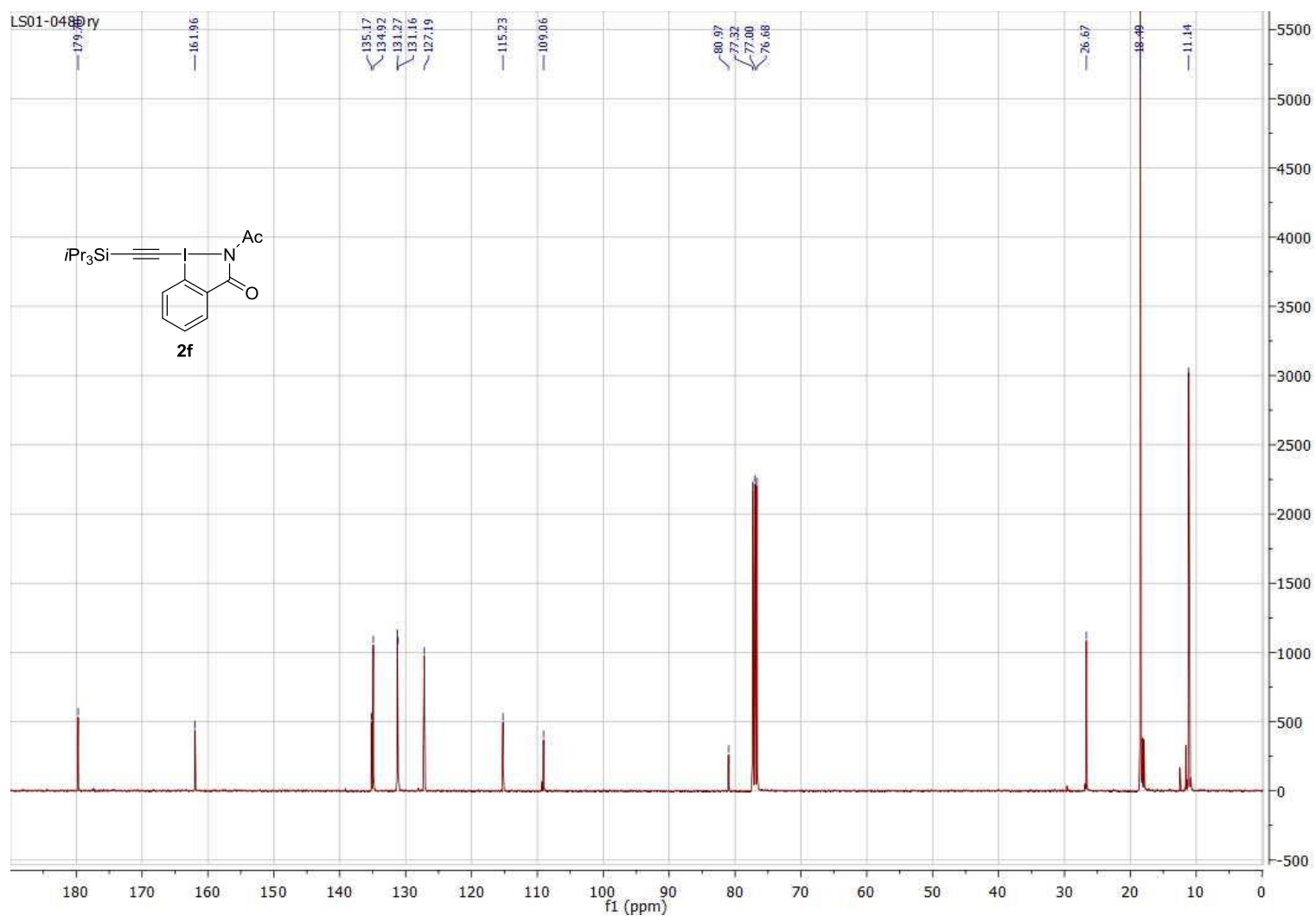


IR of compound $\mathbf{2 f}$

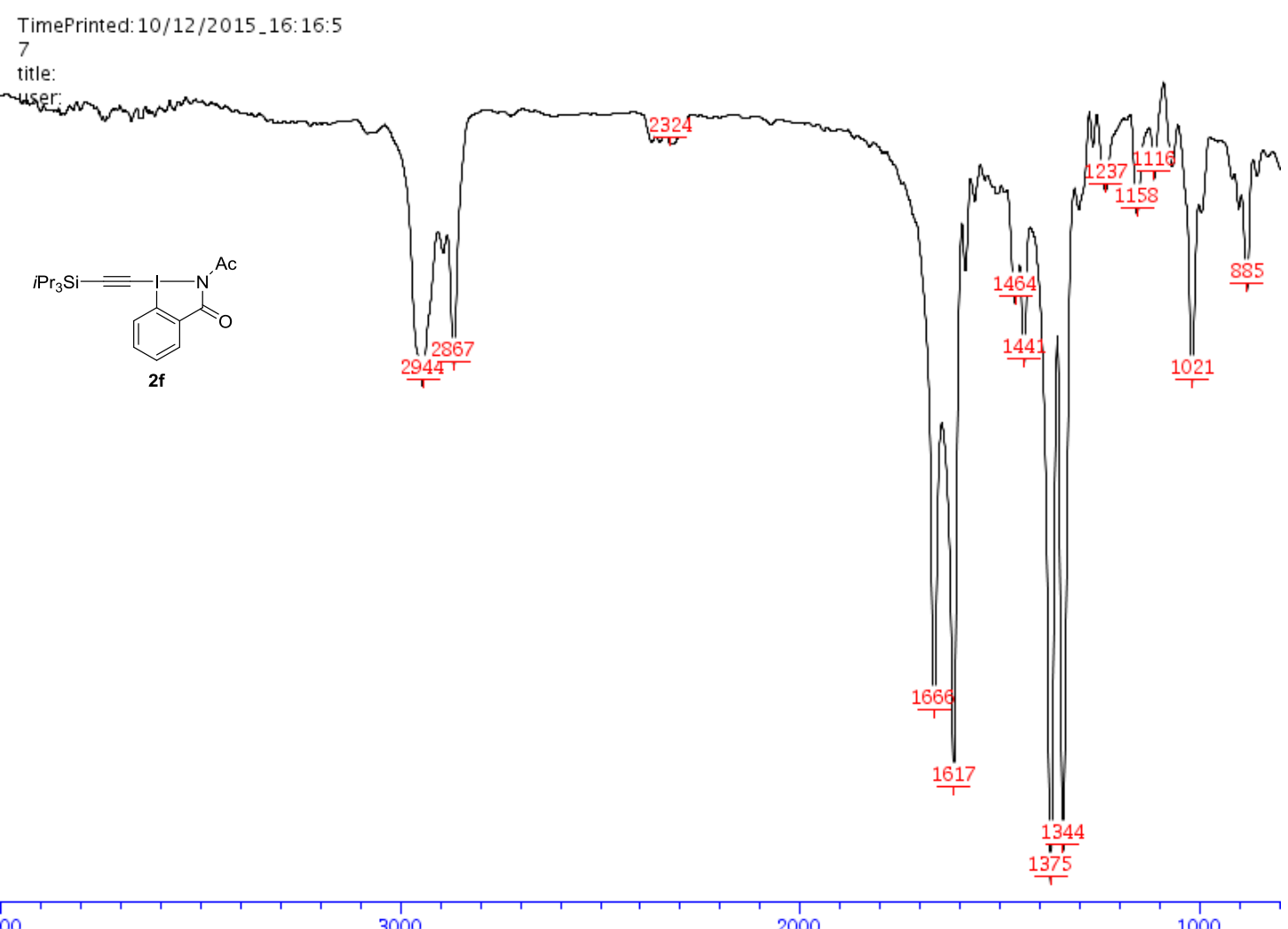


${ }^{\mathbf{1}} \mathbf{H}$-NMR (400 MHz, $\mathrm{CDCl}_{3}$ ) of compound $\mathbf{2 g}$

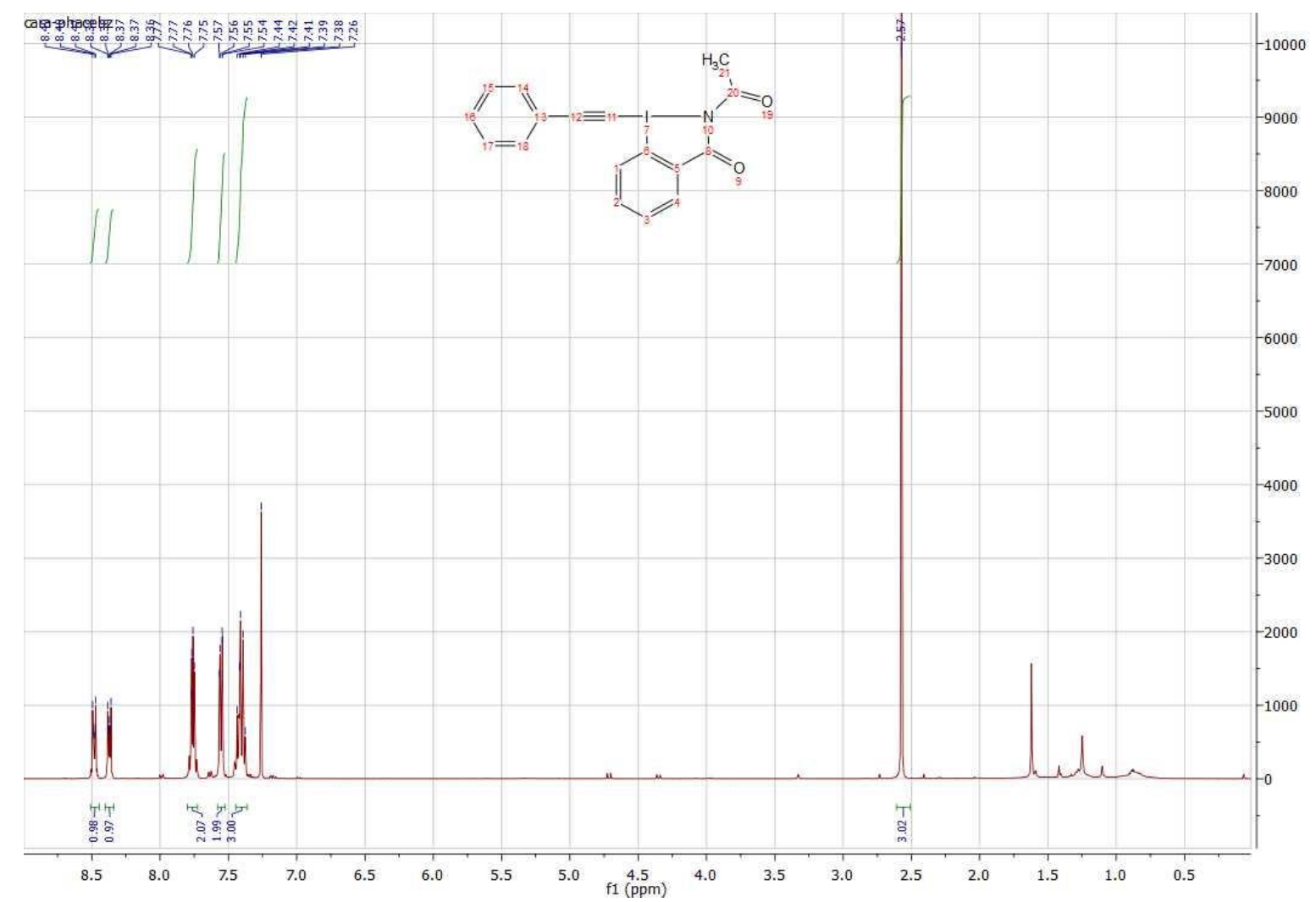

${ }^{13} \mathbf{C}$-NMR $\left(100 \mathrm{MHz}, \mathrm{CDCl}_{3}\right)$ of compound $\mathbf{2 g}$

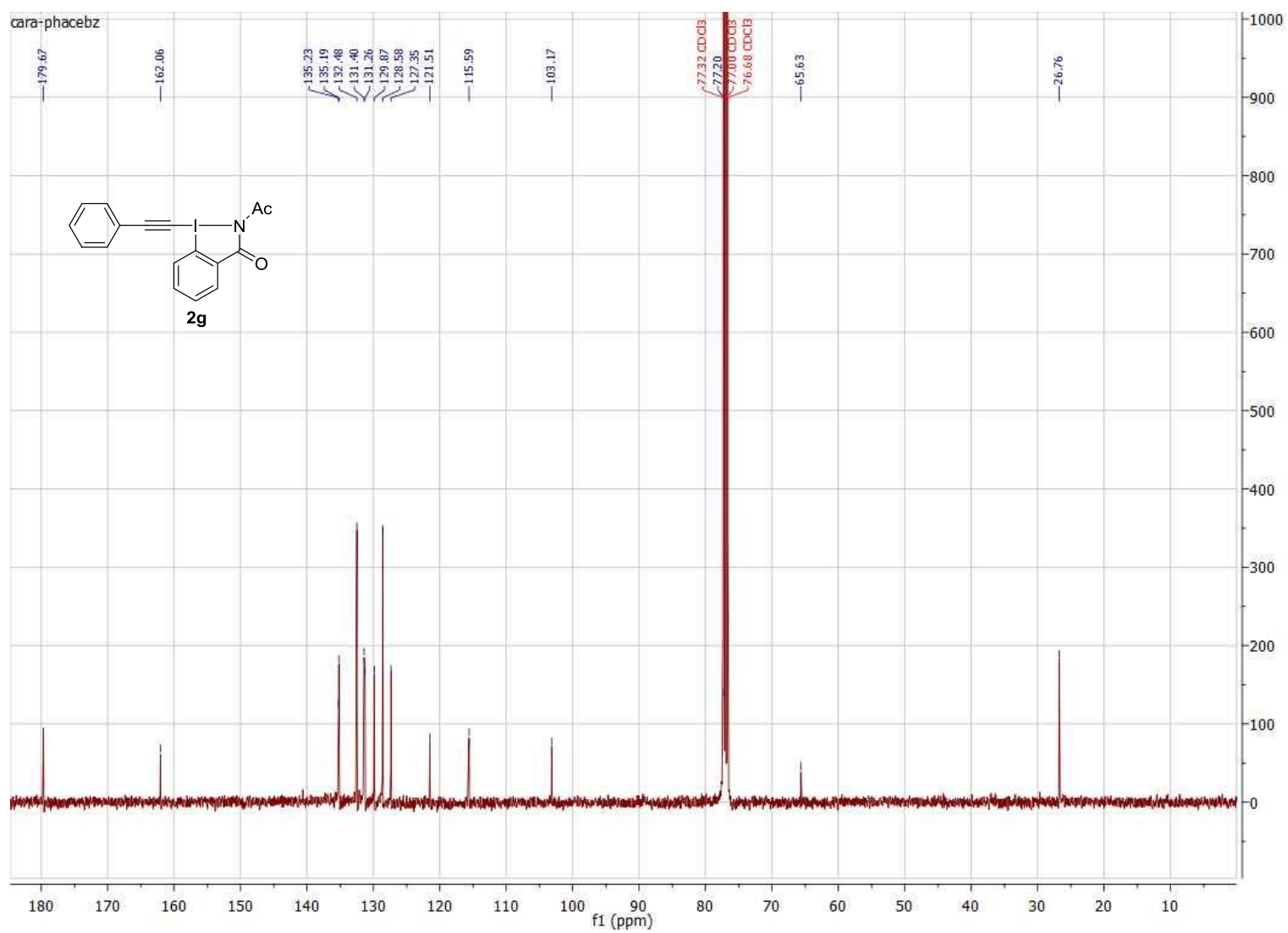




\section{IR of compound $\mathbf{2 g}$}

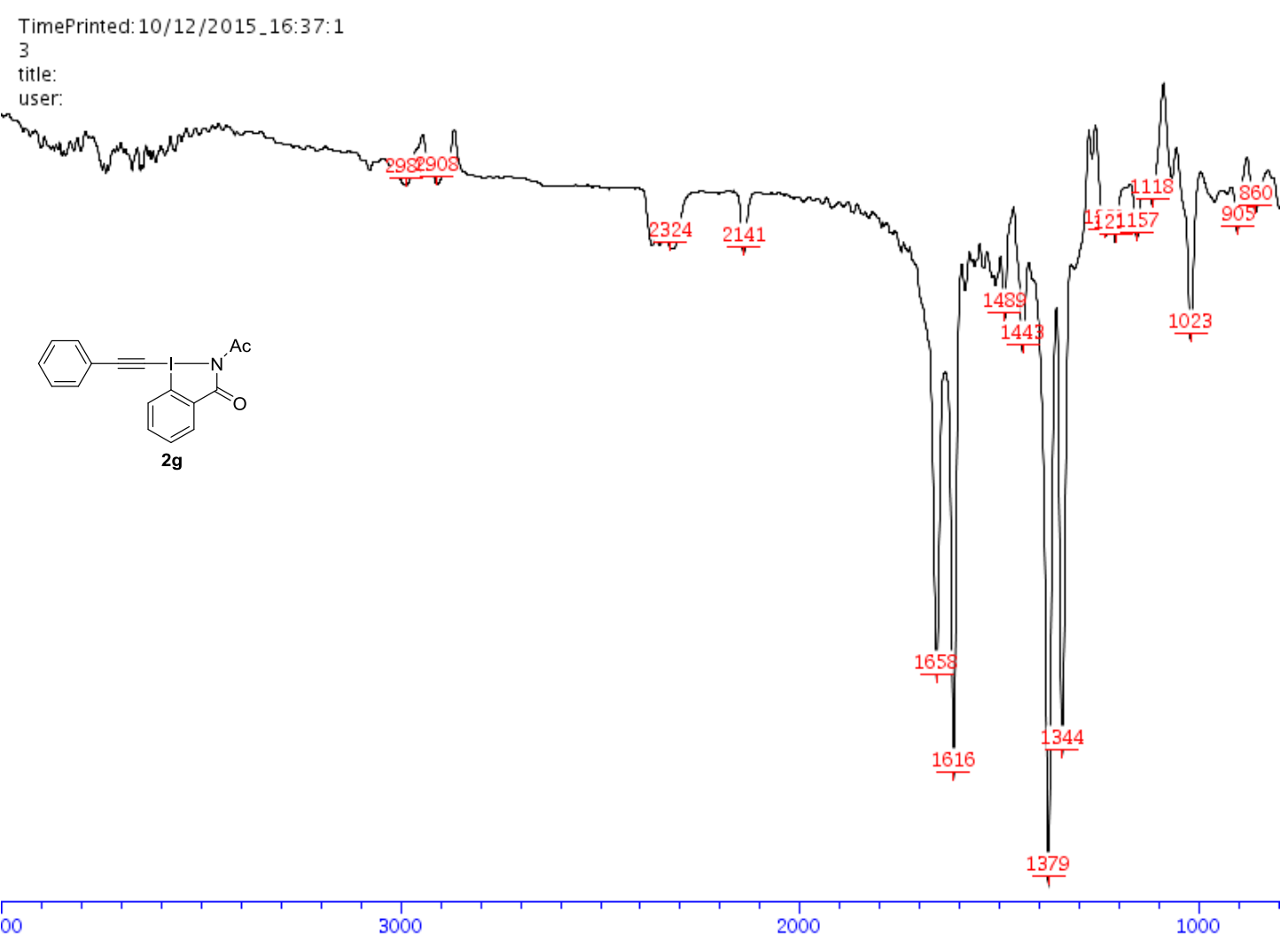


${ }^{\mathbf{1}} \mathbf{H}-\mathbf{N M R}\left(400 \mathrm{MHz}, \mathrm{CDCl}_{3}\right)$ of compound $\mathbf{2 h}$

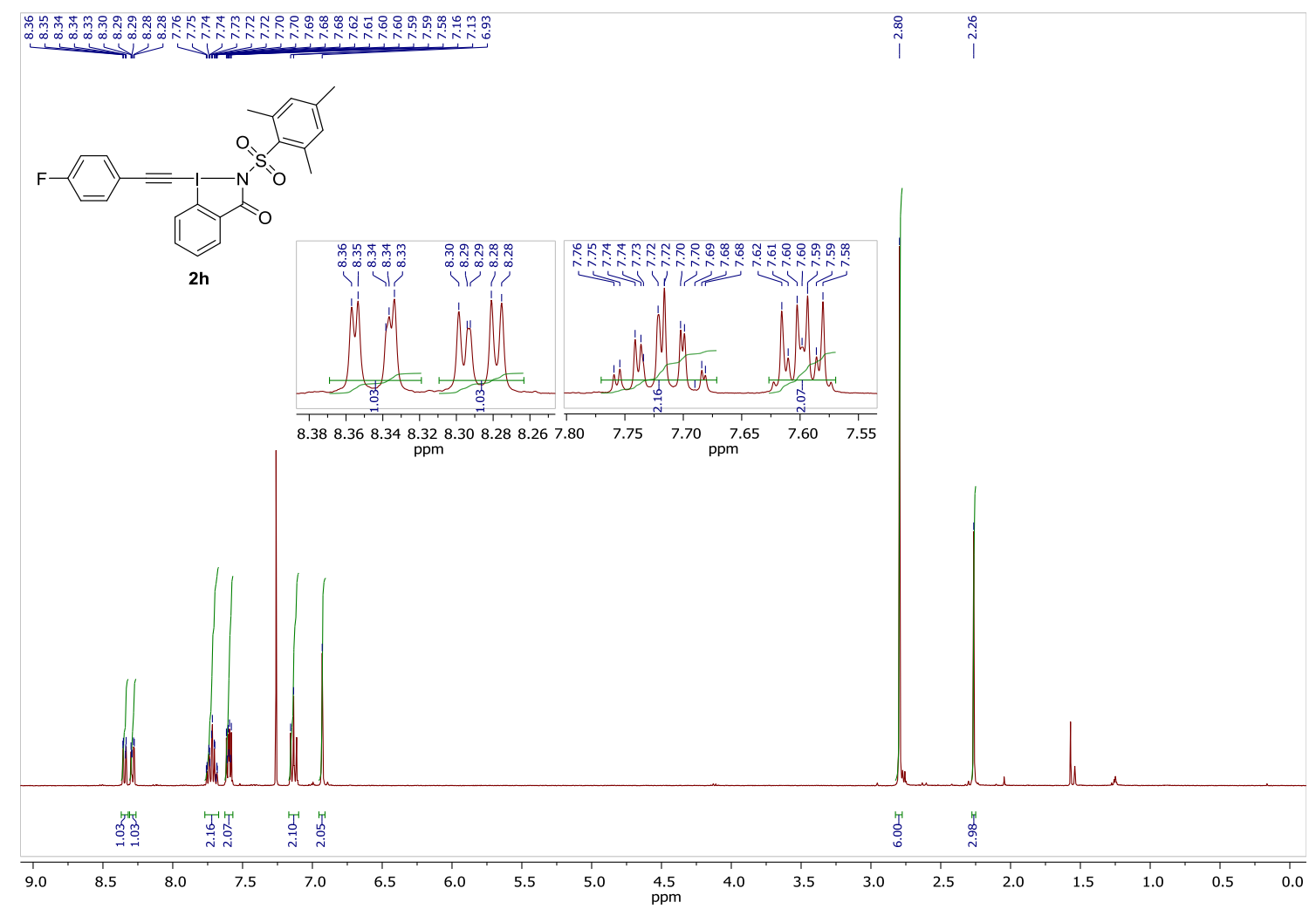

${ }^{13} \mathbf{C}-\mathbf{N M R}\left(100 \mathrm{MHz}, \mathrm{CDCl}_{3}\right)$ of compound $\mathbf{2 h}$

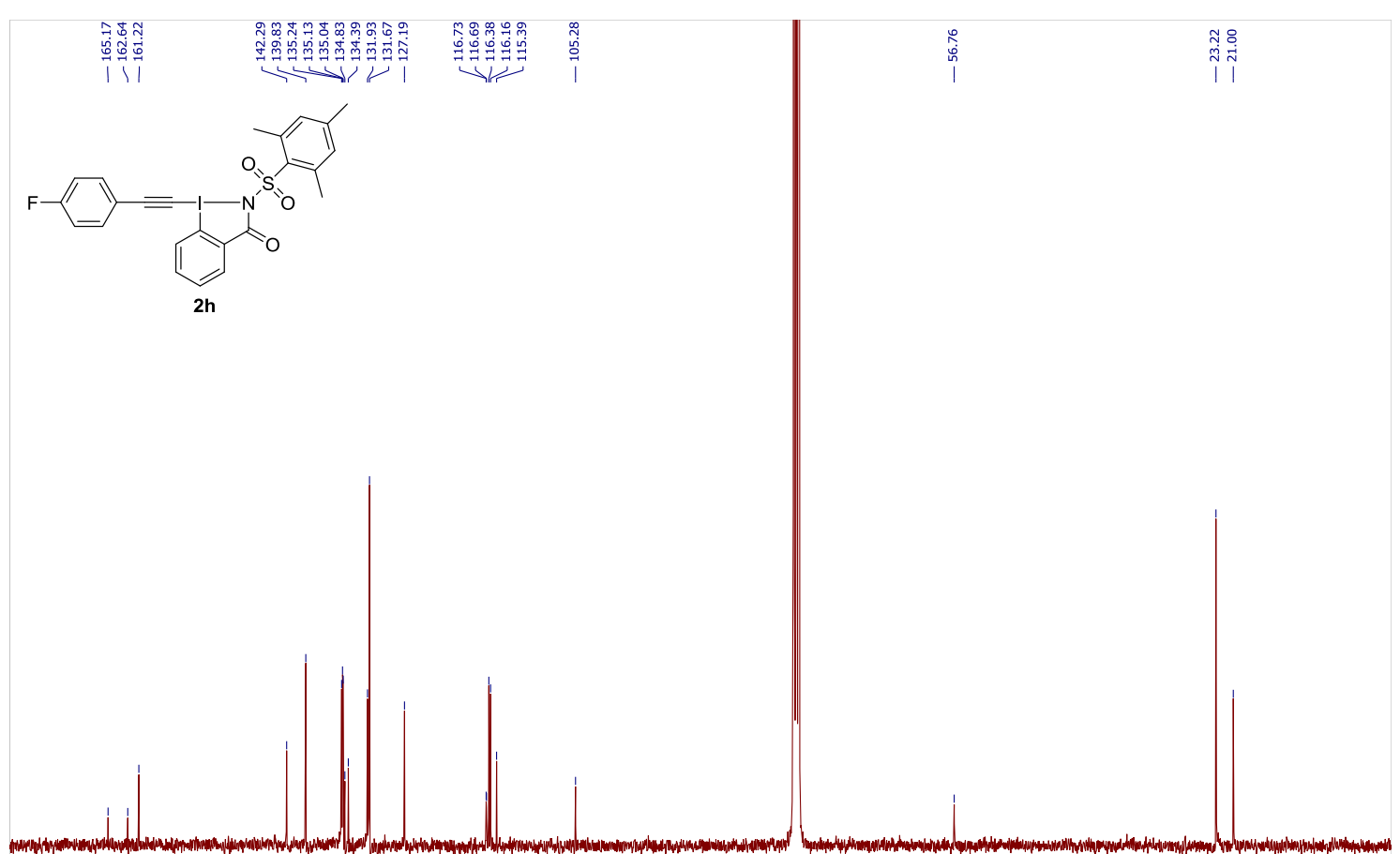

$\begin{array}{lllllllll}170 & 160 & 150 & 140 & 130 & 120 & 110 & 100 & 90\end{array}$ 
IR of compound $\mathbf{2 h}$

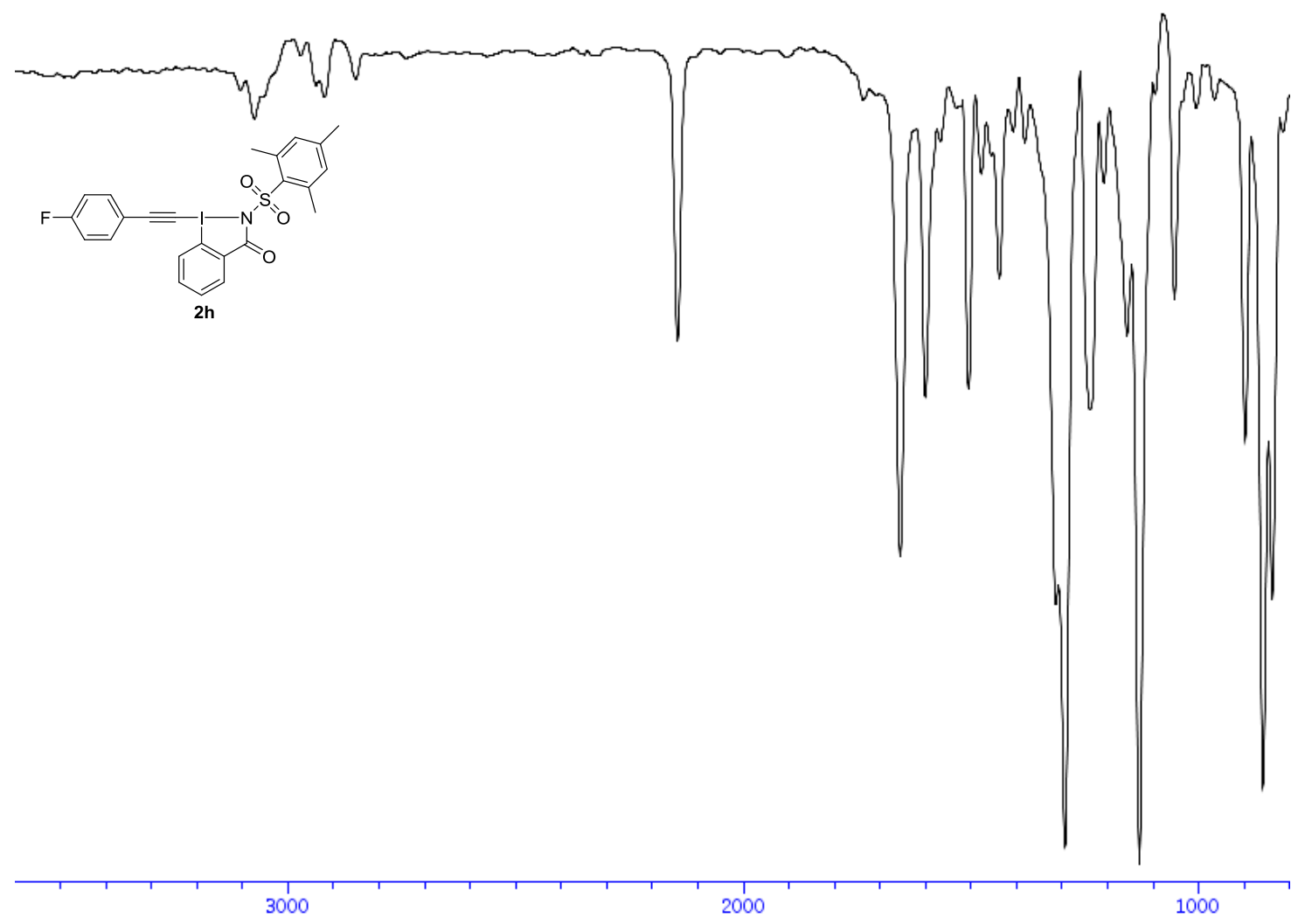


${ }^{1} \mathbf{H}-\mathbf{N M R}\left(400 \mathrm{MHz}, \mathrm{CDCl}_{3}\right)$ of compound $\mathbf{2 i}$

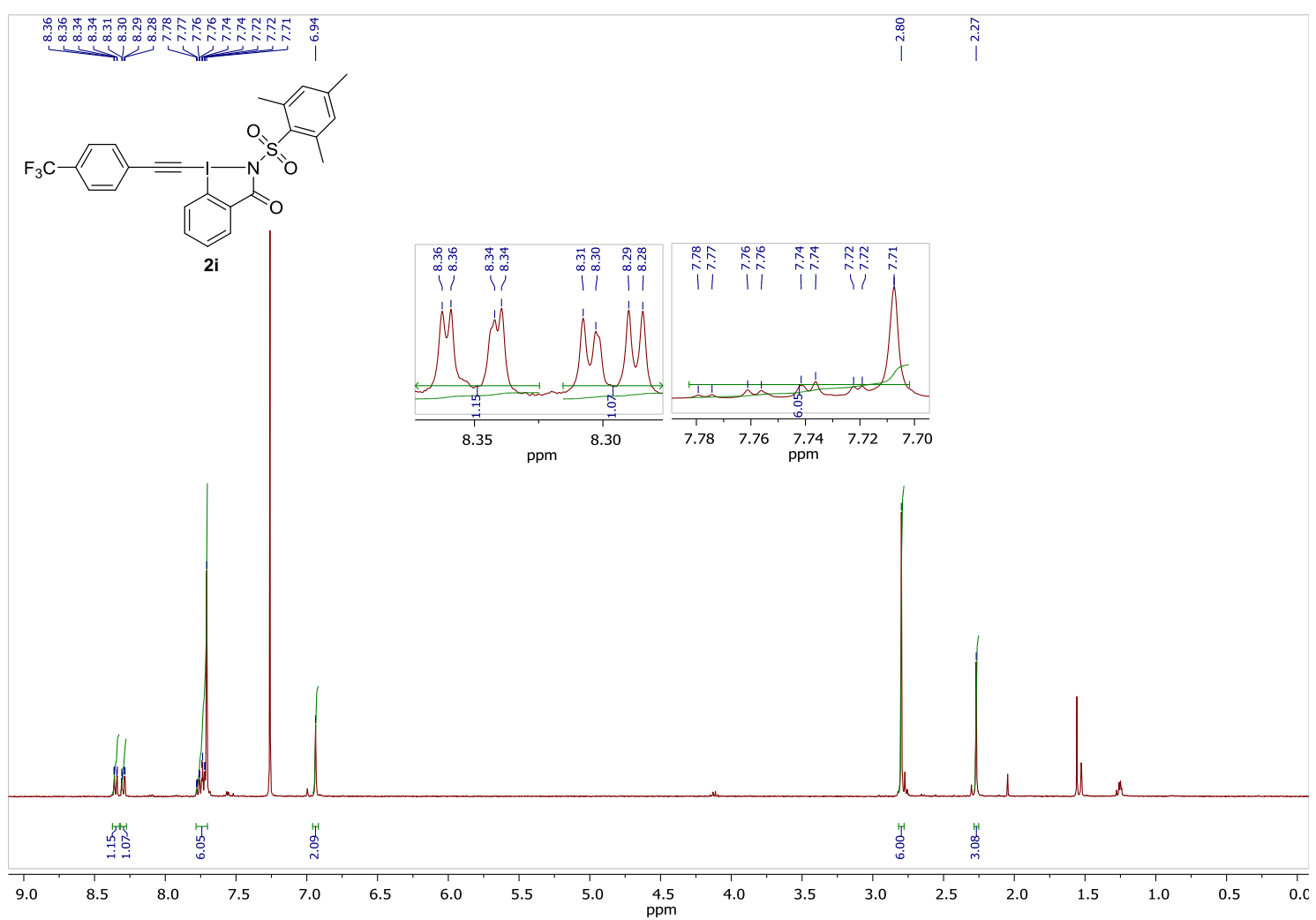

${ }^{13} \mathbf{C}-\mathbf{N M R}\left(100 \mathrm{MHz}, \mathrm{CDCl}_{3}\right)$ of compound $\mathbf{2 i}$
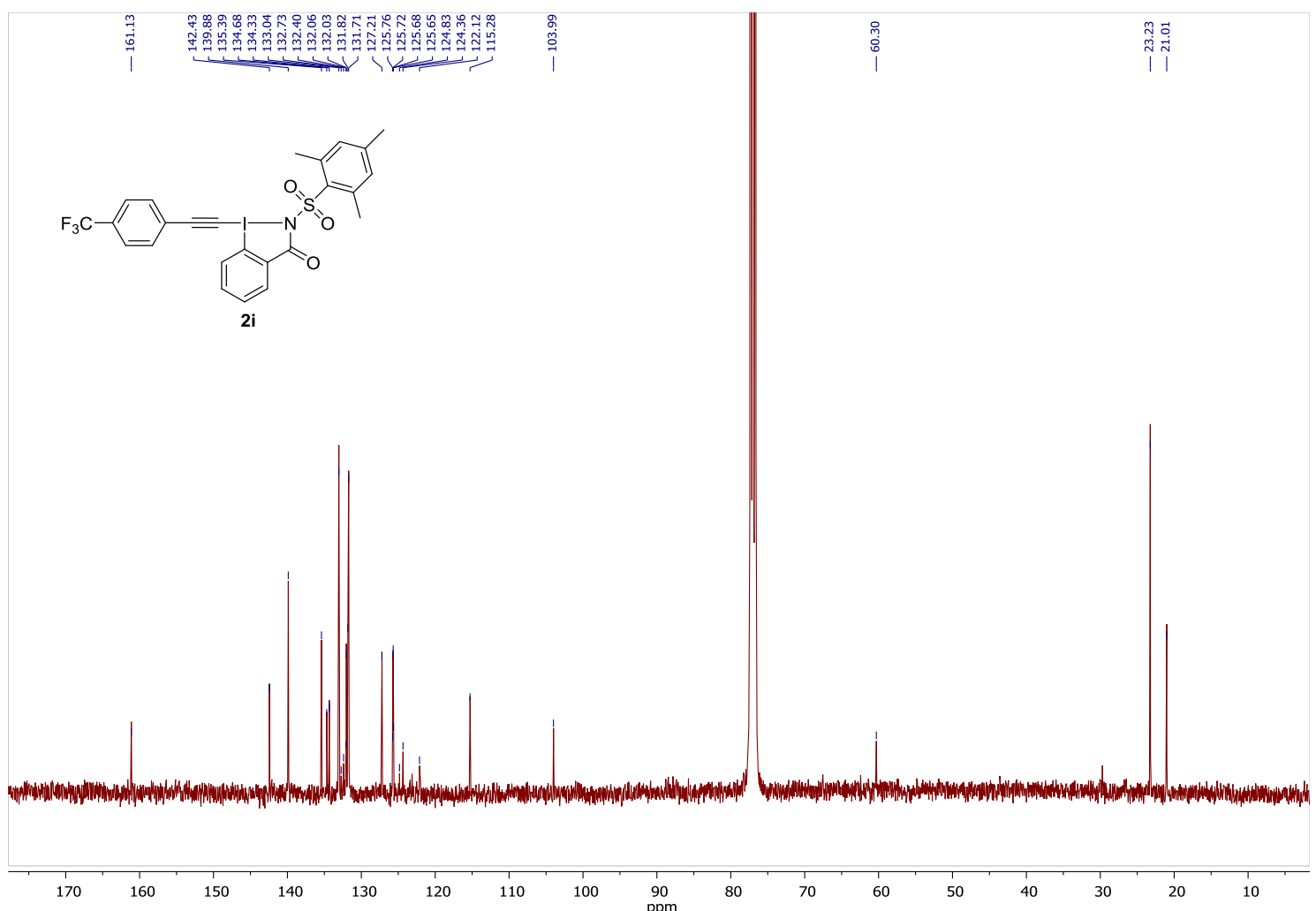
IR of compound $\mathbf{2} \mathbf{i}$

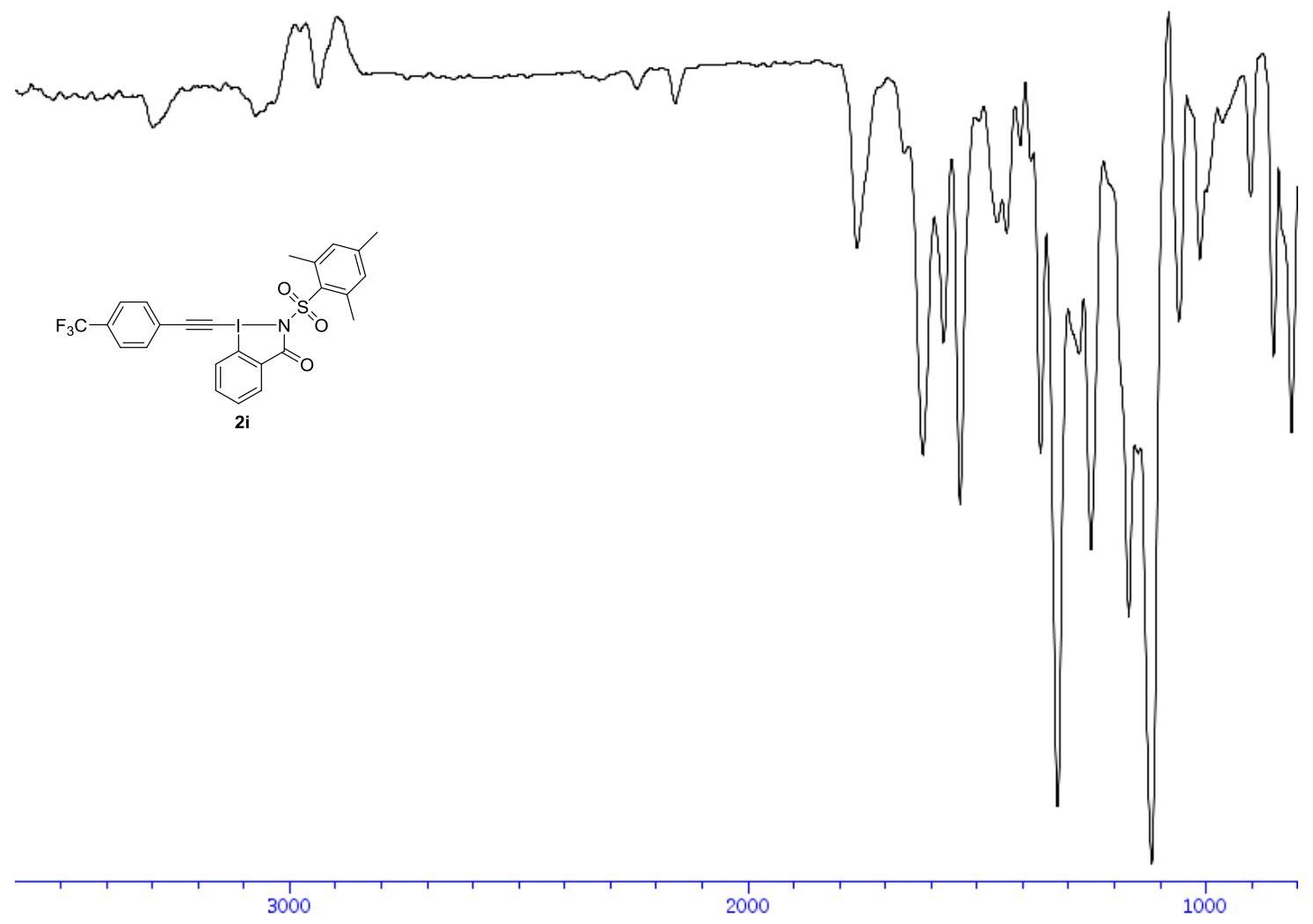


${ }^{\mathbf{1}} \mathbf{H}-\mathbf{N M R}\left(400 \mathrm{MHz}, \mathrm{CDCl}_{3}\right)$ of compound $\mathbf{2 j}$

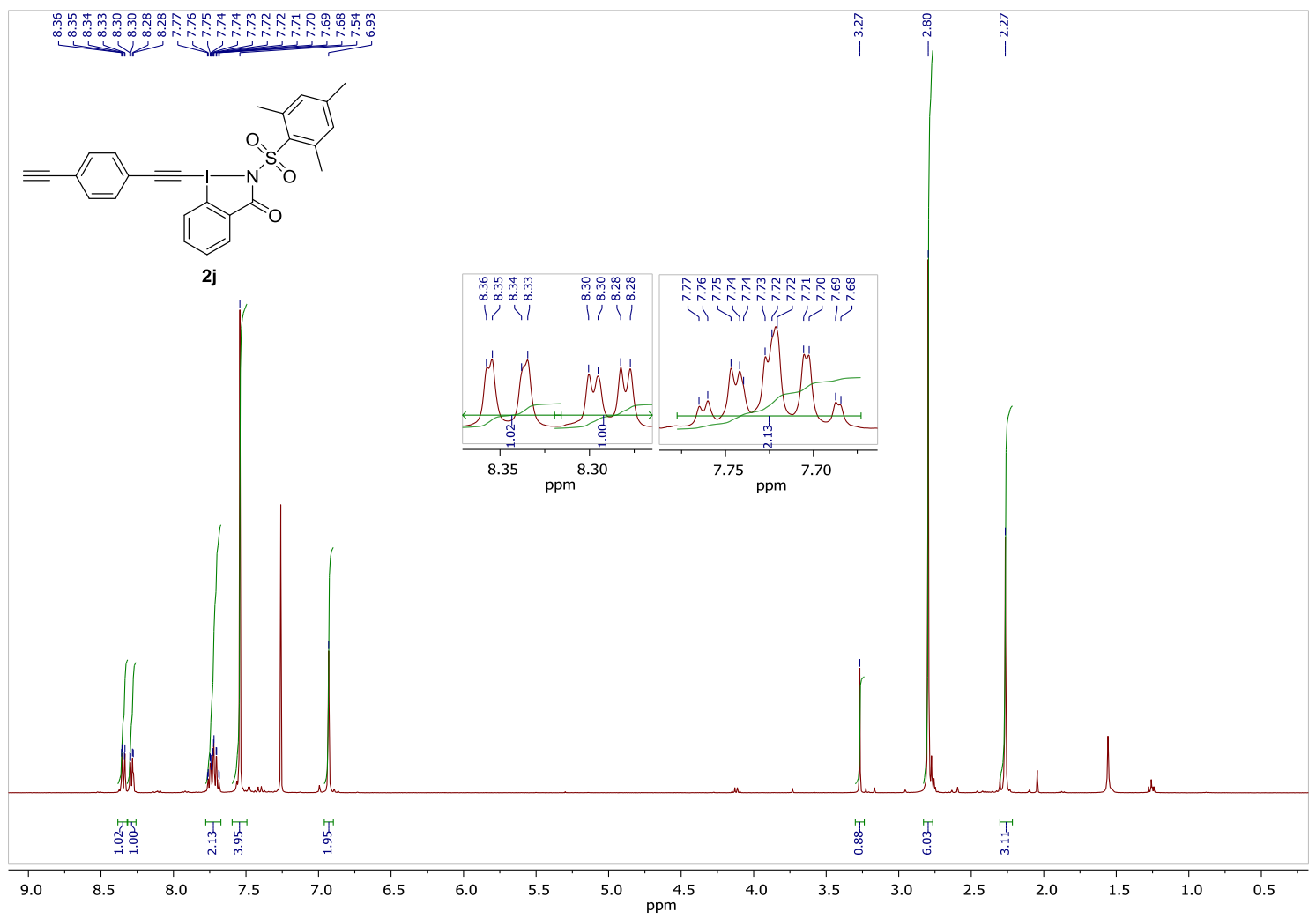

${ }^{13} \mathbf{C}-\mathbf{N M R}\left(100 \mathrm{MHz}, \mathrm{CDCl}_{3}\right)$ of compound $\mathbf{2} \mathbf{j}$

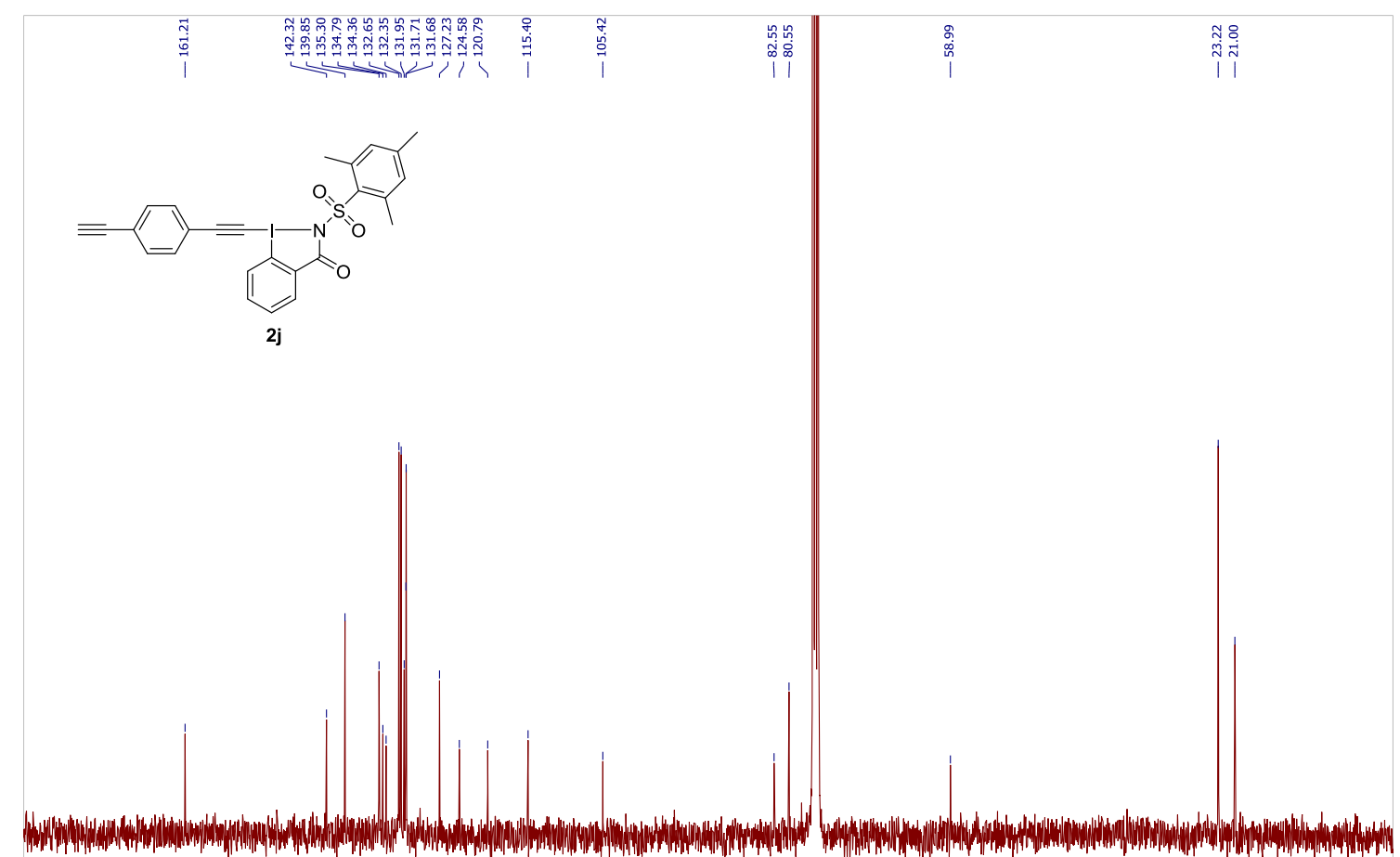

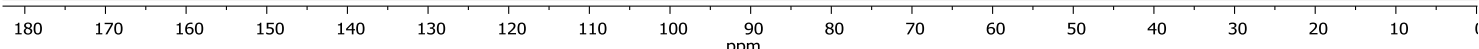


IR of compound $\mathbf{2} \mathbf{j}$

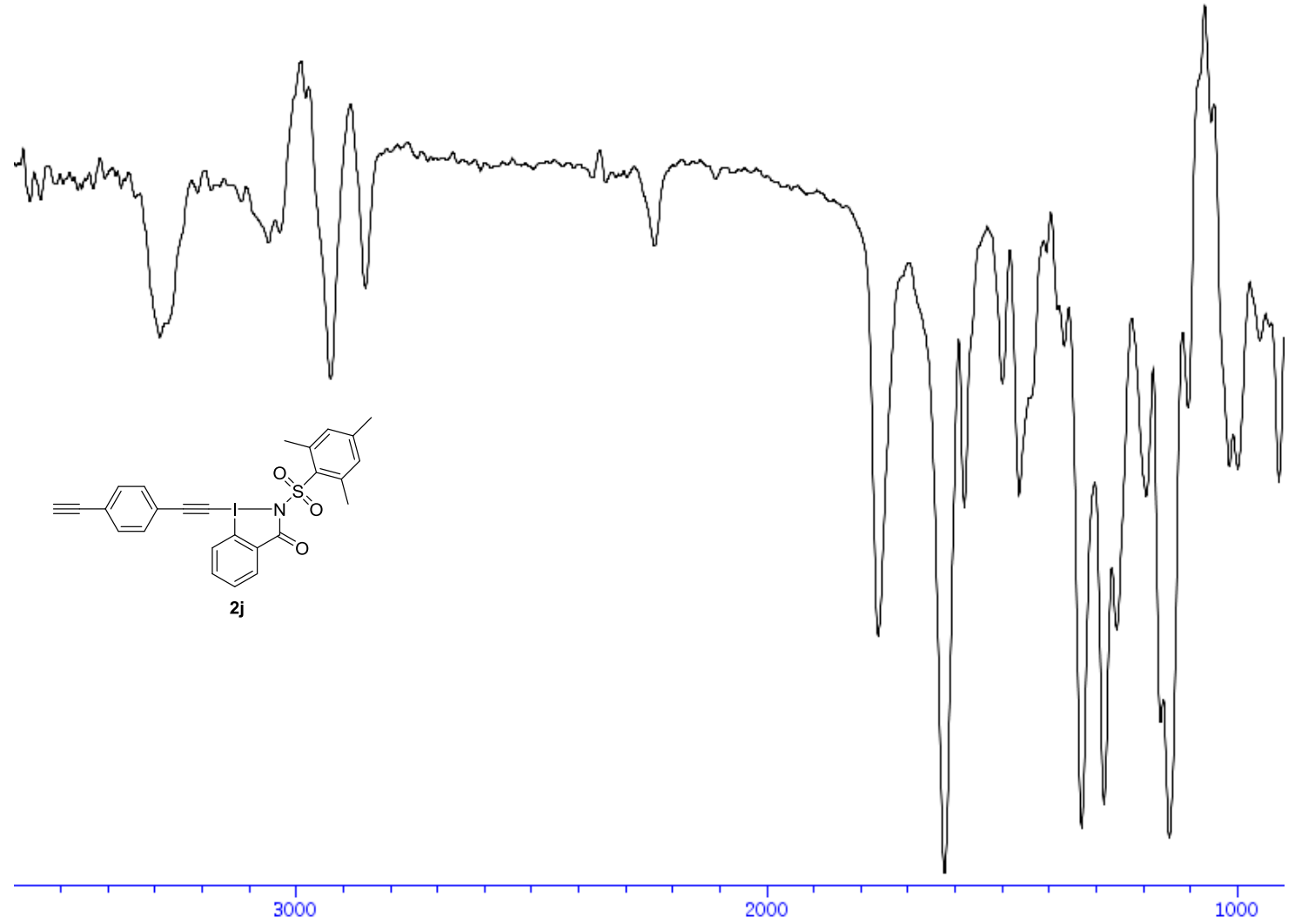


${ }^{\mathbf{1}} \mathbf{H}-\mathbf{N M R}\left(400 \mathrm{MHz}, \mathrm{CDCl}_{3}\right.$ ) of compound $\mathbf{2 k}$

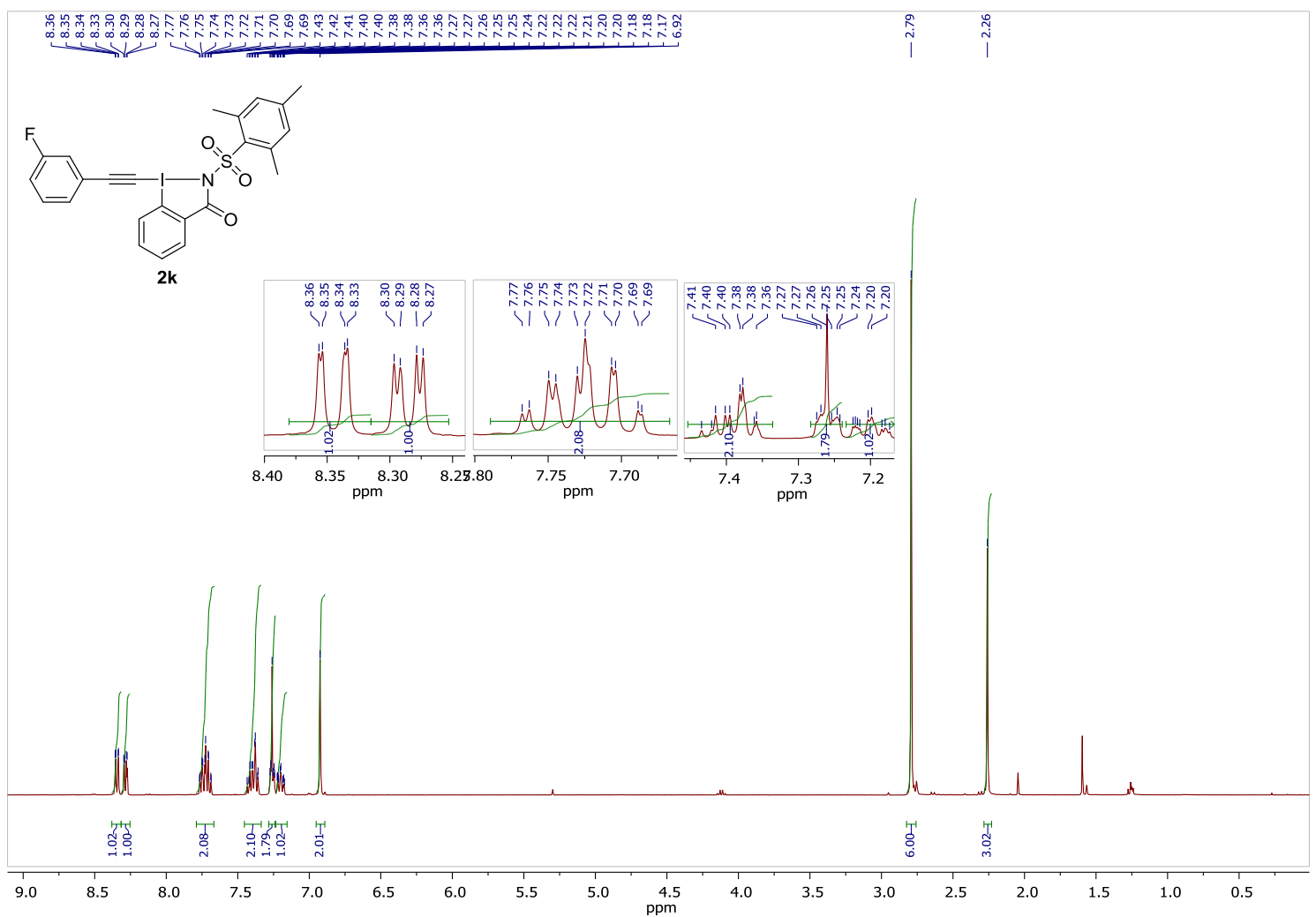

${ }^{13} \mathbf{C}$-NMR (100 MHz, $\left.\mathrm{CDCl}_{3}\right)$ of compound $\mathbf{2 k}$

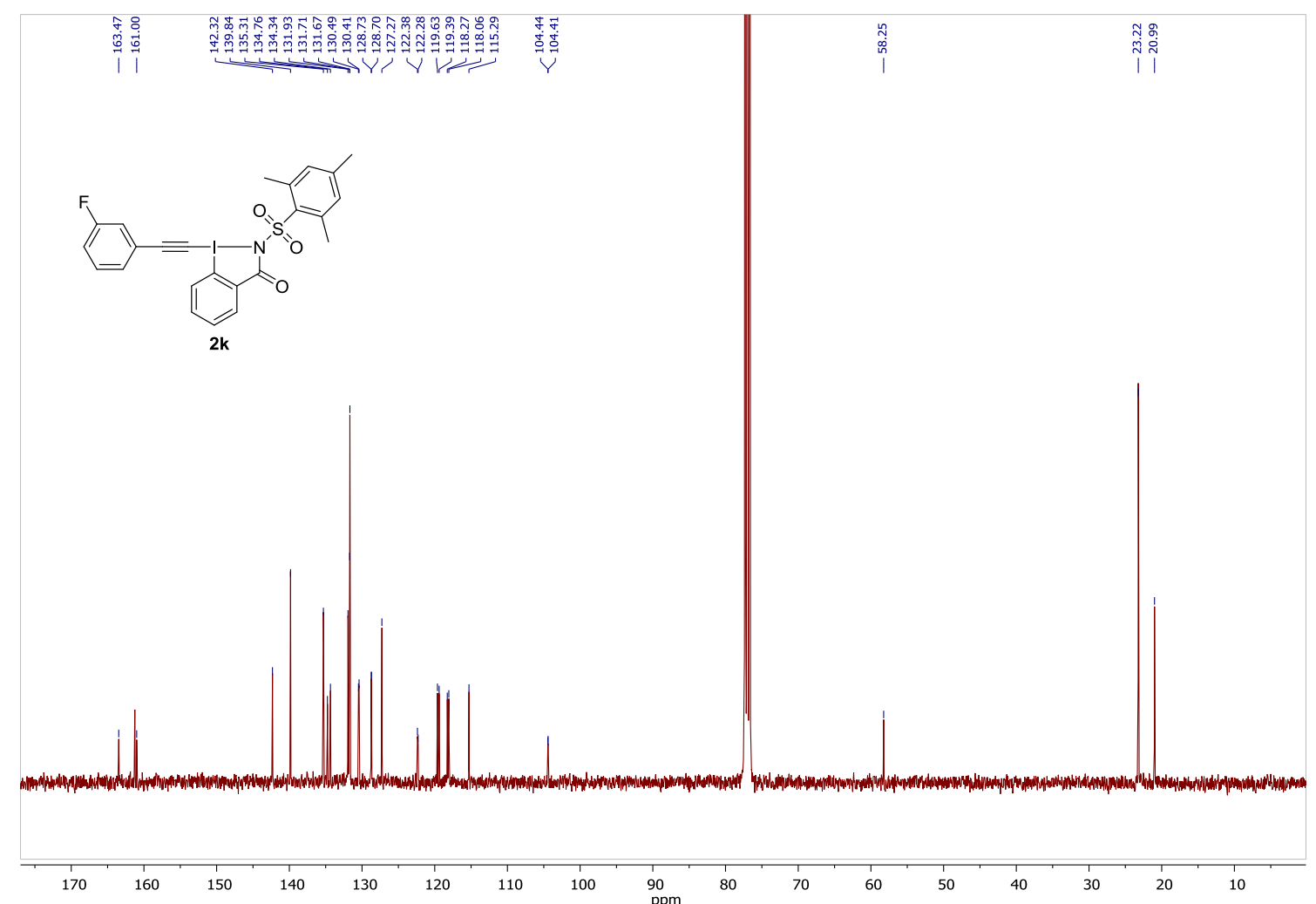


IR of compound $\mathbf{2 k}$

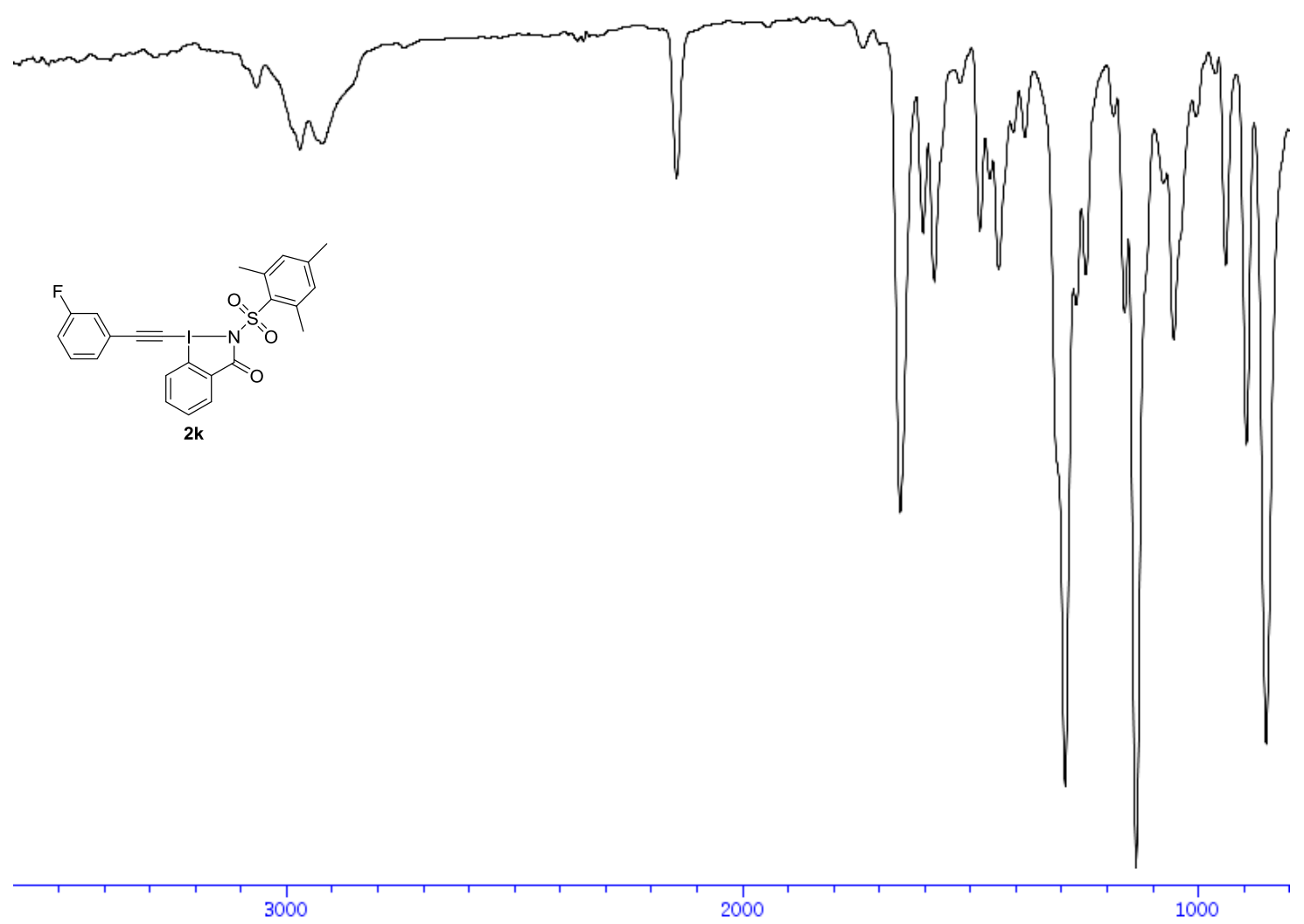


${ }^{1} \mathbf{H}-\mathbf{N M R}\left(400 \mathrm{MHz}, \mathrm{CDCl}_{3}\right)$ of compound $2 \mathbf{I}$

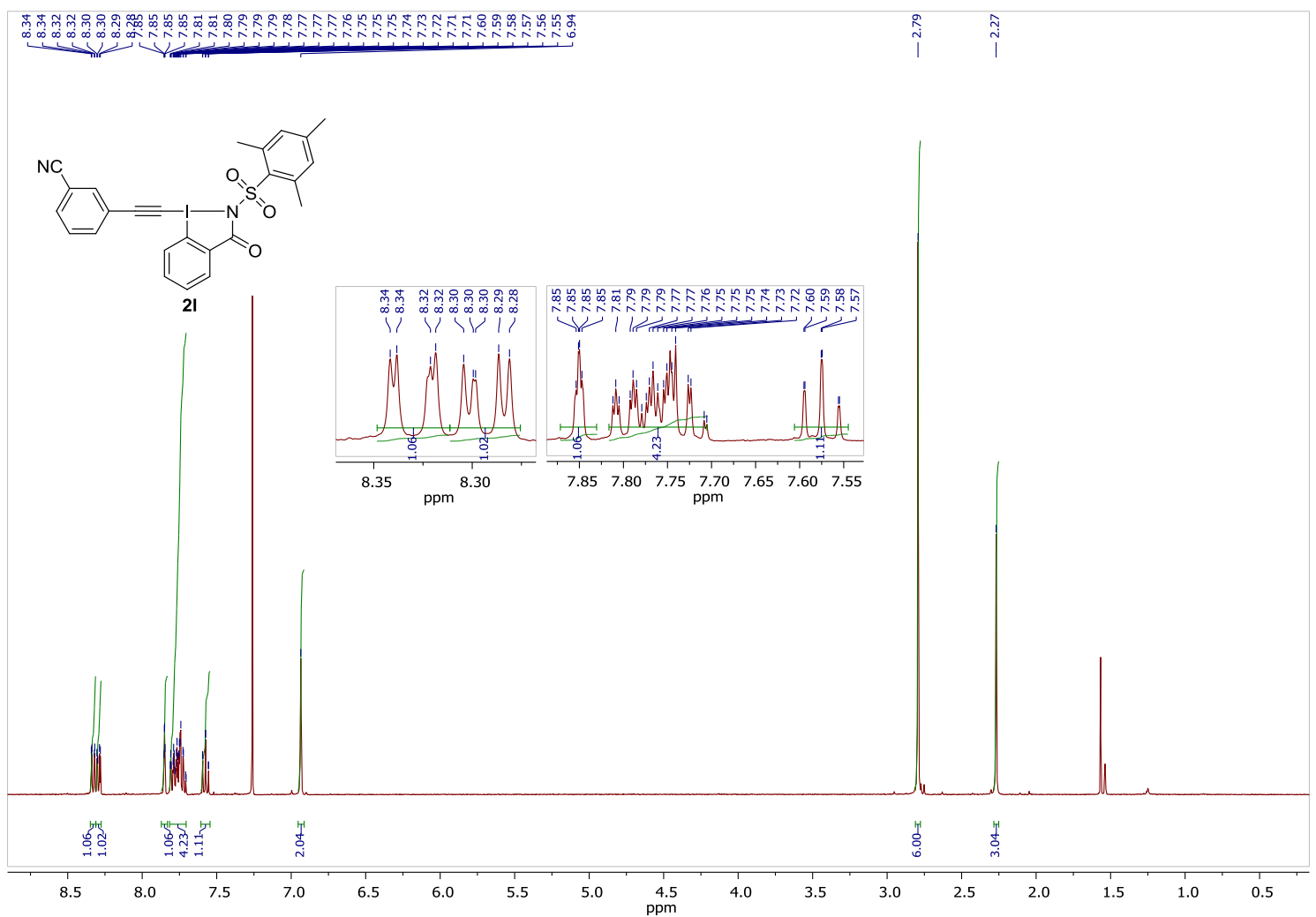

${ }^{13} \mathbf{C}$-NMR (100 MHz, $\left.\mathrm{CDCl}_{3}\right)$ of compound $\mathbf{2 l}$

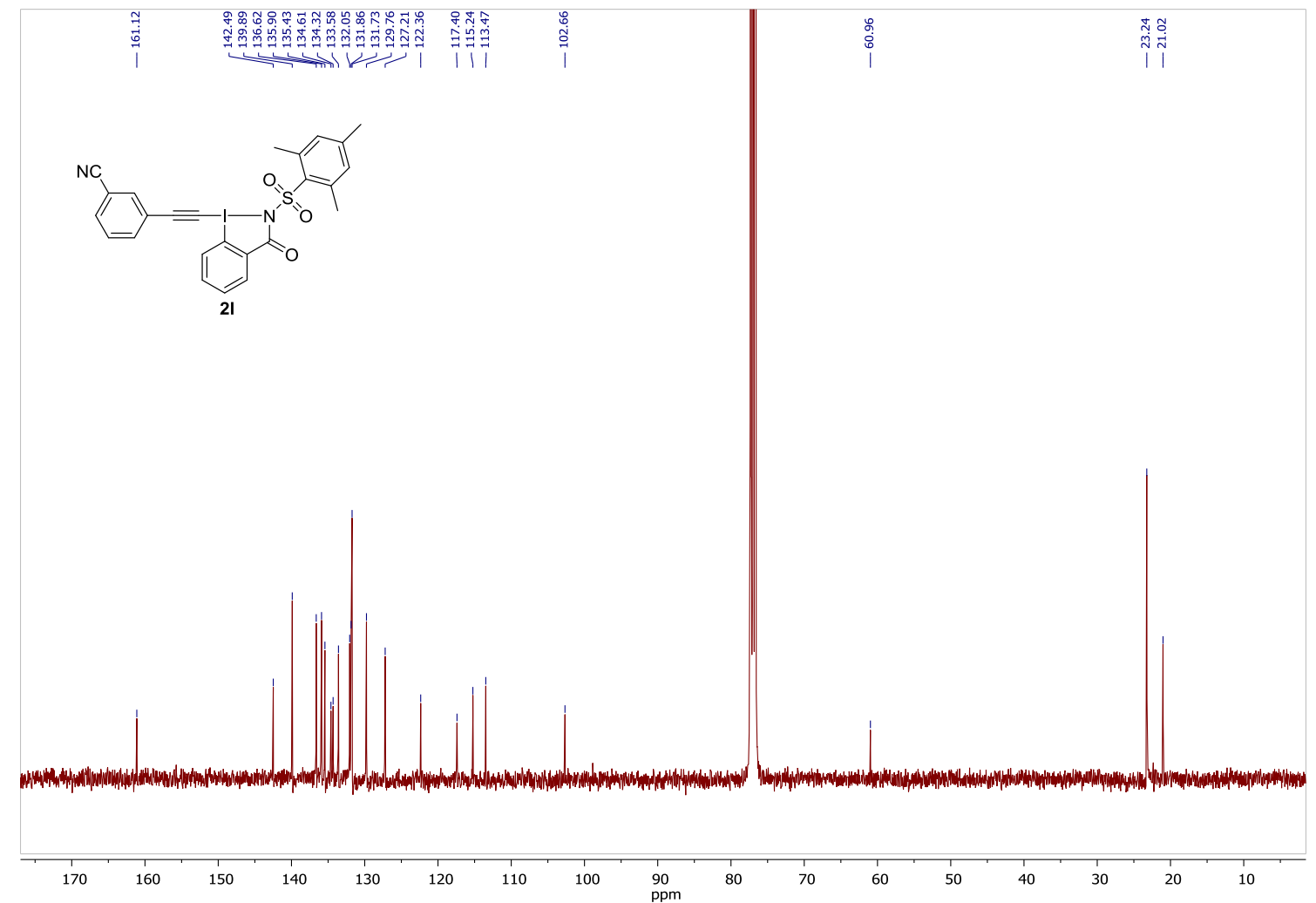


IR of compound $\mathbf{2 l}$

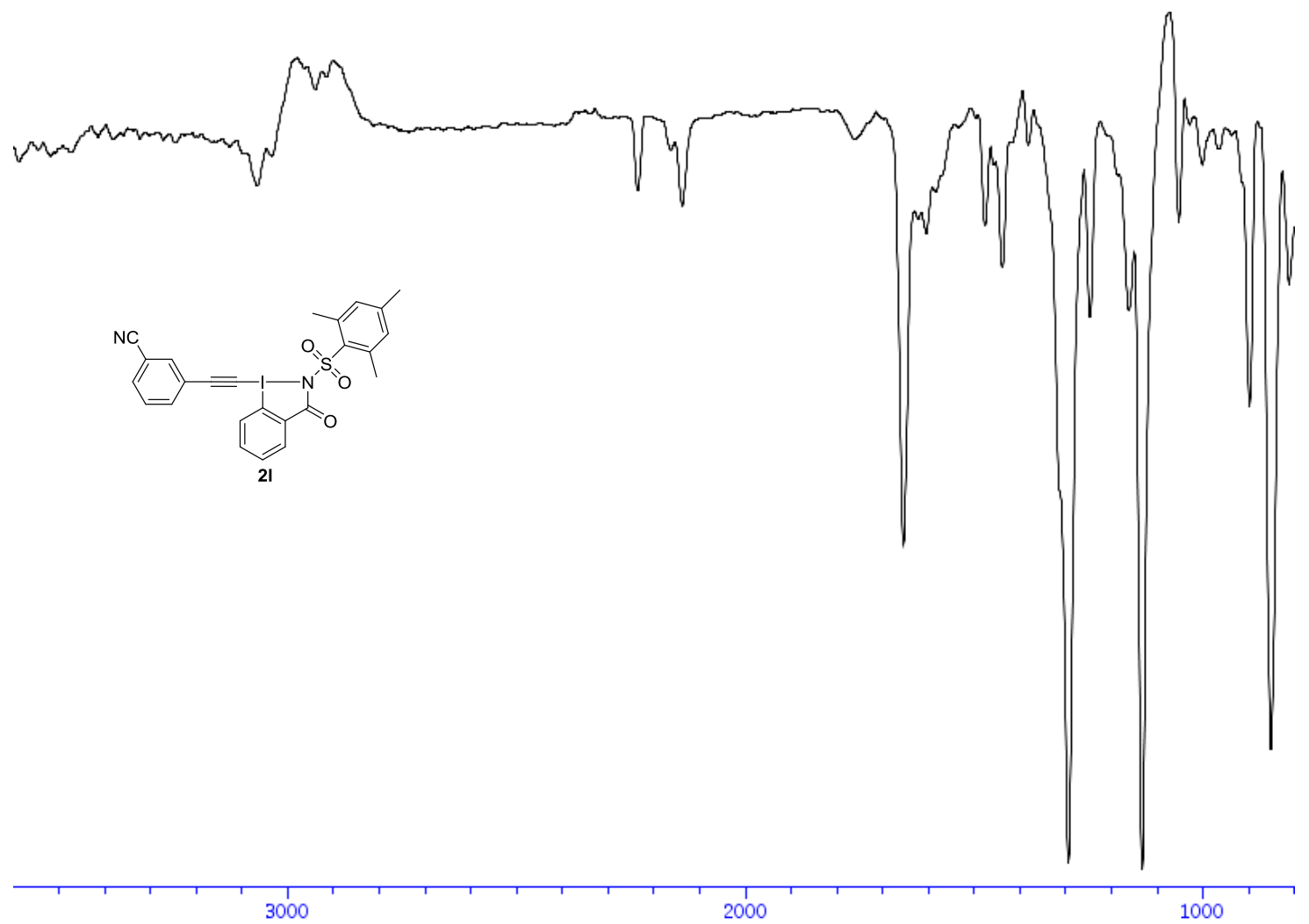


${ }^{\mathbf{1}} \mathbf{H}-\mathbf{N M R}\left(400 \mathrm{MHz}, \mathrm{CDCl}_{3}\right)$ of compound $\mathbf{2 m}$

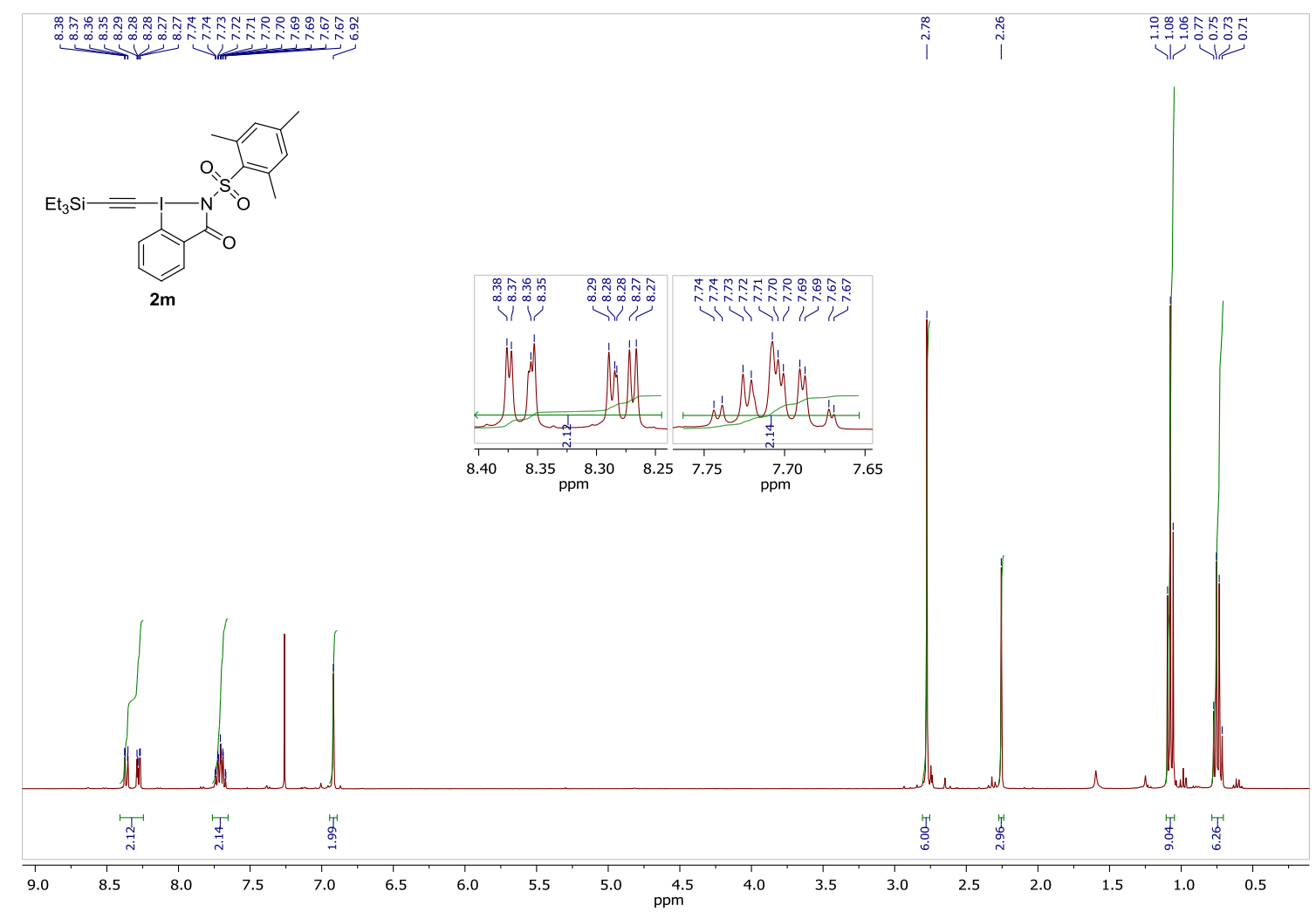

${ }^{13} \mathbf{C}-$ NMR $\left(100 \mathrm{MHz}, \mathrm{CDCl}_{3}\right)$ of compound $\mathbf{2 m}$

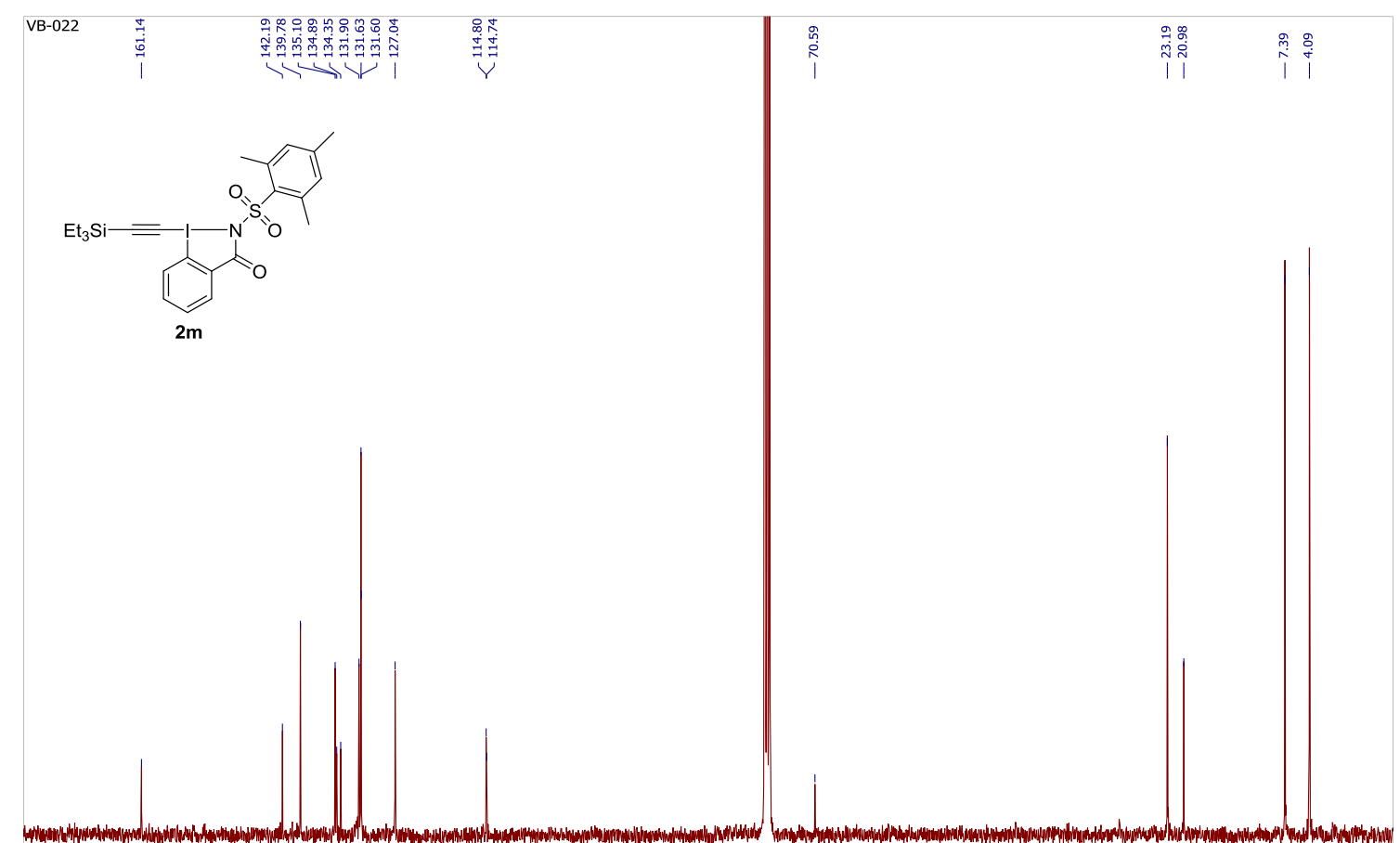

$\begin{array}{lllllllll}170 & 160 & 150 & 140 & 130 & 120 & 110 & 100 & 90 \\ & & & & & & & & \end{array}$ 
IR of compound $\mathbf{2 m}$

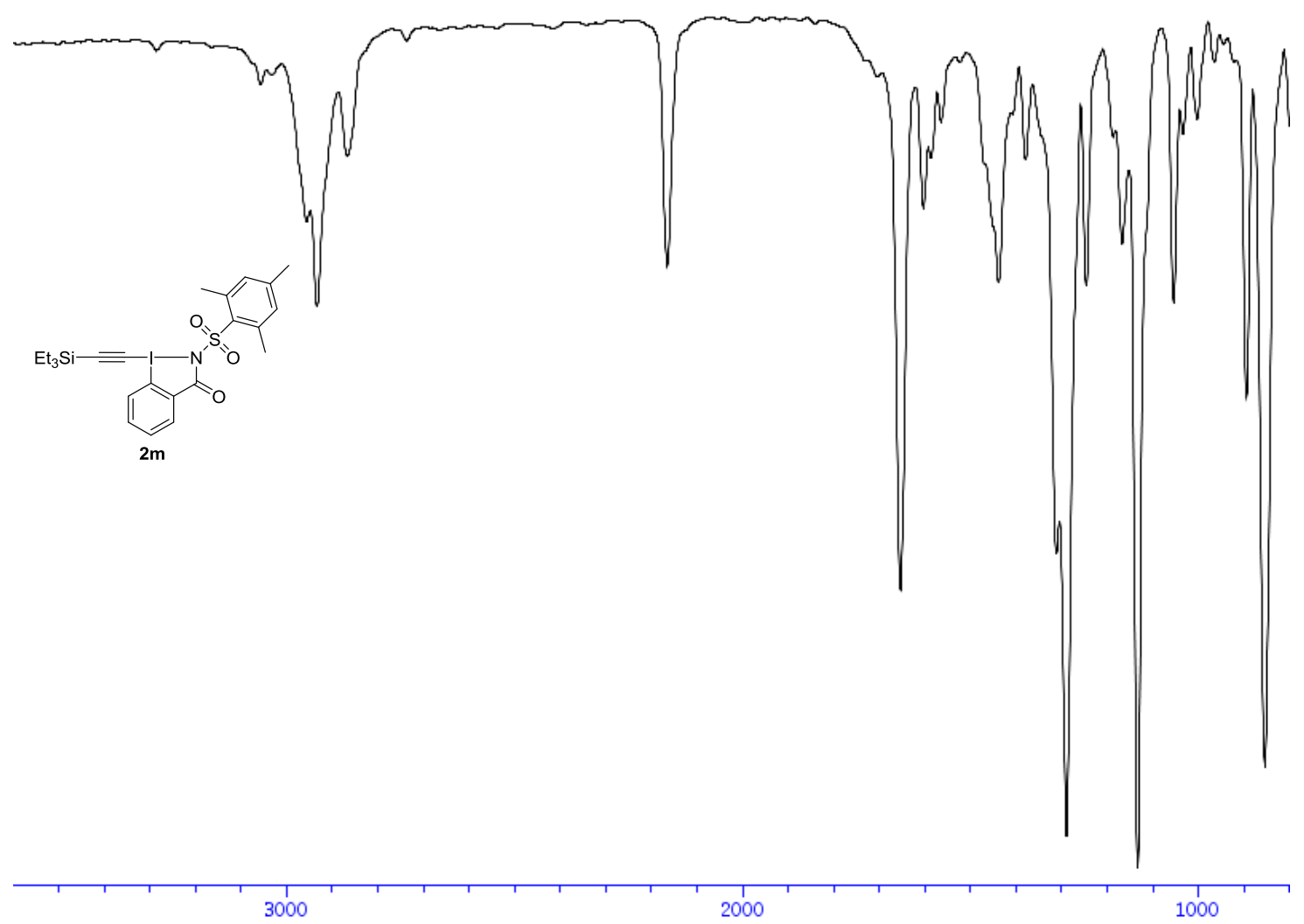


${ }^{1} \mathbf{H}-\mathbf{N M R}\left(400 \mathrm{MHz}, \mathrm{CDCl}_{3}\right)$ of compound $\mathbf{2 n}$

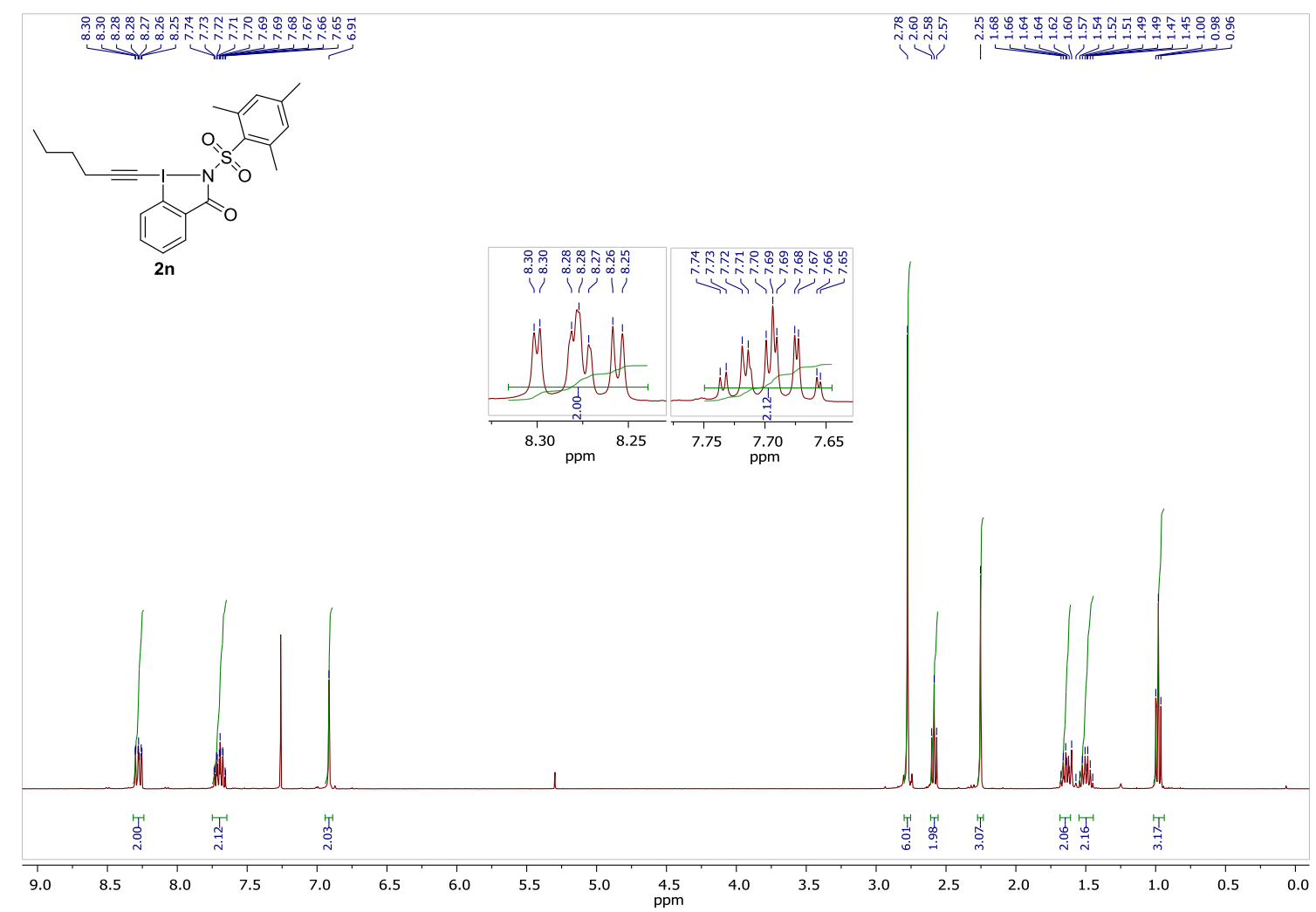

${ }^{13} \mathbf{C}$-NMR (100 MHz, $\left.\mathrm{CDCl}_{3}\right)$ of compound 2n

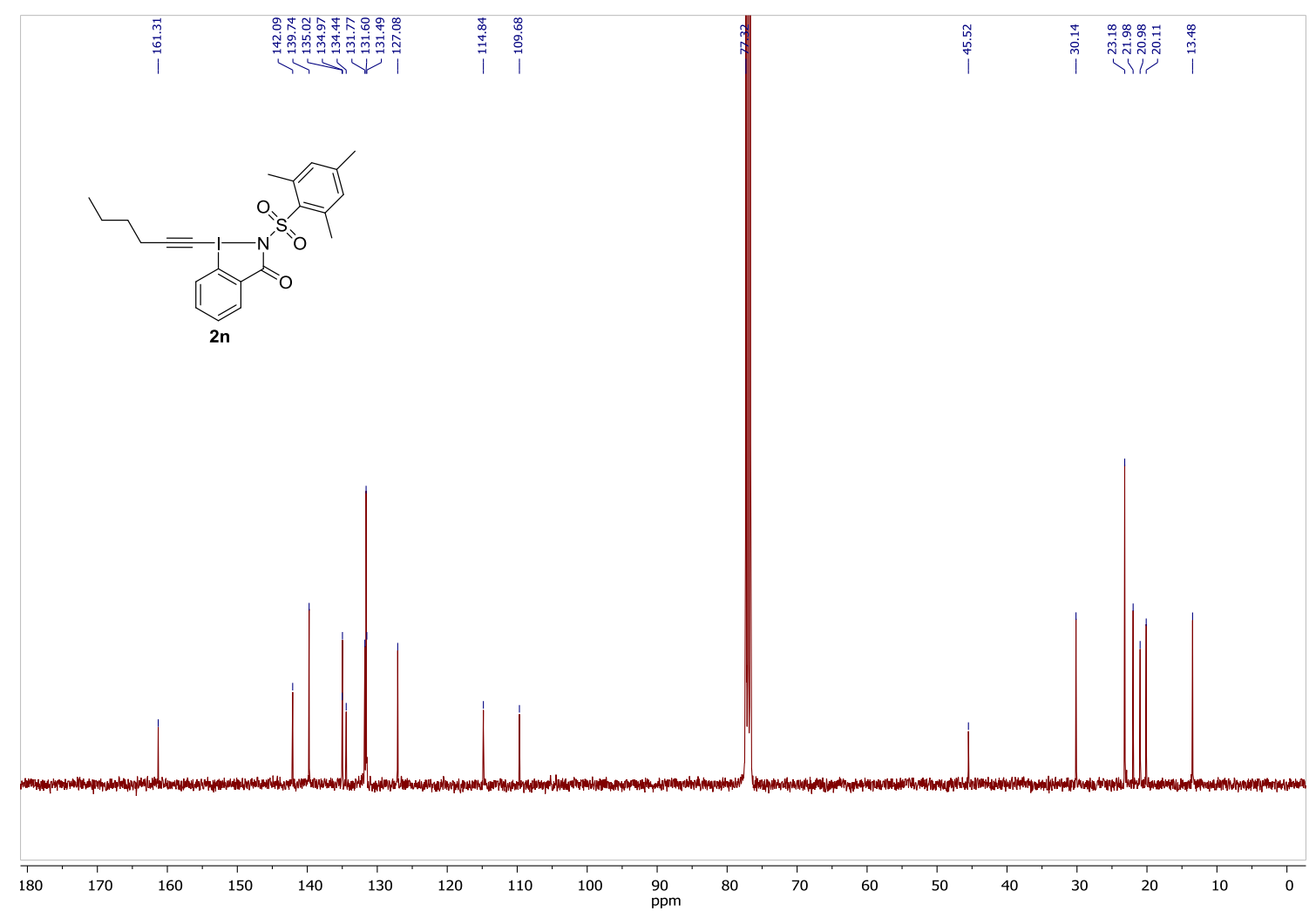


IR of compound $\mathbf{2 n}$

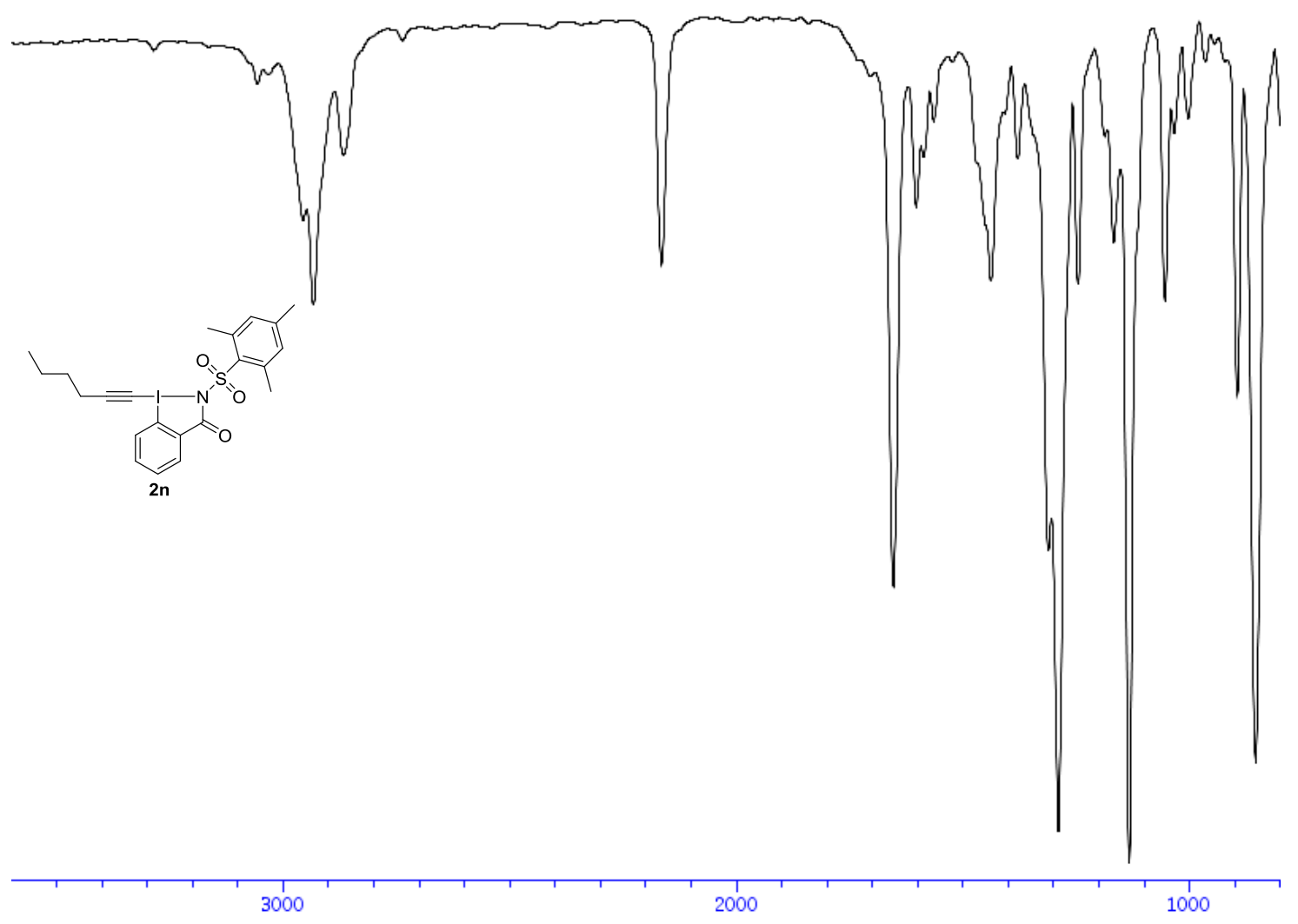


${ }^{1} \mathbf{H}-\mathbf{N M R}\left(400 \mathrm{MHz}, \mathrm{CDCl}_{3}\right)$ of compound $\mathbf{2 0}$

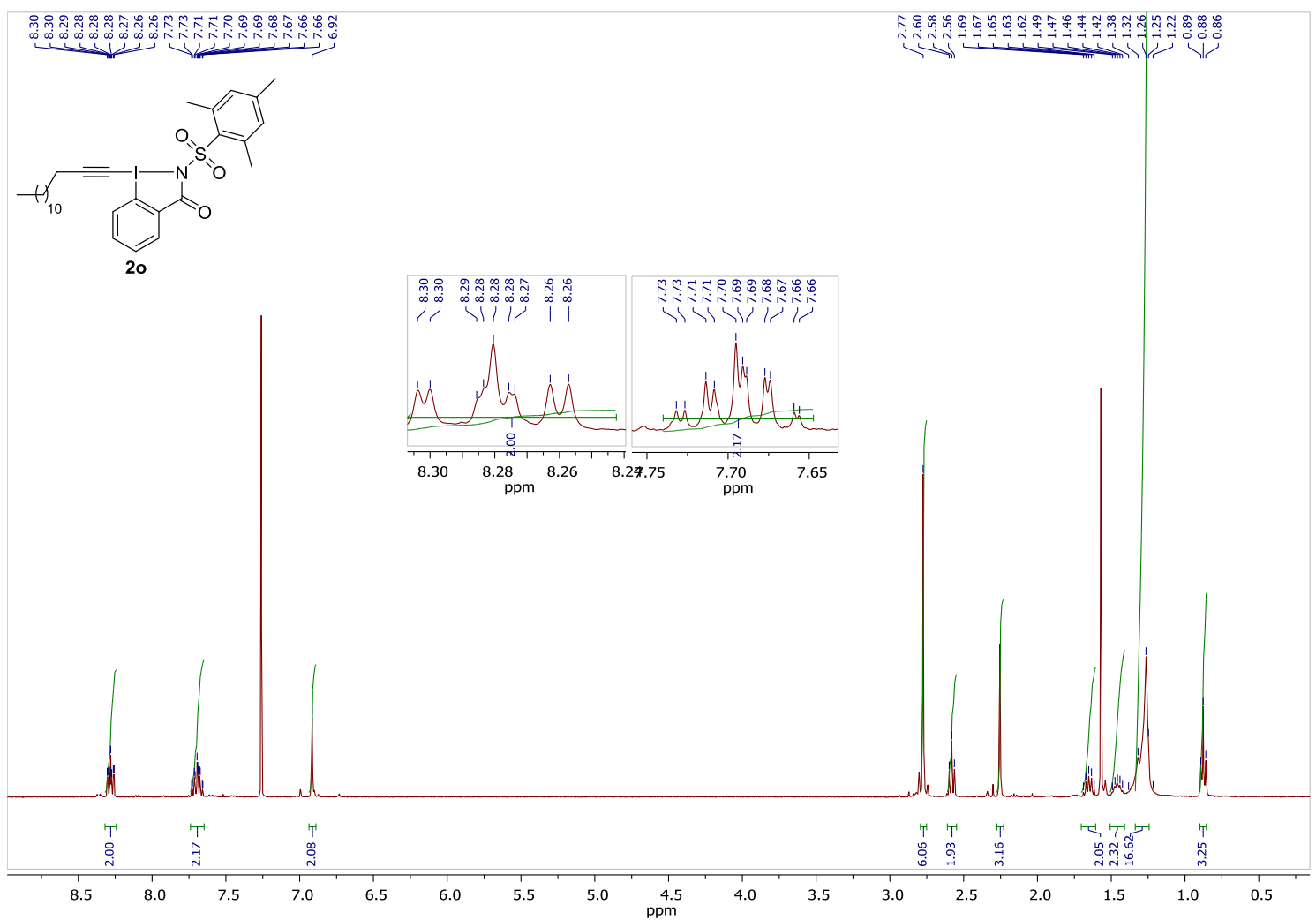

${ }^{13} \mathbf{C}$-NMR (100 MHz, $\left.\mathrm{CDCl}_{3}\right)$ of compound $\mathbf{2 0}$

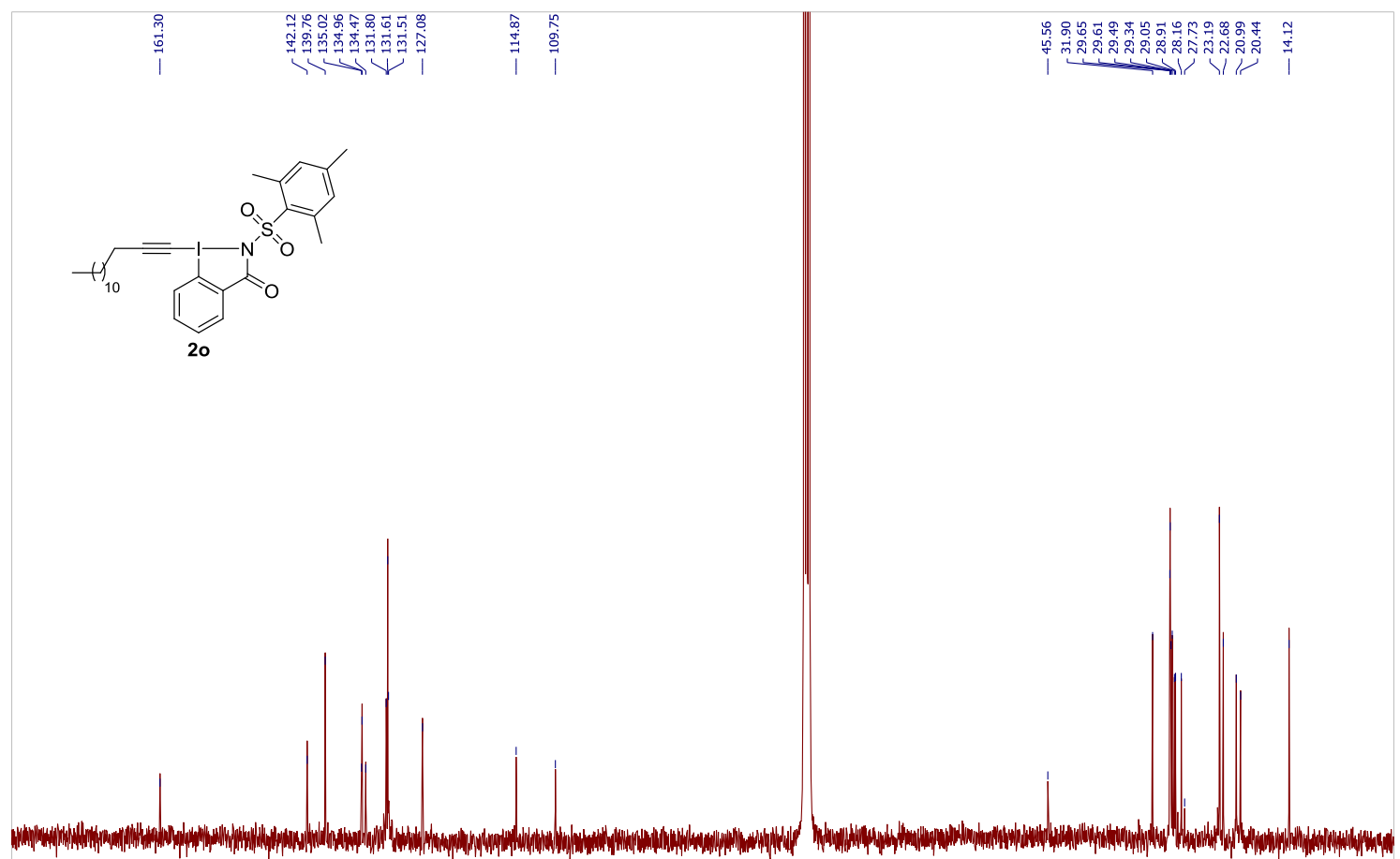

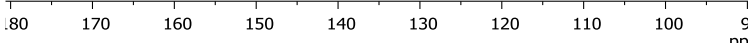


IR of compound 20

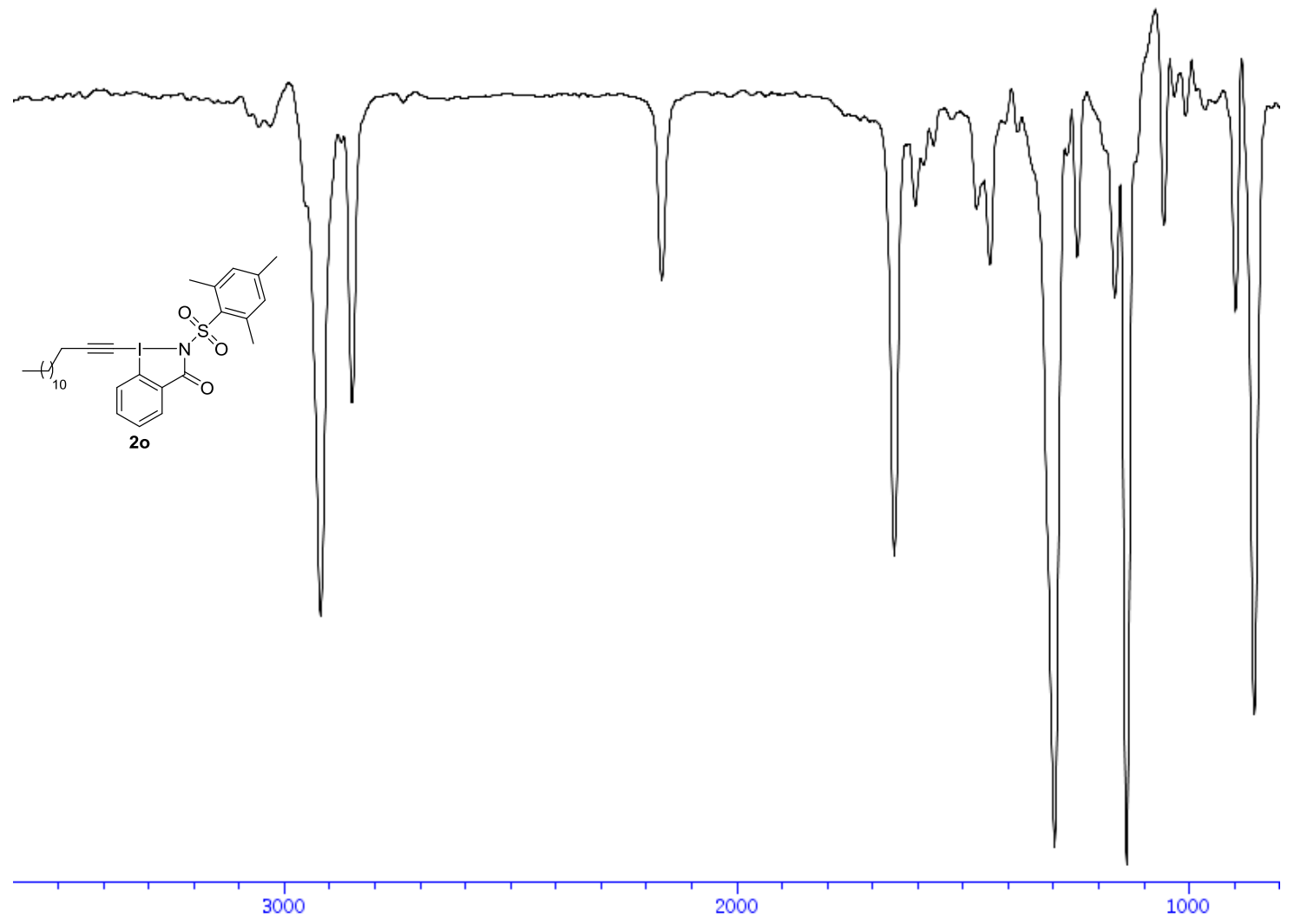


${ }^{\mathbf{1}} \mathbf{H}$-NMR (400 MHz, $\mathrm{CDCl}_{3}$ ) of compound $\mathbf{2 p}$

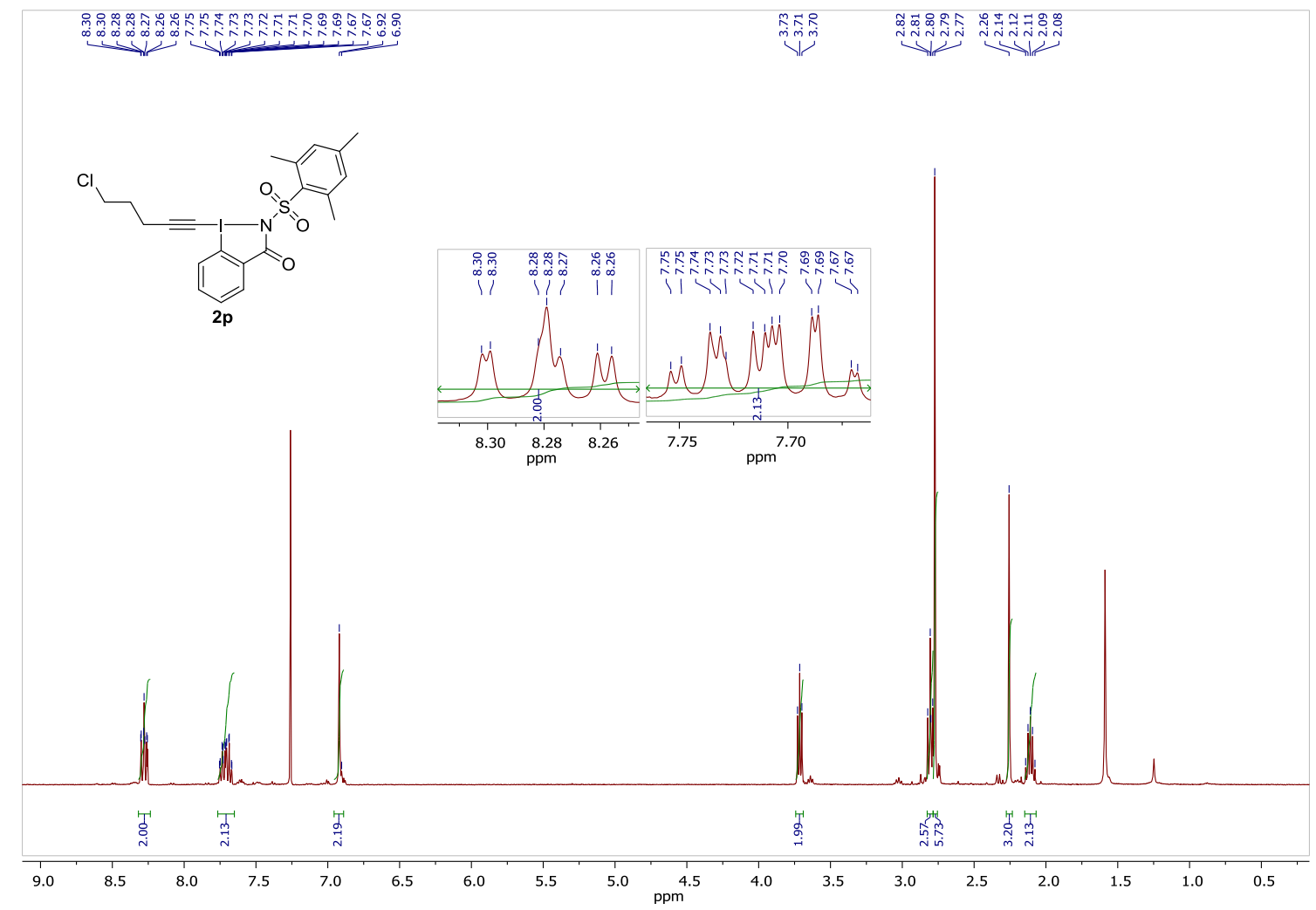

${ }^{13} \mathbf{C}$-NMR (100 MHz, $\left.\mathrm{CDCl}_{3}\right)$ of compound 2p

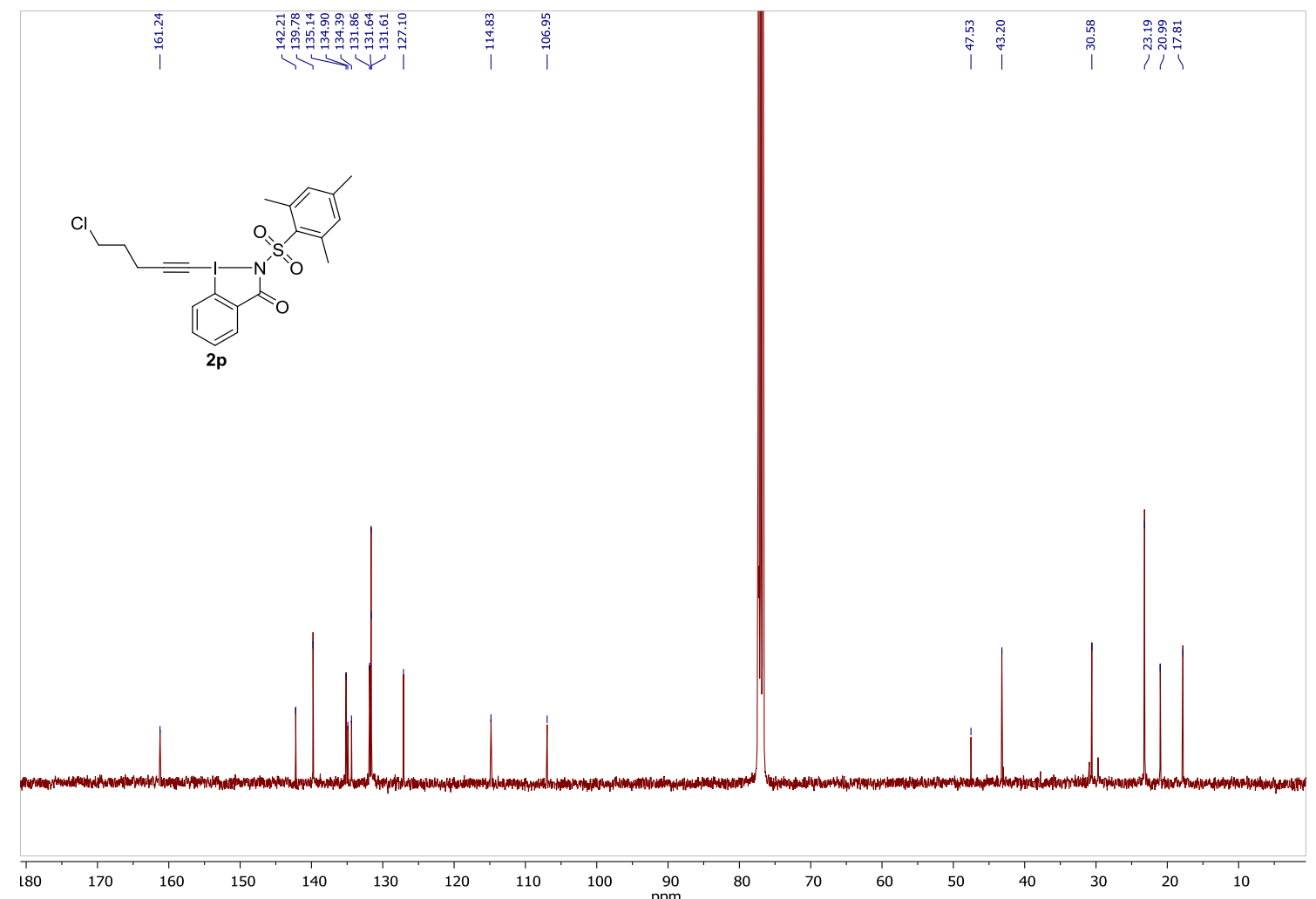


IR of compound $\mathbf{2 p}$

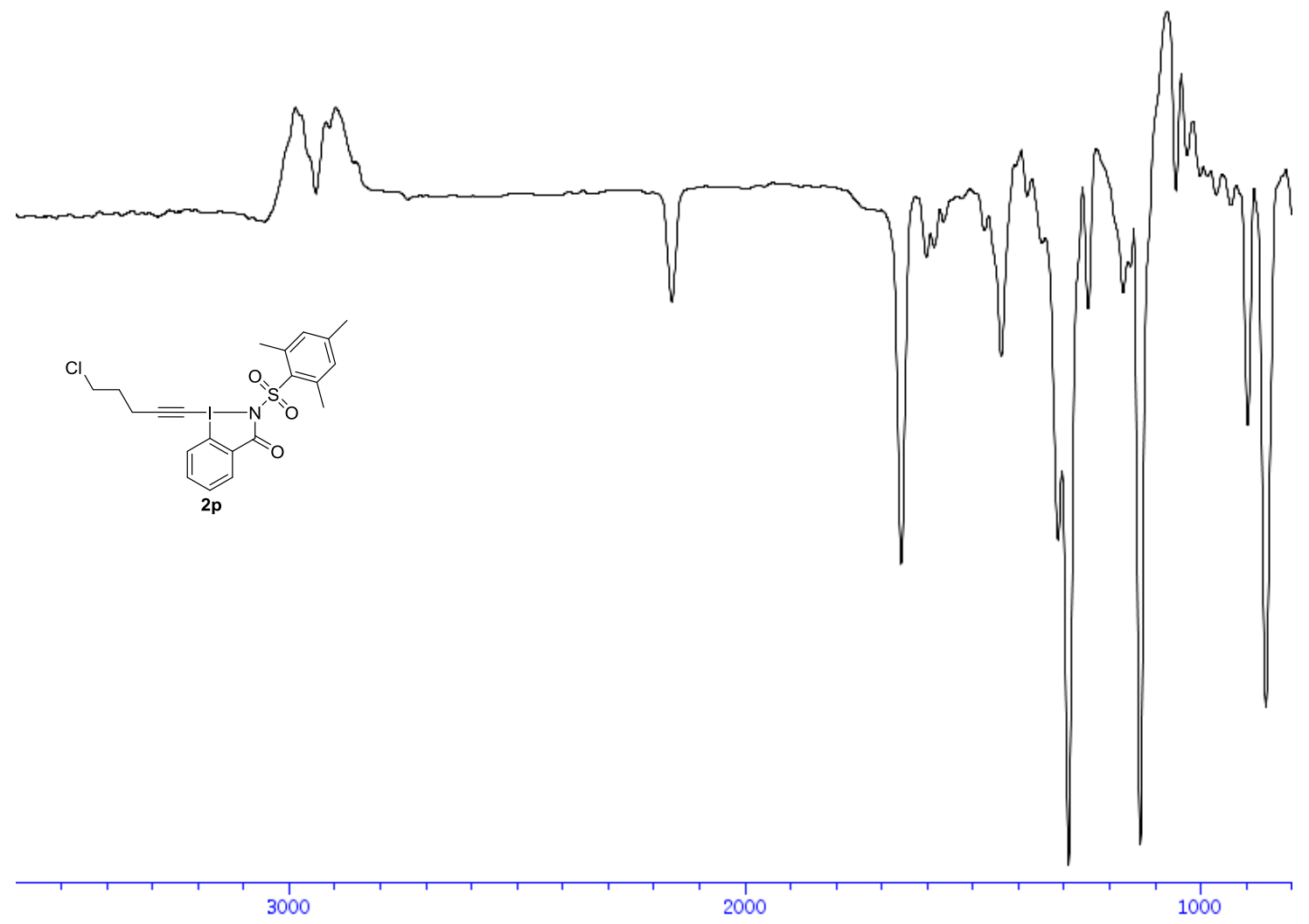


${ }^{\mathbf{1}} \mathbf{H}$-NMR (400 MHz, $\mathrm{CDCl}_{3}$ ) of compound $\mathbf{2 q}$

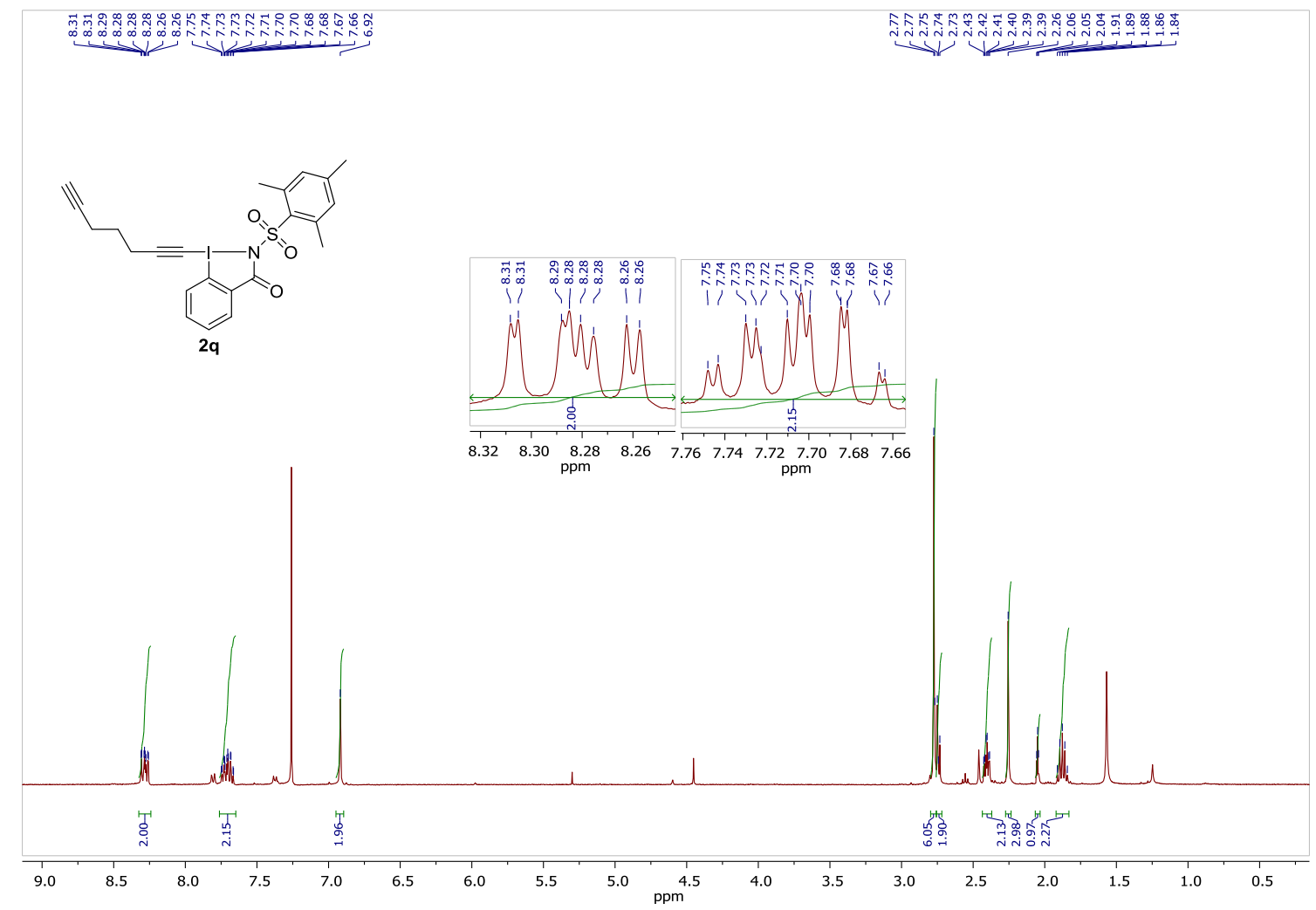

${ }^{13} \mathbf{C}$-NMR (100 MHz, $\left.\mathrm{CDCl}_{3}\right)$ of compound $\mathbf{2 q}$

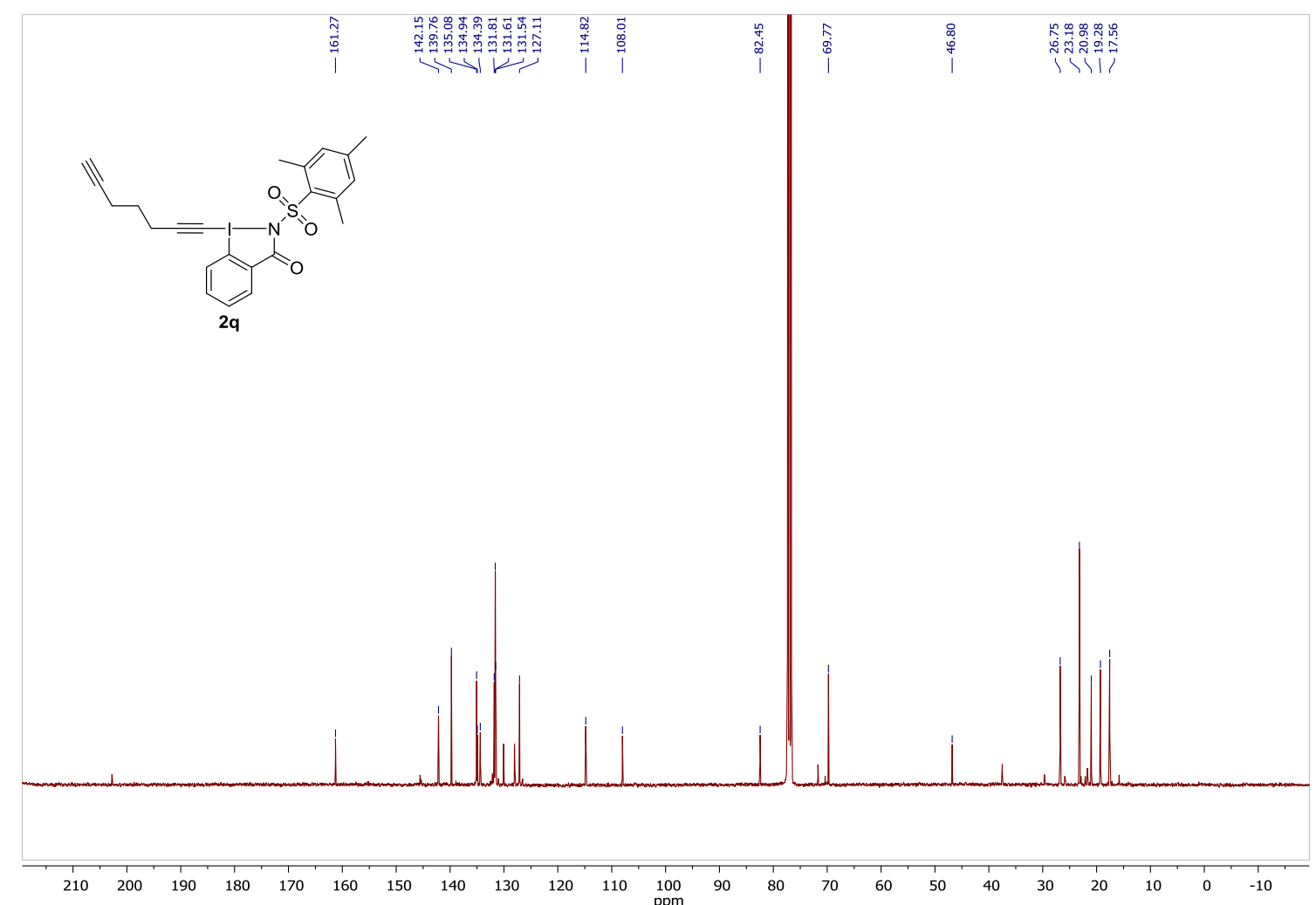


IR of compound $\mathbf{2 q}$

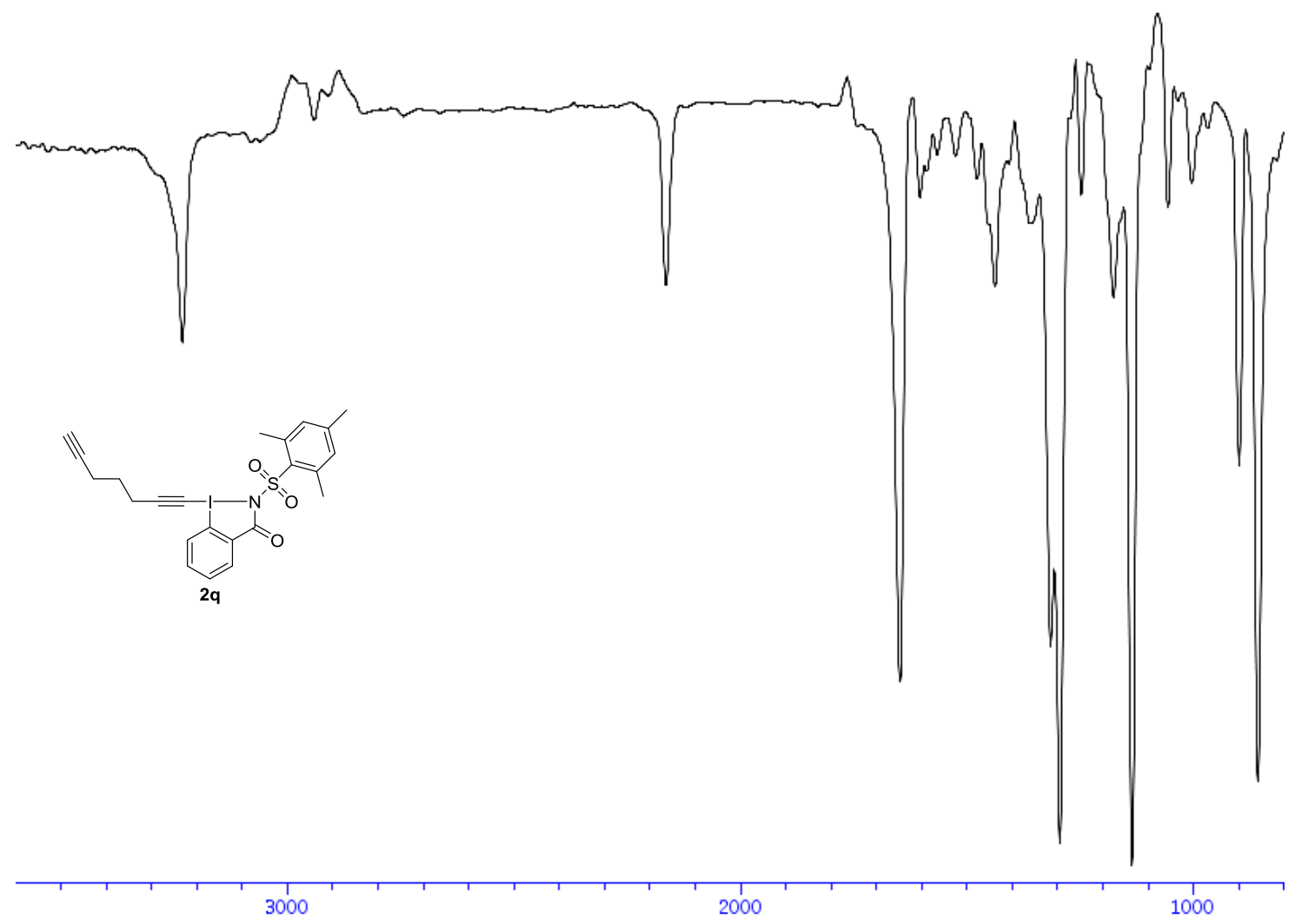


${ }^{1} \mathbf{H}-\mathbf{N M R}\left(400 \mathrm{MHz}, \mathrm{CDCl}_{3}\right)$ of compound $\mathbf{2 r}$

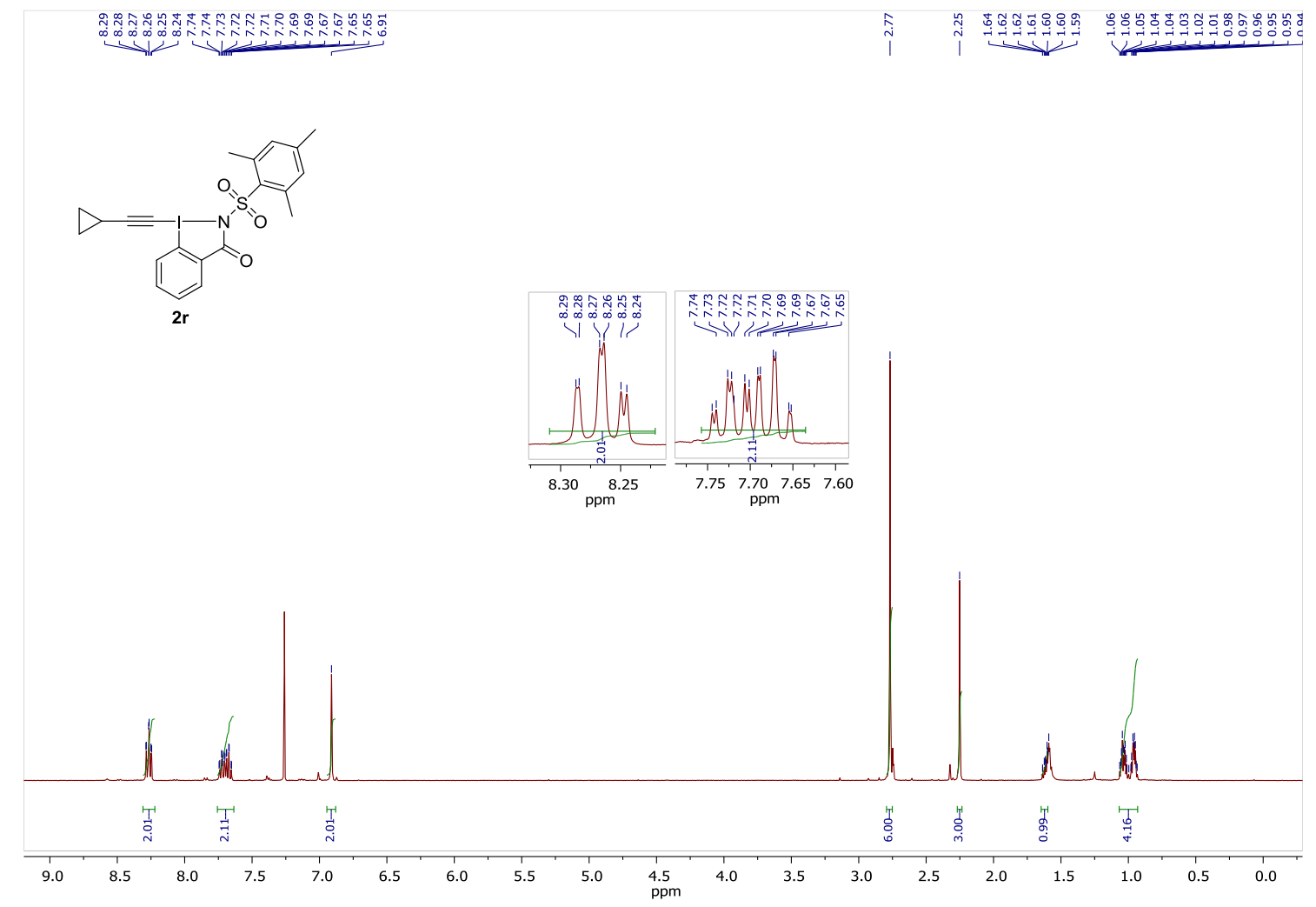

${ }^{13} \mathbf{C}-\mathbf{N M R}\left(100 \mathrm{MHz}, \mathrm{CDCl}_{3}\right)$ of compound $\mathbf{2 r}$

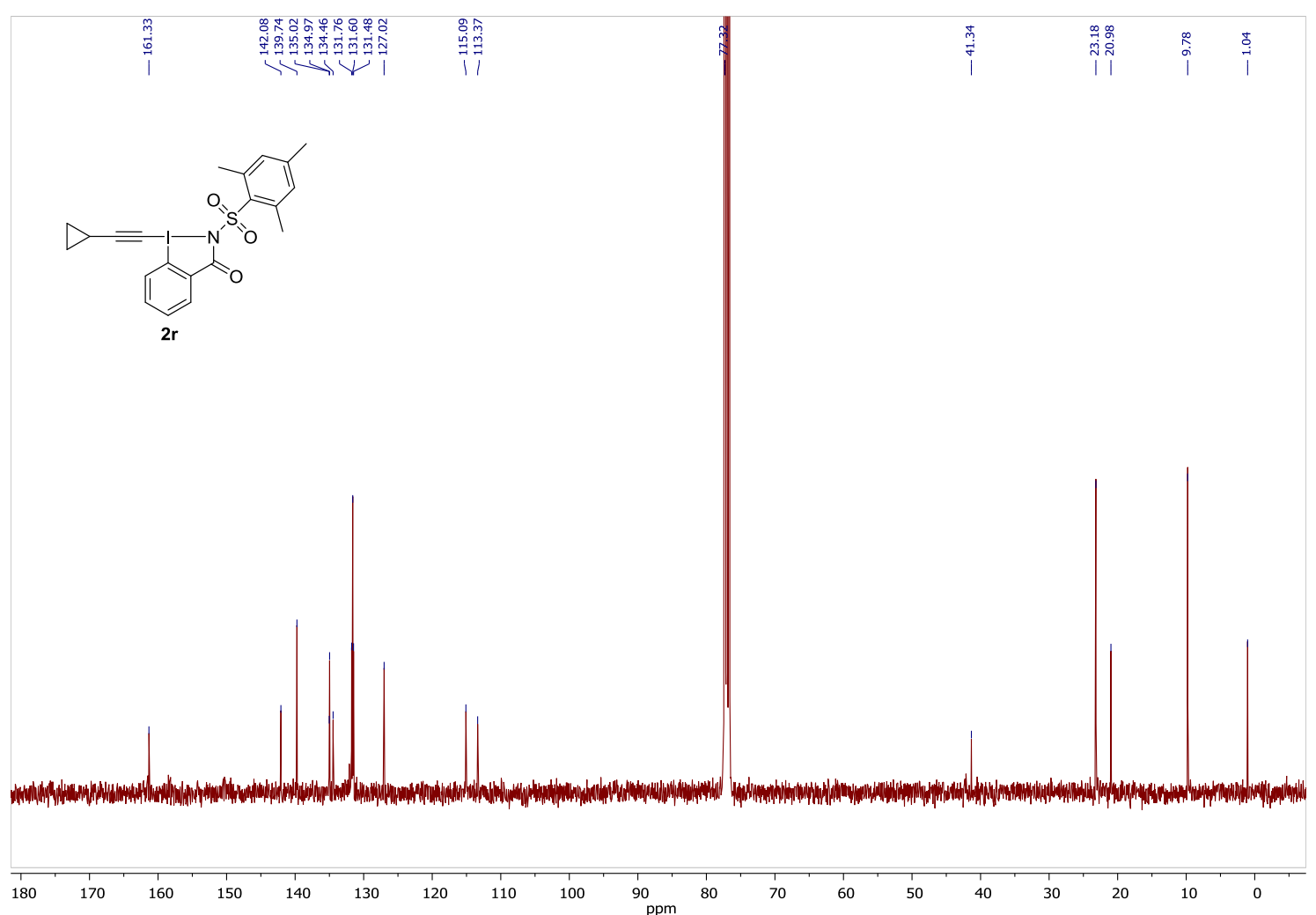


IR of compound $\mathbf{2 r}$

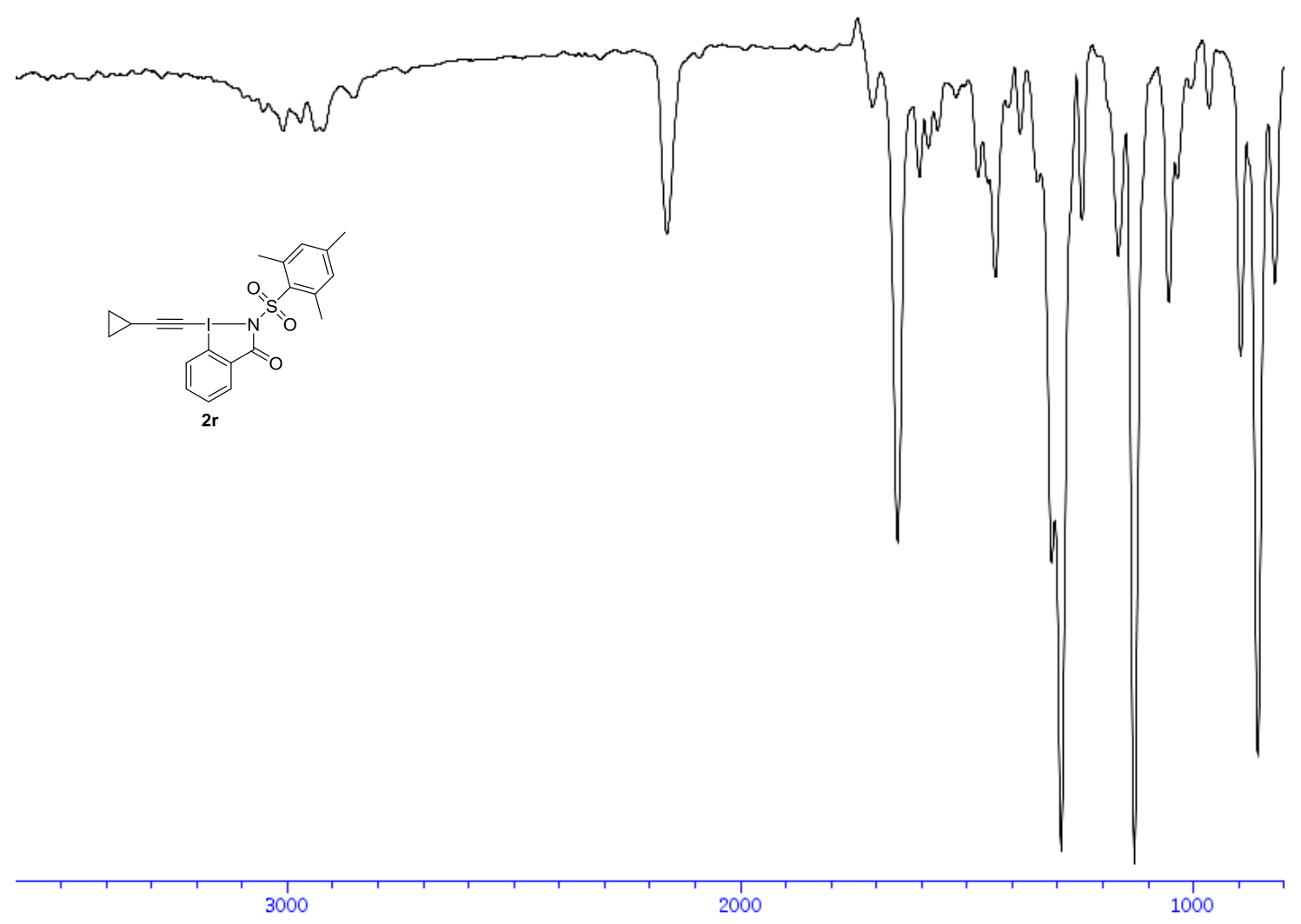


${ }^{1} \mathbf{H}-\mathbf{N M R}\left(400 \mathrm{MHz}, \mathrm{CDCl}_{3}\right)$ of compound $\mathbf{2 s}$

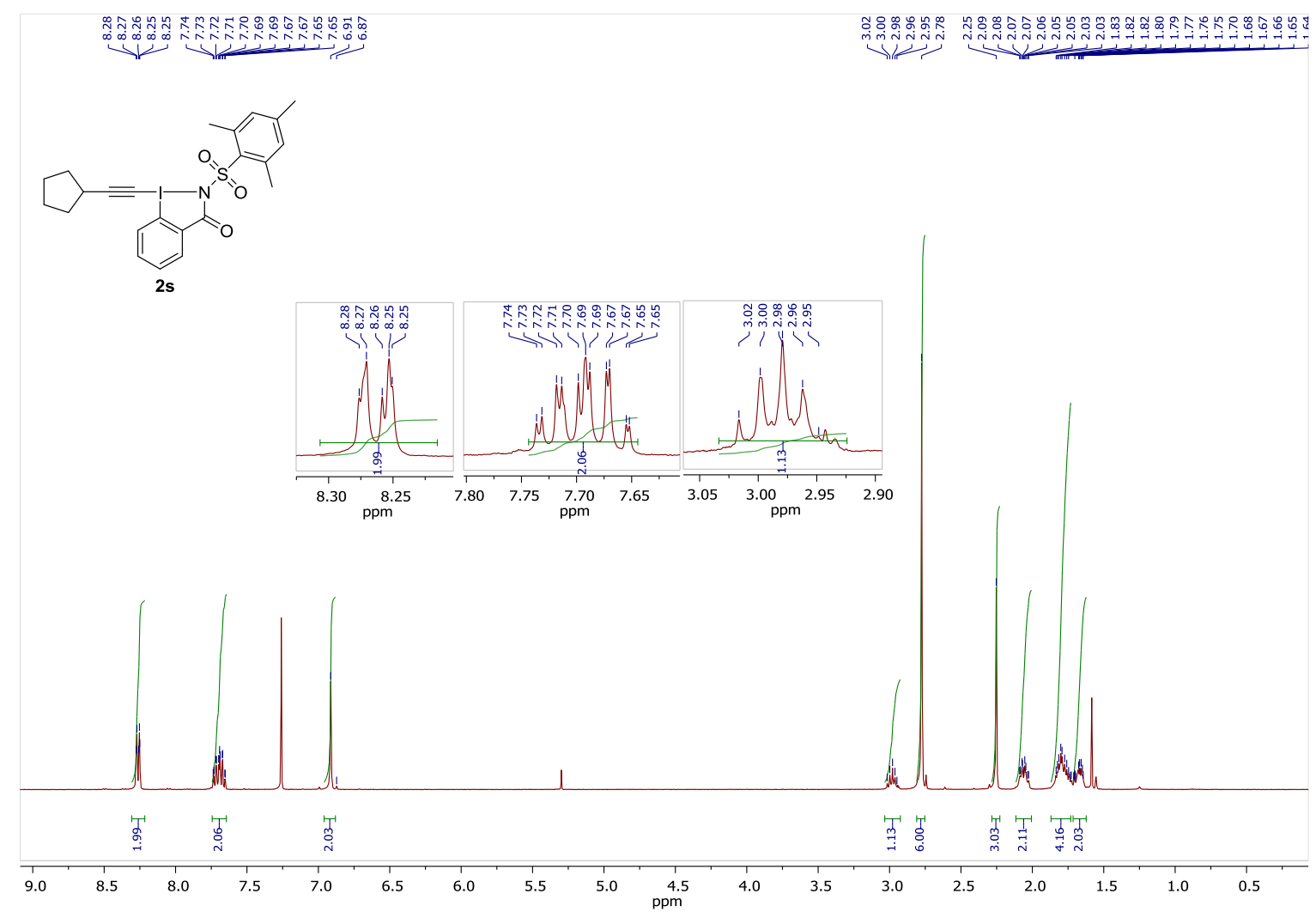

${ }^{13} \mathbf{C}-\mathbf{N M R}\left(100 \mathrm{MHz}, \mathrm{CDCl}_{3}\right)$ of compound 2s

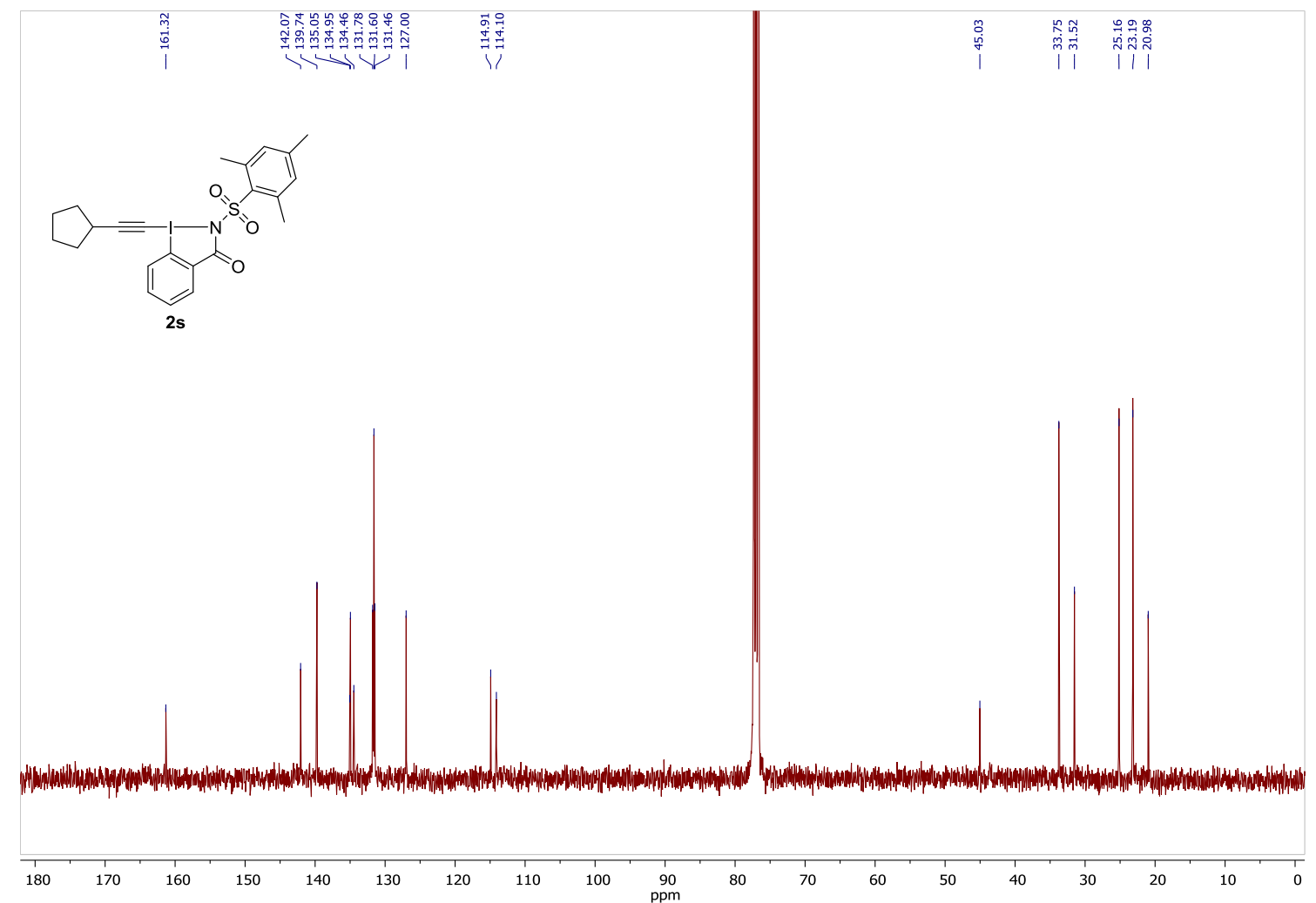


IR of compound $\mathbf{2 s}$

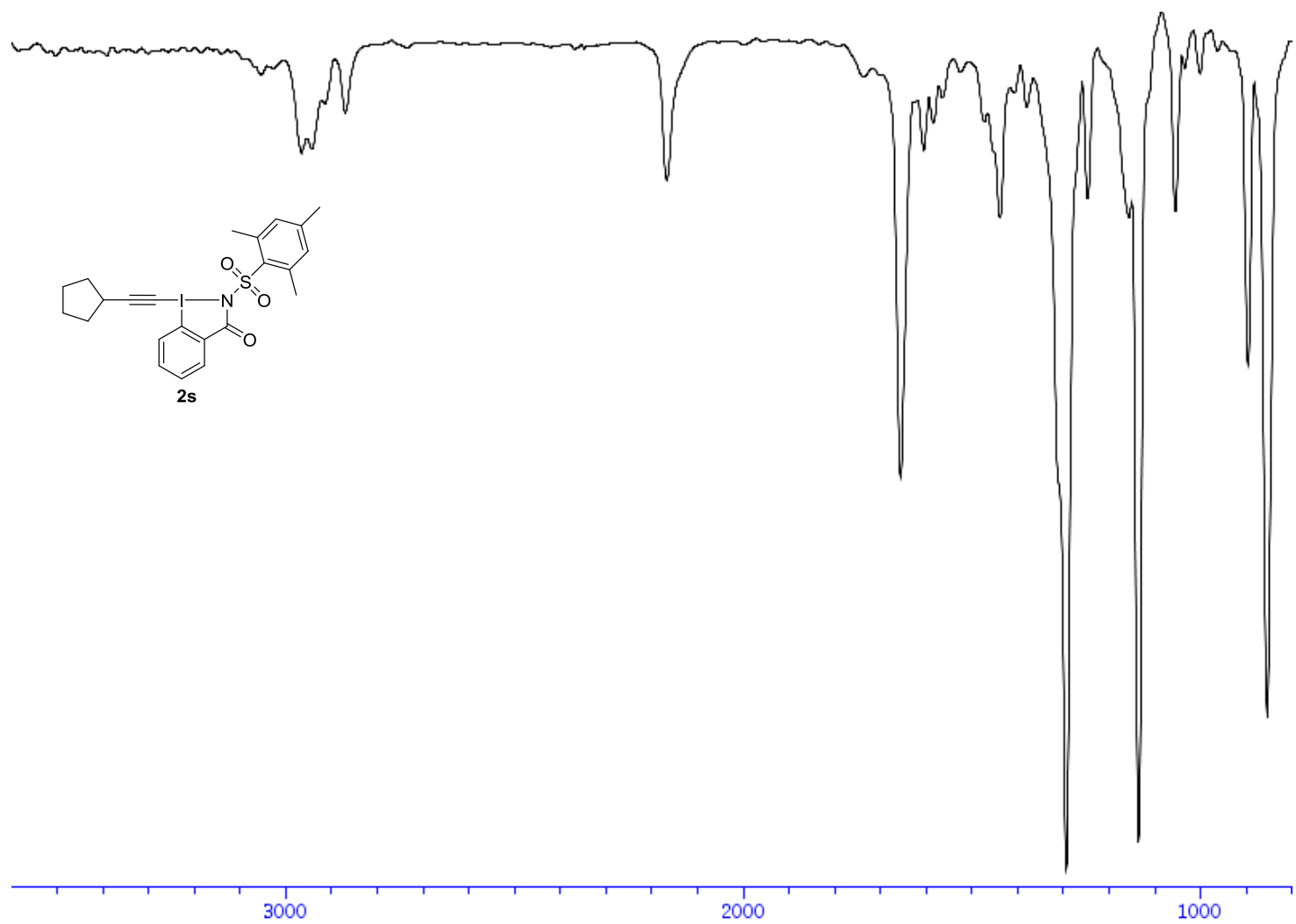


${ }^{\mathbf{1}} \mathbf{H}-\mathbf{N M R}\left(400 \mathrm{MHz}, \mathrm{CDCl}_{3}\right)$ of compound $\mathbf{2 u}$

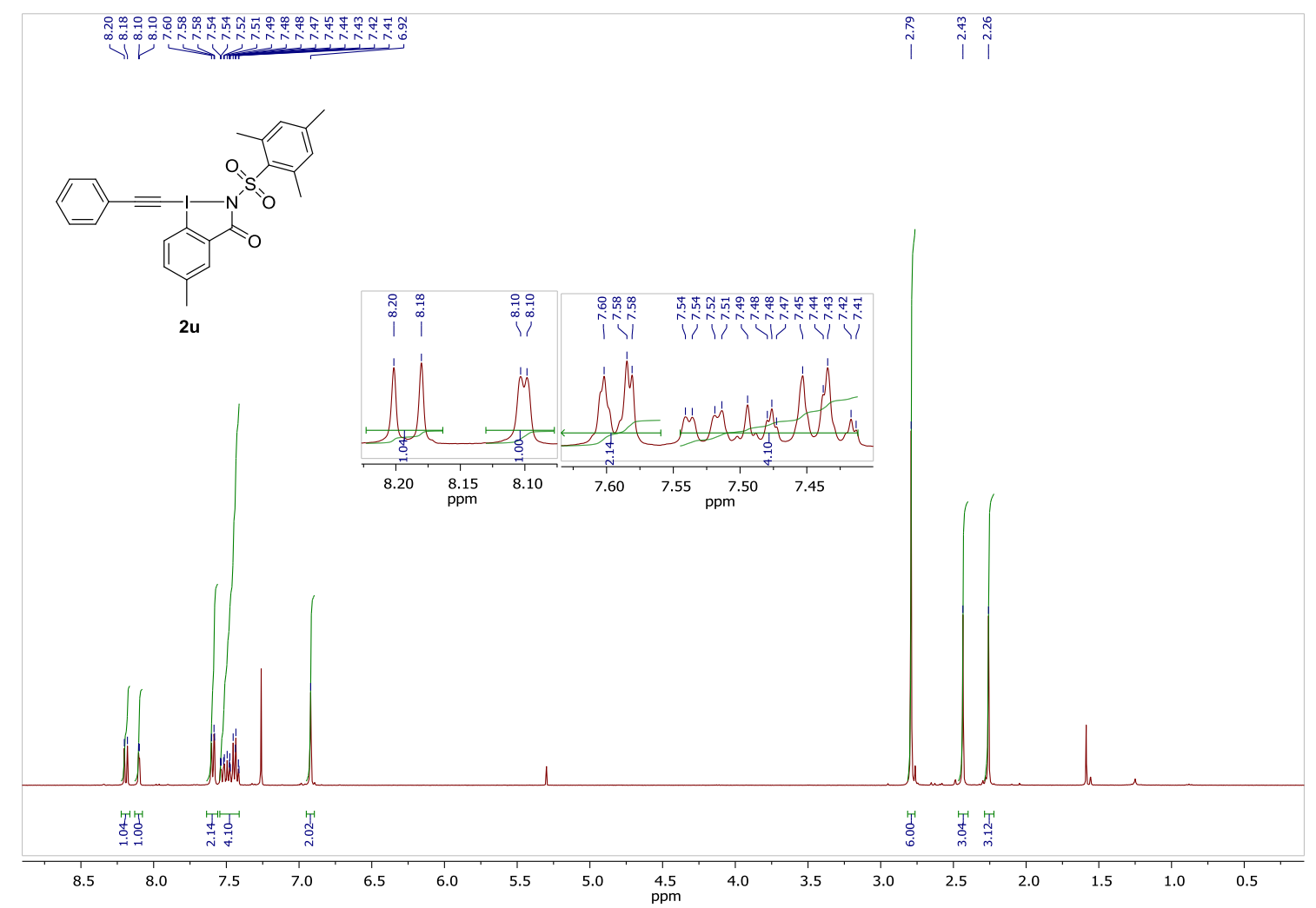

${ }^{13} \mathbf{C}$-NMR (100 MHz, $\left.\mathrm{CDCl}_{3}\right)$ of compound $\mathbf{2 u}$

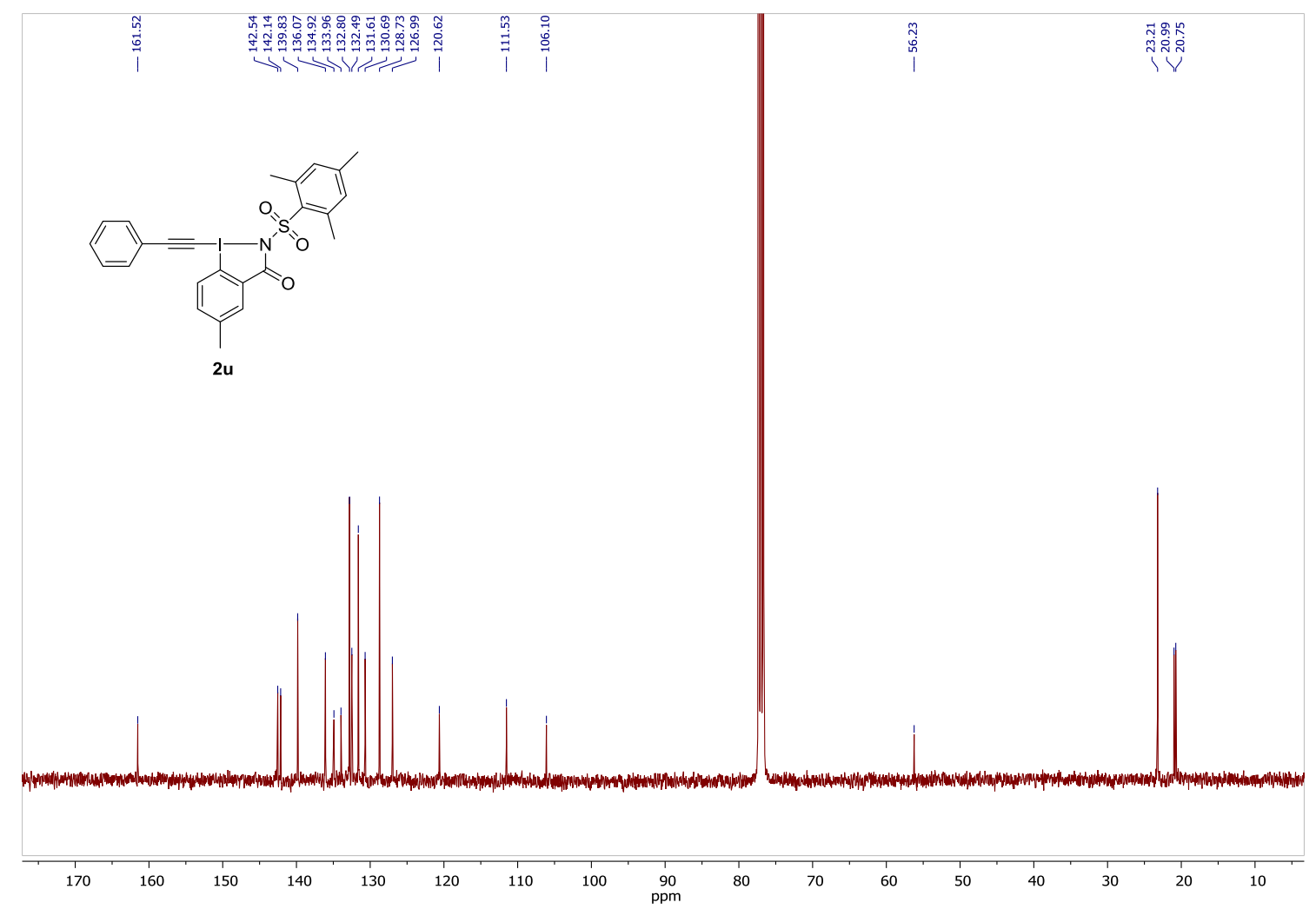


IR of compound $\mathbf{2 u}$

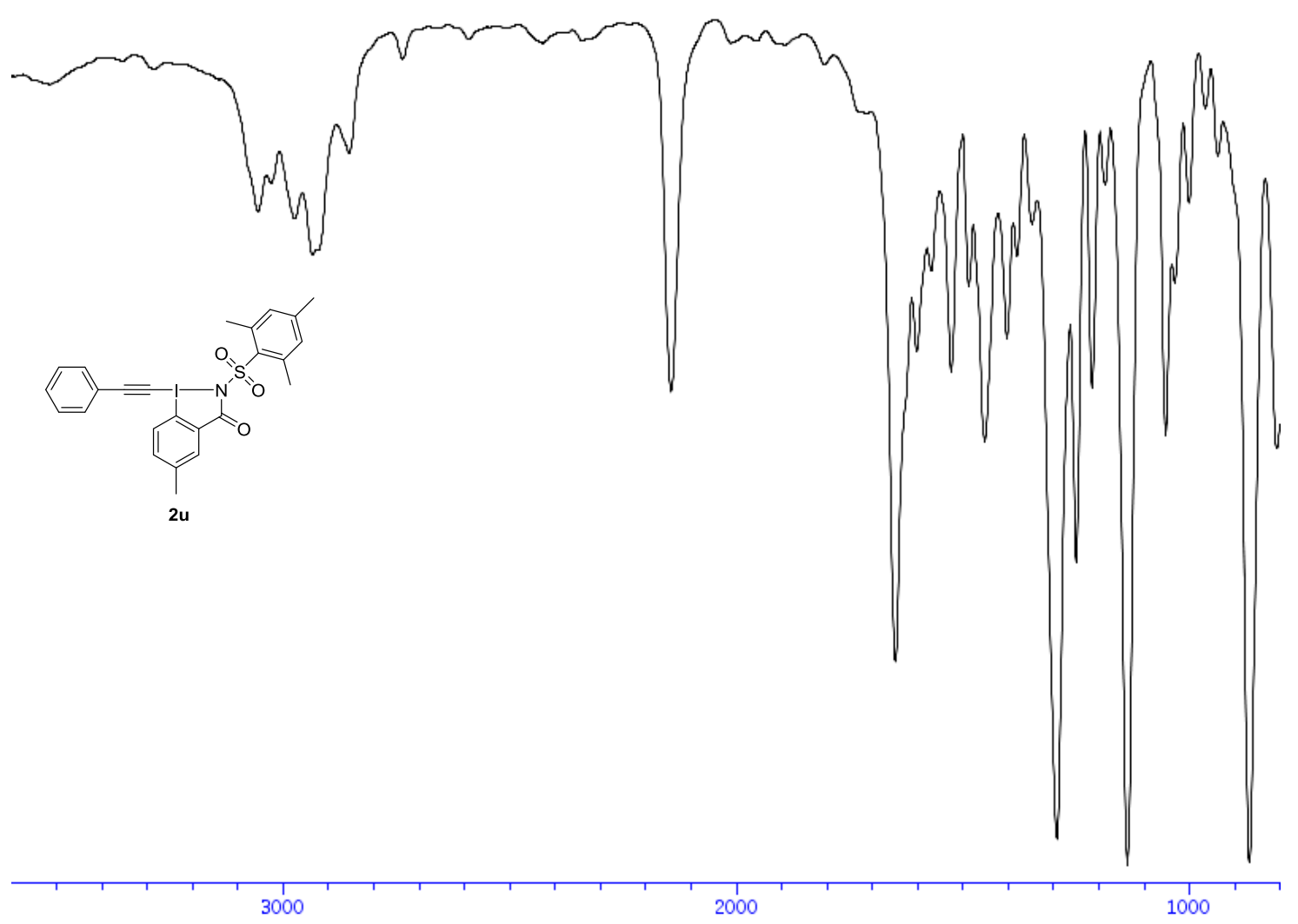


${ }^{1} \mathbf{H}-\mathbf{N M R}\left(400 \mathrm{MHz}, \mathrm{CDCl}_{3}\right.$ ) of compound $\mathbf{2 v}$

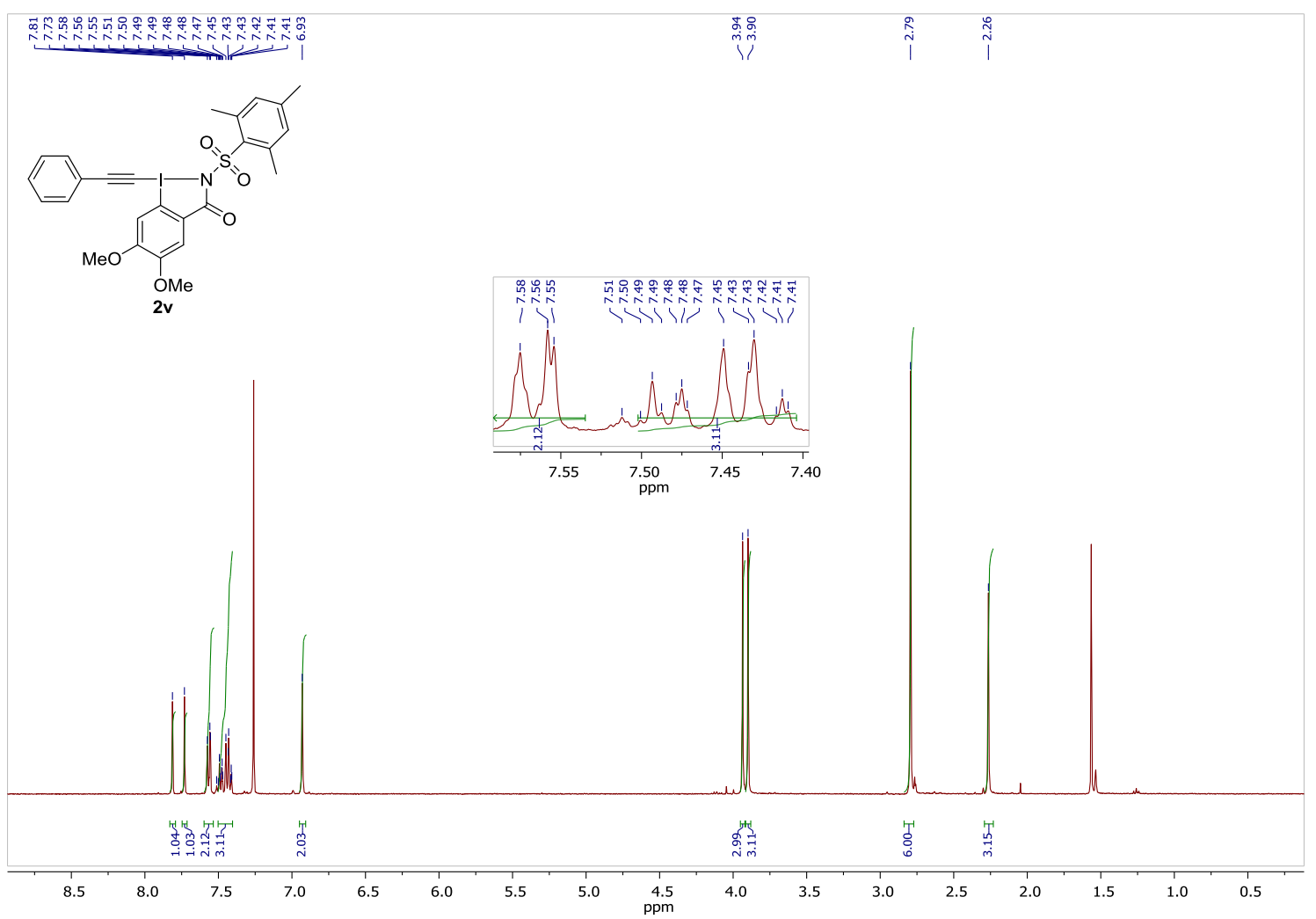

${ }^{13} \mathbf{C}$-NMR (100 MHz, $\left.\mathrm{CDCl}_{3}\right)$ of compound 2v

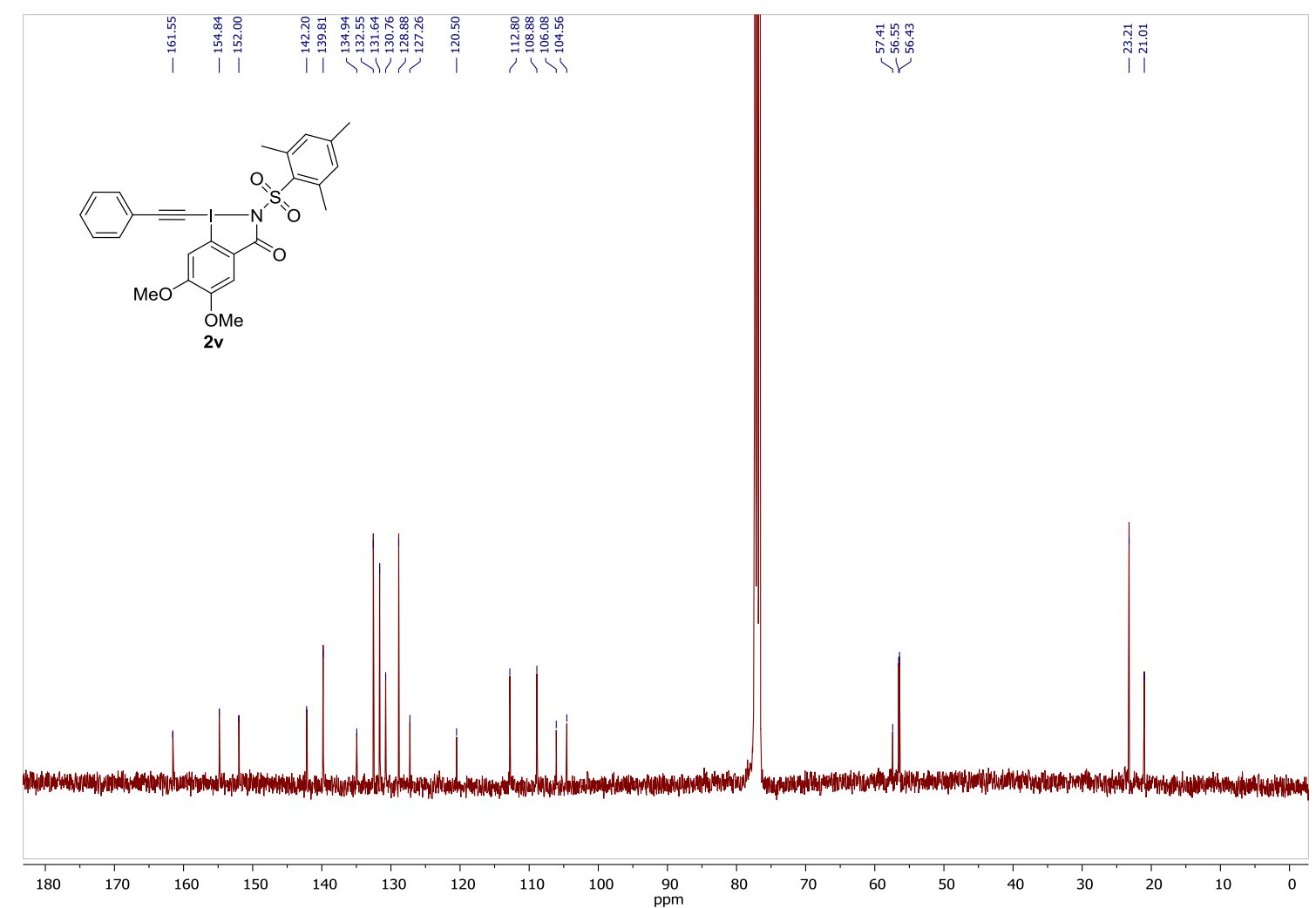


IR of compound $\mathbf{2 v}$

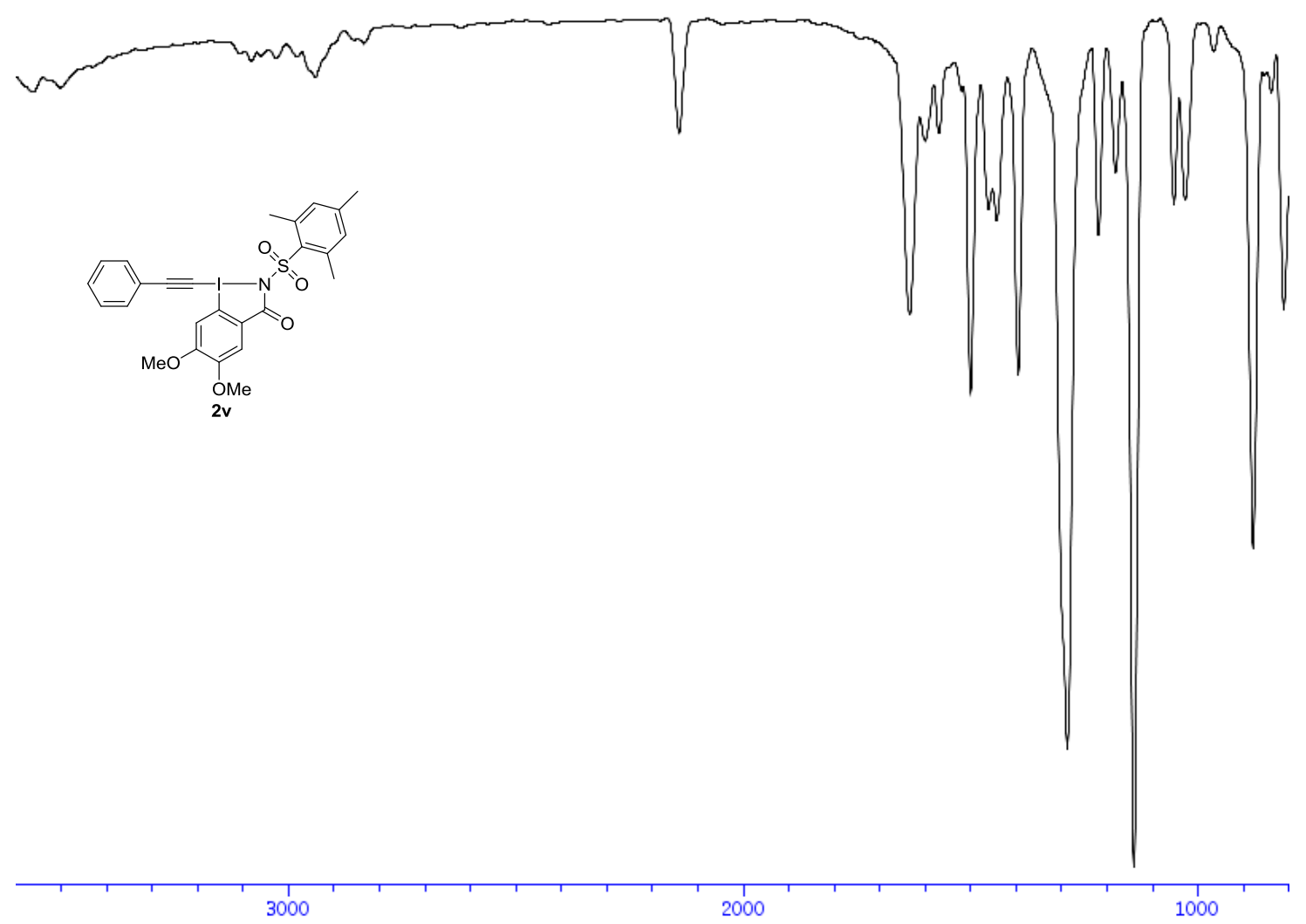


${ }^{\mathbf{1}} \mathbf{H}-\mathbf{N M R}\left(400 \mathrm{MHz}, \mathrm{CDCl}_{3}\right.$ ) of compound 14a

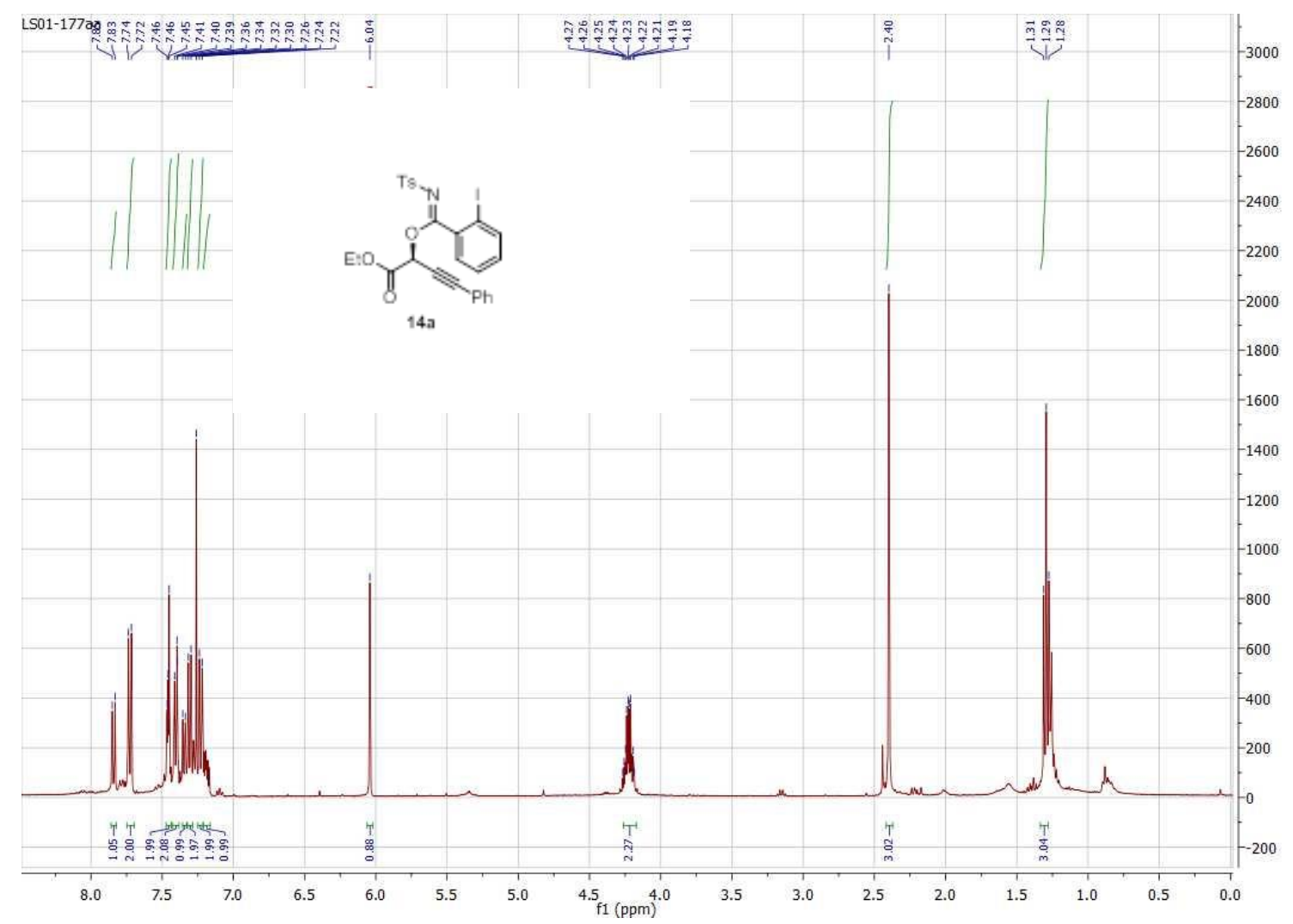

${ }^{13} \mathbf{C}-\mathbf{N M R}\left(100 \mathrm{MHz}, \mathrm{CDCl}_{3}\right)$ of compound 14a

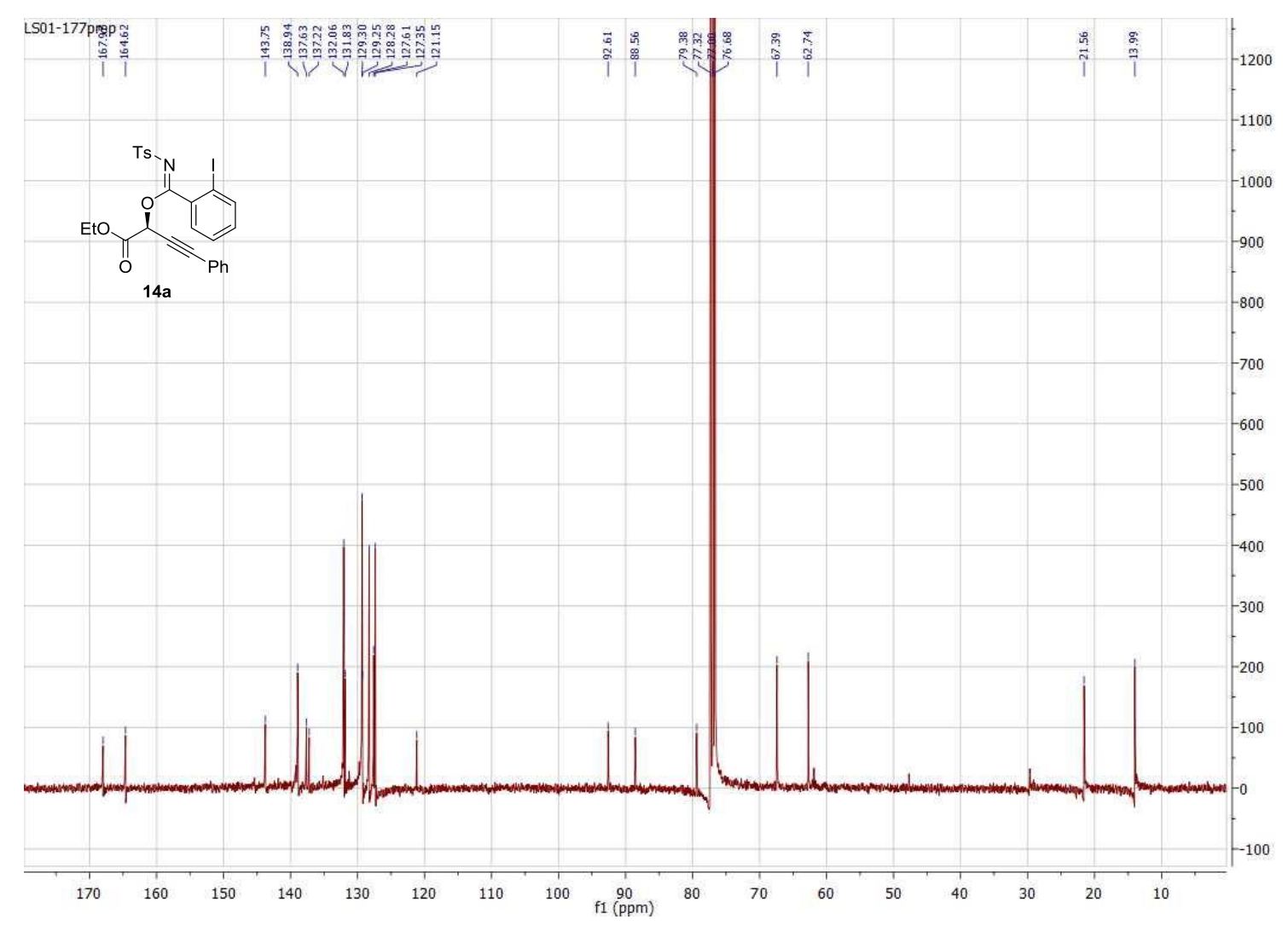


IR of compound 14a

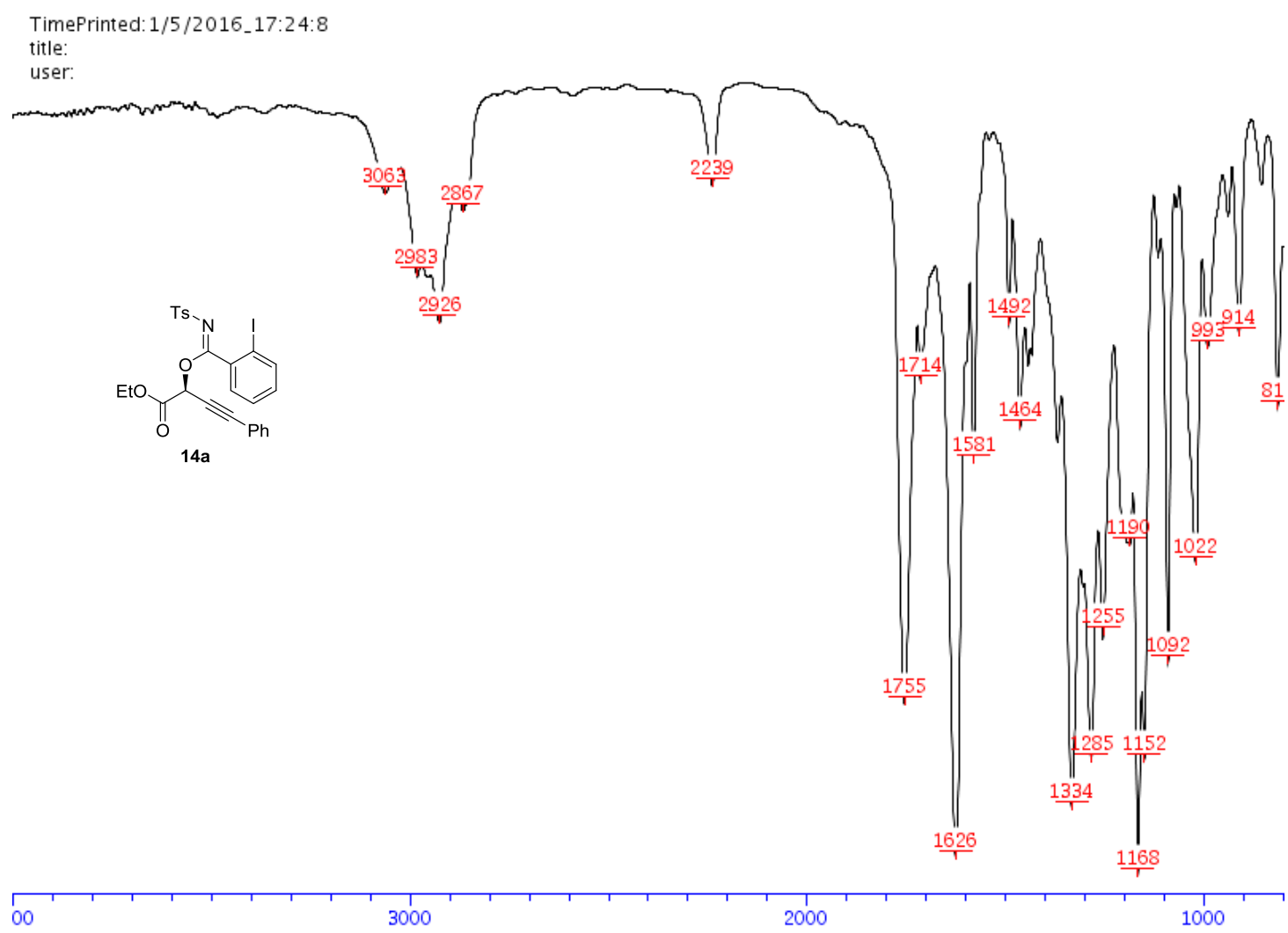


${ }^{\mathbf{1}} \mathbf{H}-\mathbf{N M R}\left(400 \mathrm{MHz}, \mathrm{CDCl}_{3}\right)$ of compound $\mathbf{1 4 b}$

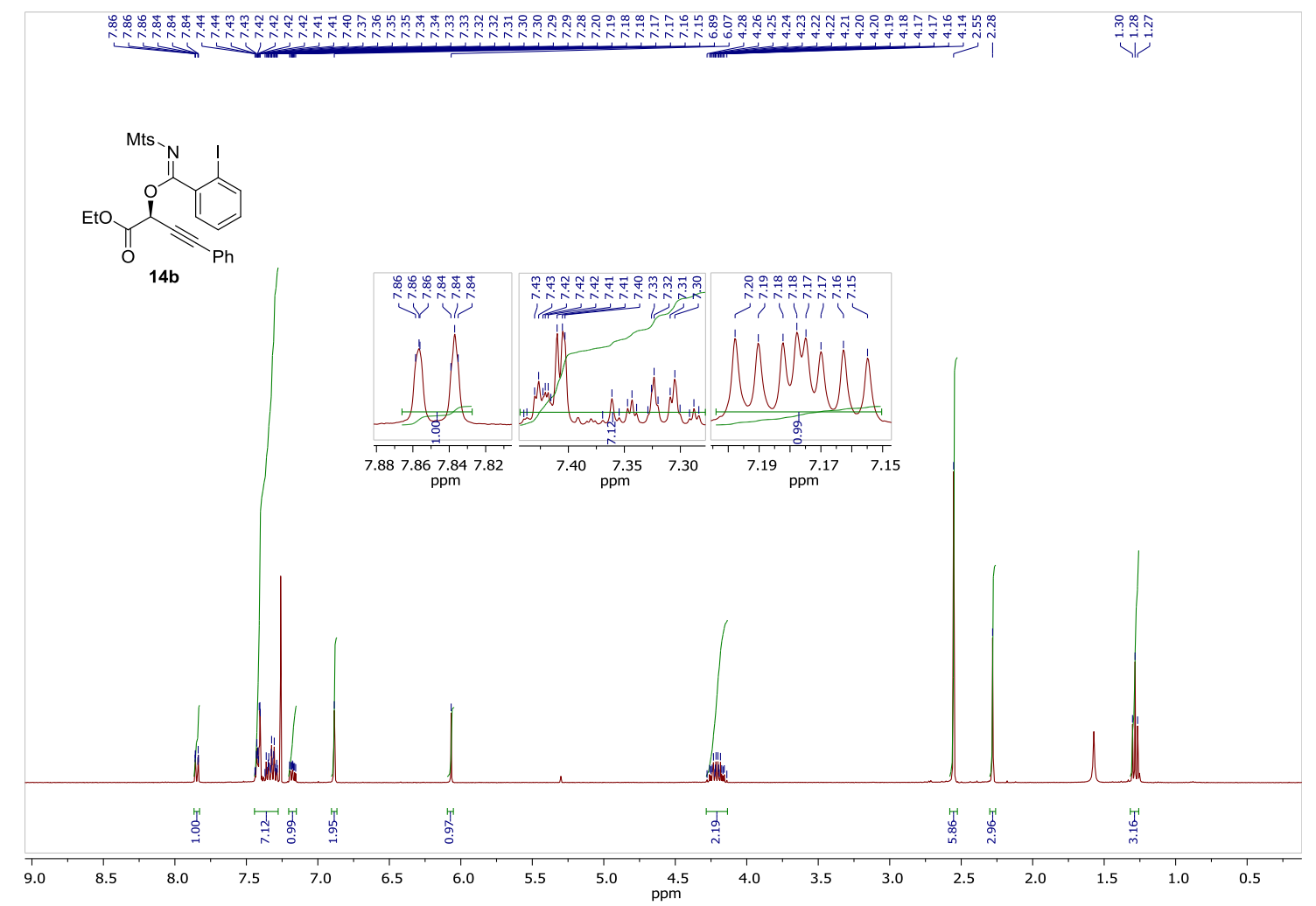

${ }^{13} \mathbf{C}-\mathbf{N M R}\left(100 \mathrm{MHz}, \mathrm{CDCl}_{3}\right)$ of compound $\mathbf{1 4 b}$

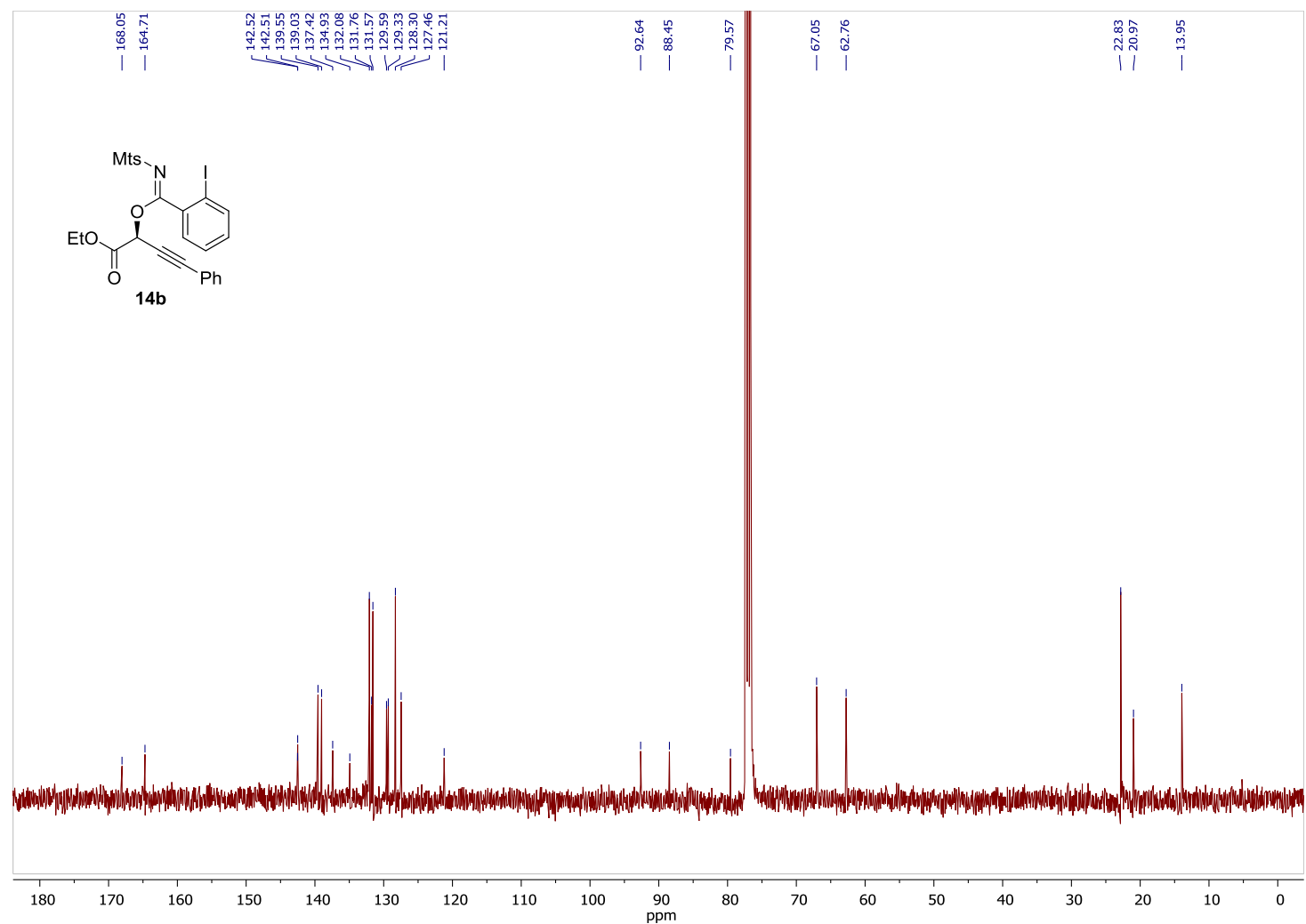


HPLC of compound 14b
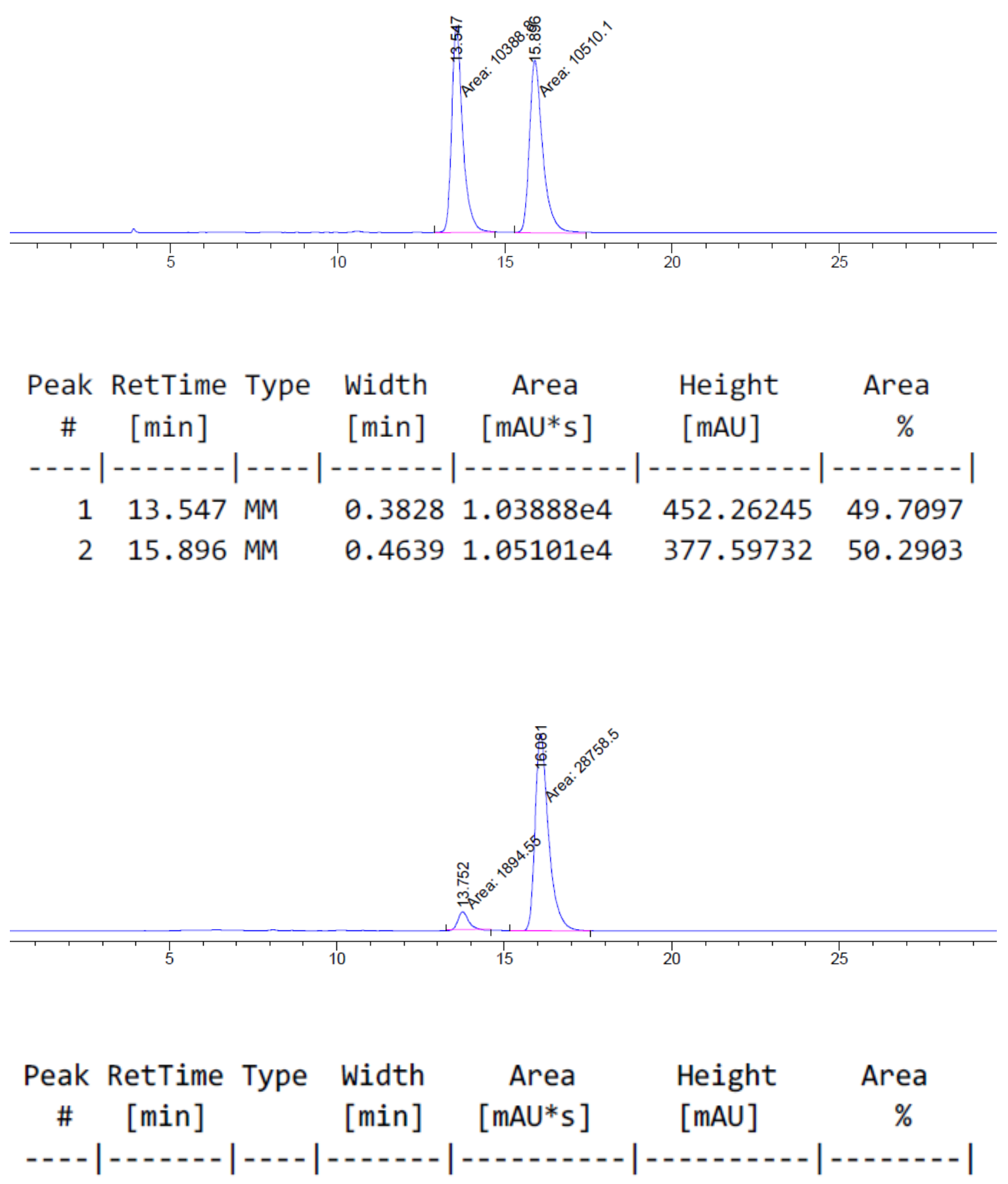

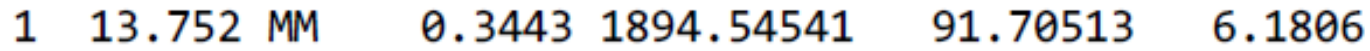

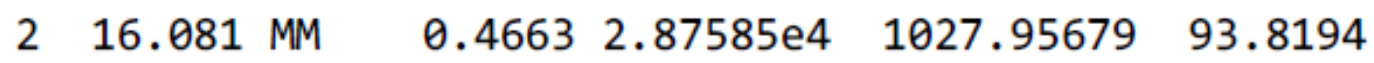


IR of compound 14b

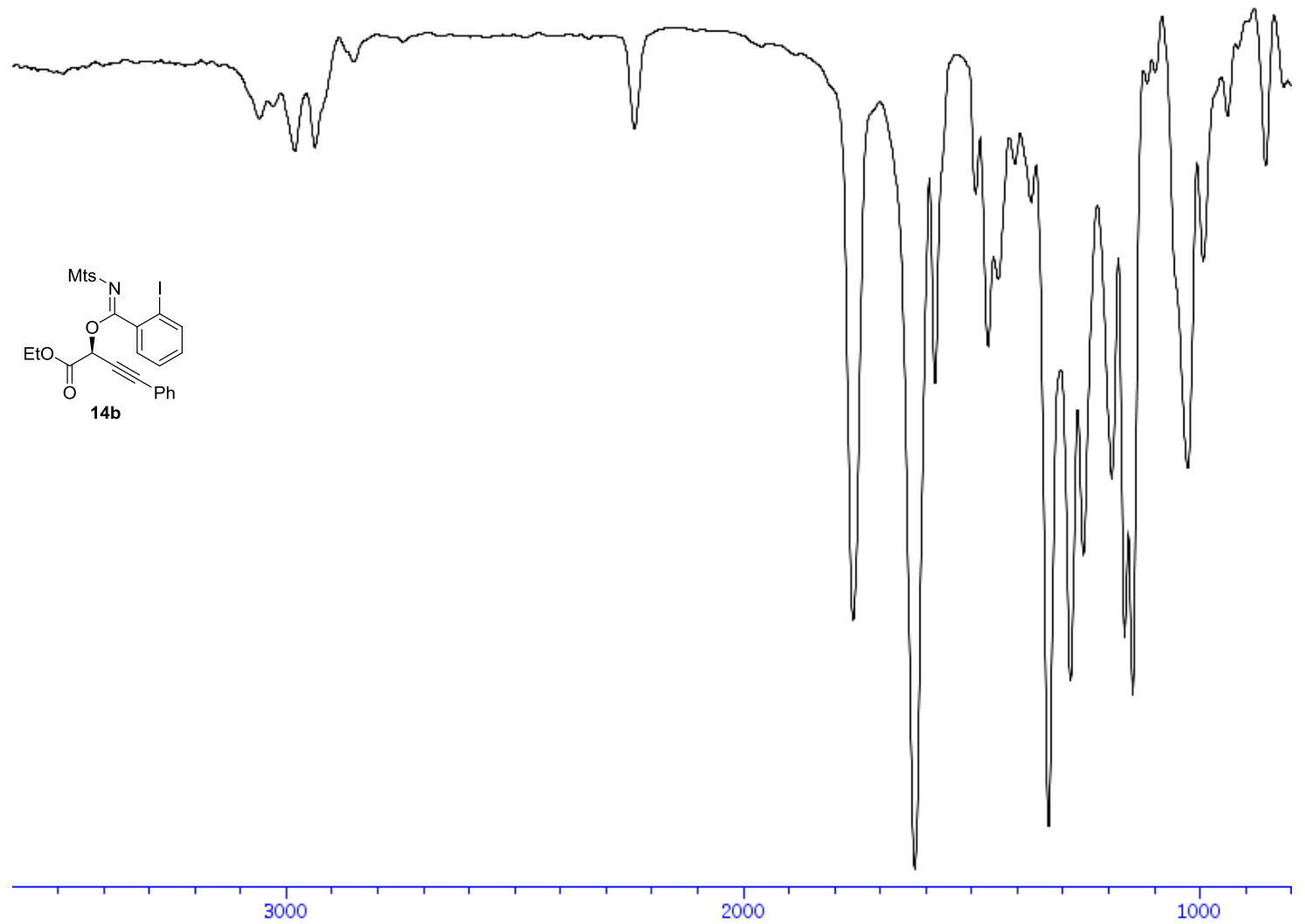


${ }^{\mathbf{1}} \mathbf{H}-\mathbf{N M R}\left(400 \mathrm{MHz}, \mathrm{CDCl}_{3}\right)$ of compound $\mathbf{1 4 c}$

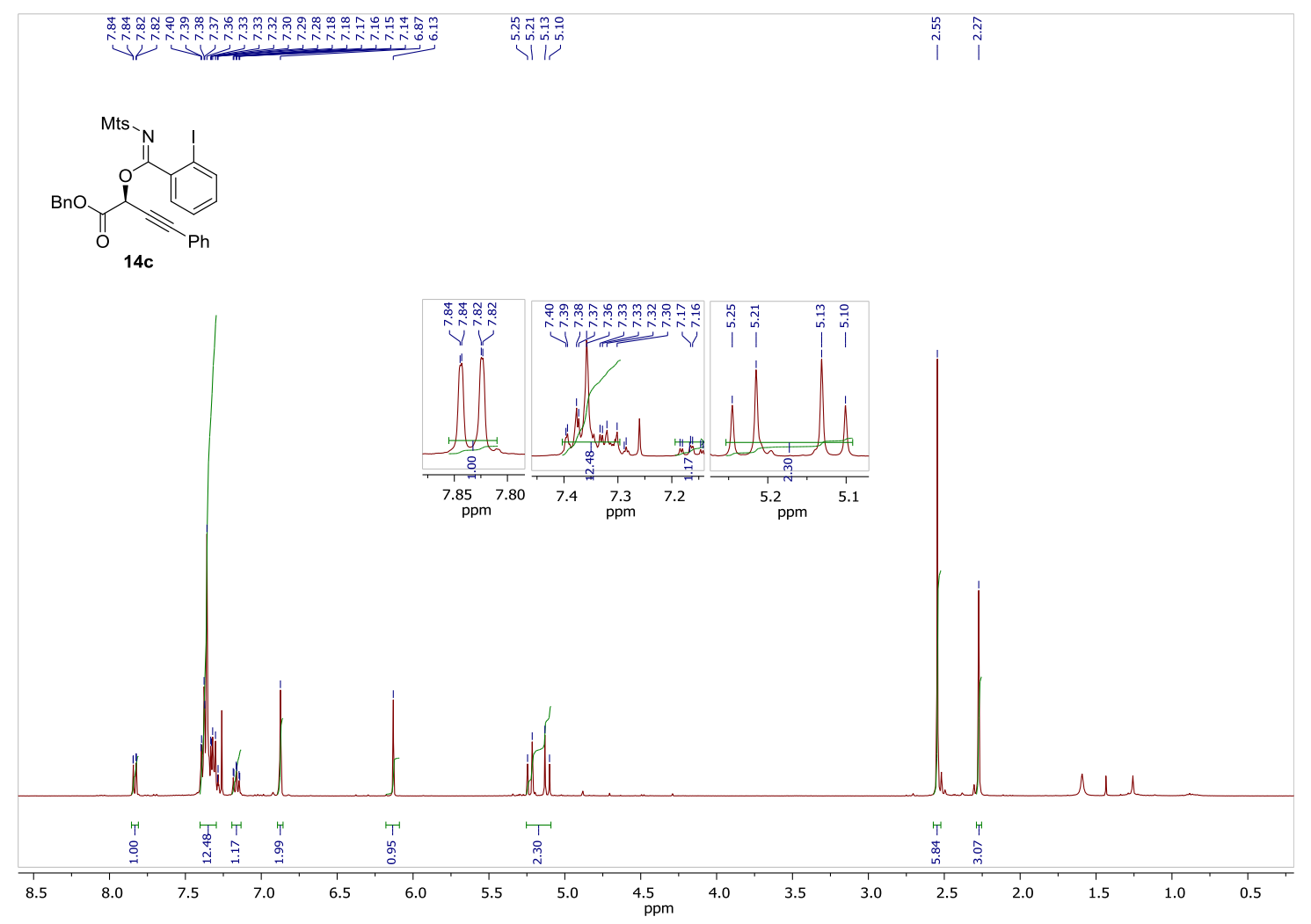

${ }^{13} \mathbf{C}$-NMR (100 MHz, $\left.\mathrm{CDCl}_{3}\right)$ of compound $\mathbf{1 4 c}$

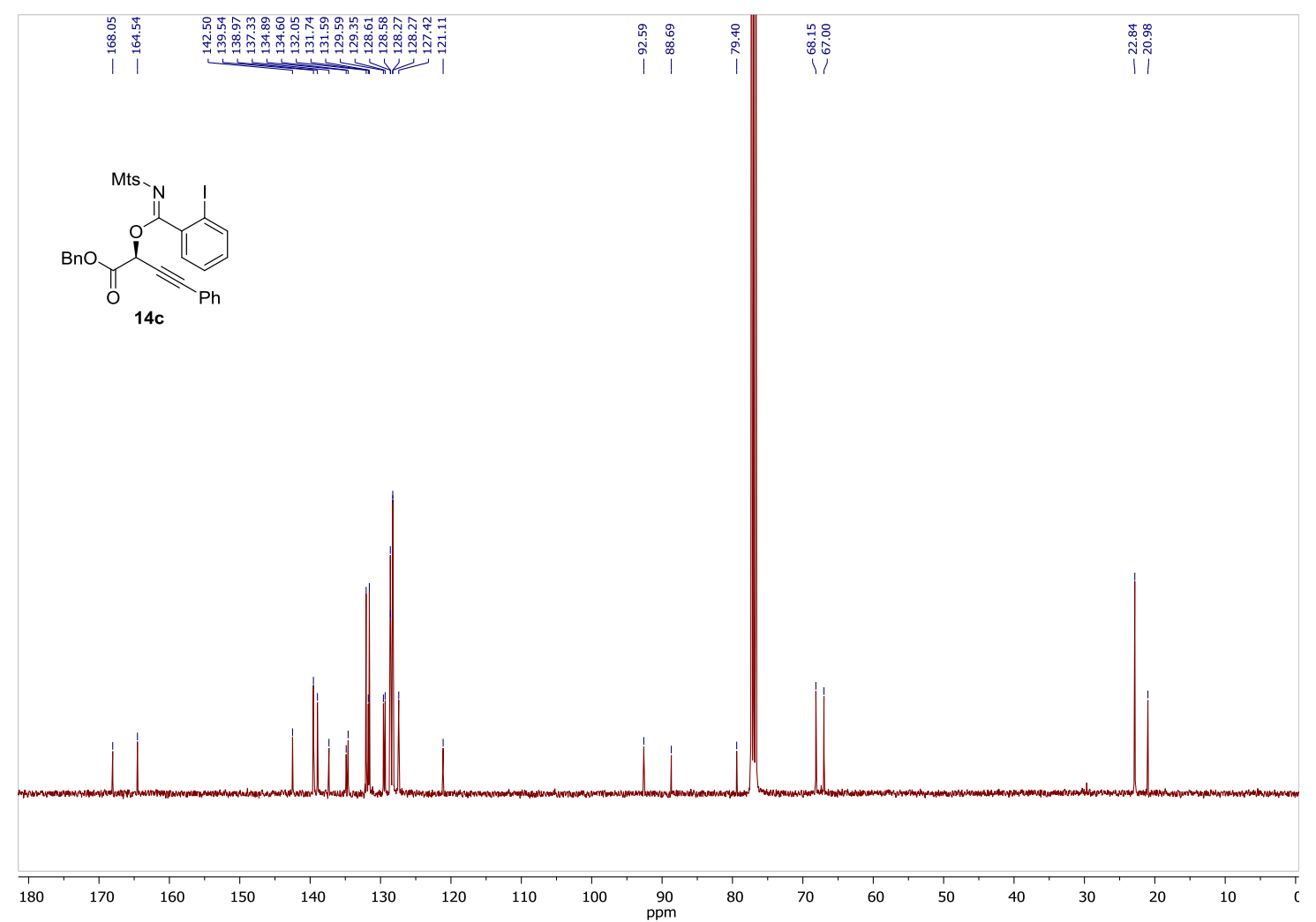


HPLC of compound 14c

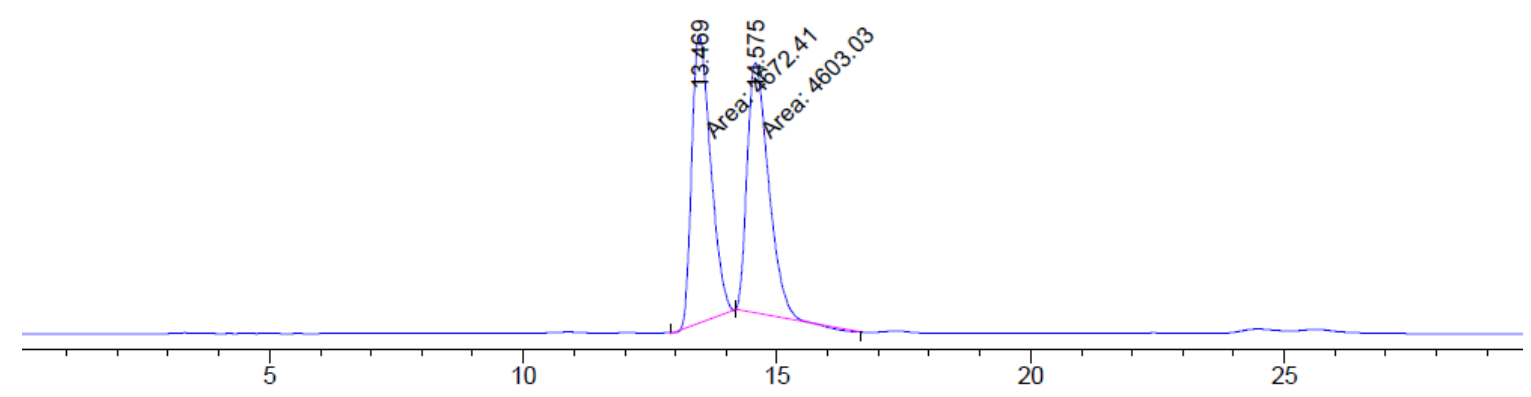

\begin{tabular}{cccccc}
$\begin{array}{c}\text { Peak RetTime Type } \\
\text { \# } \\
{[\text { min] }}\end{array}$ & $\begin{array}{c}\text { Width } \\
{[\mathrm{min}]}\end{array}$ & $\begin{array}{c}\text { Area } \\
{[\mathrm{mAU} \text { s] }}\end{array}$ & $\begin{array}{c}\text { Height } \\
{[\mathrm{mAU}]}\end{array}$ & $\begin{array}{c}\text { Area } \\
\%\end{array}$ \\
\hline 1 & $13.469 \mathrm{MM}$ & 0.4316 & 4672.41211 & 180.43593 & 50.3740 \\
2 & $14.575 \mathrm{MM}$ & 0.4915 & 4603.03125 & 156.08658 & 49.6260
\end{tabular}

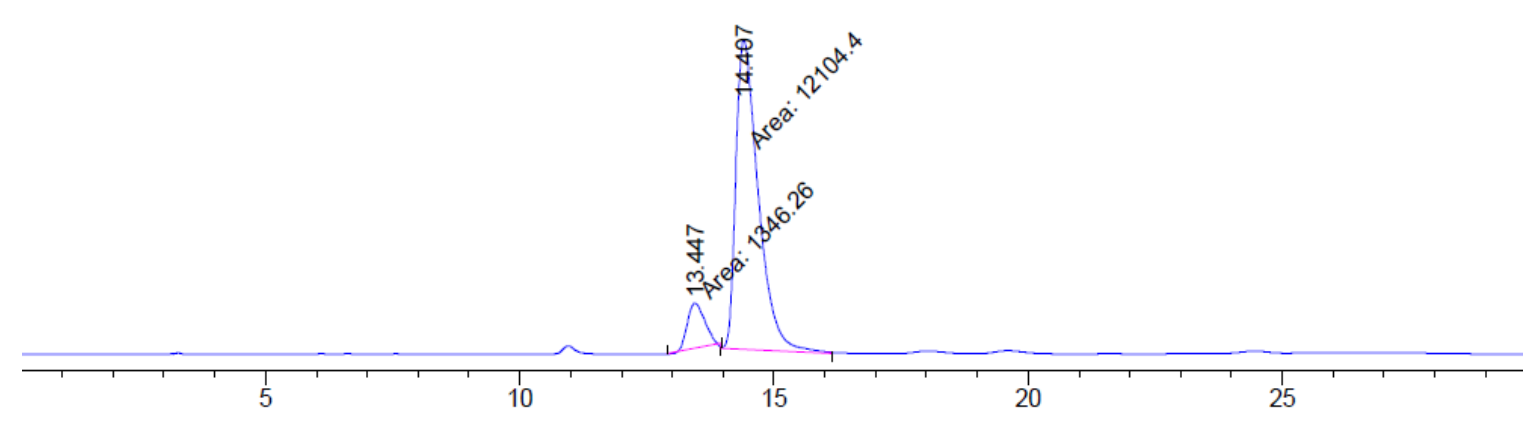

\begin{tabular}{cccccc}
$\begin{array}{c}\text { Peak RetTime Type } \\
\text { \# }\end{array}$ & $\begin{array}{c}\text { Width } \\
{[\text { min] }}\end{array}$ & $\begin{array}{c}\text { Area } \\
{[\text { min] }}\end{array}$ & $\begin{array}{c}\text { Height } \\
{[\text { mAU*s }]}\end{array}$ & $\begin{array}{c}\text { Area } \\
{[\mathrm{mAU}]}\end{array}$ & $\%$ \\
\hline 1 & 13.447 MM & 0.3915 & 1346.25659 & 57.30646 & 10.0089 \\
2 & 14.407 MM & 0.5109 & $1.21044 \mathrm{e} 4$ & 394.85516 & 89.9911
\end{tabular}


IR of compound $14 c$

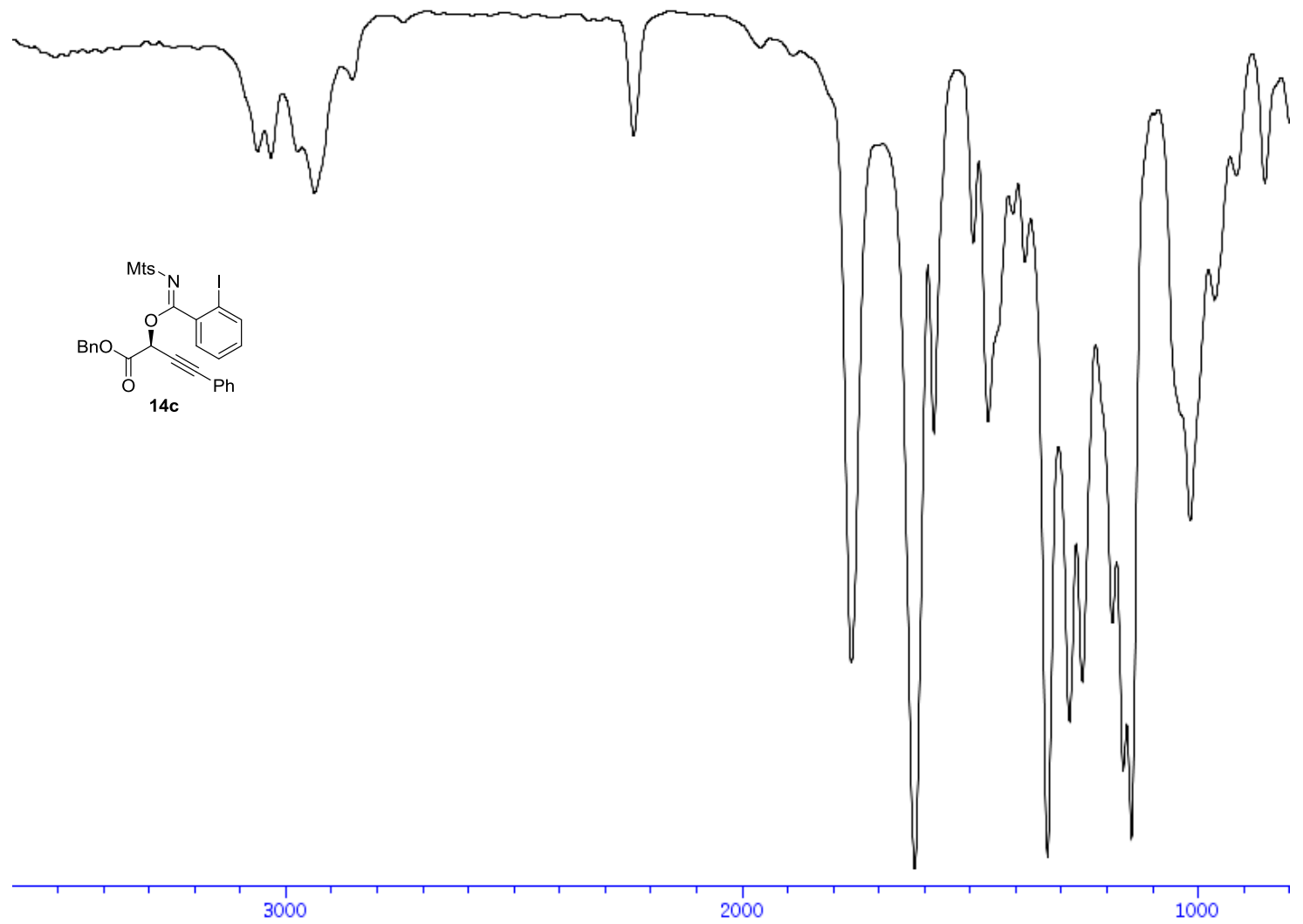


${ }^{\mathbf{1}} \mathbf{H}-\mathbf{N M R}\left(400 \mathrm{MHz}, \mathrm{CDCl}_{3}\right)$ of compound 14d

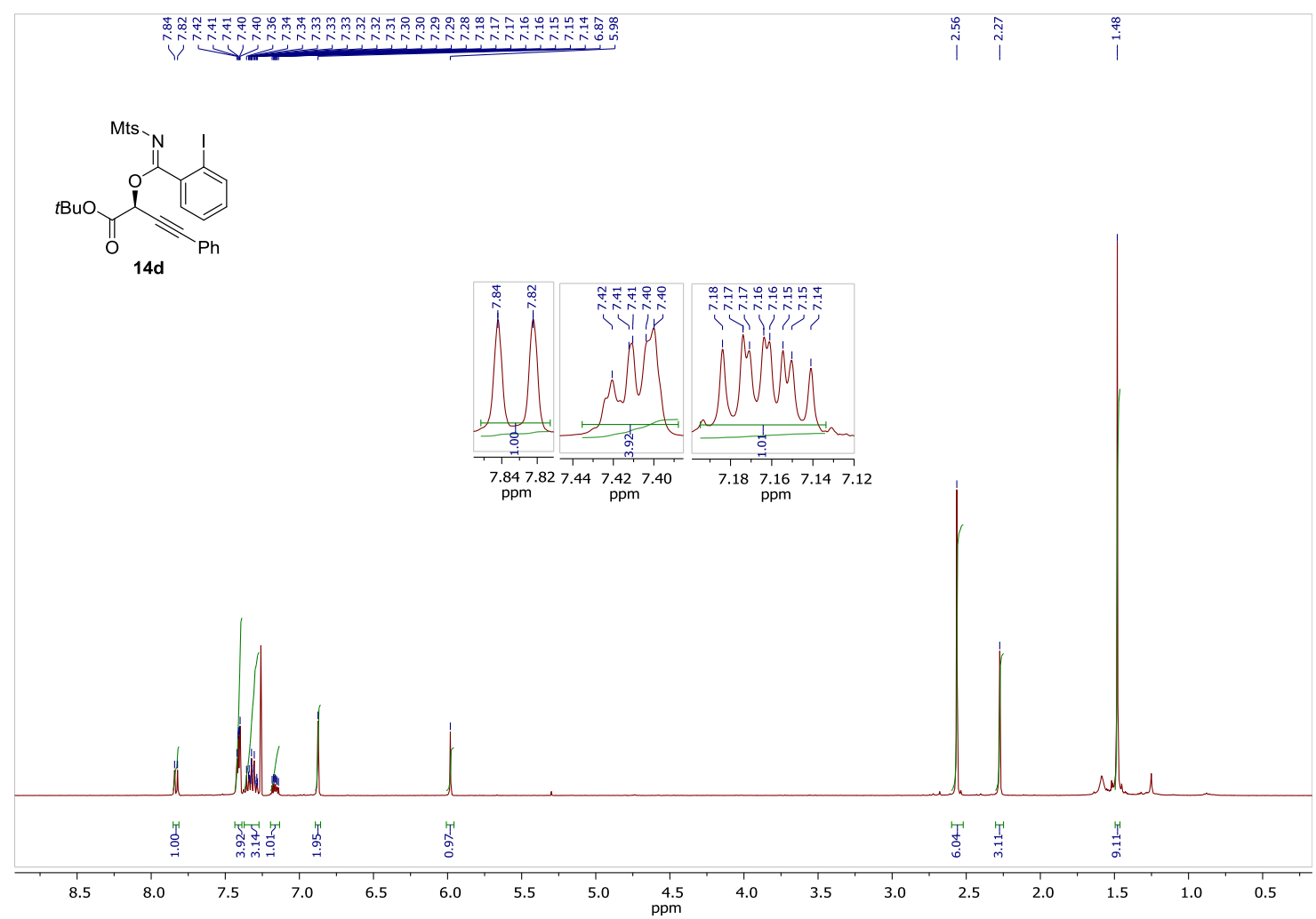

${ }^{13} \mathbf{C}-\mathbf{N M R}\left(100 \mathrm{MHz}, \mathrm{CDCl}_{3}\right)$ of compound $\mathbf{1 4 d}$

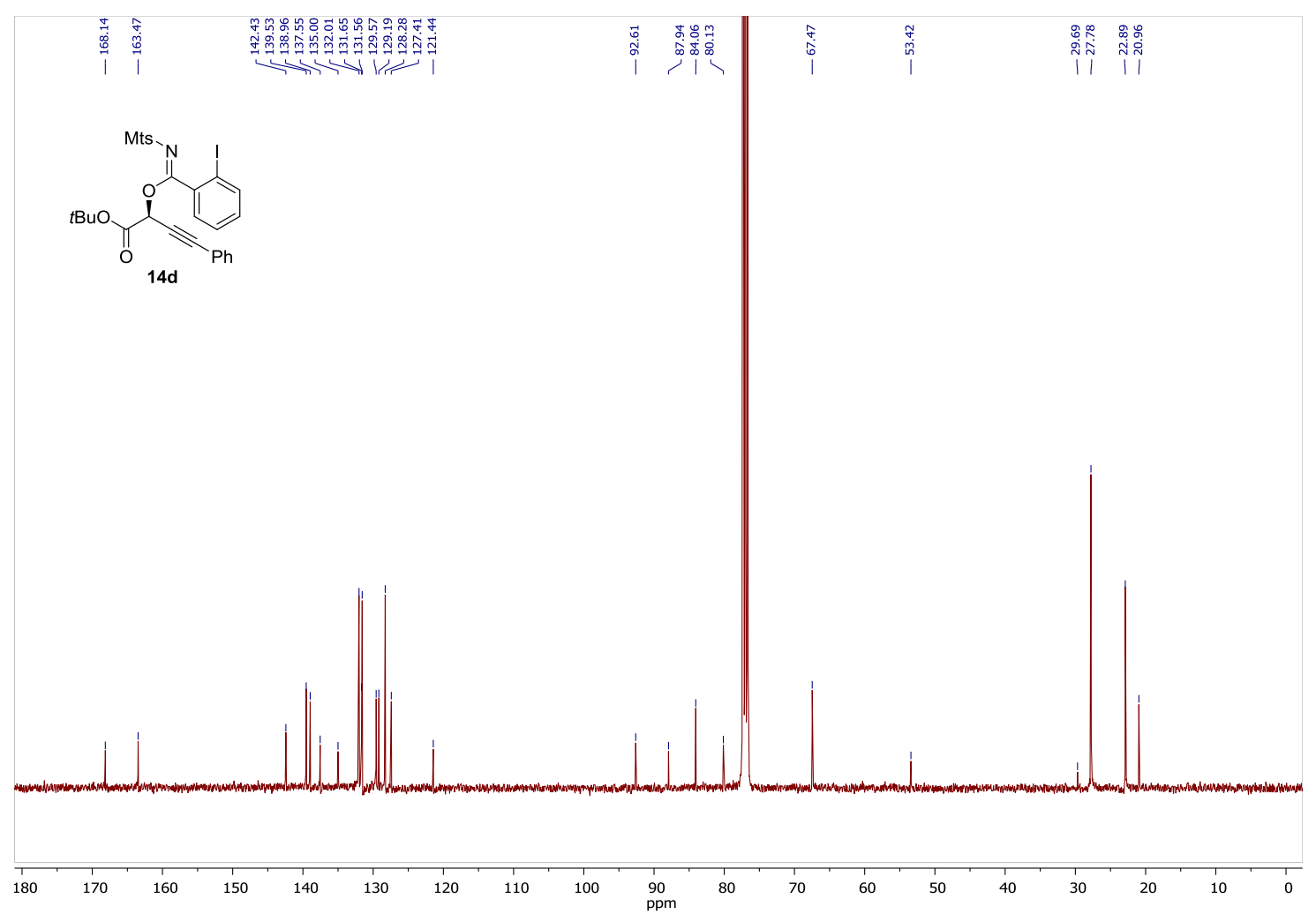


HPLC of compound 14d

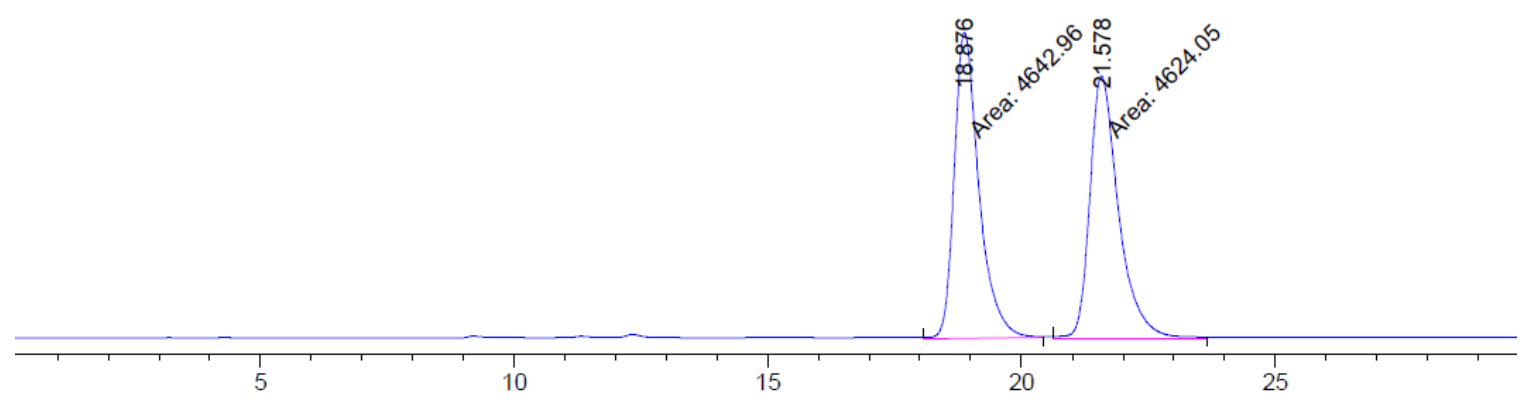

$$
\begin{aligned}
& \text { Peak RetTime Type Width Area Height Area }
\end{aligned}
$$

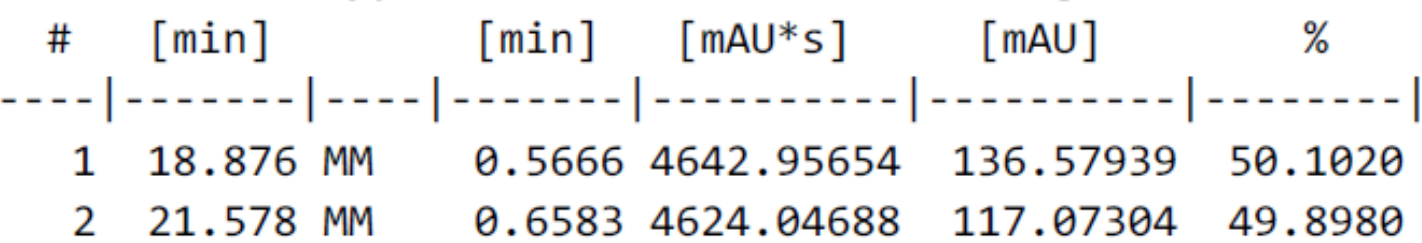

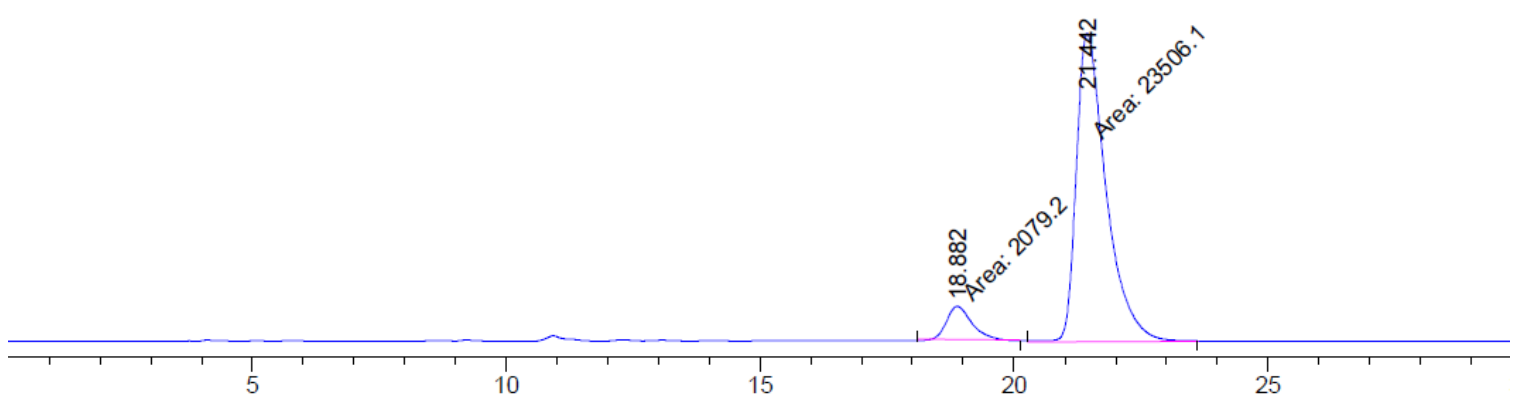

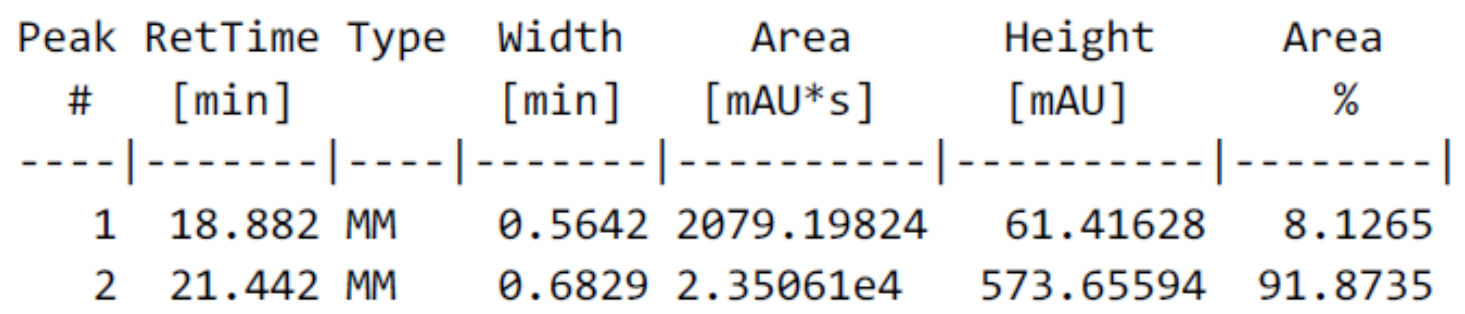


IR of compound 14d

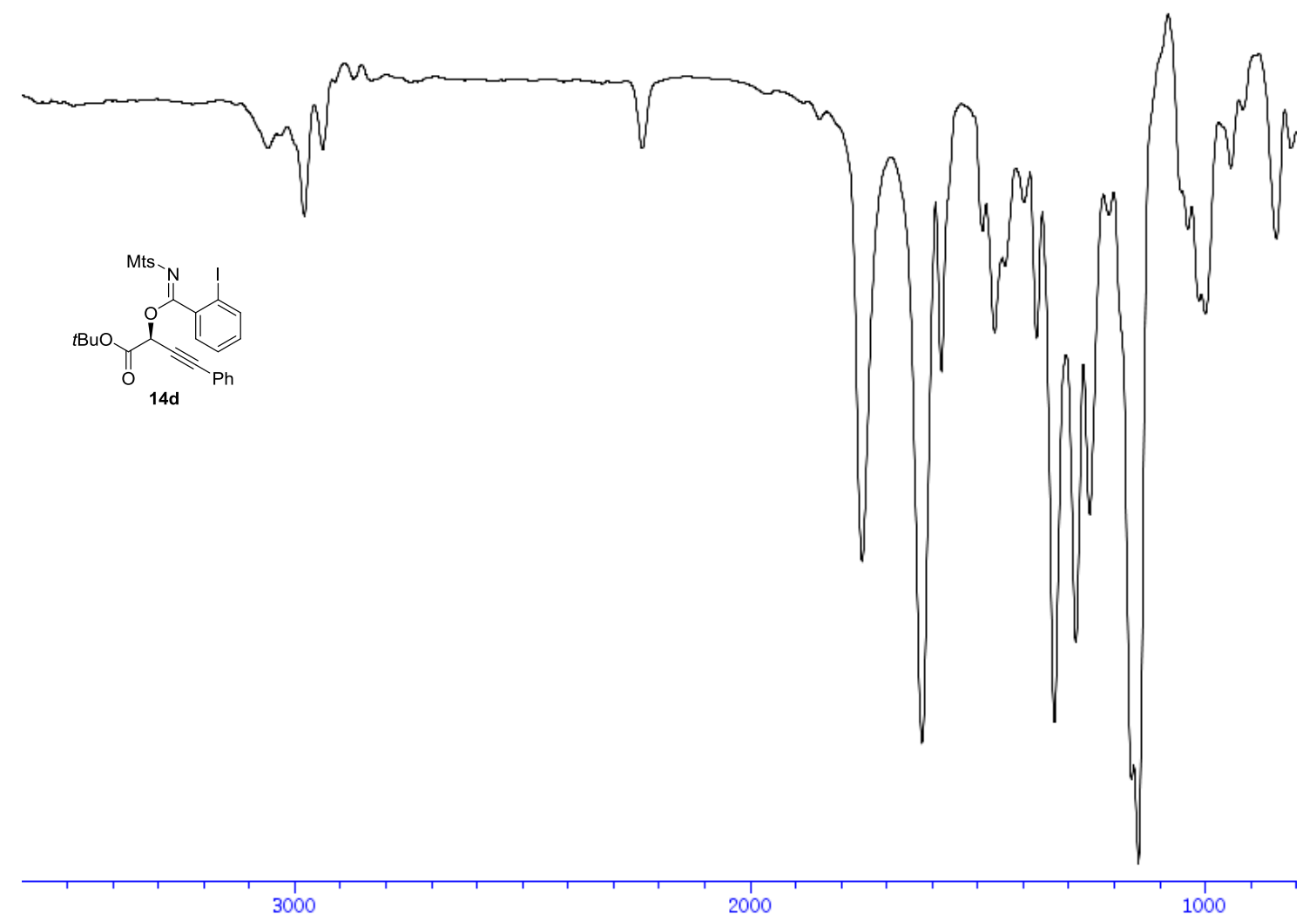


${ }^{\mathbf{1}} \mathbf{H}$-NMR $\left(400 \mathrm{MHz}, \mathrm{CDCl}_{3}\right)$ of compound $\mathbf{1 4 e}$

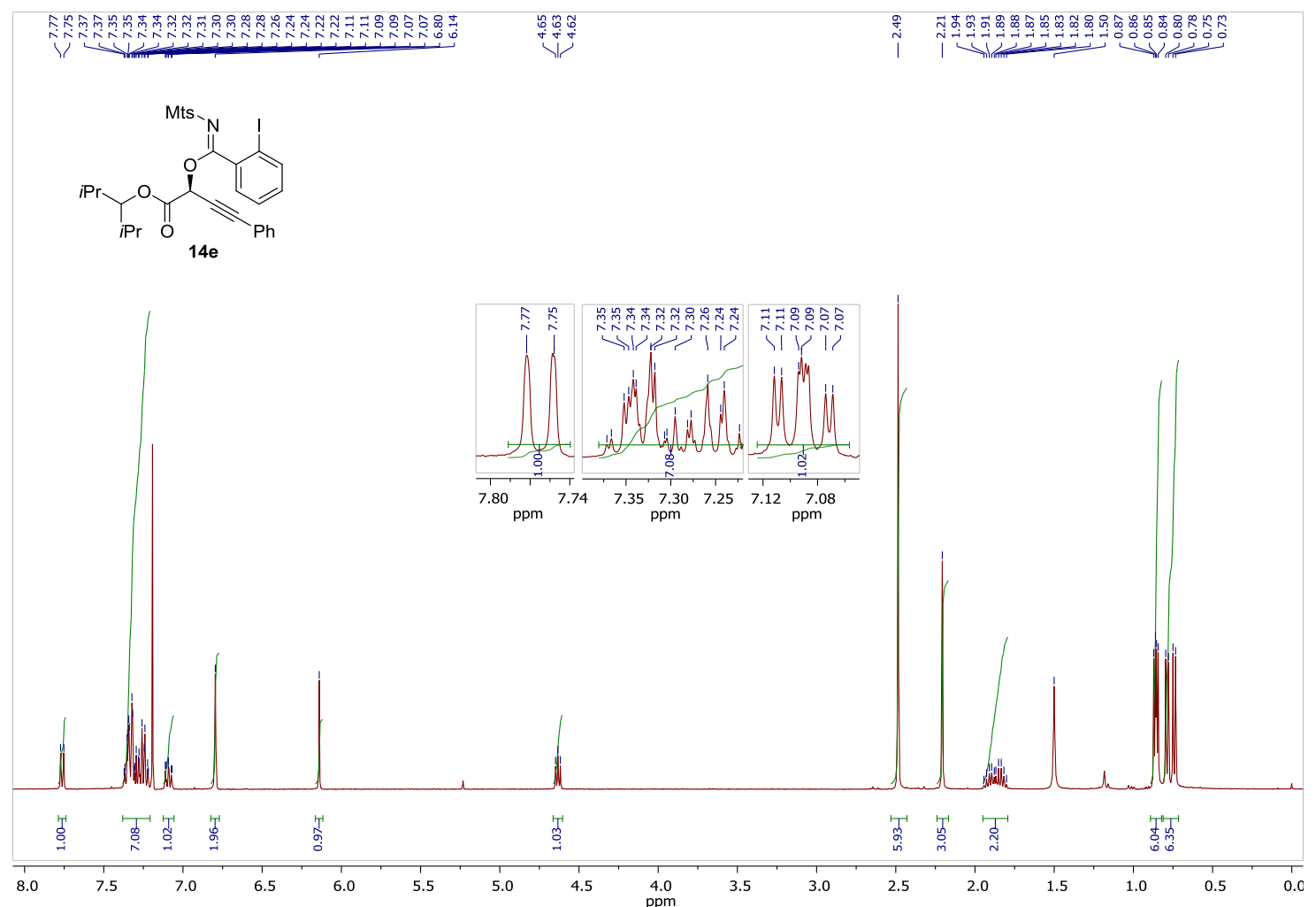

${ }^{13} \mathbf{C}-\mathbf{N M R}\left(100 \mathrm{MHz}, \mathrm{CDCl}_{3}\right)$ of compound $\mathbf{1 4 e}$

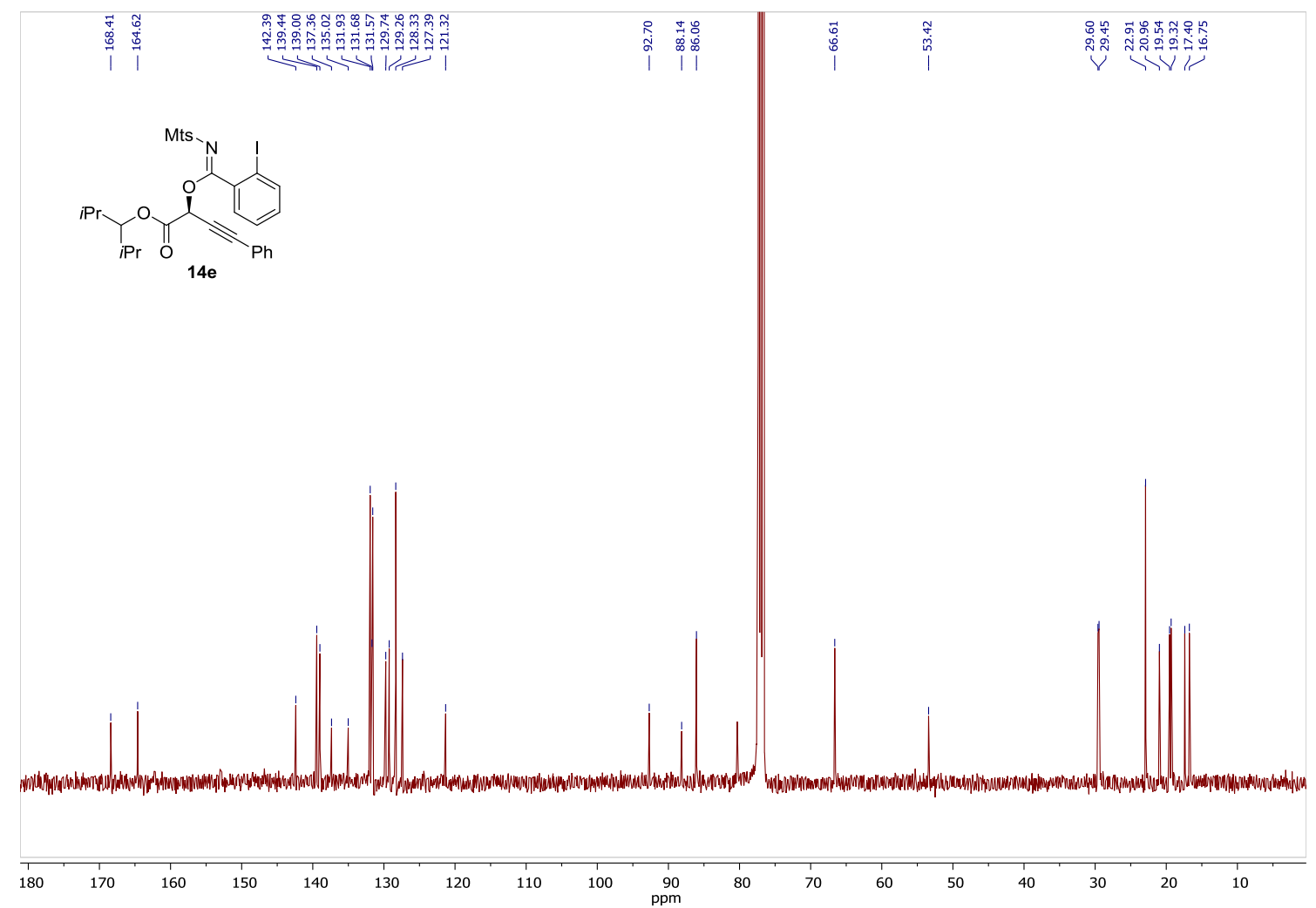


HPLC of compound 14e

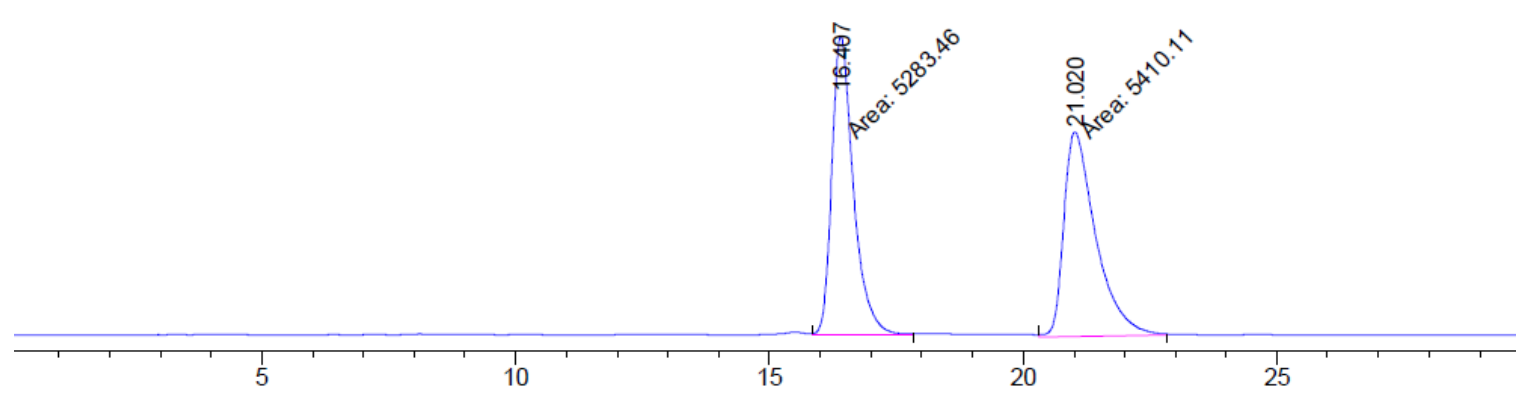

\begin{tabular}{|c|c|c|c|c|c|c|}
\hline $\begin{array}{c}\text { Peak } \\
\#\end{array}$ & $\begin{array}{c}\text { RetTime } \\
\text { [min] }\end{array}$ & Type & $\begin{array}{l}\text { Width } \\
\text { [min] }\end{array}$ & $\begin{array}{c}\text { Area } \\
{\left[\mathrm{mAU}^{*} \mathrm{~s}\right]}\end{array}$ & $\begin{array}{l}\text { Height } \\
\text { [mAU] }\end{array}$ & $\begin{array}{c}\text { Area } \\
\%\end{array}$ \\
\hline & & & & 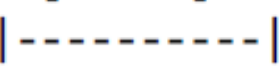 & -- & -- \\
\hline 1 & 16.407 & MM & 0.4906 & 5283.46338 & 179.49147 & 49.4079 \\
\hline 2 & 21.020 & MM & 0.7325 & 5410.10693 & 123.09530 & 50.5921 \\
\hline
\end{tabular}

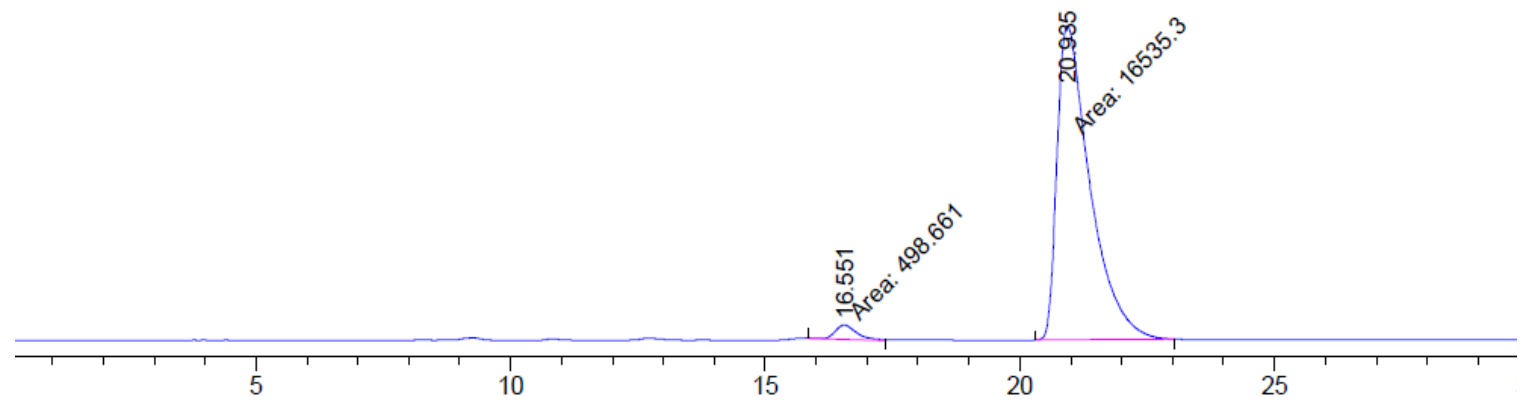

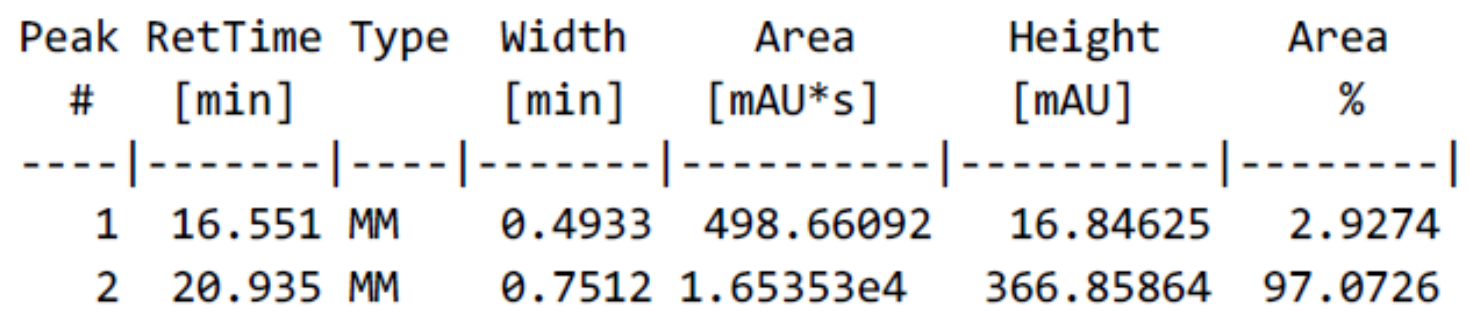


IR of compound $14 e$

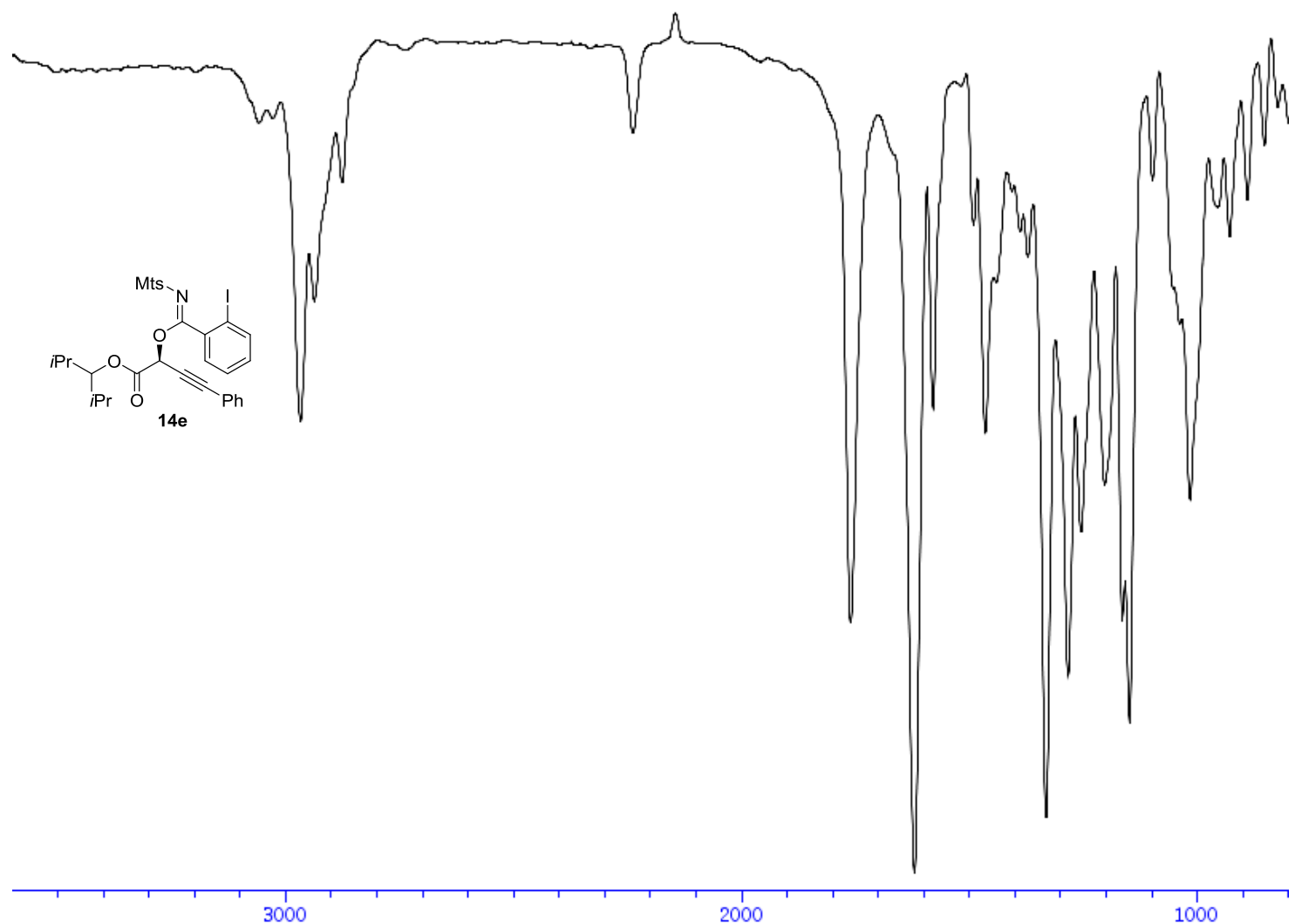


${ }^{\mathbf{1}} \mathbf{H}-\mathbf{N M R}\left(400 \mathrm{MHz}, \mathrm{CDCl}_{3}\right)$ of compound $\mathbf{1 4 f}$

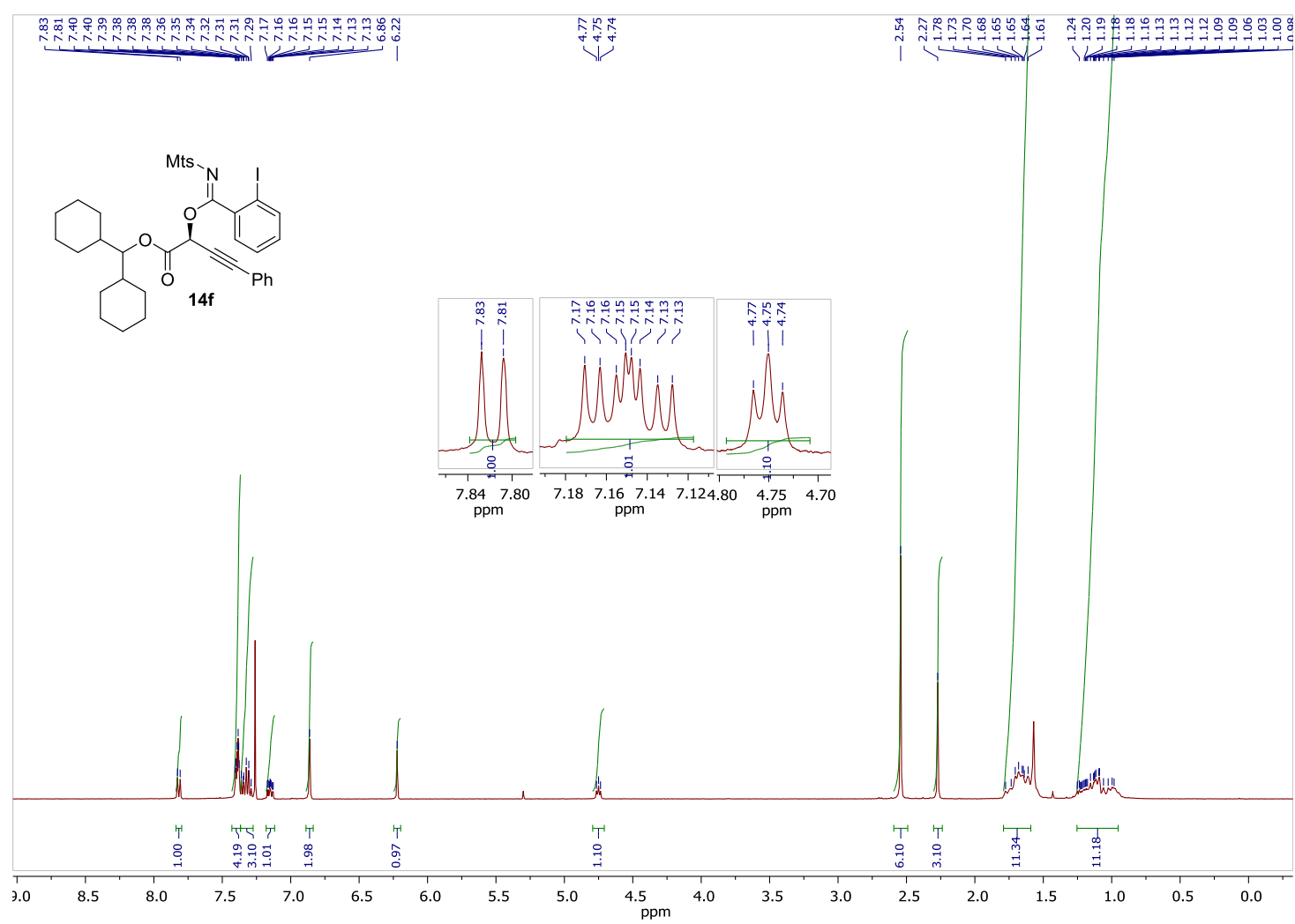

${ }^{13} \mathbf{C}-\mathrm{NMR}\left(100 \mathrm{MHz}, \mathrm{CDCl}_{3}\right)$ of compound $\mathbf{1 4 f}$

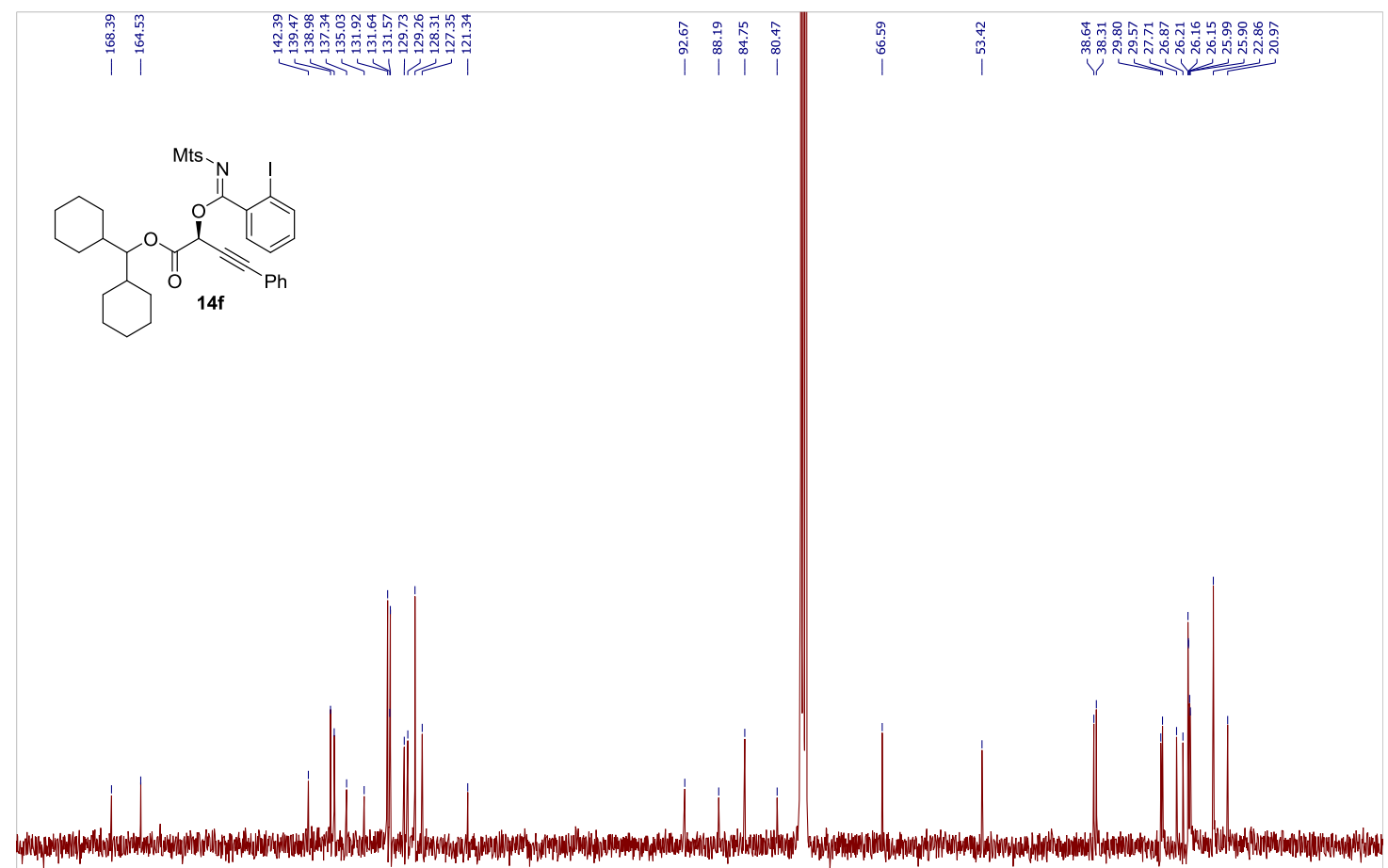

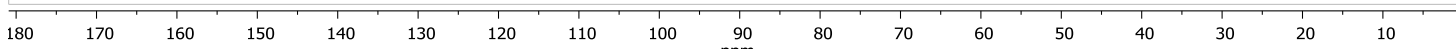


HPLC of compound $\mathbf{1 4 f}$

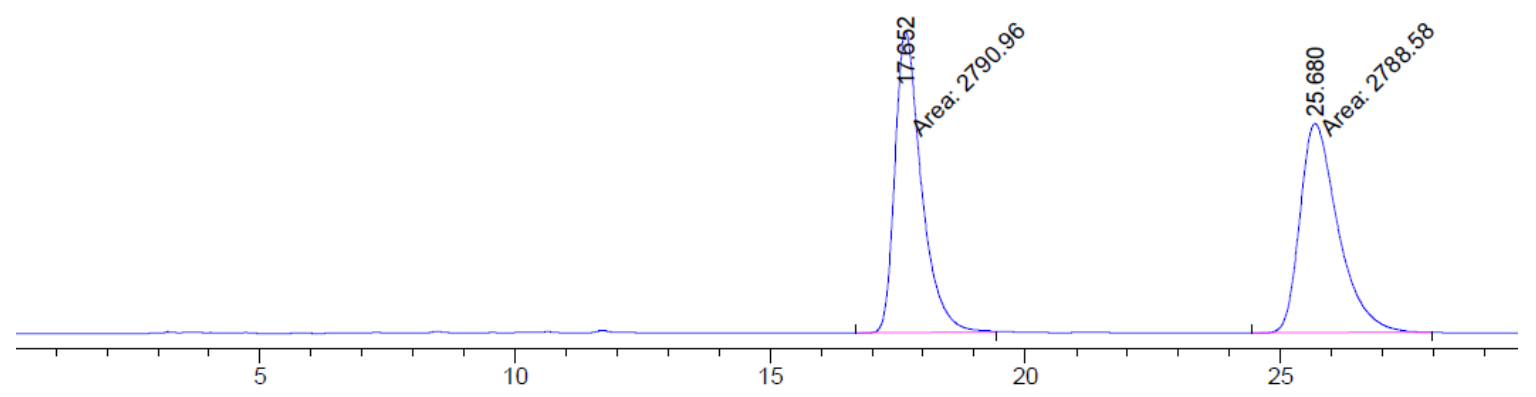

\begin{tabular}{|c|c|c|c|c|c|c|}
\hline $\begin{array}{c}\text { Peak } \\
\#\end{array}$ & $\begin{array}{c}\text { RetTime } \\
\text { [min] }\end{array}$ & Type & $\begin{array}{l}\text { Width } \\
\text { [min] }\end{array}$ & $\begin{array}{c}\text { Area } \\
{\left[\mathrm{mAU}^{*} \mathrm{~s}\right]}\end{array}$ & $\begin{array}{l}\text { Height } \\
{[\mathrm{mAU}]}\end{array}$ & $\begin{array}{c}\text { Area } \\
\%\end{array}$ \\
\hline & & & & & & \\
\hline 1 & 17.652 & MM & 13 & 2790.95996 & 77.36147 & 50.0213 \\
\hline 2 & 25.680 & MM & .8653 & 2788.58301 & 53.71205 & 49.9787 \\
\hline
\end{tabular}

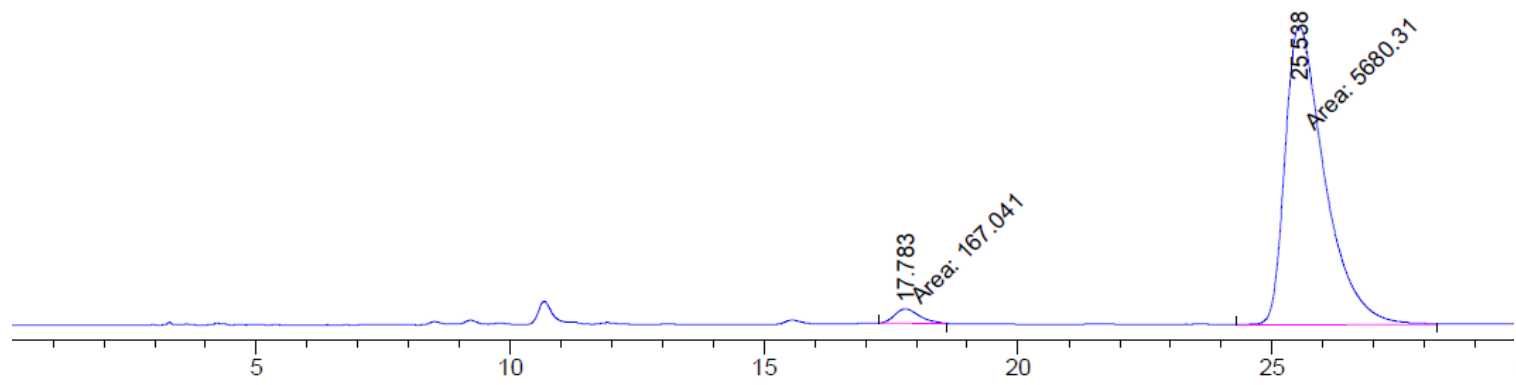

\begin{tabular}{|c|c|c|c|c|c|c|}
\hline $\begin{array}{c}\text { Peak } \\
\#\end{array}$ & $\begin{array}{c}\text { RetTime } \\
\text { [min] }\end{array}$ & Type & $\begin{array}{l}\text { Width } \\
\text { [min] }\end{array}$ & $\begin{array}{c}\text { Area } \\
{\left[\mathrm{mAU}^{*} \mathrm{~s}\right]}\end{array}$ & $\begin{array}{l}\text { Height } \\
{[\mathrm{mAU}]}\end{array}$ & $\begin{array}{c}\text { Area } \\
\%\end{array}$ \\
\hline & & & & & & \\
\hline 1 & 17.783 & MM & 0.5595 & 167.04128 & 4.97625 & 2.8567 \\
\hline 2 & 25.538 & MM & 0.9092 & 5680.30566 & 104.12856 & 97.1433 \\
\hline
\end{tabular}


IR of compound $14 f$

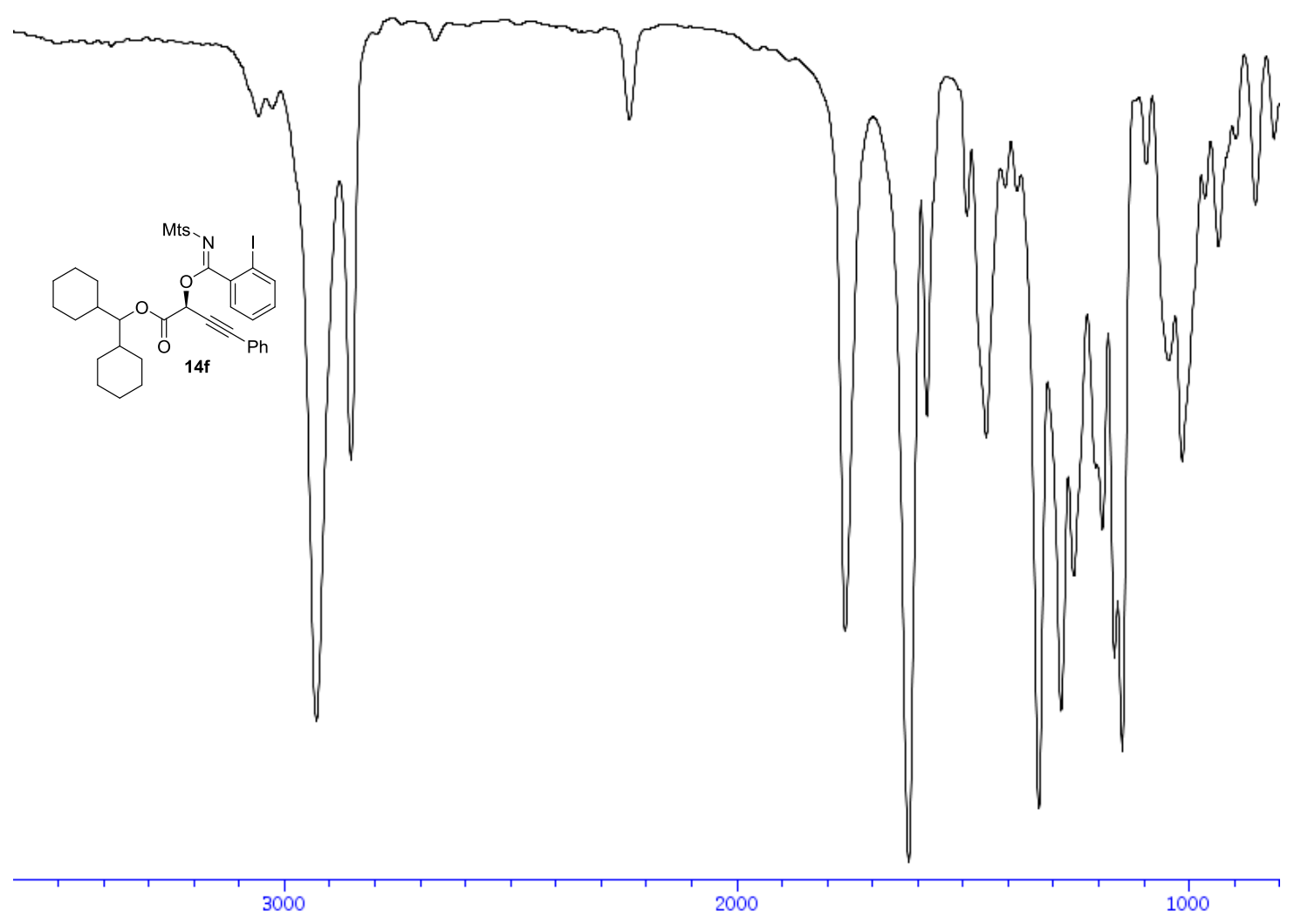


${ }^{\mathbf{1}} \mathbf{H}-\mathbf{N M R}\left(400 \mathrm{MHz}, \mathrm{CDCl}_{3}\right)$ of compound $\mathbf{1 4 g}$

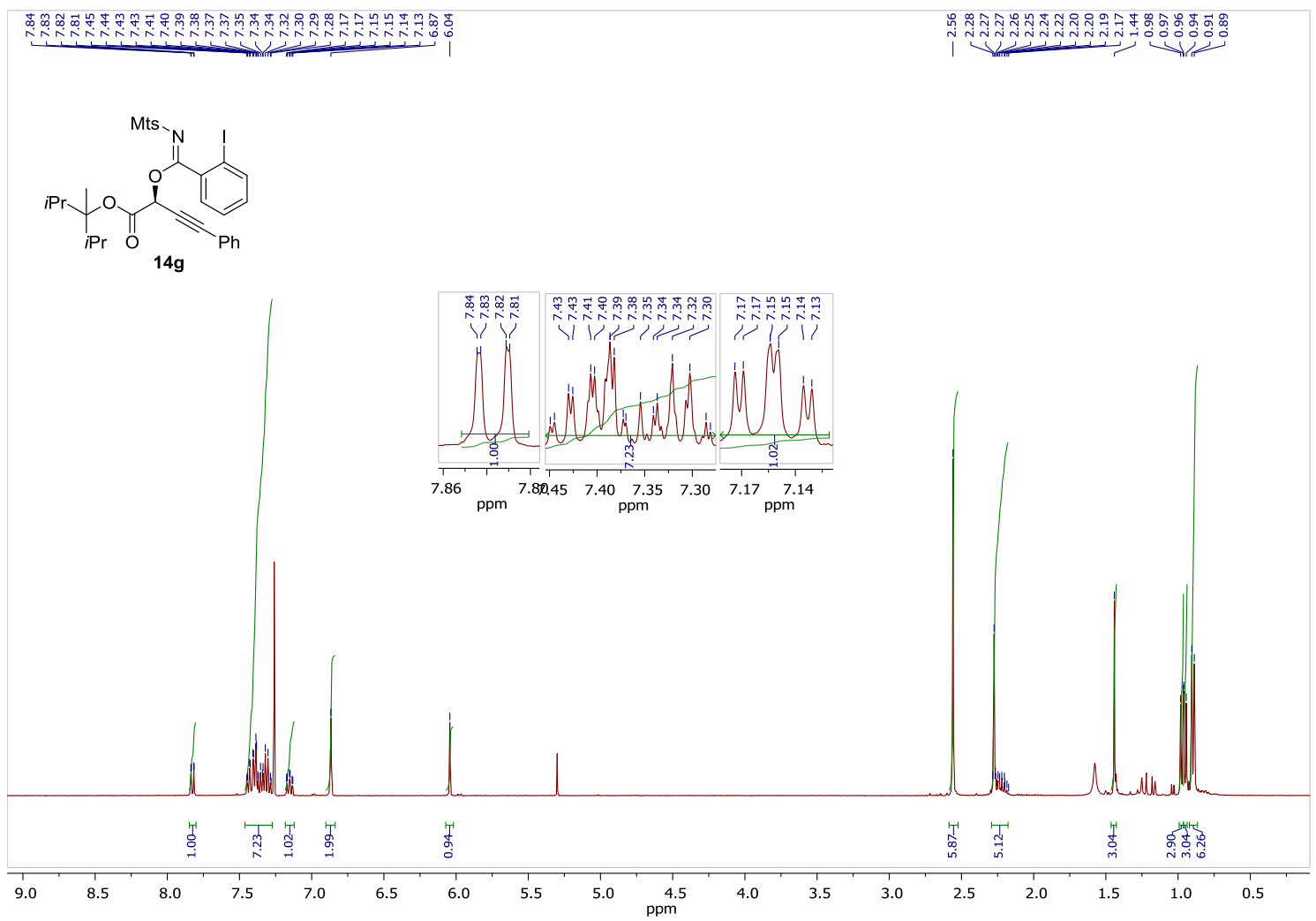

${ }^{13} \mathbf{C}-\mathrm{NMR}\left(100 \mathrm{MHz}, \mathrm{CDCl}_{3}\right)$ of compound $\mathbf{1 4 g}$

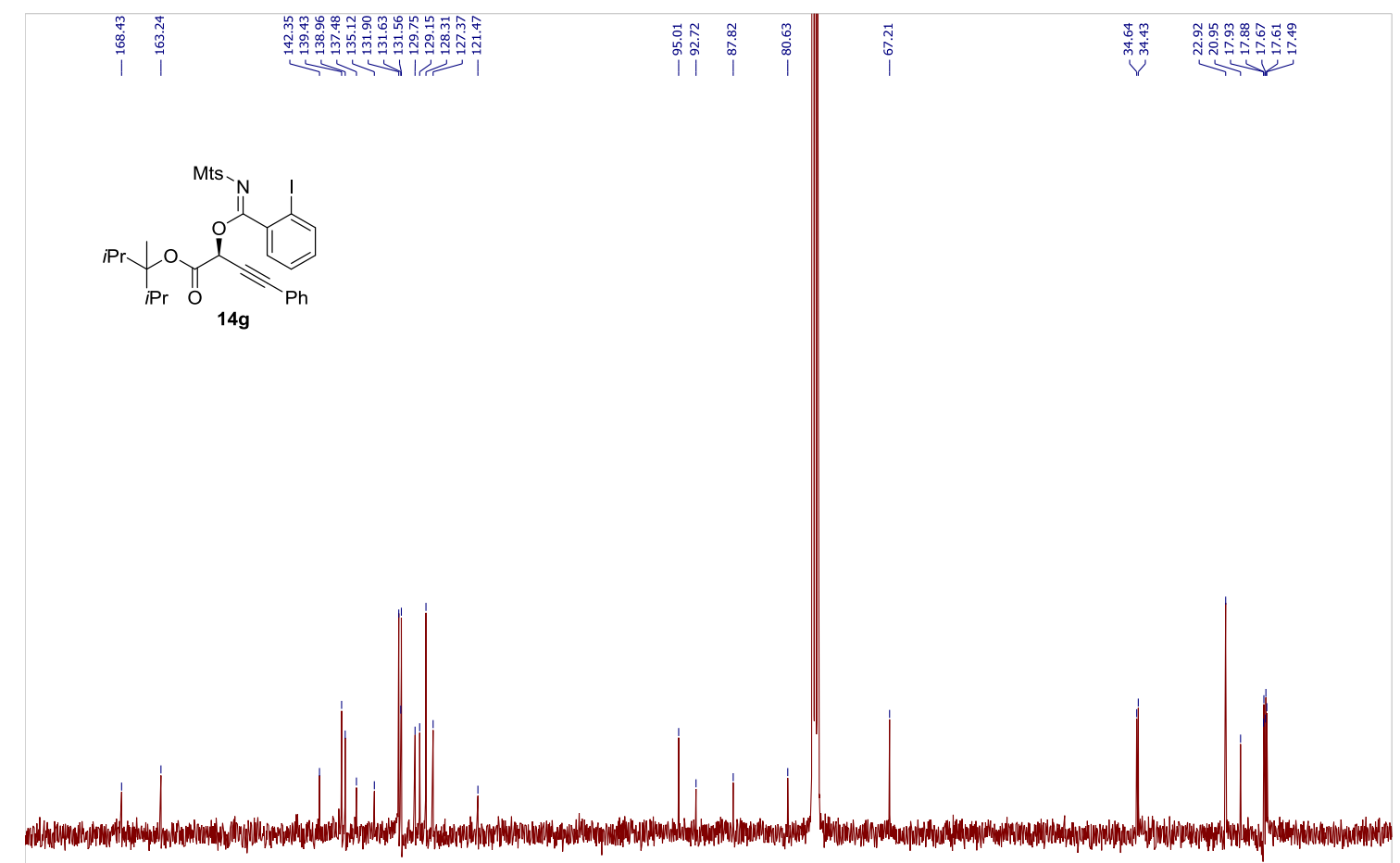

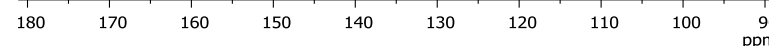


HPLC of compound 14g
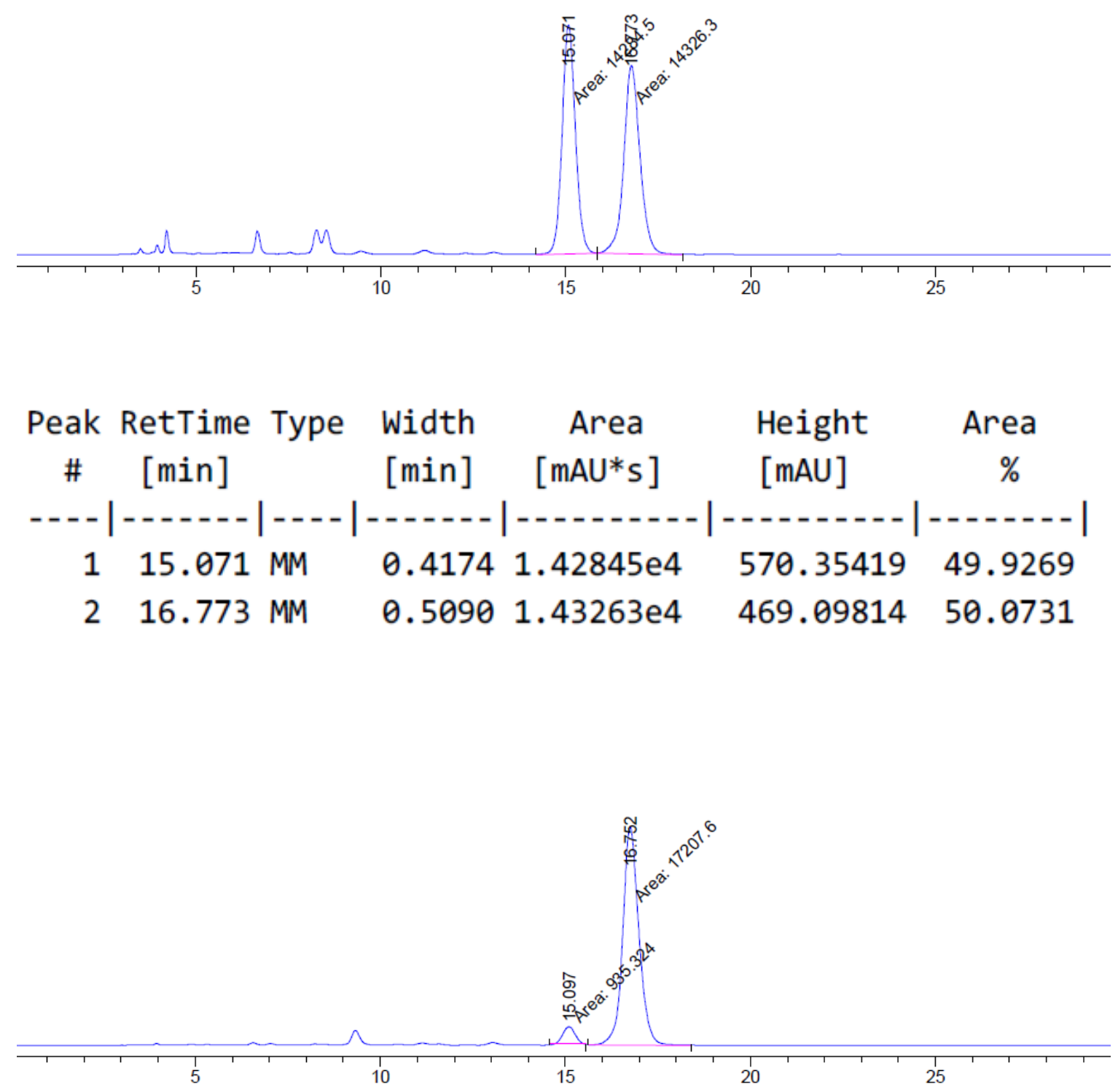

\begin{tabular}{cccccc}
$\begin{array}{c}\text { Peak RetTime Type } \\
\text { \# }\end{array}$ & $\begin{array}{c}\text { Width } \\
{[\mathrm{min}]}\end{array}$ & $\begin{array}{c}\text { Area } \\
{[\mathrm{min}]}\end{array}$ & $\begin{array}{c}\text { Height } \\
{[\mathrm{mAU} \text { s }]}\end{array}$ & $\begin{array}{c}\text { Area } \\
{[\mathrm{mAU}]}\end{array}$ & $\%$ \\
\hline 1 & 15.097 MM & 0.3620 & 935.32355 & 43.05840 & 5.1553 \\
2 & 16.752 MM & 0.5046 & $1.72076 \mathrm{~s}$ & 568.34613 & 94.8447
\end{tabular}


IR of compound $\mathbf{1 4 g}$

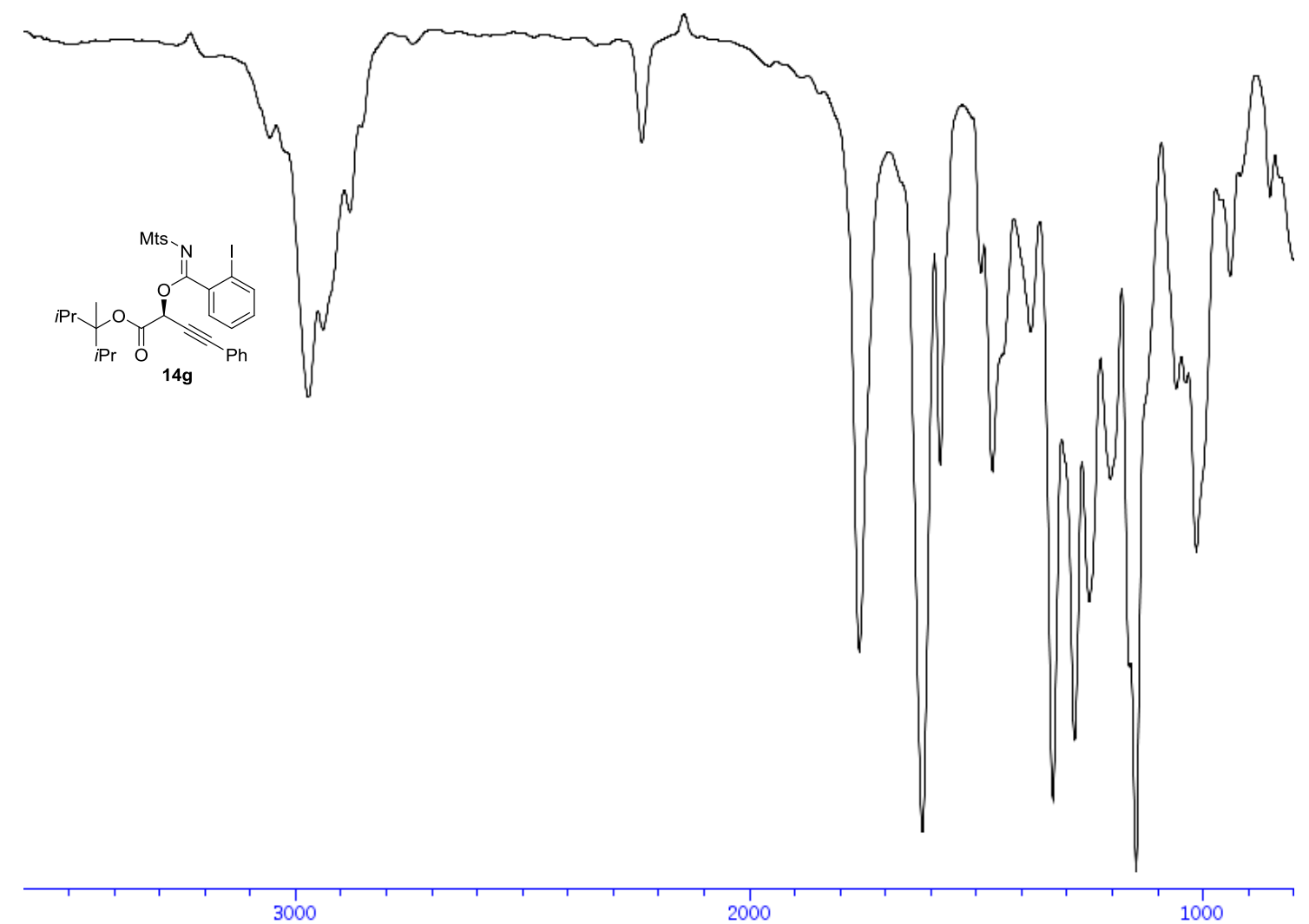


${ }^{\mathbf{1}} \mathbf{H}$-NMR (400 MHz, $\mathrm{CDCl}_{3}$ ) of compound $\mathbf{1 4 h}$

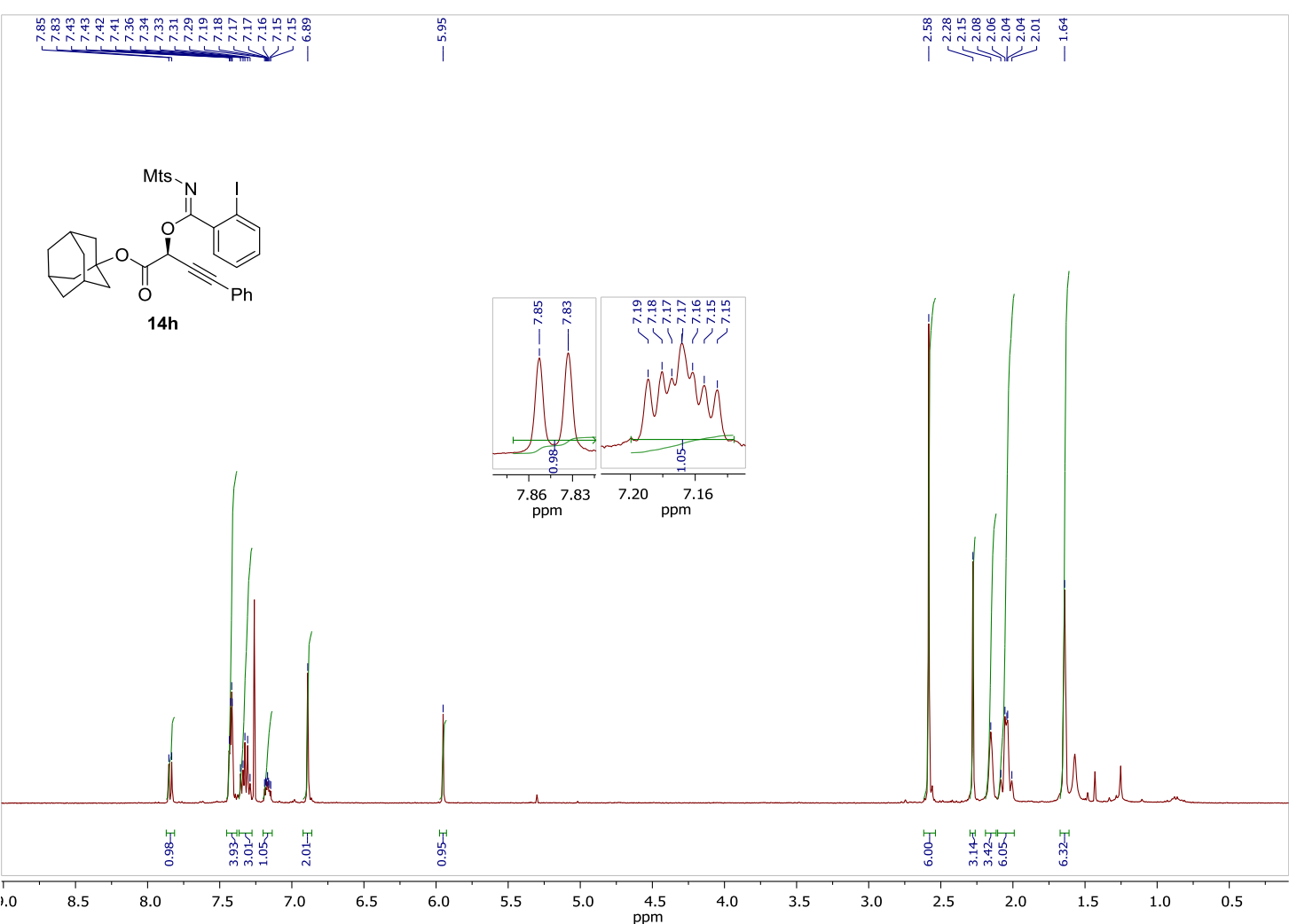

${ }^{13} \mathbf{C}$-NMR (100 MHz, $\mathrm{CDCl}_{3}$ ) of compound $\mathbf{1 4 h}$

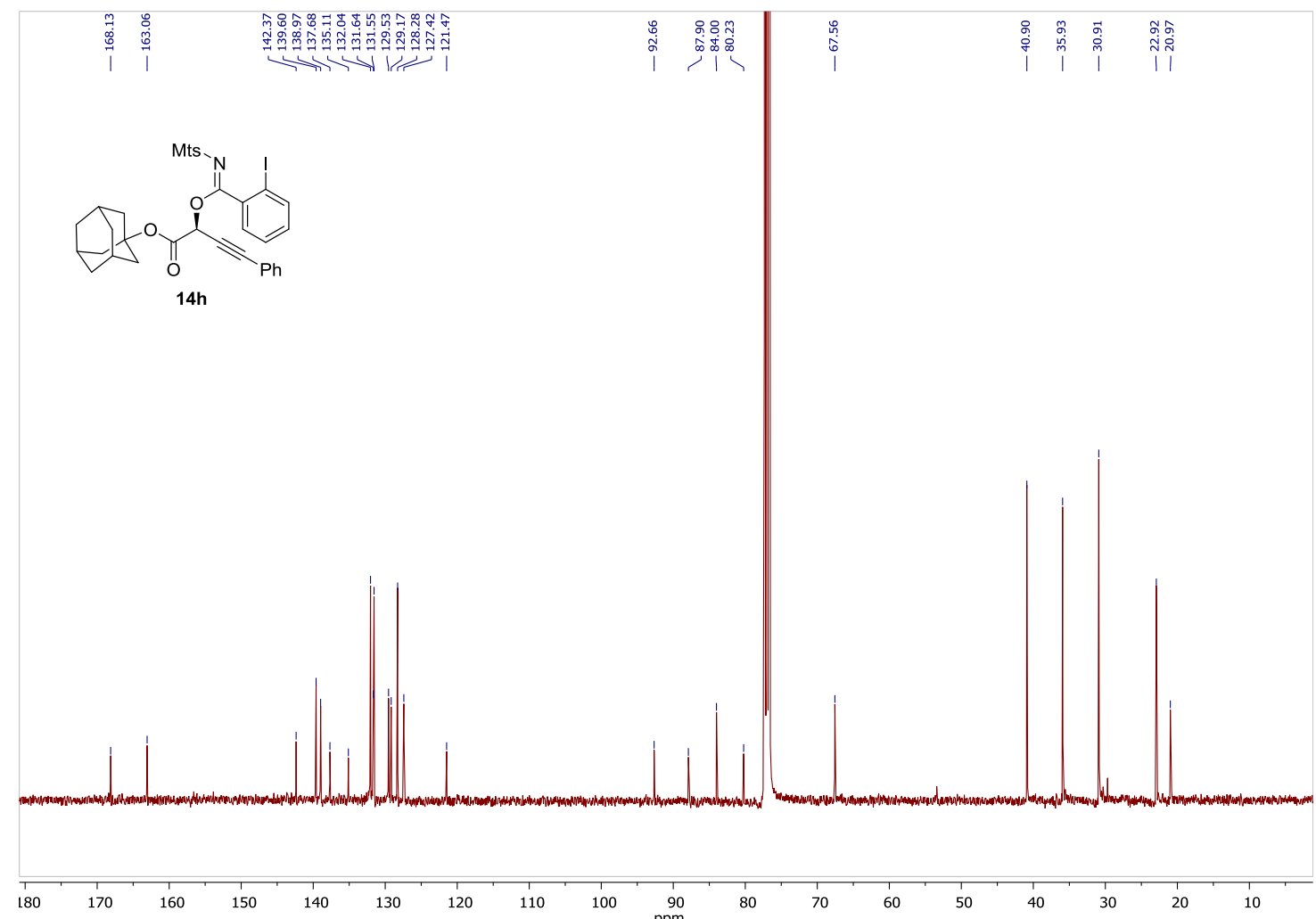


HPLC of compound $\mathbf{1 4 h}$

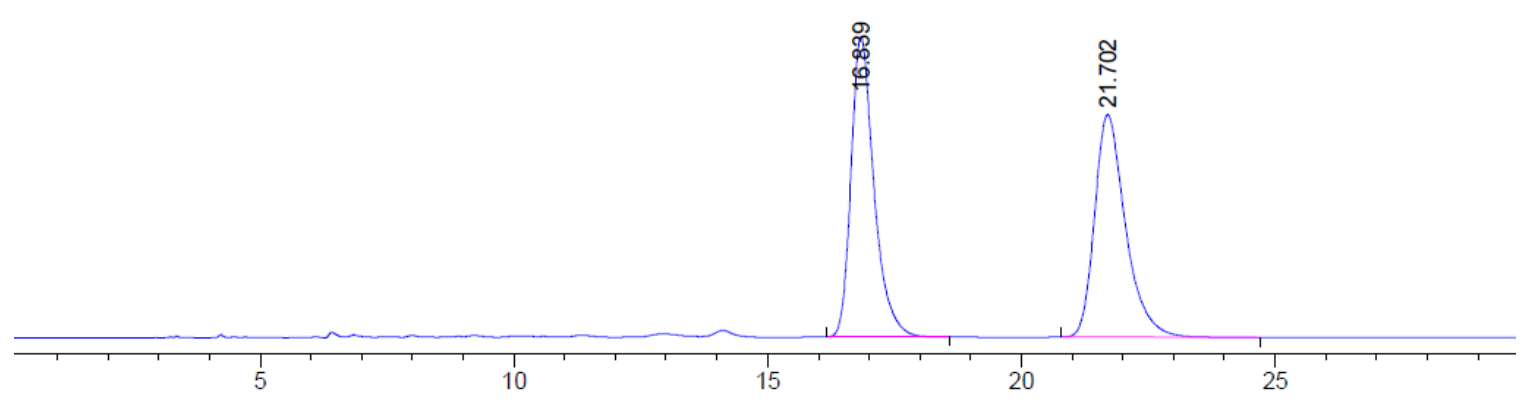

$$
\begin{aligned}
& \text { Peak RetTime Type Width Area Height Area }
\end{aligned}
$$

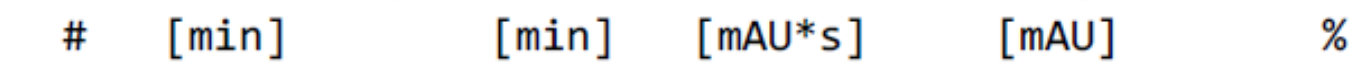

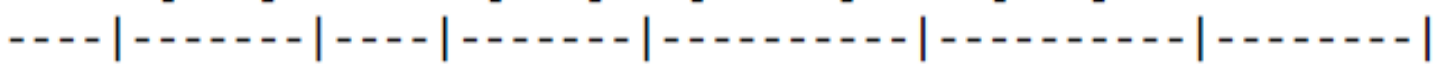

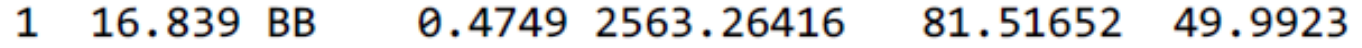

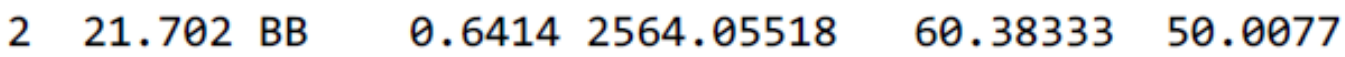

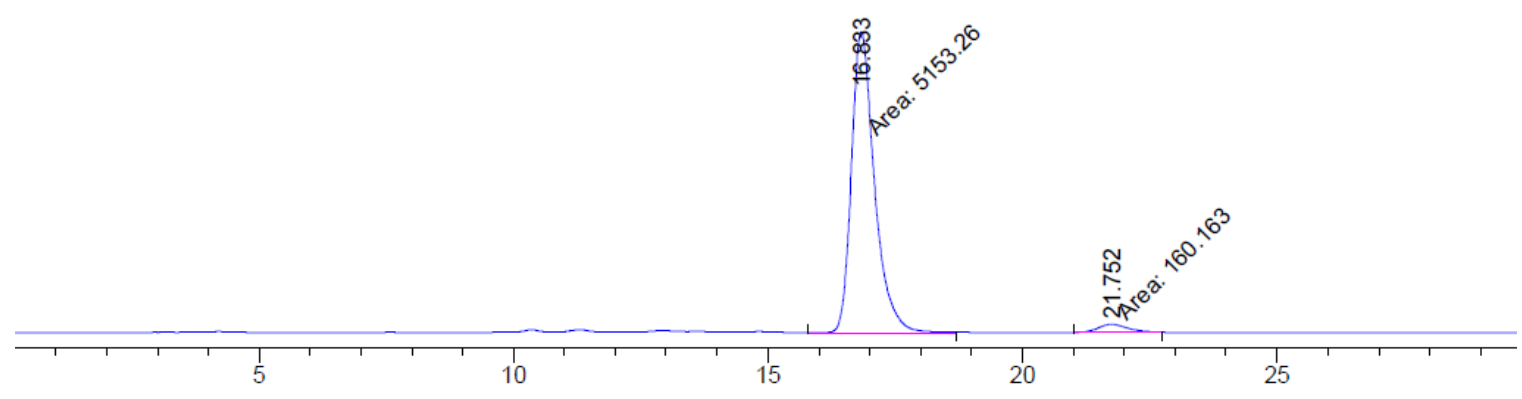

\begin{tabular}{cccccc}
$\begin{array}{c}\text { Peak RetTime Type } \\
\text { \# }\end{array}$ & Width & Area & Height & Area \\
[min] & {$[$ mAU*s $]$} & {$[$ mAU] } & $\%$ \\
\hline 1 & 16.833 MM & 0.5266 & 5153.25586 & 163.09471 & 96.9857 \\
2 & 21.752 MM & 0.6356 & 160.16348 & 4.19962 & 3.0143
\end{tabular}


IR of compound $14 \mathrm{~h}$

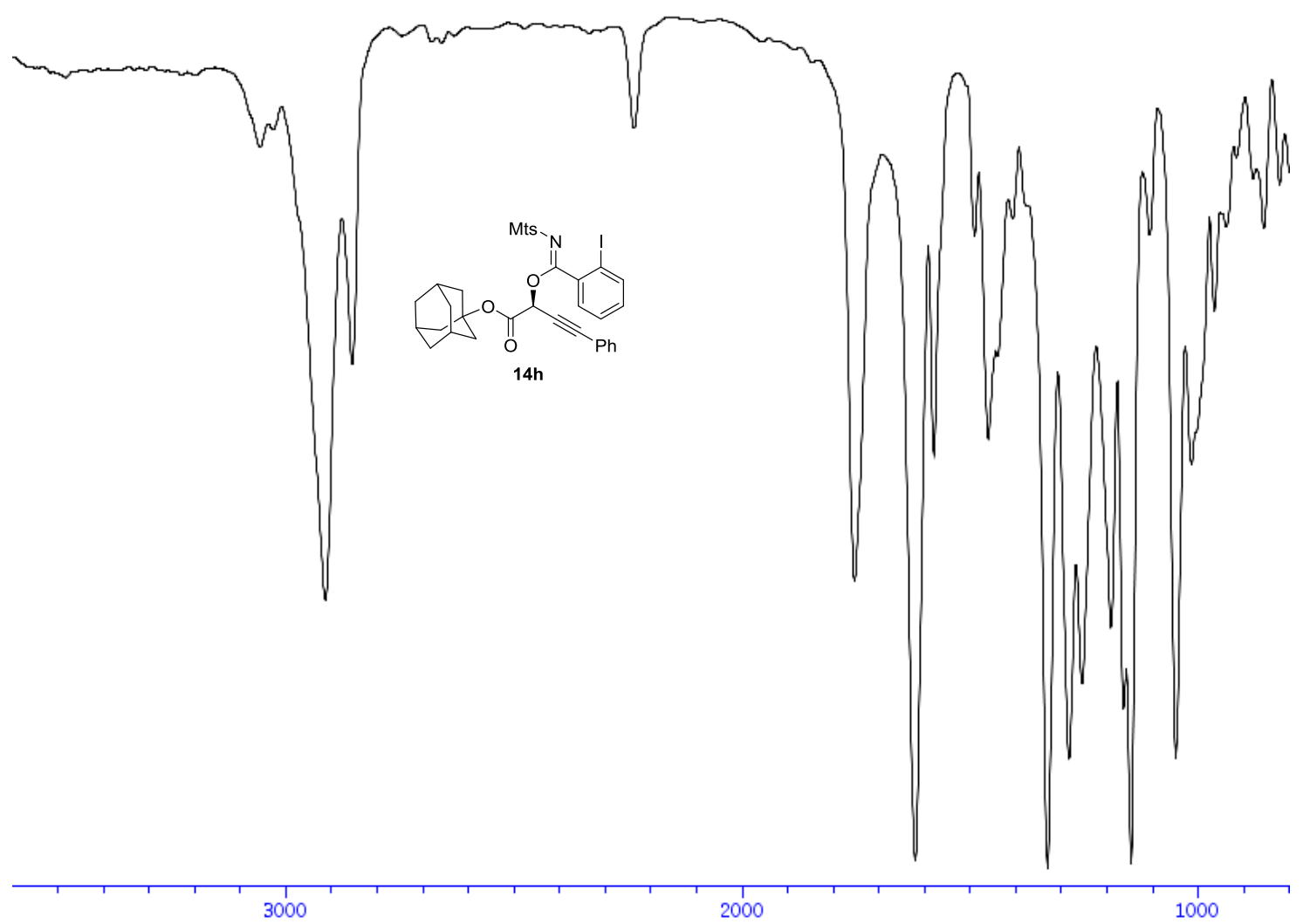


${ }^{1} \mathbf{H}-\mathbf{N M R}\left(400 \mathrm{MHz}, \mathrm{CDCl}_{3}\right.$ ) of compound 14i

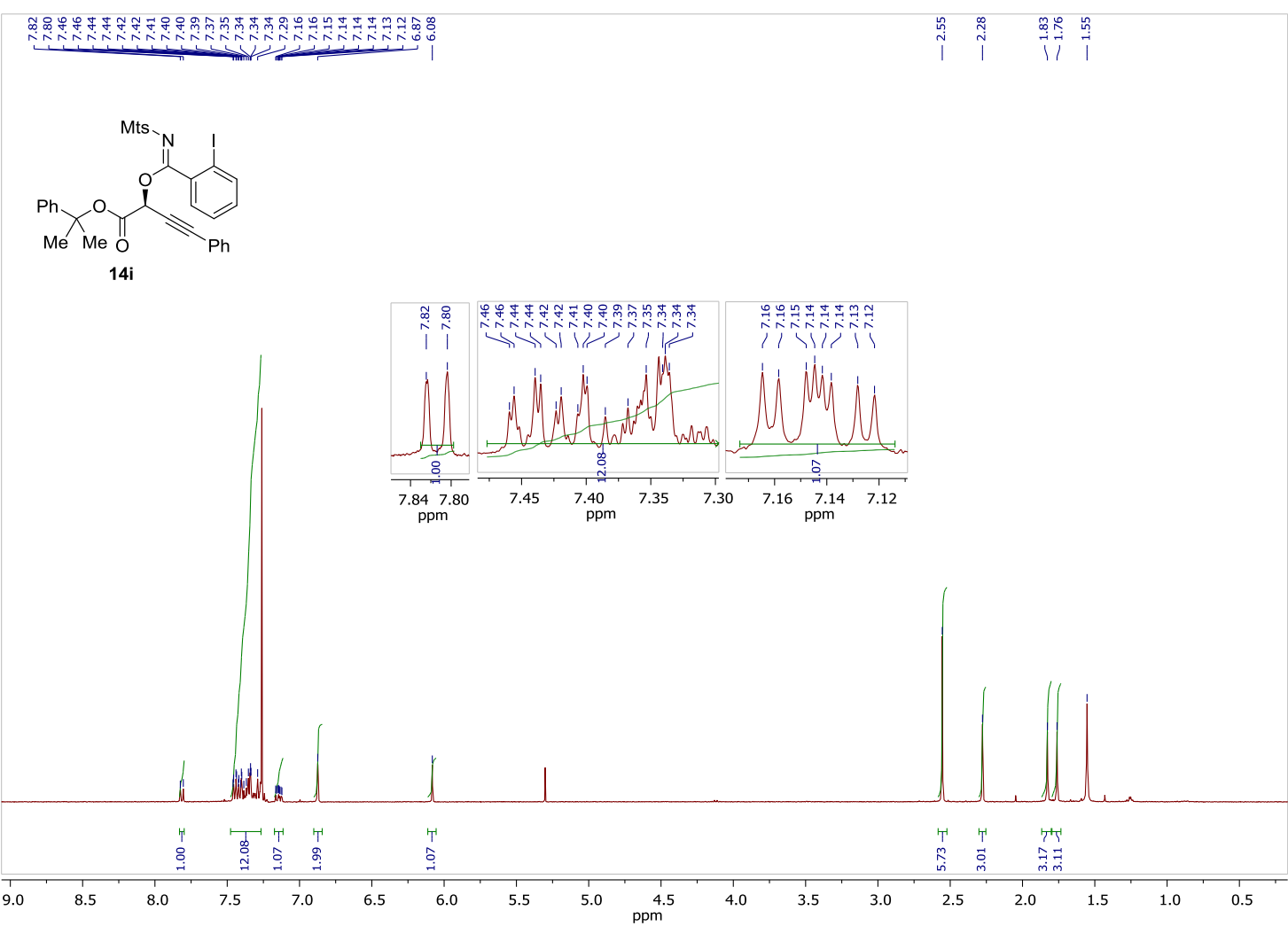

${ }^{13} \mathbf{C}$-NMR (100 MHz, $\left.\mathrm{CDCl}_{3}\right)$ of compound 14i

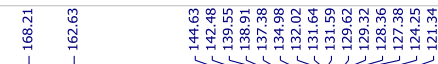

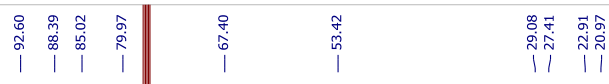

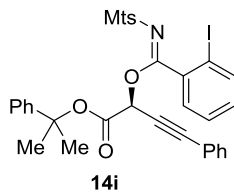

$14 i$
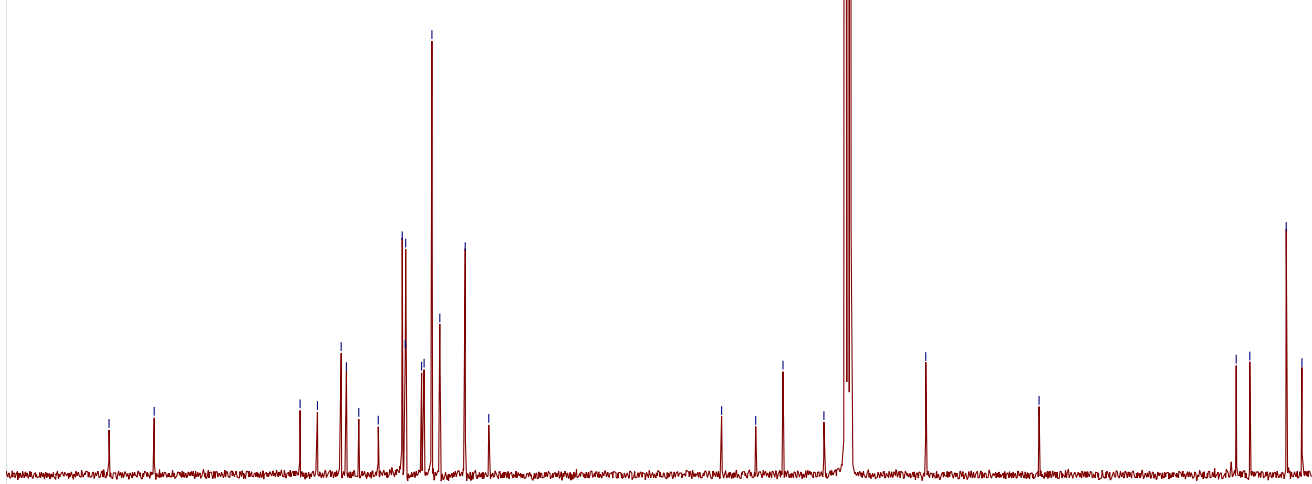

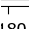

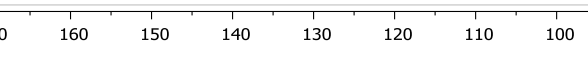

90
$\mathrm{ppm}$ 
HPLC of compound 14i
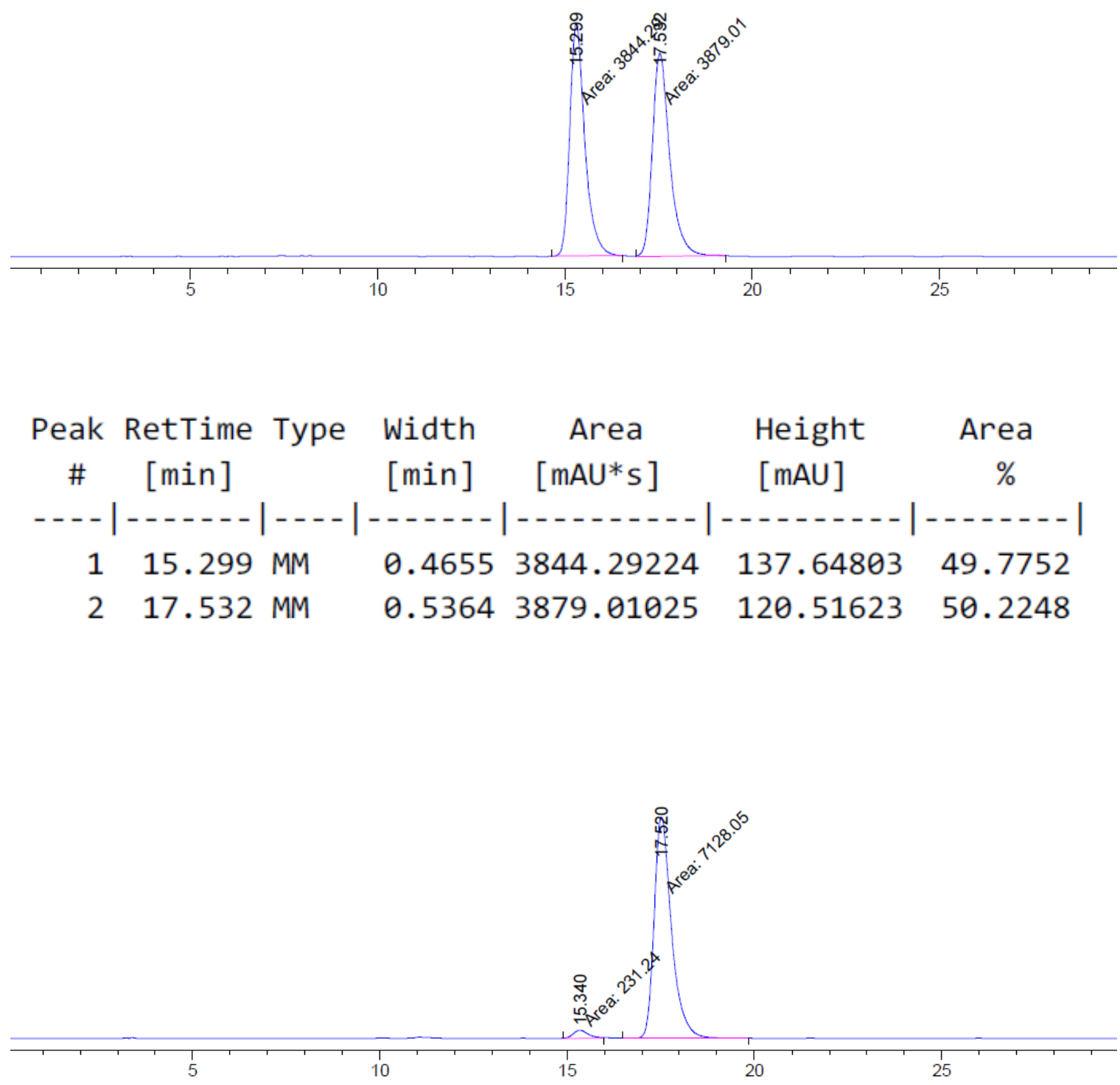

$\begin{array}{cccccc}\begin{array}{c}\text { Peak RetTime Type } \\ \#\end{array} & \text { width } & \text { Area } & \text { Height } & \text { Area } \\ & {[\mathrm{min}]} & {[\mathrm{mAU} \text { s }]} & {[\mathrm{mAU}]} & \% \\ 1 & 15.340 \mathrm{MM} & 0.4658 & 231.24036 & 8.27390 & 3.1422 \\ 2 & 17.520 \mathrm{MM} & 0.5354 & 7128.05273 & 221.89154 & 96.8578\end{array}$


IR of compound 14i

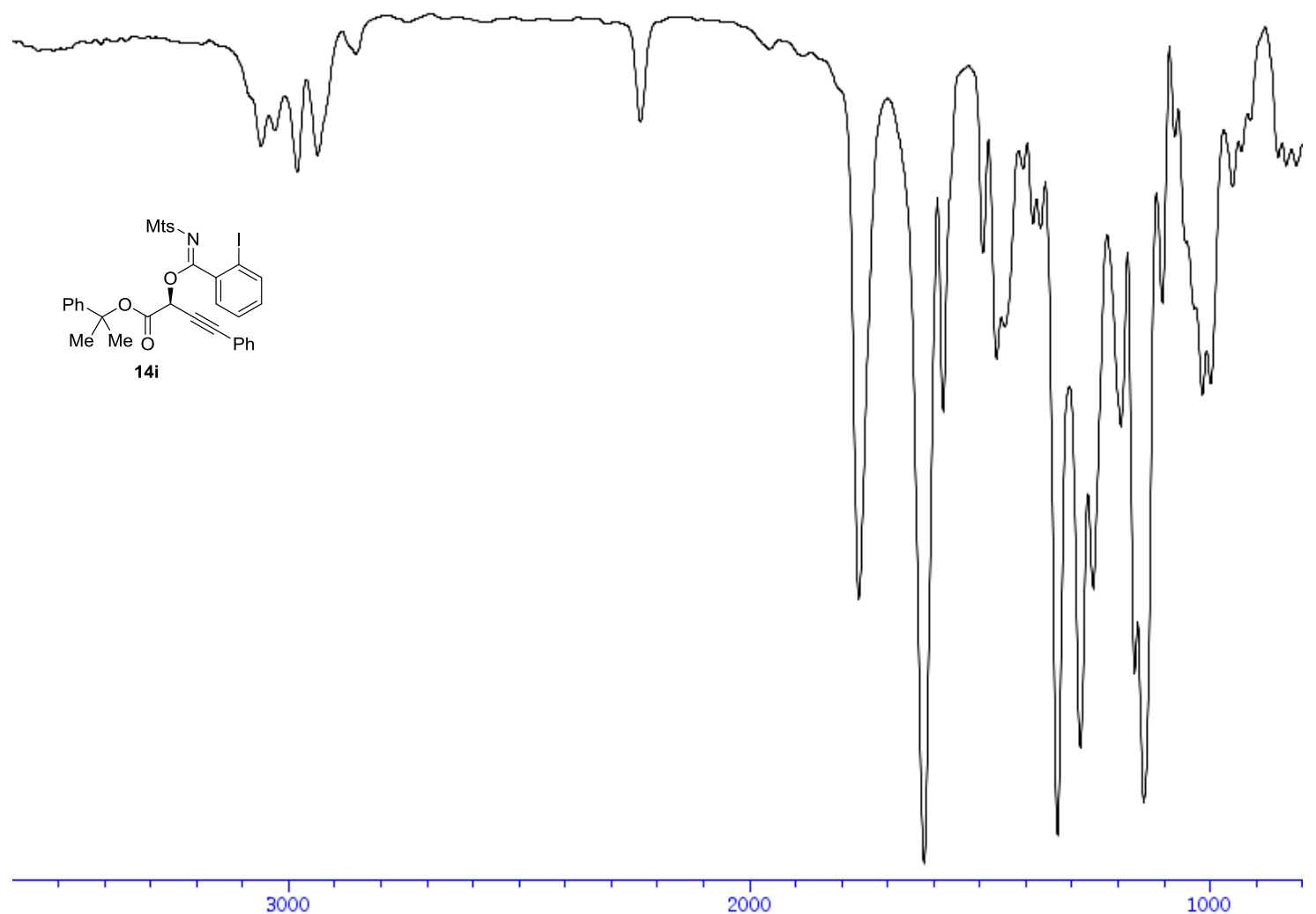


${ }^{\mathbf{1}} \mathbf{H}-\mathbf{N M R}\left(400 \mathrm{MHz}, \mathrm{CDCl}_{3}\right)$ of compound $\mathbf{1 4 j}$

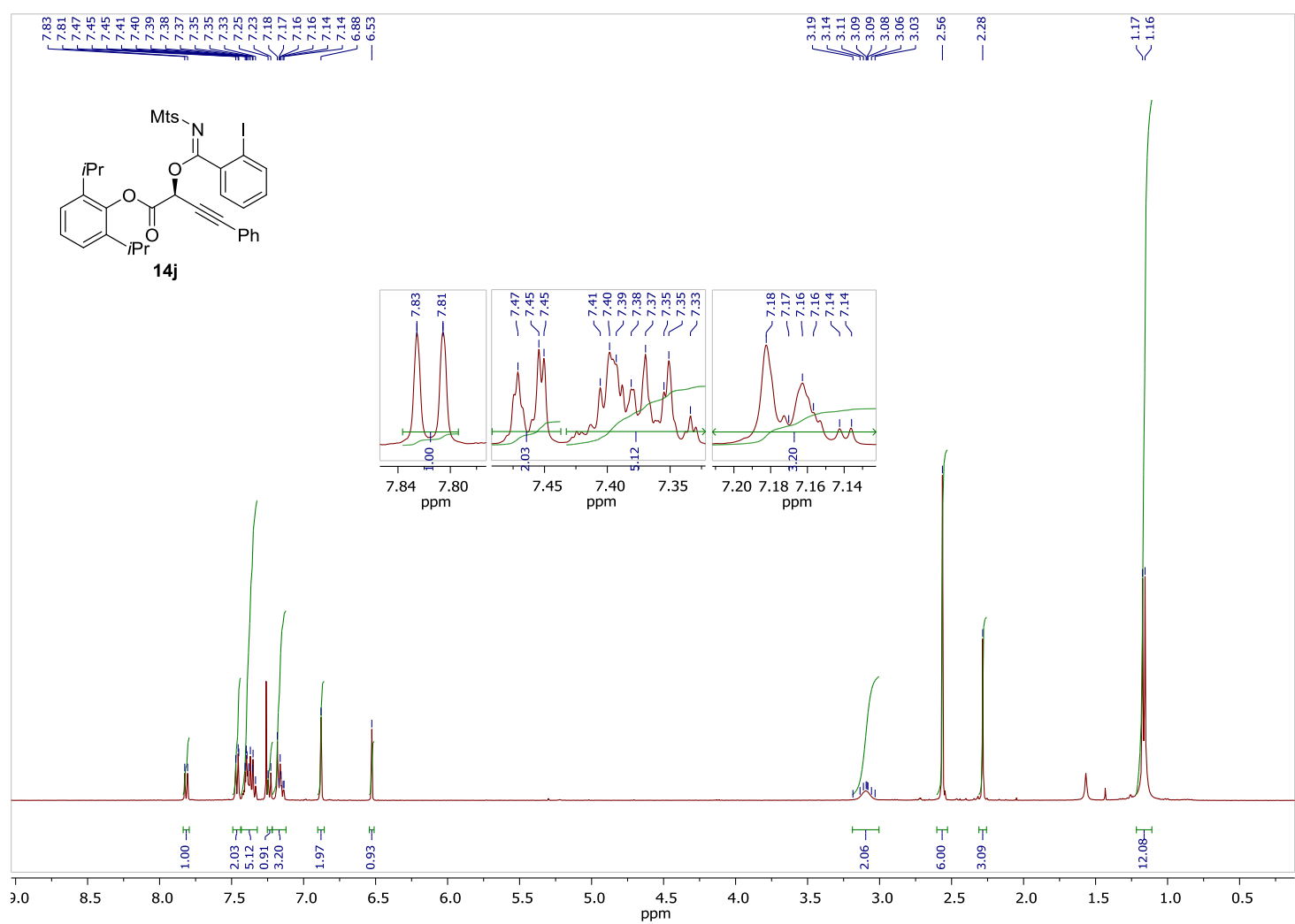

${ }^{\mathbf{1 3}} \mathbf{C}$-NMR (100 MHz, $\left.\mathrm{CDCl}_{3}\right)$ of compound $\mathbf{1 4 j}$

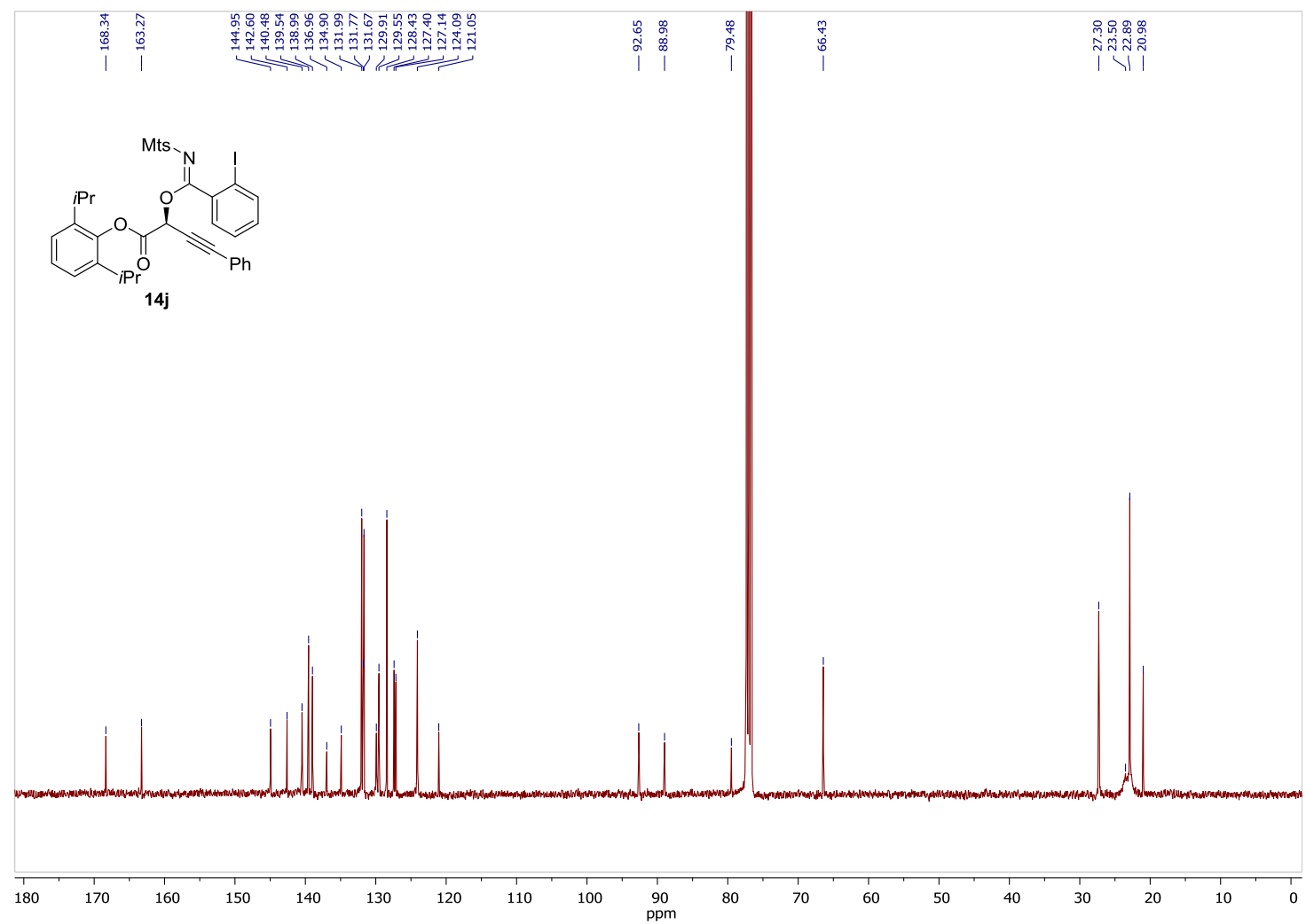


HPLC of compound $\mathbf{1 4 j}$

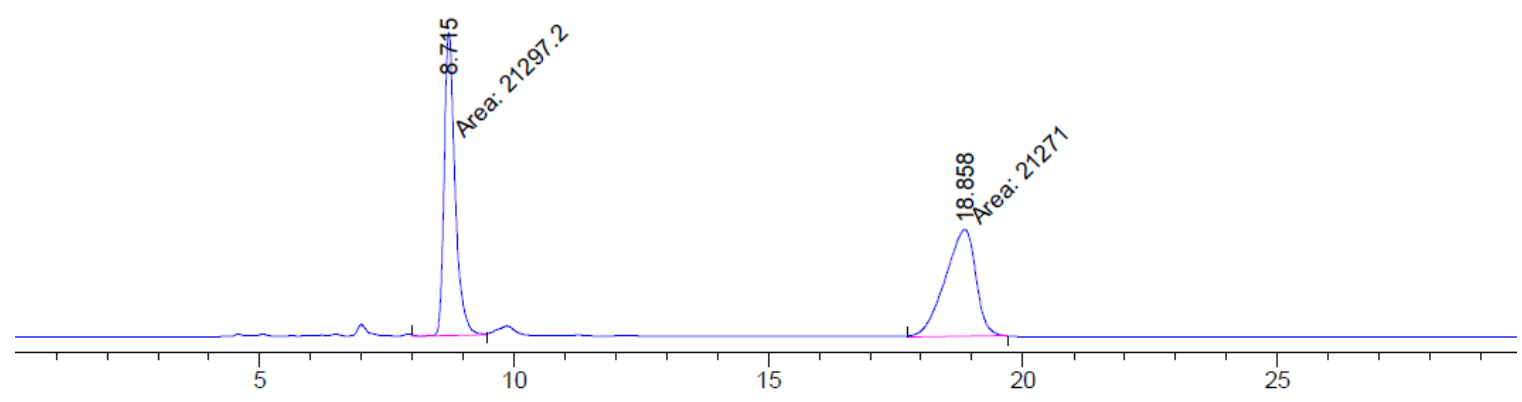

\begin{tabular}{|c|c|c|c|c|c|c|}
\hline $\begin{array}{c}\text { Peak } \\
\#\end{array}$ & $\begin{array}{c}\text { RetTime } \\
\text { [min] }\end{array}$ & Type & $\begin{array}{l}\text { Width } \\
\text { [min] }\end{array}$ & $\begin{array}{c}\text { Area } \\
{\left[\mathrm{mAU}^{*} \mathrm{~s}\right]}\end{array}$ & $\begin{array}{l}\text { Height } \\
{[\mathrm{mAU}]}\end{array}$ & $\begin{array}{c}\text { Area } \\
\%\end{array}$ \\
\hline & . & & & $\ldots-\ldots$ & $\mid--$ & . \\
\hline 1 & 8.715 & MM & 0.2469 & $2.12972 \mathrm{e} 4$ & 1437.39636 & 50.0307 \\
\hline 2 & 18.858 & MM & 0.7014 & $2.12710 \mathrm{e} 4$ & 505.46405 & 49.9693 \\
\hline
\end{tabular}

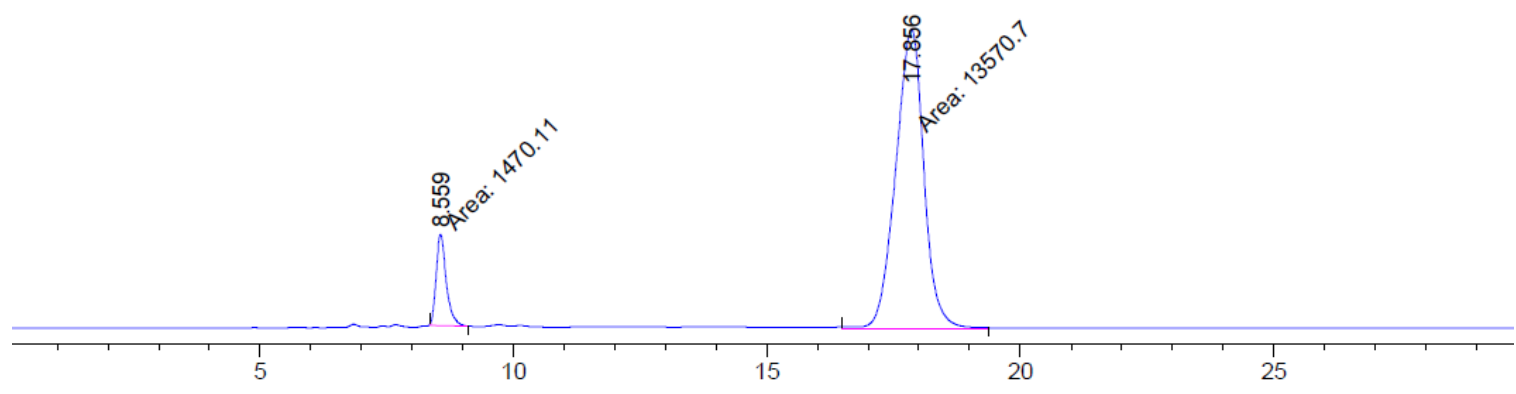

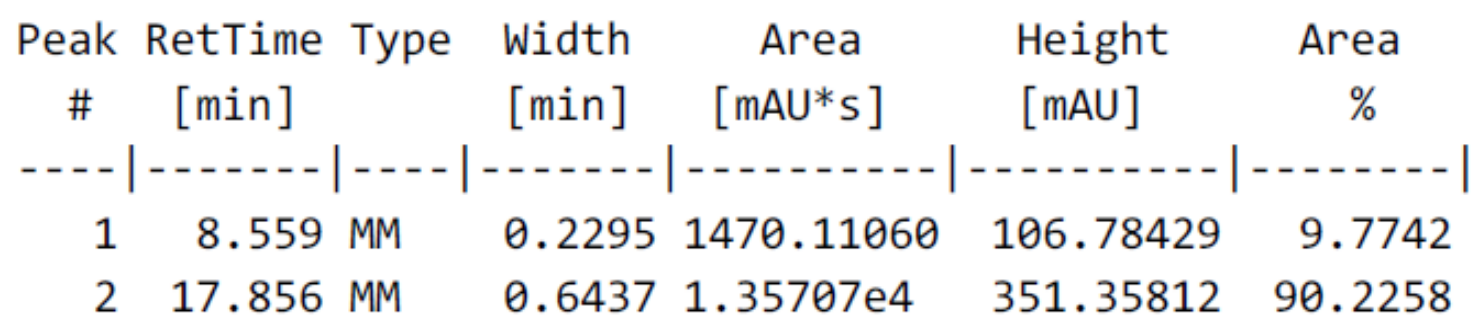


IR of compound $\mathbf{1 4} \mathbf{j}$

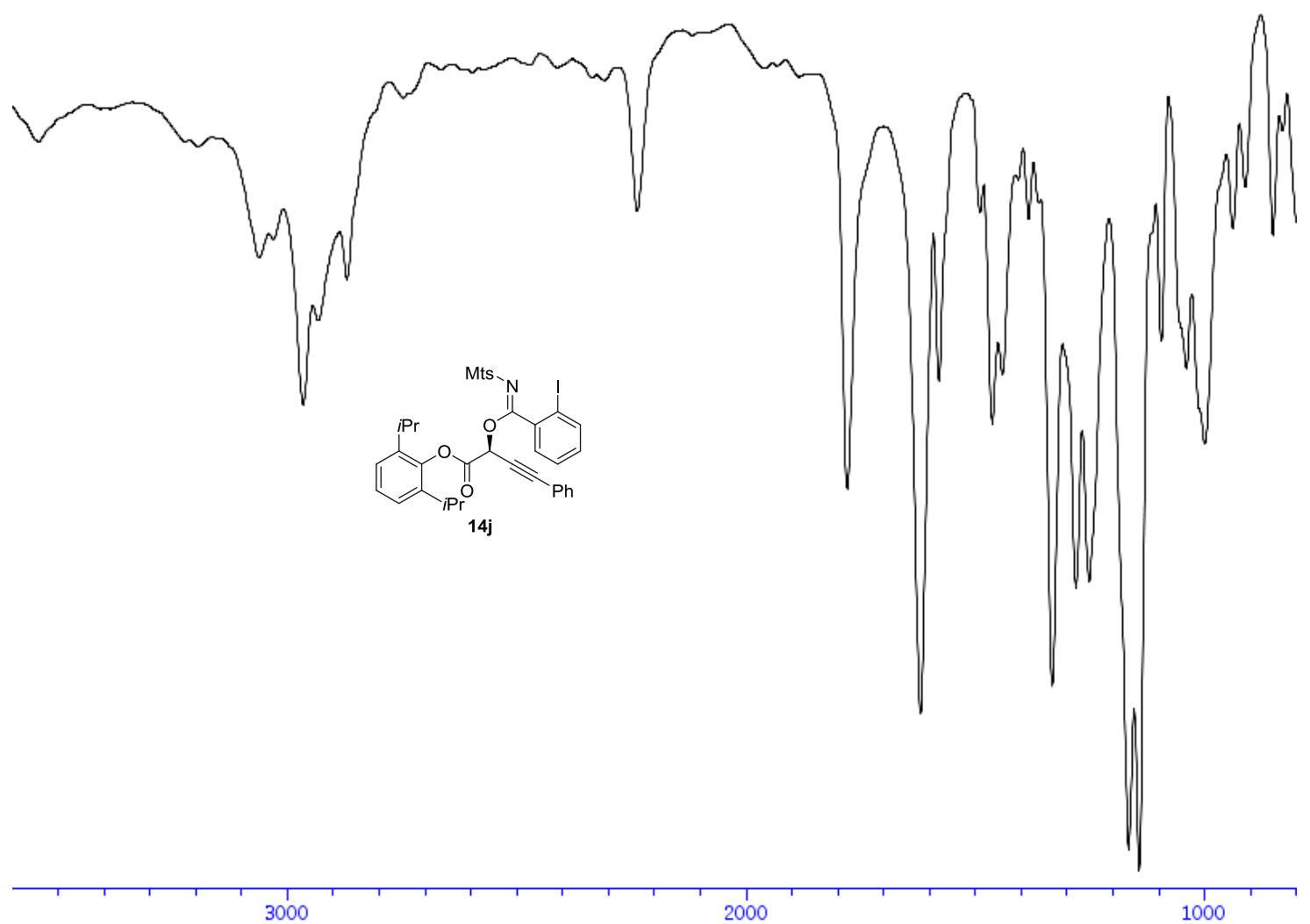


${ }^{\mathbf{1}} \mathbf{H}-\mathbf{N M R}\left(400 \mathrm{MHz}, \mathrm{CDCl}_{3}\right)$ of compound $\mathbf{1 4 k}$

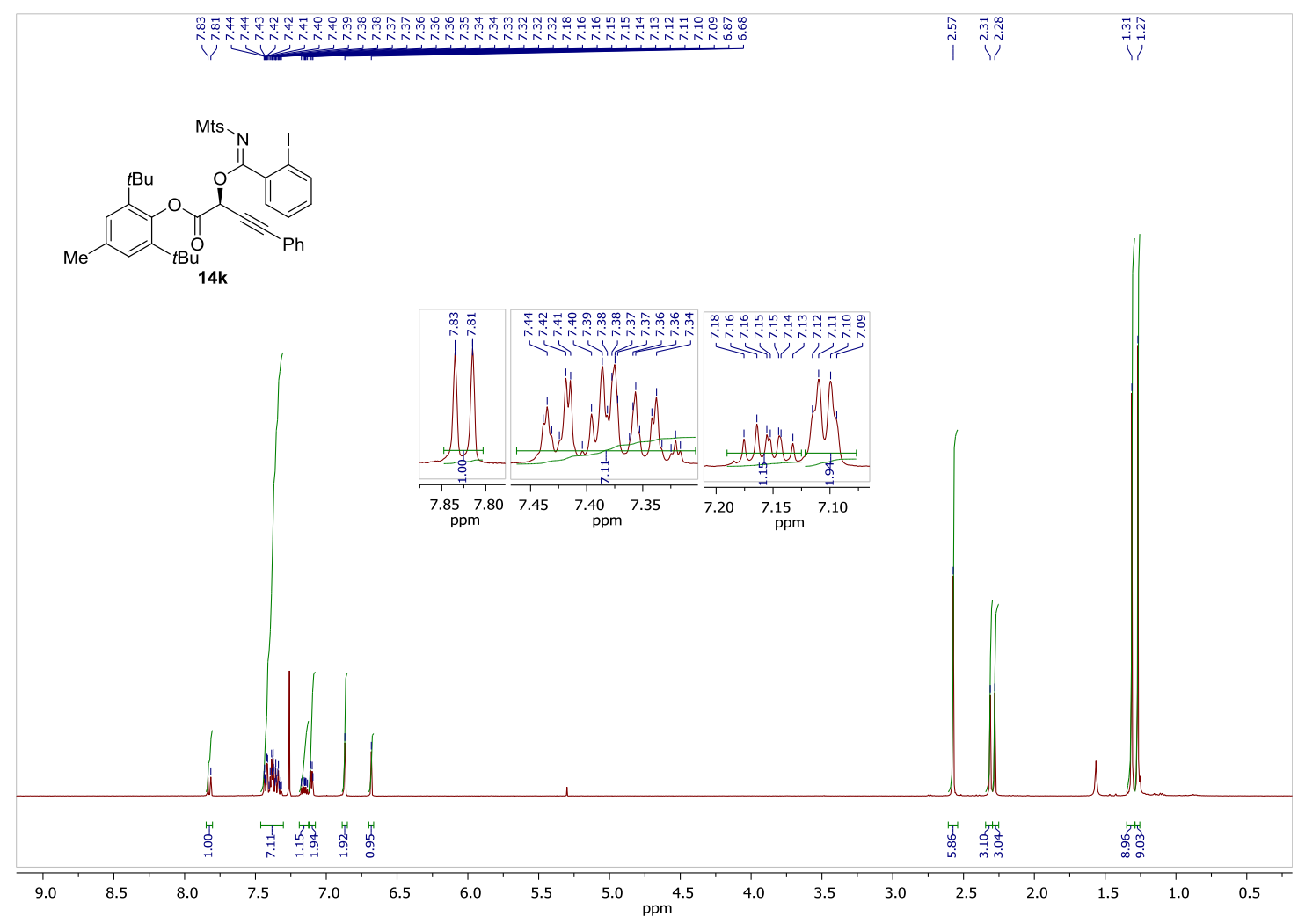

${ }^{13} \mathbf{C}$-NMR (100 MHz, $\left.\mathrm{CDCl}_{3}\right)$ of compound 14k

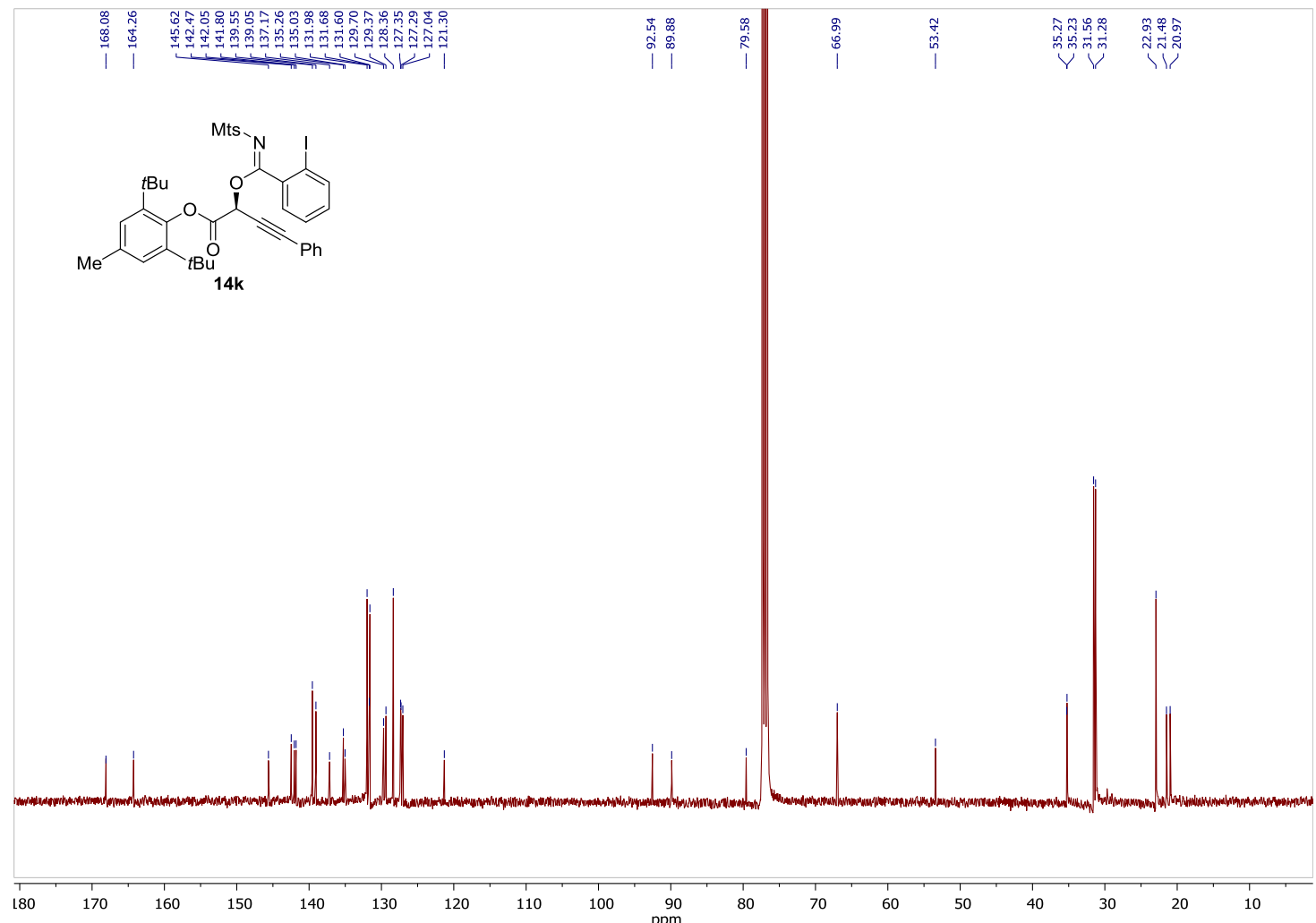


HPLC of compound 14k

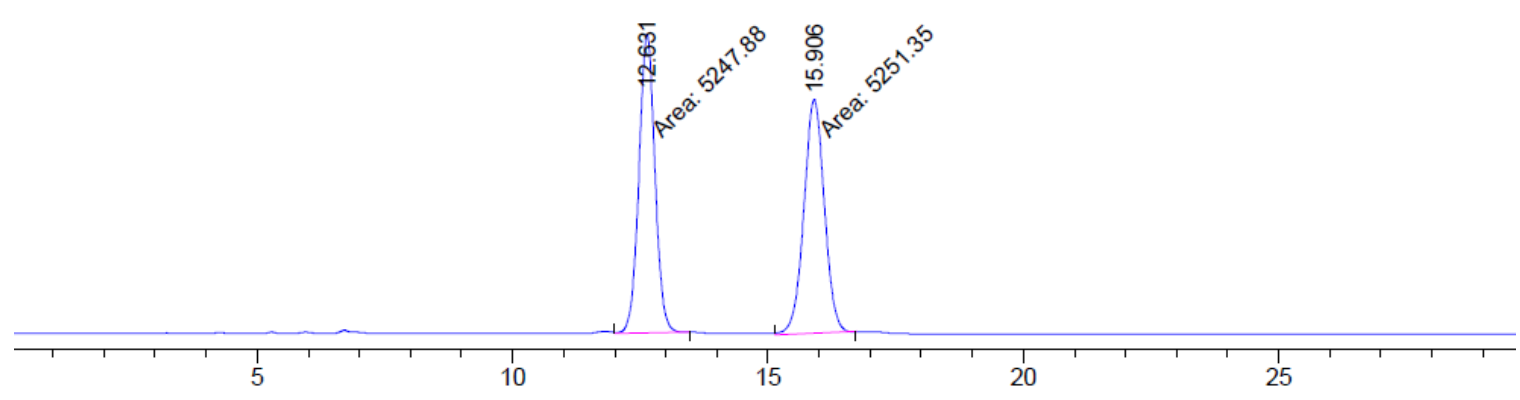

Peak RetTime Type Width Area Height Area

$\#[\mathrm{~min}]$
$[\mathrm{min}][\mathrm{mAU} * \mathrm{~s}] \quad[\mathrm{mAU}]$

$\begin{array}{lllllll}1 & 12.631 & \text { MM } & 0.3637 & 5247.87939 & 240.48375 & 49.9835\end{array}$

$\begin{array}{lllllll}2 & 15.906 \text { MM } & 0.4653 & 5251.34766 & 188.09402 & 50.0165\end{array}$

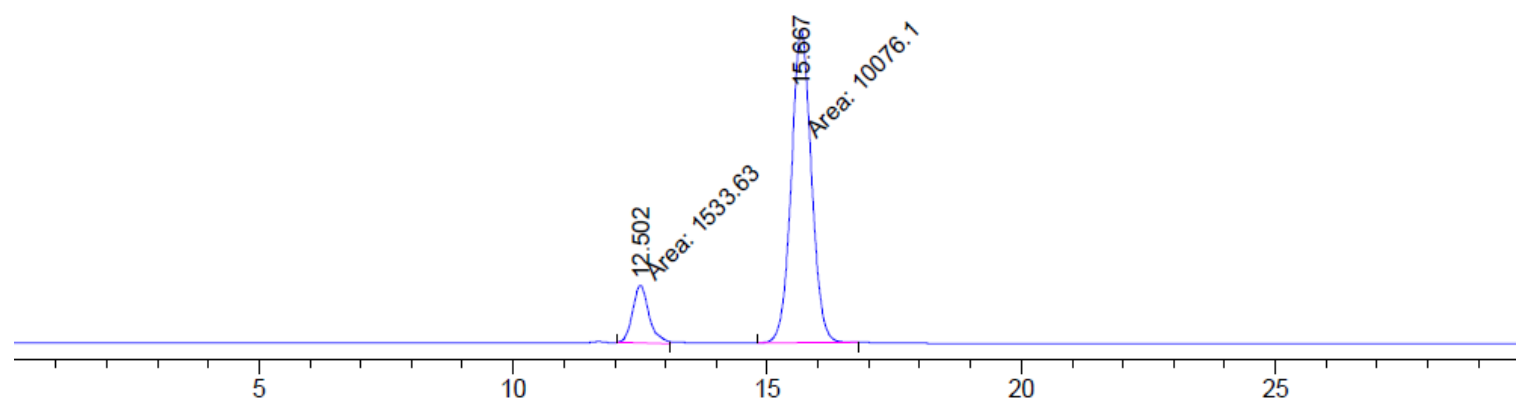

Peak RetTime Type Width Area Height Area \# [min] [min] [mAU*s] [mAU] $\%$

$\begin{array}{lllllll}1 & 12.502 & \text { MM } & 0.3786 & 1533.63477 & 67.50656 & 13.2100\end{array}$

$\begin{array}{lllllll}2 & 15.667 & \text { MM } & 0.4580 & 1.00761 \mathrm{e} 4 & 366.67584 & 86.7900\end{array}$ 
IR of compound 14k

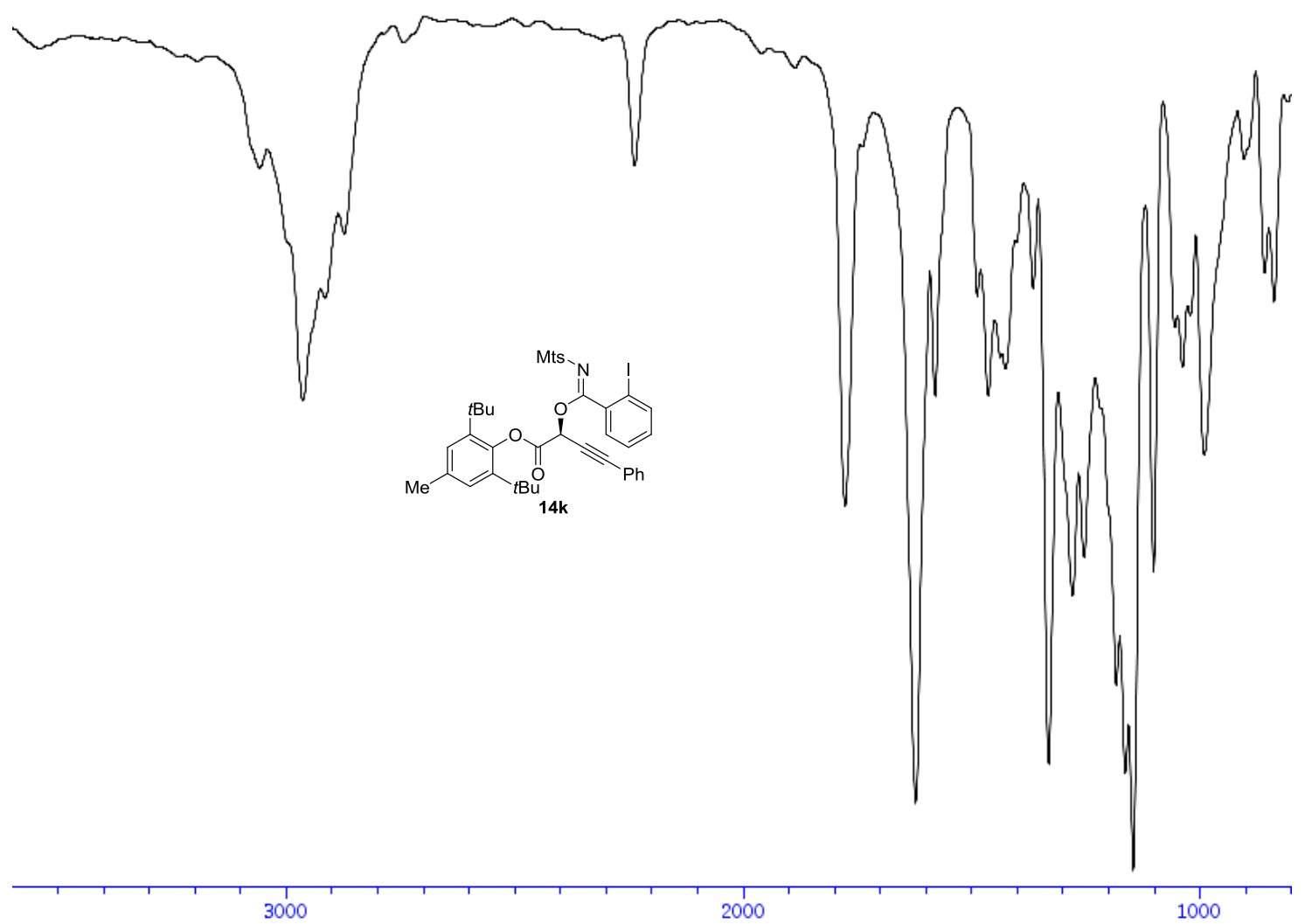


${ }^{\mathbf{1}} \mathbf{H}-\mathbf{N M R}\left(400 \mathrm{MHz}, \mathrm{CDCl}_{3}\right)$ of compound $14 \mathbf{I}$

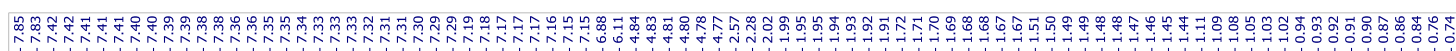
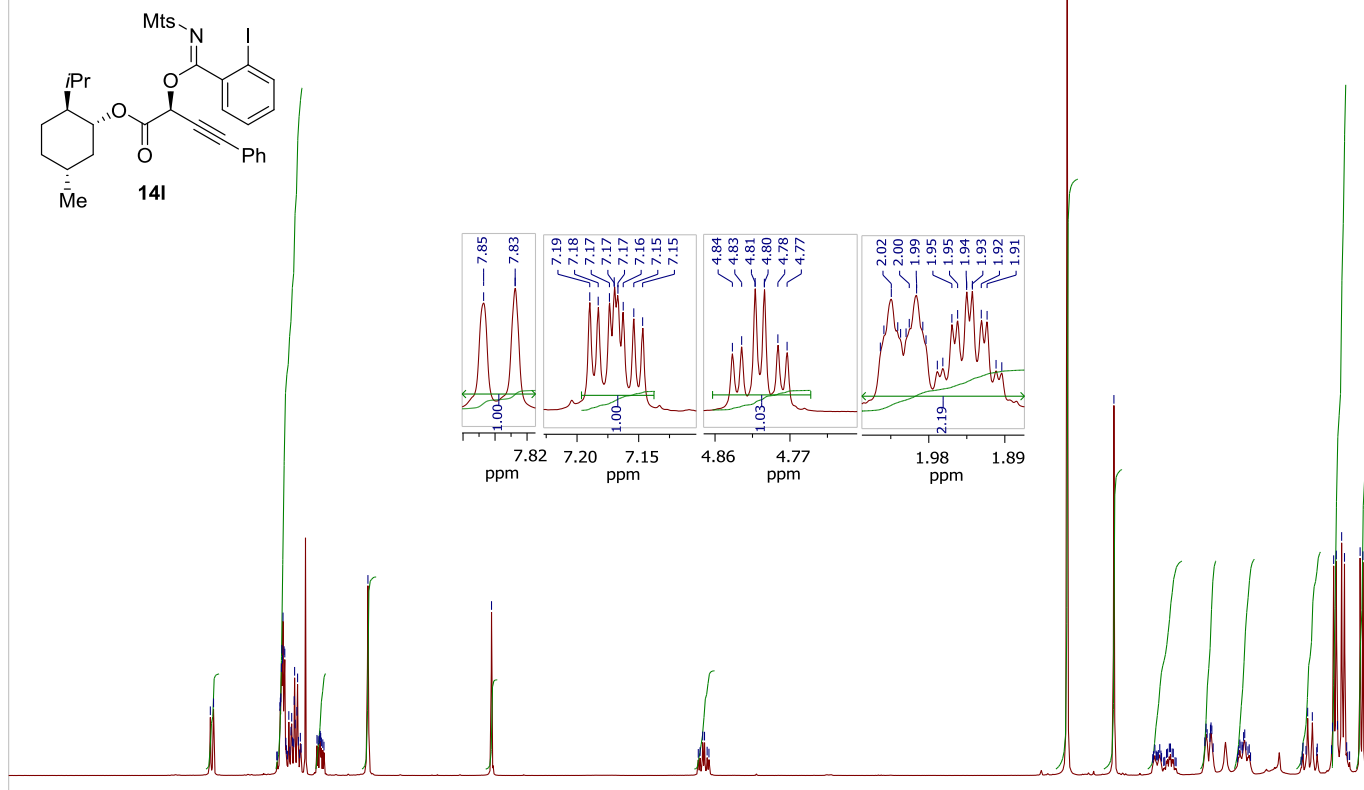

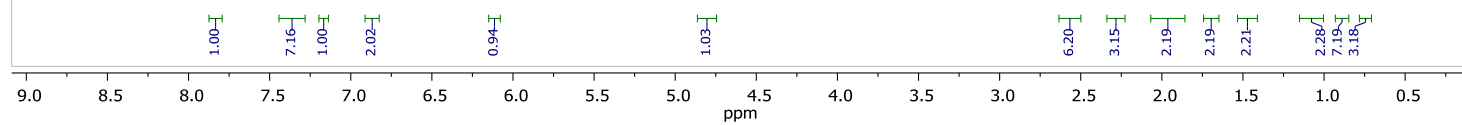

${ }^{13}$ C-NMR (100 MHz, $\left.\mathrm{CDCl}_{3}\right)$ of compound $\mathbf{1 4 1}$

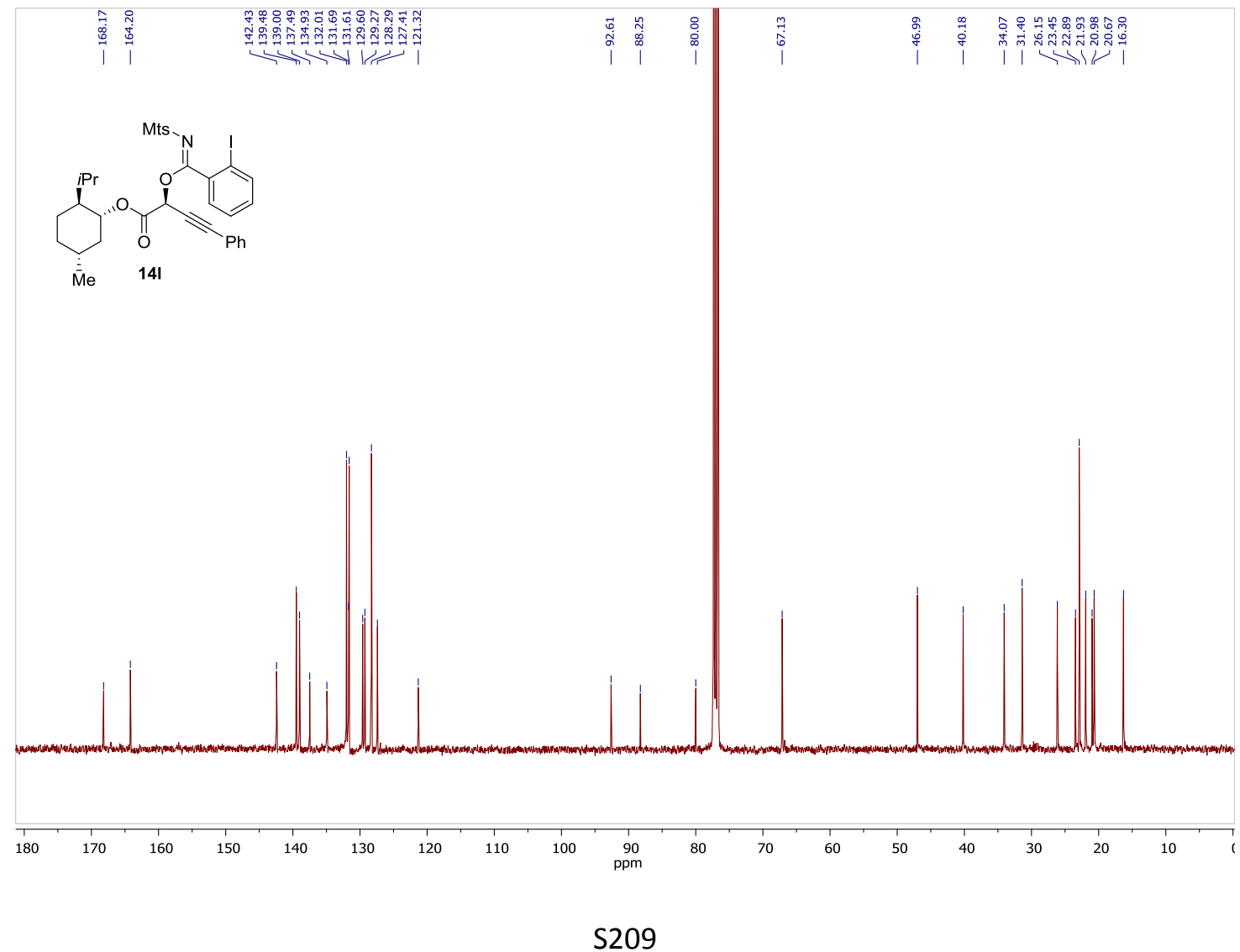


HPLC of compound 141
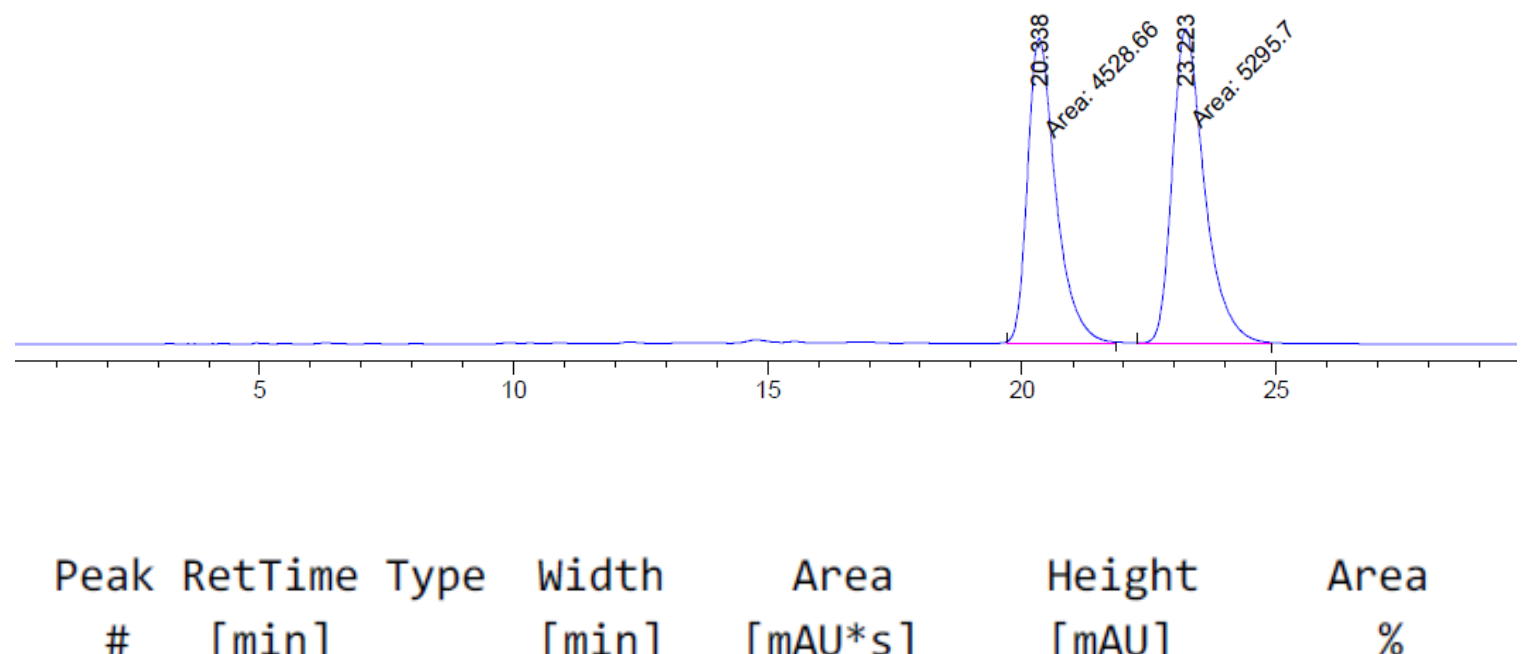

$\begin{array}{ccccccc}\# & {[\mathrm{~min}]} & {[\mathrm{min}]} & {[\mathrm{mAU} * \mathrm{~s}]} & {[\mathrm{mAU}]} & \% \\ 1 & 20.338 \text { MM } & 0.6434 & 4528.65771 & 117.31321 & 46.0962 \\ 2 & 23.223 \text { MM } & 0.7301 & 5295.69922 & 120.88832 & 53.9038\end{array}$

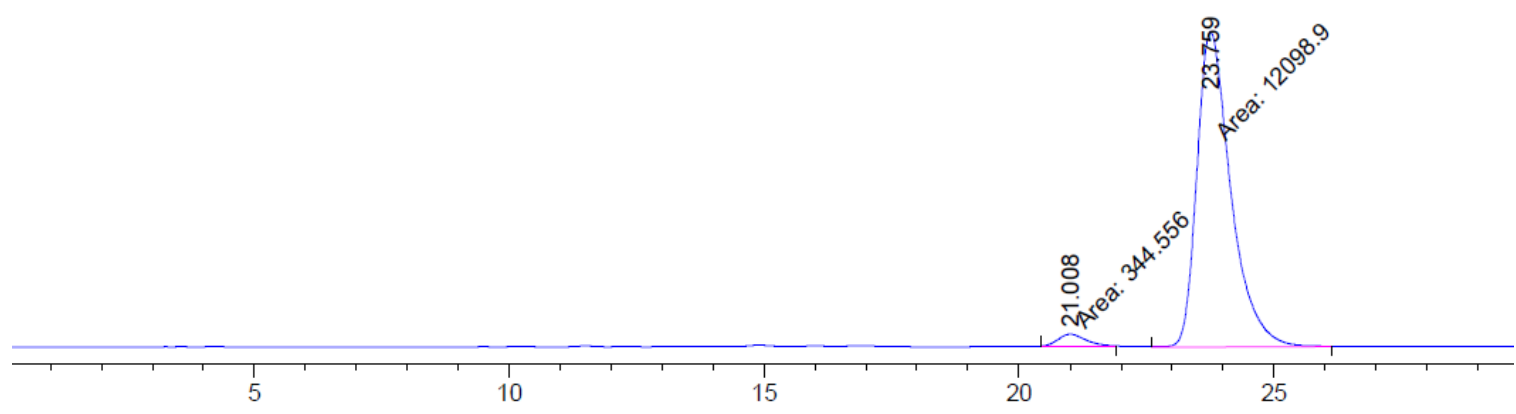

\begin{tabular}{cccccc}
$\begin{array}{c}\text { Peak RetTime Type } \\
\text { \# }\end{array}$ & $\begin{array}{c}\text { Width } \\
{[\mathrm{min}]}\end{array}$ & $\begin{array}{c}\text { Area } \\
{[\mathrm{min}]}\end{array}$ & $\begin{array}{c}\text { Height } \\
{\left[\mathrm{mAU} \mathbf{U}^{*}\right]}\end{array}$ & $\begin{array}{c}\text { Area } \\
{[\mathrm{mAU}]}\end{array}$ & $\%$ \\
\hline 1 & 21.008 MM & 0.5967 & 344.55612 & 9.62341 & 2.7690 \\
2 & 23.759 MM & 0.7727 & $1.20989 \mathrm{e} 4$ & 260.97653 & 97.2310
\end{tabular}


IR of compound 14 I

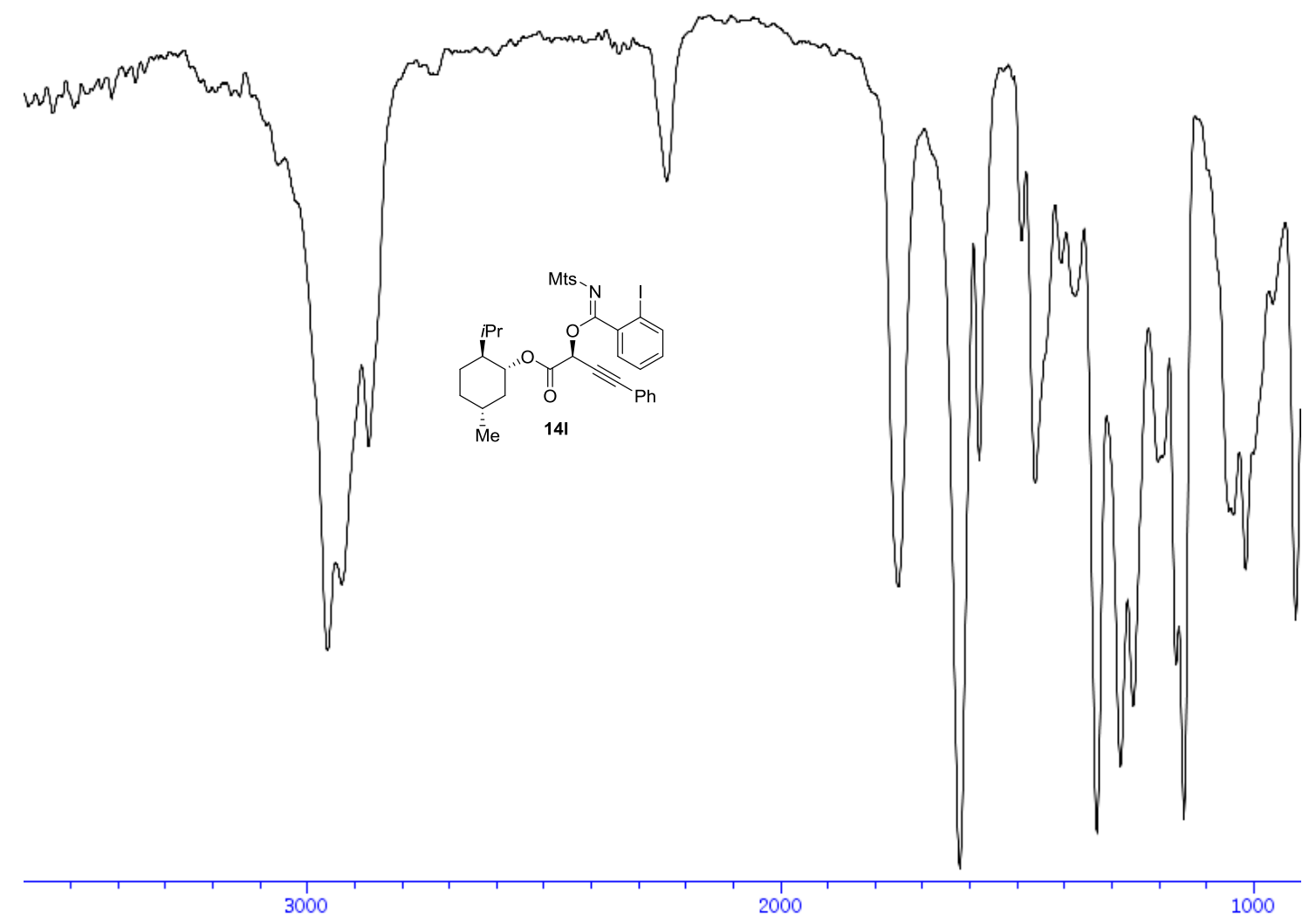


${ }^{\mathbf{1}} \mathbf{H}-\mathbf{N M R}\left(400 \mathrm{MHz}, \mathrm{CDCl}_{3}\right)$ of compound $\mathbf{1 4 m}$

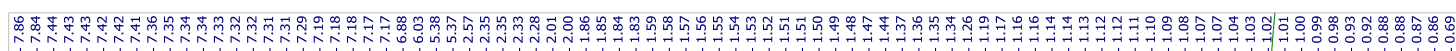

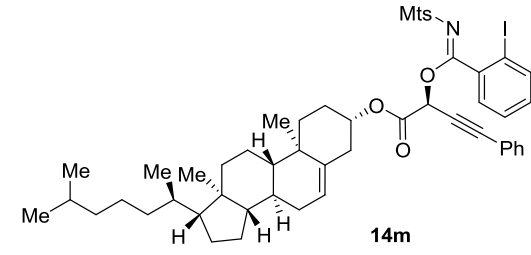
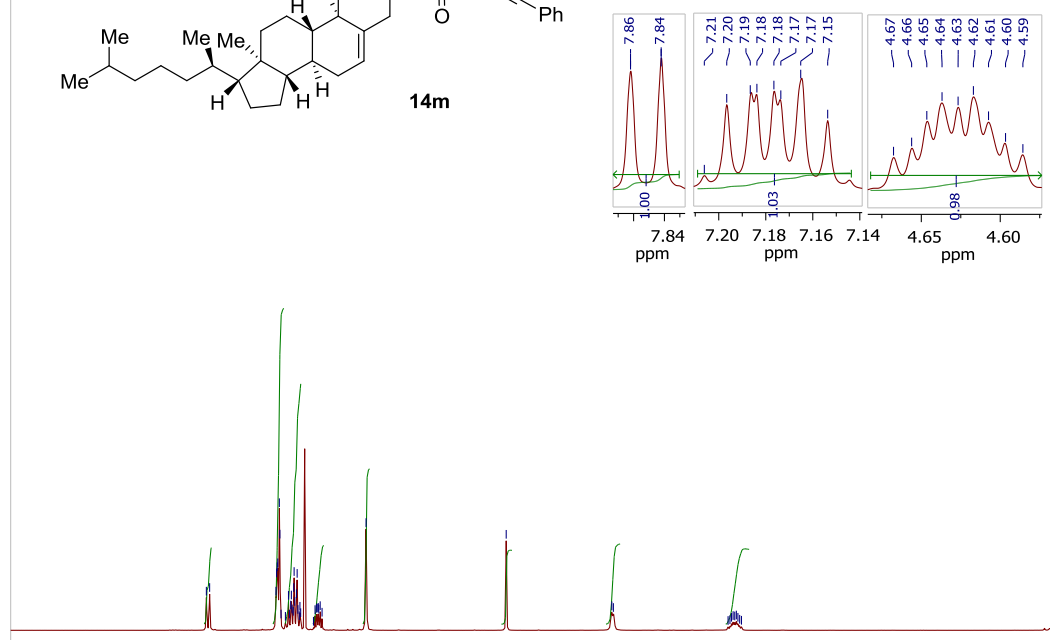

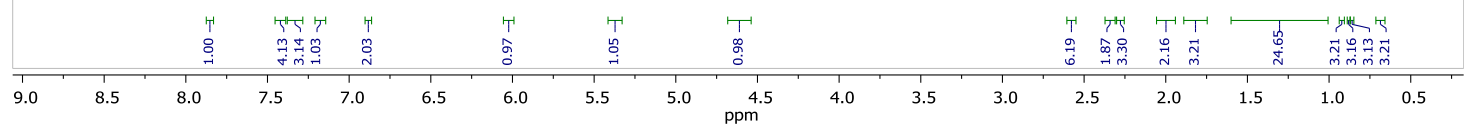

${ }^{13}$ C-NMR (100 MHz, $\left.\mathrm{CDCl}_{3}\right)$ of compound $\mathbf{1 4 m}$

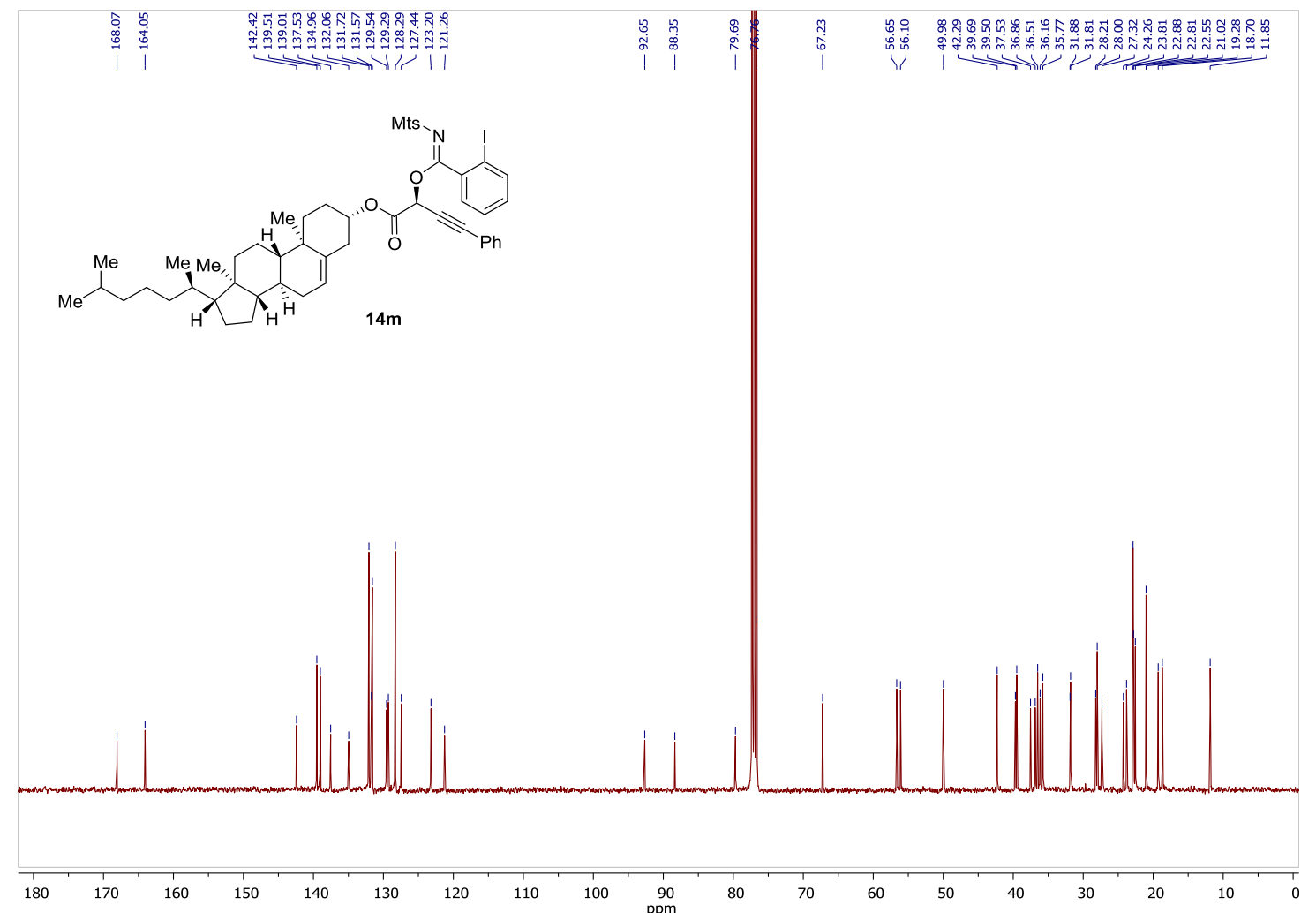


HPLC of compound 14m

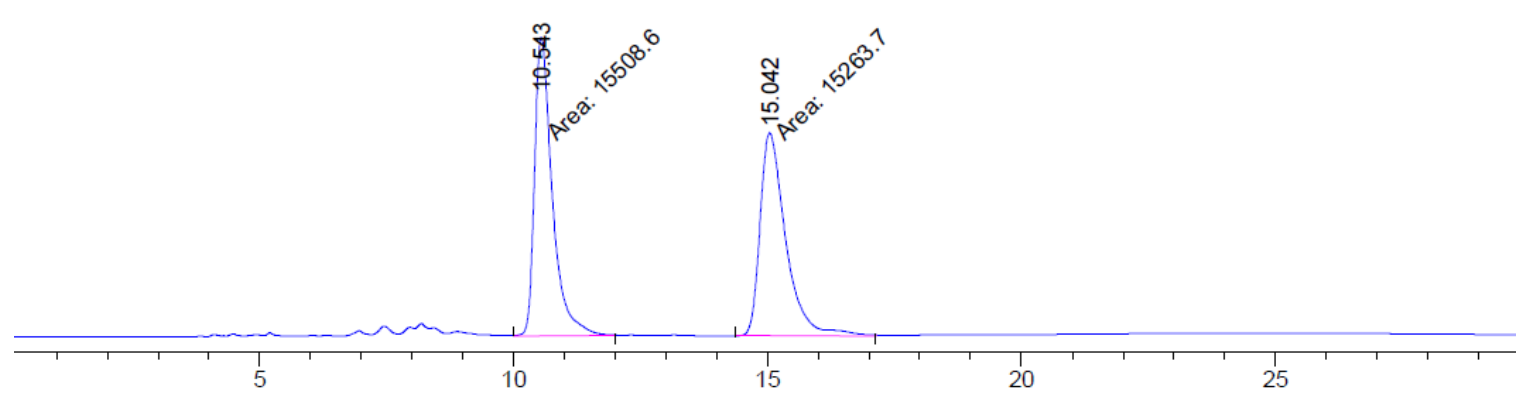

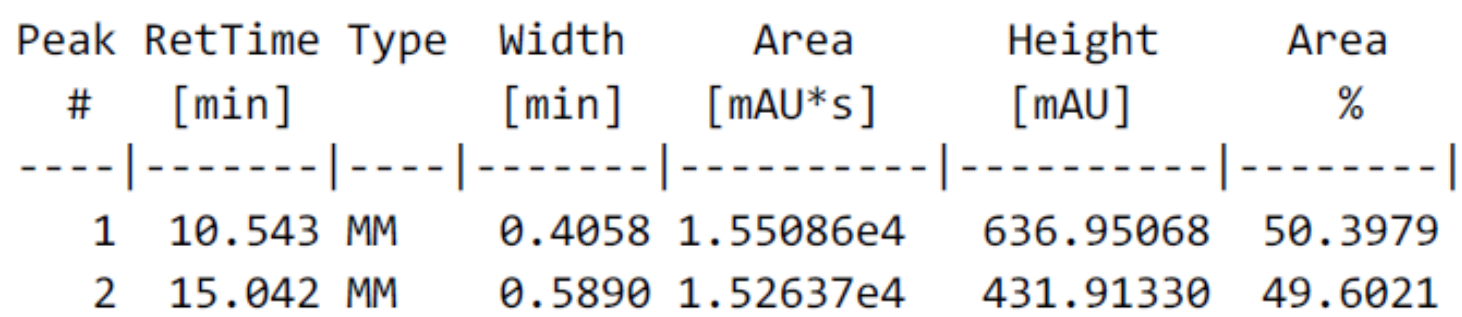

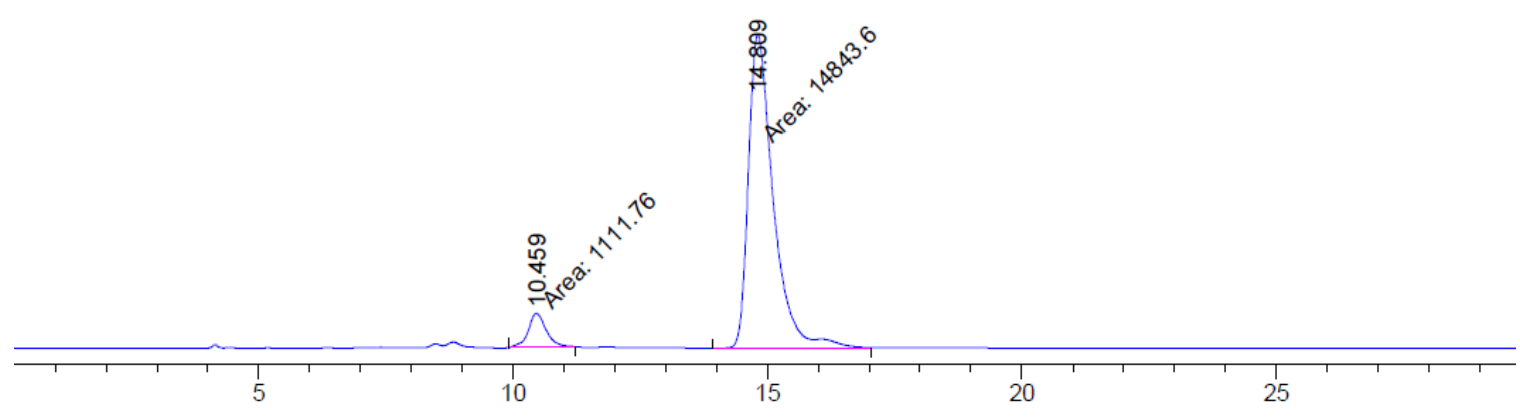

\begin{tabular}{cccccc}
$\begin{array}{c}\text { Peak RetTime Type } \\
\#\end{array}$ & $\begin{array}{c}\text { Width } \\
{[\text { min }]}\end{array}$ & $\begin{array}{c}\text { Area } \\
{[\text { min }]}\end{array}$ & {$[$ mAU*s $]$} & $\begin{array}{c}\text { Height } \\
{[\text { mAU }]}\end{array}$ & $\begin{array}{c}\text { Area } \\
\%\end{array}$ \\
\hline 1 & 10.459 MM & 0.3993 & 1111.75940 & 46.40980 & 6.9679 \\
2 & 14.809 MM & 0.5719 & $1.48436 e 4$ & 432.58713 & 93.0321
\end{tabular}


IR of compound $\mathbf{1 4 m}$

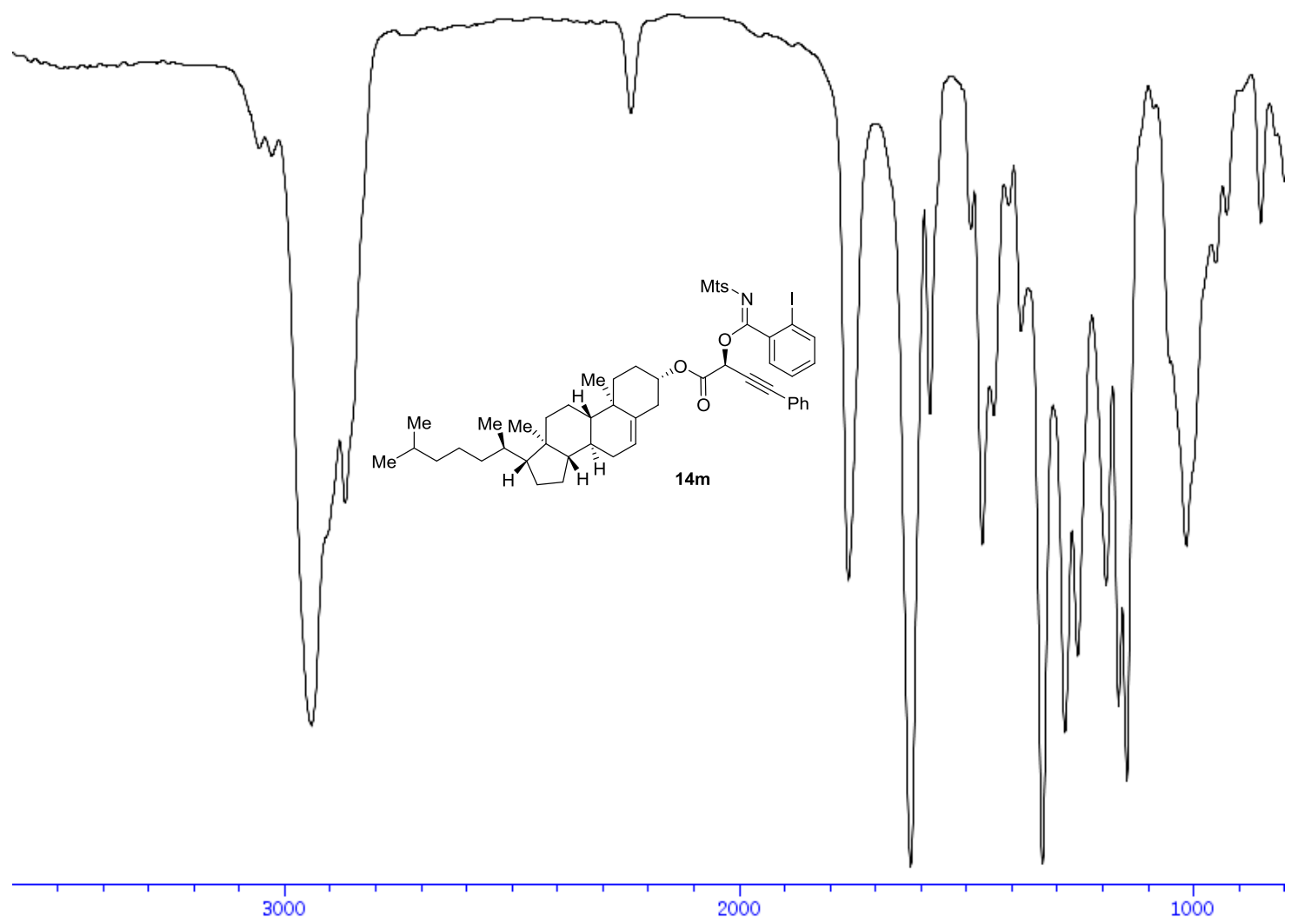


${ }^{\mathbf{1}} \mathbf{H}-\mathbf{N M R}\left(400 \mathrm{MHz}, \mathrm{CDCl}_{3}\right)$ of compound $\mathbf{1 4 n}$

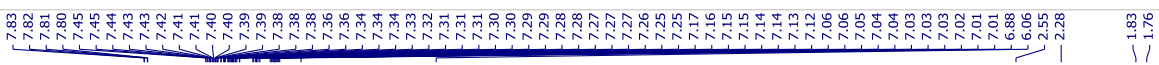<smiles>Cc1ccccc1C(=O)OC(=NS(=O)(=O)c1ccccc1)C(=O)OC(C)(C)c1ccc(F)cc1</smiles>

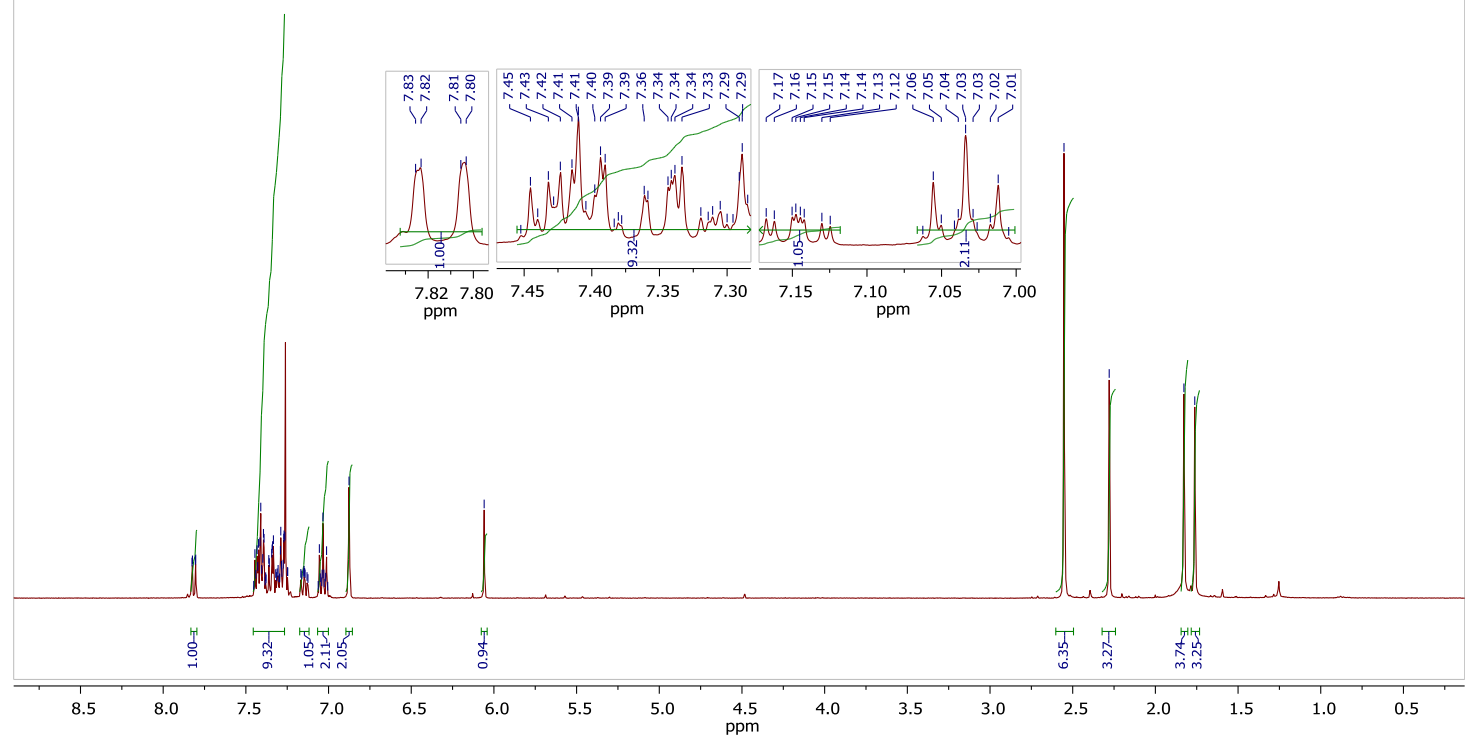

${ }^{13} \mathbf{C}-\mathbf{N M R}\left(100 \mathrm{MHz}, \mathrm{CDCl}_{3}\right)$ of compound $\mathbf{1 4 n}$

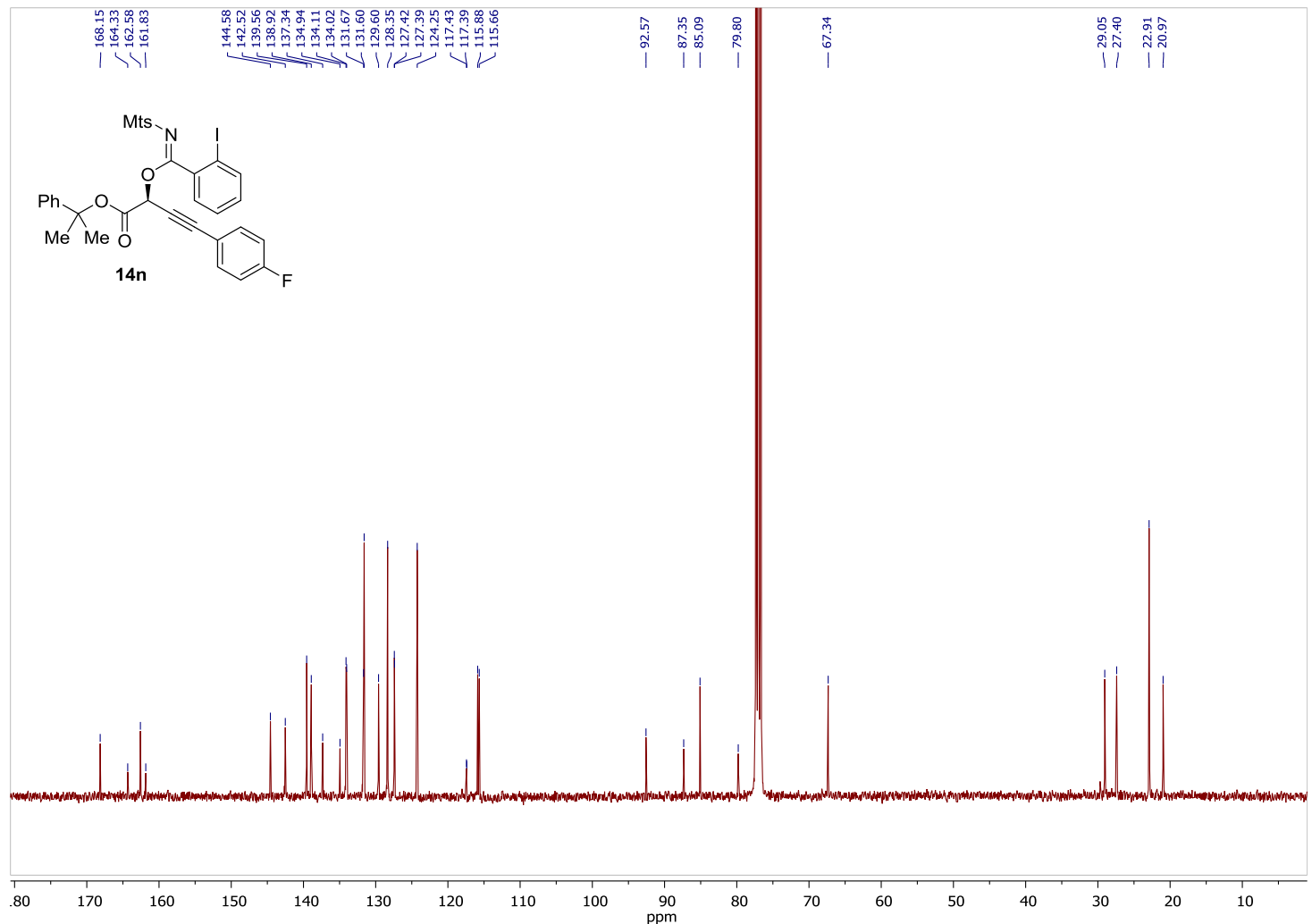


HPLC of compound 14n

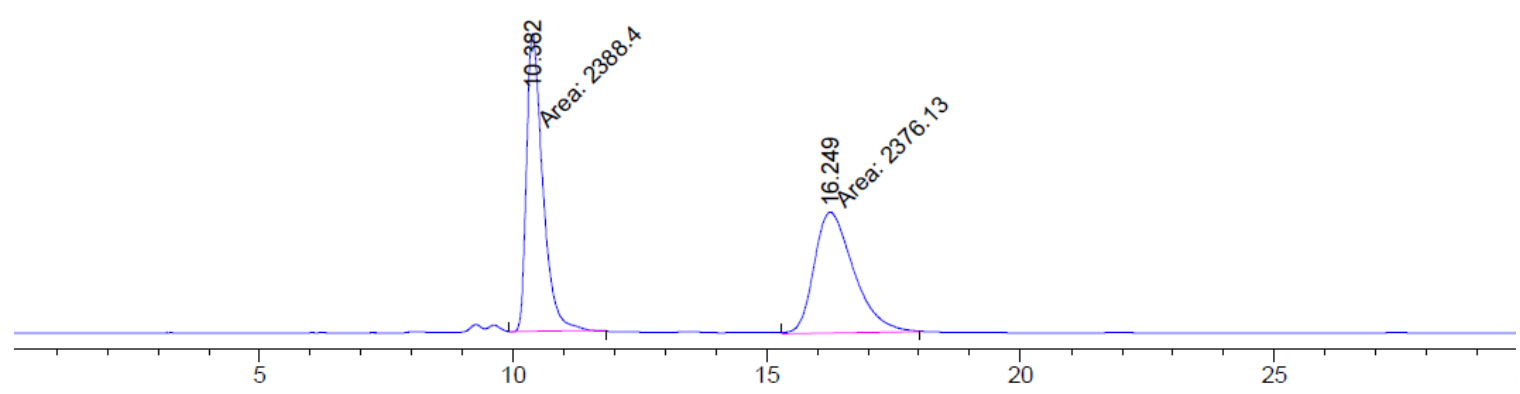

\begin{tabular}{cccccc}
$\begin{array}{c}\text { Peak RetTime Type } \\
\#\end{array}$ & width & Area & Height & Area \\
& {$[\mathrm{min}]$} & {$[\mathrm{min}]$} & {$[\mathrm{mAU}$ s $]$} & {$[\mathrm{mAU}]$} & $\%$ \\
\hline 1 & $10.382 \mathrm{MM}$ & 0.3713 & 2388.39575 & 107.19816 & 50.1287 \\
2 & $16.249 \mathrm{MM}$ & 0.9090 & 2376.12988 & 43.56669 & 49.8713
\end{tabular}

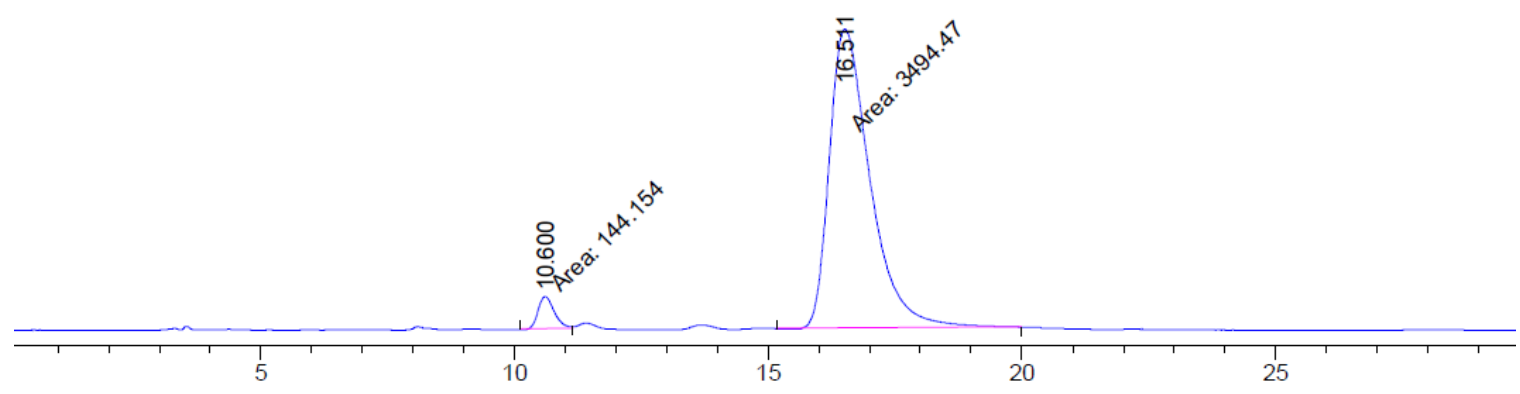

\begin{tabular}{cccccc}
$\begin{array}{c}\text { Peak RetTime Type } \\
\text { Width }\end{array}$ & $\begin{array}{c}\text { Area } \\
{[\mathrm{min}]}\end{array}$ & $\begin{array}{c}\text { Height } \\
{[\mathrm{mAU} \text { s }]}\end{array}$ & $\begin{array}{c}\text { Area } \\
{[\mathrm{mAU}]}\end{array}$ & $\%$ \\
\hline 1 & $10.600 \mathrm{MM}$ & 0.3594 & 144.15353 & 6.68413 & 3.9618 \\
2 & $16.511 \mathrm{MM}$ & 0.9283 & 3494.47144 & 62.73778 & 96.0382
\end{tabular}


IR of compound $\mathbf{1 4 n}$

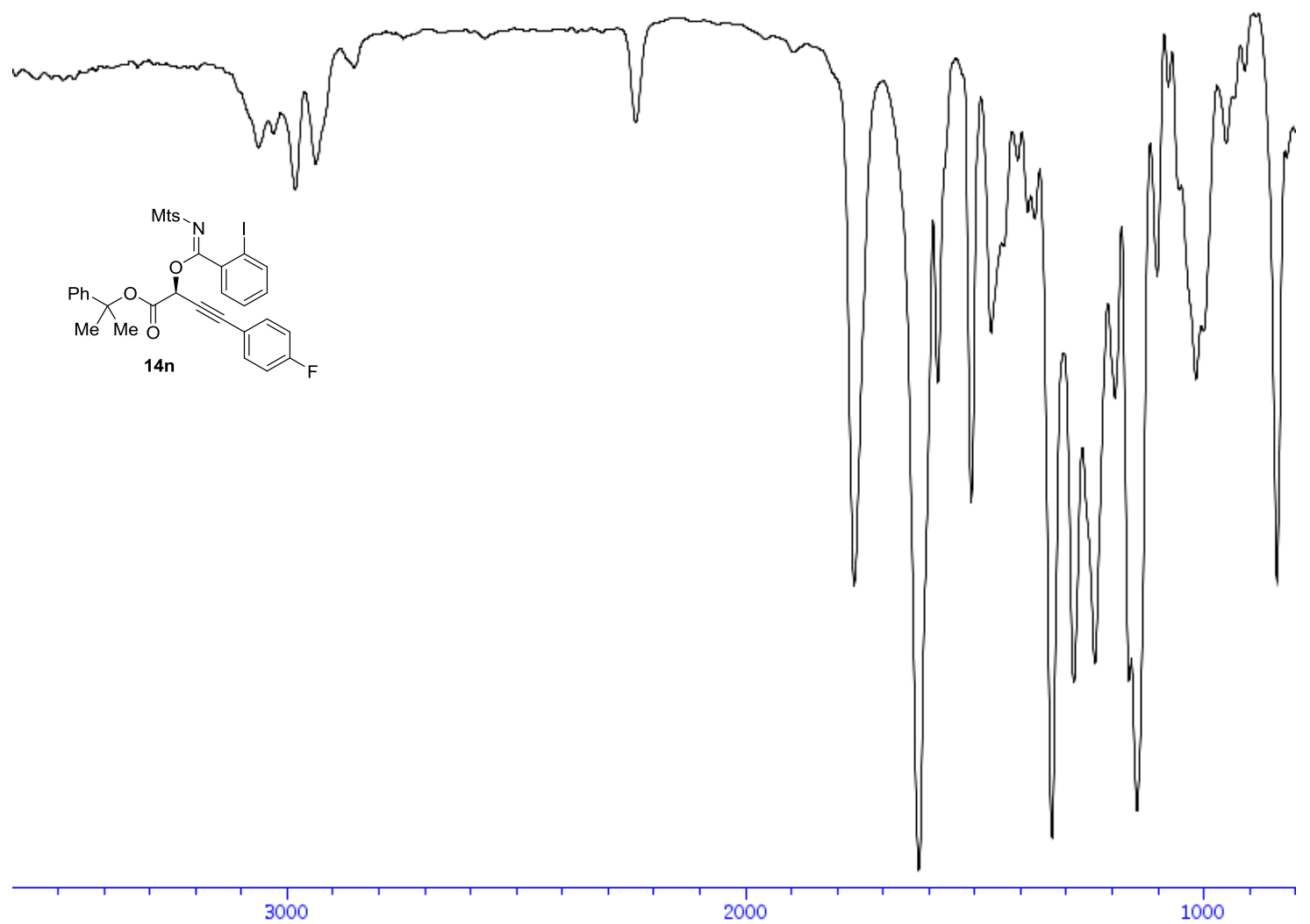


${ }^{\mathbf{1}} \mathbf{H}-\mathbf{N M R}\left(400 \mathrm{MHz}, \mathrm{CDCl}_{3}\right)$ of compound $\mathbf{1 4 0}$

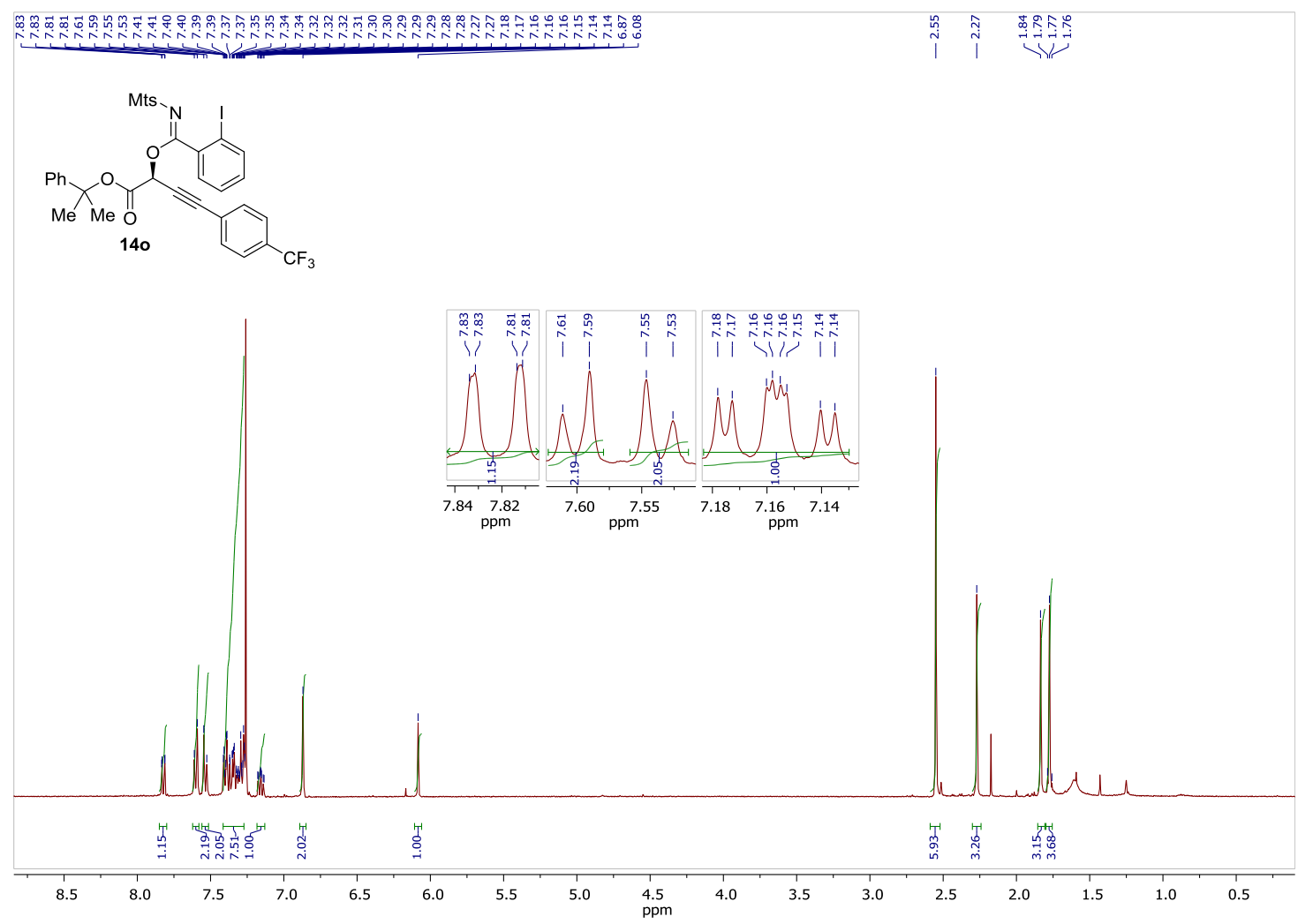

${ }^{13}$ C-NMR (100 MHz, $\left.\mathrm{CDCl}_{3}\right)$ of compound $\mathbf{1 4 0}$

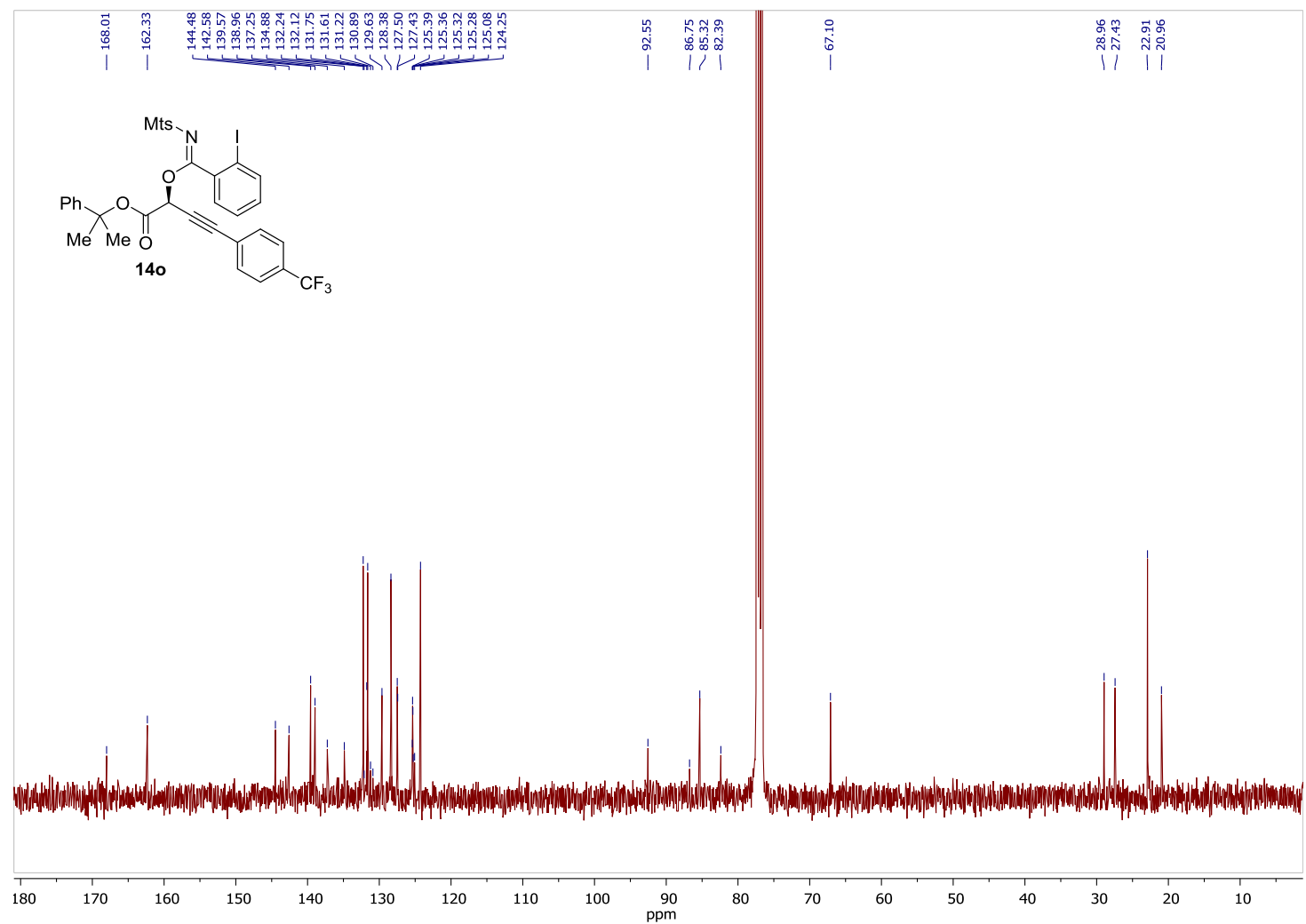


HPLC of compound 140
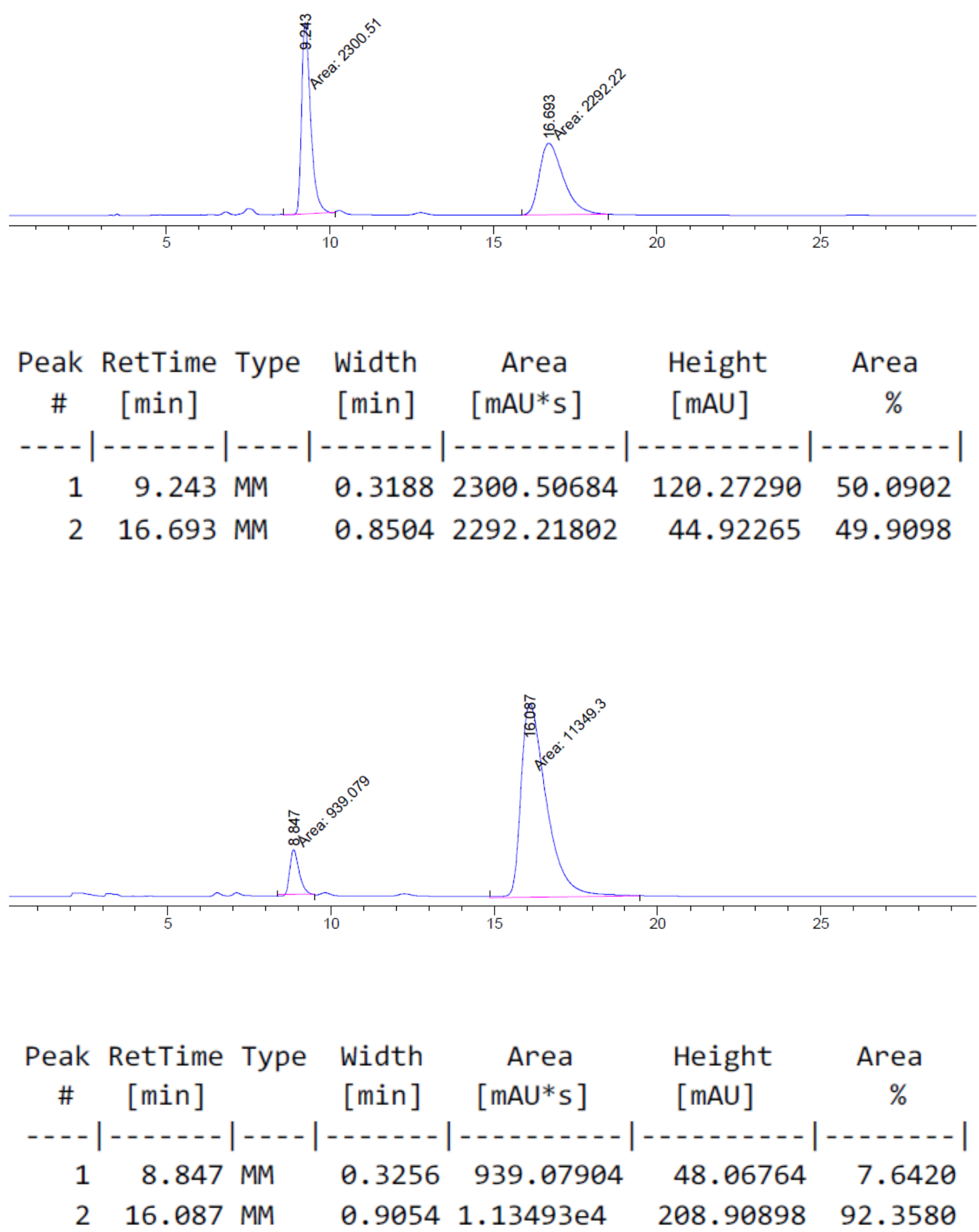
IR of compound 14o

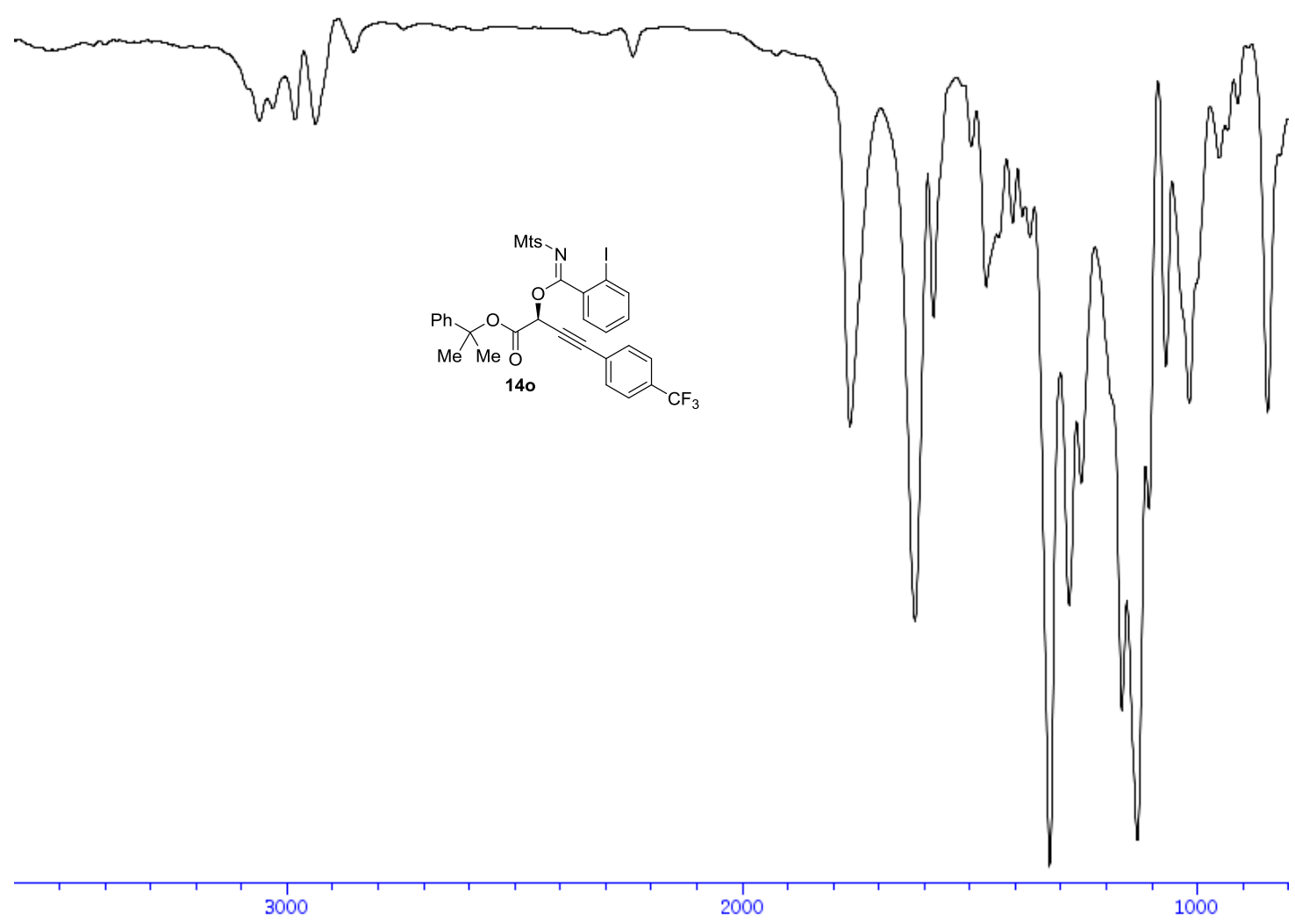


${ }^{\mathbf{1}} \mathbf{H}-\mathbf{N M R}\left(400 \mathrm{MHz}, \mathrm{CDCl}_{3}\right)$ of compound $\mathbf{1 4 p}$

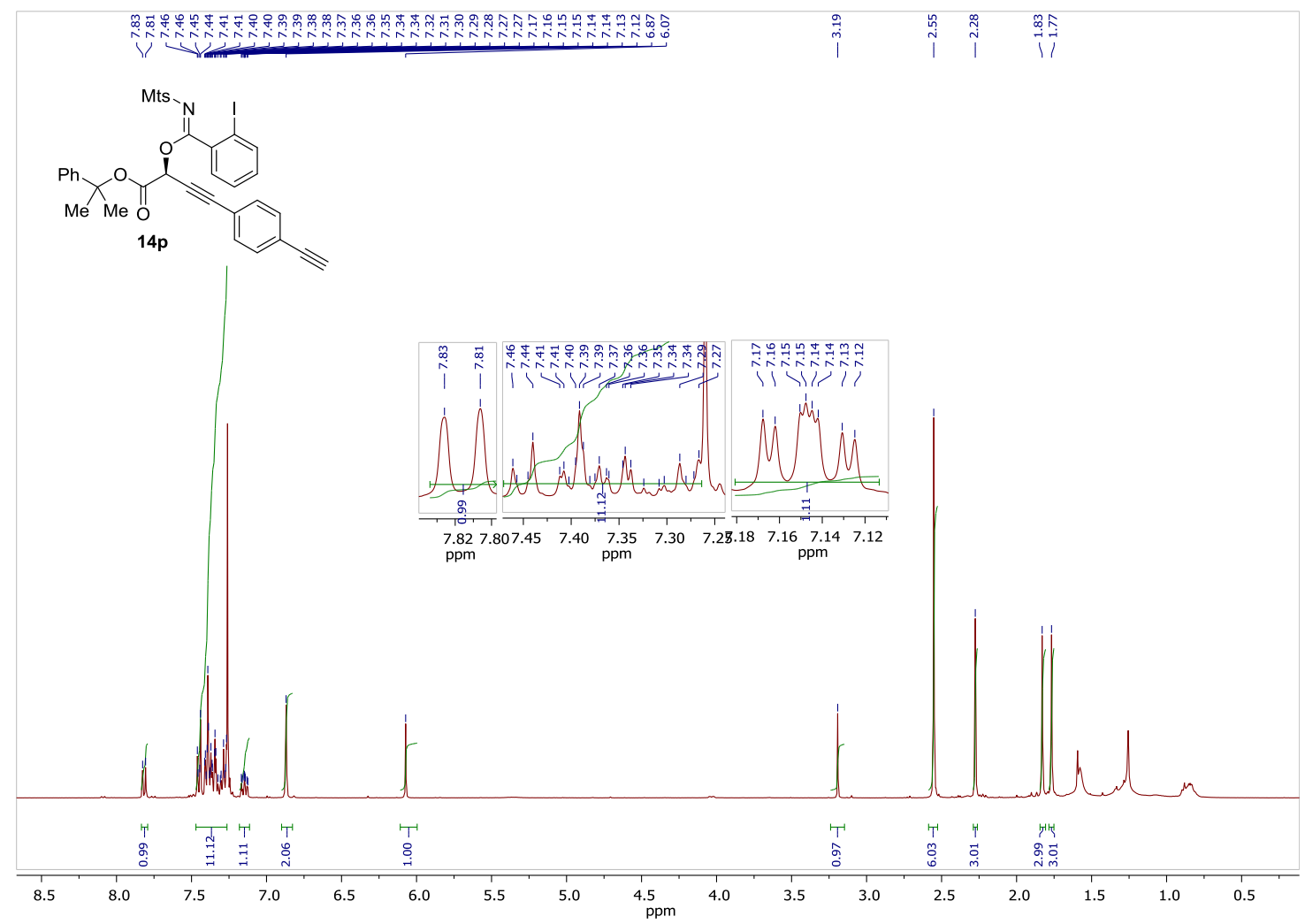

${ }^{13} \mathbf{C}-\mathbf{N M R}\left(100 \mathrm{MHz}, \mathrm{CDCl}_{3}\right)$ of compound 14p

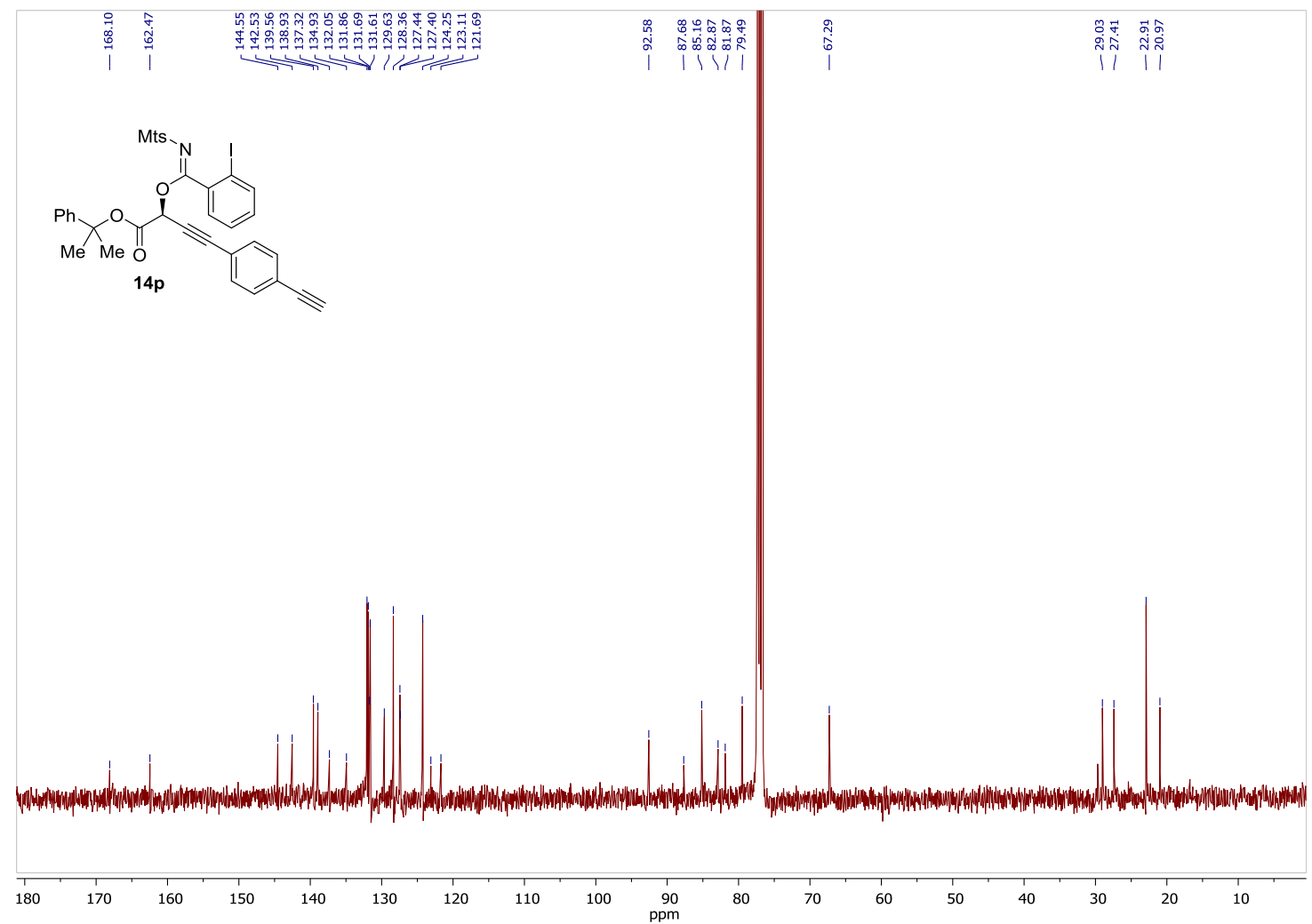


HPLC of compound 14p

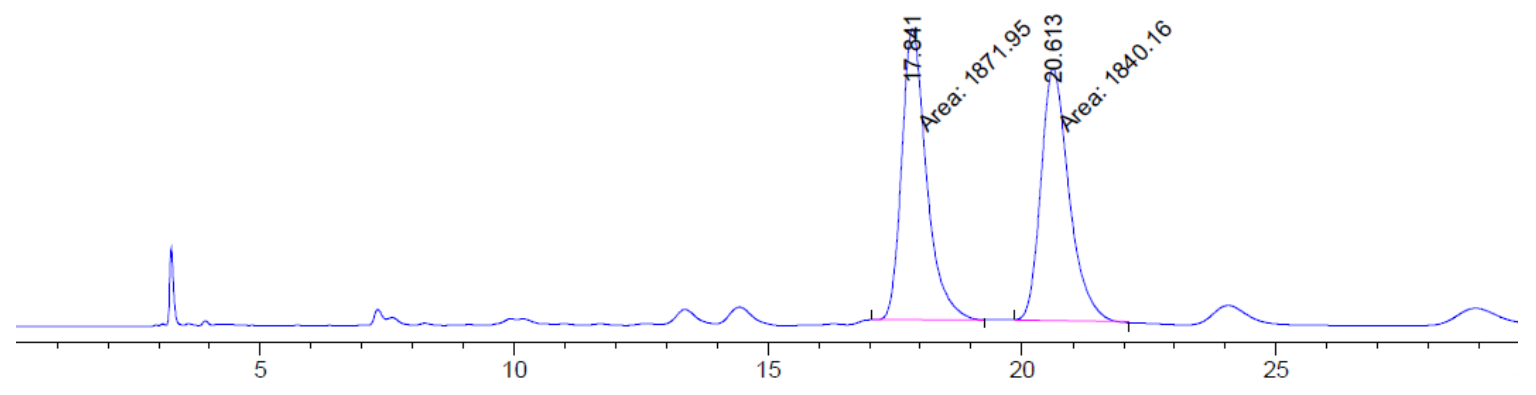

Peak RetTime Type Width Area Height Area

\# $[\mathrm{min}] \quad[\mathrm{min}] \quad\left[\mathrm{mAU}^{*} \mathrm{~s}\right] \quad[\mathrm{mAU}] \quad \%$

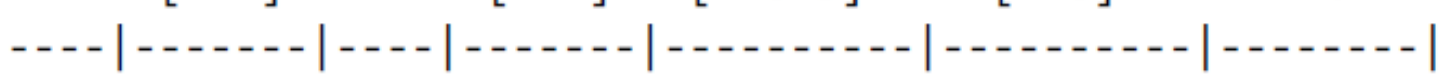

$\begin{array}{lllllll}1 & 17.841 & \text { MM } & 0.5671 & 1871.95227 & 55.01223 & 50.4282\end{array}$

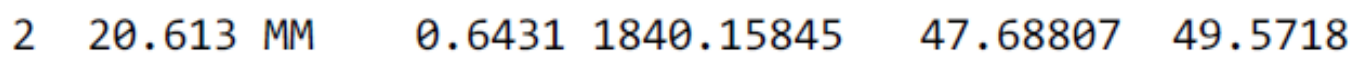

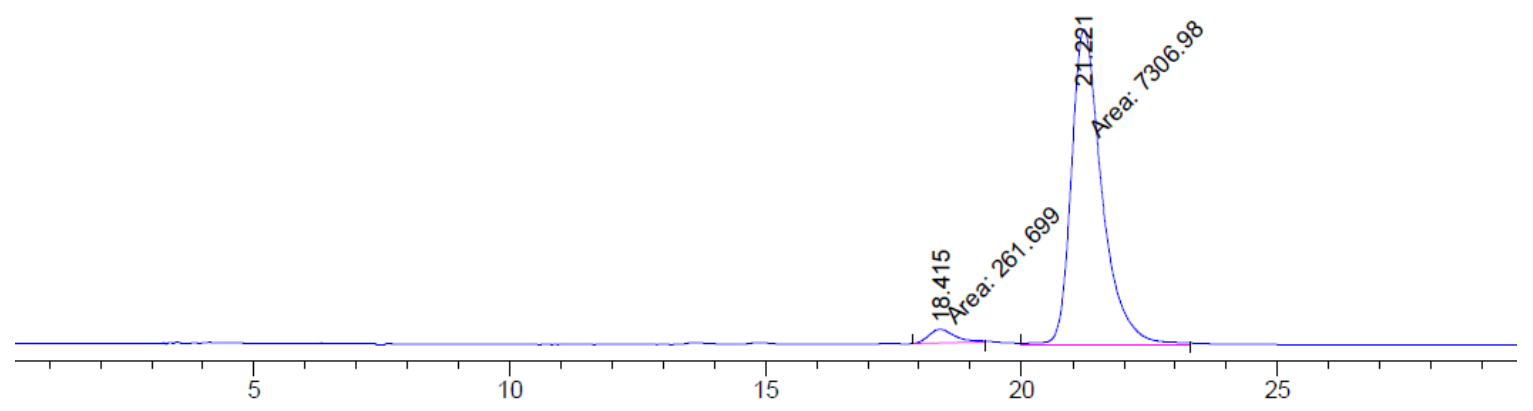

\begin{tabular}{|c|c|c|c|c|c|c|}
\hline $\begin{array}{c}\text { Peak } \\
\quad \#\end{array}$ & $\begin{array}{c}\text { RetTime } \\
\text { [min] }\end{array}$ & Type & $\begin{array}{l}\text { Width } \\
\text { [min] }\end{array}$ & $\begin{array}{c}\text { Area } \\
{\left[\mathrm{mAU}^{*} \mathrm{~s}\right]}\end{array}$ & $\begin{array}{l}\text { Height } \\
{[\mathrm{mAU}]}\end{array}$ & $\begin{array}{c}\text { Area } \\
\%\end{array}$ \\
\hline & & & & $1-$ & -1 & $-1 .-1$ \\
\hline 1 & 18.415 & MM & 0.5903 & 261.69855 & 7.38915 & 3.4577 \\
\hline 2 & 21.221 & MM & .6919 & 7306.97656 & 176.01575 & 96.5423 \\
\hline
\end{tabular}


IR of compound 14p

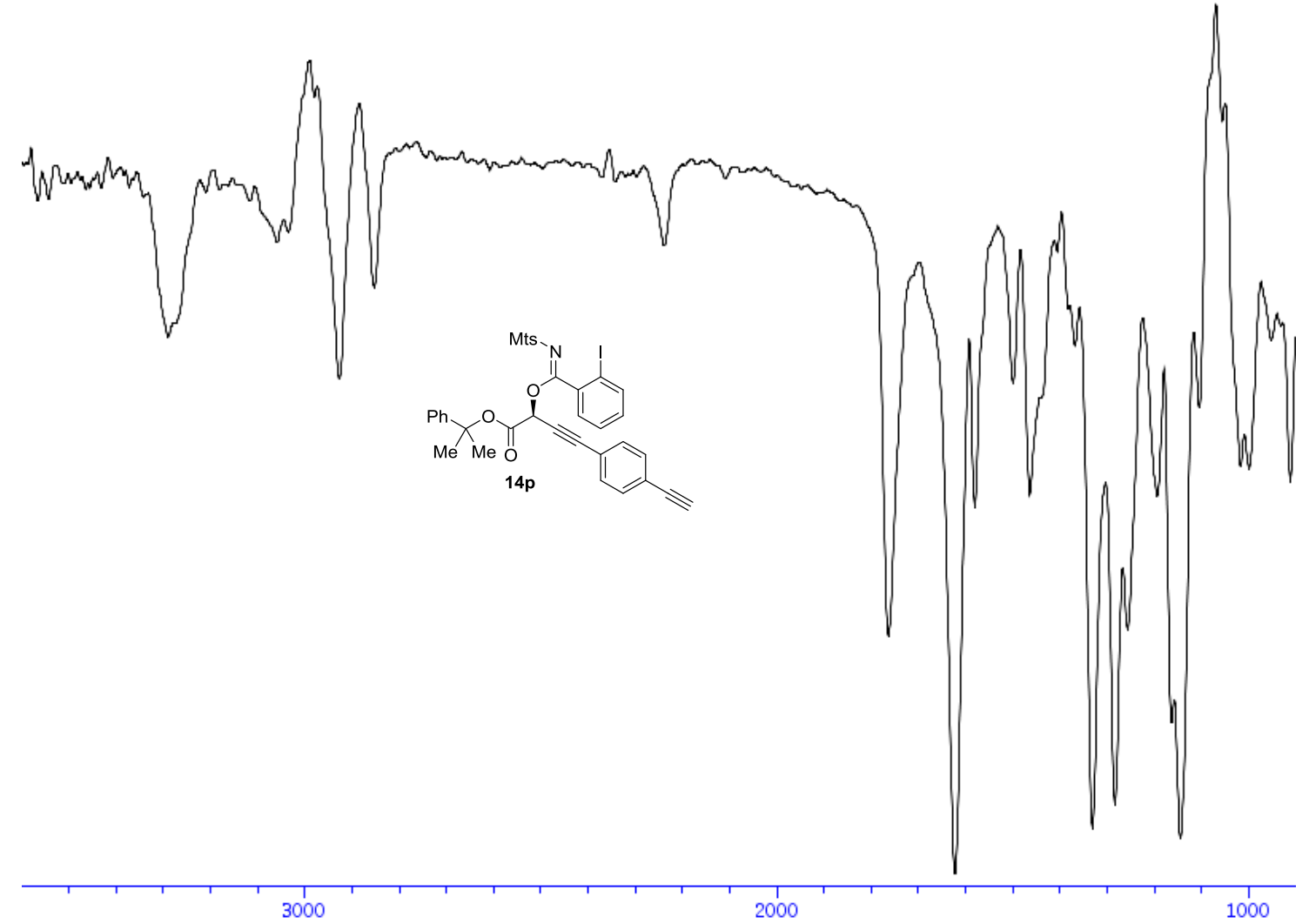


${ }^{\mathbf{1}} \mathbf{H}-\mathrm{NMR}\left(400 \mathrm{MHz}, \mathrm{CDCl}_{3}\right)$ of compound 14q

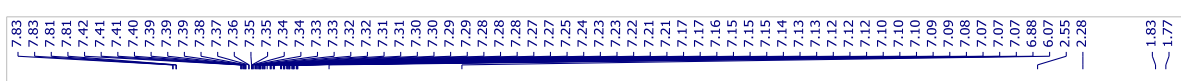
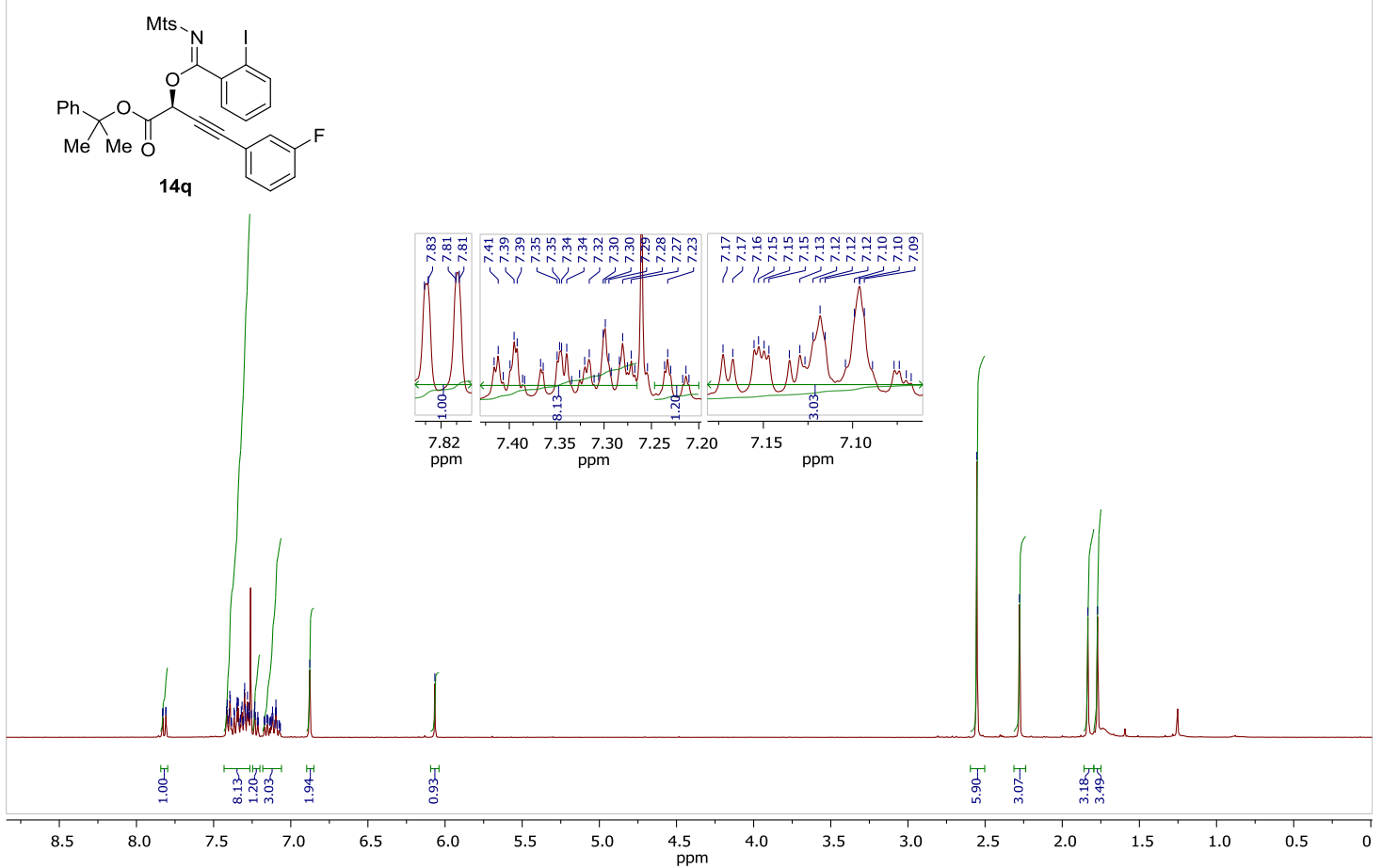

${ }^{13}$ C-NMR $\left(100 \mathrm{MHz}, \mathrm{CDCl}_{3}\right)$ of compound 14q

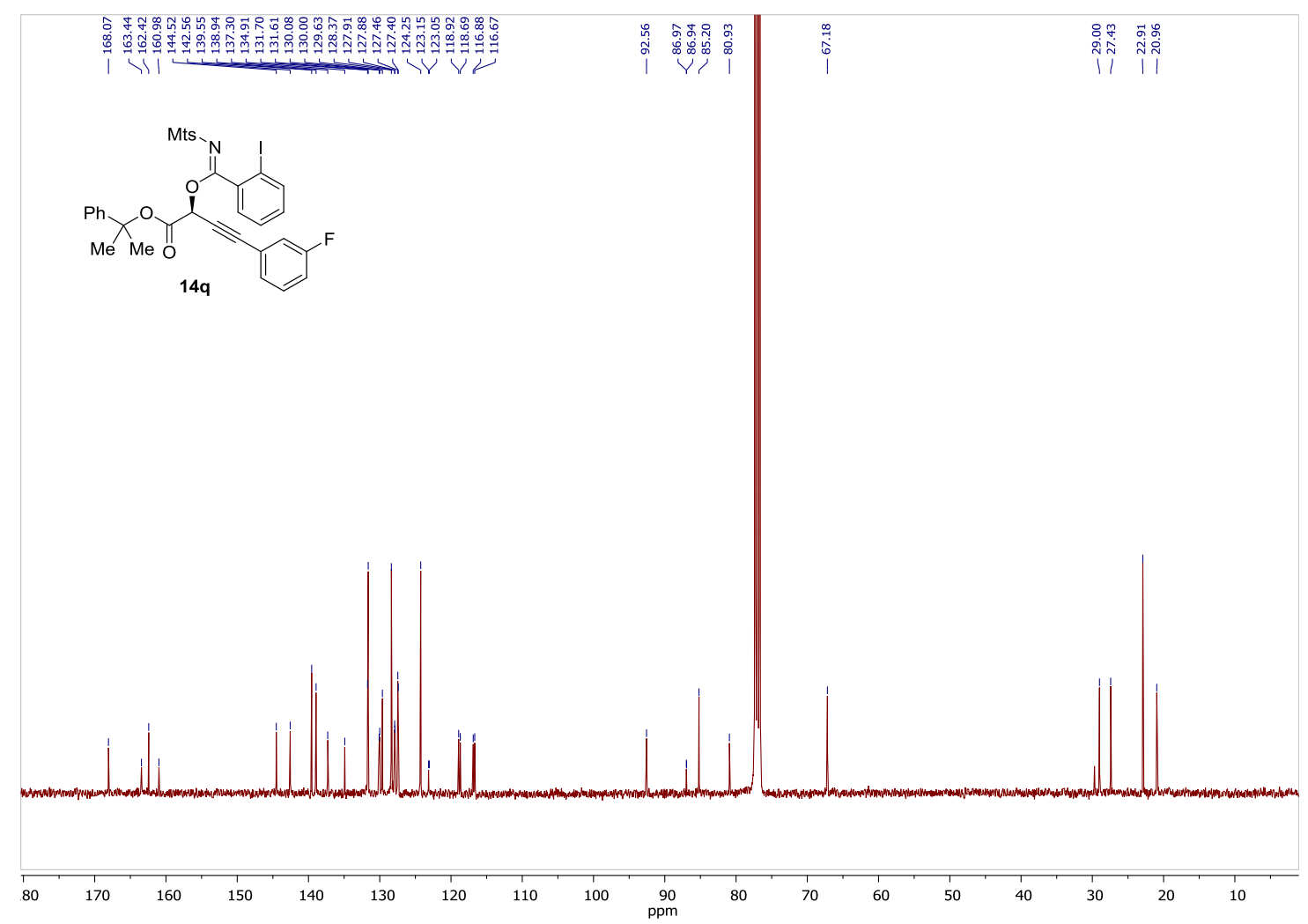

S224 
HPLC of compound 14q

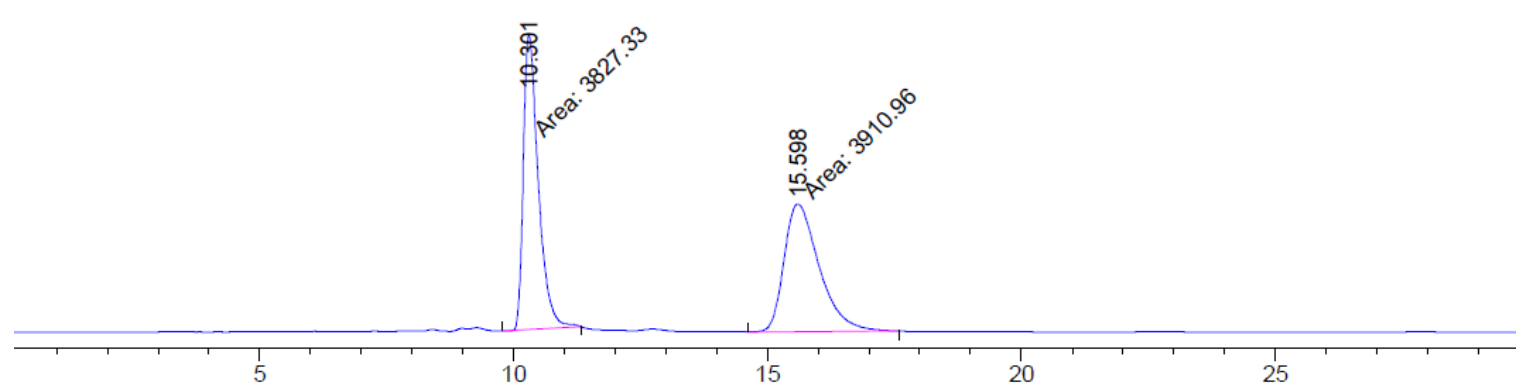

Peak RetTime Type Width Area Height Area

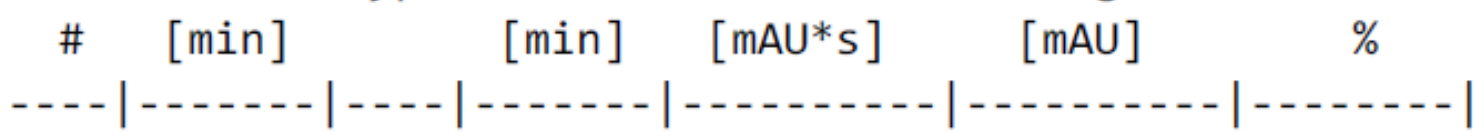

$\begin{array}{lllllll}1 & 10.301 & \text { MM } & 0.3411 & 3827.33472 & 187.01433 & 49.4597\end{array}$

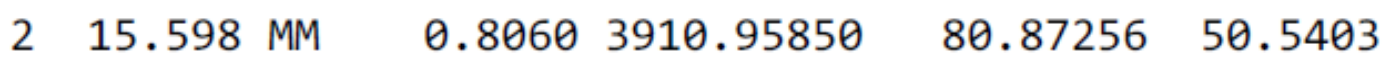

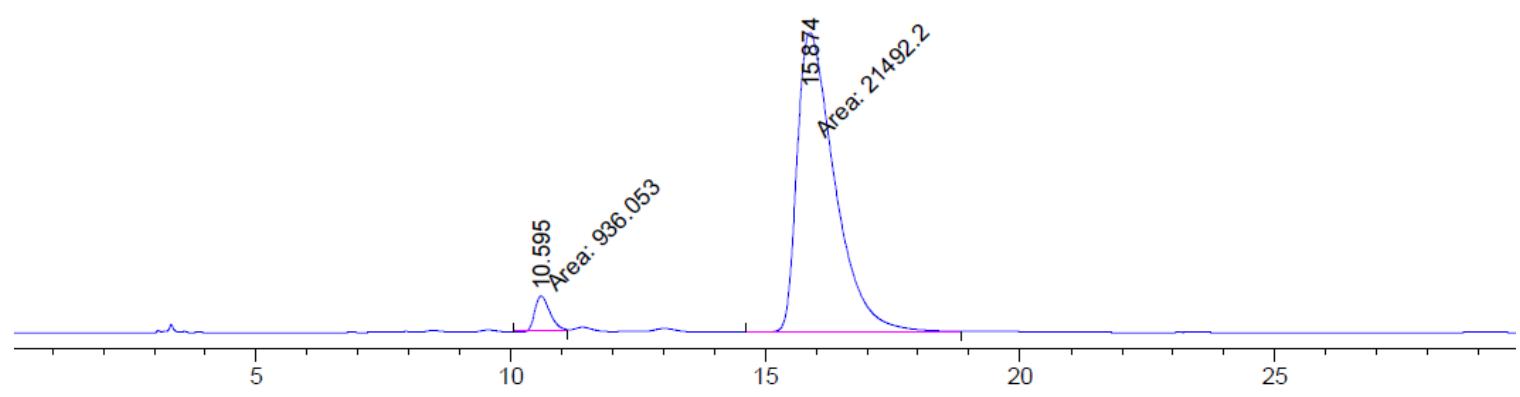

\begin{tabular}{|c|c|c|c|c|c|c|}
\hline $\begin{array}{c}\text { Peak } \\
\#\end{array}$ & $\begin{array}{c}\text { RetTime } \\
\text { [min] }\end{array}$ & Type & $\begin{array}{l}\text { Width } \\
\text { [min] }\end{array}$ & $\begin{array}{c}\text { Area } \\
{\left[\mathrm{mAU}^{*} \mathrm{~s}\right]}\end{array}$ & $\begin{array}{l}\text { Height } \\
{[\mathrm{mAU}]}\end{array}$ & $\begin{array}{c}\text { Area } \\
\%\end{array}$ \\
\hline & & & & -- & -- & --- \\
\hline 1 & 10.595 & MM & 0.3264 & 936.05255 & 47.79220 & 4.1735 \\
\hline 2 & 15.874 & MM & 0.8506 & $2.14922 \mathrm{e} 4$ & 421.11047 & 95.8265 \\
\hline
\end{tabular}


IR of compound $\mathbf{1 4 q}$

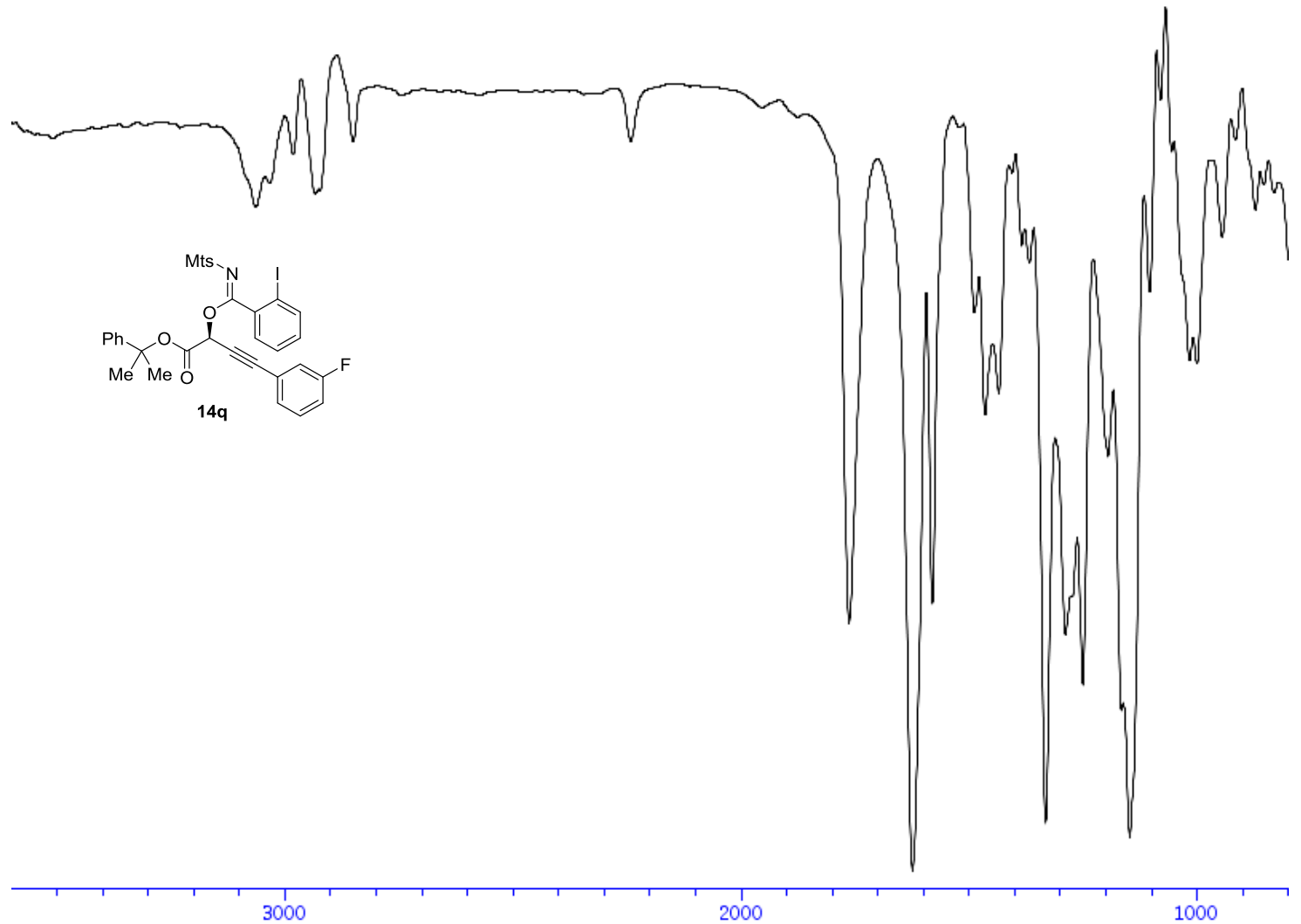


${ }^{\mathbf{1}} \mathbf{H}-\mathbf{N M R}\left(400 \mathrm{MHz}, \mathrm{CDCl}_{3}\right)$ of compound $\mathbf{1 4 r}$

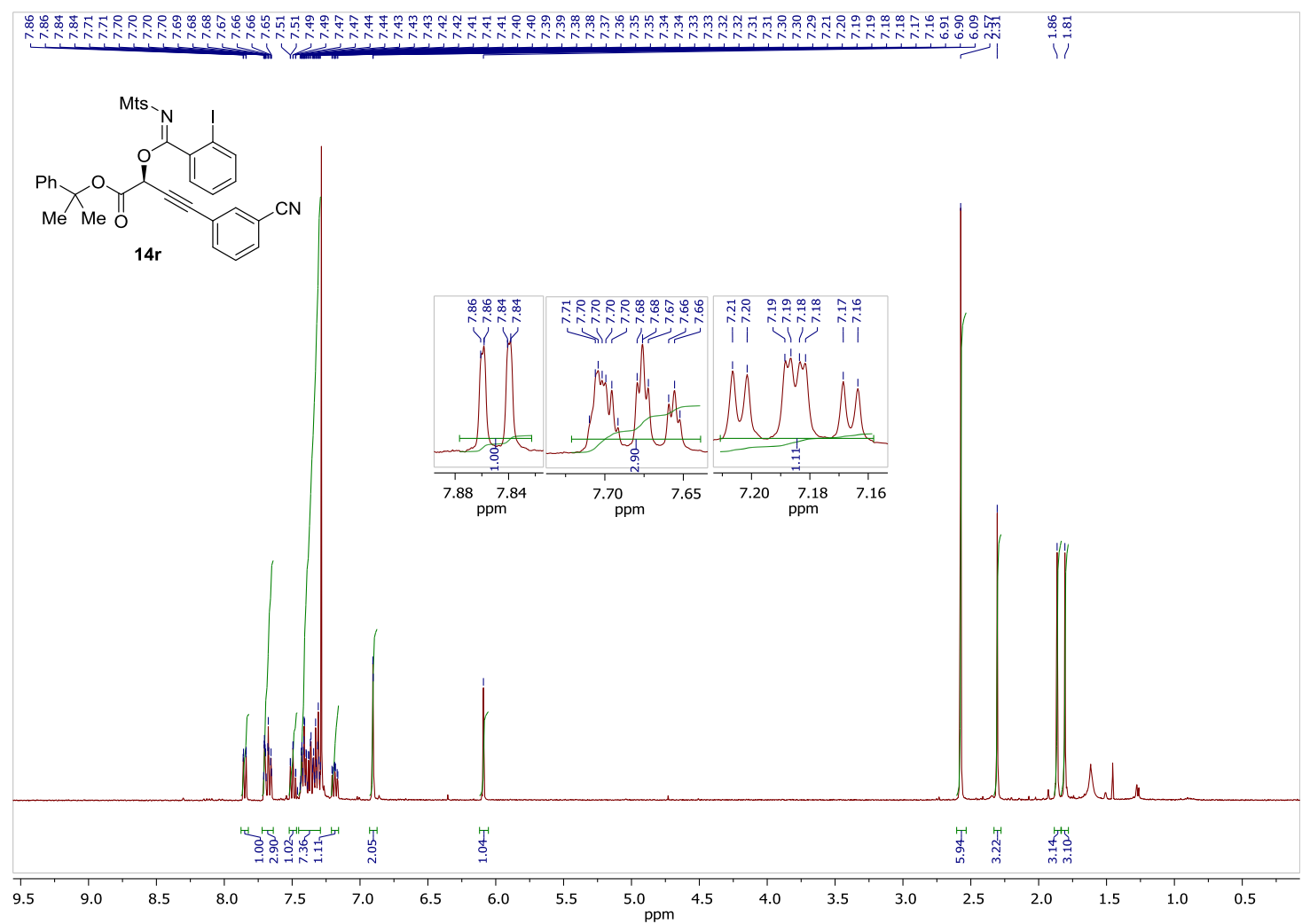

${ }^{13} \mathbf{C}-\mathbf{N M R}\left(100 \mathrm{MHz}, \mathrm{CDCl}_{3}\right)$ of compound $\mathbf{1 4 r}$

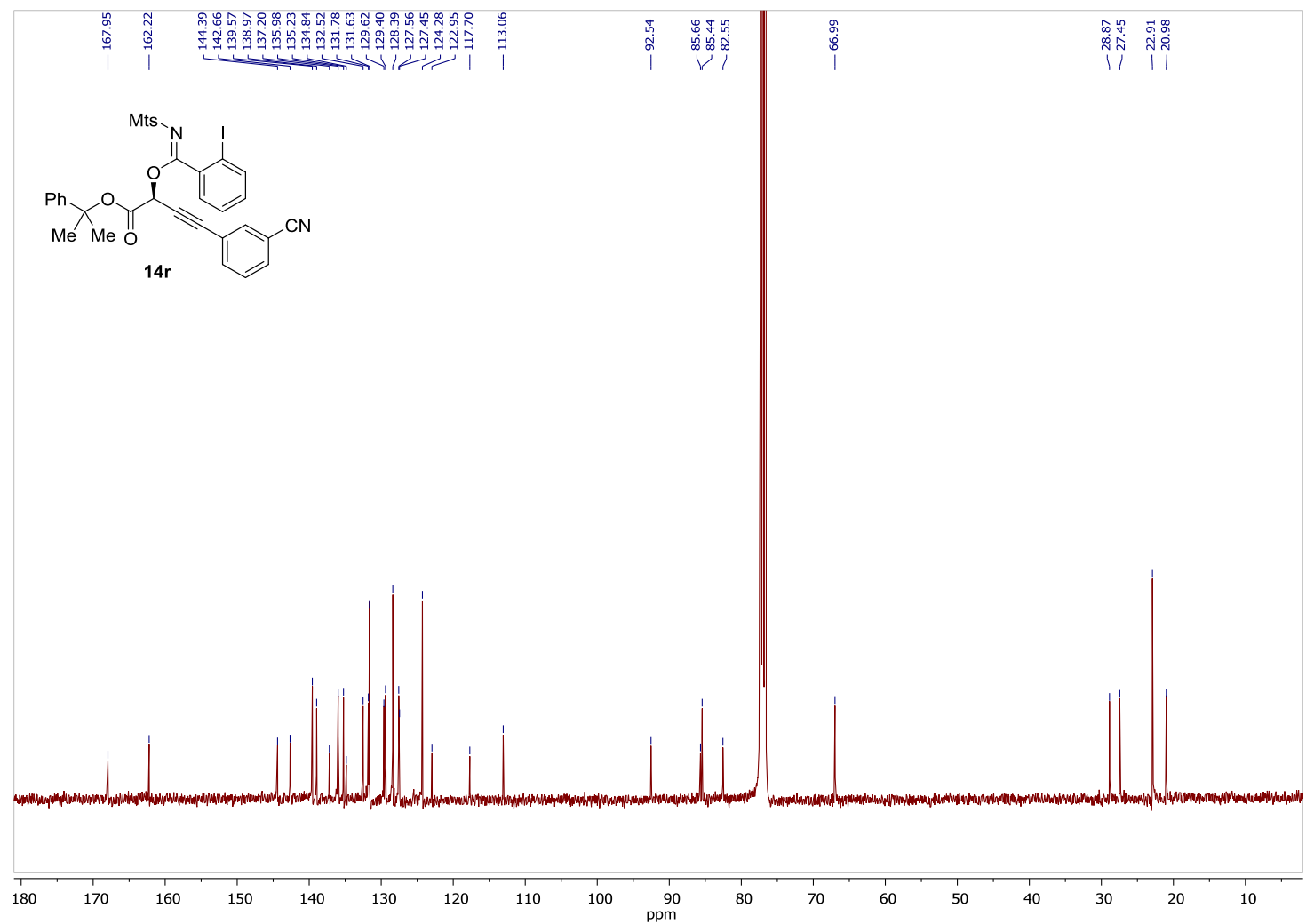


HPLC of compound 14r

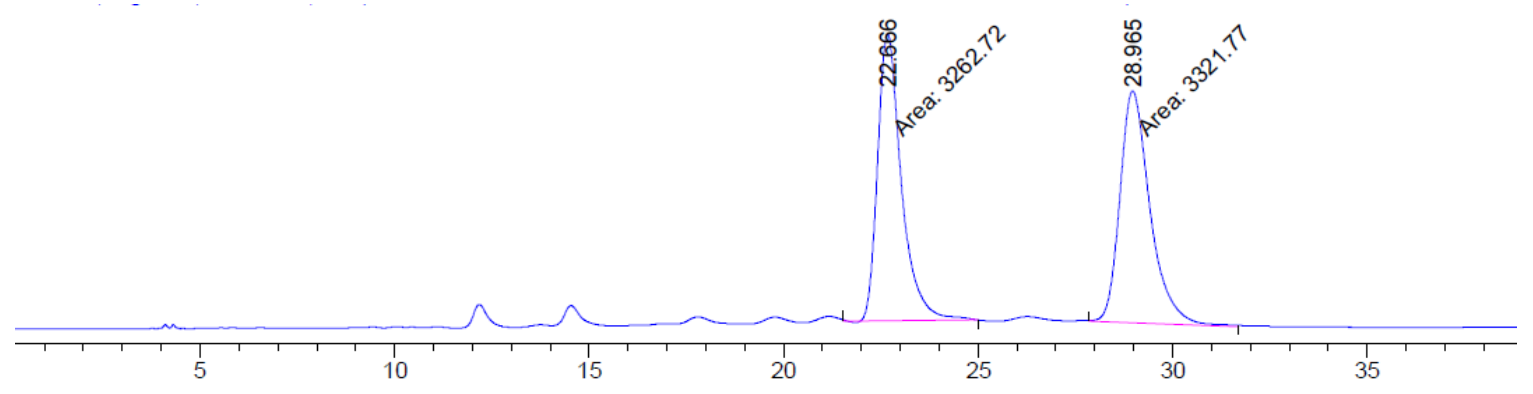

\begin{tabular}{|c|c|c|c|c|c|c|}
\hline $\begin{array}{c}\text { Peak } \\
\#\end{array}$ & $\begin{array}{c}\text { RetTime } \\
\text { [min] }\end{array}$ & Type & $\begin{array}{l}\text { Width } \\
\text { [min] }\end{array}$ & $\begin{array}{c}\text { Area } \\
{\left[\mathrm{mAU}^{*} \mathrm{~s}\right]}\end{array}$ & $\begin{array}{l}\text { Height } \\
{[\mathrm{mAU}]}\end{array}$ & $\begin{array}{c}\text { Area } \\
\%\end{array}$ \\
\hline & & & & & & \\
\hline 1 & 22.666 & MM & 097 & 3262.72363 & 76.61926 & 49.5516 \\
\hline 2 & 28.965 & MM & 953 & 3321.76831 & 3421 & 50.4484 \\
\hline
\end{tabular}

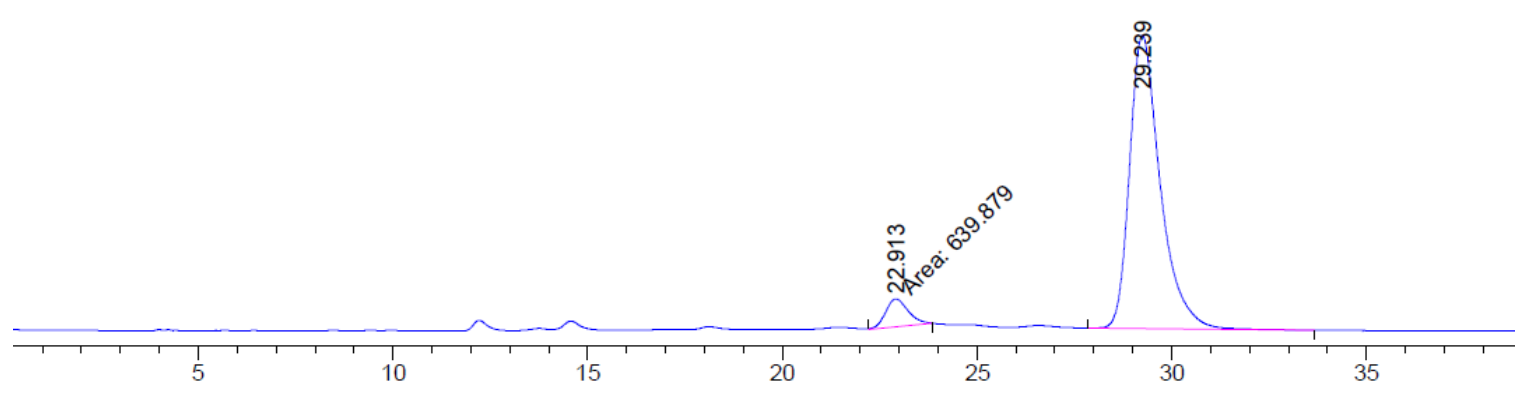

\begin{tabular}{|c|c|c|c|c|c|c|}
\hline $\begin{array}{c}\text { Peak } \\
\#\end{array}$ & $\begin{array}{c}\text { RetTime } \\
\text { [min] }\end{array}$ & Type & $\begin{array}{l}\text { Width } \\
\text { [min] }\end{array}$ & $\begin{array}{c}\text { Area } \\
{\left[\mathrm{mAU}^{*} \mathrm{~s}\right]}\end{array}$ & $\begin{array}{l}\text { Height } \\
{[\mathrm{mAU}]}\end{array}$ & $\begin{array}{c}\text { Area } \\
\%\end{array}$ \\
\hline & & & & - & -- & -- \\
\hline 1 & 22 & MM & 0.6632 & 639.87933 & 16.08115 & 491 \\
\hline 2 & 29.239 & BB & 0.8275 & 9282.15332 & 169.34767 & 93.5509 \\
\hline
\end{tabular}


IR of compound $\mathbf{1 4 r}$

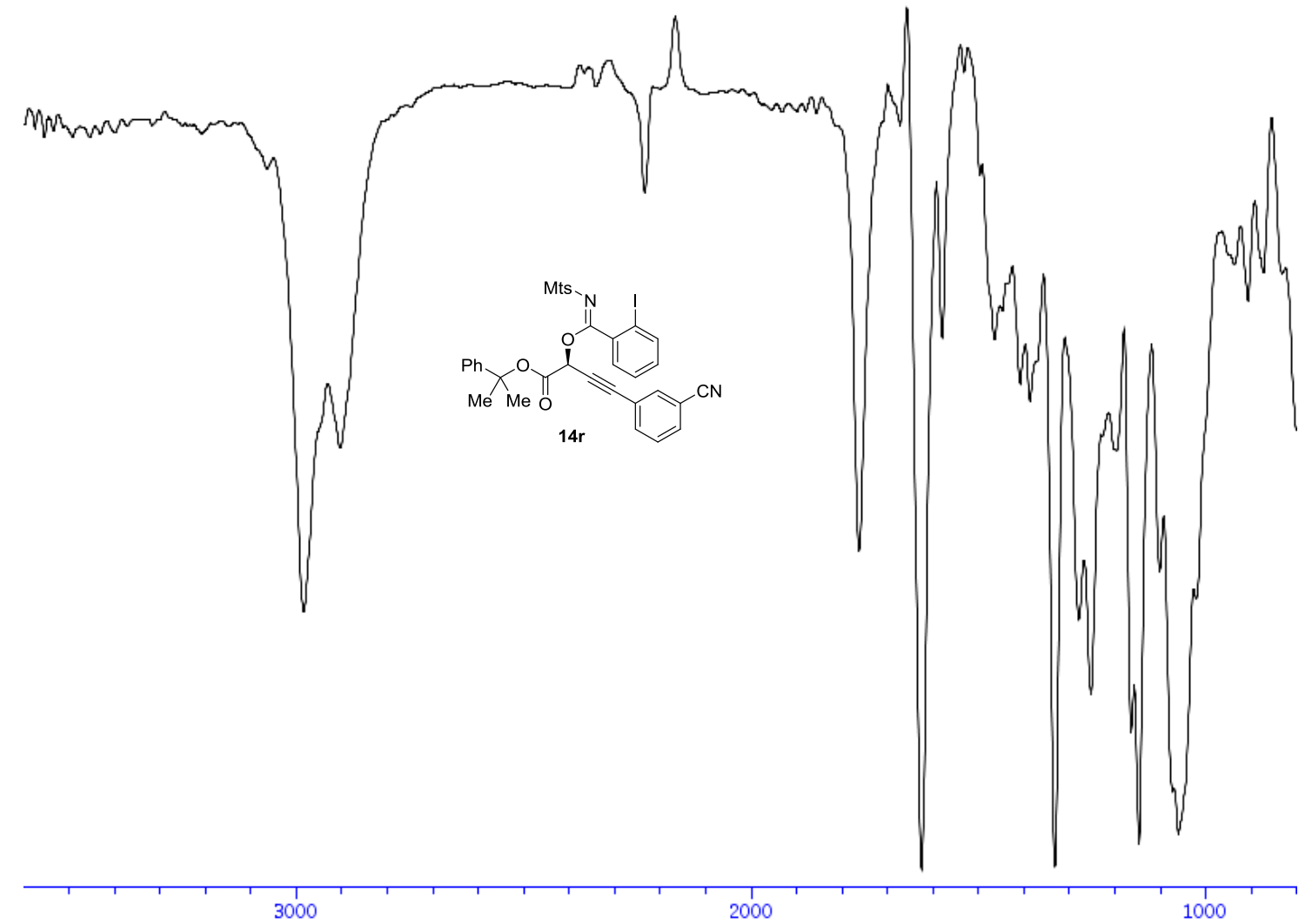


${ }^{\mathbf{1}} \mathbf{H}-\mathbf{N M R}\left(400 \mathrm{MHz}, \mathrm{CDCl}_{3}\right)$ of compound $\mathbf{1 4 s}$

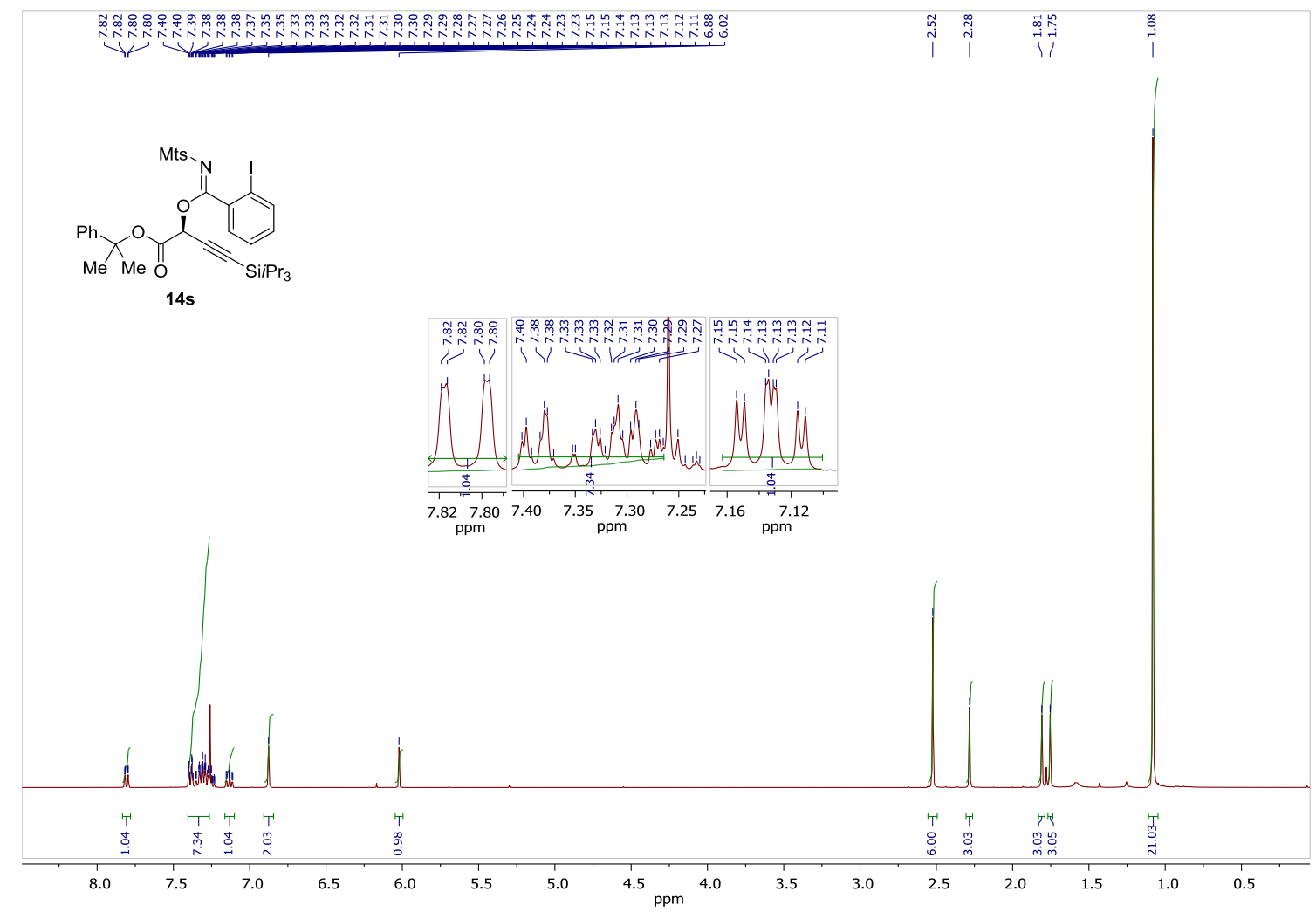

${ }^{13} \mathbf{C}-\mathbf{N M R}\left(100 \mathrm{MHz}, \mathrm{CDCl}_{3}\right)$ of compound $\mathbf{1 4 s}$

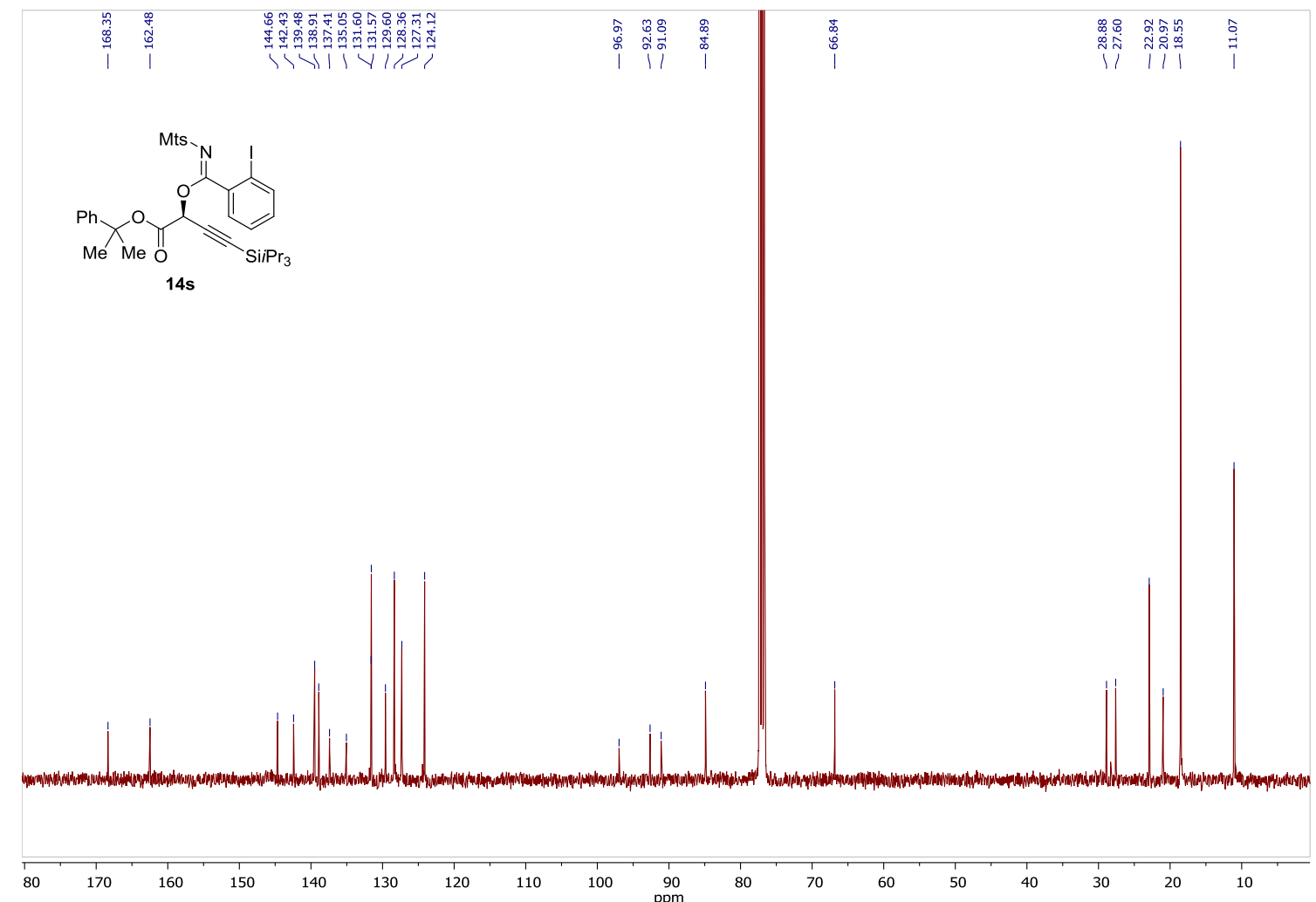


HPLC of compound 14s

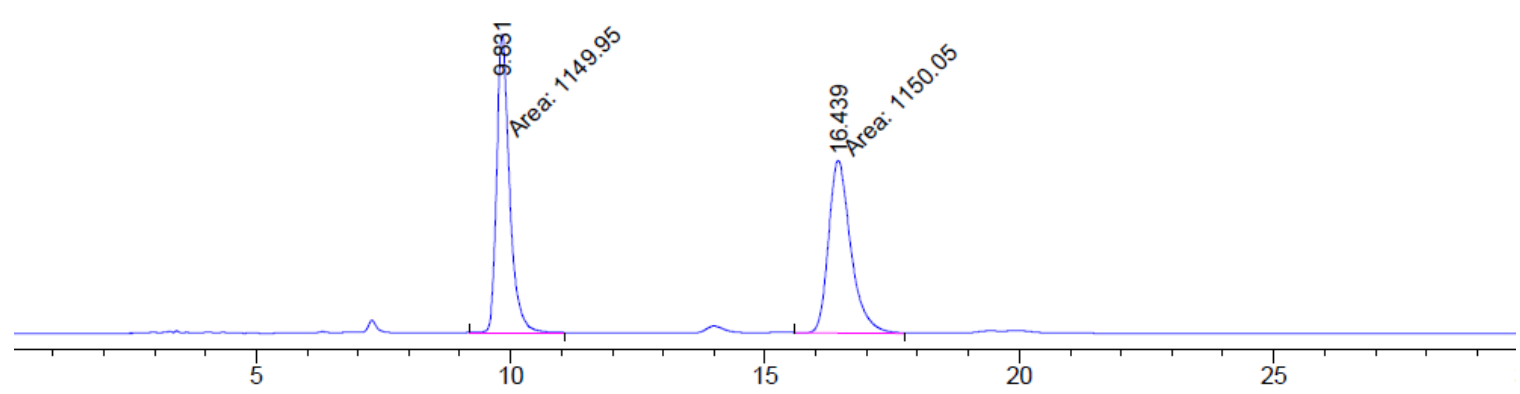

\begin{tabular}{|c|c|c|c|c|c|c|}
\hline $\begin{array}{c}\text { Peak } \\
\#\end{array}$ & $\begin{array}{c}\text { RetTime } \\
\text { [min] }\end{array}$ & Type & $\begin{array}{l}\text { Width } \\
\text { [min] }\end{array}$ & $\begin{array}{c}\text { Area } \\
{\left[\mathrm{mAU}^{*} \mathrm{~s}\right]}\end{array}$ & $\begin{array}{l}\text { Height } \\
\text { [mAU] }\end{array}$ & $\begin{array}{c}\text { Area } \\
\%\end{array}$ \\
\hline & & & & | - & & -- \\
\hline 1 & 9.83 & MM & 0.2956 & 1149.95093 & 64.82918 & 49.9979 \\
\hline 2 & 16.439 & MM & 0.5114 & 1150.04749 & 37.48198 & 50.0021 \\
\hline
\end{tabular}

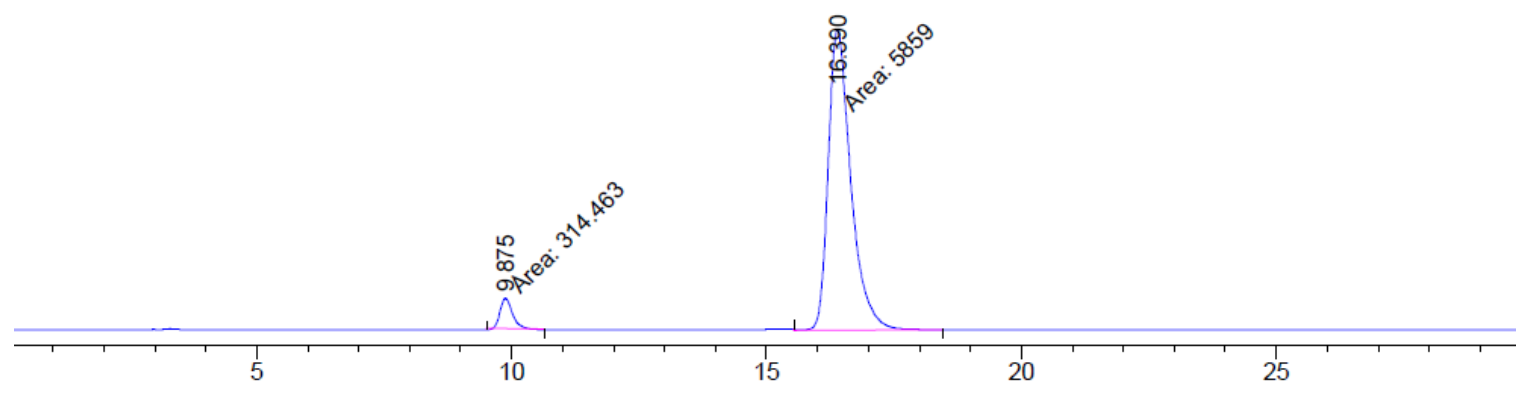

\begin{tabular}{cccccc}
$\begin{array}{c}\text { Peak RetTime Type } \\
\text { \# }\end{array}$ & $\begin{array}{c}\text { width } \\
{[\mathrm{min}]}\end{array}$ & $\begin{array}{c}\text { Area } \\
{[\mathrm{min}]}\end{array}$ & $\begin{array}{c}\text { Height } \\
{[\mathrm{mAU} \text { s }]}\end{array}$ & $\begin{array}{c}\text { Area } \\
{[\mathrm{mAU}]}\end{array}$ & $\%$ \\
\hline 1 & 9.875 MM & 0.2759 & 314.46310 & 18.99383 & 5.0938 \\
2 & 16.390 MM & 0.5181 & 5858.99707 & 188.49220 & 94.9062
\end{tabular}


IR of compound $14 s$

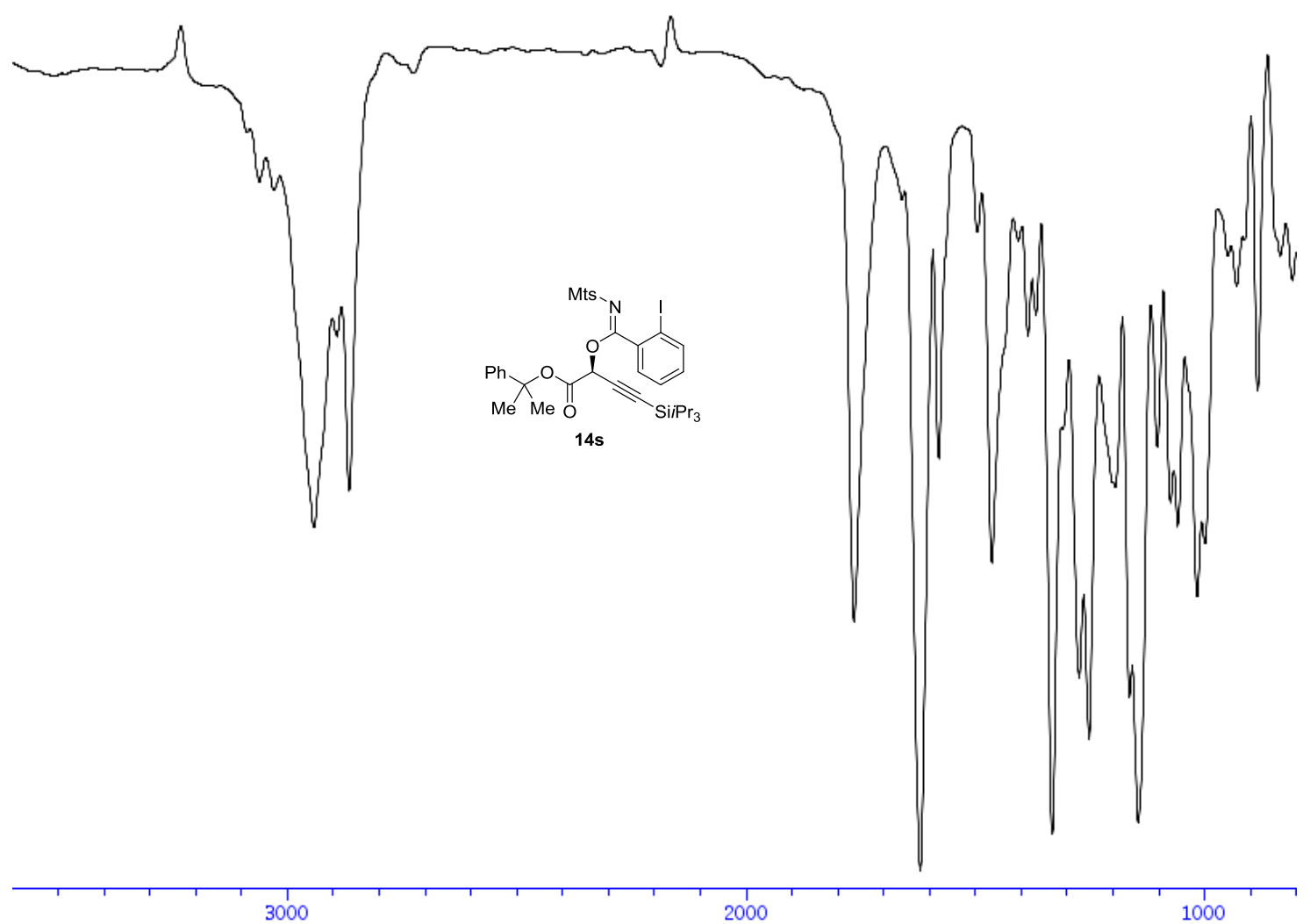


${ }^{\mathbf{1}} \mathbf{H}-\mathbf{N M R}\left(400 \mathrm{MHz}, \mathrm{CDCl}_{3}\right)$ of compound 14t

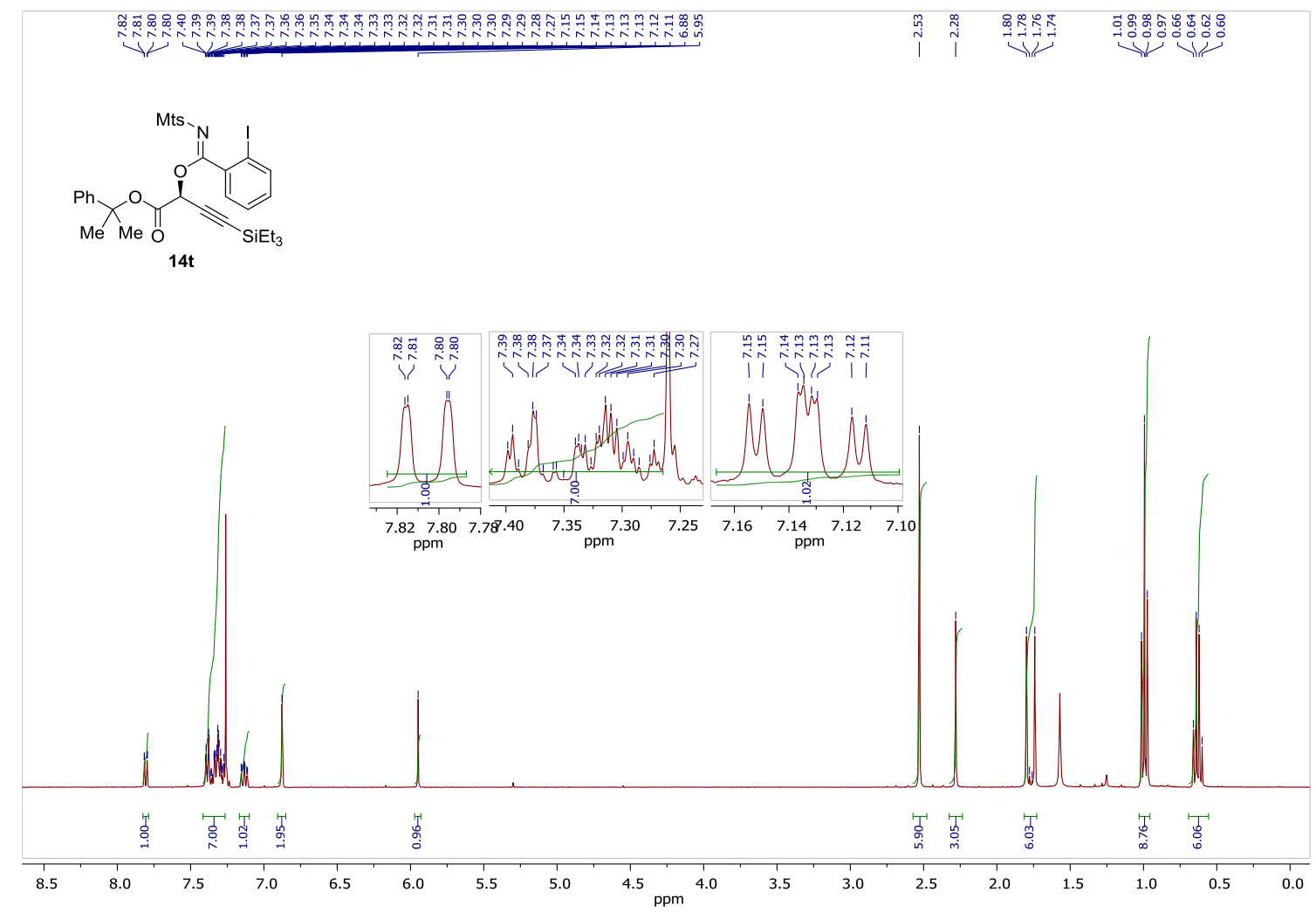

${ }^{13} \mathbf{C}-$ NMR (100 MHz, $\left.\mathrm{CDCl}_{3}\right)$ of compound $\mathbf{1 4 t}$

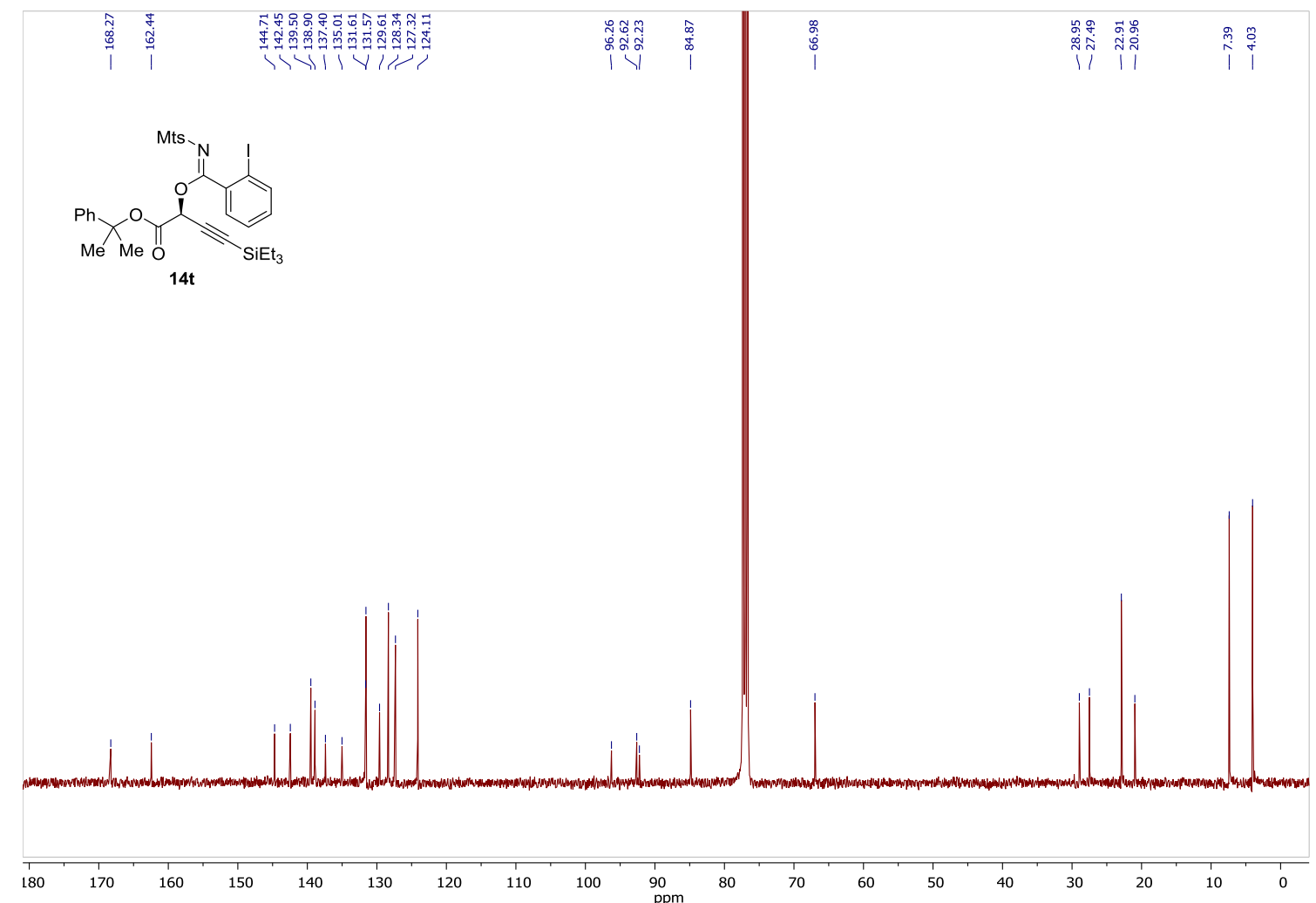


HPLC of compound 14t

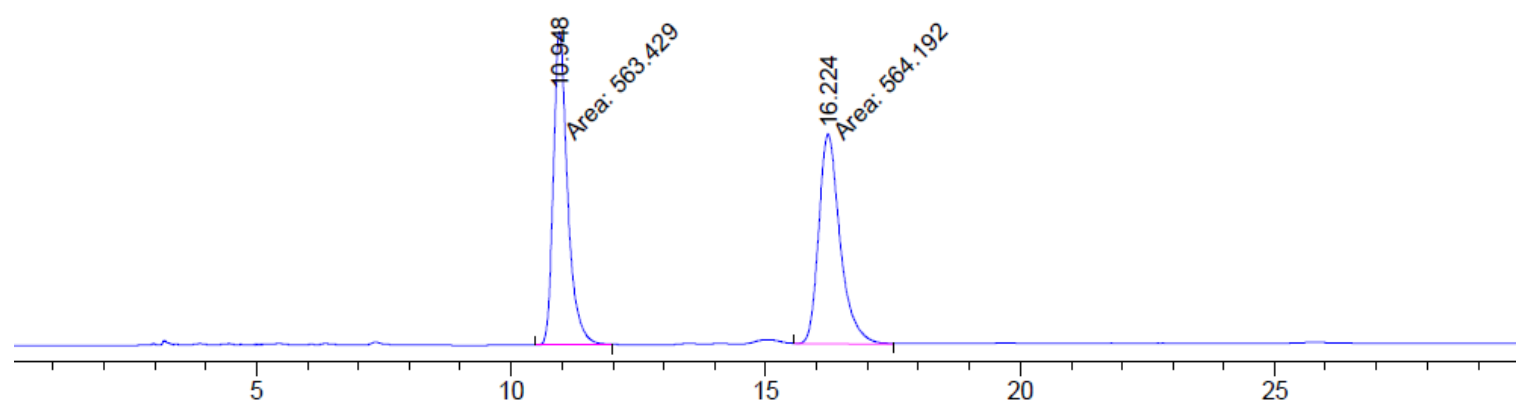

Peak RetTime Type width Area Height Area

$\begin{array}{ccccccc}\# & {[\mathrm{~min}]} & {[\mathrm{min}]} & {[\mathrm{mAU} * \mathrm{~s}]} & {[\mathrm{mAU}]} & \% \\ 1 & 10.948 \text { MM } & 0.3254 & 563.42926 & 28.85790 & 49.9662 \\ 2 & 16.224 \text { MM } & 0.4871 & 564.19244 & 19.30488 & 50.0338\end{array}$
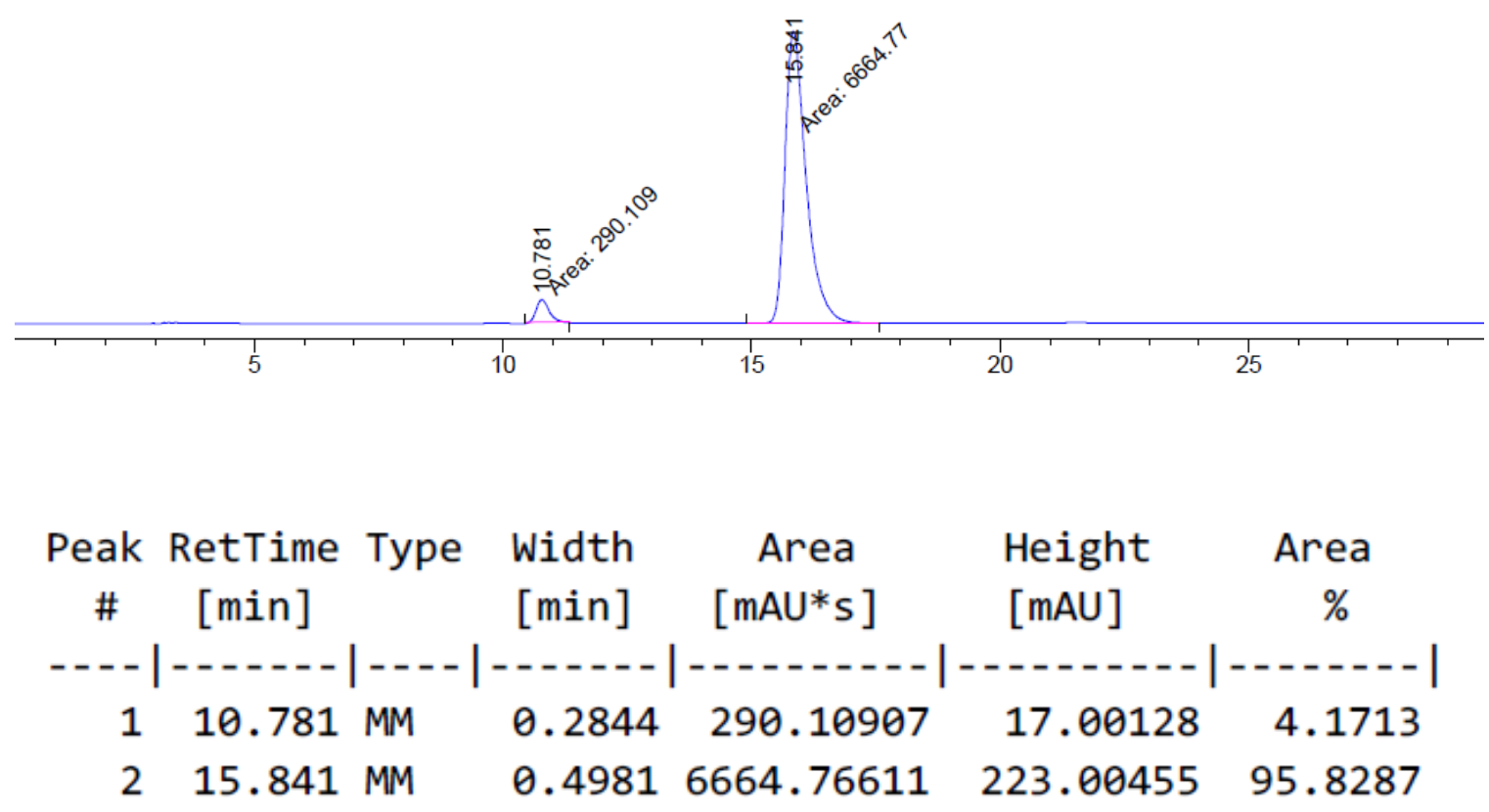
IR of compound $\mathbf{1 4 t}$

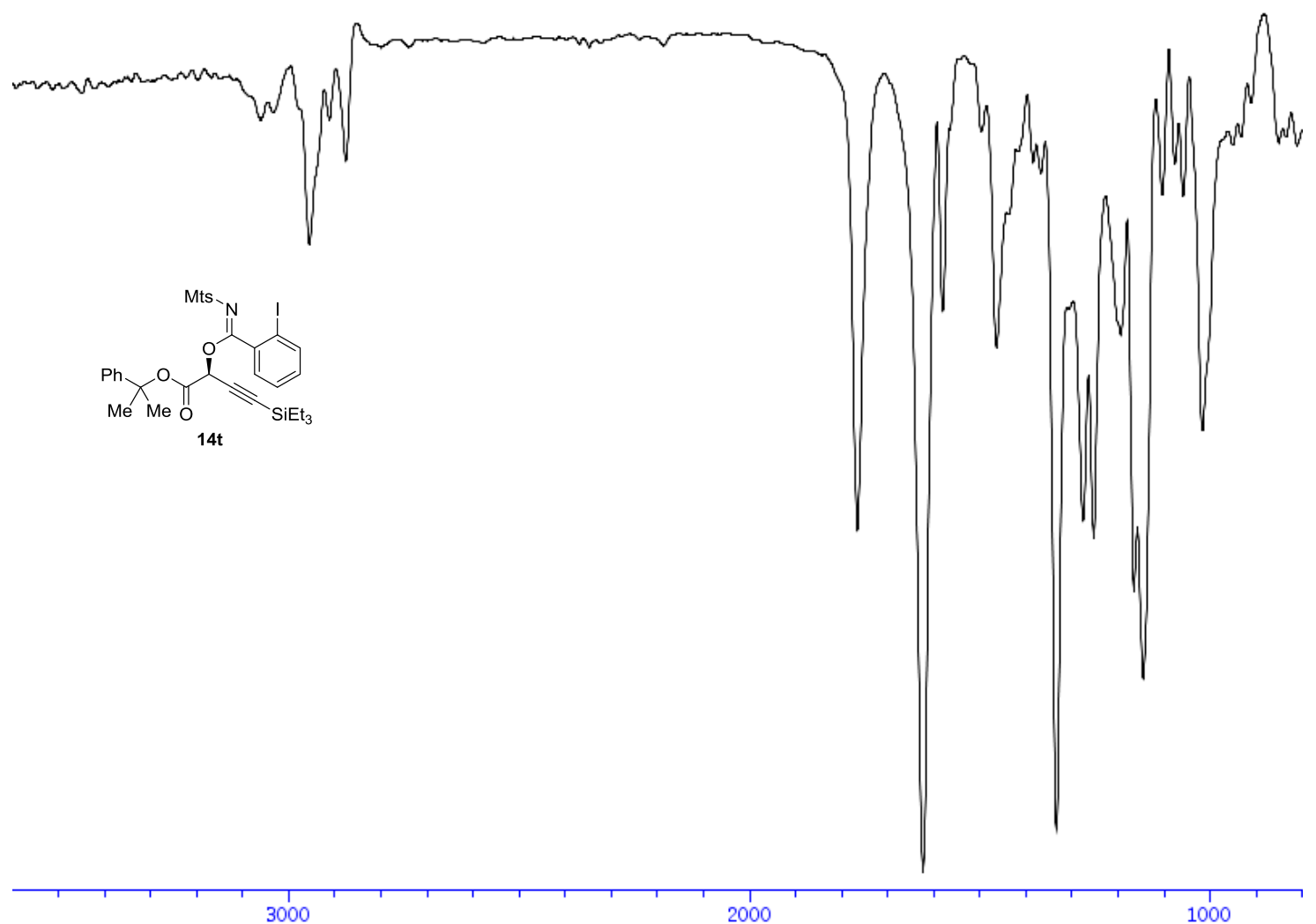


${ }^{\mathbf{1}} \mathbf{H}-\mathbf{N M R}\left(400 \mathrm{MHz}, \mathrm{CDCl}_{3}\right)$ of compound $\mathbf{1 4 u}$

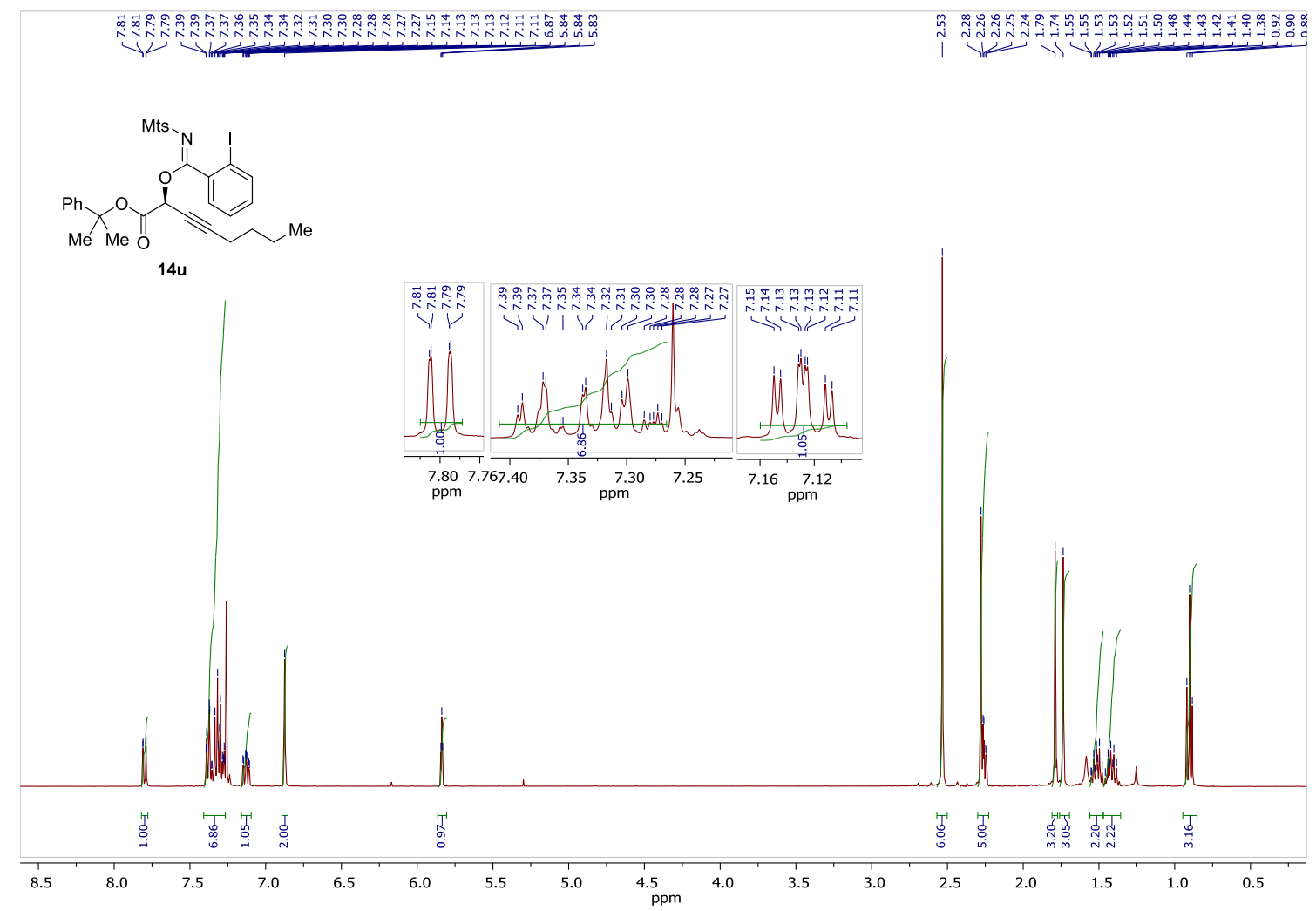

${ }^{13} \mathbf{C}-\mathbf{N M R}\left(100 \mathrm{MHz}, \mathrm{CDCl}_{3}\right)$ of compound $\mathbf{1 4 u}$

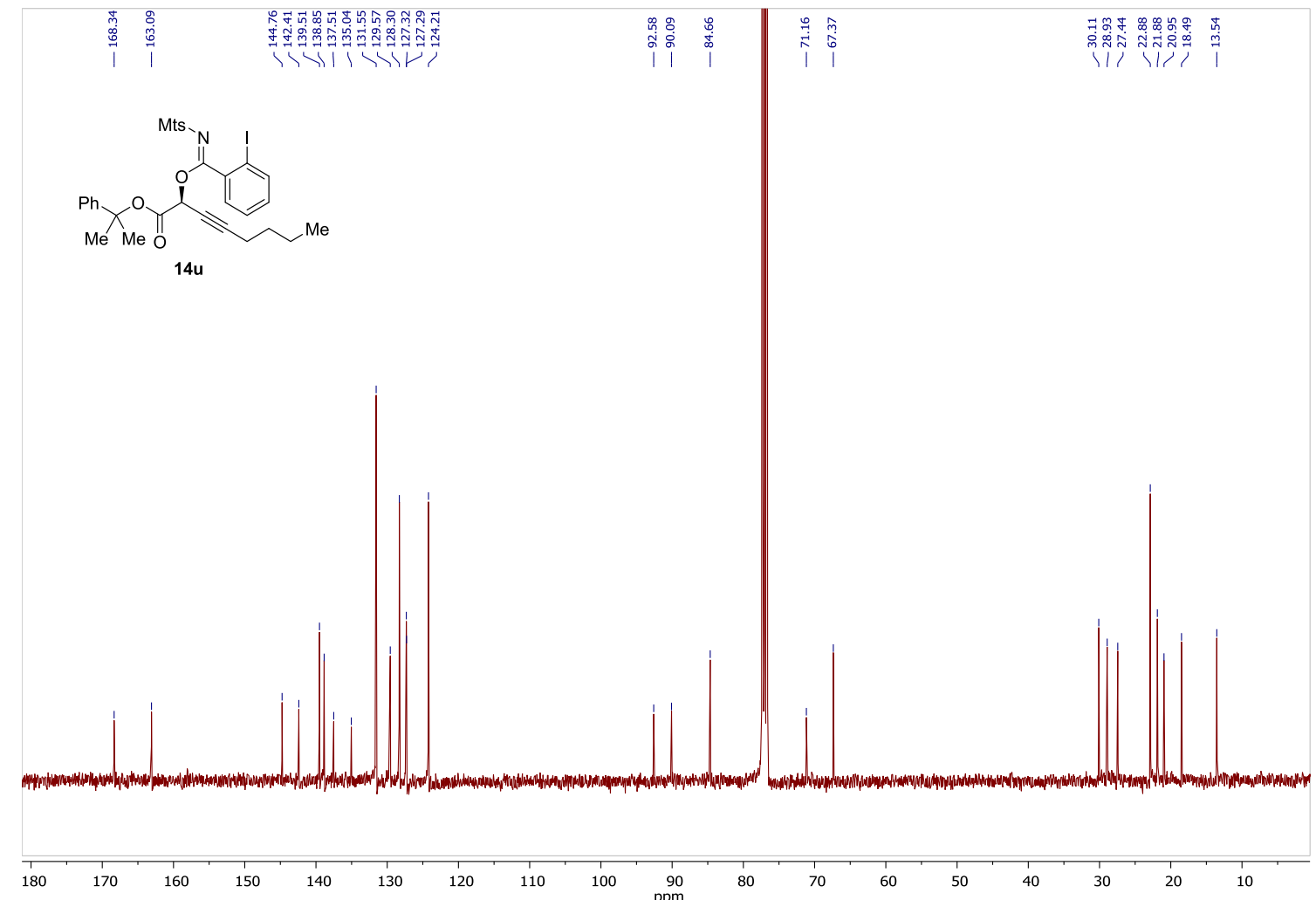


HPLC of compound 14u
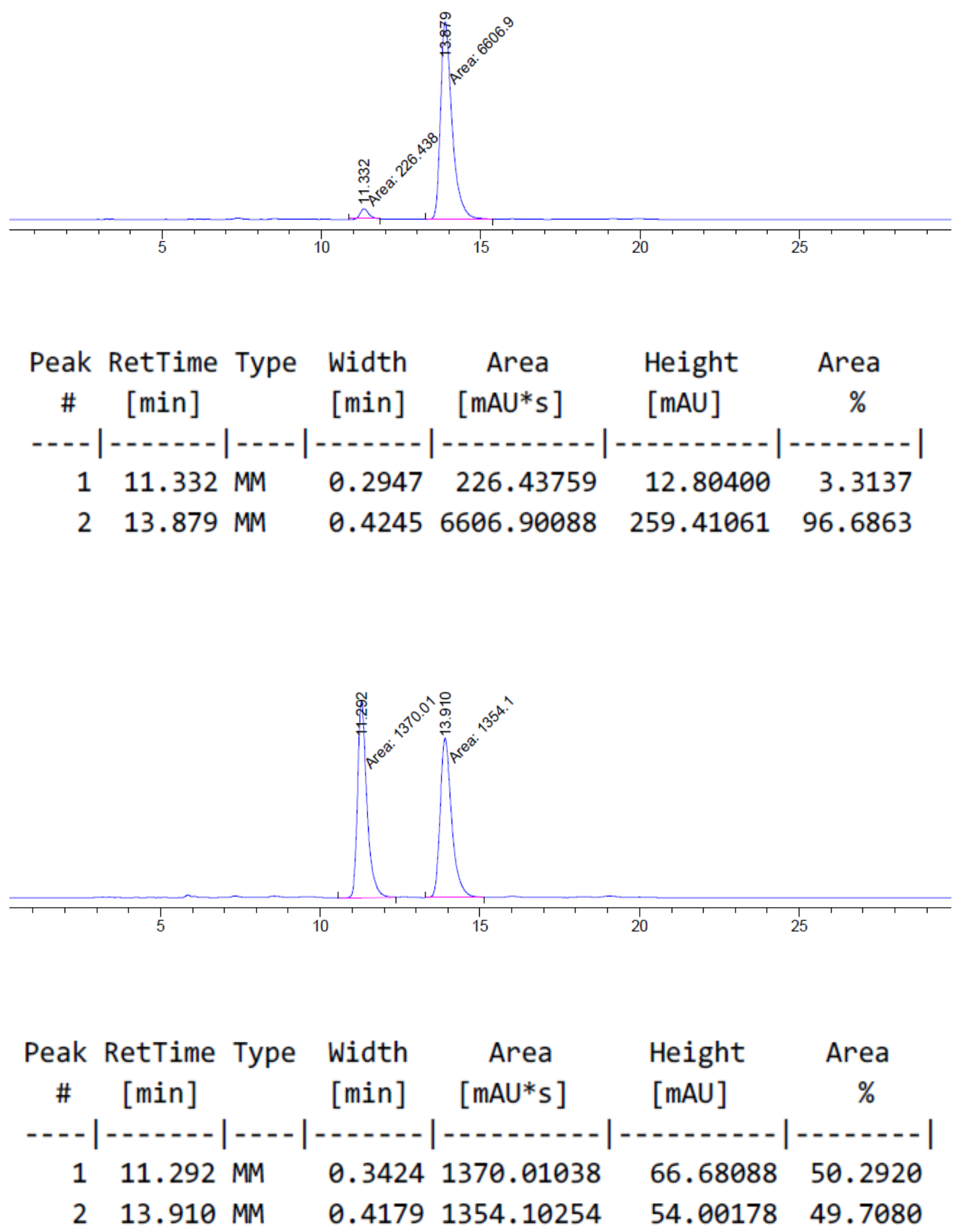
IR of compound $\mathbf{1 4 u}$

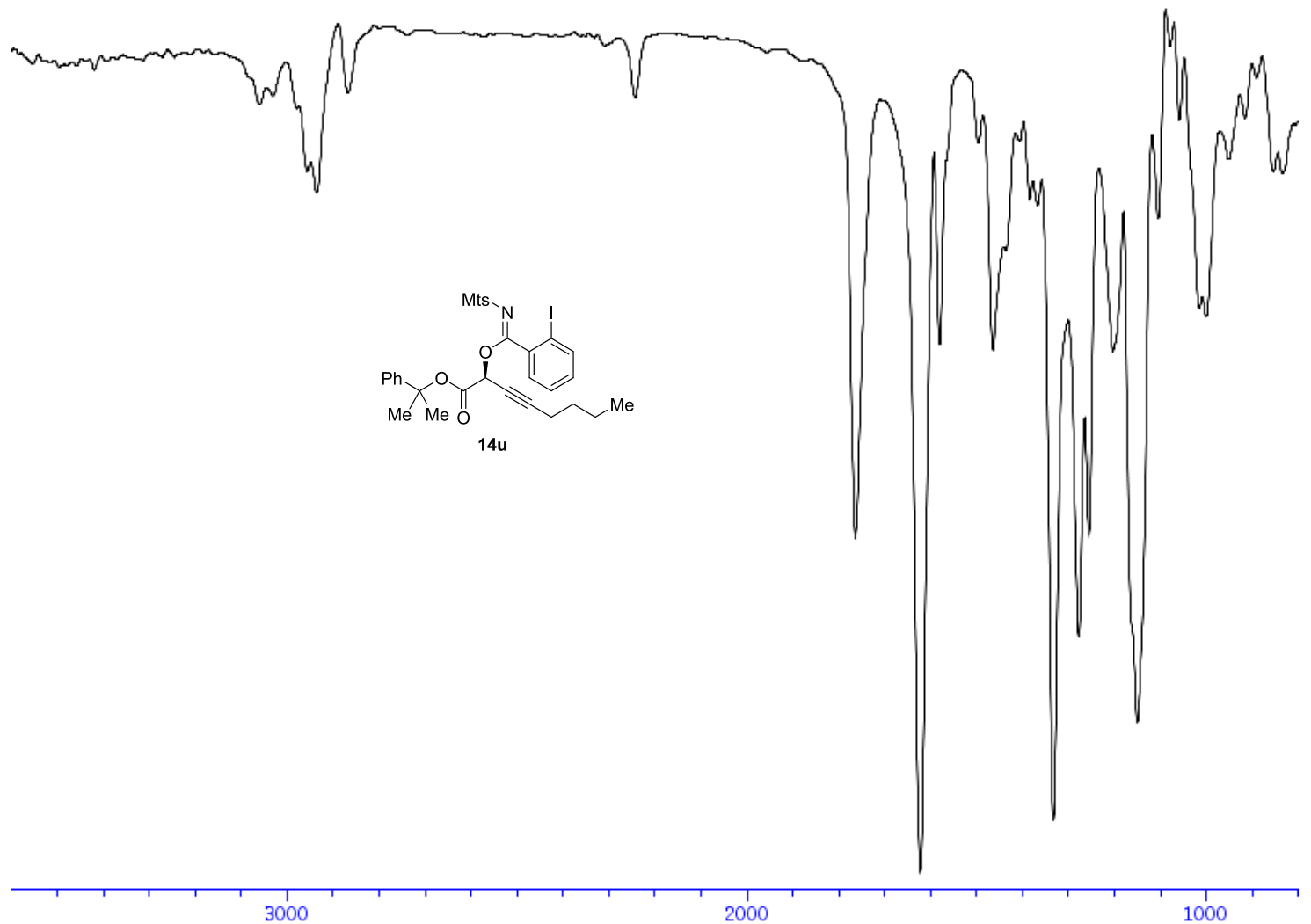


${ }^{\mathbf{1}} \mathbf{H}-\mathbf{N M R}\left(400 \mathrm{MHz}, \mathrm{CDCl}_{3}\right)$ of compound $\mathbf{1 4 v}$

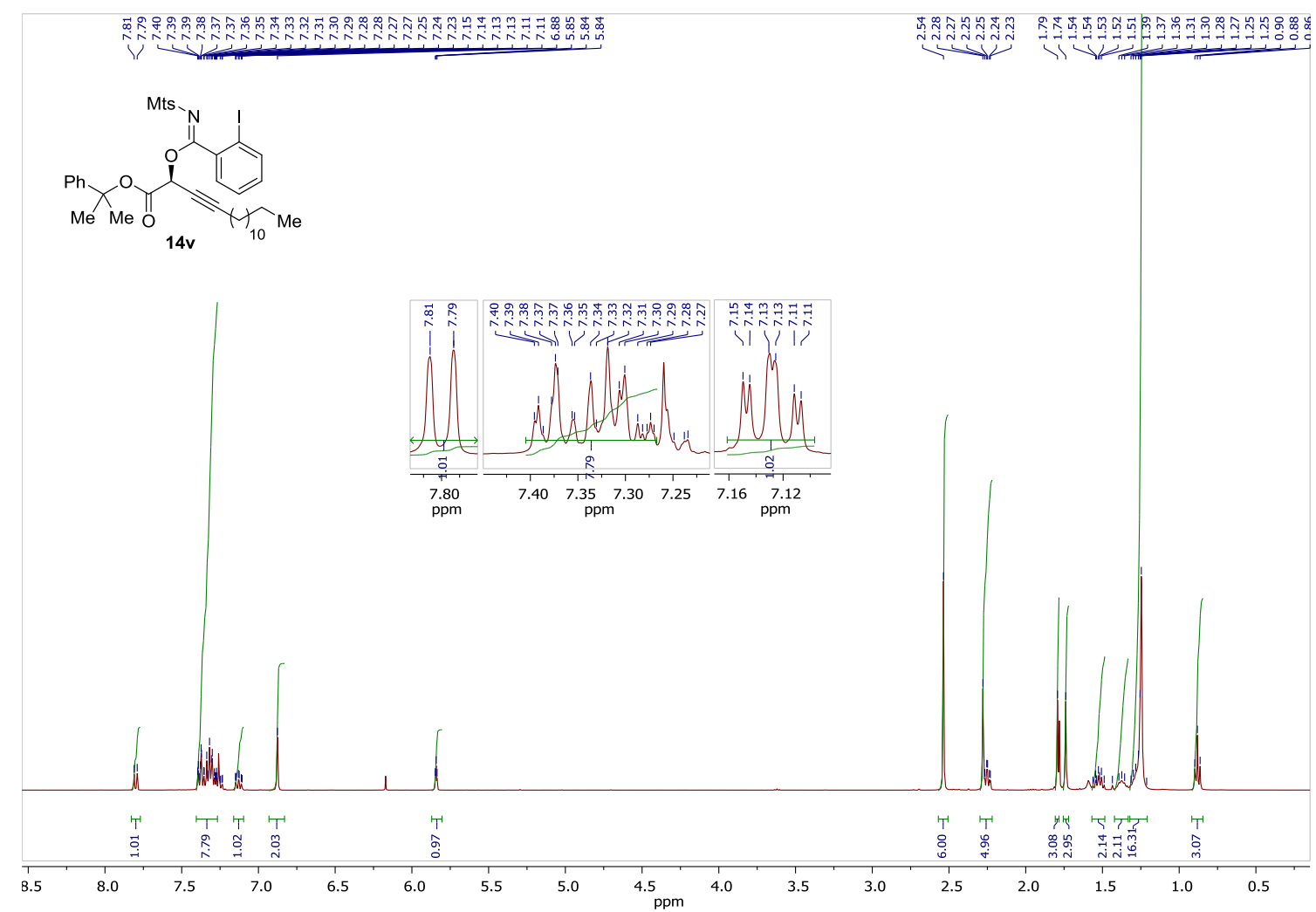

${ }^{13} \mathbf{C}-\mathrm{NMR}\left(100 \mathrm{MHz}, \mathrm{CDCl}_{3}\right)$ of compound $\mathbf{1 4 v}$

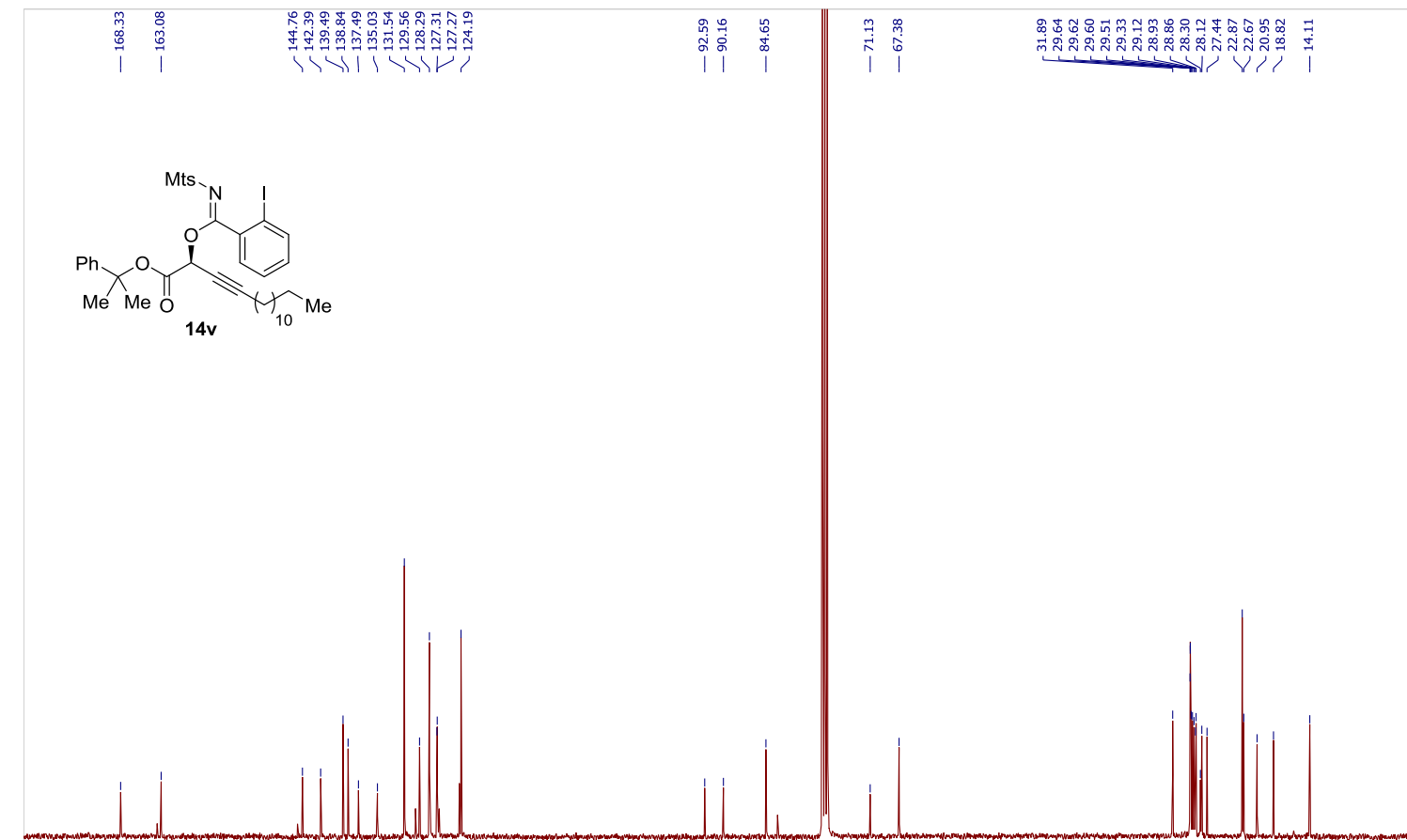

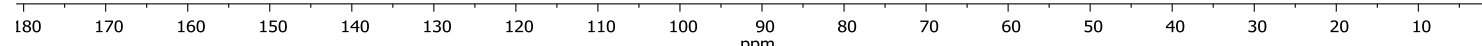


HPLC of compound 14v
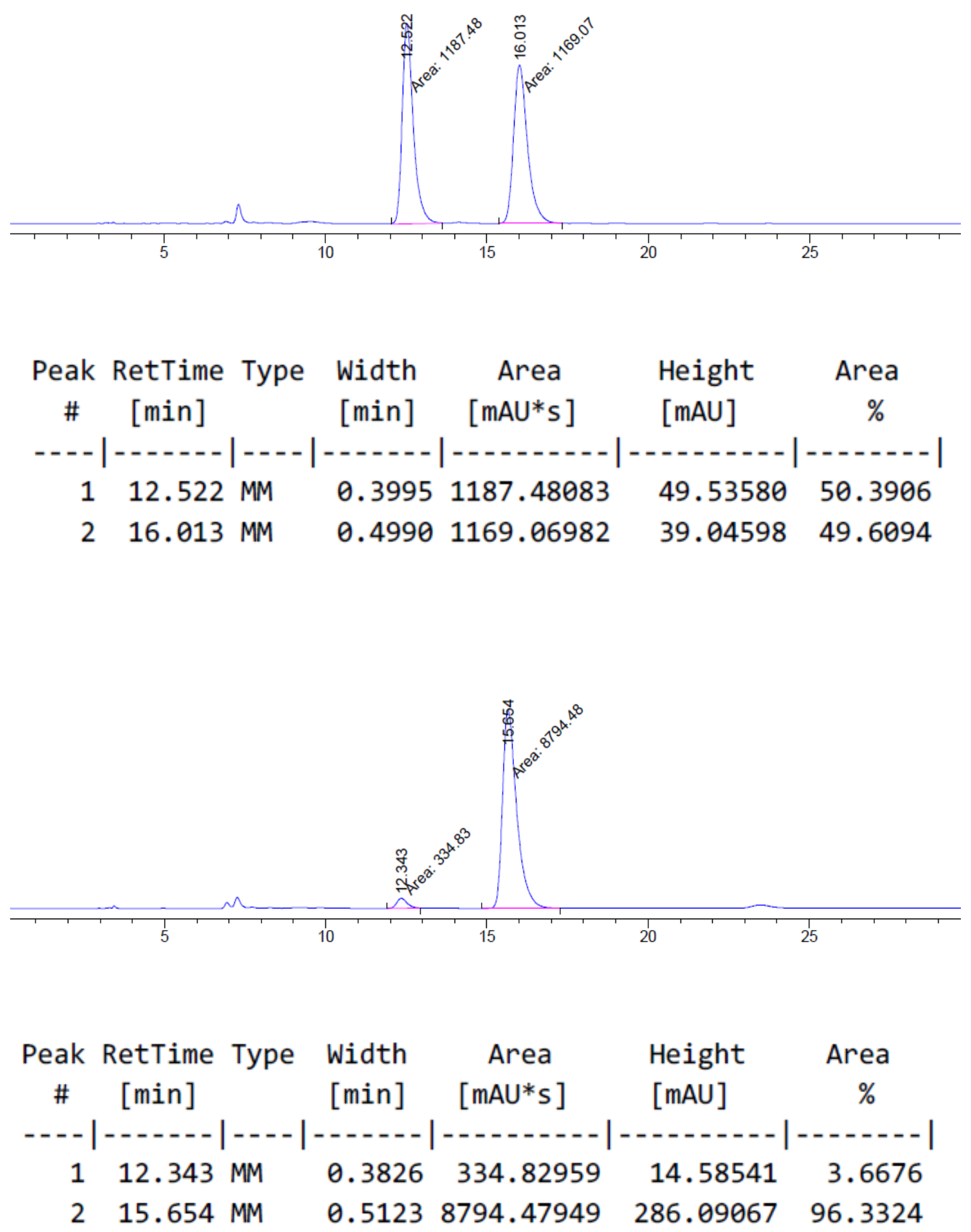
IR of compound $\mathbf{1 4} \mathbf{v}$

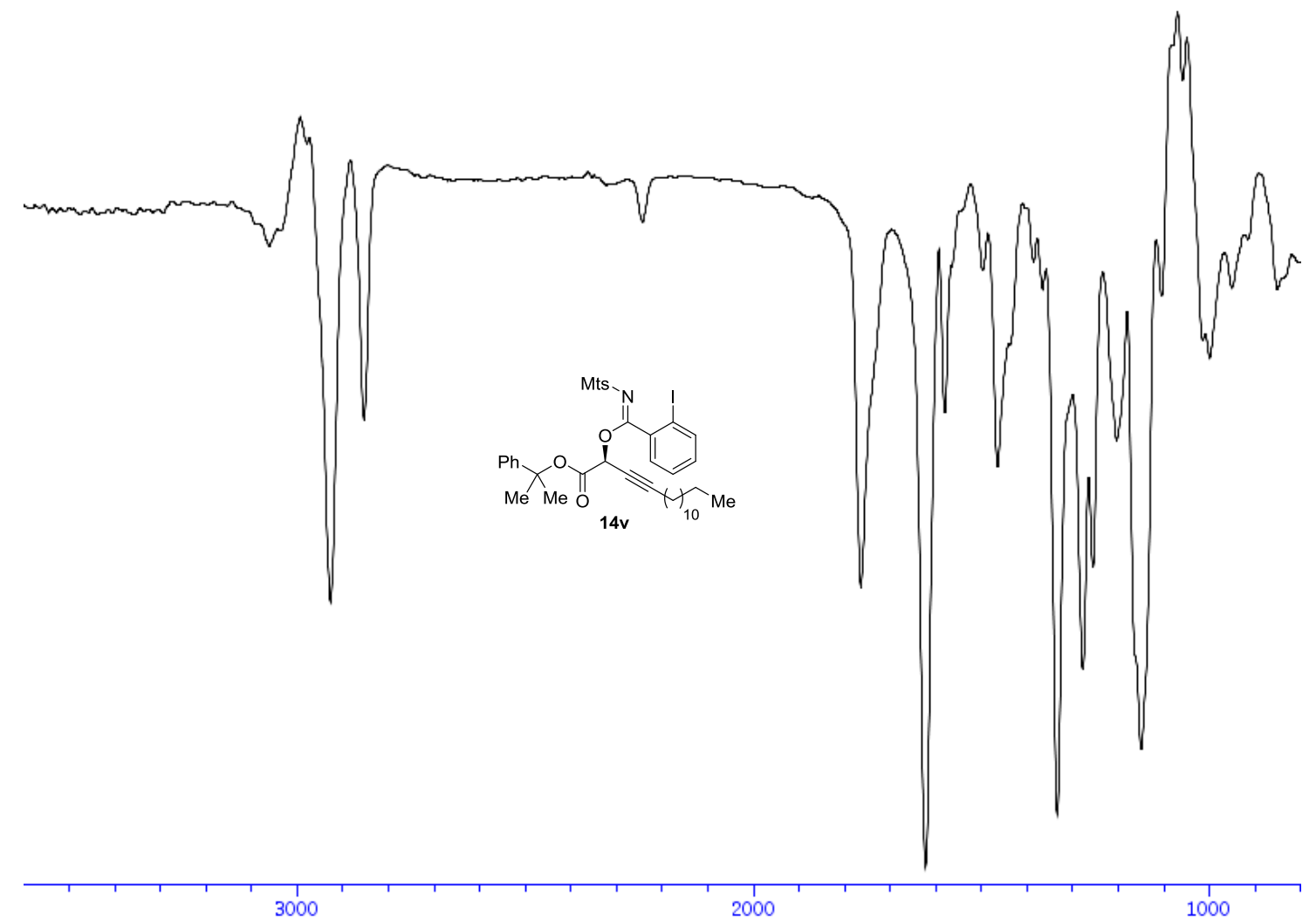


${ }^{1} \mathbf{H}-\mathbf{N M R}\left(400 \mathrm{MHz}, \mathrm{CDCl}_{3}\right)$ of compound $\mathbf{1 4 w}$

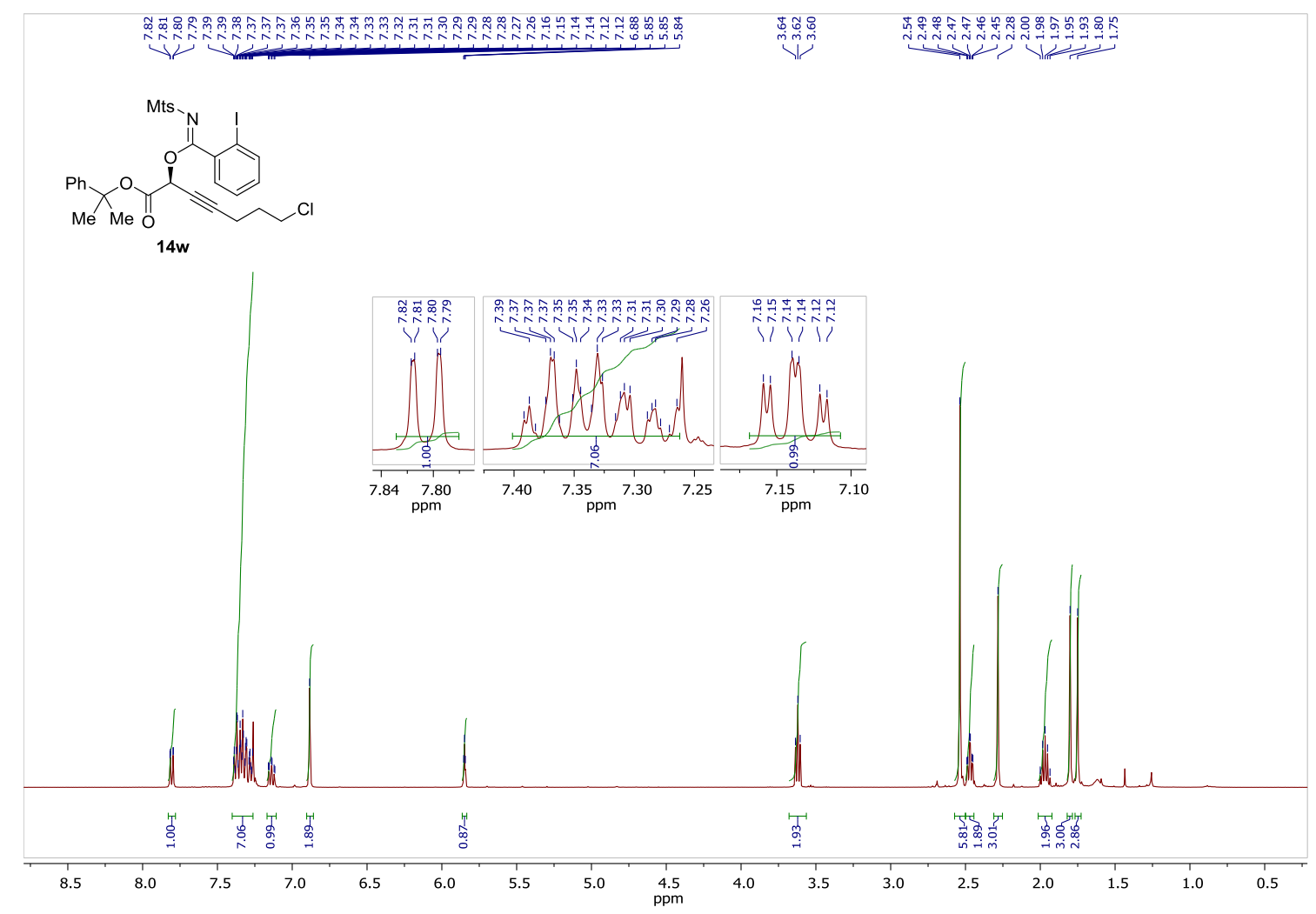

${ }^{13} \mathbf{C}-\mathbf{N M R}\left(100 \mathrm{MHz}, \mathrm{CDCl}_{3}\right)$ of compound $\mathbf{1 4 w}$

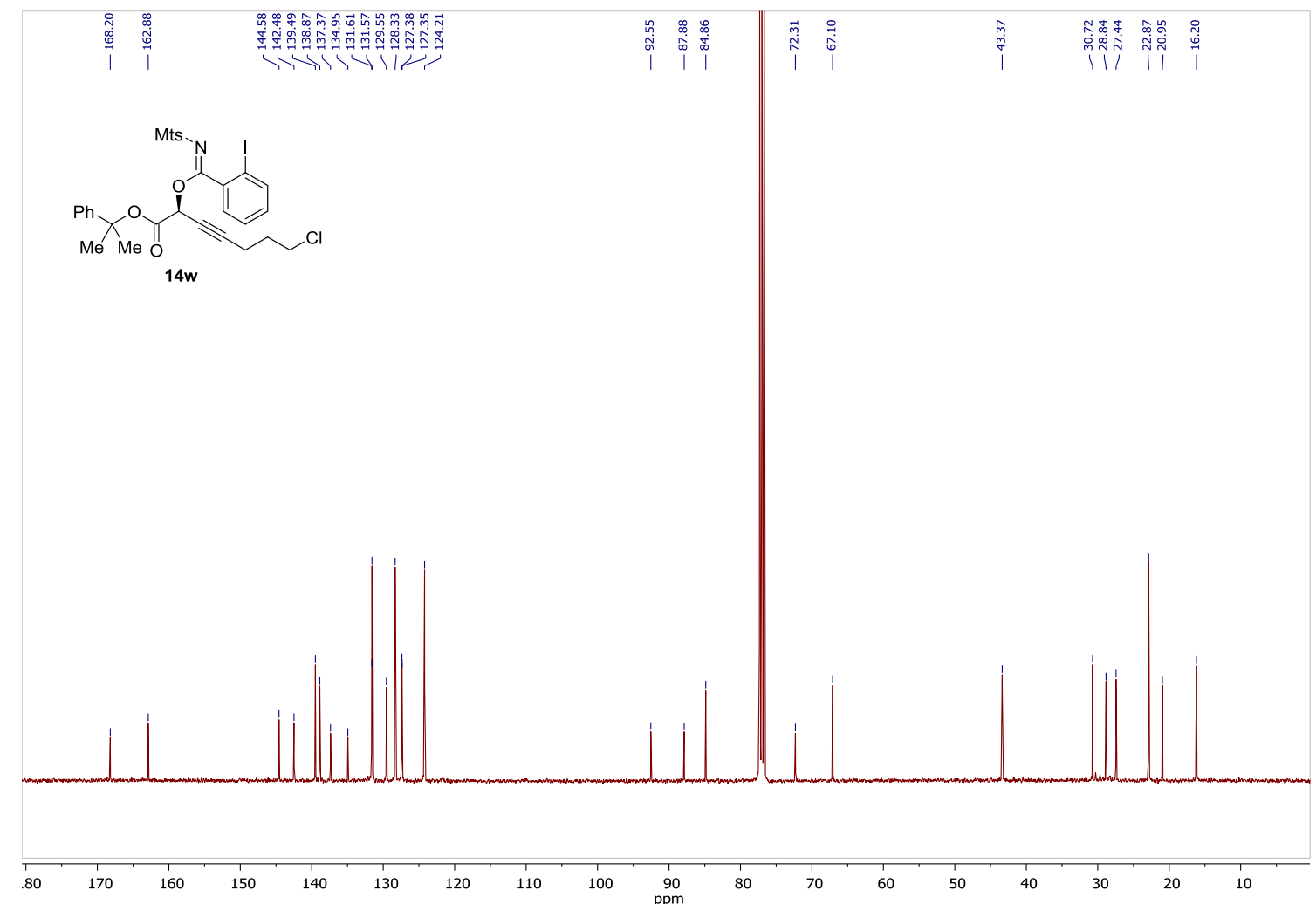


HPLC of compound 14w

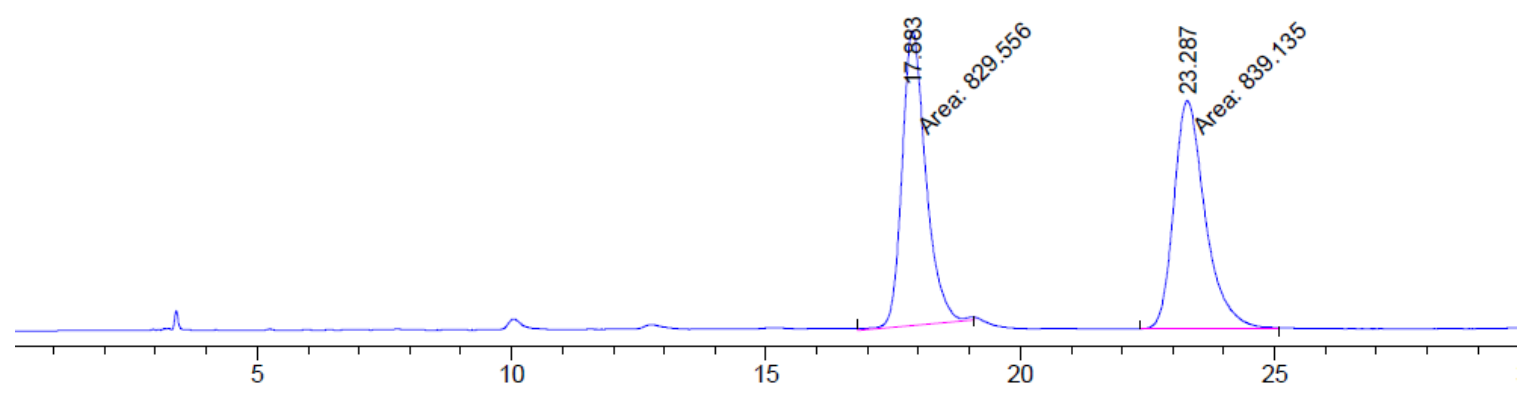

\begin{tabular}{|c|c|c|c|c|c|c|}
\hline $\begin{array}{c}\text { Peak } \\
\#\end{array}$ & $\begin{array}{c}\text { RetTime } \\
\text { [min] }\end{array}$ & Type & $\begin{array}{l}\text { Width } \\
\text { [min] }\end{array}$ & $\begin{array}{c}\text { Area } \\
{\left[\mathrm{mAU}{ }^{*} \mathrm{~s}\right]}\end{array}$ & $\begin{array}{l}\text { Height } \\
{[\mathrm{mAU}]}\end{array}$ & $\begin{array}{c}\text { Area } \\
\%\end{array}$ \\
\hline & & & & $=-$ & -1 & -1 \\
\hline 1 & 17.883 & MM & 0.5439 & 829.55560 & 25.41787 & 49.7130 \\
\hline 2 & 23.287 & MM & 0.7131 & 839.13501 & 19.61322 & 50.2870 \\
\hline
\end{tabular}

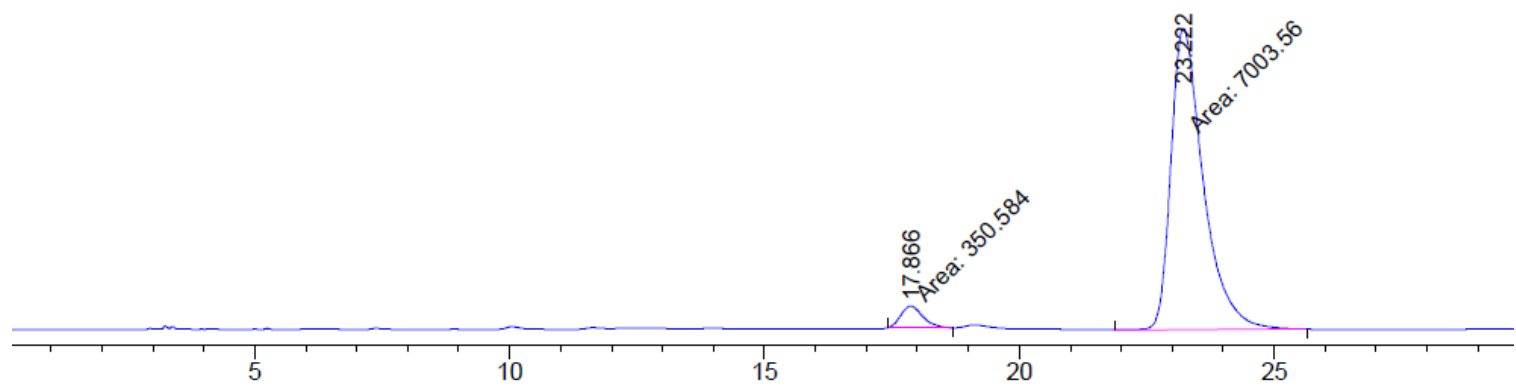

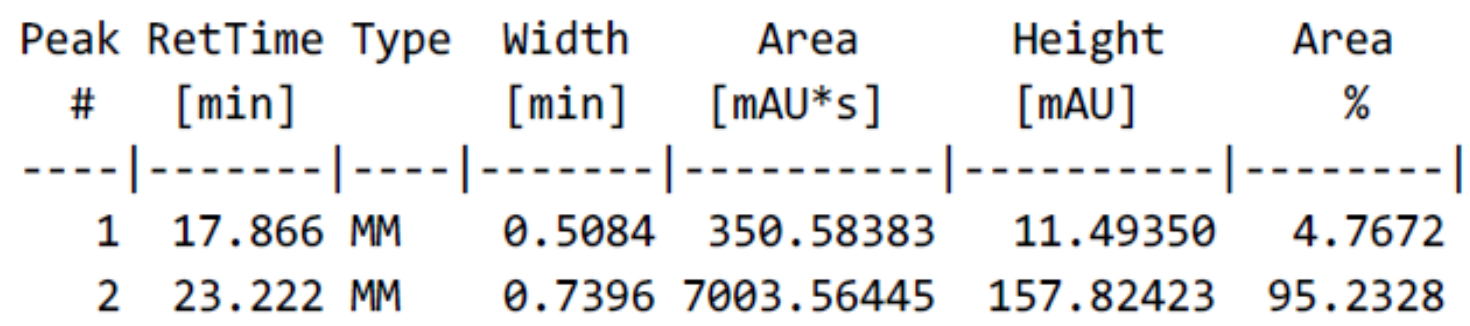


IR of compound $\mathbf{1 4 w}$

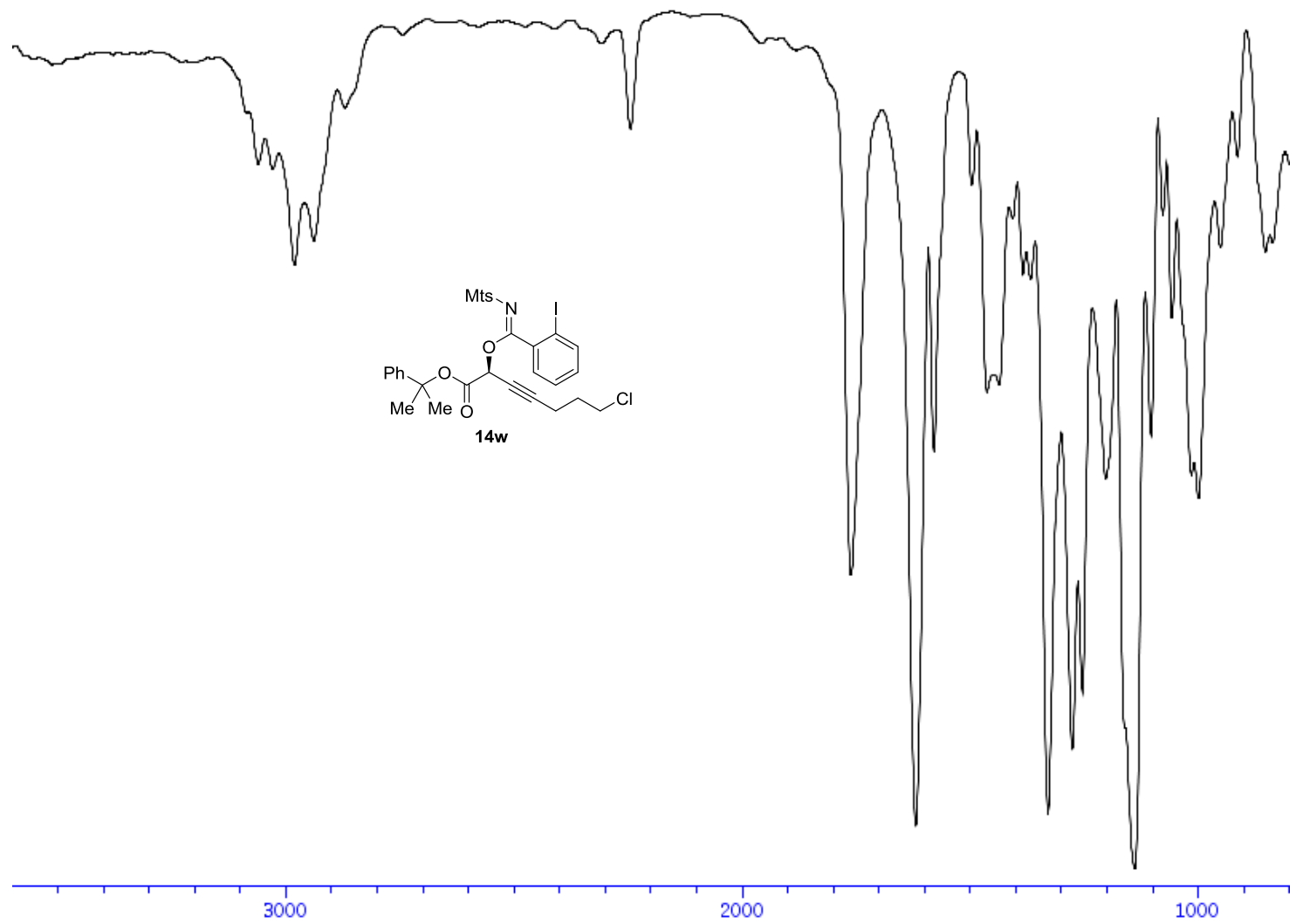


${ }^{\mathbf{1}} \mathbf{H}-\mathbf{N M R}\left(400 \mathrm{MHz}, \mathrm{CDCl}_{3}\right)$ of compound $\mathbf{1 4 x}$

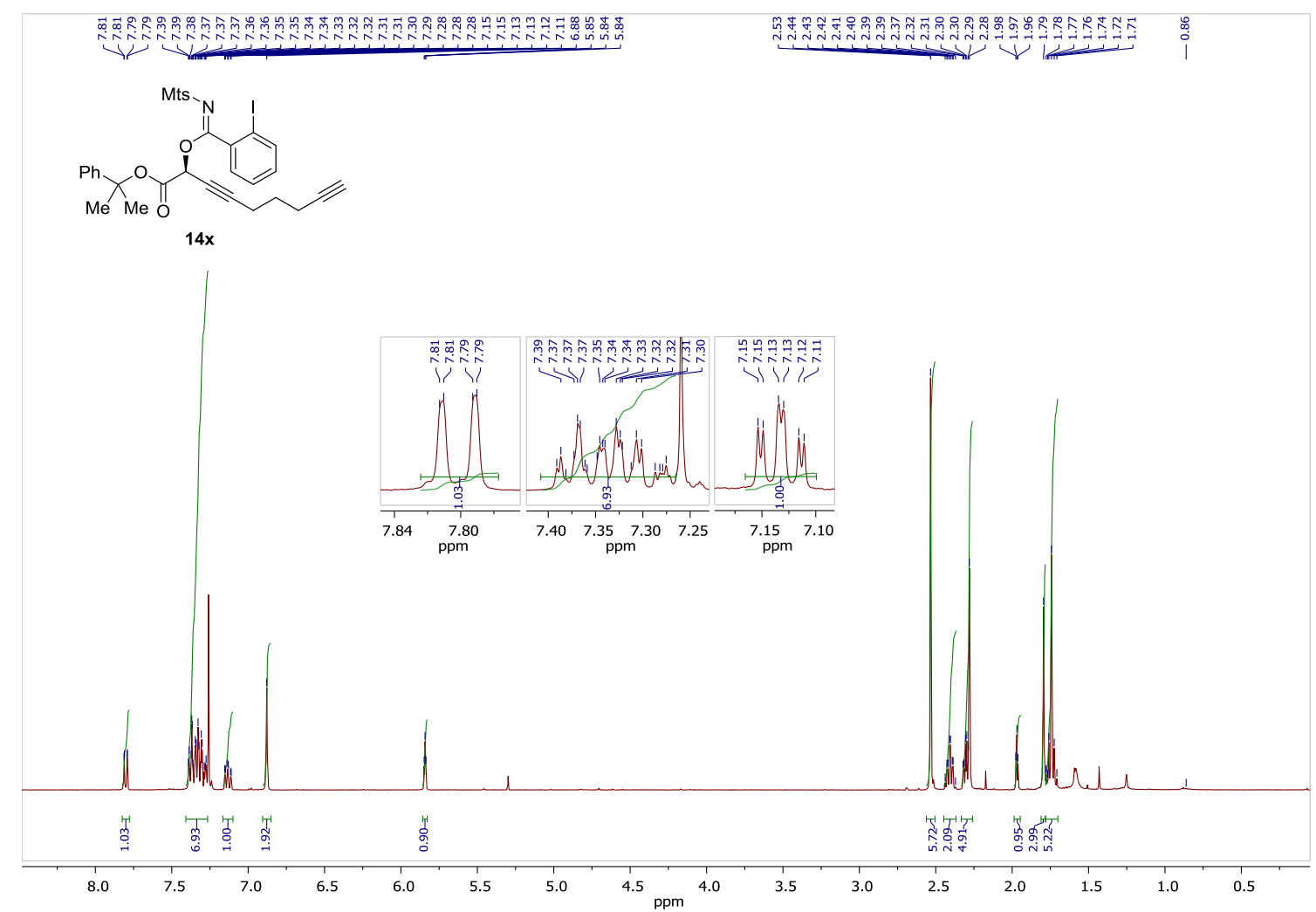

${ }^{13} \mathbf{C}-\mathbf{N M R}\left(100 \mathrm{MHz}, \mathrm{CDCl}_{3}\right)$ of compound $\mathbf{1 4 x}$

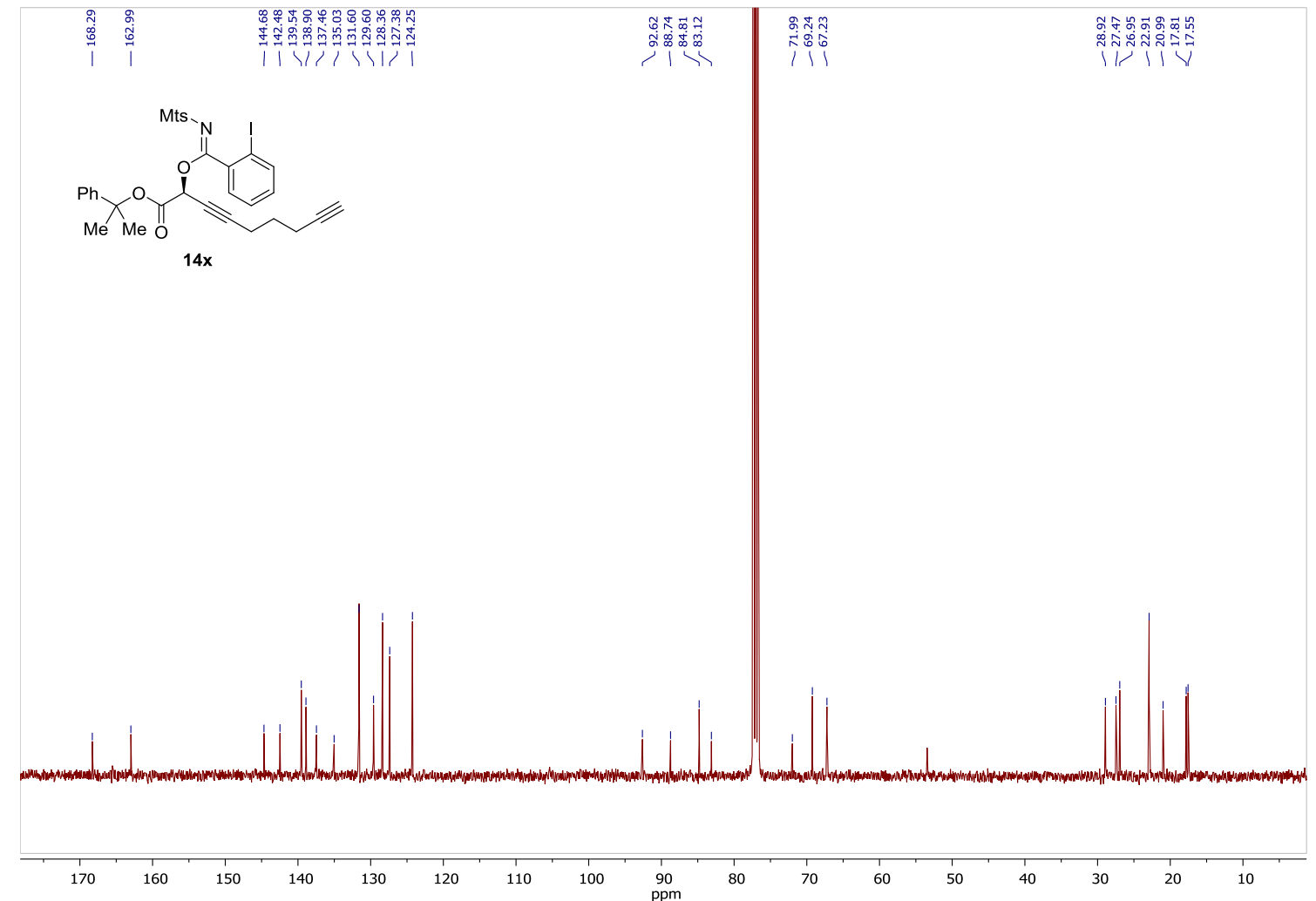


HPLC of compound 14x

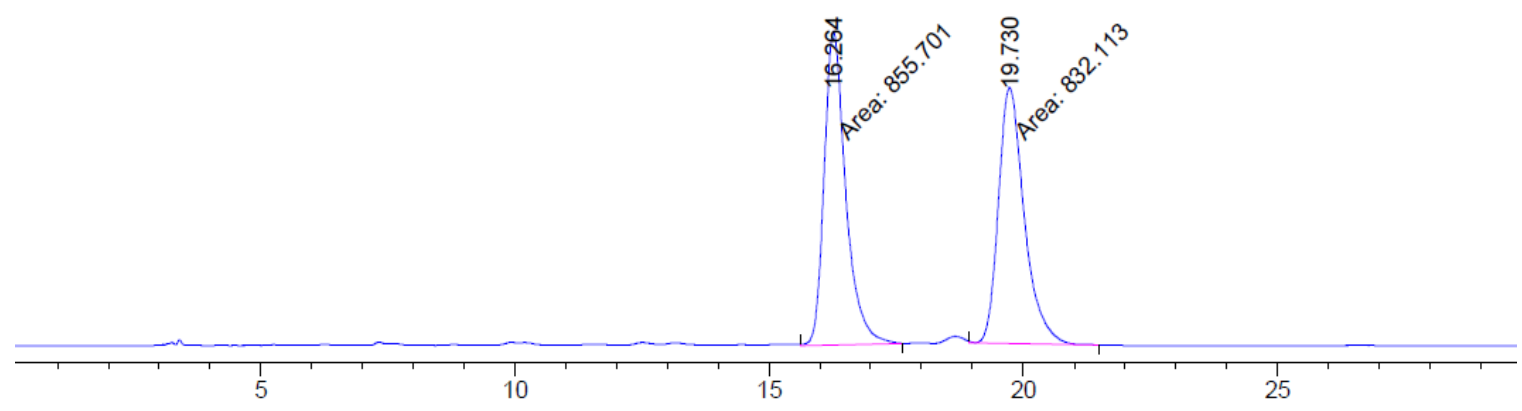

\begin{tabular}{cccccc}
$\begin{array}{c}\text { Peak RetTime Type } \\
\#\end{array}$ & $\begin{array}{c}\text { width } \\
{[\mathrm{min}]}\end{array}$ & $\begin{array}{c}\text { Area } \\
{[\mathrm{min}]}\end{array}$ & $\begin{array}{c}\text { Height } \\
{[\mathrm{mAU} \text { s }]}\end{array}$ & $\begin{array}{c}\text { Area } \\
{[\mathrm{mAU}]}\end{array}$ & $\%$ \\
\hline 1 & 16.264 MM & 0.4934 & 855.70081 & 28.90598 & 50.6988 \\
2 & 19.730 MM & 0.5889 & 832.11328 & 23.54800 & 49.3012
\end{tabular}

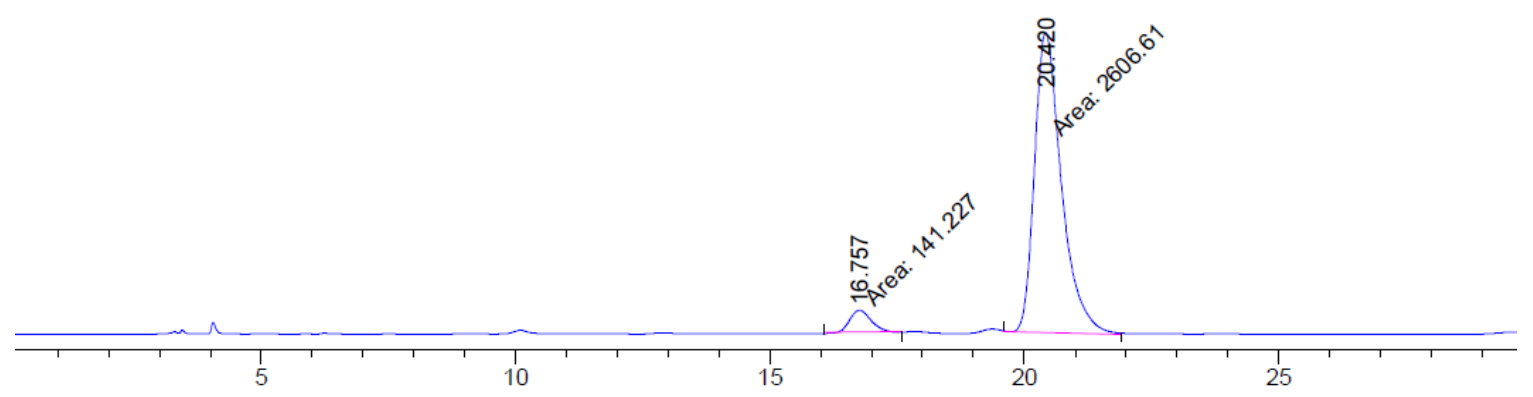

\begin{tabular}{cccccc}
$\begin{array}{c}\text { Peak RetTime Type } \\
\text { \# }\end{array}$ & $\begin{array}{c}\text { Width } \\
{[\mathrm{min}]}\end{array}$ & $\begin{array}{c}\text { Area } \\
{[\mathrm{min}]}\end{array}$ & $\begin{array}{c}\text { Height } \\
{[\mathrm{mAU} \text { s }]}\end{array}$ & $\begin{array}{c}\text { Area } \\
{[\mathrm{mAU}]}\end{array}$ & $\%$ \\
\hline 1 & 16.757 MM & 0.4662 & 141.22705 & 5.04856 & 5.1396 \\
2 & 20.420 MM & 0.6148 & 2606.61377 & 70.65749 & 94.8604
\end{tabular}


IR of compound $\mathbf{1 4 x}$

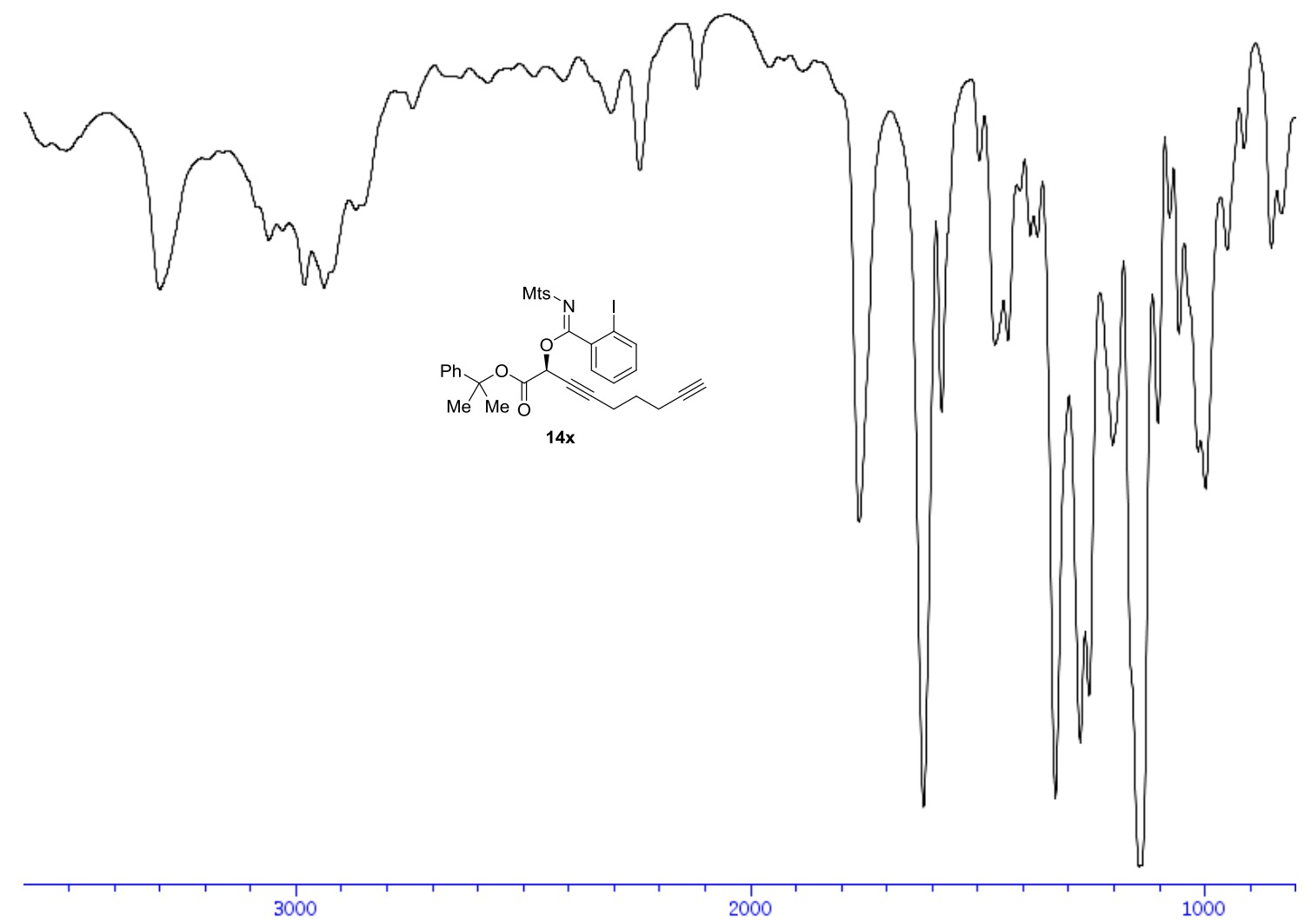


${ }^{\mathbf{1}} \mathbf{H}-\mathbf{N M R}\left(400 \mathrm{MHz}, \mathrm{CDCl}_{3}\right)$ of compound $\mathbf{1 4 y}$

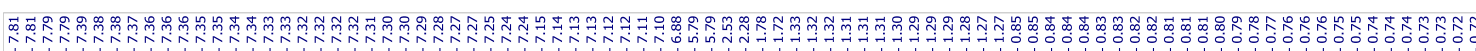

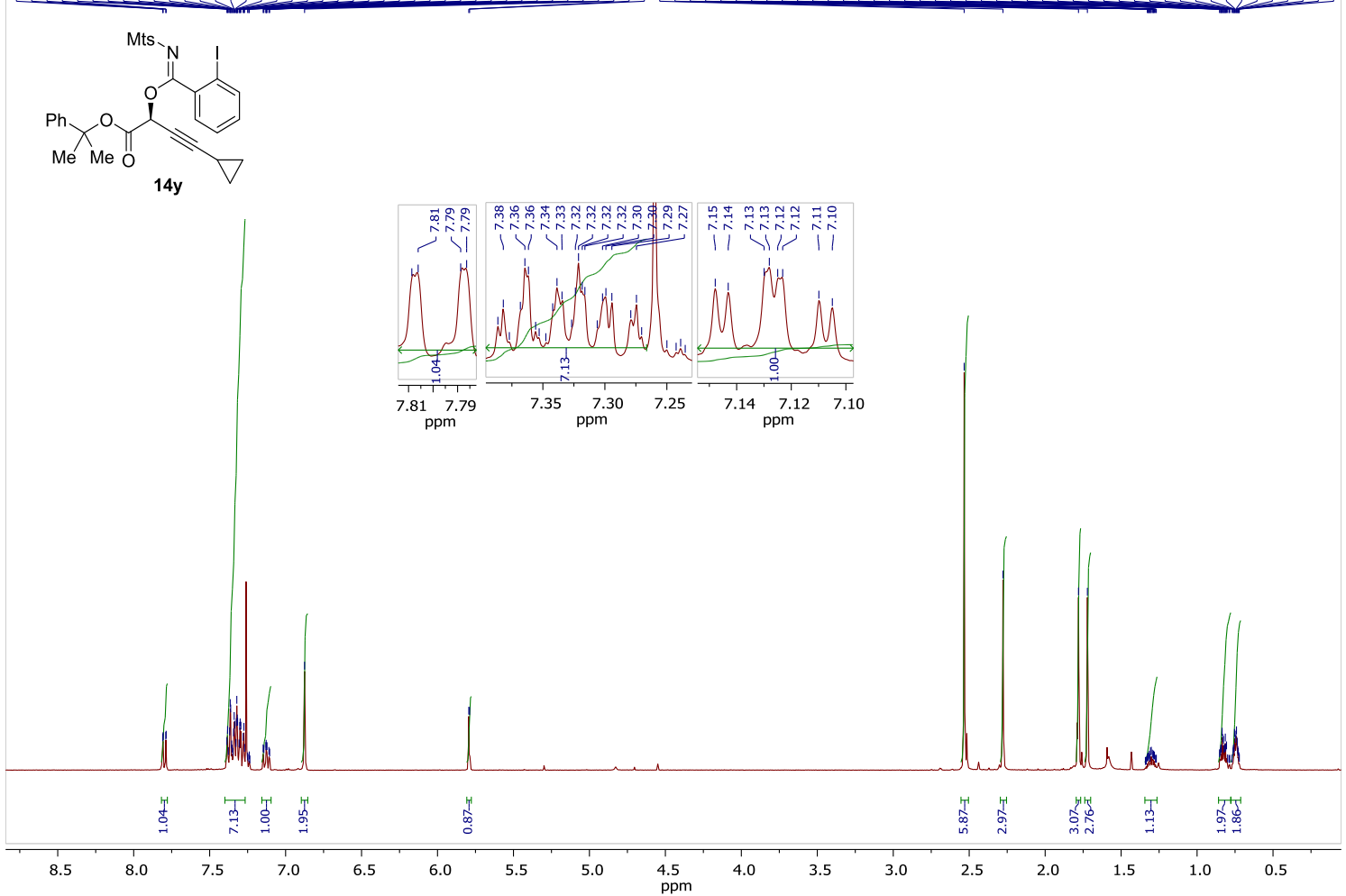

${ }^{13} \mathbf{C}-\mathbf{N M R}\left(100 \mathrm{MHz}, \mathrm{CDCl}_{3}\right)$ of compound $\mathbf{1 4 y}$

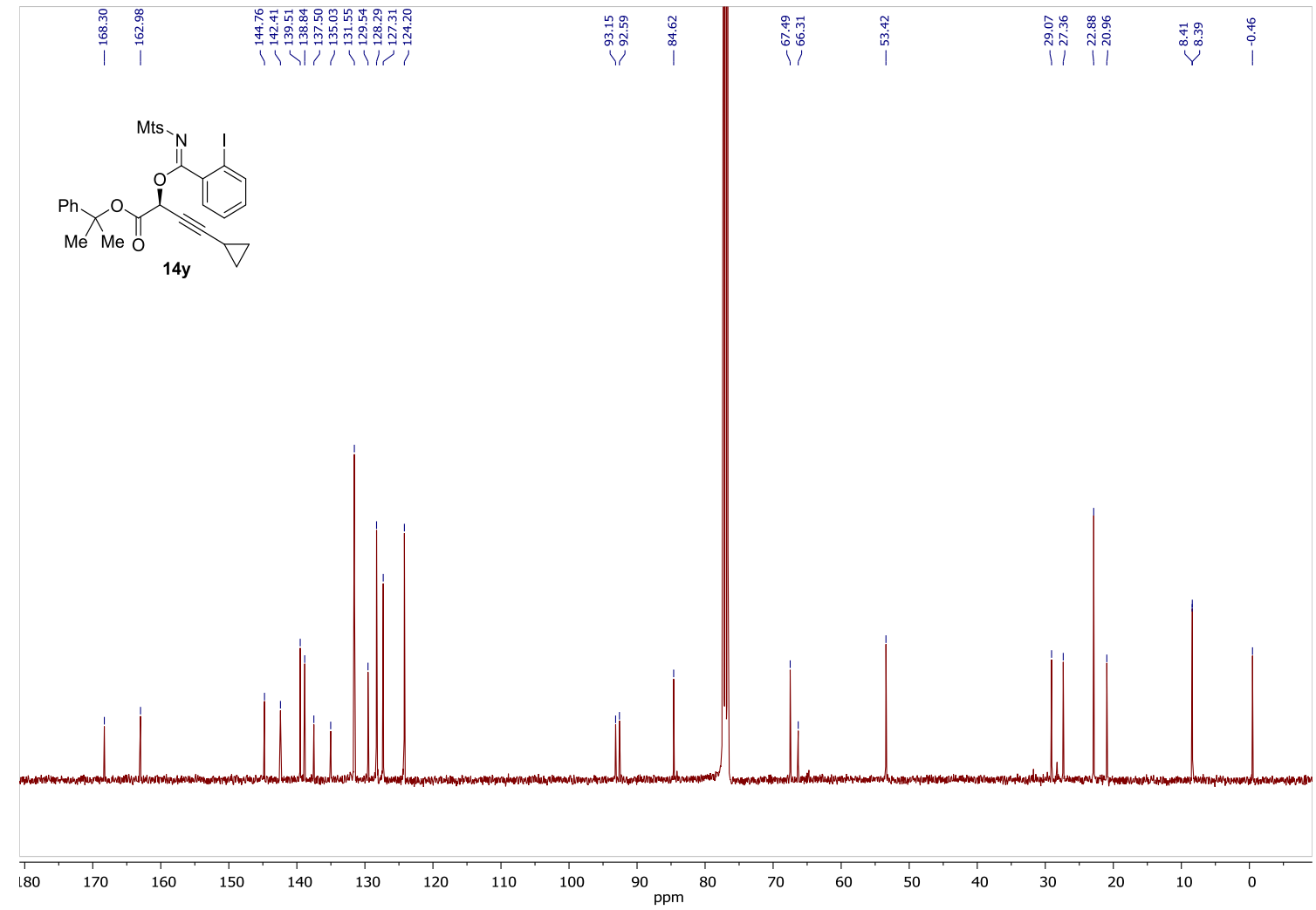


HPLC of compound $\mathbf{1 4 y}$
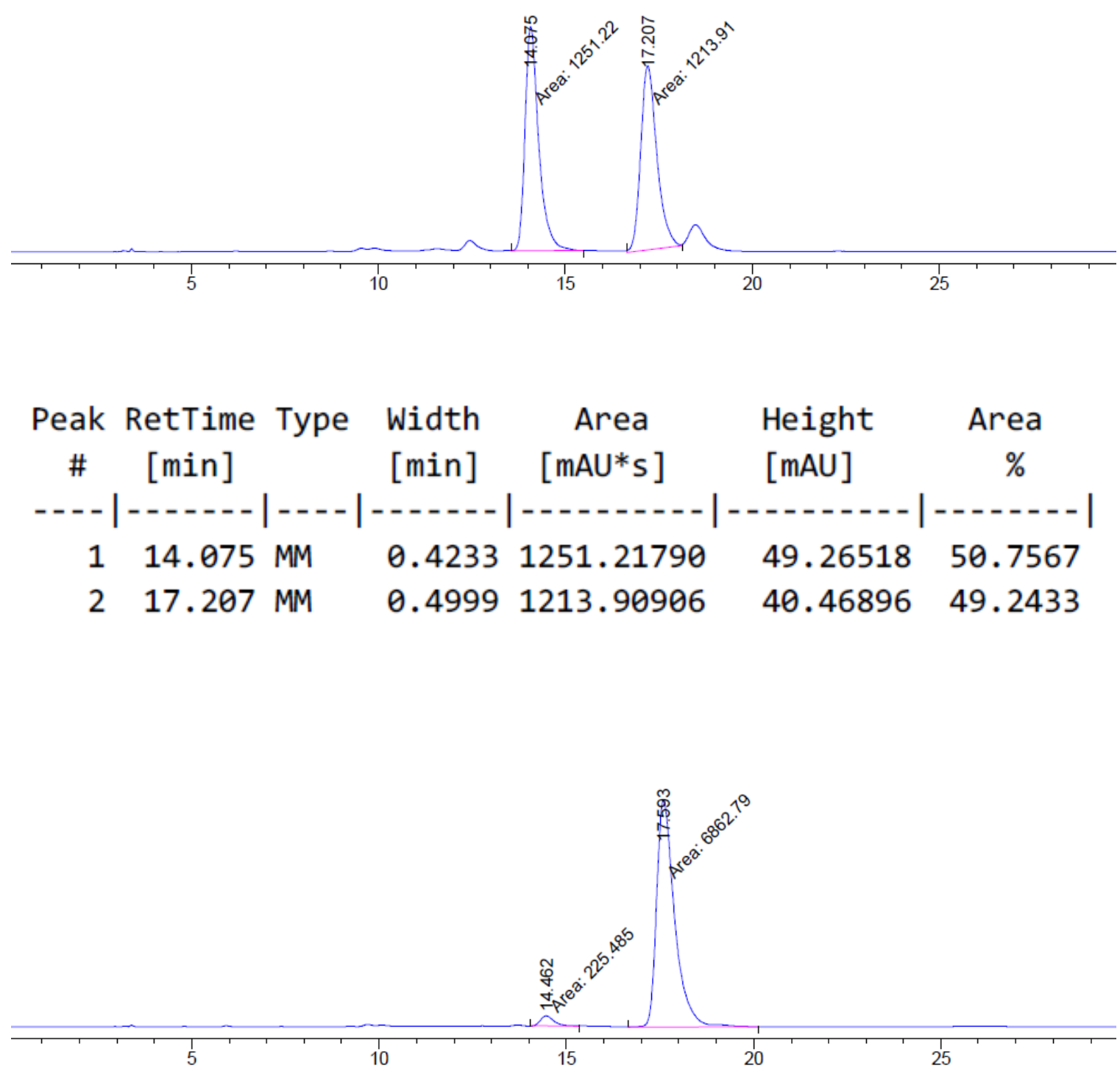

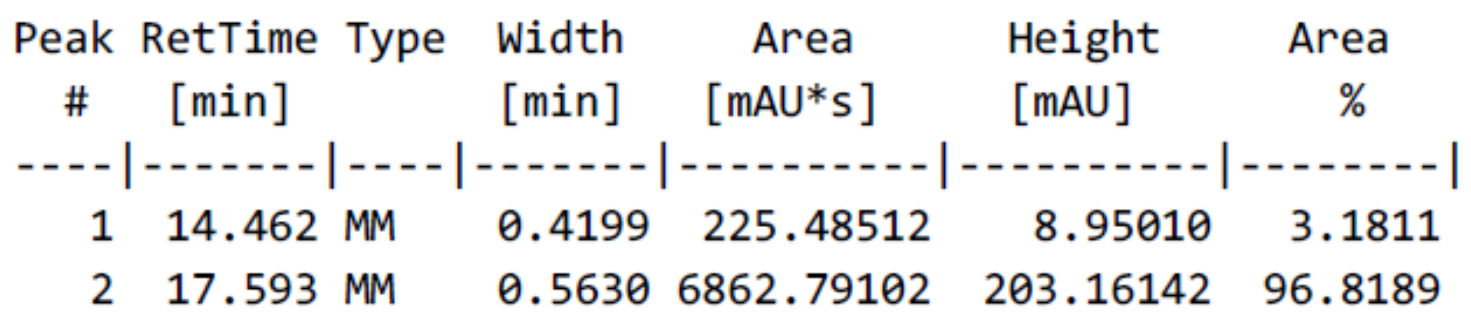


IR of compound $\mathbf{1 4 y}$

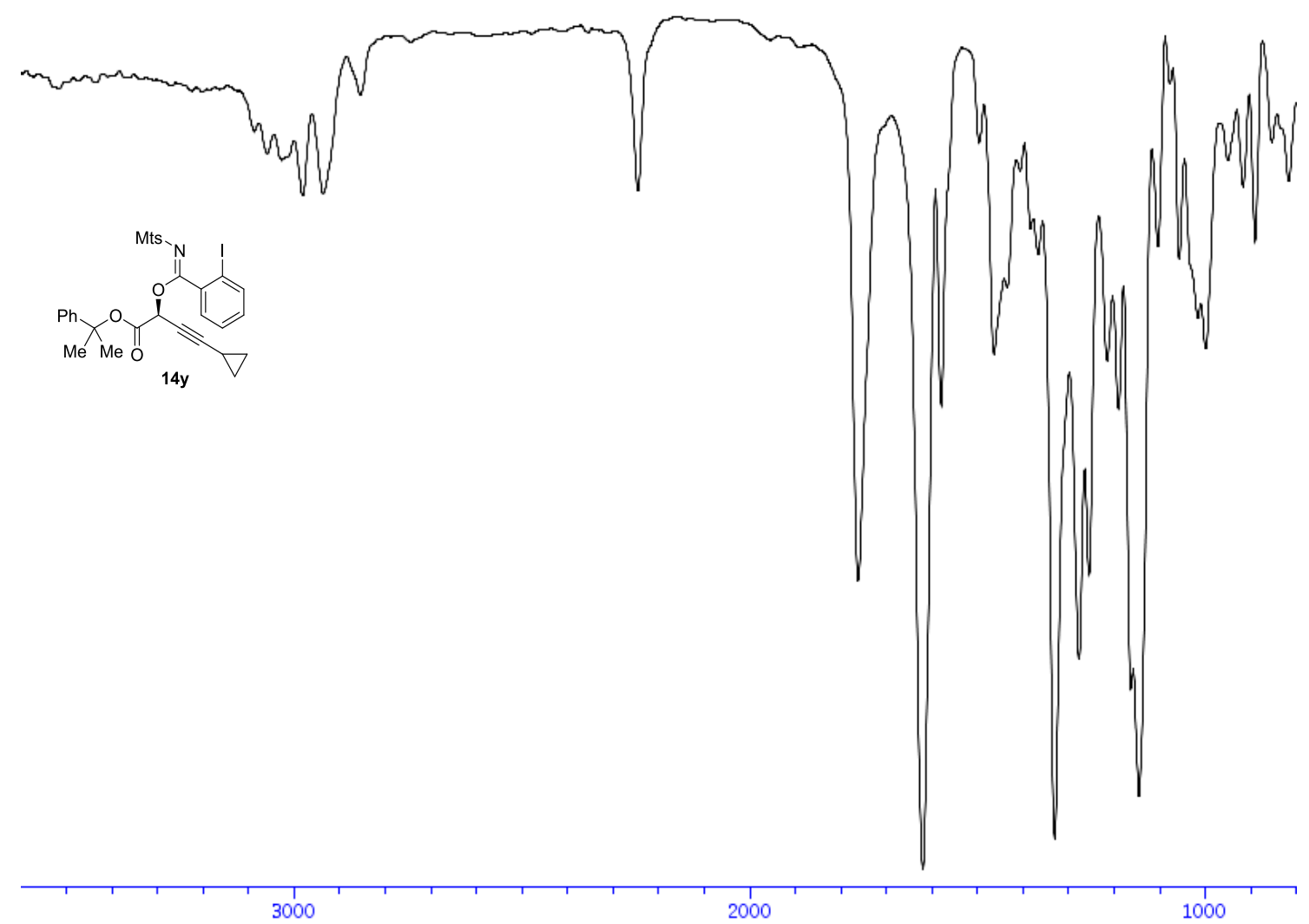


${ }^{\mathbf{1}} \mathbf{H}-\mathbf{N M R}\left(400 \mathrm{MHz}, \mathrm{CDCl}_{3}\right)$ of compound $\mathbf{1 4 z}$

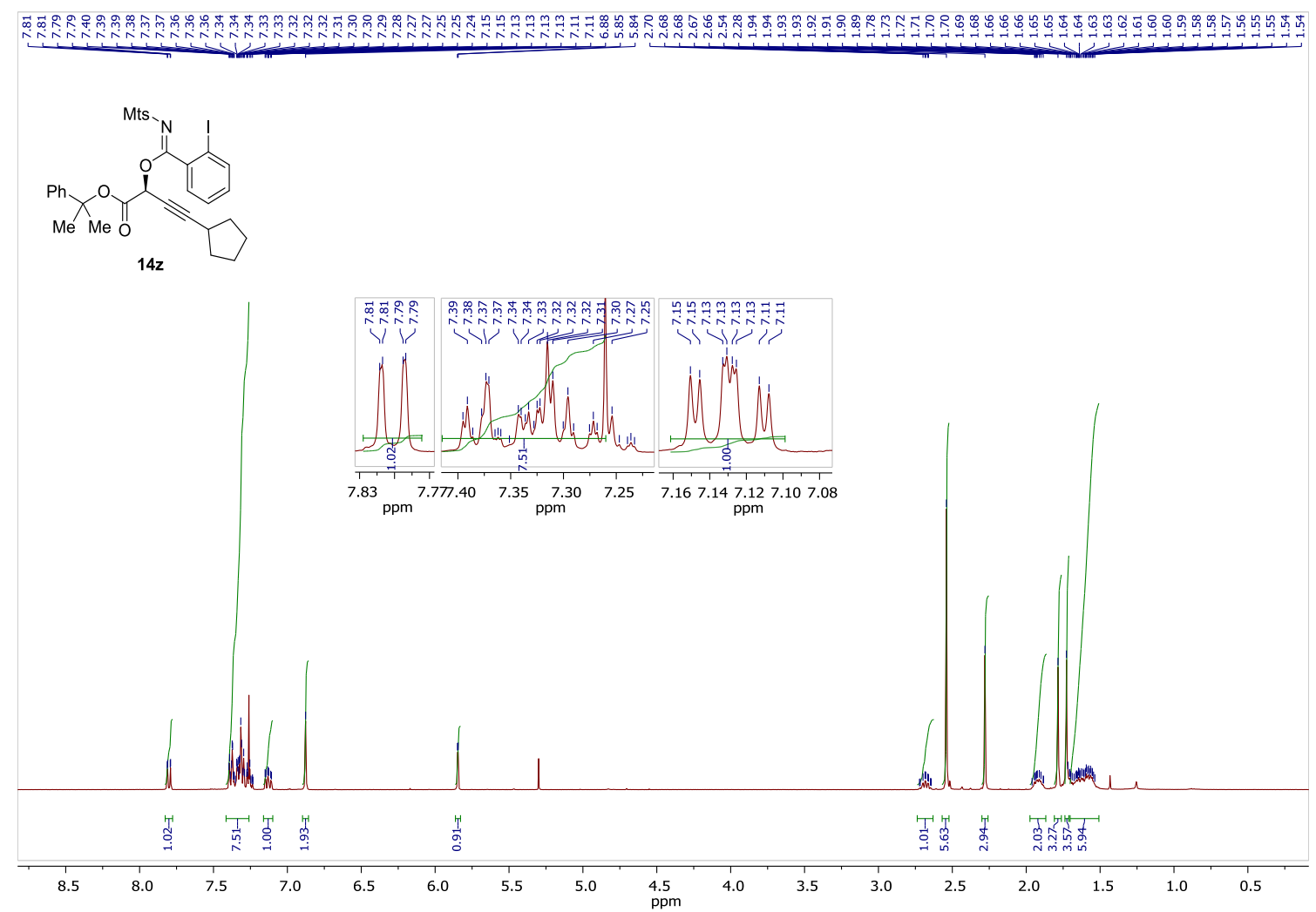

${ }^{13} \mathbf{C}-\mathbf{N M R}\left(100 \mathrm{MHz}, \mathrm{CDCl}_{3}\right)$ of compound $\mathbf{1 4 z}$

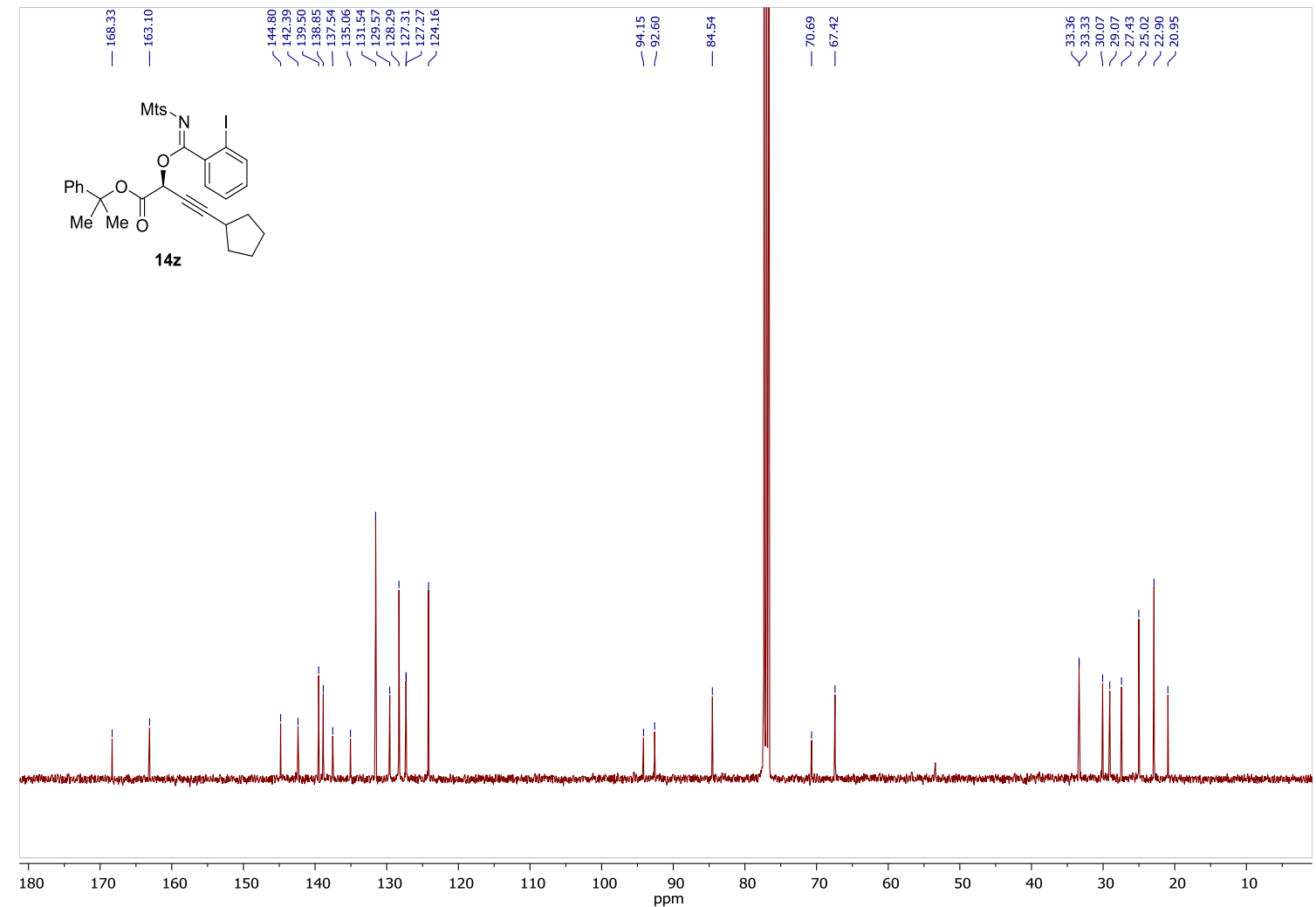


HPLC of compound $14 z$

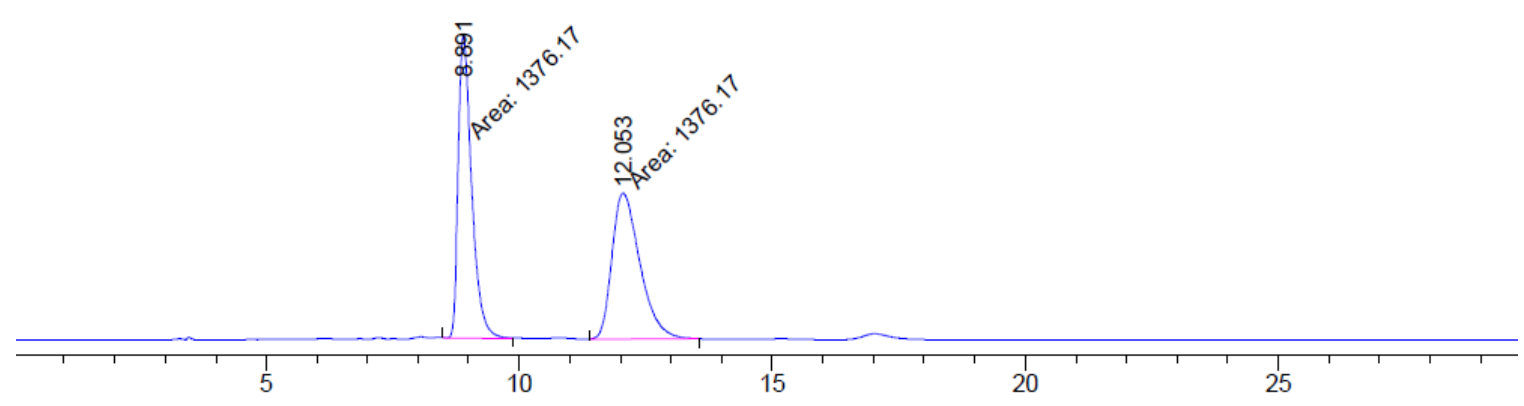

\begin{tabular}{cccccc}
$\begin{array}{c}\text { Peak RetTime Type } \\
\text { \# }\end{array}$ & $\begin{array}{c}\text { Width } \\
{[\mathrm{min}]}\end{array}$ & $\begin{array}{c}\text { Area } \\
{[\mathrm{min}]}\end{array}$ & $\begin{array}{c}\text { Height } \\
{[\mathrm{mAU} \text { s }]}\end{array}$ & $\begin{array}{c}\text { Area } \\
{[\mathrm{mAU}]}\end{array}$ & $\%$ \\
\hline 1 & 8.891 MM & 0.3081 & 1376.16699 & 74.44983 & 50.0000 \\
2 & 12.053 MM & 0.6410 & 1376.16748 & 35.78159 & 50.0000
\end{tabular}

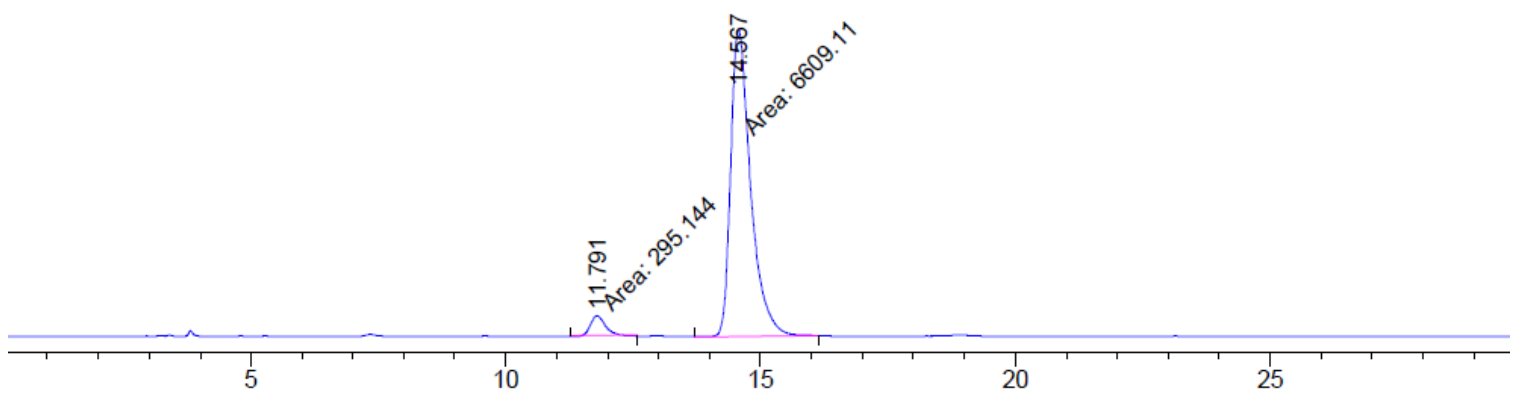

\begin{tabular}{cccccc}
$\begin{array}{c}\text { Peak RetTime Type } \\
\text { \# }\end{array}$ & $\begin{array}{c}\text { Width } \\
{[\mathrm{min}]}\end{array}$ & $\begin{array}{c}\text { Area } \\
{[\mathrm{min}]}\end{array}$ & $\begin{array}{c}\text { Height } \\
{[\mathrm{mAU} \text { * }]}\end{array}$ & $\begin{array}{c}\text { Area } \\
{[\mathrm{mAU}]}\end{array}$ & $\%$ \\
\hline 1 & 11.791 MM & 0.3212 & 295.14435 & 15.31630 & 4.2748 \\
2 & 14.567 MM & 0.4552 & 6609.11328 & 241.99719 & 95.7252
\end{tabular}


IR of compound $14 z$

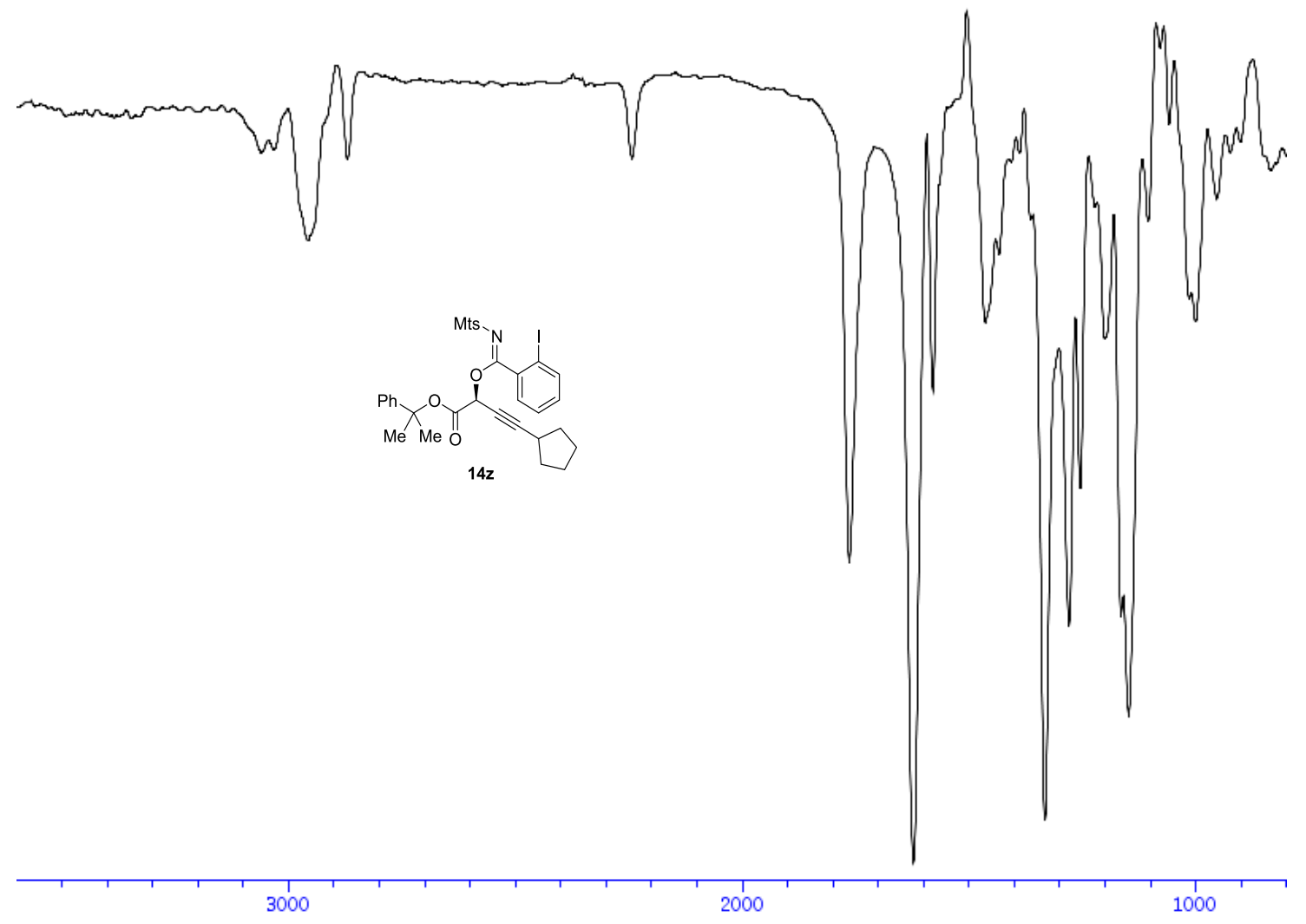


${ }^{1} \mathbf{H}-\mathbf{N M R}\left(400 \mathrm{MHz}, \mathrm{CDCl}_{3}\right.$ ) of compound 14aa

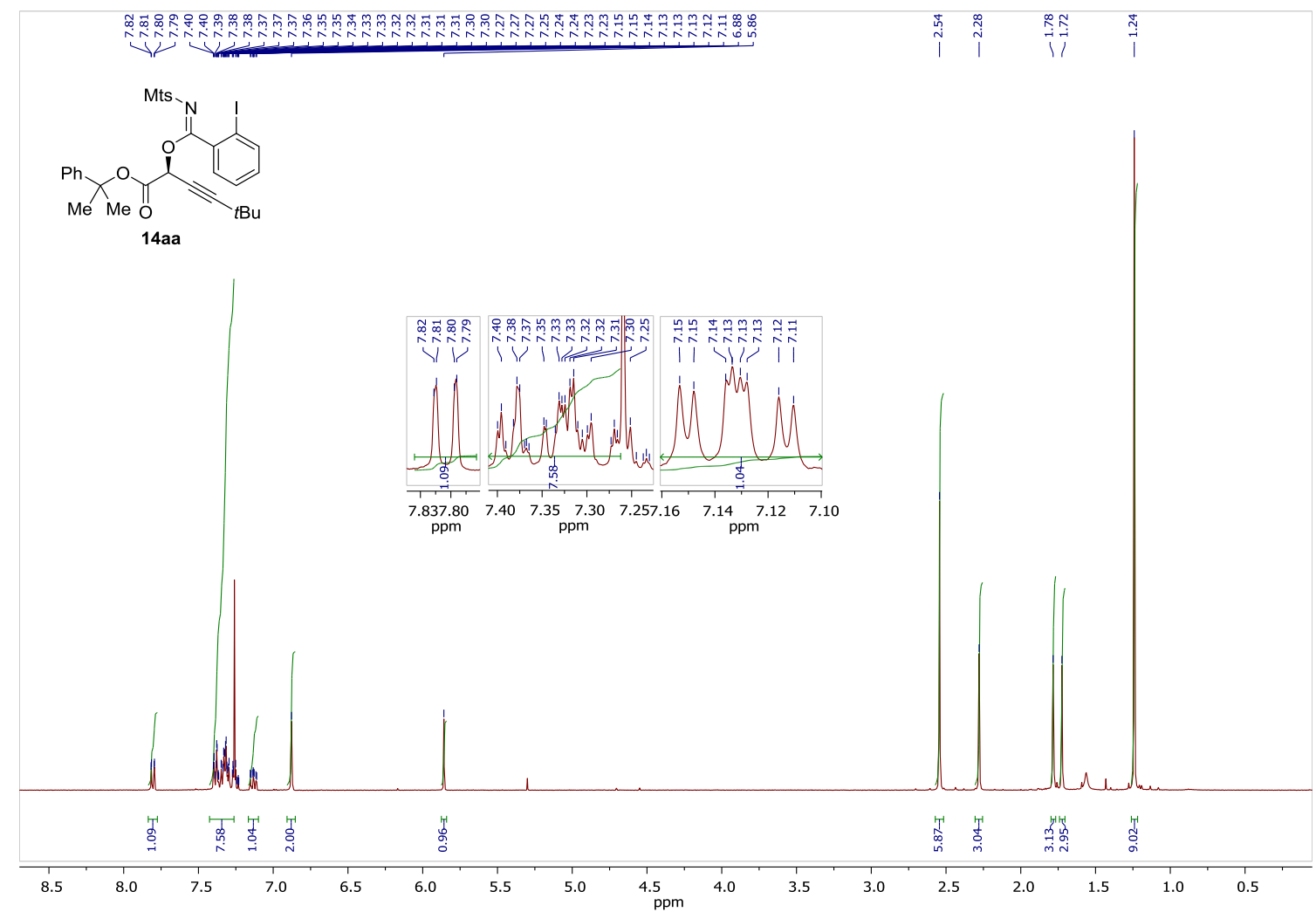

${ }^{13} \mathbf{C}-\mathbf{N M R}\left(100 \mathrm{MHz}, \mathrm{CDCl}_{3}\right)$ of compound 14aa
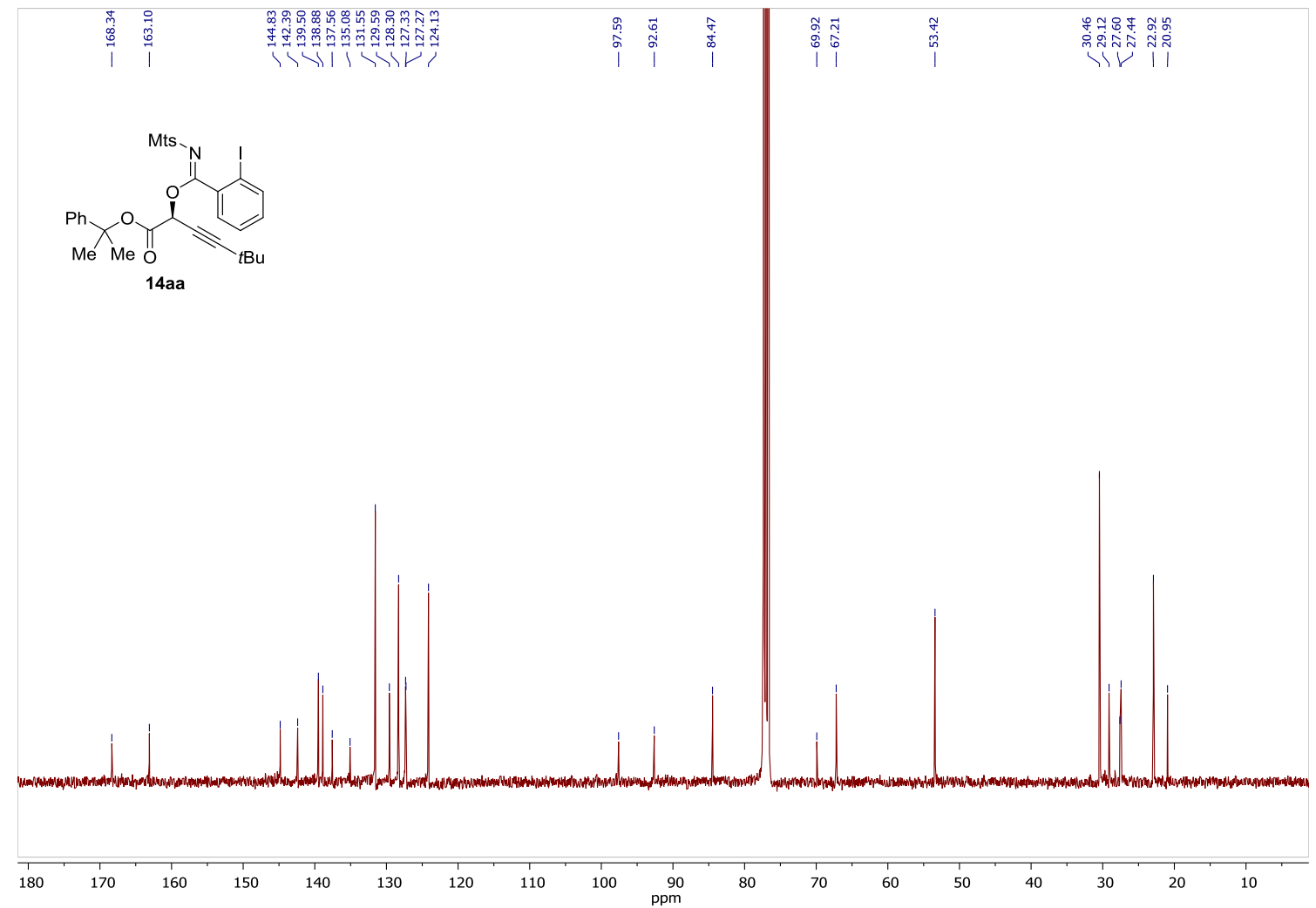
HPLC of compound 14aa
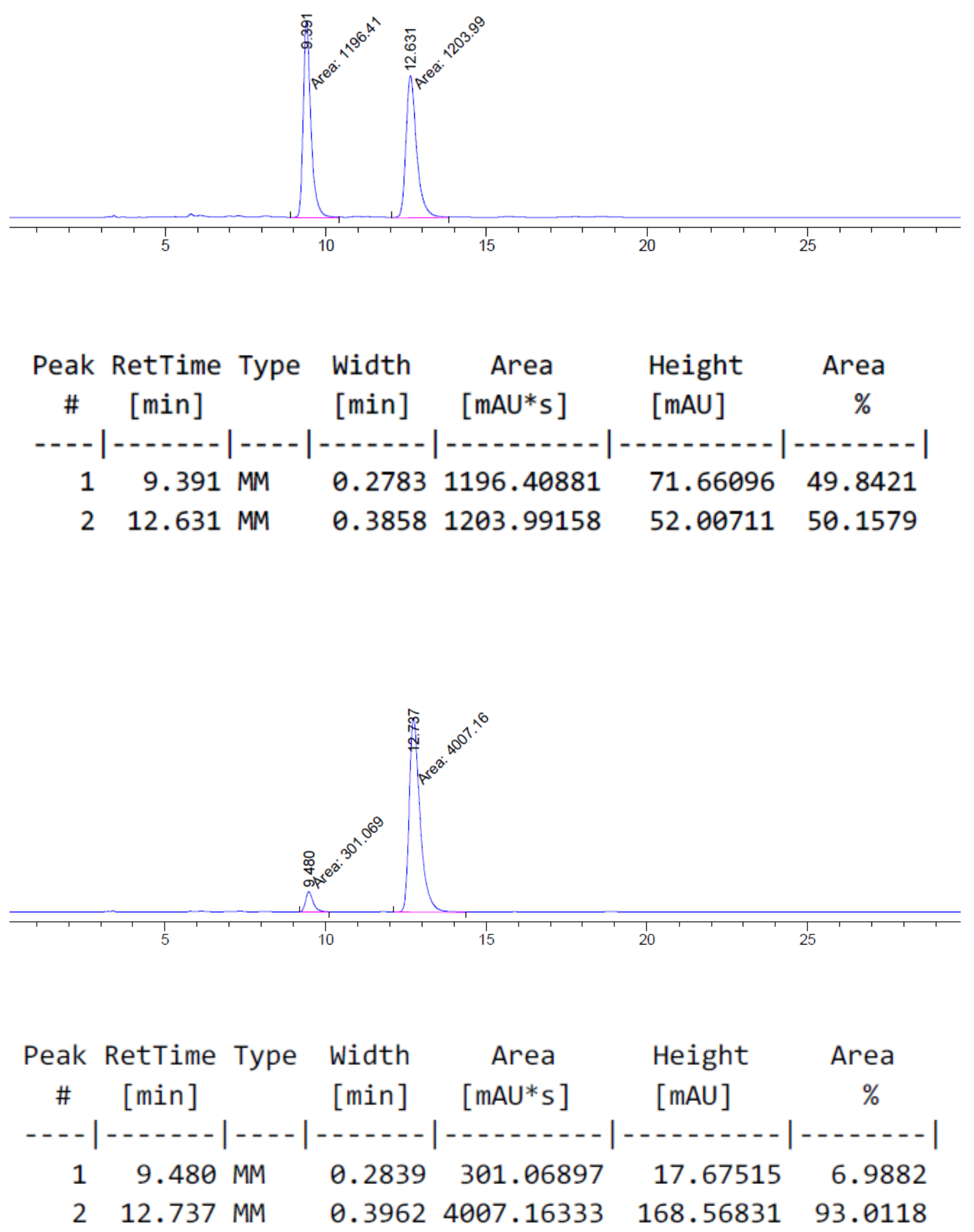
IR of compound 14aa

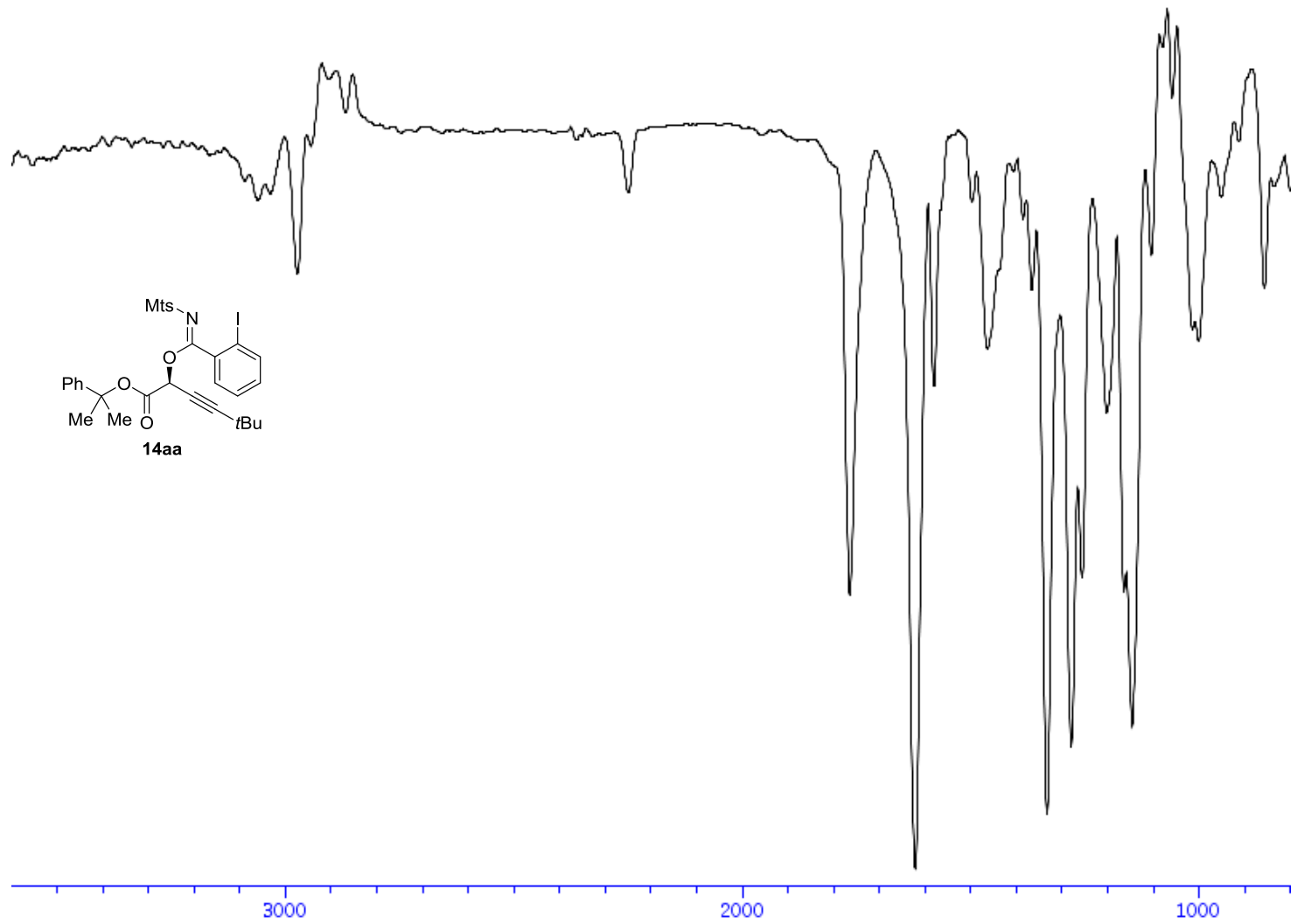


${ }^{1} \mathbf{H}-\mathbf{N M R}\left(400 \mathrm{MHz}, \mathrm{CDCl}_{3}\right.$ ) of compound 14ab

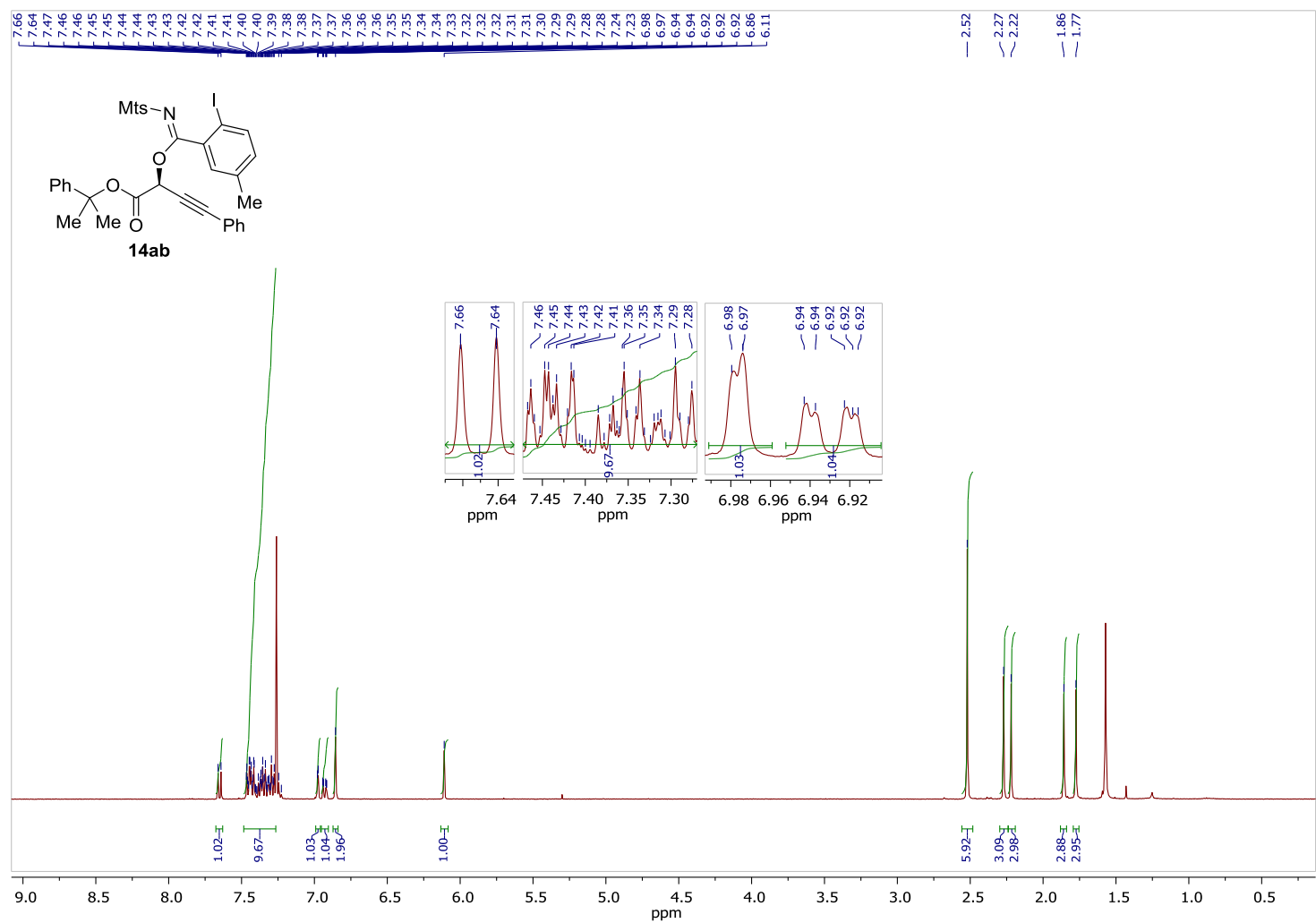

${ }^{13} \mathbf{C}-\mathbf{N M R}\left(100 \mathrm{MHz}, \mathrm{CDCl}_{3}\right)$ of compound 14ab

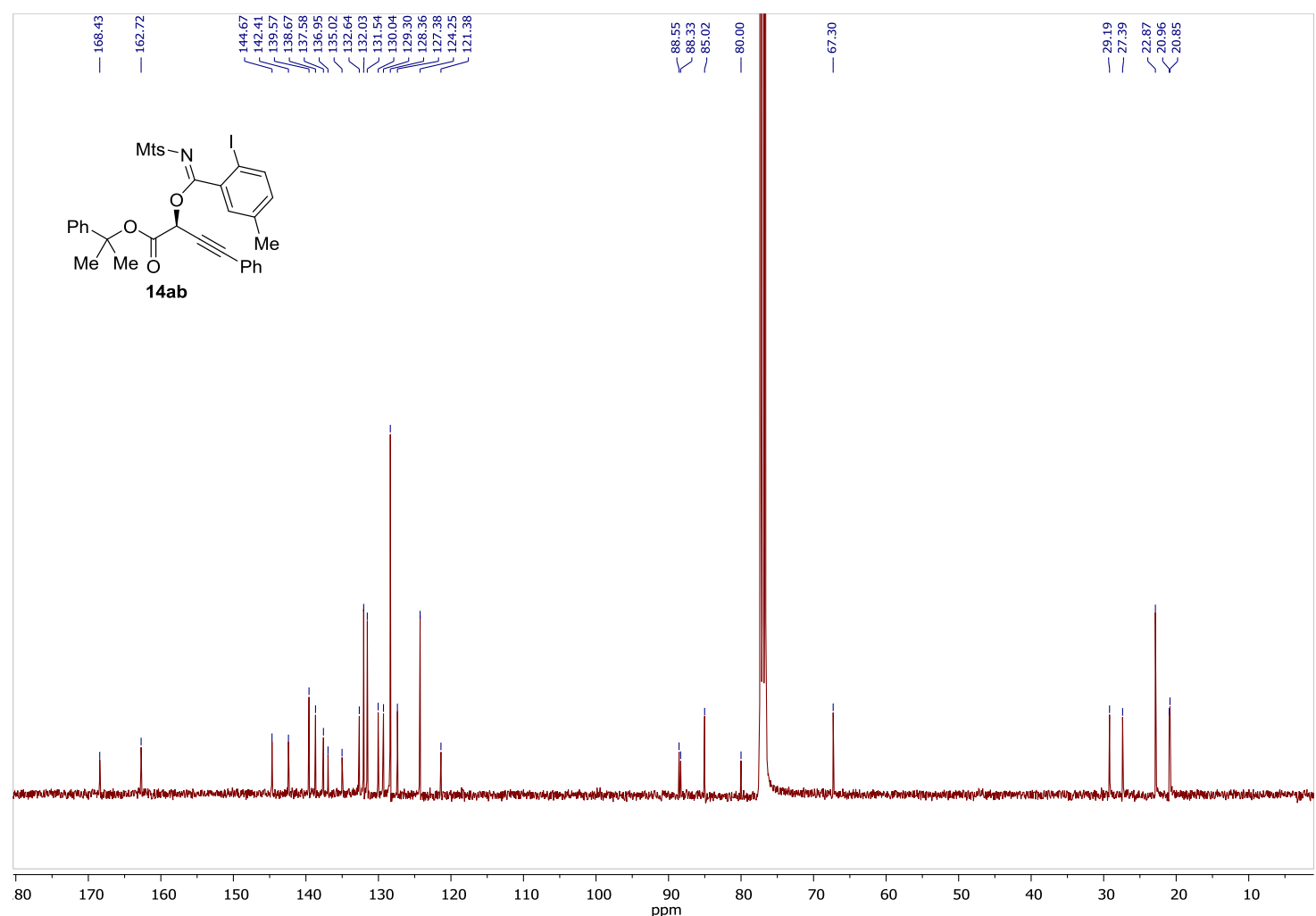


HPLC of compound 14ab

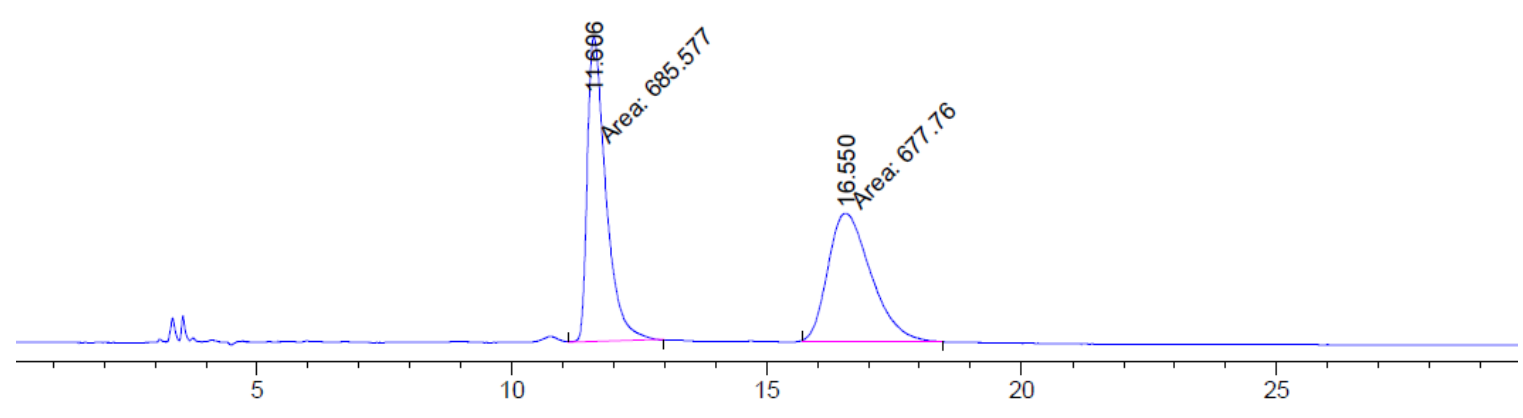

$$
\begin{aligned}
& \text { Peak RetTime Type width Area Height Area }
\end{aligned}
$$

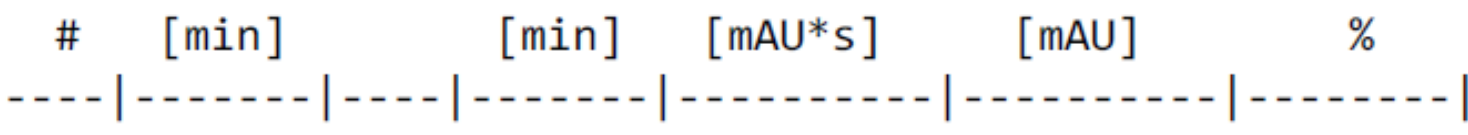

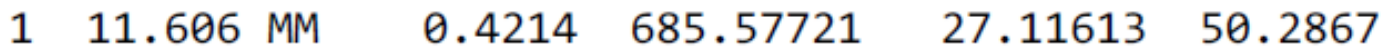

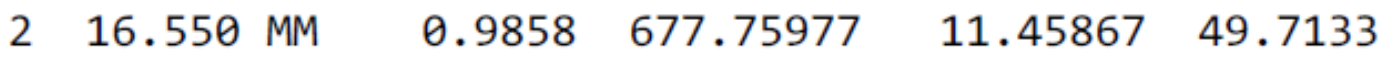

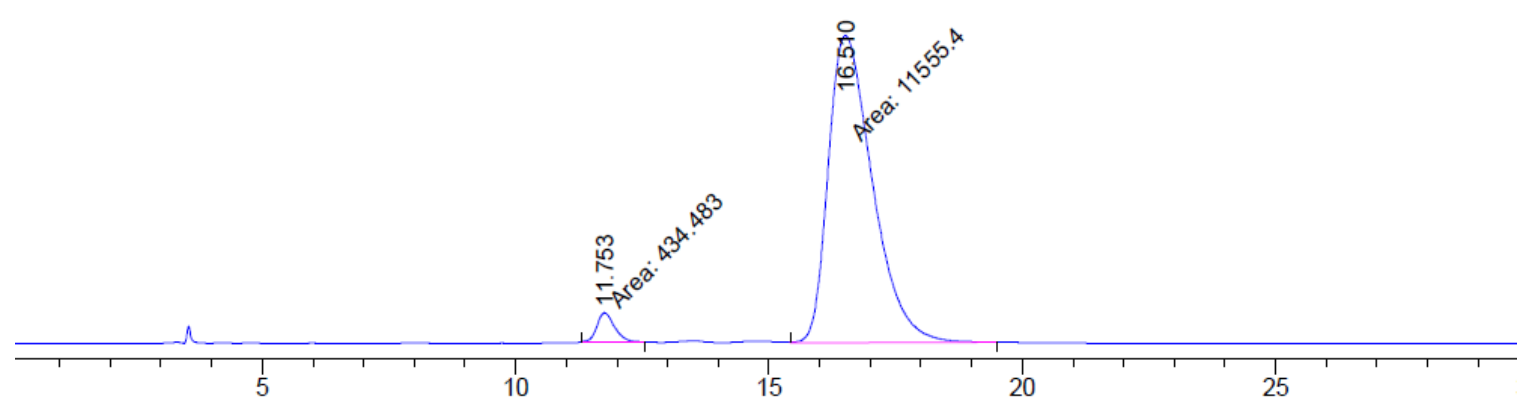

\begin{tabular}{|c|c|c|c|c|c|c|}
\hline $\begin{array}{c}\text { Peak } \\
\#\end{array}$ & $\begin{array}{c}\text { RetTime } \\
\text { [min] }\end{array}$ & Type & $\begin{array}{l}\text { Width } \\
\text { [min] }\end{array}$ & $\begin{array}{c}\text { Area } \\
{\left[\mathrm{mAU}^{*} \mathrm{~s}\right]}\end{array}$ & $\begin{array}{l}\text { Height } \\
{[\mathrm{mAU}]}\end{array}$ & $\begin{array}{c}\text { Area } \\
\%\end{array}$ \\
\hline & & & & - - & 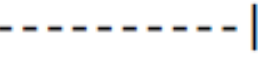 & 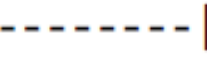 \\
\hline 1 & 11.753 & MM & 0.4029 & 434.48349 & 17.97125 & 3.6237 \\
\hline 2 & 16.510 & MM & 1.0126 & $1.15554 \mathrm{e} 4$ & 190.18657 & 96.3763 \\
\hline
\end{tabular}


IR of compound 14ab

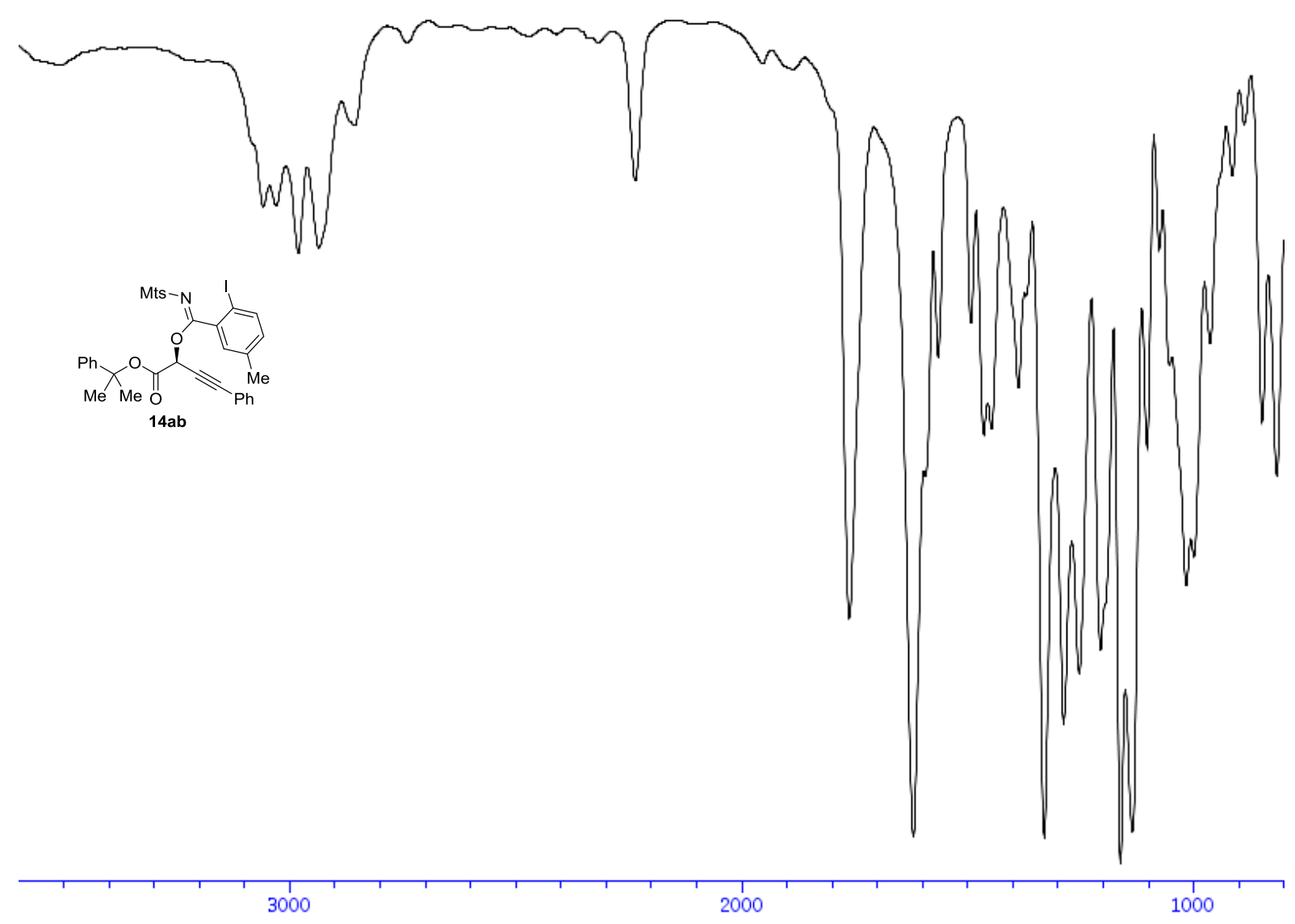


${ }^{1} \mathbf{H}-\mathbf{N M R}\left(400 \mathrm{MHz}, \mathrm{CDCl}_{3}\right.$ ) of compound $\mathbf{1 4 a c}$

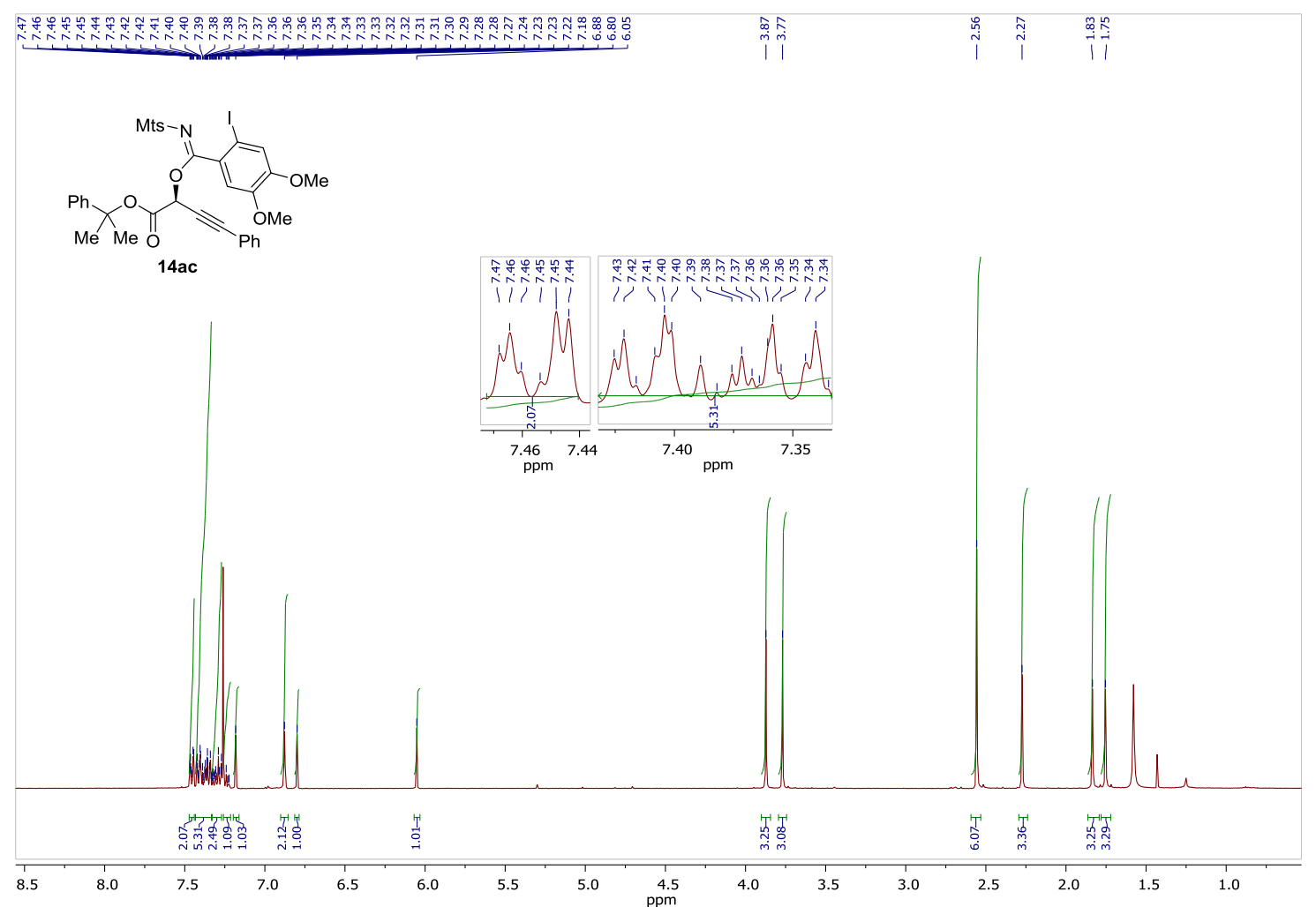

${ }^{13} \mathbf{C}$-NMR $\left(100 \mathrm{MHz}, \mathrm{CDCl}_{3}\right)$ of compound 14ac

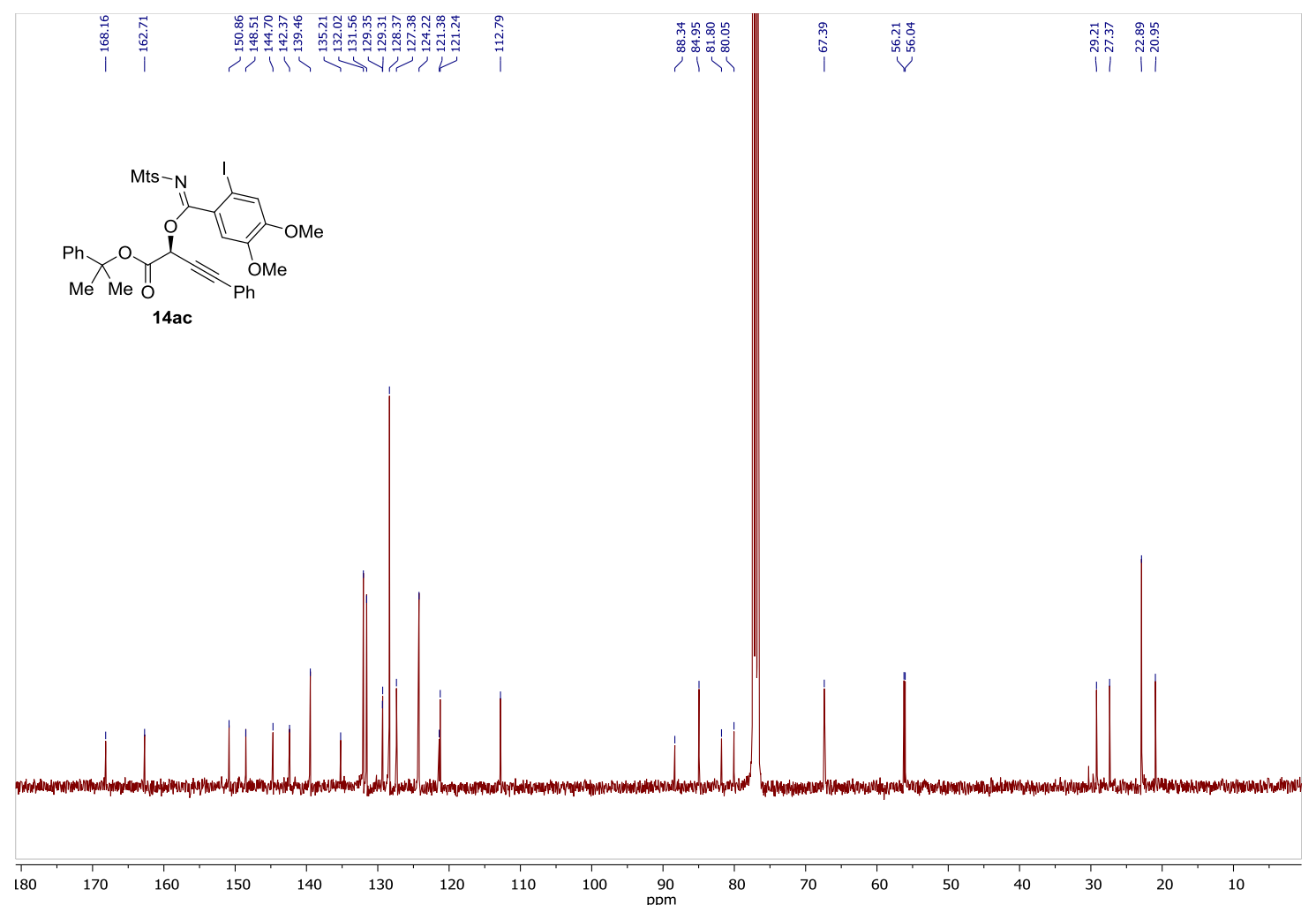


HPLC of compound 14ac
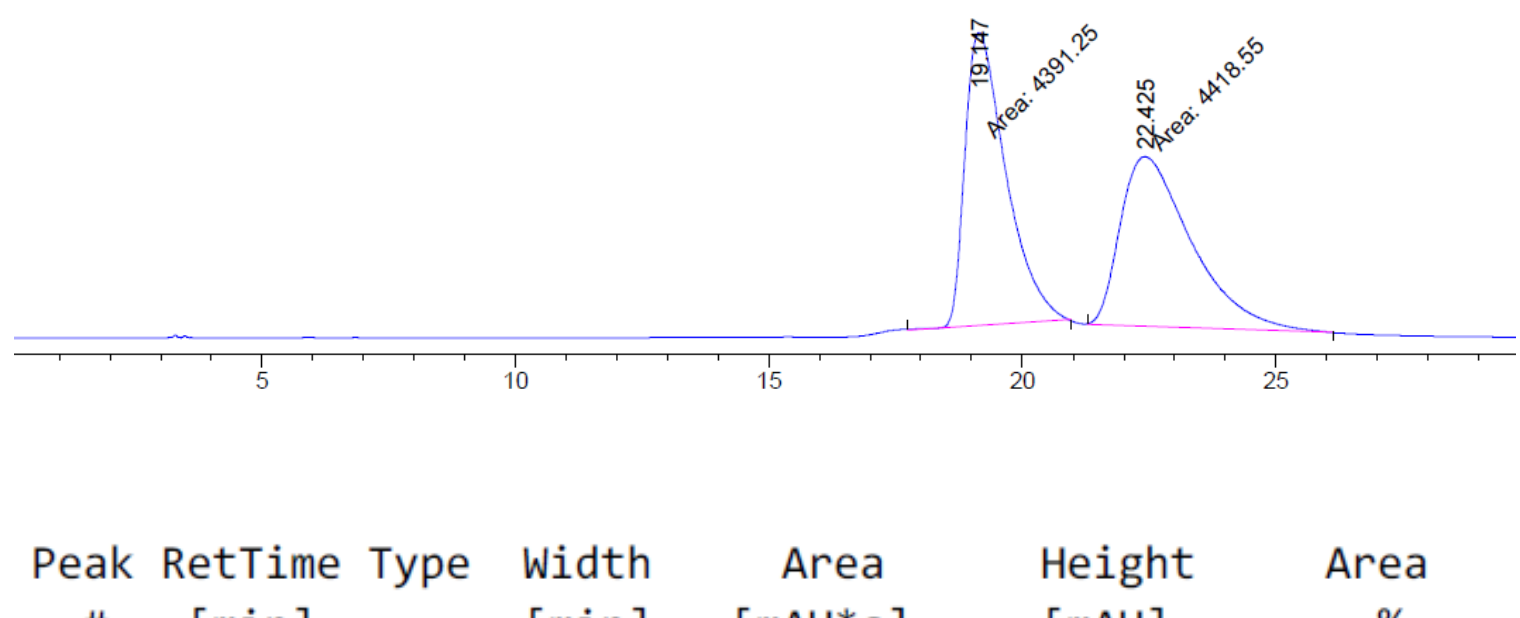

$\begin{array}{ccccccc}\# & {[\mathrm{~min}]} & {[\mathrm{min}]} & {[\mathrm{mAU} * \mathrm{~s}]} & {[\mathrm{mAU}]} & \% \\ 1 & 19.147 & \text { MM } & 0.9367 & 4391.25244 & 78.13583 & 49.8450 \\ 2 & 22.425 & \text { MM } & 1.6254 & 4418.55420 & 45.30606 & 50.1550\end{array}$

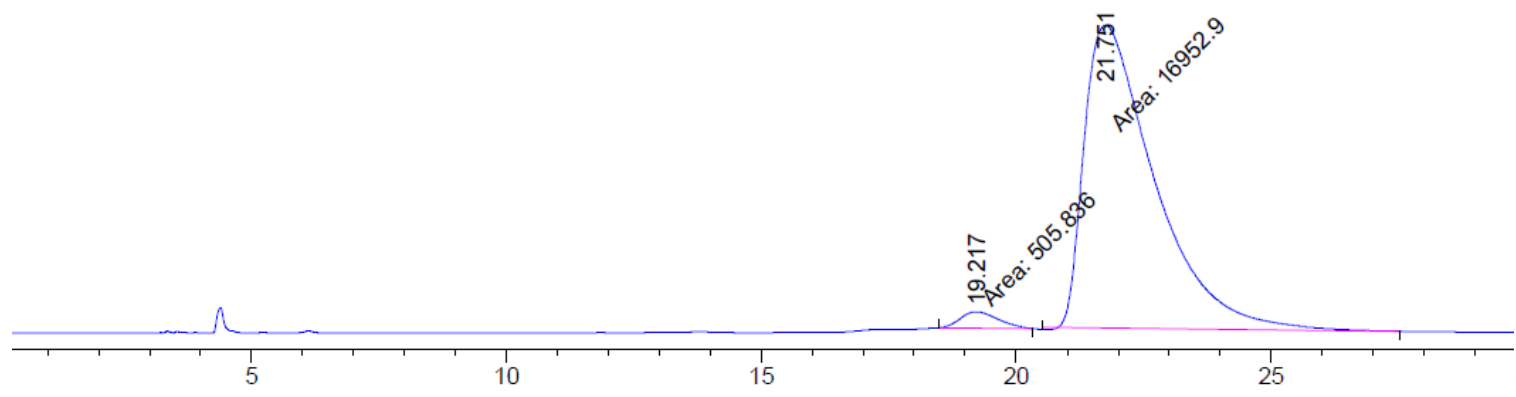

\begin{tabular}{cccccc}
$\begin{array}{c}\text { Peak RetTime Type } \\
\#\end{array}$ & Width & Area & Height & Area \\
& {$[\mathrm{min}]$} & {$[\mathrm{min}]$} & {$[\mathrm{mAU}$ s $]$} & {$[\mathrm{mAU}]$} & $\%$ \\
\hline 1 & 19.217 MM & 0.8671 & 505.83563 & 9.72240 & 2.8973 \\
2 & 21.751 MM & 1.6050 & $1.69529 \mathrm{e} 4$ & 176.03938 & 97.1027
\end{tabular}


IR of compound 14ac

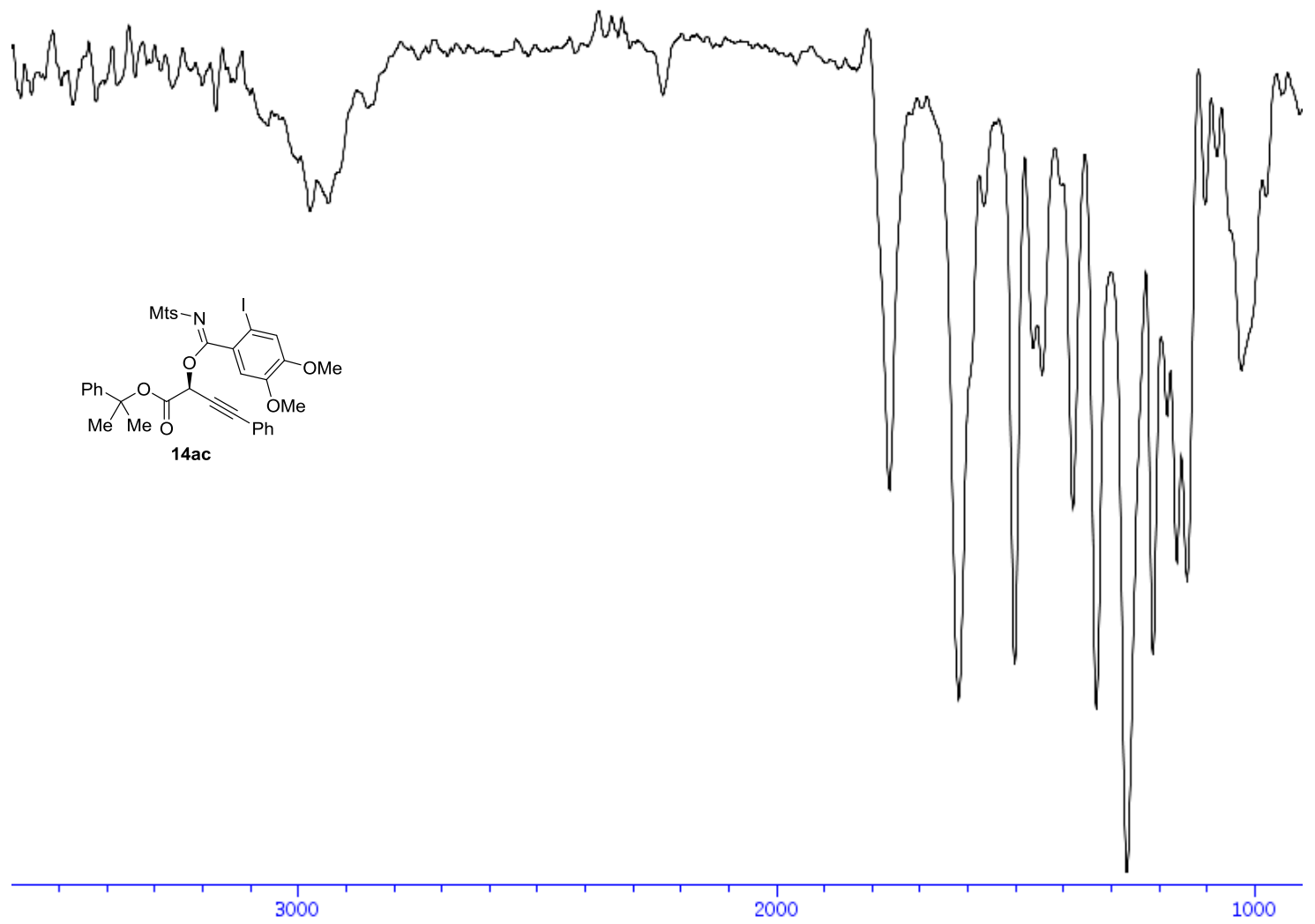

